This item was submitted to Loughborough's Research Repository by the author.

Items in Figshare are protected by copyright, with all rights reserved, unless otherwise indicated.

\title{
CPM/LOB : new methodology to integrate CPM and line of balance
}

PLEASE CITE THE PUBLISHED VERSION

\section{PUBLISHER}

(C) Saad A. Suhail

\section{PUBLISHER STATEMENT}

This work is made available according to the conditions of the Creative Commons Attribution-NonCommercialNoDerivatives 4.0 International (CC BY-NC-ND 4.0) licence. Full details of this licence are available at: https://creativecommons.org/licenses/by-nc-nd/4.0/

\section{LICENCE}

CC BY-NC-ND 4.0

REPOSITORY RECORD

Suhail, Saad A.. 2019. "CPM/LOB : New Methodology to Integrate CPM and Line of Balance". figshare. https://hdl.handle.net/2134/25619. 


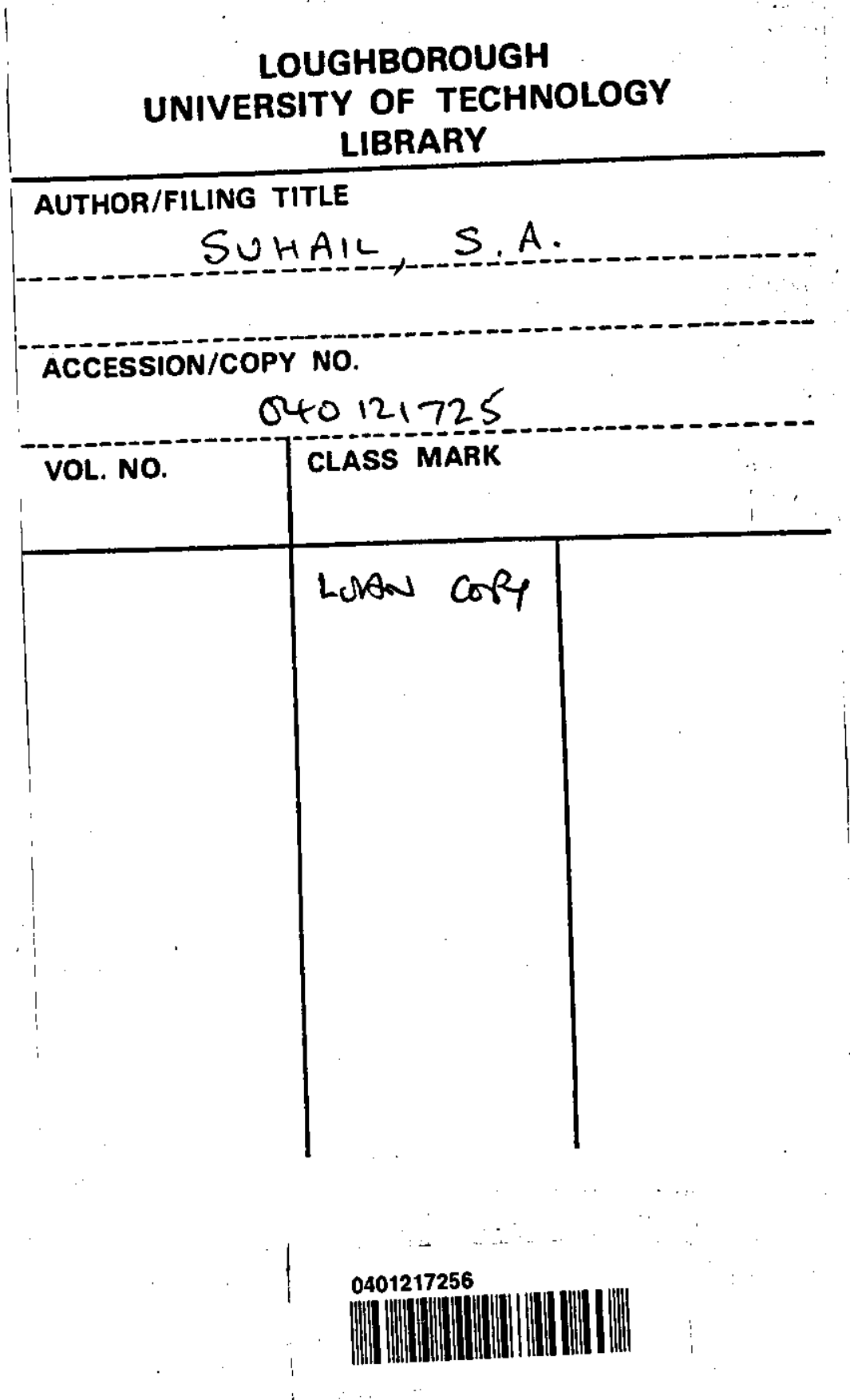





\title{
CPM/LOB: New Methodology to Integrate CPM and Line of Balance
}

\author{
by
}

Saad A. Suhail, BSc, MSc, PE, PMP

A Doctoral Thesis Submitted in Partial Fulfillment of the Requirements for the Award of Doctor of Philosophy of the Loughborough University, Loughborough, United Kingdom.

November, 1995

(C) Saad A. Suhail, 1995 


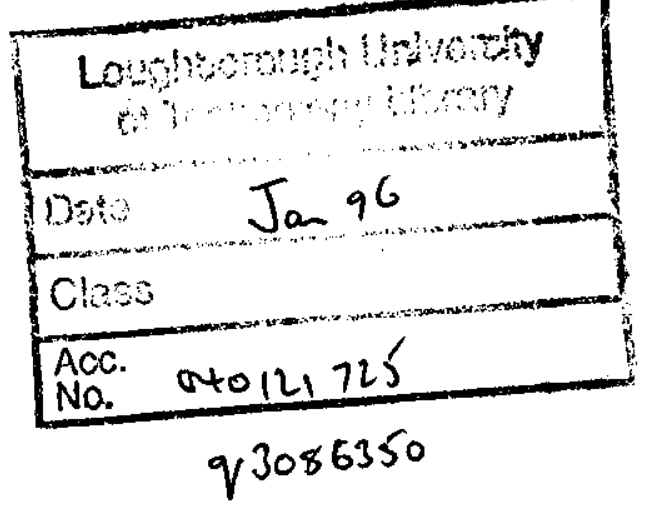




\begin{abstract}
In 1963 the United States Federal Government established a Line of Balance Coordinating Committee to study LOB's applicability and its ties with network scheduling. Ever since many researchers and practitioners attempted to integrate the merits of CPM and LOB in graphical, operational research, and activity-dominated network scheduling. Nevertheless, the obstacles were not truly eliminated in a simple and practical way that was good enough to be accepted and adopted by the construction industry. This work presents CPM/LOB, a new methodology integrating both methods in a network context. It is simple to comprehend and apply using available commercial CPM computer programs, and does not require elaborate training.
\end{abstract}

The method overcomes the vulnerability of CPM to changes in the sequence of work and inability to maintain work continuity for the working squads of the repetitive activities. It introduces float into LOB and revives LOB by creating access to it by commercially available and popular CPM packages.

Several additional features are introduced to facilitate the management of planning and control of repetitive projects, such as identifying and quantifying progress that contradicts network logic, evaluating the effect of discrete activities, and measuring the progress regularity on multiple large housing contracts as well as single and small repetitive projects.

The principles of the method have been published in Journal of Constructing Engineering and Management of the American Society of Civil Engineers in September 1994. Its practical application on projects in Kuwait and the United States is demonstrated by three case studies. 


\section{ACKNOWLEDGEMENTS}

It has been an honor and privilege to be educated at Lougborough University, UK. Certainly my first thanks goes to my prudent supervisor Mr. Richard $\mathrm{H}$. Neale for the continuous support and encouragement. My regards and thanks are extended to my director of research Professor Ronald McCaffer for his support and guidance. Drs. M. J. Mawdesley, A. N. Baldwin and A. Thorpe offered helpful opinions and expressed much appreciated interests for which I cordially thank them.

I am appreciative of all the help my previous colleagues at Loughborough University have offered in starting and finishing this work.

Deep appreciation and love embraces all my family members for their infinite help and support despite the crucial time they are undergoing.

I sincerely thank those who indirectly strengthened my perseverance. 


\section{NOTE}

This work, being written in the United States of America and submitted in the United Kingdom, had to confront the issue of using British versus American English. The initial intention was to write the thesis in British English, but due to logistical obstacles emanating partly from software availabilities, it was agreed to write it in American English.

This thesis was solely written by the author and reviewed by the supervisor of research. No other party was involved in editing or correcting it. 
ABSTRACT

iii

ACKNOWLEDGEMENTS

iv

NOTE

TABLE OF CONTENTS

ix

LIST OF FIGURES

xiv

LIST OF TABLES

CHAPTER 1 - INTRODUCTION $\ldots \ldots \ldots \ldots \ldots$ 1-1

1.1 General Description of the Subject $\ldots \ldots \ldots \ldots \ldots$ 1-1

1.2 Significance of the Problem ............ 1-1

Objectives of the Research $\ldots \ldots \ldots 1-2$

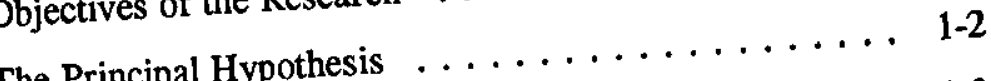

1.4 The Principal Hypothesis $\ldots \ldots \ldots \ldots \ldots \ldots \ldots$

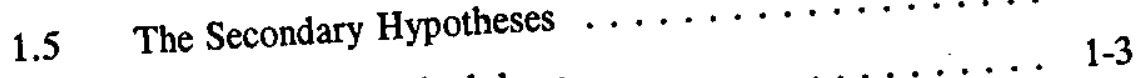

1.6 The Research Methodology $\ldots \ldots \ldots \ldots \ldots$. . . . . . . . . . .

1.7 Originality and Contributions to Knowledge $\ldots \ldots \ldots \ldots 1-5$

1.8 Organization of the Thesis $\ldots \ldots \ldots \ldots \ldots \ldots \ldots$

CHAPTER 2 - THE CRITICAL PATH METHOD . . . . . . . 2-1

2.1 Introduction . . . . . . . . . . . . . . . . . .

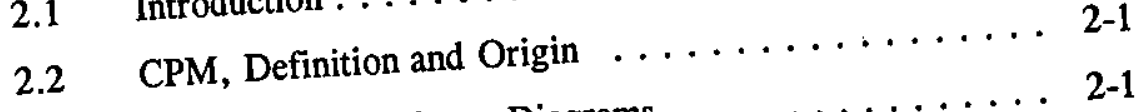

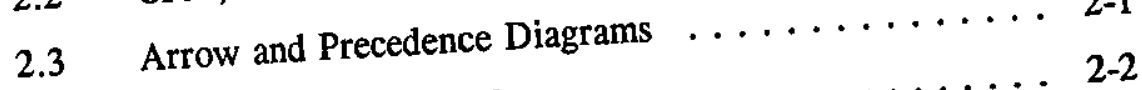

2.4 Time Analysis in CPM . ................. 2-2

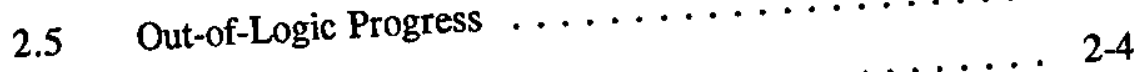

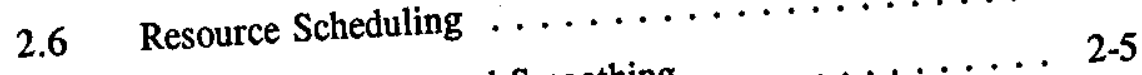

2.7 Resource Leveling and Smoothing ........... 2-6

2.8 Prioritizing in Resource Leveling ......... . . . 2-7

2.9 The Utilization of Resource Scheduling in Practice . . . . . 2-8

2.10 Out-of-Sequence Progress $\ldots \ldots \ldots \ldots \ldots \ldots \ldots \ldots$ 
2.11 Float Ownership $\ldots \ldots \ldots \ldots \ldots \ldots \ldots \ldots, 2-9$

2.12 Challenges in Using CPM on Repetitive Projects .... . 2-11

CHAPTER 3 - THE LINE OF BALANCE METHOD $\ldots \ldots \ldots$. 3-1

3.1 Background and Development $\ldots \ldots \ldots \ldots \ldots$ 3-1

3.2 The Essence of the LOB Technique ......... 3-1

3.3 The Natural Rhythm in Construction ......... 3-1

3.4 The British Approach to Formulating the LOB $\ldots \ldots$ 3-2

3.5 The US Approach to Formulating the LOB $\ldots \ldots \ldots$ 3-3

3.6 Irrelevance of Out-of-Sequence Progress in LOB . . . . 3-5

3.7 LOB Drawbacks and Limitations $\ldots \ldots \ldots \ldots \ldots$ 3-6

CHAPTER 4 - LITERATURE REVIEW . . . . . . . . . 4-1

$4.1 \quad$ Introduction $\ldots \ldots \ldots \ldots \ldots \ldots \ldots \ldots \ldots$ 4-1

4.2 Previous Attempts of Combining CPM and LOB .... . 4-1

4.3 Relevant Recommendations in Recent Literature ..... 4-5

4.4 Pertinent Methods and Theories $\ldots \ldots \ldots \ldots \ldots$ 4.5

4.4.1 Location-Time Diagrams $\ldots \ldots \ldots \ldots \ldots .4-5$

4.4.2 Linear Programming $\ldots \ldots \ldots \ldots \ldots \ldots$ 4-7

4.4.3 Dynamic Programming $\ldots \ldots \ldots \ldots \ldots .4 .8$

4.4 .4 Simulation $\ldots \ldots \ldots \ldots \ldots \ldots \ldots \ldots, 4-10$

4.4.5 Queuing Theory $\ldots \ldots \ldots \ldots \ldots \ldots \ldots .4-11$

4.4.6 Artificial Intelligence $\ldots \ldots \ldots \ldots \ldots \ldots$ 4-12

4.4.6.1 Knowledge-Based Expert

Systems .......... 4-12

4.4.6.2 Neural Networks $\ldots \ldots \ldots$ 4 4-13

4.5 Closing Remarks $\ldots \ldots \ldots \ldots \ldots \ldots \ldots .4-14$

CHAPTER 5 - CURRENT APPLICATIONS IN PLANNING REPETITIVE PROJECTS $\ldots \ldots \ldots \ldots \ldots \ldots \ldots$ 5-1

$5.1 \quad$ Introduction $\ldots \ldots \ldots \ldots \ldots \ldots \ldots \ldots \ldots$ 5-1

5.2 LOB Applications $\ldots \ldots \ldots \ldots \ldots \ldots \ldots \ldots$ 5-1

5.3 CPM Applications $\ldots \ldots \ldots \ldots \ldots \ldots \ldots .55$

5.4 Future Expectation $\ldots \ldots \ldots \ldots \ldots \ldots \ldots .5-10$ 
CHAPTER $6-$ CPM/LOB NEW METHODOLOGY . . . . . . . 6-1

6.1 Introduction $\ldots \ldots \ldots \ldots \ldots \ldots \ldots \ldots \ldots$

6.2 Parallel Scheduling $\ldots \ldots \ldots \ldots \ldots \ldots$ 6 $\mathrm{i}$

6.3 Float Utilization Concept $\ldots \ldots \ldots \ldots$ 6-2

6.4 The Methodology of CPM/LOB . . . . . . . 6-3

6.5 Example 6.1, Developing the Initial Schedule $\ldots \ldots$. . 6-5

6.6 Example 6.2, Updating and Accelerating by $\mathrm{CPM} / \mathrm{LOB} \ldots \ldots \ldots \ldots \ldots \ldots . \ldots \ldots$. . . . . . . . . .

6.7 Example 6.3, Initial Schedule with SS and FF Relationships . . . . . . . . . . . . . . 6-22

6.8 Example 6.4, Initial Schedule with Multiple Calendars . . 6-26

6.9 Example 6.5, Pipeline Project $\ldots \ldots \ldots \ldots$. . . . . . .

6.10 Example 6.6, High-rise Building . . . . . . . . . 6-34

6.11 Practical Implications . . . . . . . . . . . 6-41

6.12 Feasibility of CPM/LOB . . . . . . . . . . . 6-42

CHAPTER 7 - COMMON ACTIVITIES IN LOB AND OTHER

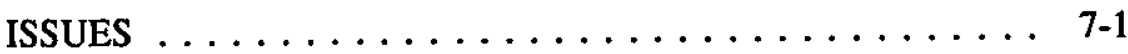

7.1 Introduction ............... 7-1

7.2 The Effect of Common Activities . . . . . . . 7-1

7.2.1 Example 7.1, Effect of a Common Activity . . . 7-4

7.3 Balancing Resources within a Particular Activity . . . . 7-18

7.4 Identifying and Quantifying Out-of-Logic Progress . . . 7-21 7.4.1 Example 7.2, Out-of-Logic Progress . . . . . 7-23

7.5 The Problem of too many Uncompleted Repetitive Activities $\ldots \ldots \ldots \ldots \ldots \ldots \ldots \ldots$ 7-28

CHAPTER 8 - MANAGING MULTIPLE CONTRACTS $\ldots \ldots \ldots 8-1$

8.1 Introduction . . . . . . . . . . . . 8-1

8.2 Comparing the Progress Status of Multiple Contractors . . 8-1

8.3 Measuring the Progress Regularity of a Repetitive Project . . . . . . . . . . . . . . . 8 8-2

8:3.1 Example 8.1, Progress Regularity ... . . . 8 8-6

8.4 Enhancing Stage Handovers Through Strategic Planning . . . . . . . . . . . . . . 8-8 
8.4.1 Example 8.2, Enhancing Stage Handovers _.. 8 8-9

8.5 Improved uniformity and Earlier Completion through

Merger ................... 8-16

8.5.1 Example 8.3, Merger . . . . . . . . 8-17

CHAPTER 9 - VALIDATION THROUGH CASE STUDIES . . . . 9 9-1

9.1 Introduction . . . . . . . . . . . 9-1

9.2 Seven Electrical Substations . . . . . . . . . 9 9-1

9.2.1 Electrical Substations, the Baseline Schedule . . 9-2

9.2.2 Electrical Substations, the Updated Schedule ... 9-4

9.2.3 Electrical Substations, Features Validated .... 9-5

9.3 A Housing Project in Kuwait . . . . . . . . . . 9-27

9.3.1 Houses, the Baseline Schedule . . . . . . . 9-27

9.3.2 Houses, the Updated Schedule . . . . . . . 9-30

9.3.3 Houses, the Revised and Decelerated Schedule . . 9-31

9.3.4 Houses, Features Validated . . . . . . . 9.33

9.4 A Prison Project in California, USA. . . . . . . . . 9-145

9.4.1 Guard Towers, the Baseline Schedule . . . . . 9-145

9.4.2 Guard Towers, the Updated Schedule . . . . . 9-147

9.4.3 Guard Towers, the Accelerated Schedule ... 9-148

9.4.4 Guard Towers, Features Validated . . . . . . 9-149

\section{CHAPTER 10 - CONCLUSIONS AND SUGGESTIONS FOR FUTURE}

RESEARCH $\ldots \ldots \ldots \ldots \ldots \ldots \ldots$ 10-1

10.1 Objectives Satisfied $\ldots \ldots \ldots \ldots \ldots \ldots \ldots$ 10-1

10.2 Test of the Principal Hypothesis . . . . . . . 10-1

10.2 Test of the Secondary Hypotheses . . . . . . . . . 10-2

10.4 Conclusions . . . . . . . . . . . . . . . 10-2

10.5 Suggestions for Future Research . . . . . . . . 10-5

APPENDIX $\ldots \ldots \ldots \ldots \ldots \ldots \ldots \ldots \ldots \ldots \ldots \ldots$ A-1

A.1 Publications from this Thesis $\ldots \ldots \ldots \ldots \ldots \ldots$ A-1

A.2 References ................. A-2 


\section{LIST OF FIGURES}

2.1 Out-of-Logic Progress on Activities C and F. . . . . . 2-3

2.2 Sequence of Operations Driven by Resource-Based Relationships. . . . . . . . . . . . . . . 2-12

3.1 British Line of Balance Schedule: Plan and Actual Progress (Neale and Neale 1989). . . . . . . . . . . . . 3-3

3.2 Handover Diagram. . . . . . . . . . . . . . 3-4

3.3 American LOB Chart: Plan and Actual Progress. . . . . . . . 3-5

3.4 Unbalanced Progress (Lumsden 1968). . . . . . . . . 3-6

5.1 Typical Unit Network Showing Lead Times for Typical Repetitive Activities. . . . . . . . . . . . 5-1

5.2 Planned Completions for Typical Repetitive Activities per the American Approach of LOB. . . . . . . . . . . . 5-2

5.3 Gradual Reduction of Out-of-Logic Progress by Varying the Rates of Completions. . . . . . . . . . . . . . . 5-6

5.4 Sequence of Operations Driven by Logical Relationships. . . . . 5-7

5.5 Durations and Logical Relationships Between Different Clusters Dictating Sequence of Operations. . . . . . . . . . . 5 5-9

6.1 Utilization of Float in Parallel Scheduling of LOB. . . . . . . 6 6-2

6.2 Steps of Developing a CPM/LOB Schedule. . . . . . . . 6-6

6.3 Example 6.1, LOB Diagram Showing Subnetworks of First and 10th Units. . . . . . . . . . . . . . . . 6-7

6.4 Example 6.1, LOB Diagram Showing Branch FG of First and 10th Unit Subnetworks. . . . . . . . . . . . . . . . . 6 6-9

6.5 Example 6.1, LOB Diagram Showing Branch FH of First and 10th Unit Subnetworks. . . . . . . . . . . . . 6-10

6.6 Example 6.1, Bar Chart. . . . . . . . . . . 6-11

6.7 Example 6.1, Flow Lines of Typical Activities Based on Early Finishes. .................. 6-12

6.8 Example 6.1, Bar Chart-Revised Sequence. . . . . . . . . 6-14

6.9 Example 6.1, CPM/LOB Chart for Day 25. . . . . . . . 6-15

6.10 Example 6.2, Bar Chart Showing Status at End of Day 10 and a Forecast Completion on Day 40. . . . . . . . . 6-17 


\section{LIST OF FIGURES (Continued)}

6.11 Example 6.2, CPM/LOB Chart at End of Day 10 Showing

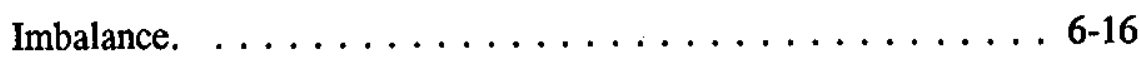

6.12 Example 6.2, Steps of Applying CPM/LOB in Adjusting a Schedule. . . . . . . . . . . . . . . . 6-18

6.13 Example 6.2, Bar Chart at End of Day 10 Showing Acceleration to Complete on Day $38 . \ldots \ldots \ldots \ldots \ldots$. . . . . . . . .

6.14 Example 6.3, Logic Diagram of a Typical Unit. . . . . . . . . 6-22

6.15 Example 6.3, Schedule Report Showing $\mathrm{T}_{2 \mathrm{a}}$ under the "Total Float" Column. . . . . . . . . . . . . . . . . 6-23

6.16 Example 6.3, Bar Chart. . . . . . . . . . . . 6-25

6.17 Example 6.4, Three Work Calendars. . . . . . . . . 6-26

6.18 Example 6.4, Schedule Report Showing $\mathrm{T}_{2 \mathrm{a}}$ under the "Total Float" Column. . . . . . . . . . . . . . . . . 6-27

6.19 Example 6.4, Bar Chart. . . . . . . . . . . . . . . 6-29

6.20 Example 6.5, Location-Time Program for Pipeline (copied from Thompson, 1981). . . . . . . . . . . . . . 6-31

6.21 Example 6.5, Bar Chart Showing Alternate Solution to Pipeline Project Using CPM/LOB. . . . . . . . . . . . . 6-32

6.22 Example 6.5, Bar Chart Showing the Impact of Eliminating One Stringing Squad. . . . . . . . . . . . . . 6-33

6.23 Example 6.6, Subnetworks of High-rise Building. . . . . . . . 6-34

6.24 Example 6.6, High-rise Building Activities Driven by Logic Relationships. . . . . . . . . . . . . 6-36

6.25 Example 6.6, CPM/LOB Application on High-rise Building. . . . 6-39

7.1 Effect of Common Activities in Repetitive Projects. . . . . . 7-2

7.2 Example 7.1, Situation 1, LOB Diagram with no Common Activities. . . . . . . . . . . . . . . . 7-5

7.3 Example 7.1, Situation 1, Bar Chart before Common Activity Effect. . . . . . . . . . . . . . . . 7-7

7.4 Example 7.1, Situation 2, LOB Diagram Showing Effect of Common Activity. . . . . . . . . . . . . 7-9

7.5 Example 7.1, Situation 2, Bar Chart Showing Effect of Common Activity. . . . . . . . . . . . . . . 7-11 


\section{LIST OF FIGURES (Continued)}

7.6 Example 7.1, Situation 2, LOB Diagram Showing Activity F Accelerated. . . . . . . . . . . . . 7-12

7.7 Example 7.1, Situation 2, Bar Chart Showing Typical Activity F Accelerated. .................... 7-13

7.8 Example 7.1, Situation 3, LOB Diagram Considering Common Activity in Rate of Project Completions. . . . . . . . . 7-15

7.9 Example 7.1, Situation 3, Bar Chart with a Revised Rate of Project Completions due to Common Activity. . . . . . . . 7-17

7.10 Four Shutter Sets and Two Carpenter Squads Performing a Roof Activity at 0.182 Roof/Day. . . . . . . . . . . . . 7-19

7.11 Roof Activity with Unbalanced Resources, Four Shutter Sets and Three Carpenter Squads. . . . . . . . . . . . . . . . 7-20

7.12 Balanced Six Shutter Sets and Three Carpenter Squads at a Higher Rate of 0.273 Roof/Day. . . . . . . . . . . 7-21

7.13 Unbalanced Resources Violating the Minimum Rate Despite Completing on Day $55 . \ldots \ldots \ldots \ldots \ldots \ldots \ldots$. . . . . . . .

7.14 Example 7.2, Logic Diagram and Initial Schedule of a House. . 7-24

7.15 Example 7.2, As-Built Bar Chart. . . . . . . . . . . 7-26

7.16 Out-of-Logic Calculation. . . . . . . . . . . . 7-27

8.1 Variance Trend Analysis. . . . . . . . . . . 8-2

8.2 Equal Completion Variances but Different Time Progress. . . . . 8-3

8.3 CPM/LOB Chart Showing Irregular Progress. . . . . . . . . 8-4

8.4 Example 8.1, Regularity Charts. . . . . . . . . . . 8 8-7

8.5 Progress Enhancing Stage Handovers. . . . . . . . . . . . 8-9

8.6 Progress not Enhancing Stage Handovers. . . . . . . . . 8-10

8.7 Example 8.2, As-Built Allocation of 260 Houses to Three Subcontractors. . . . . . . . . . . . . . 8-11

8.8 Example 8.2, Linear Program for Three Subcontractors. . . . . 8 8-13

8.9 Example 8.2, Alternative Allocation of Houses Enhancing Stage Handovers. . . . . . . . . . . . . . . . . 8-15

8.10 Example 8.3, Bar Chart of North Sector. . . . . . . . . 8 8-18

8.11 Example 8.3, CPM/LOB Chart and Regularity Measure of North Sector at End of Day 19. . . . . . . . . . . 8-19 


\section{LIST OF FIGURES (Continued)}

8.12 Example 8.3, Bar Chart of South Sector.

$8-20$

8.13 Example 8.3, CPM/LOB Chart and Regularity Measure of South

Sector at End of Day $19 . \ldots \ldots \ldots \ldots \ldots$. . . . . . . .

8.14 Example 8.3, Bar Chart of Merged Sectors. . . . . . . . 8-22

8.15 Example 8.3, CPM/LOB Chart and Regularity Measure of Merged Sectors at End of Day 19. . . . . . . . . . . 8-24

8.16 Example 8.3, Regularity Charts at End of Day $19 . \ldots \ldots$. . . 8-25

9.1 Case 1, Work Calendar. . . . . . . . . . . . . . 9-7

9.2 Case 1, Logic Diagram of a Typical Substation. . . . . . . . 9-10

9.3 Case 1, Schedule of a Typical Substation Network Showing $\mathbf{T}_{2 a}$ under the "Total Float" Column. . . . . . . . . . . . . . 9-11

9.4 Case 1, Baseline Schedule. . . . . . . . . . . . 9-13

9.5 Case 1, Original Schedule of Squad CR26. . . . . . . . . 9-18

9.6 Case 1, Full Continuity for Squad CR26. . . . . . . . . . . 9-19

9.7 Case 1, Project Status and Schedule Update as of February 2, 1988. . . . . . . . . . . . . . . . . . . . . 9-20

9.8 Case 1, Progress Regularity as of February 2, 1988. . . . . . 9-25

9.9 Case 1, Critical Activities as of February 2, 1988. . . . . . 9-26

9.10 Case 2, 260-House Project Layout. . . . . . . . . . 9-35

9.11 Case 2, Project Calendar. . . . . . . . . . . . . . 9-36

9.12 Case 2, Typical House Network. . . . . . . . . . . 9-44

9.13 Case 2, Baseline Schedule. . . . . . . . . . . . . . 9-50

9.14 Case 2, Current Sequence of Operation, as Indicated by House Projected Completions. . . . . . . . . . . . . . . . 9-95

9.15 Case 2, Status and Schedule Update as of January 31, 1988 . . . 9-96

9.16 Case 2, Correcting Out-of-Sequence Progress Versus Ignoring it in Leveling Resources. . . . . . . . . . . . . . . . . . . . 9 9-98

9.17 Case 2, Progress Regularity as of January 31, 1988 . . . . . . . 9-99

9.18 Case 2, New Sequence after Correcting Out-of-Logic Progress. 9-100

9.19 Case 2, Revised and Decelerated Schedule. . . . . . . . . . 9-104

9.20 Case 3, Typical Logic Diagram. . . . . . . . . . . . 9-150

9.21 Case 3, Linear Program of Guard Towers. . . . . . . . . 9 9-152

9.22 Case 3, Project Calendar. . . . . . . . . . . . . 9-153 


\section{LIST OF FIGURES (Continued)}

9.23 Case 3, Schedule of Tower 307 Showing $\mathrm{T}_{\mathrm{aa}}$ for MS\#8 \& MS\#12 towers under "Total Float" Column. . . . . . . . . . . . 9-163

9.24 Case 3, Schedule of Tower 307 after Leveling Serves as a First Unit Schedule for Balance of Towers. . . . . . . . . . . 9-165

9.25 Case 3, Latest Dates of the Last Tower, 312 . . . . . . . . 9-166

9.26 Case 3, Baseline Schedule. . . . . . . . . . . . 9-168

9.27 Case 3, Squad Histograms Showing Work Continuity. . . . . 9-174

9.28 Case 3, Status as of December 18, 1994. . . . . . . . . 9-175

9.29 Case 3, Progress Regularity as of December 18, 1994 . . . . 9-179

9.30 Case 3, Earliest EF and Latest LF for $T_{2 a}$ Computations. . . . 9-183

9.31 Case 3, Accelerated Schedule. . . . . . . . . . . 9.192 


\section{LIST OF TABLES}

5.1 Schedule of Activity Completions. $\ldots \ldots \ldots \ldots \ldots$. . . . . .

6.1 Example 6.1, Calculation of the Quantity of Squads. . . . . . 6-8

6.2 Example 6.2, Calculation for Adjusting Available Time for Activity Completions. . . . . . . . . . . . . . . . . . . . 6-19

6.3 Example 6.2, Calculation of Minimum Quantity of Squads to Complete Project on Original Completion, Day 38. . . . . . . . 6-20

6.4 Example 6.3, Calculation of Minimum Quantity of Squads to Complete by December 1, 1994. . . . . . . . . . . . . . . . . . . 6-24

6.5 Example 6.4, Calculation of Minimum Quantity of Squads to Complete by December 1, 1994. . . . . . . . . . . . . . . . 6 6-28

6.6 Example 6.6, Activities of a Typical Floor. . . . . . . . . . . 6-35

7.1 Example 7.1, Situation 1, Calculation of the Quantity of Squads . . . . . . . . . . . . . . . . . . 7-6

7.2 Example 7.1, Situation 3, Calculation of the Quantity of Squads . . . . . . . . . . . . . . . . . 7-14

7.3 Example 7.2, Out-of-Logic Analysis $\ldots \ldots \ldots \ldots \ldots \ldots$ 7-25

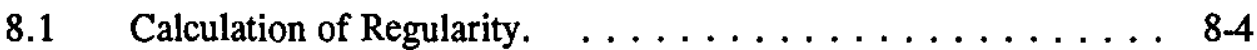

8.2 Example 8.1, Calculations of Time Variance for Progress Regularity. . . . . . . . . . . . . . . . . 8-6

8.3 Example 8.2, Allocation of Houses to Multiple Subcontractors. . 8-14

9.1 Case 1, Calculation of Minimum Quantity of Resources to Complete by August $31,1988 . \ldots \ldots$. . . . . . . . . . 9-12

9.2 Case 2, Calculation of Min. Quantity of Resources to Complete by August 17, $1988 . \ldots \ldots \ldots$. . . . . . . . . . . . . 9-47

9.3 Case 2, Calculation of Min. Quantity of Resources to Complete by March 30, 1989. . . . . . . . . . . . . . . . . 9 9-101

9.4 Case 3, Calculation of Minimum Quantity of Resources to Complete MS\#8 \& MS\#12 Towers. . . . . . . . . . . . . . 9-164

9.5 Case 3, Calculation of Minimum Quantity of Resources to Complete MS\#18 \& MS\#23 Towers. . . . . . . . . . . . 9-167

9.6 Case 3, Calculation of Minimum Quantity of Resources to Complete by June 22, 1995. 


\section{CHAPTER 1}

\section{INTRODUCTION}

\subsection{General Description of the Subject}

The line of balance technique (LOB) for planning and scheduling repetitive projects such as houses, jetty work, high-rise buildings, precast concrete production, etc., was devised in 1941 . It had provided unique and useful applications in capitalising on the economy of balancing repetitive tasks and identifying negative deviations thereof. However, it is still not suitable enough for repetitive projects where the Critical Path Method (CPM) network of the typical unit is complex and has many branching paths. It is unwieldy in large projects, difficult to update or accelerate, and does not immediately give an accurate measure of the time progress of a project. On the other hand, CPM is developed to an extent that it predominates in the industry, but its use on repetitive projects is challenged by its inability to react promptly to the incident problem of changing the sequence of work on the typical units and to maintain work continuity for the working squads. This work presents a new methodology that adequately integrates the merits of CPM and LOB by a novel introduction of float in the LOB and using resource leveling in CPM. The integrated methodology gives a prompt and accurate indication of the time progress of a repetitive project. Its virtue lies in its invulnerability to changes in the sequence of work and ability to maintain work continuity for the working squads on the repetitive activities.

\subsection{Significance of the Problem}

By definition, most repetitive projects in construction are large projects. Housing projects are repetitive projects that have indisputable importance because of their impact on people. Consequently, these projects become high-profile and draw considerable public and political attention especially on housing programs subsidized by governments after disasters. Owners as well as contractors face pressures to generate information for the effective management of these projects and for the public about the progress of these projects. While CPM has gained the acceptance and trust of the legal and the construction professions on non-repetitive construction projects, to date CPM and $\mathrm{LOB}$ have failed to schedule these projects to provide reasonably quick and accurate measures of the time progress of these projects and their projected completion dates. The abundance of research and the persistent attempts are genuine proofs on the existence of a 
dire need to solve the problem because of its impact. Those involved in the details and day-to-day planning and scheduling of repetitive construction projects know the challenge.

\subsection{Objectives of the Research}

1. Identify and evaluate past and current attempts of integrating CPM and LOB.

2. Investigate practical methods and techniques used to overcome the problems identified.

3. Develop a practical method for the integration of CPM and LOB.

4. Evaluate the effectiveness of the combined CPM and LOB in planning and scheduling repetitive projects by testing it on real life projects.

\subsection{The Principal Hypothesis}

The independent application of LOB and CPM on repetitive type projects has serious problems, in the LOB, complicated unit networks can not be handled and have to be reduced to grouped activities and control points to schedule the project. The technique does not show readily the impact of delays or changes on the project completion date. Effects of common activities are not known. The inevitable existence of float times is neglected.

The past and current use of CPM on repetitive projects confront major challenges due to the inability of CPM to react promptly to the frequent problem of changing the sequence of work on the repetitive units. This is because even relatively simple CPM networks have to contain many interfaces between the subnetworks of the repetitive units that complicate its utilization. Also, the application of CPM does not consider the work continuity of the working squads as an issue of central importance.

The major argument of this research is that if the two techniques can be adequately integrated to counteract each others weaknesses, the combination will be a more efficient planning, scheduling and monitoring technique for a wide range of projects with repetitive and non-repetitive activities. 
The principal hypothesis is:

CPM and LOB can be integrated to combine the powerful features of LOB in repetitive project controls and the popular and well known characteristics of CPM in planning, scheduling and impact analysis, creating a more effective scheduling tool. The combined technique could overcome the confusion usually associated with a large number of CPM activities required to represent repetitive type projects in an adequate and meaningful detail.

\subsection{The Secondary Hypotheses}

The integrated CPM and LOB would complement each other to be able to identify, quantify and overcome the following effects incident to repetitive type projects:

1. Common activities between some repetitive units.

2. The realties of out-of-logic progress: progress contradicting the logical relationships between the CPM activities.

3. Excessive quantities of partly completed typical activities.

\subsection{The Research Methodology}

1. Identification of past and current theories through literature review.

2. Investigation of the methods and techniques in use through site visits to selected construction projects to review project documents and conduct interviews.

3. Development of the algorithm by utilizing a popular project planning and scheduling software called Primavera Project Planner ${ }^{\star}$ (P3) with a general purpose spreadsheet software.

4. Objective evaluation of the combined CPM and LOB through interviews and interactive communications with construction professionals and academics.

5. Testing the integrated CPM and LOB through using real and assumed progress data to schedule selected representative projects and compare the results with those from alternate methods used by the project team. 


\subsection{Originality and Contributions to Knowledge}

1. Achieving the integration of the LOB and CPM techniques by introducing float into LOB and utilizing resource leveling in CPM.

2. The new methodology does not need sophisticated knowledge and training for the current average personnel usually involved in the planning and scheduling process, hence it is immediately applicable and expected to be widely accepted.

3. The new CPM/LOB methodology enables scheduling resources more efficiently than existing techniques. Repetitive activities with float in the unit network need less resources to achieve the same project completion date.

4. The integration of CPM and LOB eliminates the problems associated with the changes in sequence of work in CPM scheduling and puts the scheduler, as well as the management at a great advantage and flexibility. There is no need to revise interfaces between subnets because they do not exist. Some of the immediate benefits are considerably quicker what-if analysis related to resources management and alternative sequences of operation, and reduced human errors in scheduling.

5. In this technique, repetitive activities maintain their continuity to a practical extent in the context of CPM.

6. Through implementing the LOB control on the balance of the project along with the CPM's explicit logic between the activities, the user acquires a better overview of the project. This helps the user to focus and overcome the overwhelming of the large number of the CPM activities inherent with representing repetitive construction projects.

7. Creating access to LOB through commercially available CPM packages. This permits LOB to routinely benefit from current as well as possible future enhancements of CPM and its commercial packages. The current enhancements include: multiple calendars, advanced resource management, standard as well as customized reports and graphics, activity constraints, summary activities, activity codes, data bases, comparisons with target schedules and interfacing with other computer programs (graphics, spreadsheets, word processors, etc.).

8. Like the typical use of CPM to prove delays through Time Impact Analysis, $\mathrm{CPM} / \mathrm{LOB}$ is capable of proving delays in repetitive projects in a prompt fashion. Additionally, CPM/LOB can readily assess resource driven delays that are 
inconspicuous though potentially big. Delays stemming from out-of-Sequence progress can also be measured easily.

9. Defining the effect of common activities (discrete and less repetitive activities) between repetitive typical activities and measuring their impact on the schedule.

10. The differentiation, identification and quantification of out-of-logic progress.

11. The development of a solution to deal with the situation of excessive quantities of uncompleted typical activities during the progress of a project.

12. Enhancing the LOB chart with a representation of float and introducing the regularity chart which is rate-sensitive and indicative of the time progress of a project.

13. Introducing a new statistical measure in LOB for comparing the degree of regularity of actual progress of multiple contractors relative to their respective baseline schedules. The same measure can be used to monitor the regularity of the actual progress of a single contractor.

\subsection{Organization of the Thesis}

This thesis is organized in ten chapters. The contents of each chapter is as follows:

Chapter 1 has explained the problems associated with using CPM and LOB on repetitive projects. The research objectives, hypotheses and methodology has been stated. The chapter has concluded with a list of the original contributions to knowledge made by this research.

Chapters 2 and 3 provide a general review of CPM and LOB and emphasize those features used later to develop the new methodology. Limitations and challenges of each method's application in repetitive work are analyzed.

Chapter 4 reviews the literature concerning previous attempts and recommendations to combine CPM and LOB. A summary of several related theories is provided under separate subheadings.

Chapter 5 reviews real life applications of CPM and LOB on projects with significant repetition. It is based on the author's personal observations on projects in Kuwait and the United States of America. 
Chapter 6 introduces CPM/LOB and demonstrates how it revives the LOB by introducing float and integrating it with CPM. Several diverse examples were given with comments on practical implications.

Chapters 7 and 8 expand on the application of CPM/LOB. They deal with the identification and solution of some essential issues to the management of repetitive projects, including: the effect of common activities, out-of-logic progress, progress regularity, geographical allocations and stage handovers and the benefits of merger.

Chapter 9 shows the application of CPM/LOB on three real life construction projects: The first two are on a housing construction project in Kuwait, and the third is on part of a prison project in California, USA.

Chapter 10 presents a review of the achieved objectives and proven hypotheses along with suggestions for future research. 


\section{CHAPTER 2}

\section{THE CRITICAL PATH METHOD}

\subsection{Introduction}

This chapter describes the principles of the critical path method (CPM), and highlights the features that will be utilized later in the research. It defines new terminology necessary for identifying application problems, and reveals relevant capabilities and limitations of commercial CPM packages. Finally it demonstrates why CPM has failed in scheduling repetitive projects.

\subsection{CPM, Definition and Origin}

In the late 1950s the Dupont Company and Remington Rand (USA) developed the critical path method (Wickwire, Driscoll and Hurlbut 1991; Teplitz and Amor 1993), which is a detailed planning and scheduling technique that represents the tasks of a process or a project by distinct activities of definite durations and links them to each other by relationships thereby forming a network. The longest chain (or chains) of activities through any CPM network is the critical path (or paths). Consequently the other chains have varying slack durations or floats and are less critical for the timely completion of the project than those on the longest chain or path. Resources can be allocated to the activities towards producing many kinds of reports to help manage the project.

\subsection{Arrow and Precedence Diagrams}

The CPM network is often called the logic diagram because it shows the interdependencies between the activities hence one logical sequence of their execution. There are two ways of showing the logic diagram network, arrow diagrams and precedence diagrams. In the arrow diagram each activity is represented by an arrow whereas in the precedence diagram, each activity is represented by a node. Arrow diagrams are the traditional way of showing a logic diagram network but recently, precedence diagrams have grown more popular for many reasons. A precedence diagram is less vulnerable to human error therefore easier to develop. It also offers more types of logical relationships between the activities. Precedence diagrams use discrete activity numbers, whereas arrow diagrams refer to start and finish events that could be shared by 
other activities hence precedence diagrams offer considerable advantage in resource leveling and developing cost models (Thompson 1981). However, for presentation purposes the arrow diagram has an advantage since the arrows of the activities can be drawn to a time scale and offer an excellent depiction of logic associated with a duration sense that graphically highlights the existence of float.

\subsection{Time Analysis in CPM}

Time analysis in CPM is the mathematical calculation of the earliest and latest start and finish times of each activity in the network based on its individual duration and interdependencies along with the applicable work calendar. It comprises two passes: the forward pass where the earliest times are calculated; and the backward pass where the latest times are calculated. The difference between the early and the late times of an activity is its float.

\subsection{Out-of-Logic Progress}

At the initial stages of a construction project, planners translate the project goals into a set of milestones and completion dates. Following this they break down the work into a number of defined activities and link them logically according to many considerations that include the following:

1. The method of construction to be followed.

2. The intended timing of giving access to certain subcontractors, suppliers, etc.

3. Safety and weather considerations.

4. Protection and neatness of the works.

5. Contractual stipulations.

6. Imposing the progress of activities in a particular way to satisfy certain requirements such as, float management, resources distribution, financial considerations, etc.

The planners thus create the baseline or the initial schedule. When all the parties agree upon the details of the initial schedule, they in fact become bound to perform their contractual responsibilities represented by the CPM activities in the same logical sequence as that of the initial 
schedule. This logic in the CPM clearly defines who gains access first and which activities follow or precede other activities to organize the relations between the parties and between their activities. Of course in many cases there would be more than one alternative to the logic of the initial schedule, some alternatives known but not chosen in preference of others and some never thought of initially.

When the progress on the activities begins, it frequently does not coincide with the initial schedule, at least for its logic. A classical situation of violating the CPM logic exists when one of the parties, e.g. a subcontractor, chooses to start earlier than scheduled on one of his activities to utilize an unexpected early release of some of his expensive and unstorable resources, and by doing so he eventually blocks or impedes the works of another subcontractor. An equally frequent case is on a delayed project where a frustrated owner decides not to wait for the contractor to complete some slowly progressing activities that precede the owner's activities. The owner starts trying to save time and "slides in the activities" and the contractor claims disruption because of this.

Activities sometimes start before the end of their predecessors and, in some cases, even before the start of their predecessors (Fig. 2.1). These violations of logic may sometimes occur without negative impacts on the project, particularly when the logic between the activities is discretionary and preferential (sometimes called soft logic), not obligatory. However, in cases
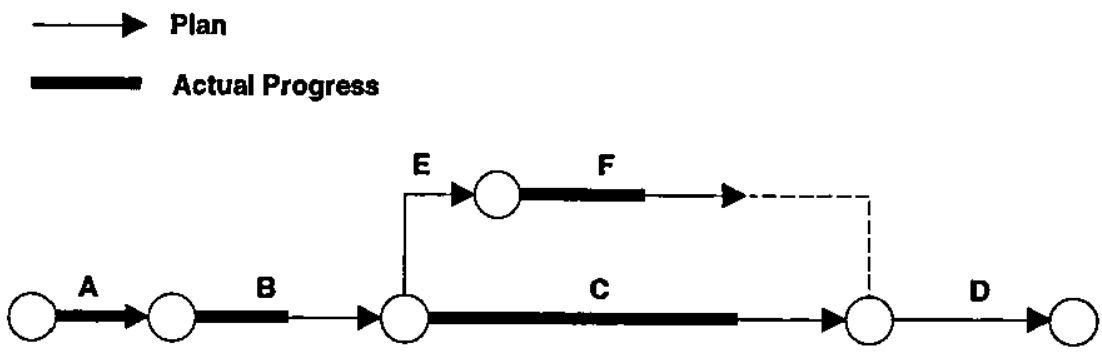

Figure 2.1 - Out-of-Logic Progress on Activities C and F.

where the logic of the initial schedule was absolute (sometimes called hard logic) and rigorously 
built based on wise reasons, complications and disputes are the expected results. Favorably, some violations happen because they represent the workable logic at the time of execution, in contrary to the initial logic which subsequently proved to be unworkable or merely inferior.

The process of identifying out-of-logic progress towards correcting it and having a workable schedule can be done by visual comparison between the logic diagram and the actual start and finish dates or the as-built bar chart, but it becomes tedious, vulnerable to human error and extremely lengthy for large and troubled projects.

\subsection{Resource Scheduling}

Resource scheduling is a methodology that takes into consideration the demand and availability of resources into time analysis. It usually has five steps:

1. Determining the limits of availability of each resource.

2. Allocating or loading individual activities with adequate resources for their completion.

3. Developing the strategy of the prioritization of activities for execution.

4. Resource leveling, which is the mathematical process that starts with the times resulting from the time analysis and delays them in line with the demand and constraints on the resources being leveled.

5. Resource smoothing, which is an extension of resource leveling aimed at reducing the fluctuation in the utilization of a particular resource between two levels of its availability, the maximum level and the normal level.

A resource, whether leveled or not, its utilization or consumption can be known through adding its quantities for each planning unit in a process called resource aggregation. 


\subsection{Resource Leveling and Smoothing}

There are many algorithms for general resource leveling within network analysis, they can be grouped in two categories: optimization-based leveling, which try to maximize or minimize specific schedule variables (e.g. shortest project duration), and require excessive computational efforts; and heuristic-based leveling, which produces practically satisfying solutions (e.g. assign resources by the minimum float rule), (Lorterapong 1994). In this work, only heuristic-based leveling was used and the issue of resource leveling techniques was considered too broad to fall within the scope of this research. Nevertheless it is necessary at this point to state what is to be expected from resource leveling and smoothing from a commercial CPM package which uses heuristic-based resource leveling.

Throughout this research and previously on the projects presented as case studies thereof, this author has utilized a CPM package called Primavera Project Planner ${ }^{\otimes}$ (P3), by Primavera Systems, Inc., Pennsylvania, USA. The reference manual of P3 has these important paragraphs about resource leveling that are self explanatory:

"..computerized resource-leveling techniques [heuristic-based] do not produce an optimal schedule. Developing an optimal application of resources for a complex network with multiple resources requires computing power far in excess of that normally available on a microcomputer. Optimization techniques using dynamic integer programming are possible but are generally too expensive for large networks.

The customary resource-leveling procedure uses [the skill and judgment of the] project planner, and supplements these with a set of decision rules carried out and reported by the computer system."

and about resource smoothing:

"The purpose of smoothing is to obtain a more uniform profile of resource usage. While it is not possible to obtain an optimum solution to resource problems (without extensive and expensive dynamic programming algorithms), it is possible to iron out some of the fluctuations in resource assignments that tend to raise staffing costs on projects." (Primavera 1990). 
It is essential to focus on how P3 handles an important feature of resource scheduling: prioritizing the activities for leveling.

"Before leveling begins, P3 arranges all project activities in a topological sequence-the order in which they must take place based on predecessor and successor relationships. In some networks, two or more activities are adjacent in the topological order and can be resequenced without affecting the network's integrity. This condition allows [the user] to assign priority codes to activities to determine the order in which they are leveled.

During resource leveling P3 establishes a list of activities in topological sequence. Then, as resources are leveled, if more than one activity [have the same topological order and] can be leveled at the same time, the priority codes are used as a tie-breaker. P3 levels the activity having the highest priority code before the ones with lower priority codes.

Priority codes are schedule parameter codes or activity codes specified on the Level Window [by the user]. The sort order can be based on any combination of priority codes: activity codes, activity ID codes, or schedule parameter codes such as late finish dates and total float. . . . for any sequential chain of activity relationships, the first activity in the chain is always leveled first regardless of the priority codes assigned.

If a project contains no relationships between activities, the priority codes determine the leveled dates of the activities (as long as enough resources are available). In the opposite case where only one chain of activities exists in the project, priorities have no effect on leveled dates because the topological sequence provides a unique order of eligible activities.

and,

"The order in which activities are leveled can result in different schedules." (Primavera 1991) 
There may be a situation where a lower priority activity gets leveled before a higher priority activity for the simple reason that the higher priority activity is not ready to start per the time analysis calculation whereas the low priority activity is ready.

\subsection{The Utilization of Resource Scheduling in Practice}

Based on this author's observations of the construction industries in both Kuwait and the United States, resource scheduling is rarely used as set out in section 2.6 above.

Due to the lack of in-depth planning and scheduling education, and relatively poor CPM programs, very few managers are using these techniques in making daily management decisions (Popescu 1991).

The frequent utilization emerges when the owner imposes the link between progress payments and the schedule. In this case contractors have their activities loaded with cost to generate and prepare payment requests. The utilization of resource leveling on materials, equipment and workers has not matured yet. And as one executive of a prominent CPM package described it:

"People ask for resource leveling when buying a CPM software, but they do not use it." (Faris, 1994)

There have been some outstanding improvements during the last ten years on the general capabilities of commercial CPM packages that are available to the general user, but the advancement in the resource scheduling capacity has been comparatively marginal. This could be a reason for the limited utilization of resource leveling: the user sets out expecting that the package will simulate for him the way he wants to manage his resources then faces some unrealistic limitations and backs off completely.

These limitations, that exist not necessarily in one package, include the inability to:

1. Prioritize the allocation of resources to the activities based on user-defined codes.

2. Make a resource follow a calendar different to the activity it is assigned to. 
3. Use a fixed resource budget quantity for one resource, hence a variable rate of utilization while using the opposite of that on another resource within the same activity: a mixed mode.

4. Show cumulative resource curves in a realistic step line format.

5. Use parallel resource scheduling where more than one activity can share a single resource and progress concurrently though alternately.

6. Associate the learning curve phenomena with the resource rather than the activity duration if at all. Also recognizing low productivity on days when the weather is harsh but work can still be progressing.

\subsection{Out-of-Sequence Progress}

Although this term has been widely used on out-of-logic progress situations as defined above, the author has alternatively chosen the expression "out-of-logic" to describe the progress of an activity that starts or finishes contrary to the pre-defined relationship with its predecessor. This is so because the term "logic diagram" has been widely used for naming CPM networks. The author is of the opinion that it is more appropriate to apply the term "out-of-sequence" to progress that does not breach the logic of the network but breaches the sequence of the priority order for resource leveling.

At the time of preparing this work, Primavera Project Planner ${ }^{\otimes}$ (P3) calls the progress of an activity that violates a relationship with one of its predecessors "out-of-sequence progress" and it can produce a report about these activities if their predecessors are not completed. If the predecessors have already been completed the violation would not be reported. In other words P3 can report only current out-of-logic progress.

From this point onward in this writing, the term out-of-logic progress shall be used as described above on logic violations, and the term out-of-sequence progress shall be applied to breaches of the priority order in resource leveling. 


\subsection{Float Ownership}

Many construction contracts do not address the issue of float ownership explicitly (Trauner 1990). It is best that this issue be addressed expressly in the contract documents and that the contractor be advised up front (de Leon 1986).

Trauner (1990), stated:

"In reality, the contract owns the float and either party may use it as long as it does not financially affect the other party. Some owners claim they own the float and, if they use it, the contractor will receive no additional compensation. This is a form of no damage for delay clause."

When the contractor sees the owner demanding sole ownership of float it is likely to present a schedule that does not reflect its true intentions and that has little float (Householder 1989). Float can be artificially dissipated by abusing preferential logic, imposing unnecessary constraints, overestimating activity durations and using resource leveling unrealistically.

Zack (1991), stated:

"In most States [USA], "float" within the project schedule is the property of the contractor unless otherwise specified in the contract documents."

Person (1991), mentioned:

"ambiguities in a float-sharing clause usually are construed against the drafter, which is normally the owner."

Zack (1993), confirmed:

"The legal precedent that, absent a contract clause to the contrary, float belongs to the contractor as one of his resources was established more than 20 years ago (Wickwire and Smith 1974). Despite current thinking that float ought to be a shared project resource, all too many public project owners [USA] continue to let contract documents that do not clarify this critical issue (Wickwire and Driscoll 1990; Wickwire et al. 1991). Thus they strengthen the argument that float belongs solely to the contractor." 
Zack (1993) observed that some take advantage of this legal rule and assert that under the equitable-adjustment language of the contract provisions they deserve time extension even though the completion date of the project is not affected. Their assertion is that, to maintain the same relative time position after issuance of the change as before (which is the equitable-adjustment "formula"), they are entitled to time extension to restore their float consumed by the change. To block this, he recommended that float was not for the exclusive use or benefit of the owner or the contractor but an expiring resource available to whoever uses it first. This may sound unfair to the party who does not have float towards the end of a contract duration after the other party has used it up earlier. Consequently, in contracts of joint-ownership of float, some owners drafted specifications that allow tracking, accounting for and banking the float generated by critical activities of one of the parties then use it to offset its delays (Zack 1993). Arditi and Patel (1989) presented a method to apportion costs between the owner and the contractor when accelerating to gain the time lost due to their delays.

In construction claims considerable attention is given to float ownership and the courts recognize the link between float and resource management:

"In Utley-James Inc., No. 5370 (1984), the General Service Board of Contract Appeals [USA] describes fundamental network analysis concepts, recognizing sophisticated CPM techniques such as resource leveling, sequence restraints, use of float and negative float, and buy-back time through acceleration".

and in

"Weaver-Bailey Constructors, Inc. v. United States, 19 Cl. Ct. 474 (1990), the landmark claims court decision on float in which the court found that the contractor had the right to plan its work in accordance with its own plans or reasonable resource usage (regardless of the fact that this utilized float)". (Wickwire, Driscoll and Hurlbut 1991)

As stated earlier in resource scheduling, leveling staggers activities to allow for more efficient use of resources, consequently it consumes float. Therefore, if a contractor wants to keep resource quantities relatively constant it should utilize resource leveling. Overtime and 
productivity losses can be reduced and sometimes avoided with a sensible utilization of float in resource leveling.

The view of Neale and Neale (1989) is that the contractor owns the float because he needs it to make the best use of resources through systematic scheduling. They stated:

"the principle that the contractor owns the float must be respected, and clients should not rob him of this important managerial asset."

Person (1991) however, was of the opinion that the courts failed to take into account whether the consumption of float has disturbed the contractor's leveling scheme or caused a delay to project completion because of limited resources. He therefore recommended including resource constraints (resource-based relationships, Suh 1993) in the schedule to lock-in the float that might otherwise be used by others. Resource constraints or dependencies are logical relationships simulating the sequence of resource movements on the activities notwithstanding that there is no physical constraint requiring such an order.

This author agrees with Person's opinion about locking-in float but qualifies it with utilizing resource leveling in lieu of resource-based relationships. Resource leveling is dynamic and a true depiction of resource availability and demand throughout the project. It produces more than one logical sequence to execute the work, more credible schedules and can be managed more easily in practice vis a vis sole time scheduling. This is especially true on projects with significant repetition.

\subsection{Challenges in Using CPM on Repetitive Projects}

Both practitioners and researchers voiced their disappointment with CPM application on repetitive projects. One of the main reasons was CPM's vulnerability to sequence changes of work between the repetitive typical units, which is, on repetitive projects, a matter of choice and strategy and is quite frequent depending on unforeseen circumstances (Rahbar and Rowings 1992). In CPM, repetitive typical activities are linked by pure logic relationships to simulate the sequence of operations (Fig. 2.2). Progressing apart from that sequence produces an error in the CPM package called out-of-sequence progress, but in fact it should be called out-of-logic progress because logic is being violated. Fig. 2.2 shows the application of CPM in repetitive construction 


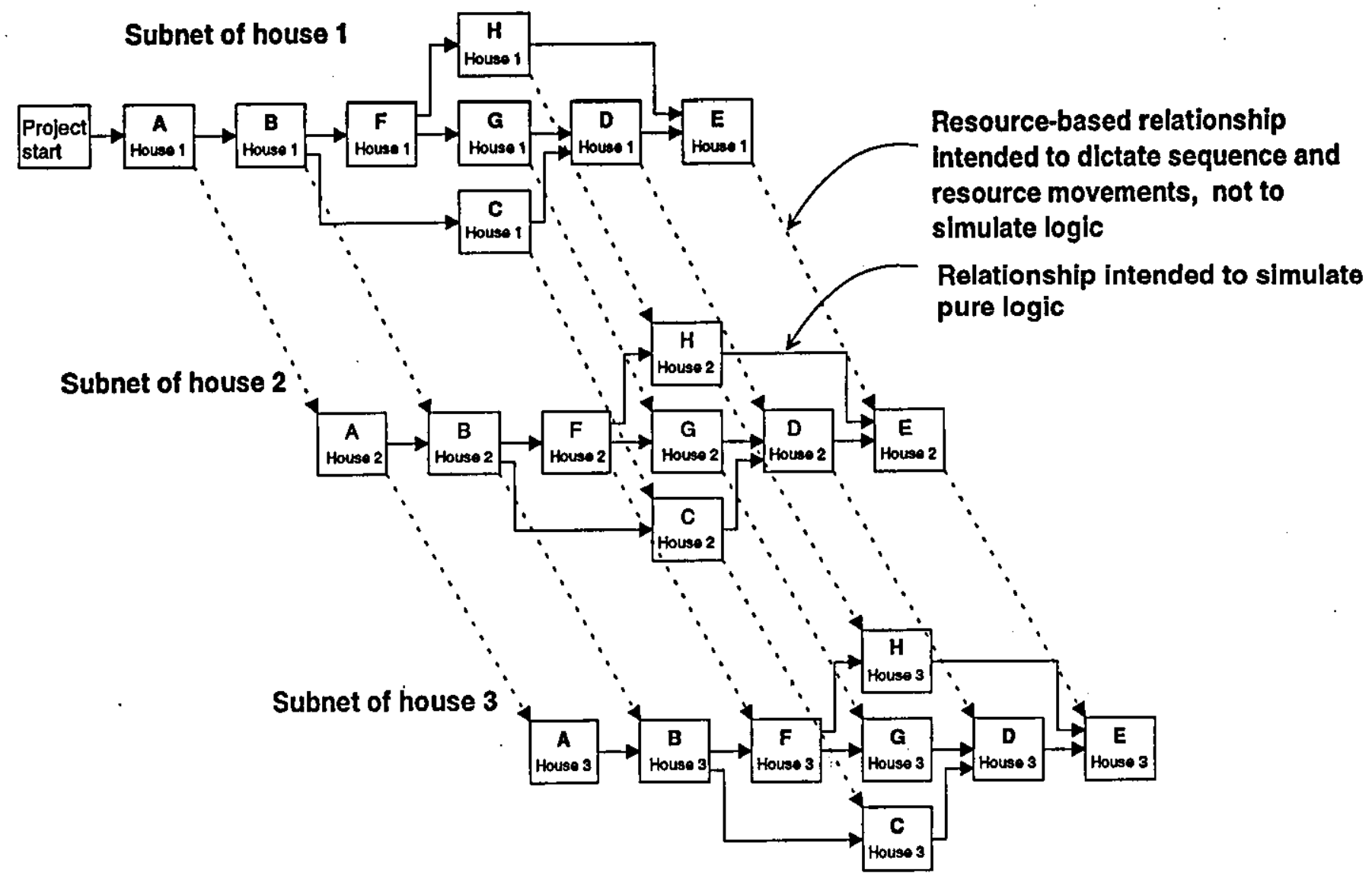


where a subnet is created for each house and linked to other subnets. This assumes that a squad working on a typical activity, for example activity "D", is supposed to move from one house to the other according to the sequence dictated by the management and represented by the dotted relationships. However, the squads will often follow another sequence and the need for revising the dotted relationships would be a must to maintain a meaningful and manageable schedule. The revision of the dotted relationships will be more tedious if the planner realistically deploys more than one squad to work on each typical repetitive activity and when there is more than one trade squad working on a single activity for only part of its duration. Temporary changes of resource availabilities add more complication to these relationships. Scheduling without correcting the error of out-of-logic progress produces unworkable schedules. Therefore each time the sequence is changed, it becomes necessary to expend many workhours to correct the dotted relationships to keep the schedule meaningful and workable (Rahbar and Rowings 1992).

Carr and Meyer (1974) in criticizing CPM on this point stated that:

"The order of progress through such a project is usually chosen by management rather than required by the dependencies of the activities."

Peer (1974) stated that the requirement by many governments and private clients to use CPM has been a main reason for its wide spread. However, CPM failed to solve practical problems of production processes on site (repetition). He argued that the gap between a CPM schedule and reality cannot be eliminated by utilizing the resource leveling techniques and that the input of the required number of resources would only be by accidental guessing.

Pilcher (1976) stated:

"For such works [repetitive projects] it is extravagant, as well as difficult, to draw and maintain critical path networks illustrating the detail of construction process for each of the units involved. The updating of such a network and its use as an instrument of control for the delivery of materials and each individual construction operation presents many problems of organization. The discipline and logic of networking can, however, be employed in a more rational way for such projects by using the line-of-balance method." 
Heyel (1979) asserted that organizations using PERT or CPM for research and development activities often switched to LOB for control of the related manufacturing processes and that, in general, the reverse was not true.

Arditi and Albulak (1979) mentioned that high repetition reduces the chances of successful planning and control by network analysis. They also stated that linking the repetitive subnetworks with relationships was an extremely tedious process.

Clough and Sears (1991) indicated that the practical application of CPM in repetitive operations was complicated, nevertheless CPM was occasionally used.

Russell and Wong (1993) stated that a significant barrier to the use of a computerized system in the planning and control of repetitive projects is posed by the tedium of describing such projects in the face of logic and work location changes. They noted that location sequences are often arbitrary and construction strategies are opportunistic. Also, updating algorithms should allow for changes to work location orderings and crewing.

The other main disappointment with the use of CPM is that it is not oriented towards providing work continuity for the squads of the repetitive activities which is fundamental in repetitive construction.

Peer (1974) said that limitations were imposed on the use of network analysis. The need for creating working continuity and balancing the whole process is completely neglected.

Selinger (1980) indicated that CPM, when used as a model of repetitive construction, had ruled out the requirement of creating work continuity to obtain maximum resource utilization.

Reda (1990) in trying to shorten the project duration and reduce the direct costs, indicated that in repetitive projects planned by CPM, this shortening would sometimes, and depending where the flow of the critical path is, cause a squad to work with a higher production rate at a particular stage or unit and then work at a normal production rate at the rest of the units. Thus the production rate for the squad, and consequently the repetitive activity duration would not be maintained throughout the project. Furthermore, by using CPM calculations the continuity of work for the squads is not maintained. 
Rahbar and Rowings (1992) exposed some disadvantages of CPM, they mentioned that CPM is unable to distinguish rates of progress of activities and that the number of units that can be completed within any period of duration is not clearly visible. They also stated:

"conventional resource allocation, smoothing, or leveling procedures are incapable of ensuring full continuity for a production crew or process which is the backbone of planning repetitive cases."

Russell and Wong (1993) stated:

"issues such as work continuity, learning effects and multiple crewing strategies make the use of traditional network approaches difficult."

Russell and McGowan (1993) stated:

"The usefulness of traditional network approaches for [repetitive] projects is limited by their inability to treat issues such as work continuity, multiple crewing strategies, and changing work location sequences."

Humphries (1993) mentioned that the typical CPM schedule provides no indication of the rate at which the work is being accomplished.

Resolving the problem of out-of-logic progress that results from inevitable changes of operations sequence on the typical repetitive units is one of the prime objectives of this research. The other prime objective is maintaining work continuity for the working squads in the CPM context. 


\section{CHAPTER 3}

\section{THE LINE OF BALANCE METHOD}

\subsection{Background and Development}

The line of balance (LOB) technique for the planning and control of repetitive operations originated from the manufacturing industry during World War II as a tool for monitoring and controlling the war production plant of the Goodyear Tire and Rubber Company (USA) in 1941. It was further developed by the US Navy during the same war and the Korean War (Turban 1968; Heyel 1979; Goodman and Love 1980). In the mid 1960s with the growth of industrialized housing development, LOB attracted the interest of the UK construction industry (Harper 1983).

Professor E. G. Trimble of Loughborough University, UK introduced P. Lumsden to LOB and developed the technique for application to building construction while at the (British) National Building Agency, the Newcastle office (Lumsden 1968; National Building Agency 1962). The technique is not well known among young practitioners in the USA (Sobhy 1995). This is probably attributed to a disenchantment among prominent US educators with it (Enrico 1995). LOB is well known in the UK and in countries educationally influenced by Britain including India, Sudan (Neale and Raju 1988) and Australia (Hinkley 1989).

\subsection{The Essence of the LOB Technique}

The LOB is a graphically represented method for scheduling repetitive work. It is concerned with the components which make up the flow of finished products (O'Brien 1969). It shows the required and actual progressive completions for each activity in equivalent end-product units. The LOB is oriented towards the required delivery of completed units, and is based on a knowledge of how many activities of any type are required to have been completed by any given day, in order that the programmed delivery of units can be met (Lumsden 1968).

\subsection{The Natural Rhythm in Construction}

In construction, tradesmen rarely work individually or in large groups. Experience and practice have established an effective size for particular squads of tradesmen. For example, to obtain the most efficient productivity, bricklayers usually work in a squad of two bricklayers 
serviced by one laborer, similarly, plumbers work in pairs. Therefore balancing calculations have to be done on the basis of output of squads rather than individuals to avoid interference. This restriction makes it difficult to achieve a common rate of work for all trades or activities in construction (Neale and Neale 1989). The normal rate of production of a squad of optimum size, as described above, or multiples thereof is the natural rhythm of that trade or squad. Consequently planning resources hence rates of production closest to the natural rhythm is nearest to optimum productivity.

To the contrary, this is rarely the case in a manufacturing environment. The rate. of progress of each activity may be adjusted quite finely; consequently, activities may be put to progress at almost the same rate (Neale and Neale 1989).

Consideration of the natural rhythm notion gave rise to two approaches in formulating the LOB schedules. These two approaches can best be distinguished as British and American (US). Both approaches start the calculation from a handover or a delivery rate, but the American approach de-emphasizes or disregards the natural rhythm notion. This was probably because the LOB technique was born in the manufacturing industry of the United States.

\subsection{The British Approach to Formulating the LOB}

The definition of a typical British application of the LOB is given by the National Building Agency in 1968: A series of inclined bar-lines at different slopes, one for each trade. The bar-lines vividly display the speed of one trade against another, highlighting the risk of collision courses (interference) and unbalanced manning. The implementation has been more of a mathematical calculation of trade manhours prior to plotting it on a time scale. This was referred to by Darlow (1968) and later by Pilcher (1992) as resource scheduling, where the calculations is dominated by the natural rhythm of each activity leading to a rate of progress that is nearest to the desired handover rate. The balancing calculations of LOB can be tedious unless a spreadsheet is used (Neale and Neale 1989). This approach is followed by all British text books if not all papers by British authors or c0-authors reviewed in this research. Fig. 3.1 is copied from Neale and Neale (1989) and represents the form of a typical British LOB schedule that was not used by any American text book covered in this research. However, Suh (1993) adopted this approach in his PhD work that was supervised by David Arditi, a former student of $\mathrm{E}$. G. Trimble. 


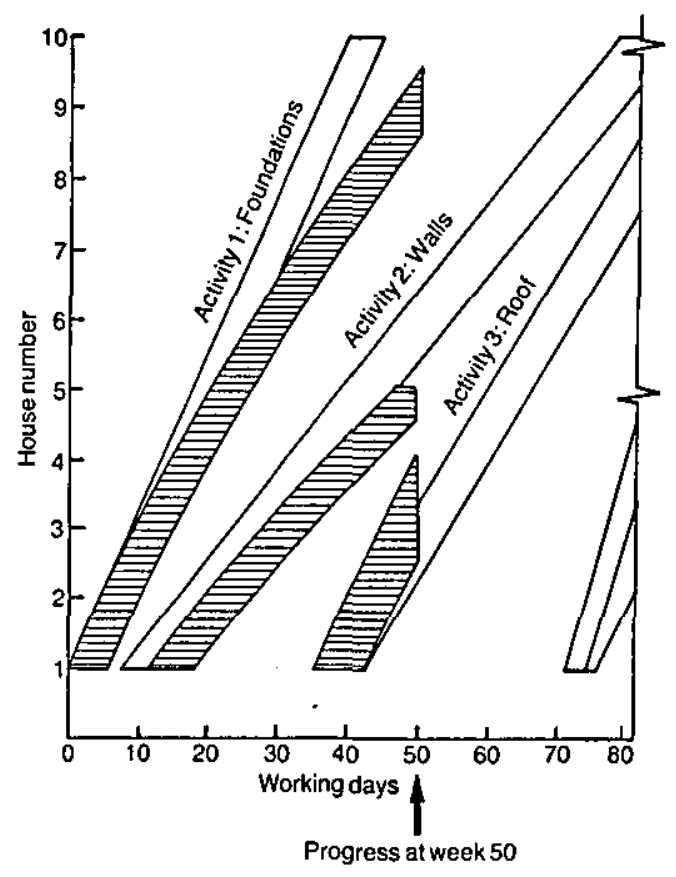

Figure 3.1 - British Line-of-Balance Schedule: Plan and Actual Progress (Neale and Neale 1989).

\subsection{The US Approach to Formulating the LOB}

LOB is frequently referred to as a graphical technique in the US. A common handover rate is imposed upon all activities in what is called parallel scheduling (Darlow 1968; Harris and McCaffer 1977; Trimble 1984; Pilcher 1992). Balancing of all activities to eliminate interference thus squad waiting time is only possible if their durations are divisible by a common factor. Parallel scheduling leads to a shorter overall duration hence savings in overheads.

The approach analyzes the activities of a typical unit network with relation to their completions rather than commencements. Lead times are established representing the duration by which particular events must precede the end event. Then completions are represented versus time in a line graph (Fig. 3.2). The number which should be completed for each activity or event, can be read off against the line of completions by going forward the lead time of that event or activity (Pilcher 1976). 


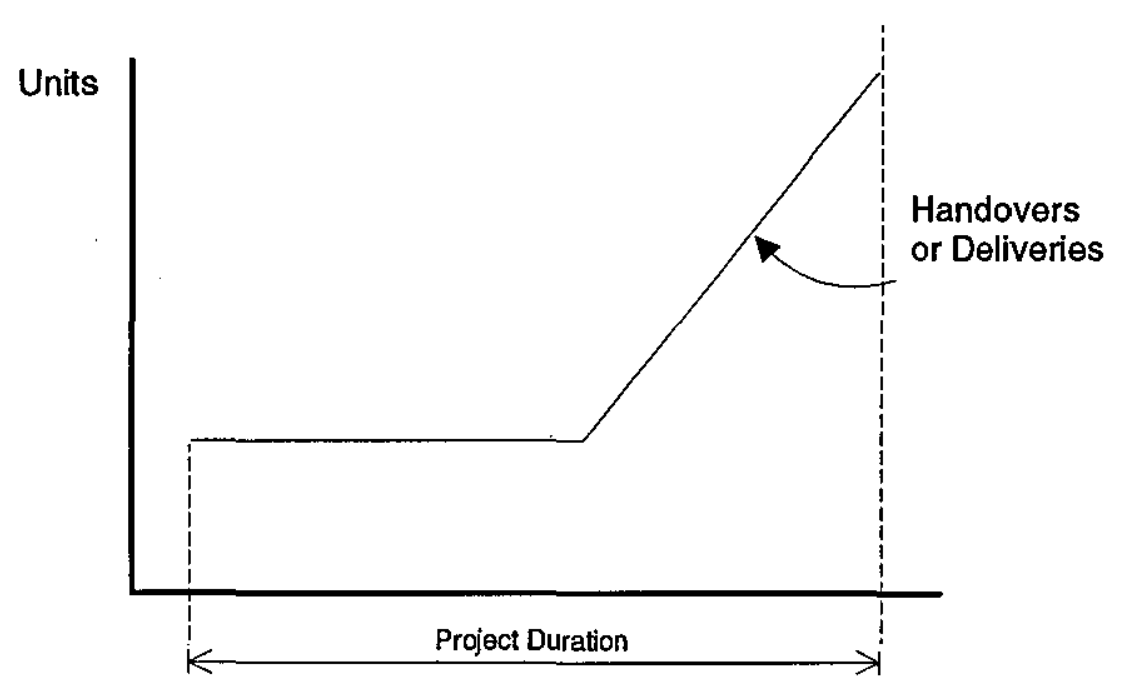

Time

Figure 3.2 - Handover Diagram.

A LOB Chart (Fig. 3.3) is then drawn by quantitatively representing the cumulative completions of activities associated with a level of planned number (line of balance) at a given point in time. It provides a vivid overview of the project overall status and reveals graphically any out of balance that suggests a deviation from the plan due to the actual uneven progress of activities. Management is promptly enabled to focus on assessing the deviation quantitatively (Khisty 1970). On the other hand, while this chart shows a delay in one or more activities it does not show the impact of this delay on the completion date of the project. Also, what the LOB Chart does not show is that not only must there be enough activity completions to the level of the line of balance at a given point in time, but an additional number of activities must be underway to meet the following interim planning goal. According to the references reviewed for this work, no British author used the LOB Chart except Pilcher (1976; 1992).

This approach eased the use of LOB on complex unit networks, however left room for the occurrence of the interference problem. Birrell (1980) cited another author called Saul Horowitz, Jr. who emphasized the practical benefit of management psychology and speed competition on the construction of New York skyscrapers where faster squads where put to work behind slower squads to pressure the earlier to work faster. However, Neale and Neale (1989) mentioned that if tradesmen cannot see work available for them for some reasonable period ahead, they may simply slow down. 


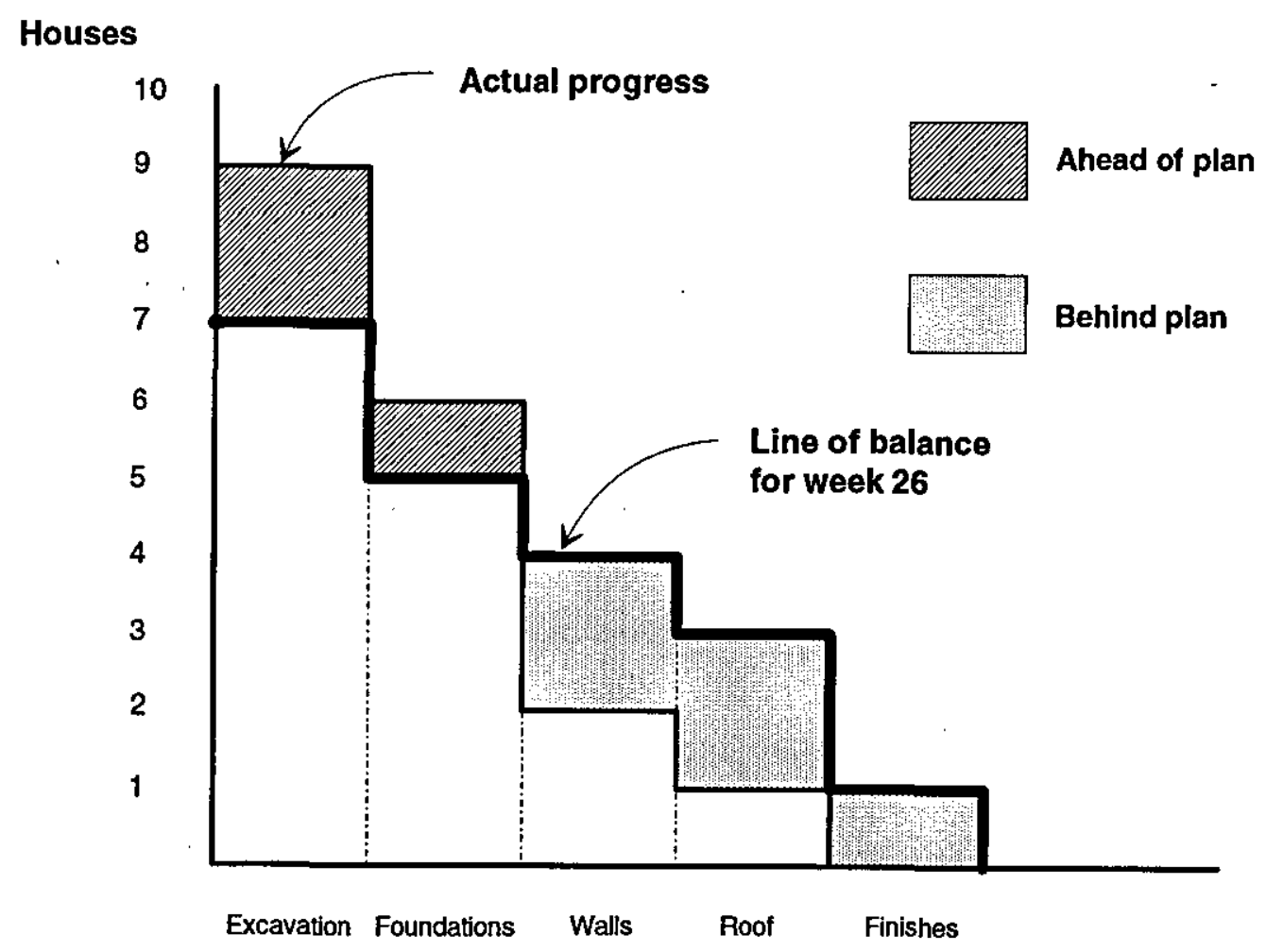

Activities

Figure 3.3 - American LOB Chart: Plan and Actual Progress.

\subsection{Irrelevance of Out-of-Sequence Progress in LOB}

The construction site environment differs substantially from the manufacturing environment in this issue of sequence. In chapter two the problems of out-of-sequence progress were addressed in the context of construction network scheduling, and concluded that many relationships between the subnetworks need to be revised each time progress sequence changes. To the contrary, in the manufacturing industry, where the LOB technique originated, change of progress sequence between the repetitive units can not exist. The manufactured repetitive components are not associated with any particular individual commodity. A component may be assembled on any individual commodity or end-product. Components and commodities do not have a unique identity or a serial number before they are completed. In construction however; a house has its identity, an address number established since the preparation of the drawings and any "component" or activity of it can not be completed and used on another house like the case 
of manufacturing a particular component of a car. The issue became a visible concern when LOB was applied in the construction industry.

\subsection{LOB Drawbacks and Limitations}

Lumsden (1968) gave a very good example on a commonly encountered situation on housing projects; unbalanced progress (Fig. 3.4, reproduced from Lumsden, 1968). In this example the work on stages or activities 1 and 2 progressed ahead of schedule (past the vertical dashed line of time now) while subsequent work on activities 3 was not started, thus behind schedule. In this case if activities 1 and 2 were intensive value activities and the progress was related entirely to income, the impression might be gained that the progress of the whole project was ahead. However, reference to the LOB diagram (Fig. 3.4) indicates that the progress is
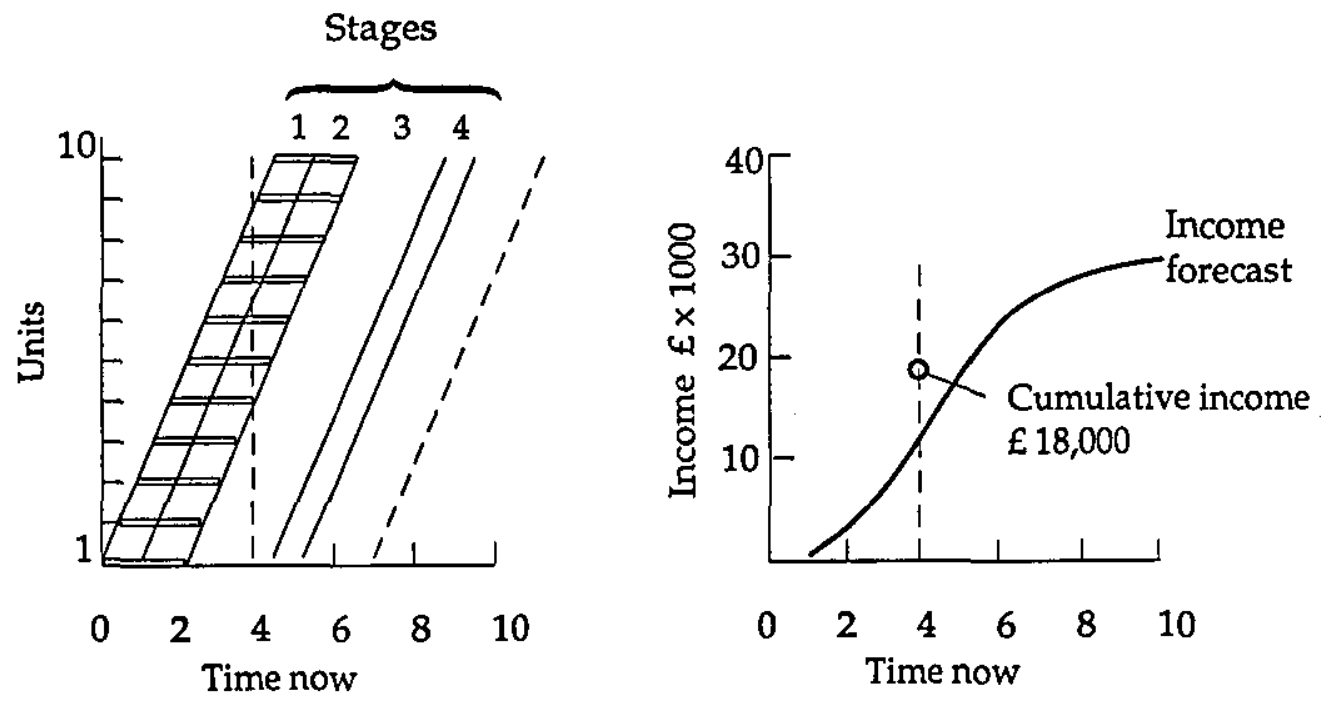

Figure 3.4 - Unbalanced Progress (Lumsden 1968).

unbalanced and the completion line of activity 4 will be shifted to the dashed line if no adjustment to resources is possible. The analysis of the LOB diagram on complex and branching networks of typical units would not be indicative of the delay and the time progress of the project. LOB can forecast the delay in the delivery of a unit but not any accompanying delay in total project completion. This output is typically produced by CPM (Carr and Meyer 1974). 
Heyel (1979) said that on projects where surplus time existed within activities, LOB did not deal directly with the reallocation of spare time as did CPM and PERT.

Artiti and Albulak (1979) mentioned that LOB was not able to generate corrective measures in cases of delay, as the degree of detail was too low. However, when LOB used more detail, hence many activities the diagram became a jungle of oblique lines with no sense even to the planner who prepared it.

Kavanagh (1985) indicated that the LOB techniques were designed to model simple repetitive production processes and, therefore, they do not transplant readily into a complex and capricious construction environment.

Arditi and Albulak (1986) commented about the readability and the visual problems with the presentation of the LOB diagram, they stated:

"there are major problems in the presentation of the LOB information: unless colored graphics are used, it is difficult to differentiate between overlapping activities that have equal rates of production."

Neale and Neale (1989) mentioned that LOB can show clearly only a limited amount of information and a limited degree of complexity, especially when using the technique to monitor the progress.

Al Sarraj (1991) indicated that a review of literature revealed that the LOB method was not formalized in acceptable form to make it suitable for general implementation in construction and as a result of that, its use in the construction project management had been limited.

Hegazy et al. (1993) drew attention to two limitations in the LOB: inability to allow for the presence of non-repetitive activities and to reveal enough about the relationships among activities of a particular unit.

Despite all of the above, LOB remains a powerful technique for control that provides concise reporting in establishing the project status. Unlike CPM, LOB can clearly show management the priorities for reallocating resources at a glance. Balancing crews with each other is a fundamental principle that is well considered in LOB. 


\section{CHAPTER 4}

\section{LITERATURE REVIEW}

\subsection{Introduction}

The task of combining the two techniques of CPM and LOB or sometimes combining their merits under different names has been a heavily researched theme for three decades. It has been a subject of interest to several researchers (Hegazy et al. 1993). This reinforces the notion that they are complementary (Turban 1968; Carr and Meyer 1974). This chapter summarizes what was published in the literature concerning the combination of the two techniques and comments on what is most close to this work.

\subsection{Previous Attempts of Combining CPM and LOB}

Schoderbek and Digman (1967) presented PERT/LOB. They worked on integrating the planning elements and discipline of PERT with the control elements of LOB; PERT in the development phase and LOB in the production phase. They realized that there was an obvious need for a single management tool to centralize the management of a project from inception to completion. Their method expanded a CPM arrow network of milestones to include one-time regular activities and repetitive activities. However, the algorithm of scheduling repetitive activities was not shown. Also, it could not be inferred that it was resource-driven.

Turban (1968) stated:

"The two techniques are complementary, though each can be used effectively by itself. ... In 1963, the federal government established a LOB Coordinating Committee, similar to the well-known PERT Coordinating Committee. Major tasks set for the committee were to study LOB's applicability and its tie with PERT."

Lumsden (1968) in his valuable publication stated:

"It has been found that the power of logical networks combined with the principles of the Line-of-Balance method provides a microscopic picture of any repetitive project without 
the administrative burden which comparable networks could create. . . By combining the logical disciplines of networks and the principles of the Line-of-Balance method, where we are concerned with the rate at which events and activities occur, the power of networks has been retained without increasing the administrative work load beyond that produced by adherence to Bar Chart control."

Lumsden kept the combination of CPM and LOB principles in the LOB graphical context.

Howell (1983) in trying to combine resource oriented scheduling (ROS) (LOB is resource oriented) with CPM, described the opinion of some practitioners as:

"believed that was an extremely ambitious concept which is unlikely to work in practice."

Trimble (1984) integrated the comments of Howell and considered the integration of ROS with CPM a daunting task, though a very powerful tool by which to schedule efficiently the complete range of project types from totally repetitive to totally non-repetitive.

Soliman (1987) and Mansur (1990) graphically emphasized the possibility of fluctuating a repetitive activity between two boundaries in the LOB context. Soliman concluded that economical resource allocation required extensive analysis to determine the number of squads for each activity. Mansur encouraged expanding network based computer packages to account for the LOB technique.

Neale and Raju (1988) tried to refine the LOB in a spreadsheet format by introducing activities that run concurrently. They then confronted the complex relationships that their spreadsheet had to express and concluded that it was practically meaningless to draw the output in the form of a diagram of incomprehensible mass of flow lines.

Al Sarraj (1990) developed a mathematical method for the LOB to facilitate finding the project duration, start and finish times for every activity at every repetitive unit, the available buffer and the information about the intersection place. He provided an alternative for drawing a LOB diagram as a means of defining the schedules. The example he presented was for a project of multiple standard units with each standard unit having a series of sequential operations 
(activities), therefore the expected effects of using CPM with LOB were aborted. Consequently no float times were generated or investigated.

Rahbar and Rowings (1992) presented a method that was insensitive to out-of-sequence progress yet detailed to be meaningful to the end user. They basically kept the CPM at the summary level of the discrete non-repetitive activities to avoid a complicated network with out-ofsequence progress errors, and then switched to implement the LOB at the further detailed level (the level of repetitive activities). The discrete activities (CPM activities) summarized repetitive "step activities" (subactivities) that were shown sequential in their logical relationships, and no attempt was shown on dealing with complicated non-sequential logic in repetitive "step activities". At the detailed level of repetitive activities the scheduler was to perform resource leveling using his personal judgement while automatic resource leveling was discredited for its pre-existing assumptions which may were in conflict with the user's intent.

Suh (1993) presented a computerized program for repetitive projects using LOB. He argued that the concept of criticality in LOB differs from that in network planning and that none of the existed methods had recognized that concept nor defined float in LOB. He advocated floats calculation in LOB as they would be in network scheduling, however, he was able to calculate free float only. He based his method on a single unit network and the traditional LOB then incrementally increased squads on selected repetitive activities in a specific order to achieve a satisfactory rate. He called this process compression analysis. This seriously underutilized LOB's power to balance the trades as a starting point rather than a finishing point and necessitated multiple iterations. Resource-based logic relationships were used and the effect of sequence changes was not addressed, however, squad work continuity was maintained.

Hegazy et al. (1993) presented a computer program for scheduling and controlling linear projects called BAL. The algorithm was based on the British approach to the LOB technique as described by Harris and McCaffer (1977). It utilized logical relationships between the repetitive activities to calculate start and finish times as well as the quantity of crews to be employed on each activity. It could show crew movements along the repetitive units in a straight flow line format. The authors asserted that alternative bar charts and networks were difficult to comprehend, particularly if large number of repetitions were involved. However, they suggested future enhancements include the development of a general planning and scheduling algorithm integrating LOB and a network technique to enable scheduling complicated logic diagrams and incorporating non-repetitive activities. 
Russell and McGowan (1993) had their initial research goal finding a way to marry network analysis and linear scheduling into one mathematical technique.

In June 1993 Russell and Wong, and separately, Russell and McGowan presented REPCON (Representing Construction) which is a project management software marrying CPM with linear scheduling. REPCON models projects with repetitive elements which are linked by logic relationships. Typical activities (repetitive) and discrete activities are called "Planning Structures". Planning Structures can be continuous, interruptible, cyclic or non-repetitive. The logic relationships are of the precedence type and can be start to start (SS), start to finish (SF), finish to finish (FF) and start to finish (SF). The updating process allows for changes of sequence and number of squads. It also handles location offsets and space buffers.

The main important and relevant feature, is that in spite of using logic relationships between repetitive activities to dictate the sequence of operation, REPCON generalizes the concept of precedence so that logic relationships amongst components of planning structures can be specified using a single relationship enabling speedy definition and revision of these relationships. Each time change is necessary there is no tedious revision of each relationship and therefore considerable time is saved on large CPM networks.

According to the authors, REPCON needed considerable training of personnel on the concept. The user defined number of squads and therefore rates of completions are the starting point. Balancing the squads in view of the available project duration was not demonstrated.

Thabet and Beliveau (1994) created a formalized scheduling procedure for multistory buildings. They computerized the logical relationships within each floor (horizontal constraints) and those between the typical networks of the multiple floors (vertical constraints). The procedure considered the continuity of activities but had a limitation in that it did not provide for "varying of the work flow direction of crews", which is the out-of-sequence progress. This is understandable on high-rise buildings since the work flow is almost always upward with the possible exception of some activities such as, final external finishes and cleaning which could go more conveniently downward. 
In the summer of 1989 , my supervisor Mr. Richard H. Neale, prudently advised to summarize my work and publish the principles of CPM/LOB in a paper to establish its intellectual property. But due to drastic events this was delayed until 1993.

Interestingly thereafter, the manuscript of the CPM/LOB paper, containing the fundamentals of this research, had already been received by ASCE for review and possible publication on June 8, 1993 when Russell and Wong (June 1993) presented "REPCON" and recommended resource leveling as an "interesting challenge" for future investigation. REPCON, is a construction management software that schedules repetitive activities driven by CPM logic relationships.

Humphries (1993) published RPM (Repetitive Production Method Scheduling Technique) in the AACE Transactions on 11 July and stated in his conclusions section:

"Some methods to consider in future papers would be the use of resource leveling in connection with an RPM schedule."

Because CPM/LOB is based on resource leveling, it is therefore clear that the above authors support is implied and that their recommendations could not have possibly been a beacon for this author.

\subsection{Pertinent Methods and Theories}

\subsubsection{Location-Time Diagrams}

Location-time diagrams are graphical representations of linear activities on axes of distance (location) and time so that the slopes of the curves represent rates of production (Calvert 1970; Thompson 1981). They can show locations of likely restrictions (Cormican 1985) and discrete activities like culverts thereby provide a good project overview. They are most suitable for roadworks where they depict in a block form the cut and fill quantities. Calvert (1970) had called the method speed diagrams. Roesch (1972) called it velocity diagrams and argued that in 
comparison with networks, logical mistakes are more noticeable in velocity diagrams. Stradal and Cacha (1982) presented time-space scheduling method which is basically identical to location-time diagrams by Calvert (1970) and Thompson (1981).

Vertical production method was introduced by O'Brien (1975) and applied to multi-story construction. It was similar to location-time diagrams and little was made to cater for nonrepetitive elements.

Johnston (1981) presented Linear Scheduling Method (LSM) and stated that any differentiation between it and the LOB may only be a question of emphasis. He encouraged using CPM to schedule discrete portions of linear projects to determine their durations and transplant them in the output on the LSM presentation. A culvert or a bridge on a highway would be an example of a discrete portion on a linear project.

Arditi and Albulak (1986) mentioned that LOB was a linear scheduling method that makes use of network technology and can adjust activities' rates of production towards smooth and efficient flow of resources. They applied LOB experimentally to a pavement construction project but faced problems in presenting overlapping activities.

Chrzanowski and Johnston (1986) applied CPM and LSM to a real life roadway construction project. They contended that even though an LSM diagram does not delineate series of critical activities it can show field personnel what work has to be done and where with ease. They acknowledged that CPM is capable of readily determining the effect of changes on following activities though can be extremely detailed on complex projects containing repetitive activities.

Mawdesley et al. (1989) developed a link between this method which they alternatively called time chainage, and a CPM package. They integrated chainage charts into a CPM computer package used by Tarmac Construction. They contended that it was a useful addition facility to the standard scheduling technique on linear projects, and that all the standard facilities in the existed package remained available with no increase in complexity. They also mentioned that it was good for projects with less than 100 activities but produced confusing diagrams on large projects with a lot of detail. 


\subsubsection{Linear Programming}

Linear programming is an analytical technique which provides a mathematically representation of a process of the first degree with respect to the variables towards optimization.

Perera (1982) presented a linear programming method with much merit. He used linear programming to analyze networks comprising repetitive activities. His method addressed multiple branching networks within a project, it was resource oriented in computing the duration of the project and accounted for float times. It also integrated cost constraints. It entailed an iterative use of an additional software for linear programming each time with revised coefficients till the optimal solution is determined. The objective was to help planners analyze networks comprising repetitive activities more accurately in a resource sharing environment.

Reda (1990) proposed RPM (Repetitive Production Method) as an alternative approach to the traditional network techniques for planning, scheduling and monitoring repetitive construction activities. RPM, a linear programming model is based on graphical or analytical techniques to model repetitive projects. It uses the activity on the node diagram and gives each activity a time cost curve and assumes a constant duration for the same activity at all stages to maintain a constant production rate. No idle time is allowed for any crew. Each activity has a time buffer with it predecessor. Any two concurrent activities may have a stage buffer of a specific number of stages between them to meet practical and/or technological purposes. The RPM model finds, for each feasible project duration, the start time of each activity at the first and last stage, the duration of each activity (which is to be set equal at all the stages to maintain a constant production rate for the crew working on the activity), and finally the project minimum direct cost that can achieve this project feasible duration. The number of constraints needed to represent the project depends on the number of activities in the typical network and is independent of the number of stages of the project.

A constraint must be written for each activity and for each predecessor it has for that stage or unit of the project. Start and finish dates of activities are given only for the first and last units. It limits the resources to only one squad for each typical activity to ensure continuity and no idle time. Since the method is based on a constant rate of production for each crew, it is expected that accommodating a varying rate of production would necessitate undesirable further complication. It would be inevitable, on complicated and branching unit networks, that the 
graphical presentation of this method, just like the traditional flow lines of LOB, will be a mass of incomprehensible lines.

Linear scheduling techniques only focus on production rates and do not provide a practical solution (Rahbar and Rowings 1992). Linear programming is sophisticated and can be too complex especially for site personnel in a real life project application. It still needs a higher level of planners than normally employed nowadays for scheduling construction projects.

One inherent disadvantage is that the effects of changes in a given activity may not be readily determined by tracing the flow of succeeding activities like that on a CPM diagram.

During construction many changes are usually considered, and it is essential that the impact that alternatives have on completion status be quickly assessed (Carr and Meyer 1974). To a large extent, the plan should contain the strength to absorb minor time variations or location variations (Birrell 1980).

\subsubsection{Dynamic Programming}

Dynamic programming was developed by Richard Bellman and his colleagues at the Rand Corporation, USA (Nemhauser 1966). It is an analytical technique similar to linear programming which improves the computational efficiency by more detail and recursive computations until a specific optimum solution is reached. It is not constrained by linear assumptions (Lutz 1990). Dynamic programming is used to solve complex optimization problems by determining optimal decisions one at a time. The problem has to be represented in a mathematical model. The amount of mental effort and analysis required to construct a mathematical model of real-world phenomena is great (Nemhauser 1966).

Peer (1974) had suggested using dynamic programming as an alternative to CPM to schedule repetitive projects. He discussed its utilizations and compared it with CPM and confirmed that network analysis was incapable of providing a practical construction schedule for site management and that resource allocation techniques were not realistic. He contended that the need dictated a procedure based on a set of algorithms suitably combined for a given project and in accordance with its specific requirements. 
CPT, Construction Planning Technique was presented by Peer and Selinger (1976). It was a linear scheduling method for repetitive projects that utilized dynamic programming. It was proposed for repetitive projects as well as projects containing discrete activities.

Selinger (1980) presented a scheduling method for linear construction projects based on dynamic programming developed with a view to satisfy work continuity and minimum project duration. It was based on labor requirement and fixed crews, rather than on predetermined activity durations.

Russell and Caselton (1988) improved on Selinger's work. Their method included precedence relationships and the ability to treat a variety of activity durations and work continuity constraints. In addition, a sensitivity analysis procedure was described to permit the identification of near-optimal alternative solutions when a specific combination of durations and continuity constraints was insisted upon. The authors stated that their contribution was directed towards the goal of having linear scheduling become a practical computer-based tool for repetitive construction projects. They suggested that future research in dynamic programming included nonserial cases (i.e. branching networks).

Moselhi and El-Rayes (1993) extended Selinger's work by incorporating cost as a decision variable in the optimization process. Unlike preceding dynamic programming models which were limited to minimization of project duration, their model could evaluate the impact of different project acceleration strategies on the overall project cost.

The application of dynamic programming on repetitive projects in practice requires a mathematician to work with a sophisticated planner to establish the construction project schedule and to revise and update it thereafter. Dynamic programming is usually not taught in universities at the master degree level in construction, hence the availability of such personnel to the construction industry is nonexisting unless an organization hires the services of a specialized researcher. None of the above paper authors mentioned that their methods were tested in practice. This author has never come across a construction project where dynamic programming was used to schedule construction work. Dynamic programming semantics and mathematical notations are too complex to appeal to the construction industry personnel for utilization in construction scheduling. There is no generic system that is applicable and transferrable from project to project that promises to yield attractive cost savings in practice. 


\subsubsection{Simulation}

Simulation is the process of designing a logical model of a real system then experimenting with it by varying the input conditions to estimate the output towards its improvement. It can illustrate deterministic as well as stochastic models (Lutz 1990). Simulation assists in testing important management decision variables before construction takes place (Birrell 1980).

Ashley (1980) contended that the analogy between assembly line and environmentally exposed operations should not be taken too far since deterministic approaches, CPM and LOB, become quite variable when weather and other factors affect them. He explored computer simulation modeling as a valuable stochastic quantitative technique that could enhance management intuition in repetitive-unit construction planning. He argued that his approach allowed a simple conversion of CPM into simulation.

Kavanagh (1985) presented a repetitive construction simulation model called SIREN which is a computer program retaining the precedence network to show the logical relationships between repetitive and non-repetitive activities. It models work squads queuing to carry out the activities instead of doing a critical path analysis. The logic of the precedence diagram is always followed and then squads que to carry out the activities that are ready to be started. The program has the ability to incorporate inclement weather days, learning curves, plant utilization and a monte-Carlo simulation. It also can apply the resource to only part of the activity duration.

However, a new program must be written for each new network or new project. Each activity must be executed by only one squad, like a concrete slab must be resource loaded by either a wooden shutter or a squad of craftsmen, not both. It can not change squad sizes (resource availability) during the project. And most importantly, no dependencies between activities in different units is allowed, however, the start of a typical unit may be dependent on one or more activities in the preceding unit by a pure logical relationship. Kavanagh stated that eliminating this restriction would make the model intolerably complicated. Of course this makes the program unsuitable for accommodating frequent changes in the sequence of progress. This disadvantage diminishes in the type of example presented by Kavanagh: a high-rise building where the order of starts of work on the floors has to follow a definite sequence from bottom to top. But in housing projects, frequently the order or sequence of starts of work on the typical units changes and can often be made in a variety of locations. 
Chang and Carr (1987) presented RESQUE (Resource Oriented Simulation System for Multiple Resource Constrained Processes). It was somewhat complex and required significant training to achieve the desired results (McCahill and Bernold 1993).

Lutz (1990) and, Lutz and Halpin (1992) presented a methodology called SIMLOB, which involved the application of simulation in a LOB context. Halpin (1976) was echoed in that linear production rates (constant slope) of the LOB might be erroneous due to the stochastic nature of the construction process. A computer program by D. Halpin called MicroCYCLONE was enhanced and applied on several case studies. Individual process production curves were plotted. Field application and validation of the program and enhancement to simulate large project networks were recommended.

McCahill and Bernold (1993) stated that there was a need for a practical simulation model before the methodology can be moved from the realm of research to regular use by practicing construction managers. In their opinion, which they mentioned was shared by recognized leaders in the field, shortcomings were: inability to respond to changing site conditions and to produce understandable results to the field personnel.

\subsubsection{Queuing Theory}

Queuing theory is an analytical technique that formulate solutions to problems involving waiting and delay in serving people or servicing machines. It has the advantage of addressing stochastic input and providing optimum solutions. Queuing can be coupled with simulation as in the case of subjecting the data to a Monte Carlo computation. The arrival of trucks at a loader to be loaded with dirt is a classical example of a queuing situation in construction.

Birrel (1980) considered CPM a failure and strongly advocated utilizing the queuing theory in repetitive construction. He presented his model in a matrix very similar to Location-time diagrams and containing diagonal paths of work squads. He mentioned that David B. Ashley carried out simulation based on his concepts and the results were positive and realistic.

The analysis of queuing problems with uncertainty in arrival or servicing time or both can become exceedingly complicated (Humphries 1991). Some limitations of queuing include its simplifying assumptions (e.g., homogeneous resources, exponential distributions, etc.) and the difficulty when formulating models for large processes with complex resource flows (Lutz 1990). 


\subsubsection{Artificial Intelligence}

Artificial Intelligence (AI) are computer based systems that attempt to solve problems by trying to imitate the reasoning process of a human brain thereby minimize user's interaction. There are two main AI systems: Knowledge-Based Expert Systems and Neural Networks.

\subsubsection{Knowledge-Based Expert Systems}

Knowledge-Based Expert Systems (ES) are software that solve problems that normally require human expertise. They can assist decision making by asking relevant questions, making suggestions and explaining the reasons for adopting certain action. They utilize pre-elicited information or know-how and preserve it. However, they cannot come up with new and novel solutions to problems because they merely reflect what the programmer has put into them in the form of a rule. Hence if a problem cannot be described it cannot be programmed (Laudon and Laudon 1991).

Artificial Intelligence Planners are Expert Systems able to generate automated project plans and schedules and to reason with, and provide explanations from, stored knowledge. Despite the optimism of some researchers (Levit et al. 1988), they are yet to be challenged and fully applied in practice on non-repetitive projects prior to projects with significant repetition and sequence changes and therefore of more sophisticated resource management requirements. Research has shown that they are by far slower than conventional network solutions (see Kartam and Levitt 1990).

Currently the objective of the construction researchers in the AI field is to make AI work in practice, they do not seem to be very concerned yet with making it more economical than the traditional methods. Their focus has been on building a feasible logic for networks based on several reasonable considerations such as: support and geometry, constructibility, safety and resource limitations. Unfortunately, resource-based relationships, not resource leveling, are used to represent resource limitations in high-rise and low rise repetitive construction (Levit et al. 1988; Kartam and Levitt 1990; Echeverry et al. 1991; Morad and Beliveau 1991 and 1994; Shaked and Warszawski 1992 and 1995). Some researchers embedded commercially available planning and scheduling software in their Expert Systems: Moselhi and Nicholas (1990) chose 
Promis and recommended Primavera; and Shaked and Warszawski (1995) chose Microsoft Project. This author thinks that integrating a commercial software is a step in the proper direction towards practical implementations. Morad and Beliveau (1991 and 1994) presented a system that integrated $\mathrm{AI}$ and $\mathrm{CAD}$ to generate $\mathrm{CPM}$ networks thereby visually simulating the construction process. The system has a good potential in improving constructability and visualization, also animation that helps in construction claims.

The relatively long time and effort needed to elicit and set up a knowledge base of an Expert System for a specific construction project renders it infeasible. This is especially true if most of the knowledge will not be transferable to other similar projects.

\subsubsection{Neural Networks}

Neural Networks (NN) are networks consisting of many computer microprocessors that are highly interconnected and can work in parallel, they are called processing elements (PE). Each $\mathrm{PE}$ attempts to emulate the biological neuron. The connections are associated with weights that modify the output signal on each path to become stronger or weaker than other paths. The intelligence is in the hardware in the form of generalized capability to learn. Learning is the process of adjusting the connection weights through thousands of cycles of example observations to make the NN produce the desired output. NNs are relatively simple to develop and modify and require less time and effort than ESs. NNs are suited to applications requiring pattern recognition and learning, recall by association and fault tolerance. NNs are designed to imitate the physical thought process of the biological brain. Unlike Expert Systems NNs do not provide explanations for the conclusions and can interpolate an output for a case even if that particular case was not observed during learning. (Laudon and Laudon 1991; Dawood 1994; Hegazy and Moselhi 1994; Ahuja et al. 1994).

NNs can supplement or complement ESs and can be more acceptable to practicing construction personnel than other non-AI techniques requiring advanced mathematics (Moselhi et al. 1991). 


\subsection{Closing Remarks}

It was found through the literature review that the gap between CPM and LOB was not bridged in a way that encourages practical application in the industry. Researchers had continually indicated the need for enhancing a network-based solution that incorporates the LOB principles and schedules repetitive projects flexibly. They also preferred including some non-repetitive activities.

In the construction industry, almost all large project contracts mandate the use of CPM. This is coupled with an availability of affordable commercial CPM software. Consequently, in this research, it was decided to work within the fundamental principles of CPM and LOB and try to find a solution for combining them that would be immediately applicable in the industry. Expanding methods that needed advanced mathematical approaches for optimization were briefly investigated and excluded because of their current inability to lead to a practical and acceptable solution to the industry.

Primavera Project Planner was selected as a tool to perform the research because it is a popular CPM software, user friendly and available. 


\section{CHAPTER 5}

\section{CURRENT APPLICATIONS IN PLANNING REPETITIVE PROJECTS}

\subsection{Introduction}

This chapter reviews real world applications of planning and scheduling techniques on projects with significant repetition. It is based on the author's personal observations on projects in Kuwait and the United States of America. Other references to projects in other countries were taken from the literature.

\subsection{LOB Applications}

The author had previously applied a method that was based on the American approach to the LOB. A typical network was established for the repetitive units as in Fig. 5.1 and lead times were calculated in a spreadsheet by subtracting the Early Finish of each typical repetitive activity from the completion date of the typical unit. Then the number which should be completed

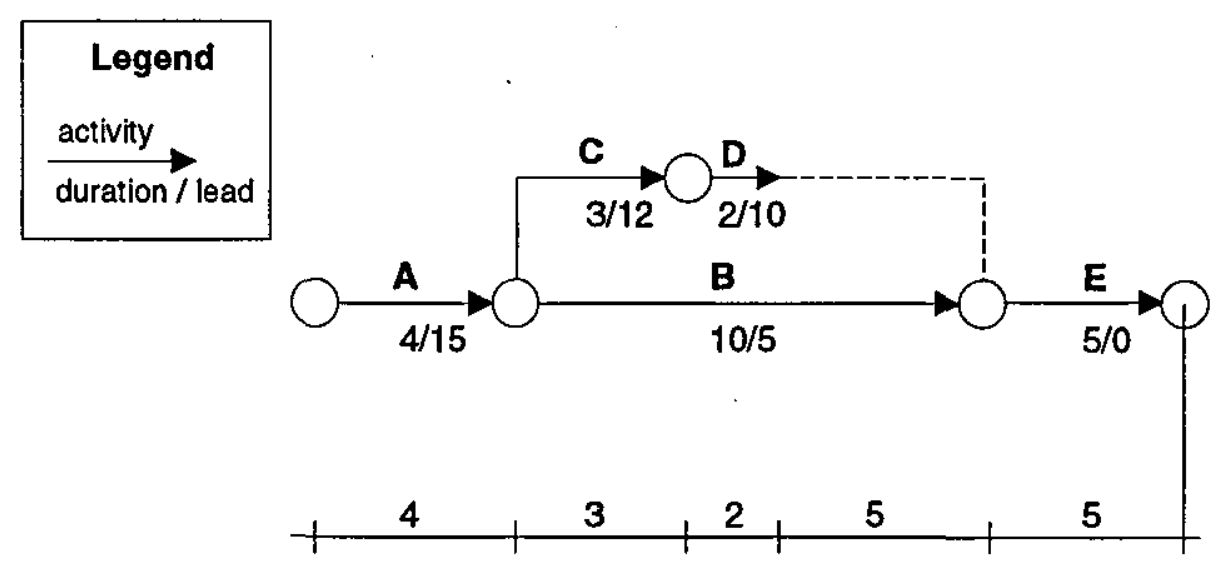

Time

Figure 5.1 - Typical Unit Network Showing Lead Times for Typical Repetitive Activities. 
for each typical activity was read off against the line of completions by going forward in time the lead time of that activity (Fig. 5.2).

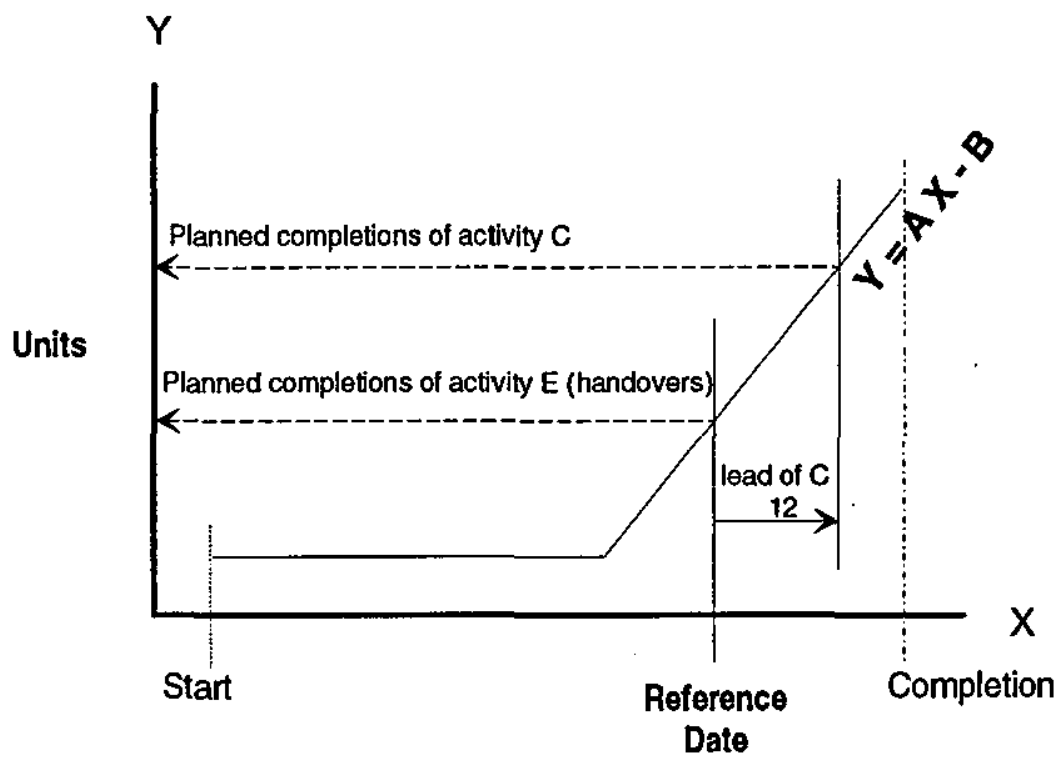

Time

Figure 5.2 - Planned Completions for Typical Repetitive Activities per the American Approach of LOB.

The application was on a 260 house project. A separate straight flow line of planned completions was established for each prime subcontractor and a single network for a typical house was developed and lead times were calculated for each typical activity. Based on the lead times calculated and the algebraic formula of the completion flow line, a program written in BASIC calculated the required number of completions of each typical activity for the end of each projected month. Monthly updates were executed based on the actual number of typical activities completed and a realistic rate of completion for each typical activity was calculated. The BASIC program was soon considered redundant due to the alternative ease in application and presentation a spreadsheet could offer. A sample output of this program is shown in Table 5.1 for 51 houses, part of the project. 
SAUD AND IBRAFIM AL ABDUL, RAZAK EST.

JAHRA HOUSING PROJECT

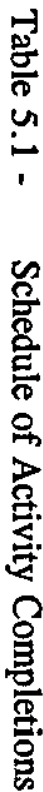

ACTIVITY DESCRIPTION

EXCAVATION \& LAYOUI

PLAIN CONC. FOR FOOTINGS

REINFORCED CONC. FOR FOOTINGS

BACKF COMCRETE FOR GROUND BEAMS

PLAIN CONCRES

Ind BACKFILL \& SLAB ON GRADE

2nd BACKr

GF LOFT WAIS \& SLABS

GF ROOF

FOFT wars a sLaBs

FF ROOF

RENTDUSE COCUMNS a

作

PRECAST WINDOWS ERDCTION

GF \& FF CDMENT BLOCK WORKS

BLOCK WORKS OF PENTHOUSE

DR PARAPEIS

SANDLIME BRICKS CLADDING

BOINDARY WALI SARY PIPES

COATE
UPDATED SCHOULE OF JOB COMPLETIONS

Subcontractor: SEVEN SEAS (51 houses)

Based on actual progress till $31 / 1 / 88$

YARD PLLWBIING PRECAST MANHOLES

DLUMBING PIPES

RICAL INSTALLATIONS:

SANITARY HOUSE CONIDCTION (Infra.)

ROOE DRAINS

MARBEL FRAMES

PLASTERING PREPARATION

ELOCK WORKS OF SHAETS

PLASTERING

PROCELAIN WORKS

PARAPEI COPING \& TANK SUPPORTS

COMON BOUNDARY WALL REIN.CONC.COLUM

XARD ELECTRICAL PIPES \& HANDHOLES

SANTIARY FICANGS (Floor Enbeded

WATERPROFING IN WET FLOR AREAS

ELEC. \& TELE. BOARDS, PANELS \& WIRING

MARBEL STARIRS \& SKIRTINGS

ROOF FOAM \& SCREED
YARD EATTH WORKS \& PLAIN CONCRETE

PRECAST UNITS OF EOUNDARY WALL

ROOF' WATER PROOEING

$\begin{array}{llll} & & & \end{array}$

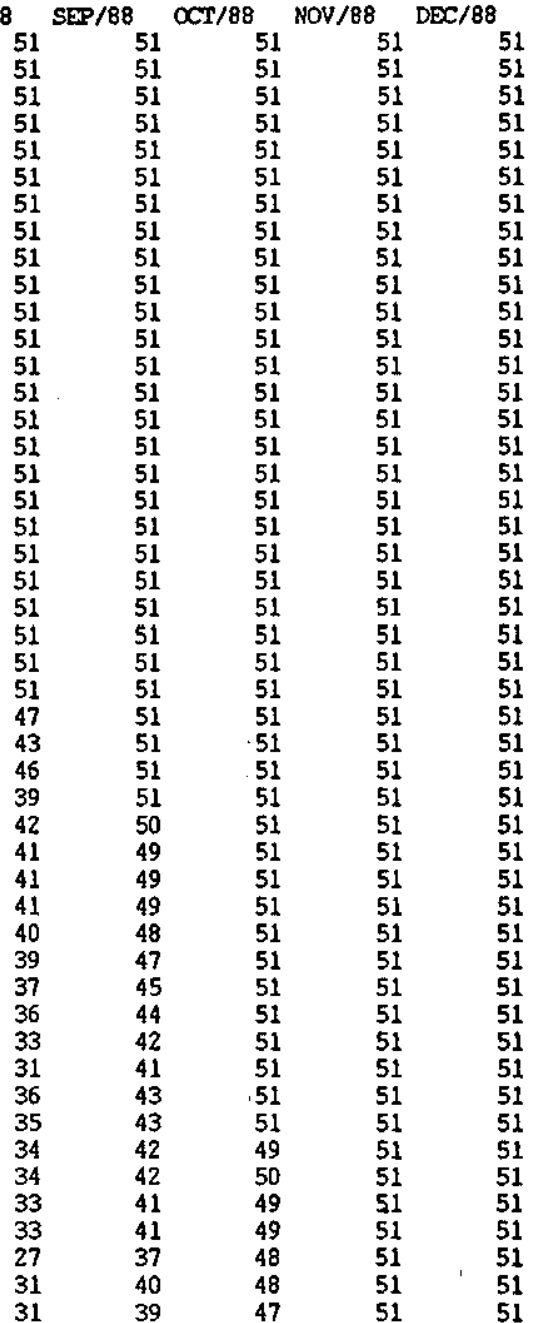


SAUD AND IBRAKIM AL ABDUL RAZAK EST. JAHRA HOUSING PROJECT

$$
260 \text { HOUSES }
$$

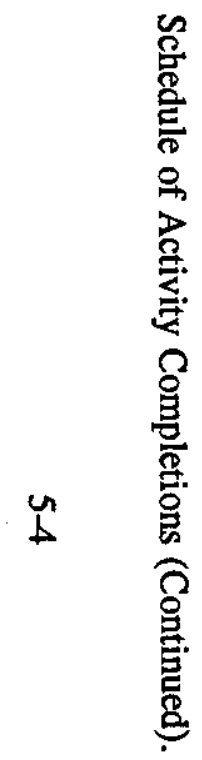

UPDATED SCHDULE OF JOB COMPLETIONS

Subcontractor: SEVEN SEAS (51 houses)

Based on actual progress till $31 / 1 / 98$

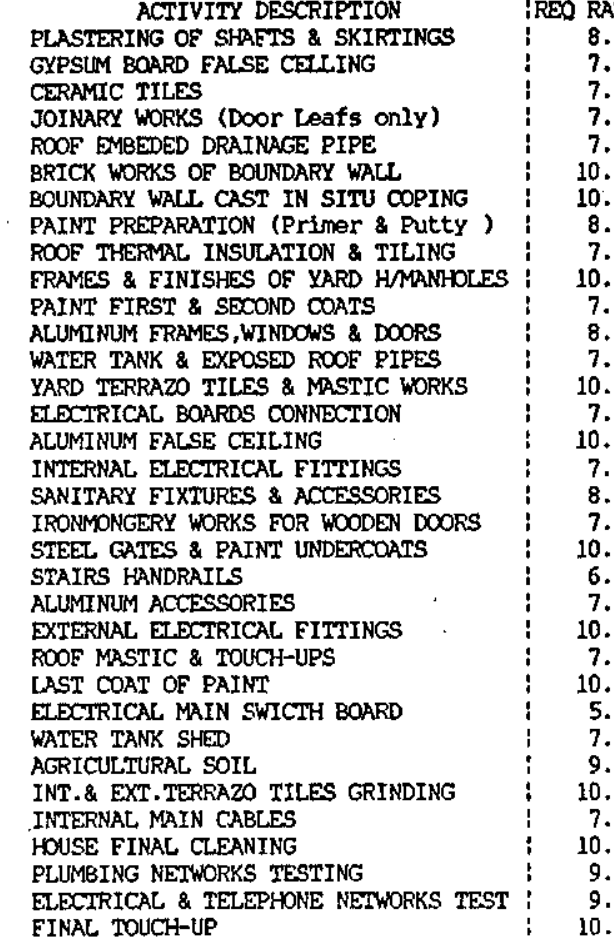

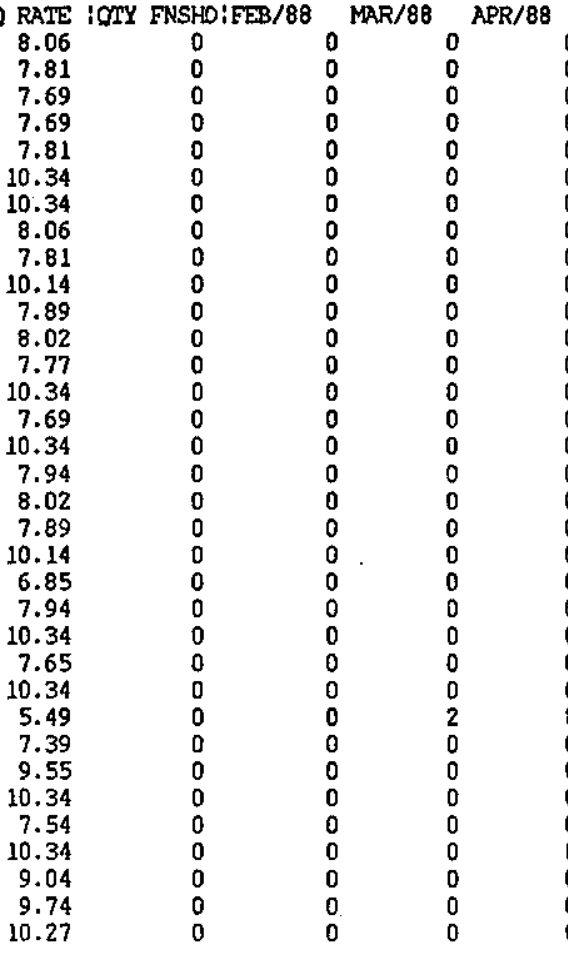

MAY/88
0
0
0
0
0
0
0
0
0
0
0
0
0
0
0
0
0
0
0
0
0
0
0
0
8
0
0
0
0

$\begin{array}{ll}31 & \text { SEP/ } 88 \\ 31 & 39 \\ 32 & 39 \\ 32 & 39 \\ 30 & 39 \\ 23 & 38 \\ 21 & 33 \\ 27 & 31 \\ 27 & 35 \\ 18 & 35 \\ 25 & 28 \\ 24 & 33 \\ 25 & 32 \\ 17 & 33 \\ 25 & 27 \\ 15 & 33 \\ 23 & 25 \\ 22 & 31 \\ 23 & 30 \\ 15 & 31 \\ 27 & 25 \\ 23 & 33 \\ 14 & 31 \\ 23 & 24 \\ 13 & 31 \\ 30 & 23 \\ 23 & 36 \\ 14 & 30 \\ 11 & 24 \\ 22 & 22 \\ 11 & 29 \\ 16 & 21 \\ 13 & 25 \\ 9 & 23 \\ & 19\end{array}$

\begin{tabular}{cccc}
\multicolumn{3}{c}{ OCT/88 } & \multicolumn{2}{c}{ NOV/88 } & \multicolumn{2}{c}{ DEC/88 } \\
39 & 47 & 51 & 51 \\
39 & 47 & 51 & 51 \\
39 & 47 & 51 & 51 \\
39 & 47 & 51 & 51 \\
38 & 46 & 51 & 51 \\
33 & 44 & 51 & 51 \\
31 & 42 & 51 & 51 \\
35 & 43 & 51 & 51 \\
35 & 43 & 51 & 51 \\
28 & 39 & 49 & 51 \\
33 & 41 & 49 & 51 \\
32 & 41 & 49 & 51 \\
33 & 41 & 49 & 51 \\
27 & 38 & 48 & 51 \\
33 & 40 & 48 & 51 \\
25 & 36 & 46 & 51 \\
31 & 39 & 47 & 51 \\
30 & 39. & 47 & 51 \\
31 & 39 & 47 & 51 \\
25 & 35 & 46 & 51 \\
33 & 40 & 47 & 51 \\
31 & 39 & 47 & 51 \\
24 & 35 & 45 & 51 \\
31 & 39 & 46 & 51 \\
33 & 34 & 44 & 51 \\
36 & 41 & 47 & 51 \\
30 & 38 & 45 & 51 \\
24 & 34 & 43 & 51 \\
22 & 32 & 43 & 51 \\
29 & 37 & 45 & 51 \\
21 & 32 & 42 & 51 \\
25 & 34 & 43 & 51 \\
23 & 33 & 43 & 51 \\
19 & 30 & 40 & 51 \\
& & &
\end{tabular} 
The program was a powerful communication tool and was invulnerable to changes in sequence of work between the houses, which were numerous. This was so because it dealt with the completed activities as bare quantities regardless to which house they belonged. The site management and engineers preferred it to CPM, but it had its shortfalls. It could not readily indicate the impact of delays on the completion date of the project. It ignored the number of typical activities that were started but not completed in the calculations of "Quantity finished" and the planned completions.

Interestingly it offered a valuable approach to rectify out-of-logic progress problems. It was found that this approach dealt with out-of-logic progress with the following philosophy: as long as out-of-logic progress happened repeatedly, the violated logical relationships must have been either preferential or wrong, so progress could be corrected gradually by accelerating the rate of the delayed predecessor until it takes over the successor before or by the last unit and the logic is respected again (Fig. 5.3).

Fig. 5.3 shows activity $\mathrm{B} 1$ and $\mathrm{C} 1$ starting one and two days late then running in out-oflogic progress due to the less-than-planned rate of $\mathrm{B}$ and higher-than-planned rate of $\mathrm{C}$. After "time now" B's rate was accelerated and C's was decelerated resulting in out-of-logic progress tapering off.

\subsection{CPM Applications}

Frequently owners specifically demand that contractors submit construction schedules based on computerized CPM. This has been a popular requirement despite the problems CPM application faces in projects with significant repetition.

The CPM-based approach is simply developing a subnetwork for each unit or house and link them together (Fig. 5.4). This assumes that a squad working on a typical activity, for example activity "D", is supposed to move from one house to the other according to the sequence dictated by the management and represented by the dotted relationships. However, the squads will frequently follow another sequence and the need for revising the dotted relationships would be a must to maintain a meaningful and a manageable schedule. A CPM software would not differentiate between logical relationships the way the scheduler would, a violation of any of them is an error even if it was preferential logic like the dotted relationships. The revision of the dotted 


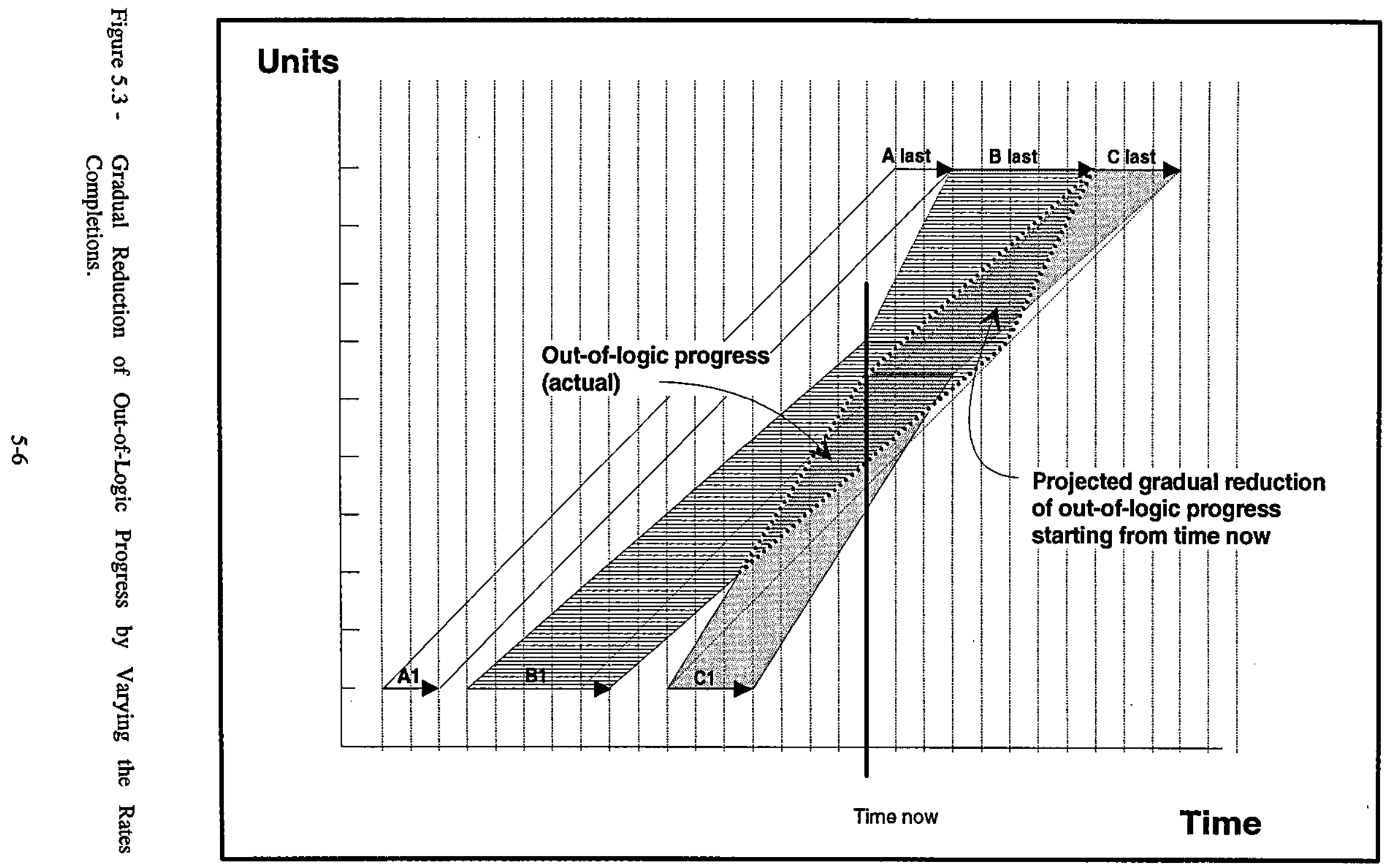

웅 


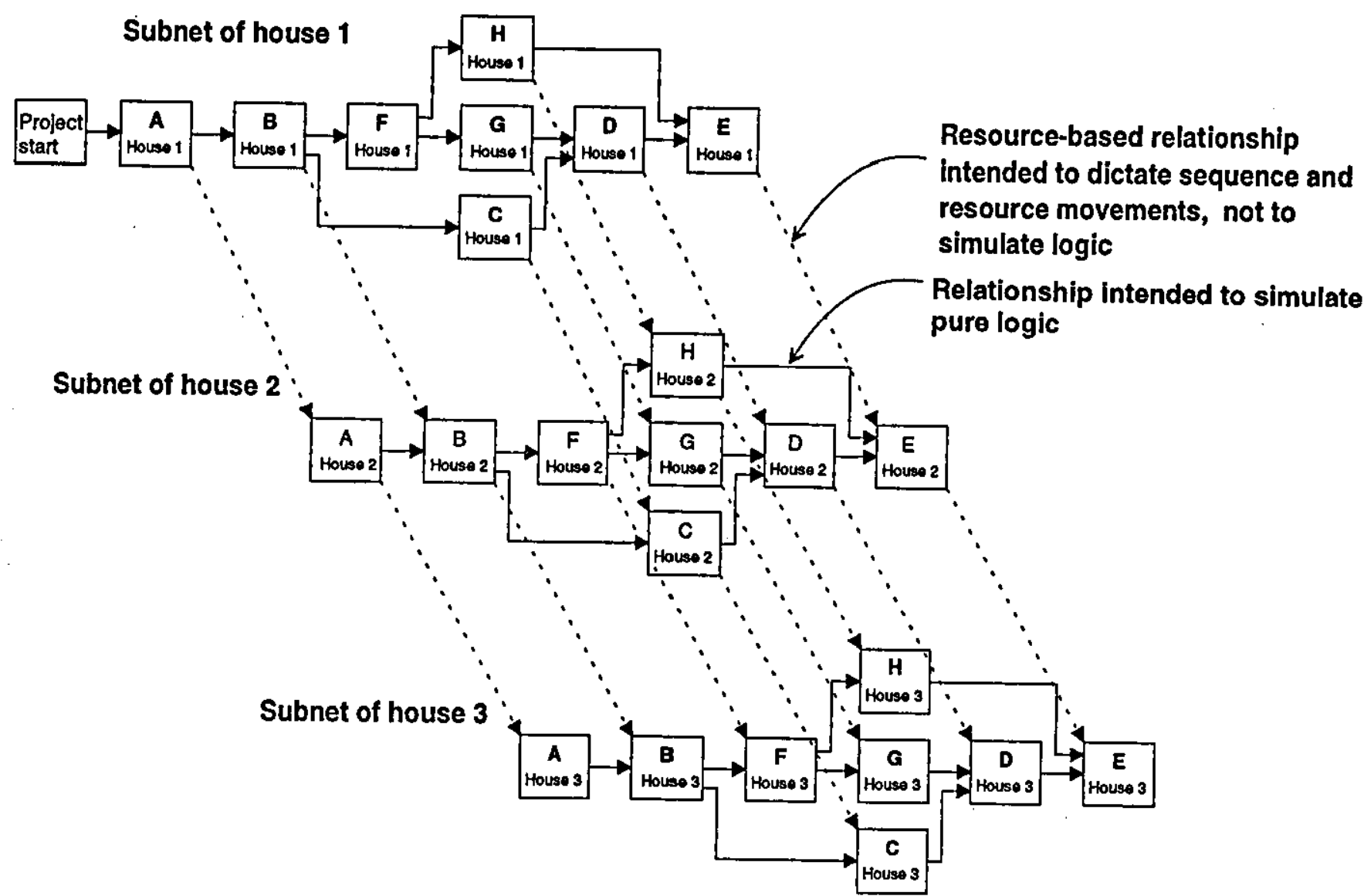


relationships would not be an easy routine on a big project. The pattern of these dotted relationships will be more tedious to correct if the planner realistically deploys more than one squad to work on each typical activity. The author witnessed the application of this approach on several different projects in Kuwait, three prisons, two high-rises, and a hospital in California, USA. The literature shows that the same approach was utilized by Pedersen (1972) on a housing projects in Bergen, Norway. Russell (1993), however, has automated this approach in a computer program called REPCON and applied it in Canada.

To reduce the frequent effort of revising the dotted relationships in the above approach, another approach was adopted. The planner would make a single subnetwork for each cluster of houses, where each cluster represents a number of houses on abutting lots. The number of subnetworks would thereby be reduced considerably, and thus the number of dotted relationships connecting them. Fig. 5.5, shows how the subnetworks representing the clusters were linked to dictate the sequence of operation; for example, cluster I comprises two houses, so typical activity "A, house 1" now becomes "A, cluster I" and summarizing two houses; 1 and 2. The dotted relationships between clusters become start to start relationships with varying lags depending on the number of squads working on that typical activity and the sequence of operation. So if we assume that the variable " $\mathrm{d}$ " is the duration of each typical activity on just one house of a cluster, the dotted relationship between any activity "X, cluster II" and activity "X, cluster III" would be start to start with a lag of $1 \mathrm{~d}$ (SS d) if four squads (a,b,c and e) are working on typical activity " $X$ ". However, if only three squads (a,b and $c$ ) are working on this typical activity " $X$ ", the relationship between activity "X, cluster II" and activity "X, cluster III" would be start to start with a lag of $2 \mathrm{~d}$ (SS 2d).

This approach also needed revising the start to start relationships that connect the typical activities in related clusters each time the sequence of work on clusters changed and each time the quantity of squads working on a typical activity was revised. Additionally, the durations of the typical activities needed revision because they were dependant on the sequence of operation as well as the number of squads. Moreover, out-of-logic progress occurred within the typical subnetworks of the clusters as a result of the very nature of representing typical activities of many houses by one typical activity of a cluster. For example, on a three house cluster, like cluster III in Fig. 5.5, if the concrete roof started on house 7 after the completion of the columns of the same house and before the total completion of the columns of houses 8 and 9 , (assuming sequential relationships) an out-of-logic progress error will take place, since columns of houses 7, 8 and 9 are represented by one CPM activity called "Columns, cluster III" and similarly for 


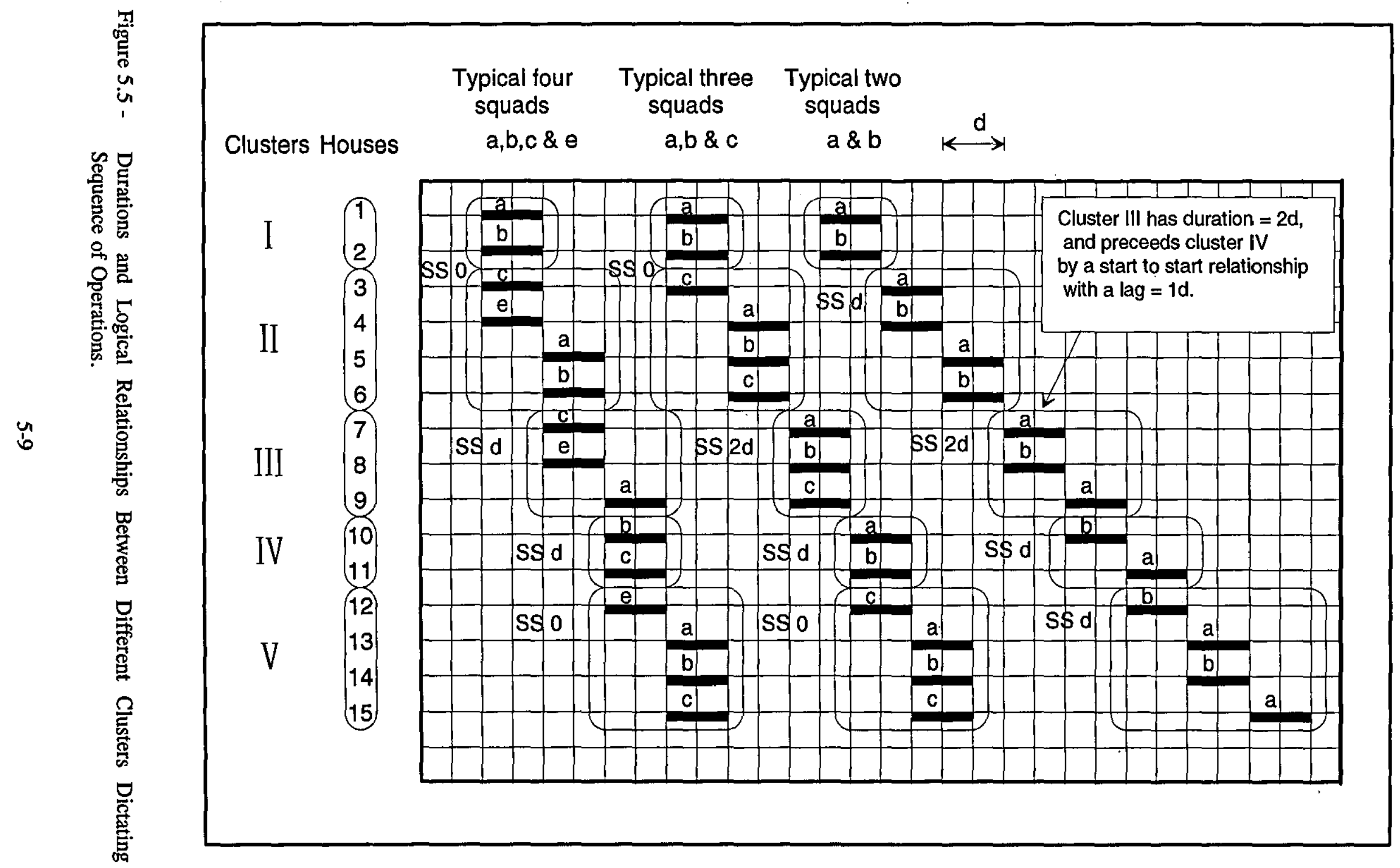


the concrete roof. This out-of-logic progress is not real, it is the result of summarizing too many CPM activities. This author calls it artificial out-of-logic progress.

A minor deviation from the above approach, and rather simplistic, was to represent all the houses of the project by subnetworks of groups that comprise a constant number of houses throughout the schedule no matter how many houses in each cluster were. But, in a cluster of say 6 houses, it would not be organizationally nor technologically convenient to standardize the group size at 4 houses. And since the size of the clusters varied, standardizing the group size was not reasonable.

There was another modification that limited the dotted relationships to interfaces between selected repetitive activities. This was noted in a housing project in Kuwait as well as a prison project in Northern California. The problem with this approach is that while it staggers the linked activities realistically based on the availability of squads it may project an unrealistic and stacked plan for those that are not linked. This stacking of the typical repetitive activities often means a requirement above the available number of squads to perform the work.

It is worth noting that none of the CPM applications mentioned above, calculated the number of squads based on the LOB technique. Surprisingly, the issue of balancing the squads did not seem to attract the attention of the users.

\subsection{Future Expectation}

The current applications in the construction industry are very much influenced by the owners and the construction managers, the typical bodies that put the schedule specifications in the contracts. This is, presumably influenced by the current available level of skill in the industry concerning managers and technicians as well. Any more sophisticated approach than the current approaches will have to consider a compatibility with the available skills. It is unlikely that the construction industry will commit additional resources in training unless it sees a reasonable chance of improving cost savings and performance.

Simplicity in solving the problems associated with planning and scheduling repetitive construction is an extremely important issue if a new methodology is intended for practical application in the industry. 


\section{CHAPTER 6}

\section{CPM/LOB NEW METHODOLOGY}

\subsection{Introduction}

In repetitive construction, there is no doubt that the independent application of the LOB and the CPM did not satisfy their users. Construction veterans downplayed the application of CPM in repetitive-construction projects which are usually large projects and provide wide fronts for resource deployment. This stimulated intensive research over the past decades to combine the merits of both methods. Unfortunately, the application of the LOB almost vanished and the application of CPM failed to respond to the frequent changes in the sequence of operation between the repetitive units and to maintain work continuity for the working squads. As will be demonstrated in this chapter, $\mathrm{CPM} / \mathrm{LOB}$ revives the $\mathrm{LOB}$ and achieves the objective of integrating the merits of the two methods. It centers around resource leveling and the utilization of float times to streamline the scheduling process. The virtue of CPM/LOB lies in its simplicity of application that enables the user to plan and control effectively using CPM, a tool with which he is already familiar.

\subsection{Parallel Scheduling}

In LOB, parallel scheduling is the strategy of compelling all the repetitive activities of a project to progress at a single rate of completion. The purposes are, ease of planning in the office and reduced overall project duration. However, when that single rate of completion denies the natural rhythm of some repetitive activities, their squads shall be forced to encounter waiting times. On the other extreme side, adhering to the natural rhythm of each repetitive activity may save direct costs but may also push the project completion date into the future thereby raising indirect costs.

It is a fact that construction projects rarely follow their initial schedules which sometimes project plans far ahead in the future. Nowadays, delays and, to a lower extent, accelerations, affect almost every project with varying degrees. Delays affect the repetitive activities and disrupt the natural rhythm when either start or end buffers between repetitive activities are exceeded. It is therefore realistic and probably safer to start with parallel scheduling and leave contingent time towards the end of a project to accommodate the inevitable changes as they unfold. Float times 
whether existing in the original plan or generated due to later on delays offer an opportunity in parallel scheduling that should not be missed to relax the rate of completions of some repetitive activities and possibly satisfy their natural rhythm without further delay to the project.

\subsection{Float Utilization Concept}
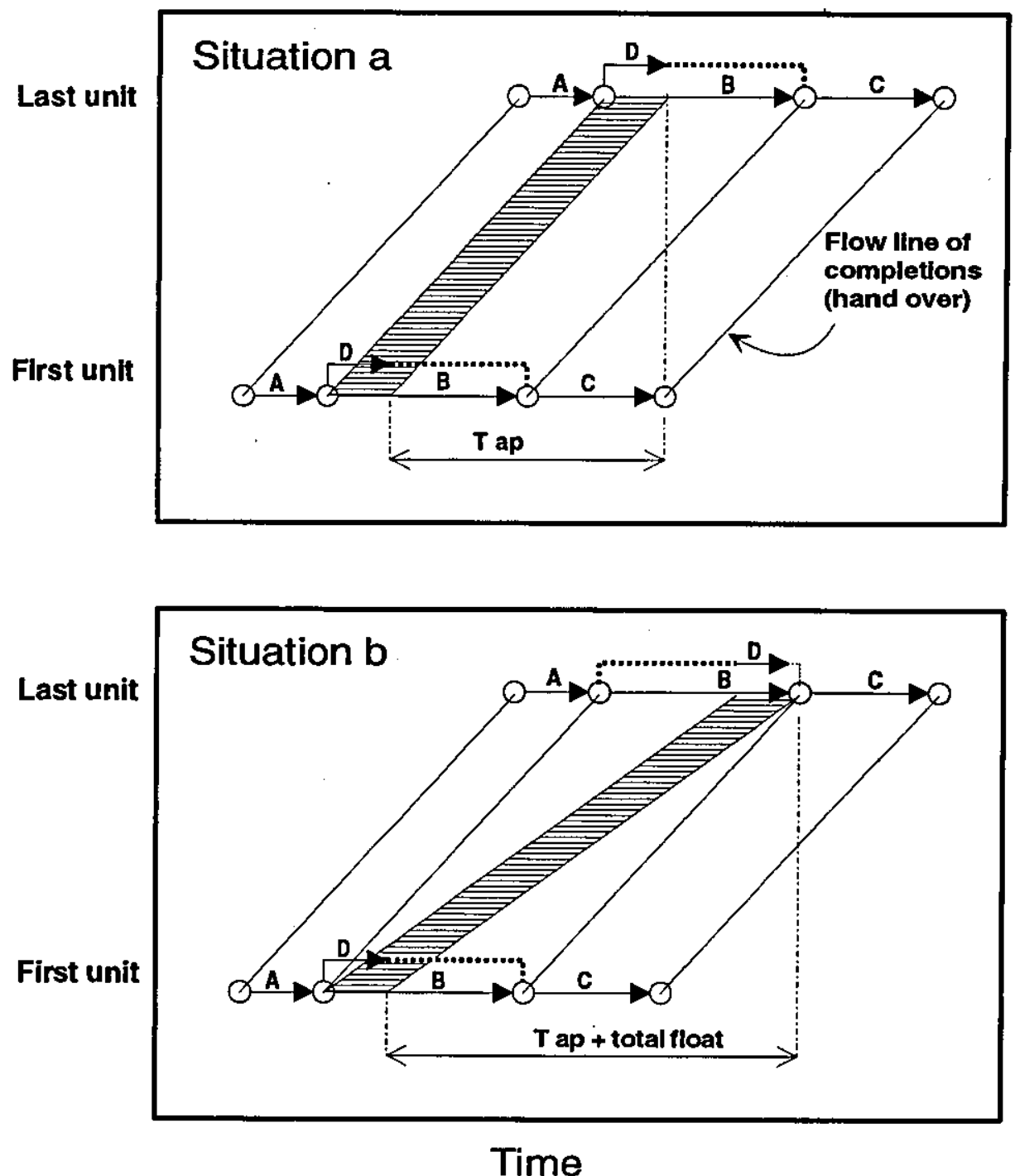

Figure 6.1 - Utilization of Float in Parallel Scheduling of LOB.

Fig. 6.1, situation a, shows a LOB diagram of a sample housing project, where each typical housing unit has four typical activities A, B, C and D. The typical network has activities $B$ and $D$ running in parallel. Activity $D$ has some float time that is represented by the dotted thick line. For clarity, only the first and last typical networks of the houses are shown. 
Each of these typical activities in the diagram has the same rate of completion, which if reduced will delay the completion of the whole project. However, typical activity D may utilize its float without delaying the project completion. Fig. 6.1 situation b, clearly shows that. Within a parallel scheduling environment, the progress of a typical activity with float maybe adjusted towards its natural rhythm through using that float without delaying the project.

\subsection{The Methodology of CPM/LOB}

The objective of this methodology is to overcome the main challenges in CPM scheduling of repetitive construction projects; changes in sequence, and the lack of control on the continuity of work through a practical combination of CPM and LOB in the already developed CPM context.

The fundamental principles of this methodology were first developed by the author on a large and troubled housing project in Kuwait in 1987 after being confronted on the field with the above mentioned challenges.

Traditionally, on a jobsite of a housing project, the main question on the mind of a project manager, is how many squads of a particular trade or sets of roof shutters, etc. are adequate to complete the project on time. Therefore, a planner must consider building up a schedule responsive to this need; in other words, making his schedule resource oriented. This understanding should encourage the scheduler to use resource leveling along with time scheduling.

In Fig. 6.1 situation a, each of the typical activities in the diagram has the same amount of time from the first unit completion to the last unit completion. This amount of time is called the available time for project completions, $T_{a p}$. Also the rate of completions, $R$, is the same for all the typical activities and is equal to

$$
R=\frac{\text { Number of units }-1}{T_{a p}}
$$

The quantity of squads required to complete each typical activity on time, $S$, is then

$$
S=R \times \text { Duration of squad work on activity }
$$

$S \leq$ Total number of units 
Here, a squad can be a combination of craftsmen working on one particular typical activity (such as typical activity D) throughout the project for a certain duration that needs not be identical to the duration of the activity. For example: on a concrete roof that has a planned activity duration of 12 days, there can be three squads working on it; carpenters, working for 11 days, iron workers, working for 4 days and concreters, working for 1 day. The carpenters' squad may comprise 2 carpenters and 4 laborers and it is considered just one squad. Similarly, the other two squads, iron workers and concreters, may comprise a combination of craftsmen.

If all the front events of the typical networks of the houses are linked to a start milestone activity such as, Project Start, and likewise the end events to activity Project Completion, all these networks will become subnetworks and can float between the Project Start and Project Completion activities. Since the quantity of the required squads for each typical activity can be calculated from the previous equations, these subnetworks can be staggered realistically by performing resource leveling of the different squads. The intended sequence of operation from one unit to the other on site, can be dictated by a simple rule in resource leveling; a sequential priority code can be assigned to each typical activity that maintains the allocation of the required squads according to the intended sequence of operation.

However, as mentioned in the previous section, some activities may have some float that can be utilized to adjust their rate of completions and relax the demand on their squads. Typical activity D, in Fig. 6.1 has a certain duration of float. If this float, is totally utilized, the progress of activity $\mathrm{D}$ will take the shape of the shaded parallelogram shown in situation $b$ without affecting any other activity in any unit. Therefore, the duration from the completion of activity $D$ on the first unit to the completion of the same typical activity on the last unit, $T_{a p}$, can be increased by up to the total float of that typical activity calculated from its original typical unit network. Thus, a more general equation for calculating the available time for completions for each typical activity, $\mathrm{T}_{\mathrm{aa}}$, becomes

$$
T_{a a}=T_{a p}+\text { total float of the typical activity }
$$

Consequently, the minimum rate of completions of a typical activity required to complete the project on time, $\mathbf{R}_{\mathrm{ma}}$, is 


$$
R_{m a}=\frac{\text { Number of units }-1}{T_{a a}}
$$

Activity $D$ may progress, in any rate of completion between $R$ and $R_{m a}$, which is anywhere between situation a and situation $b$. The other activities that have no float in the typical unit network, must progress at a rate $R$ that is equal to $R_{\mathrm{ma}}$ in their case.

Fig. 6.2 details the steps to develop the initial or the as-planned schedule in CPM/LOB.

Clearly, any change in the sequence of operation between the typical units can be accommodated by simply revising the values of the priority codes of the affected activities. This process is easier than revising the logical relationships to dictate a new sequence of operation. Also, work continuity for each squad is maintained to a practical extent through resource leveling of the squads that imposes such rates of completions that do not cut in on other activities.

\subsection{Example 6.1, Developing the Initial Schedule}

Fig. 6.3 shows a LOB diagram for planning the execution of 10 units in 38 days. Each unit is represented by one typical subnetwork that contains 8 activities and needs 27 days to be completed. Only the subnetworks of the first and tenth units are shown for clarity. All the subnetworks start from event 11 and end by event 17 . There are no logical relationships between the typical activities of the subnetworks. The inclined lines represent the flow lines of activity completions.

The available time for project completions, $\mathrm{T}_{\mathrm{ap}}$, is therefore

$$
\begin{aligned}
& T_{a p}=\text { target completion date of project }-E F \text { date of } 1 \text { st unit } \\
& \mathrm{T}_{\mathrm{ap}}=38-27=11 \text { days }
\end{aligned}
$$

Scheduling a typical unit network will produce the following total float durations; activities F \& G, 7 days; activity H, 12 days; A, B, C, D \& E, no float. The minimum quantity of squads to complete the project in 38 days can be calculated using equations $6.4,6.5$, and 6.2 and as shown in Table 6.1. 


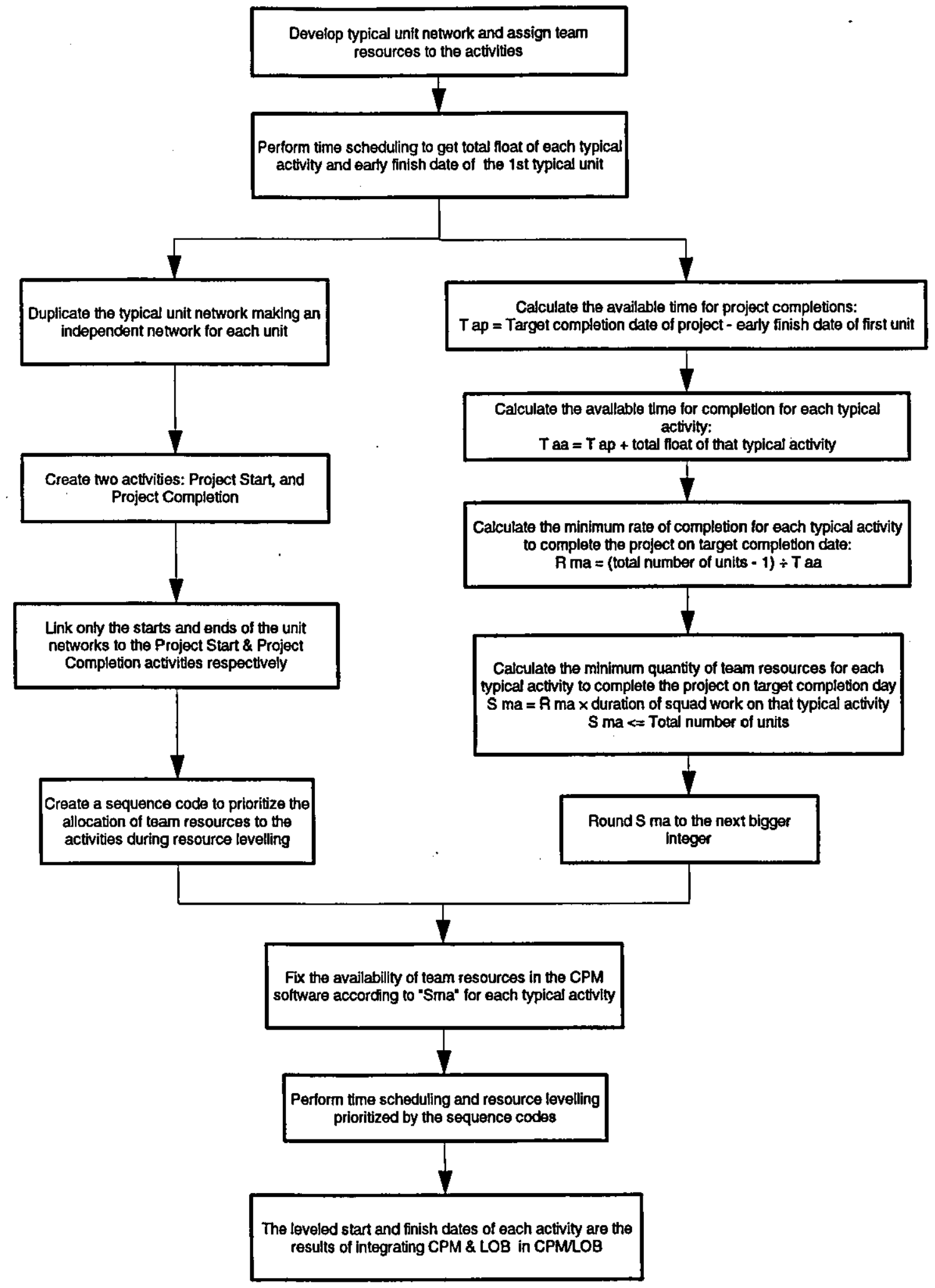

Figure 6.2 - Steps of Developing a CPM/LOB Schedule. 


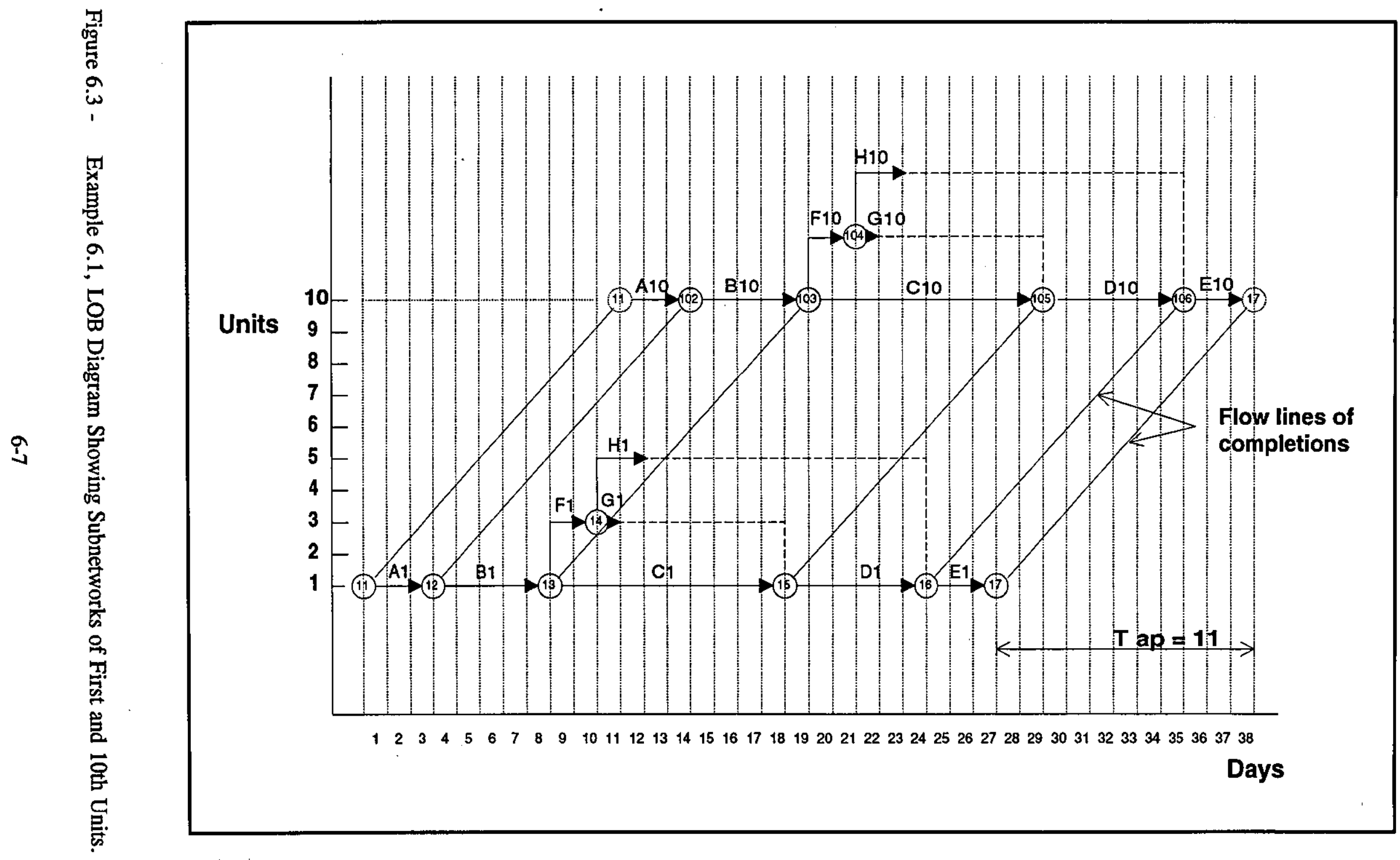




\begin{tabular}{|c|c|c|c|c|c|c|}
\hline $\begin{array}{l}\text { Typical } \\
\text { activity }\end{array}$ & $\begin{array}{c}\begin{array}{c}\text { Resource } \\
\text { duration }\end{array} \\
\text { D } \\
\text { (2) }\end{array}$ & $\begin{array}{r}\text { Total } \\
\text { float } \\
\text { TF } \\
\text { (3) } \\
\end{array}$ & $\begin{array}{c}\text { Available } \\
\text { time for } \\
\text { completions } \\
T_{\mathrm{a}}= \\
\left\{\mathrm{T}_{\mathrm{ap}}+\mathrm{TF}\right\} \\
(4) \\
\end{array}$ & $\begin{array}{c}\text { Minimum rate } \\
\text { of completions } \\
\mathrm{R}_{\mathrm{ma}}= \\
\left\{(10-1) \div \mathrm{T}_{\mathrm{w}}\right\} \\
(5)\end{array}$ & $\begin{array}{c}\text { Minimum } \\
\text { quantity of } \\
\text { squads } \\
\mathrm{S}_{\mathrm{max}}= \\
\left\{\mathrm{D} \times \mathrm{R}_{\mathrm{max}}\right\} \\
(\text { () } \\
\end{array}$ & $\begin{array}{l}\text { Rounded } \\
\mathbf{S}_{\mathrm{ma}} \\
\quad(7) \\
\end{array}$ \\
\hline A & 3 & 0 & 11 & 0.818 & 2.454 & 3 \\
\hline B & 5 & 0 & 11 & 0.818 & 4.090 & 5 \\
\hline C & 10 & 0 & 11 & 0.818 & 8.180 & 9 \\
\hline D & 6 & 0 & 11 & 0.818 & 4.908 & 5 \\
\hline E & 3 & 0 & 11 & 0.818 & 2.450 & 3 \\
\hline $\mathbf{F}$ & 2 & 7 & 18 & 0.500 & 1.000 & 1 \\
\hline $\mathrm{G}$ & 1 & 7 & 18 & 0.500 & 0.500 & 1 \\
\hline $\mathrm{H}$ & 2 & 12 & 23 & 0.391 & 0.782 & 1 \\
\hline
\end{tabular}

Table 6.1 - Example 6.1, Calculation of the Quantity of Squads.

Figs. 6.4 and 6.5 show, symbolically, how the total floats of the noncritical activities were used to spread the work over a longer period and reduce the demand on the quantity of squads needed. The quantity of squads listed on the last column of Table 6.1 can now be entered into the CPM software as resource availability. A priority code should now be assigned to each activity to impose the sequence or order of operation from one unit to the following one. In this example, the work is planned to proceed on the units in the order: $1,2,3,4,5,6,7,8,9$ and 10. This means that typical activity $A$ of unit $1, A 01$, will have a priority code of 1 and activity A 02 priority 2 and so on until the last unit, and similarly for the other typical activities. Upon performing time scheduling and resource leveling prioritized by the priority codes, the results are immediately obtained as in Fig. 6.6. The bars represent the early work and the dashed lines represent the late work. The difference between them is the total float. The total float gives a measure of both the time and the resource criticality of the activities.

Fig. 6.7, shows a more realistic version than Figs. 6.3-6.5 of the activity completions flow lines. This is derived from the CPM software. Note that due to the nongradual (stepline) starts of the squads on the typical activities in contrast with the way it is traditionally shown on a LOB diagram (straight line), an early completion may be realized. In this example, the full utilization of the squad working on typical activity $F$ could not have been less gradual and 


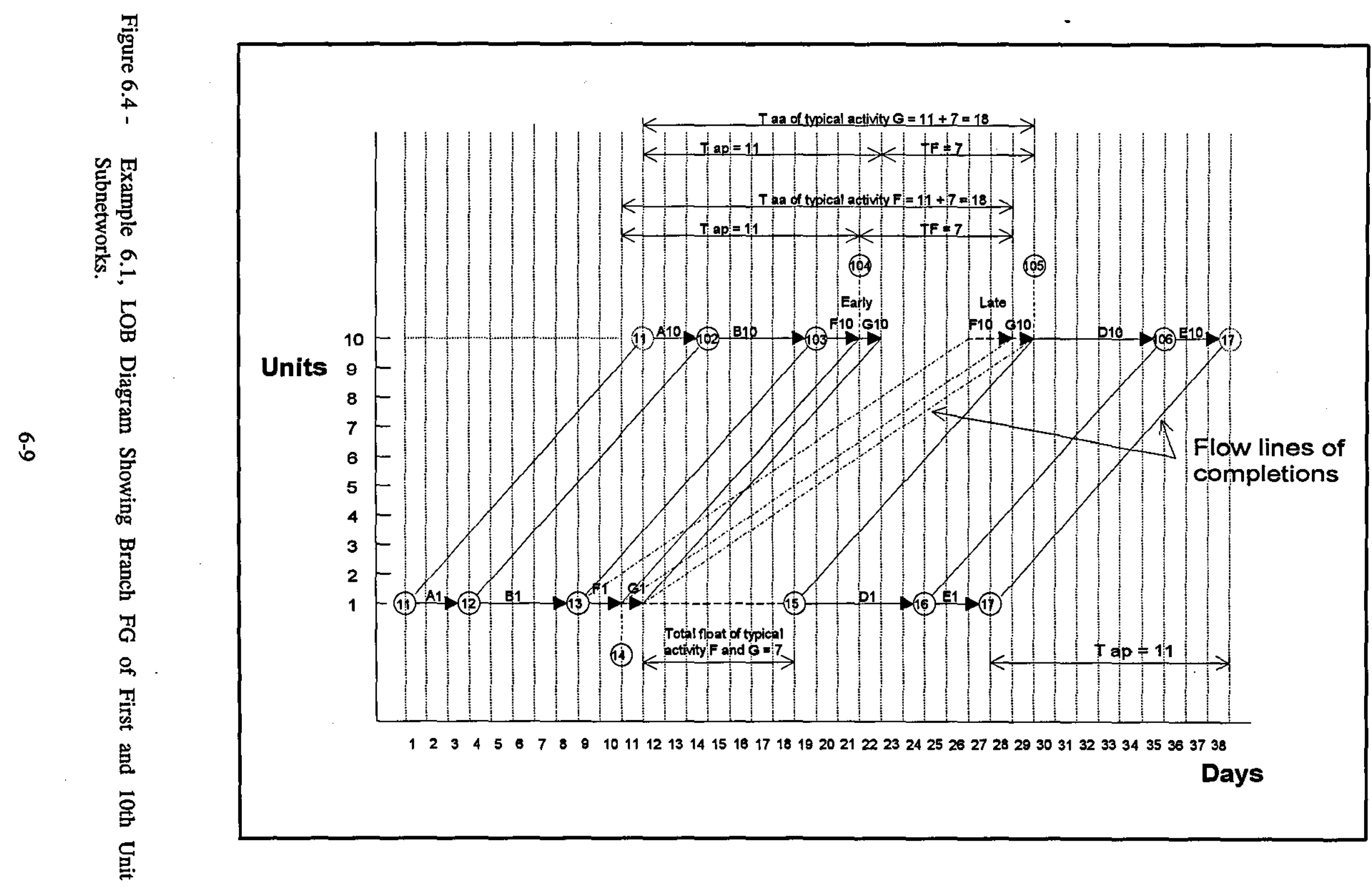




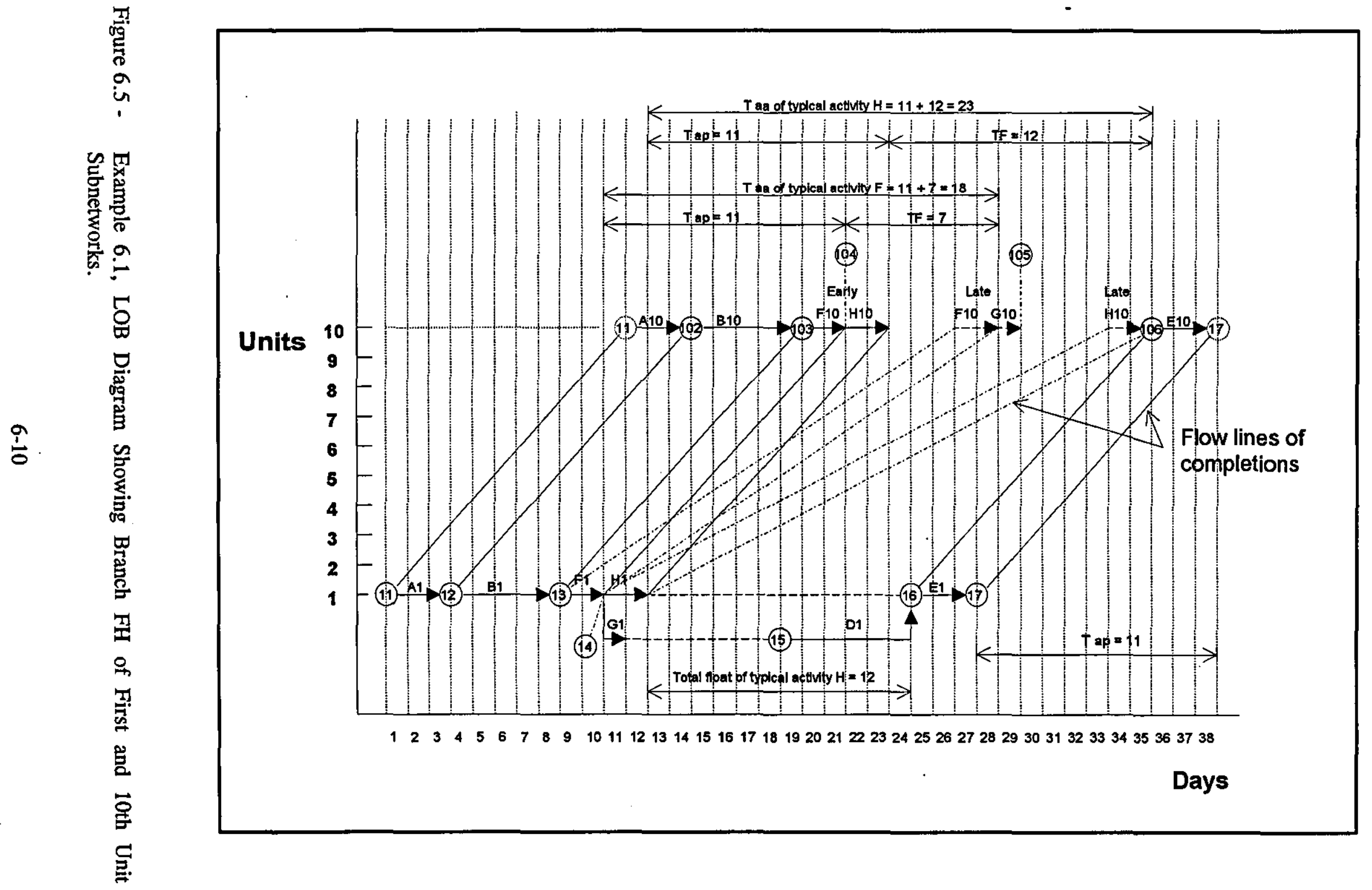




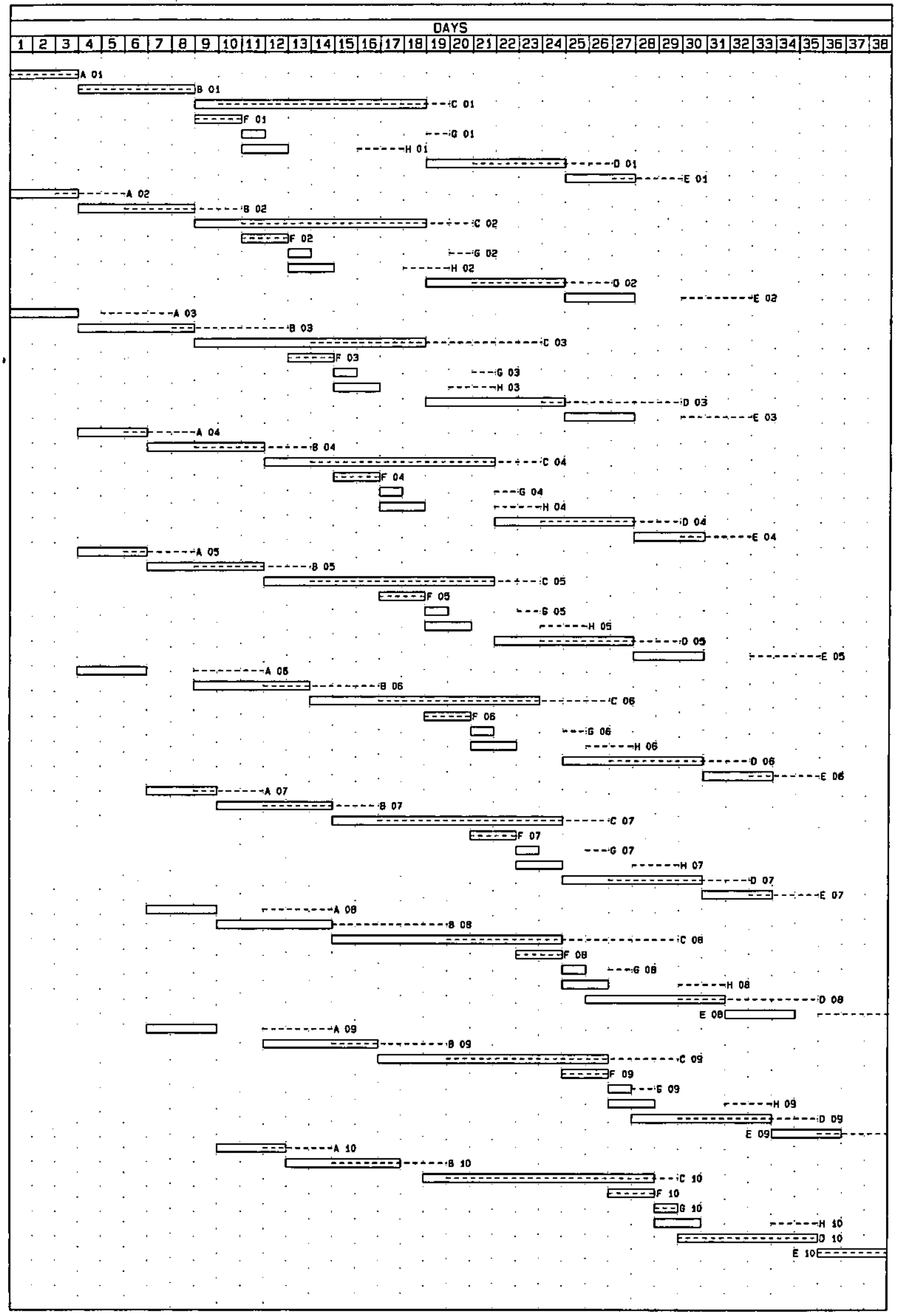

Figure 6.6 - Example 6.1, Bar Chart. 


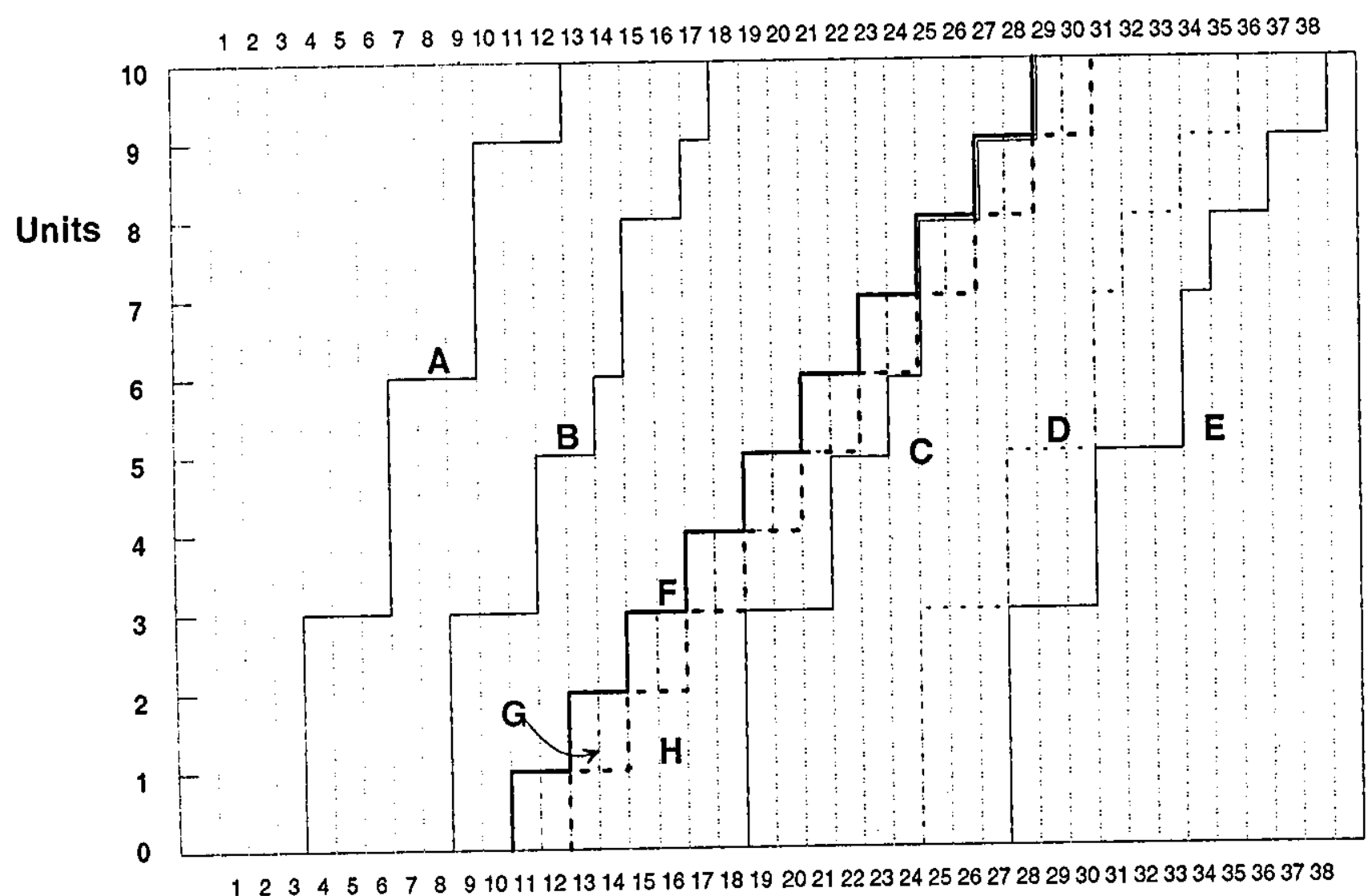

Time 
therefore typical activity $F$ paced the project and made it complete precisely on day 38 and not earlier.

Note that if the total floats of typical activities $\mathrm{F}$ and $\mathrm{H}$ were not utilized, a higher and unnecessary resource requirement would be demanded, which, if provided would not make the project complete earlier

$$
\begin{aligned}
& T_{2 a} \text { of activities } F \text { and } H=11 \text { days }+0 \text { total float } \\
& \mathrm{R}_{\mathrm{ma}}=(10-1) \div 11=0.818 \text { units } / \text { day } \\
& \mathrm{S}_{\mathrm{ma}}=0.818 \times 2=1.636 \text { squads }
\end{aligned}
$$

and

$$
S_{\mathrm{ma}} \text { (rounded) }=2 \text { squads, instead of one squad previously calculated }
$$

To demonstrate how easy it is to change the sequence of operation, assume that the project manager decides to put house 9 third in order and house 3 ninth in order. The scheduler can accordingly revise the priority codes of the activities of houses 3 and 9 , and perform resource leveling thereafter. The process of revising the sequence and getting the output in Fig. 6.8, can be as quick as the computer and the CPM software. It requires minimal and negligible effort from the scheduler.

Fig. 6.9, shows the lines of balance for an arbitrary point in time, end of day 25. Clearly, for each typical activity the difference between the levels of the solid line and the dotted line represents the allowable quantity of completions beyond which the completion date of the project will be delayed. 


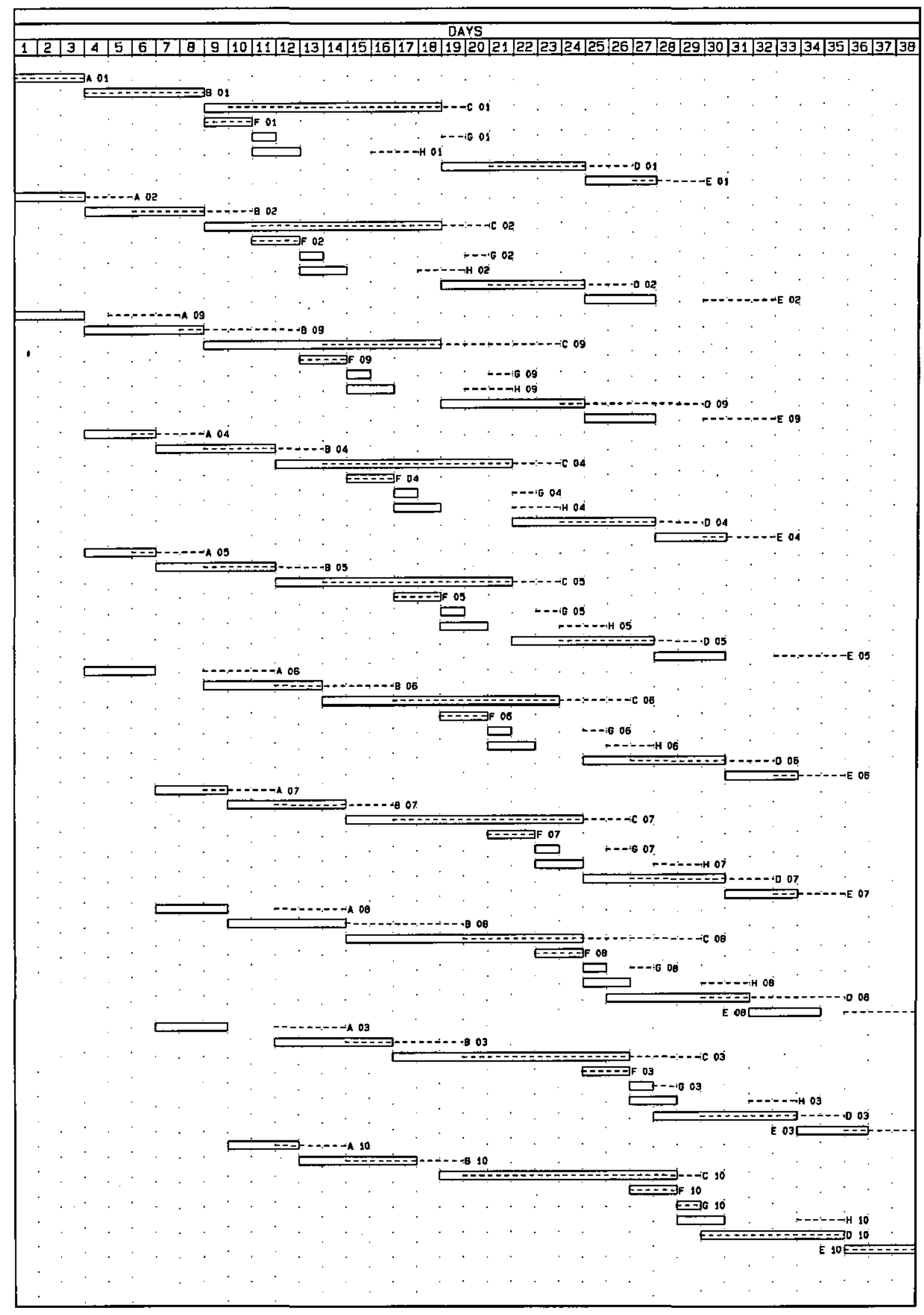

Figure 6.8 - Example 6.1, Bar Chart-Revised Sequence. 


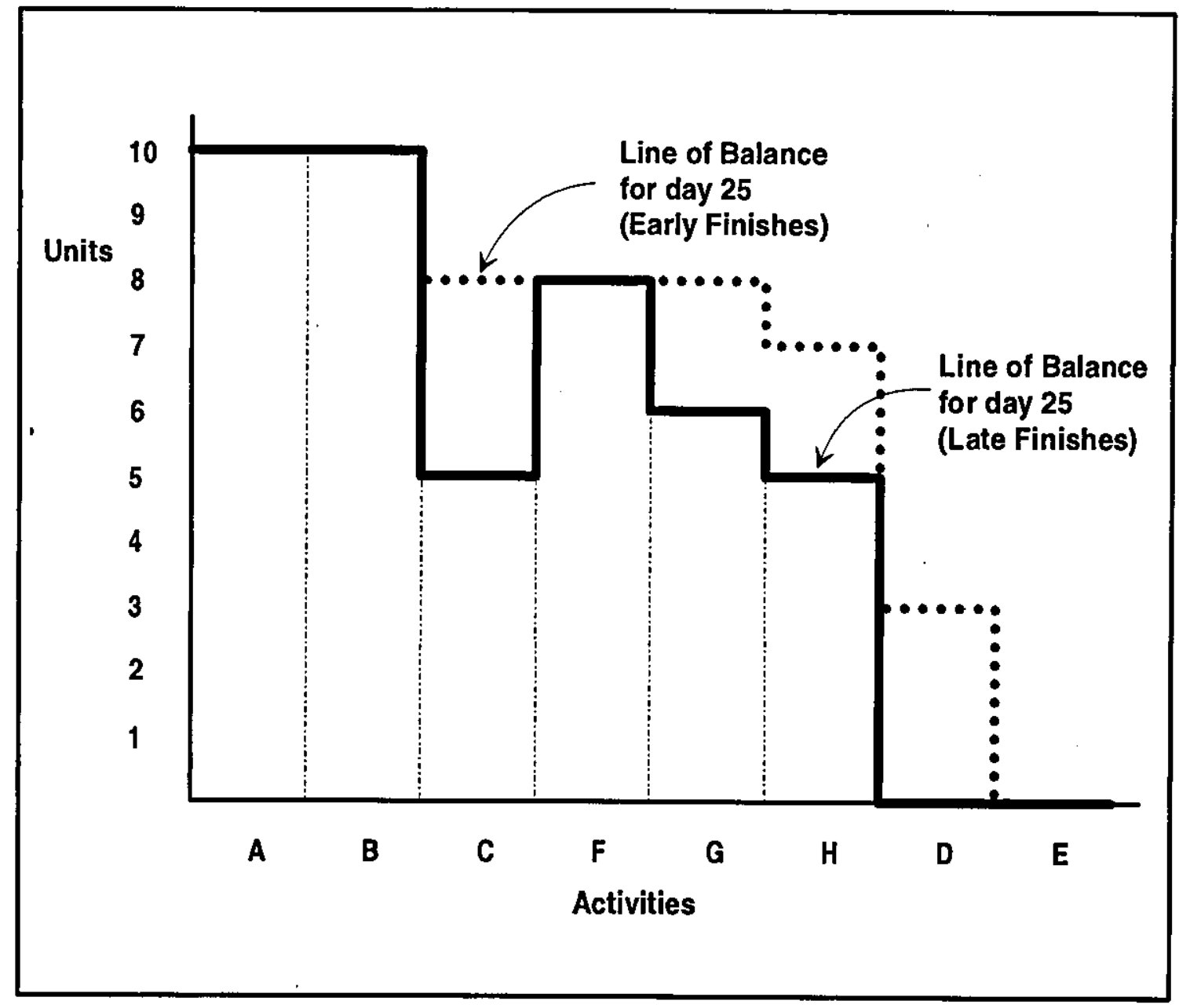

Figure 6.9 - Example 6.1, CPM/LOB Chart for Day 25. 


\subsection{Example 6.2, Updating and Accelerating by CPM/LOB}

This is based on the ten unit project of example 6.1. The assumption is that it is now end of day 10 and the CPM is showing the project forecast completion two days behind schedule. The objective is recovering the delay and exchanging the sequence of unit 4 and unit 7 due to a certain problem on site.

Fig. 6.10 shows the actual progress until end of day 10 and the forecast dates of the future progress based on the same quantity of squads allocated in example 6.1. The calculation of this updated schedule in Fig. 6.10, is obtained after entering the assumed actual dates and progress percentages on example 6.1, revising the sequence, performing time scheduling, and resource leveling the squads. The quantities of squads are kept unchanged from the beginning of the project according to example 6.1. The current forecast completion is day 40 which is two days behind the initial target completion, day 38.

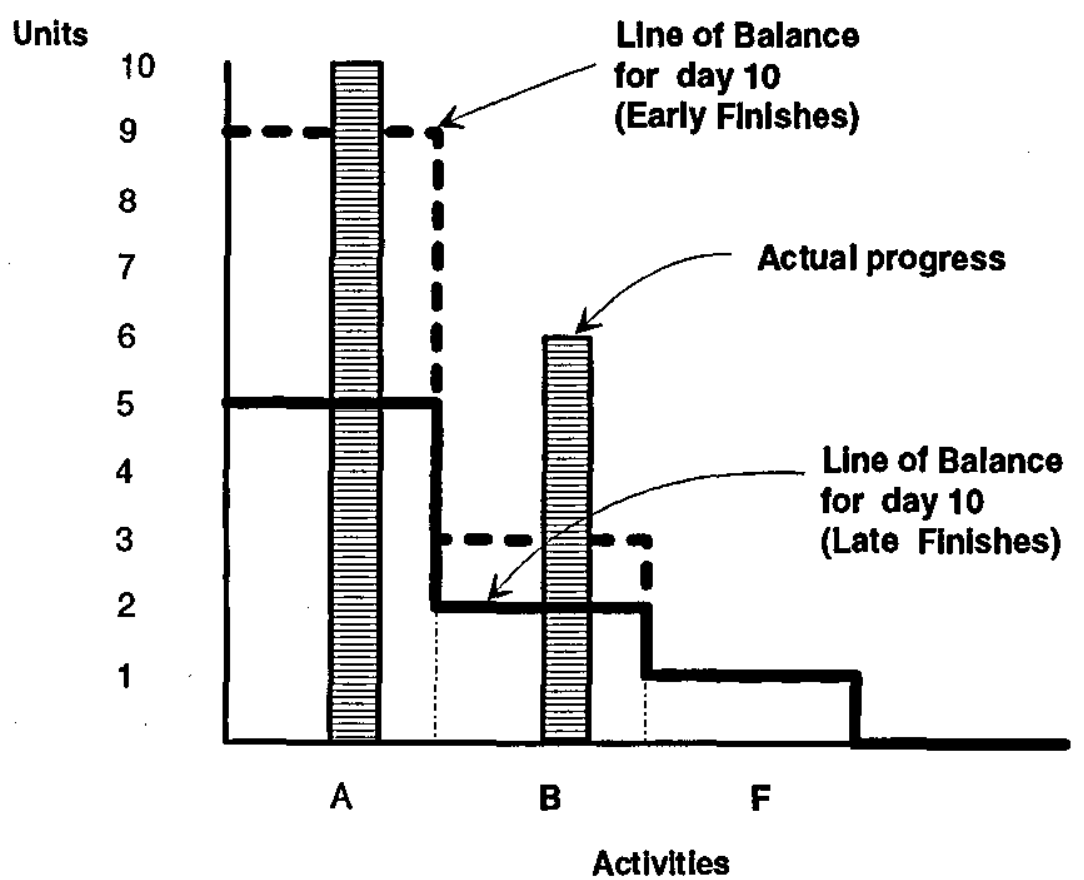

Figure 6.11 - Example 6.2, CPM/LOB Chart at End of Day 10 Showing Imbalance.

Fig. 6.11 shows the CPM/LOB chart at the end of day 10. Clearly the progress of the project is unbalanced. While typical activities A and B are ahead of the early schedule, typical activity $F$ is behind the late schedule. 


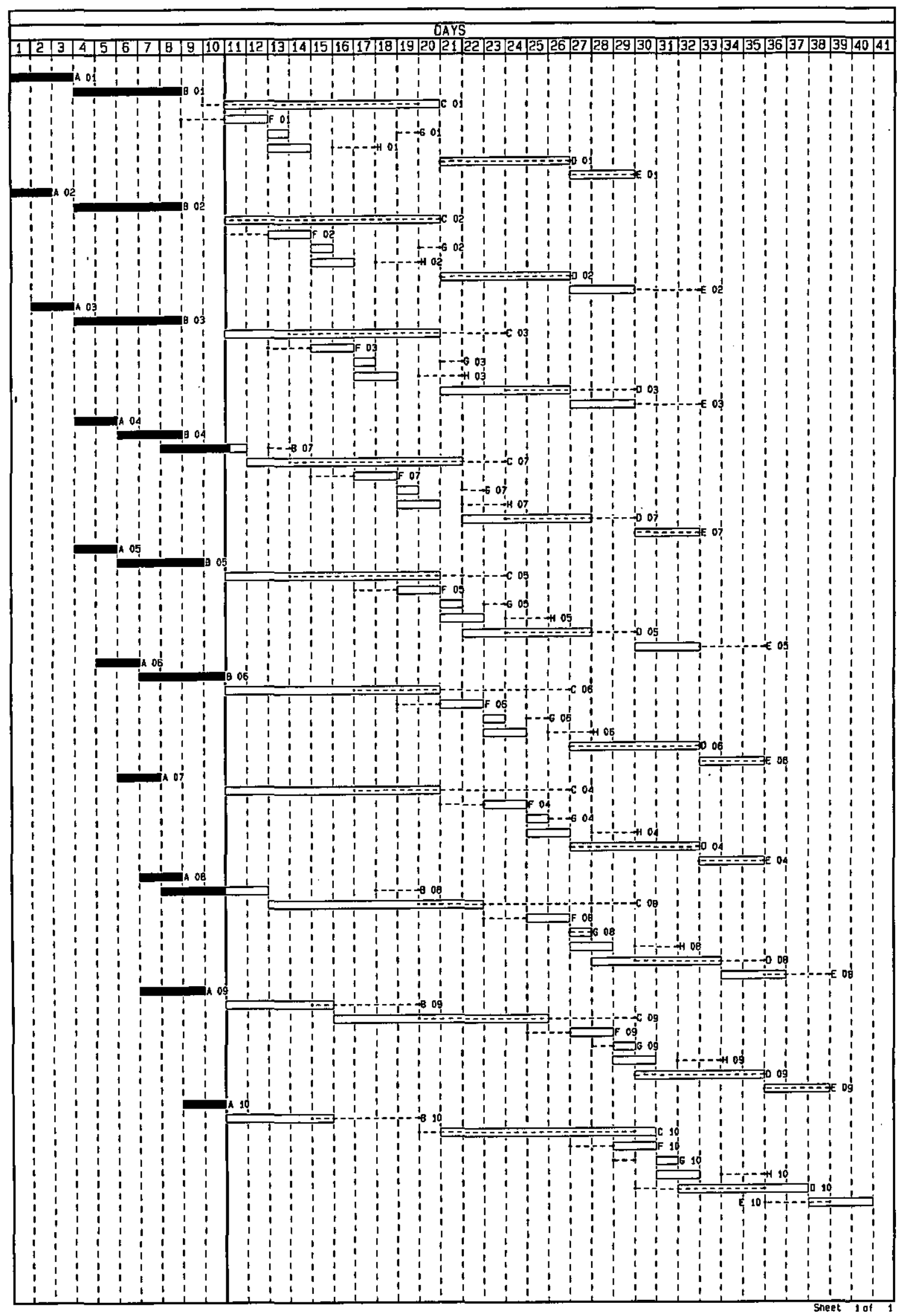

Figure 6.10 - Example 6.2, Bar Chart Showing Status at End of Day 10 and a Forecast Completion on Day 40. 


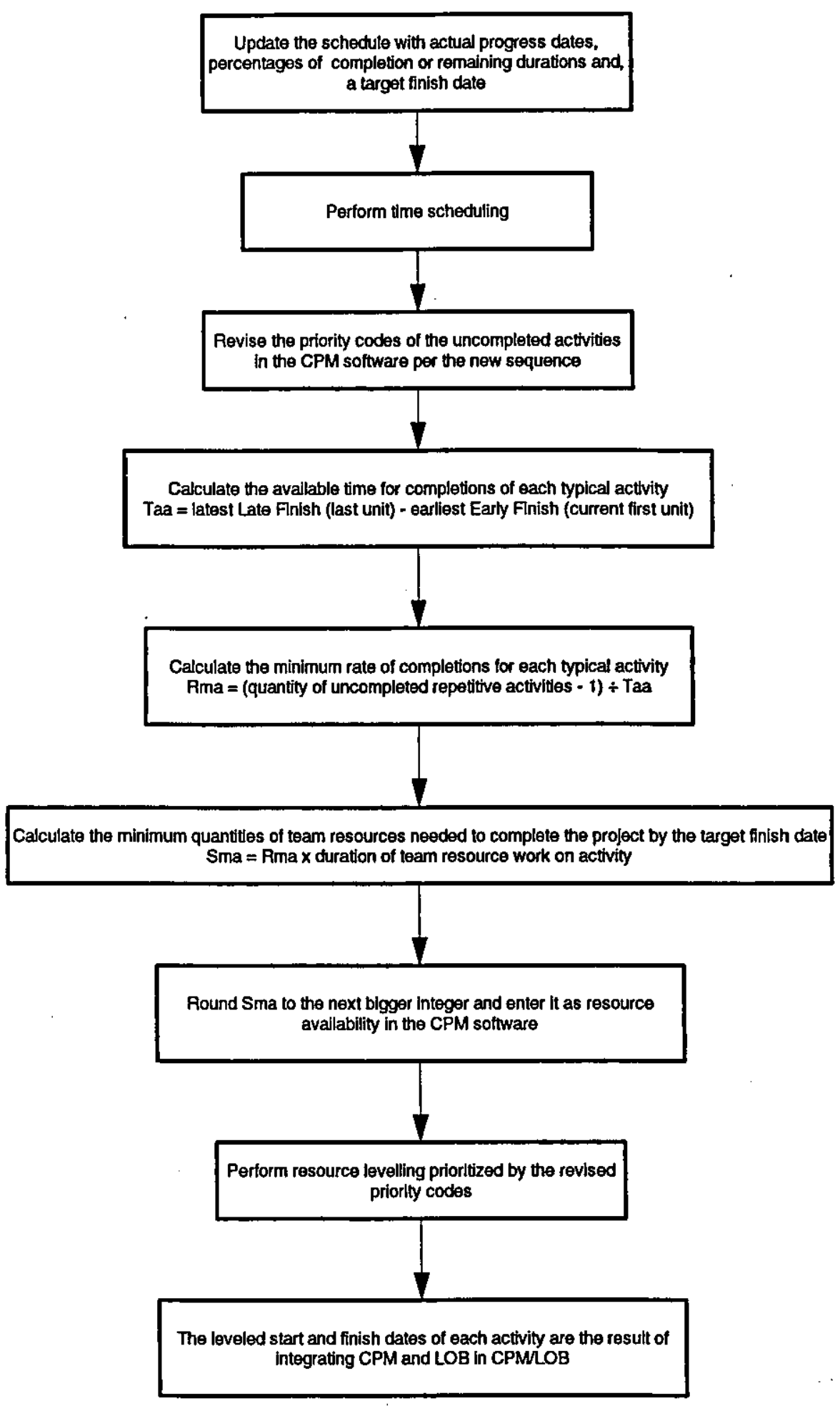

Figure 6.12 - Example 6.2, Steps of Applying CPM/LOB in Adjusting a Schedule. 
CPM/LOB shall now be applied to change the sequence of progress on the units and accelerate the schedule to recover the 2 day delay. Fig. 6.12 shows the steps of this application. The current step is the calculation of the minimum quantities of squads to complete the project on time, day 38 .

To get the quantity of squads it is necessary to calculate the available time for activity completions, $T_{a \mathrm{a}}$ for each typical activity and, $\mathrm{R}_{\mathrm{ma}}$, the minimum rate of completions for each typical activity

$$
\text { ' } T_{a a}=\text { latest LF (last unit) - earliest EF (the current first unit) }
$$

Where LF is late finish and EF is early finish. Therefore $\mathbf{T}_{\mathrm{aa}}$ for the seven uncompleted typical activities is as shown in Table 6.2.

\begin{tabular}{c|c}
\hline \hline Typical activity & $\mathrm{T}_{\mathrm{aa}}$ \\
\hline B & $19-11=8$ \\
\hline C & $29-20=9$ \\
\hline D & $35-26=9$ \\
\hline E & $38-29=9$ \\
\hline F & $28-12=16$ \\
\hline G & $29-13=16$ \\
\hline H & $35-14=21$ \\
\hline \hline
\end{tabular}

Table 6.2 - Example 6.2, Calculation for Adjusting Available Time for Activity Completions.

The minimum rate of completions for each typical activity to complete the whole project on day $38, R_{\operatorname{ma}}$, can be obtained from the following equation:

$$
R_{m a}=\frac{\text { number of uncompleted repetitive activities }-1}{T_{a a}}
$$

The minimum quantity of squads, $S_{\mathrm{ma}}$, can now be calculated from equation (6.2) 
Table 6.3 shows the calculation of the adjusted minimum quantity of squads of the typical activities to complete the project on the original completion target, day 38 .

\begin{tabular}{c|c|c|c|c|c}
\hline \hline $\begin{array}{c}\text { Typical } \\
\text { activity }\end{array}$ & $\begin{array}{c}\text { Initial } \\
\mathrm{S}_{\mathrm{ma}} \\
\text { (from example 6.1) }\end{array}$ & $\begin{array}{c}\text { Quantity of } \\
\text { uncompleted } \\
\text { repetitive activities } \\
(1)\end{array}$ & $\begin{array}{c}\mathrm{R}_{\mathrm{mm}}= \\
\{(3)-1\} \div \mathrm{T}_{\mathbf{u}} \\
(2)\end{array}$ & $\begin{array}{c}\mathrm{S}_{\mathrm{mm}}= \\
\left\{\text { Duration } \times \mathrm{R}_{\mathrm{ma}}\right\}\end{array}$ & $\begin{array}{c}\text { Rounded } \\
\mathrm{S}_{\mathrm{mu}} \\
(5)\end{array}$ \\
\hline $\mathrm{B}$ & 5 & 4 & 0.375 & 1.875 & 2 \\
\hline $\mathrm{C}$ & 9 & 10 & 1.000 & 10.000 & 10 \\
\hline $\mathrm{D}$ & 5 & 10 & 1.000 & 6.000 & 6 \\
\hline $\mathrm{E}$ & 3 & 10 & 1.000 & 3.000 & 3 \\
\hline $\mathrm{F}$ & 1 & 10 & 0.563 & 1.125 & 2 \\
\hline $\mathrm{G}$ & 1 & 10 & 0.563 & 0.563 & 1 \\
\hline $\mathrm{H}$ & 1 & 10 & 0.429 & 0.858 & 1 \\
\hline \hline
\end{tabular}

Table 6.3 - Example 6.2, Calculation of Minimum Quantity of Squads to Complete Project on Original Completion, Day 38.

The following step is entering the quantity of squads listed on the last column of table 6.3 into the CPM software as the resource availability necessary to complete the project on day 38 . The priority codes in this example are revised; units 4 and 7 exchanged priorities. After performing time scheduling and resource leveling the results are obtained and shown in Fig. 6.13.

It is noted that there was a significant change in the minimum quantity of squads required for some activities to complete the project on day 38 . While the demand of typical activity $B$ on squads was reduced from 5 to 2 because of its over completions, some of the following typical activities needed more squads due to the critical delay of typical activity $\mathrm{F}$. 


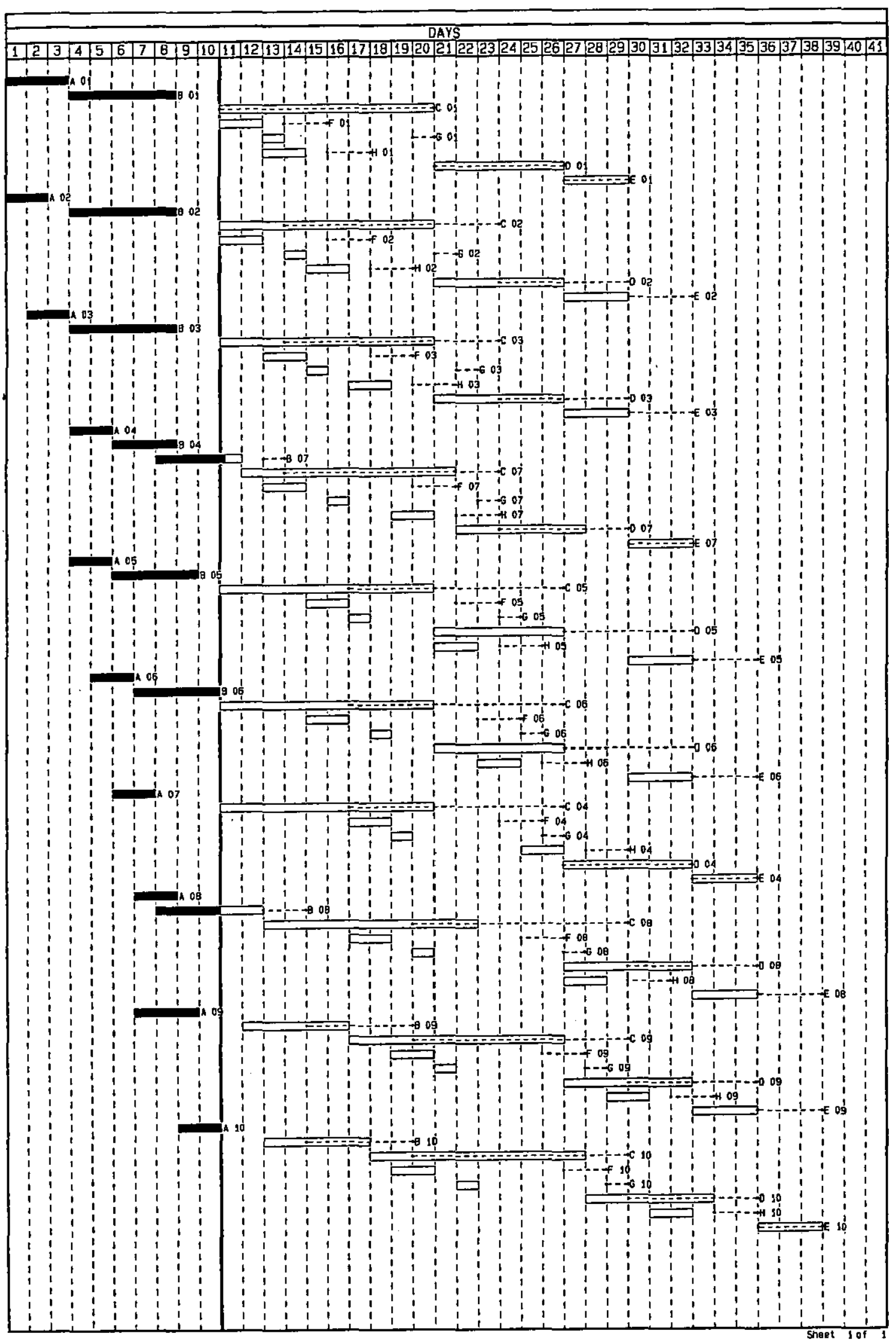

Figure 6.13 - Example 6.2, Bar Chart at End of Day 10 Showing Acceleration to Complete on Day 38. 


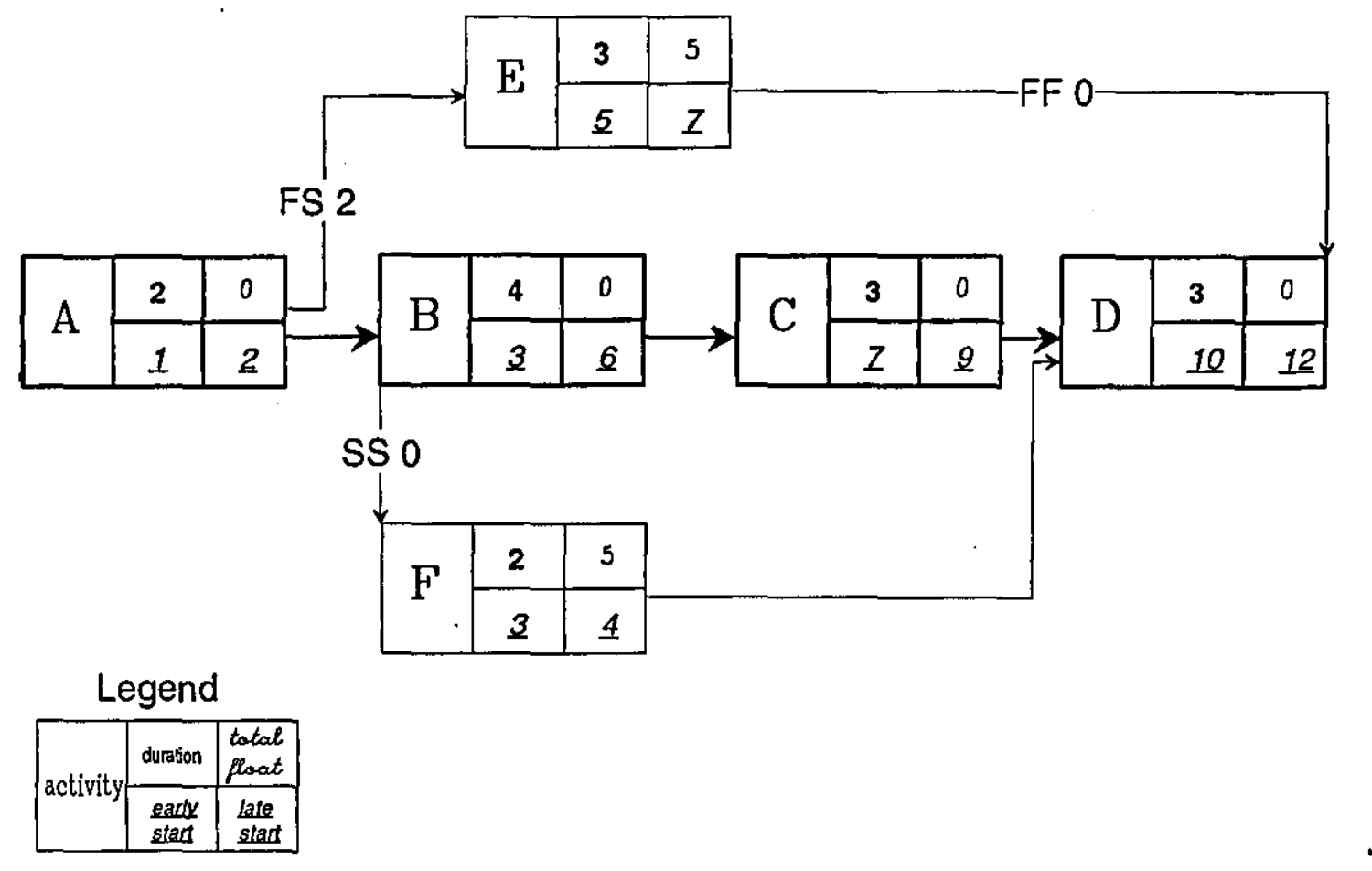

Figure 6.14 - Example 6.3, Logic Diagram of a Typical Unit.

This example exhibits the usage of the precedence diagram method to show nontraditional relationships between the activities: start to start SS, finish to finish FF and finish to start FS with a lag. Fig. 6.14 shows the logic diagram of one unit of ten that make up the project. A single calendar is used which has 7 workdays a week. The objective is to create a schedule to begin the work on November 1, 1994 and complete it by December 1, 1994.

The available time for completions for each typical activity, $T_{a 2}$, can be calculated from equation (6.4) or alternatively obtained from the CPM software. In the CPM software, when the schedule of one unit network is run independently, the total floats of the activities are calculated based on the early finish date of one unit network, or the assumed first typical unit network. However, if for this same schedule, a date after the unit early finish date is imposed as a required completion date, the time difference between the two dates will become the $T_{\text {ap }}$ of the project, the available time for project completions. Consequently the individual total floats of each activity 
in the typical unit network are increased by an amount equal to $T_{a p}$ which make them equal to $T_{a a}$, the available time for completions for each typical activity per equation (6.4).

The above concept was applied on the typical unit network of Fig. 6.14 by imposing December 1, 1994 as a completion date and running the schedule to start on November 1, 1994. Fig. 6.15 shows the results where the total floats equal the $T_{2 a}$ 's of the activities.

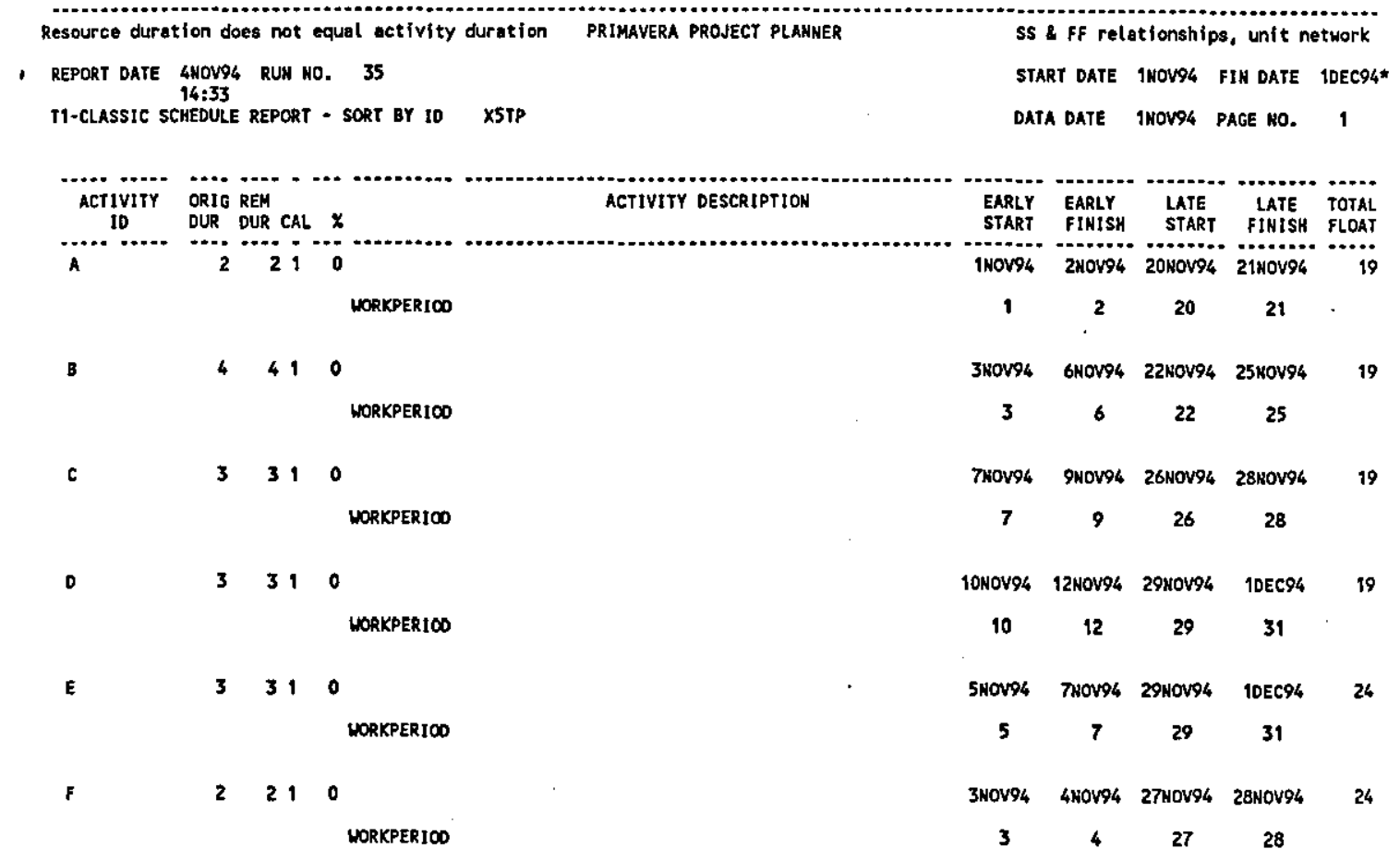

Figure 6.15 - Example 6.3, Schedule Report Showing $\mathrm{T}_{2 \mathrm{a}}$ under the "Total Float" Column.

Table 6.4 shows the calculations of the minimum quantity of squads or resources to achieve a December 1, 1994 completion. It is noted that the duration of typical activity $C$ is three days and that it has two squads assigned to it, S1C and S2C. Squad S2C is partially applied to the activity duration since it is required for only one day. 


\begin{tabular}{|c|c|c|c|c|c|c|}
\hline $\begin{array}{l}\text { Typical } \\
\text { activity }\end{array}$ & $\begin{array}{l}\text { Squad } \\
\\
\text { (2) } \\
\end{array}$ & $\begin{array}{c}\begin{array}{c}\text { Squad } \\
\text { duration }\end{array} \\
\text { D } \\
\text { (3) } \\
\end{array}$ & $\begin{array}{c}\text { Available time for } \\
\text { completions } \\
\mathrm{T}_{\mathrm{eu}} \\
\text { (4) } \\
\end{array}$ & $\begin{array}{c}\text { Minimum rate } \\
\text { of completions } \\
\\
\mathrm{R}_{\mathrm{ma}}= \\
\left\{(10-1) \div \mathrm{T}_{\mathrm{u}}\right\} \\
(5)\end{array}$ & $\begin{array}{c}\text { Minimum } \\
\text { quantity of } \\
\text { squads } \\
S_{\mathrm{man}}= \\
\left\{\mathrm{D} \times \mathrm{R}_{\mathrm{mes}}\right\} \\
(6) \\
\end{array}$ & $\begin{array}{l}\text { Rounded } \\
\qquad \mathrm{S}_{\mathrm{me}} \\
\text { (7) }\end{array}$ \\
\hline A & SA & 2 & 19 & 0.474 & 0.95 & 1 \\
\hline B & SB & 4 & 19 & 0.474 & 1.90 & 2 \\
\hline \multirow{2}{*}{$\mathrm{C}$} & S1C & 3 & 19 & 0.474 & 1.42 & 2 \\
\hline & S2C & 1 & 19 & 0.474 & 0.47 & 1 \\
\hline D & SD & 3 & 19 & 0.474 & 1.42 & 2 \\
\hline $\mathrm{E}$ & $S E$ & 3 & 24 & 0.375 & 1.13 & 2 \\
\hline $\mathrm{F}$ & SF & 2 & 24 & 0.375 & 0.75 & 1 \\
\hline
\end{tabular}

Table 6.4 - Example 6.3, Calculation of Minimum Quantity of Squads to Complete by December 1, 1994.

The other nine unit networks are created after duplicating the typical unit network. Resource availability is entered in the CPM software per Table 6.4, column (7). Following that, time scheduling and squads leveling result in the bar chart of Fig. 6.16 where the early finish date is November 30th. A one day gain was realized due to the rounding up of the squad quantities. 


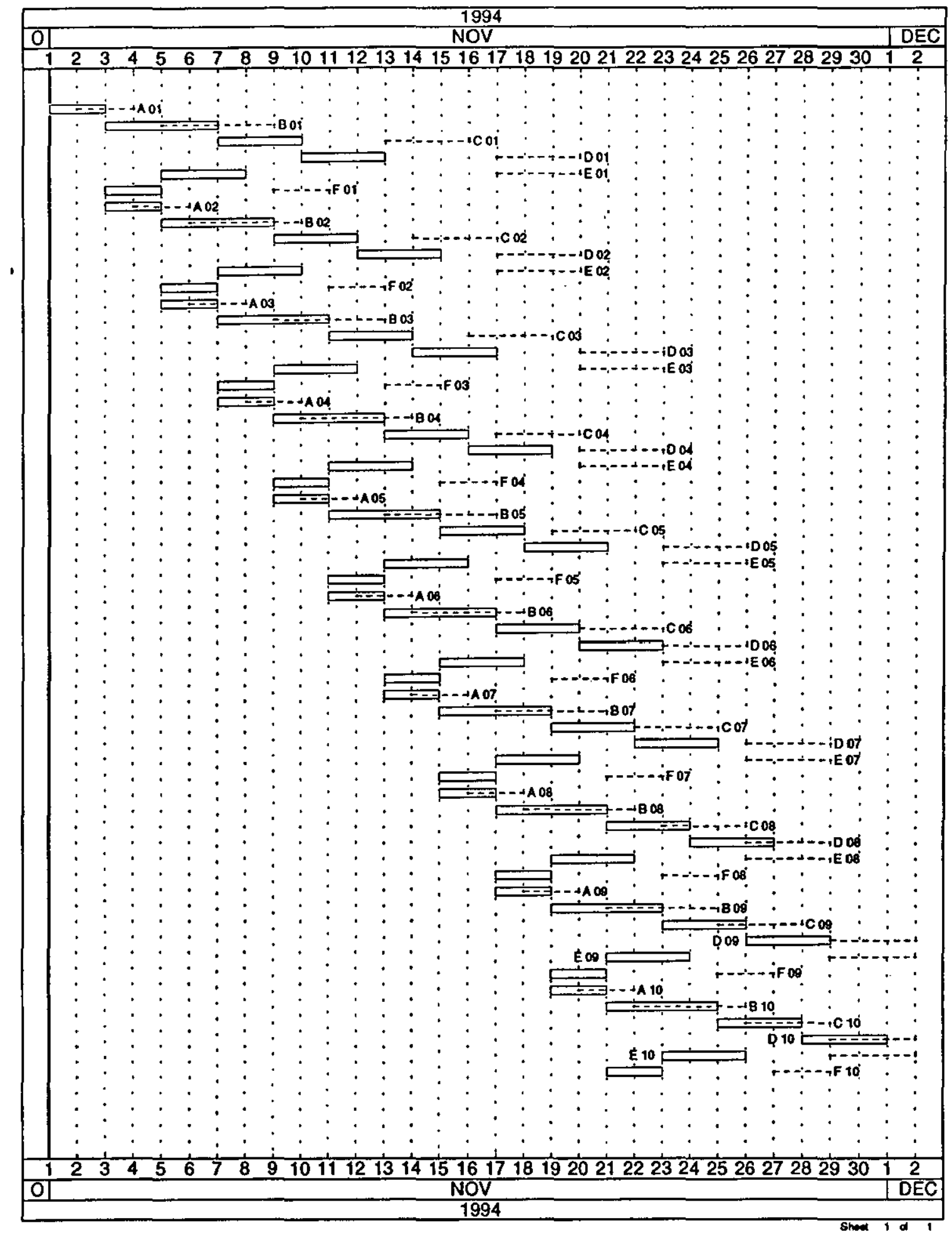

Figure 6.16 - Example 6.3, Bar Chart. 


\subsection{Example 6.4, Initial Schedule with Multiple Calendars}

This is based on all the details of example 6.3 with the exception of the calendar. There will be two additional calendars to the exiting 7 workday calendar of example 6.3: a second calendar of 6 workday weeks and a third calendar of 5 workday weeks. Fig. 6.17 shows the details of the project calendars.

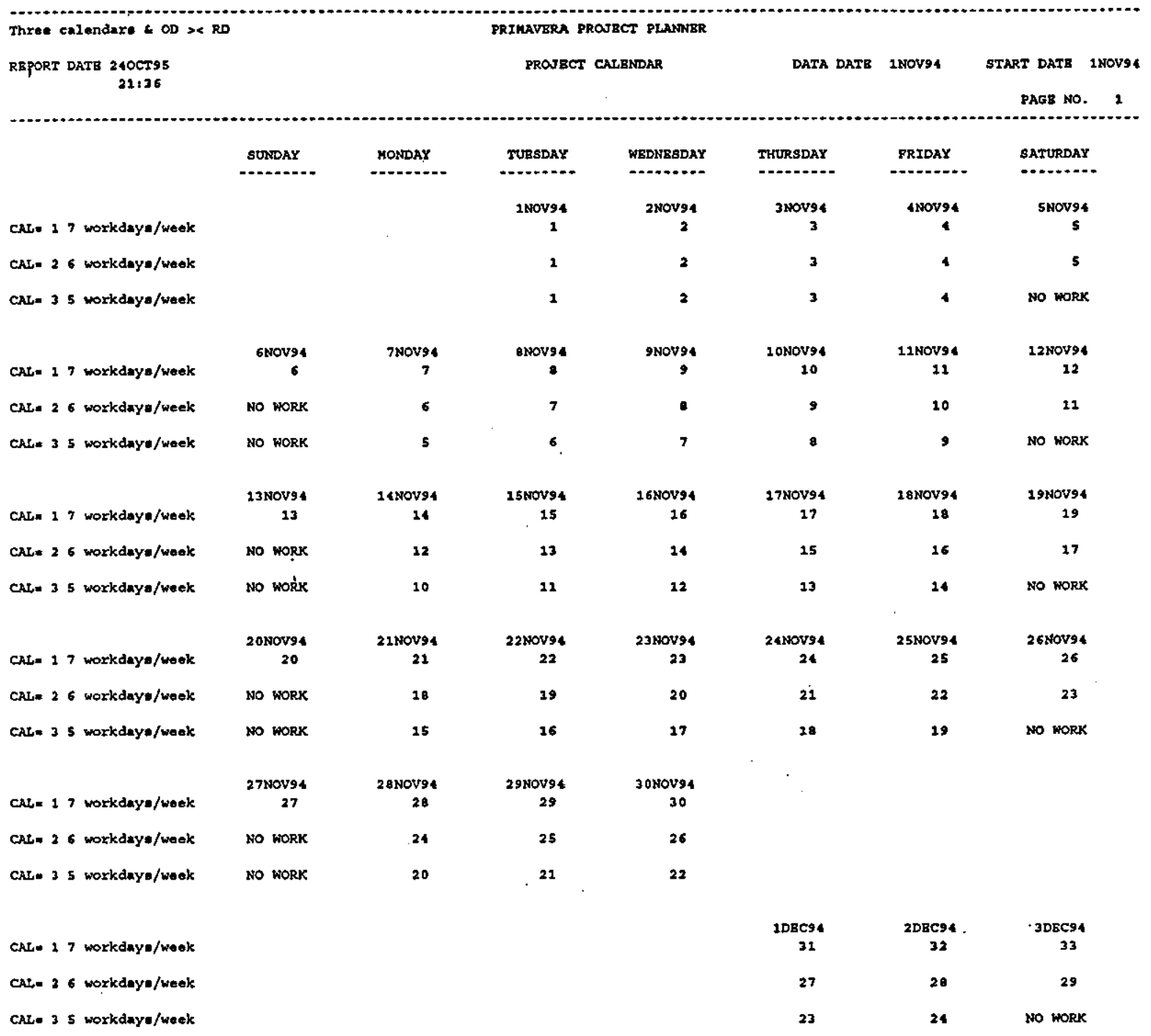

Figure 6.17 - Example 6.4, Three Work Calendars.

Typical activities $\mathrm{C}$ and $\mathrm{E}$ will follow a 6 workday week calendar (calendar 2), and typical activity $\mathrm{F}$ will follow a 5 workday week calendar (calendar 3). Fig. 6.18 shows the 


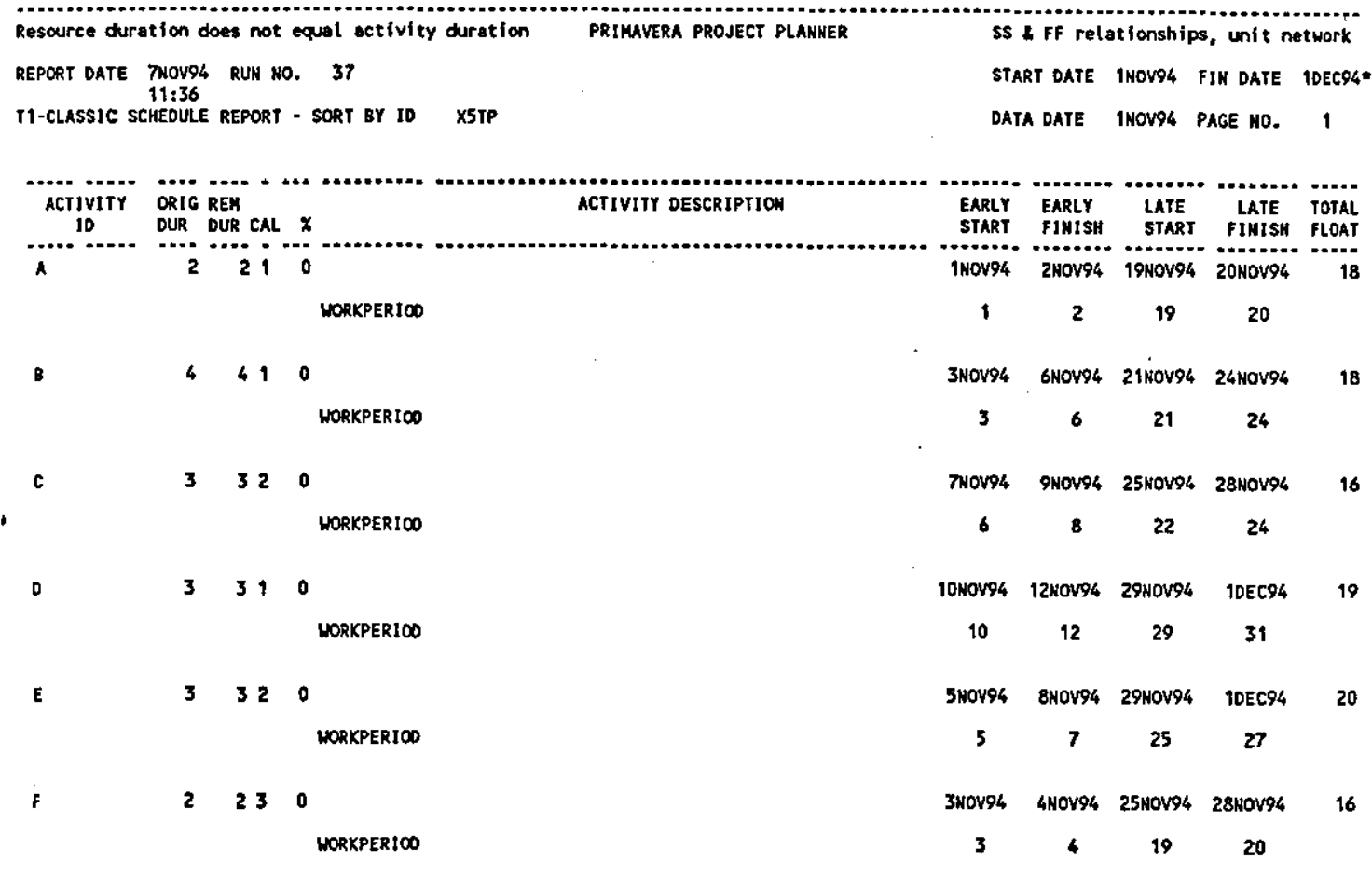

Figure 6.18 - Example 6.4, Schedule Report Showing $\mathrm{T}_{\mathrm{aa}}$ under the "Total Float" Column.

schedule report of one typical unit network having activities on different calendars. So for example, work period 6 means two different dates on two different calendars. The total floats of the activities are based on their relevant work periods per their calendars, hence they represent their individual available time for completions, $\mathrm{T}_{\mathrm{aa}}$ in workdays.

Table 6.5 displays the calculation of the minimum quantity of squads based on a December 1, 1994 completion and three different calendars. Column (7) demonstrates that only the quantity of squads of typical activity $F$ need to be double that of example 6.3 to finish on target. Fig. 6.19 shows a bar chart of all the project activities. It is noted that some activity bars are "necked" on nonwork periods and that the completion date of December 1, 1994 is achieved. 


\begin{tabular}{|c|c|c|c|c|c|c|}
\hline $\begin{array}{c}\text { Typical } \\
\text { activity } \\
\text { and calendar } \\
\\
\text { (1) } \\
\end{array}$ & Squad & $\begin{array}{c}\text { Squad } \\
\text { duration } \\
\text { D } \\
\text { (3) } \\
\end{array}$ & $\begin{array}{l}\text { Available time } \\
\text { for completions } \\
\qquad \mathrm{T}_{\mathbf{u}} \\
\text { (4) }\end{array}$ & $\begin{array}{c}\text { Minimum rate of } \\
\text { completions } \\
R_{\mathrm{m}}= \\
\left\{(10-1) \div \mathrm{T}_{\mathrm{an}}\right\} \\
(5)\end{array}$ & $\begin{array}{c}\text { Minimum } \\
\text { quantity of } \\
\text { squads } \\
S_{\mathrm{ma}}= \\
\left\{D \times \mathrm{R}_{\mathrm{mas}}\right\} \\
(6)\end{array}$ & $\begin{array}{c}\text { Rounded } \\
S_{\mathrm{m}} \\
(7) \\
\end{array}$ \\
\hline A, 1 & SA & 2 & 18 & 0.500 & 1 & 1 \\
\hline B, 1 & SB & 4 & 18 & 0.500 & 2 & 2 \\
\hline \multirow{2}{*}{ C, 2} & S1C & 3 & 16 & 0.563 & 1.69 & 2 \\
\hline & $\mathrm{S} 2 \mathrm{C}$ & 1 & 16 & 0.563 & 0.56 & 1 \\
\hline $\mathrm{D}, 1$ & SD & 3 & 19 & 0.474 & 1.42 & 2 \\
\hline E, 2 & $\mathrm{SE}$ & 3 & 20 & 0.450 & 1.35 & 2 \\
\hline$F, 3$ & SF & 2 & 16 & 0.563 & 1.13 & 2 \\
\hline
\end{tabular}

Table 6.5 - Example 6.4, Calculation of Minimum Quantity of Squads to Complete by December 1, 1994.

The following two examples were selected from the literature to challenge the application of CPM/LOB. In the surveyed literature, there is an abundance of examples that can substantiate the advantages of CPM/LOB application in a straightforward fashion. However, the author's purpose goes beyond exhibiting examples readily showing the strength of CPM/LOB to draw more credence from its applicability on sophisticated examples published by experts in the field. Examples 6.5 and 6.6 represent the nature of complex construction processes as experienced by two authorities in this area of knowledge. 


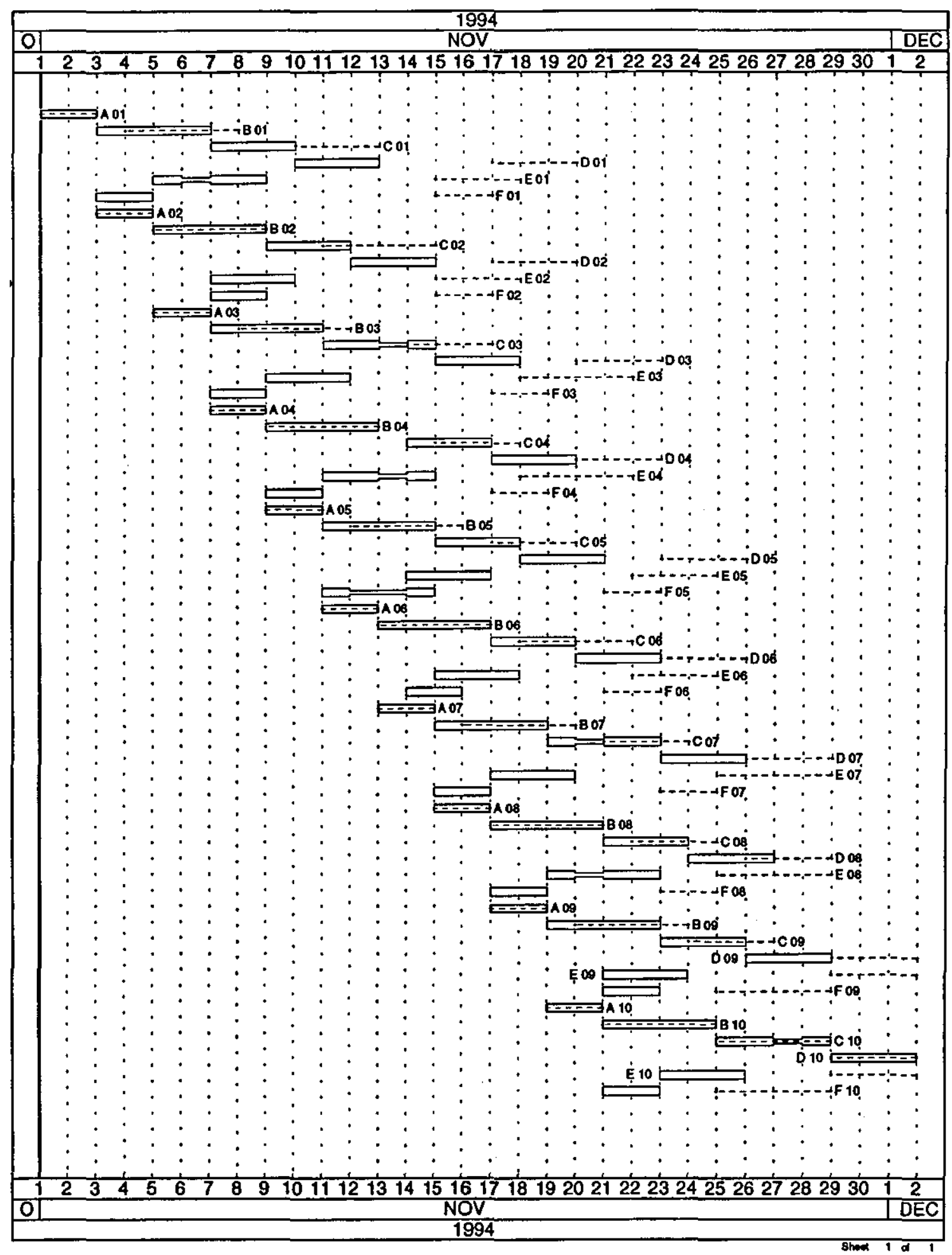

Figure 6.19 - Example 6.4, Bar Chart. 
This example is taken from Thompson (1981), Organization and Economics of Construction. A pipeline project comprising the laying of 26 Kilometers of a water pipe (Fig. 6.20). There is hard rock from chainage 10 to $13 \mathrm{Km}$, and bridgings at chainages $2,7,14,18$ and $22 \mathrm{Km}$. Commencing week 1, pipes are available at $1000 \mathrm{~m} / \mathrm{wk}$. Two stringing squads are mobilized and each can handle $1000 \mathrm{~m} / \mathrm{wk}$. Five pipelaying squads are employed with each having a rate of $300 \mathrm{~m} / \mathrm{wk}$ in normal ground. However, in hard rock their rates are much slower. There is one bridging squad working at a rate of four weeks per bridge or crossing. Testing water would be available during week 33. Testing takes two weeks for each three Kilometer segment and can proceed concurrently on alternate segments. Pipelaying and stringing can not be done concurrently on one Kilometer due to space constraints. Squads can not be increased and their continuity is to be maintained. Fig. 6.19 shows the application of the location-time method to achieve the project goals of minimum cost and completion by end of the 37th week. It was inferred from Thompson's solution that the work followed a calendar of six workdays per week.

Clearly this is a repetitive construction project where CPM/LOB should be applicable. Fig. 6.20 demonstrates the certainty of incorporating the aforementioned details of the pipeline project into a CPM network utilizing CPM/LOB. The logical relationships and constraints are respected while squads continuity is maintained to achieve the same completion date (end of week 37). Certain squads were directed to specific chainages to follow the location-time solution presented by Thompson. This was achieved by allocating these particular squads to the corresponding locations then performing time scheduling and prioritized resource leveling. Squads availability was allowed in the CPM software in conformity with the constraints and availabilities set by Thompson.

The first finding is that the project duration was optimum, therefore could not be reduced. However, CPM/LOB has shown an advantage through the identification of some floats.

Another advantage of the application of CPM/LOB on this example can be demonstrated by the ability to perform quick what-if analyses: assume that only one stringing squad was available to the project and find the impact. Fig. 6.22 shows the effect of eliminating one stringing squad on the arrangement of the work and on the completion date. The delay is about two weeks and a half and the criticality of some activities changed, notably the testing activities. 
Distance from $\mathrm{A}, \mathrm{km}$

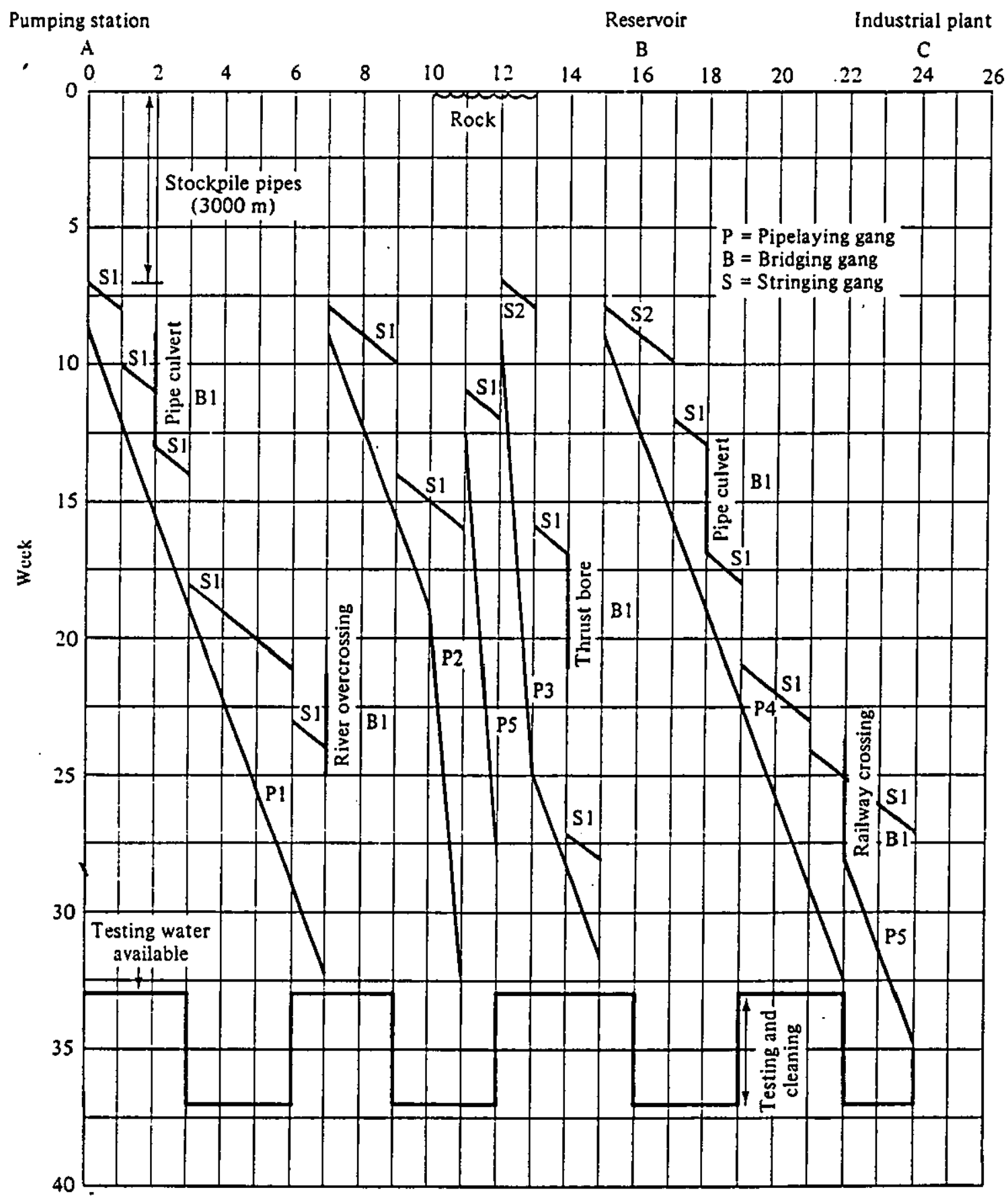

Figure 6.20 - Example 6.5, Location-Time Program for Pipeline (copied from Thompson, 1981). 


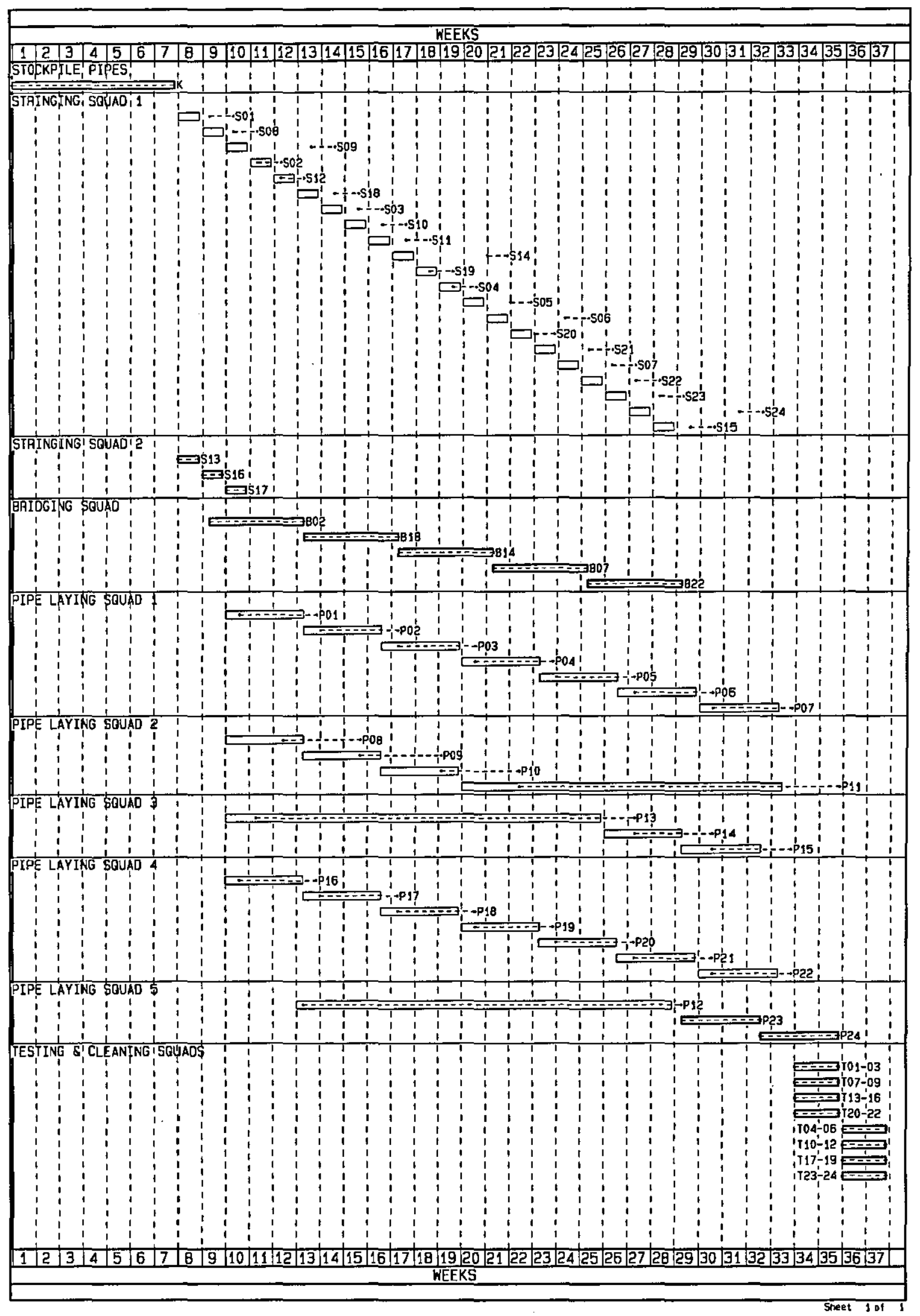

Figure 6.21 - Example 6.5, Bar Chart Showing Alternate Solution to Pipeline Project Using CPM/LOB. 


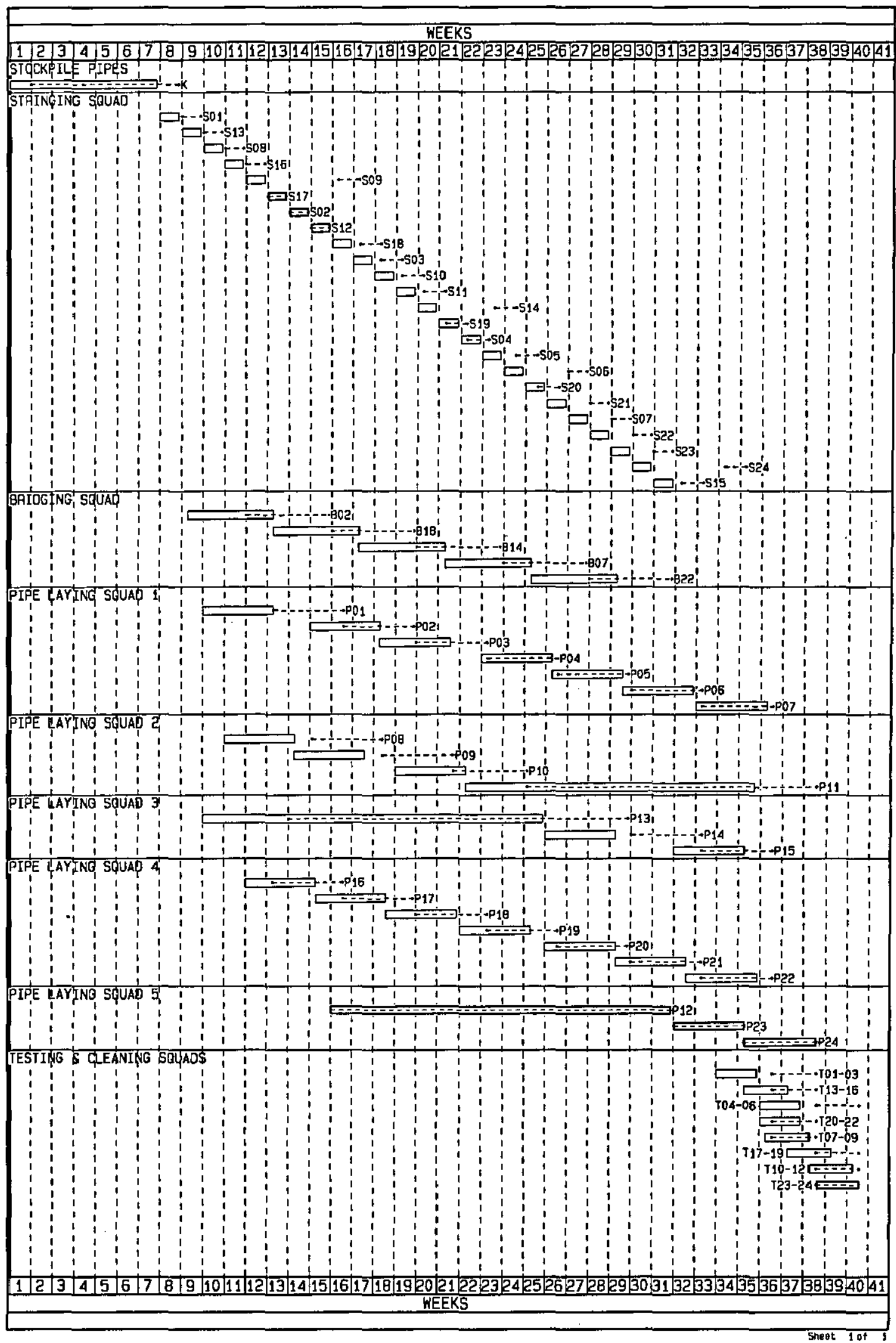

Figure 6.22 - Example 6.5, Bar Chart Showing the Impact of Eliminating One Stringing Squad. 


\subsection{Example 6.6, High-rise Building}

This follows an example published in a paper authored by Russell and Wang (1993), New Generation of Planning Structures, Journal of Construction Engineering and Management, ASCE. The authors presented the application of a construction management software called REPCON on a high-rise building; a ten story concrete building with large floor plans which must be poured in two stages per floor, zone one and zone two. The core, walls and columns are crane intensive.

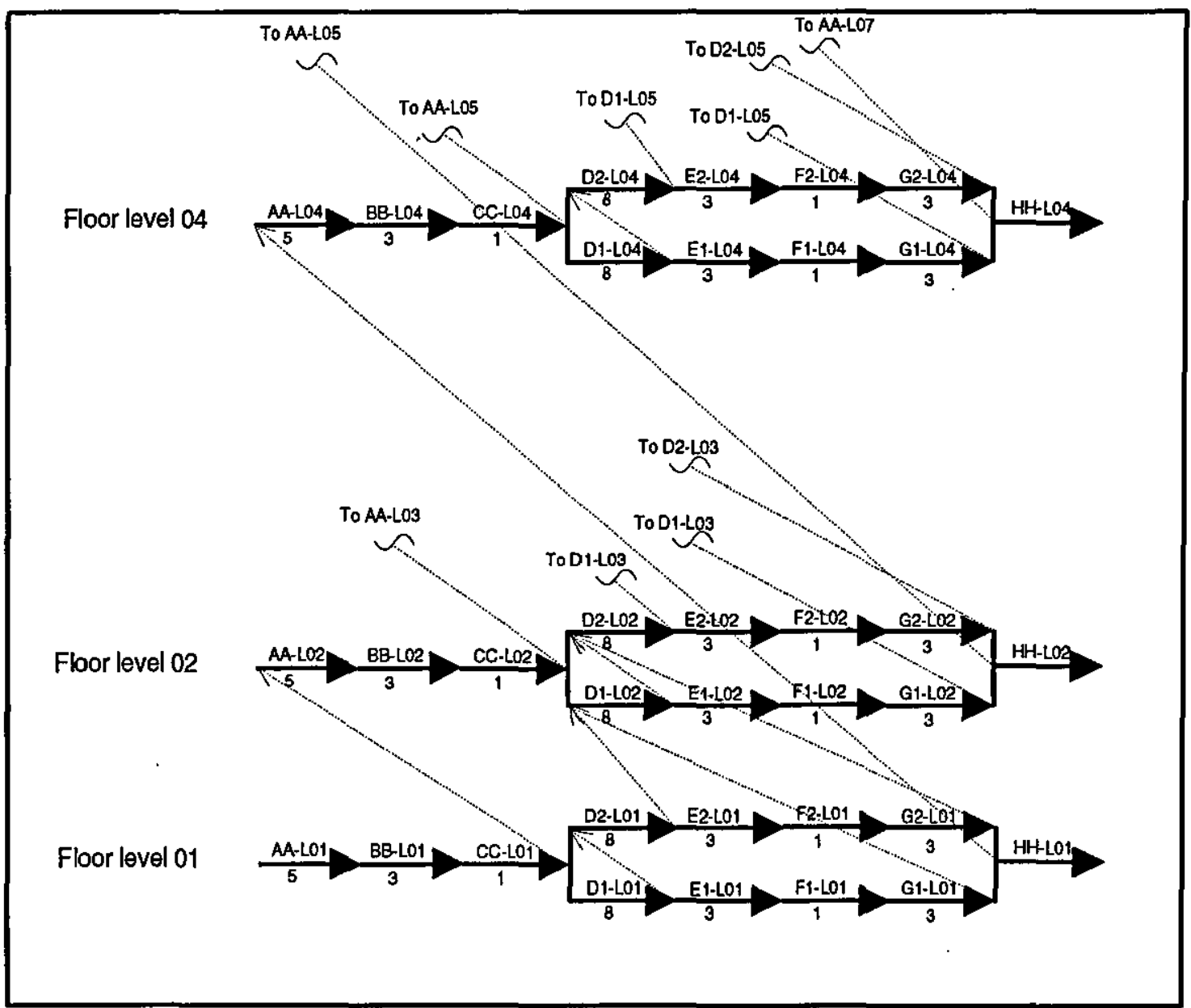

Figure 6.23 - Example 6.6, Subnetworks of High-rise Building.

Work continuity for the slab forming squad has to be facilitated. The core should not lead the floors construction by more than three floors to avoid obstructing the tower crane rotation. A selfclimbing form is used on the core. Fig. 6.23 represents some of the typical floor subnetworks. Table 6.6 lists the description of the typical activities. Activity HH, Overhead HVAC rough in, 


\begin{tabular}{l|l}
\hline $\begin{array}{c}\text { Typical } \\
\text { activity } \\
(1)\end{array}$ & \multicolumn{1}{|c}{ Description } \\
\hline AA & Form core \\
\hline BB & Reinforcement of core \\
\hline CC & Place concrete of core \\
\hline D1 & Form slab zone 1 \\
\hline D2 & Form slab zone 2 \\
\hline E1 & Reinforcement of slab zone 1 \\
\hline E2 & Reinforcement of slab zone 2 \\
\hline F1 & Place concrete of slab zone 1 \\
\hline F2 & Place concrete of slab zone 2 \\
\hline G1 & Form, reinf. \& pour walls and columns, zone 1 \\
\hline G2 & Form, reinf. \& pour walls and columns, zone 2 \\
\hline HH & Overhead HVAC rough in \\
\hline \hline
\end{tabular}

Table 6.6 - Example 6.6, Activities of a Typical Floor.

was added by this author to expose and highlight the distinction in the application of CPM/LOB. The rest of the activities were presented by Russell and Wang and were driven by logic relationships as shown in Fig. 6.23. Activity durations had to be assumed by this author to exclude the effect of the learning curve on the schedule calculations.

The finish to start relationships between typical activity $\mathrm{CC}$ (place concrete of core) and typical activity AA (form core) on the floor above denotes an absolute technological constraint where the form of the core of a particular floor can not be set up before placing the core concrete of the level below. Similarly, typical activities D1 and D2 (form slabs) have to succeed G1 and G2 (form, reinforcement \& pour walls and columns) of the floor below respectively. D1 and D2 are planned sequentially since one forming squad is employed. In order to limit the progress gap between the core and the floors, and give leeway to the tower crane, typical activity AA should not start before the completion of typical activities G1 and G2 three floors below.

It is obvious that the sequence of the planned progress is absolute and can not be changed. This sequence is mandated by the logical relationships between the activities. REPCON, the 


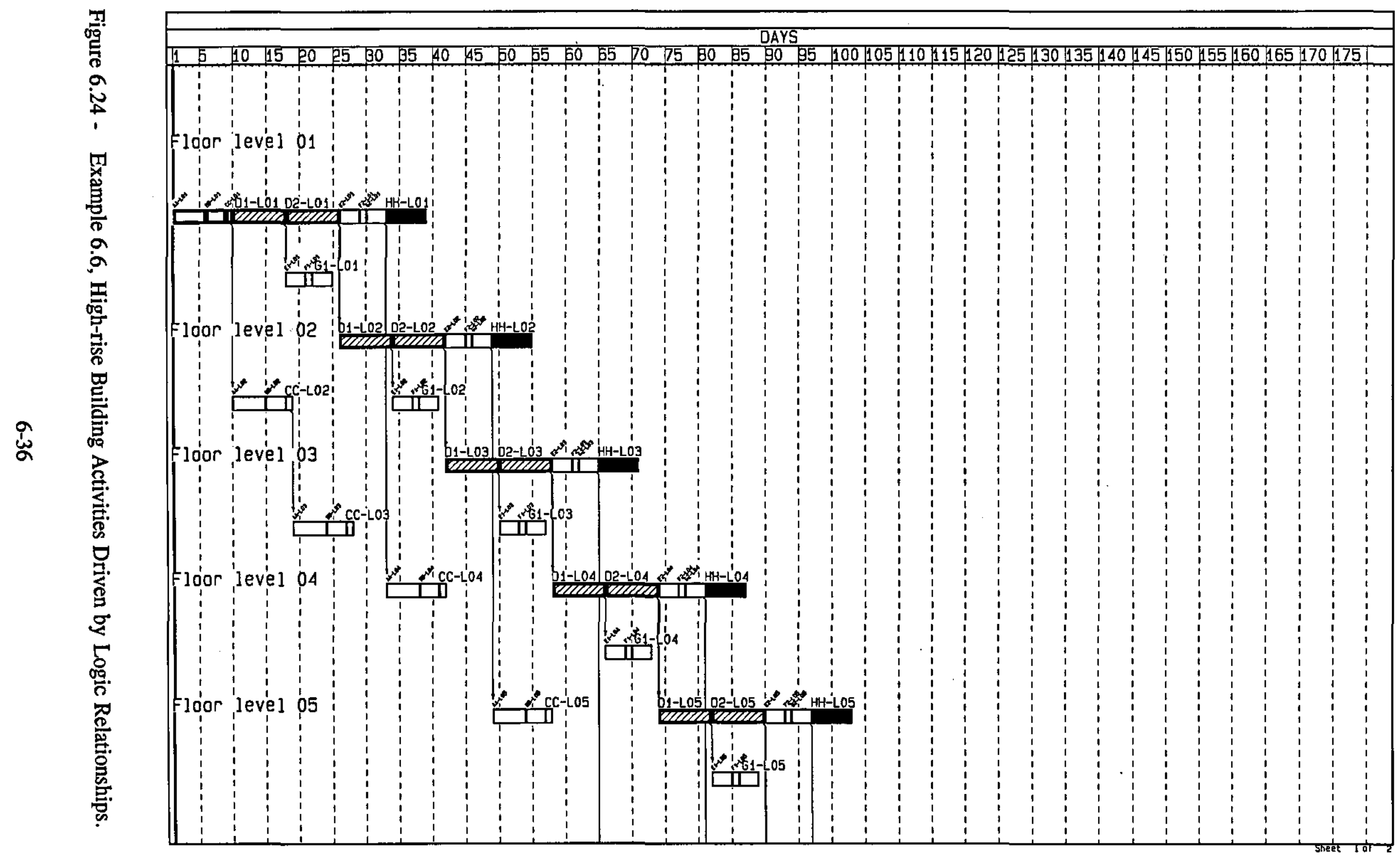




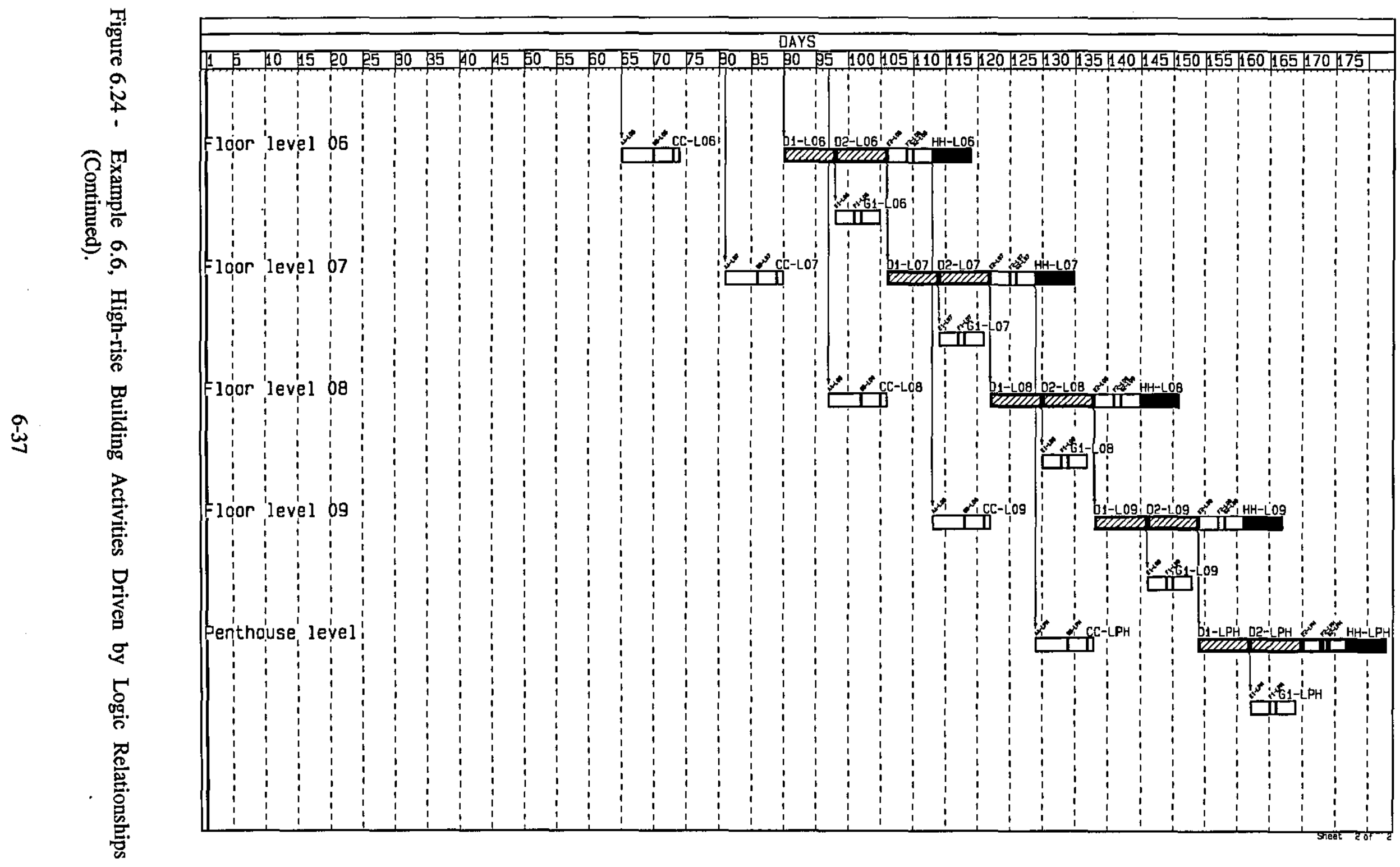


project management software presented by the authors, summarizes these logic relationships in a global and general form that enables speedy development and revision. Each time change is necessary it is done globally instead of an otherwise tedious revision of each relationship and therefore considerable time is saved on large CPM networks. REPCON does not however, utilize resource leveling. As of this writing none of the published literature surveyed gave adequate details on how its mechanism develops and revises logic relationships globally.

Alternatively, this author utilized Primavera Project Planner ${ }^{\otimes}$ to calculate the schedule of the example presented by the authors while adhering to the details in Fig. 6.23. The output of Primavera is shown in Fig. 6.24 in a linked bar chart form. The subnetworks are linked by interface logic relationships and no resource scheduling is used initially in imitating REPCON. For better clarity only driving relationships are shown. The end-to-end placement of activities denotes a finish to start relationship in conformity with Fig. 6.23. It is noted that the continuity of the forming squad on typical activities D1 and D2 is maintained.

CPM/LOB using resource leveling as a vehicle to maintain continuity of the repetitive activities and drive their sequence of execution, it does not aim at interfering with the interface logic relationships between the repetitive subnetworks of a project, in this case between the subnetworks of the multiple floor levels. The main reason is interface logic relationships become redundant where prioritized resource leveling offers superior and dynamic alternatives. However, in this case, the added constraint of the tower crane freedom to rotate and the objectionable start of typical activities D1 and D2 before the completion of G1 and G2 on the floor below necessitate supplementing CPM/LOB with some interface logic relationships. In CPM/LOB these interface logic relationships are completely redundant on repetitive projects where the subnetworks are independent and the sequence of progress can start and change independent on previous subnetworks (e.g. housing projects).

Fig. 6.25 shows the output of Primavera after the application of CPM/LOB. The logic relationships between typical activity D1 and D2 were deleted, however, the same continuity was effected through employing and leveling one forming squad. Of course the priority codes for typical activities D1 and D2 followed the obvious succession from zone to zone then from floor to floor going upward.

Typical activity HH (overhead HVAC rough in) is a finishing activity and does not have to follow the same sequence of progress of the concrete structure. Like many other finishing 


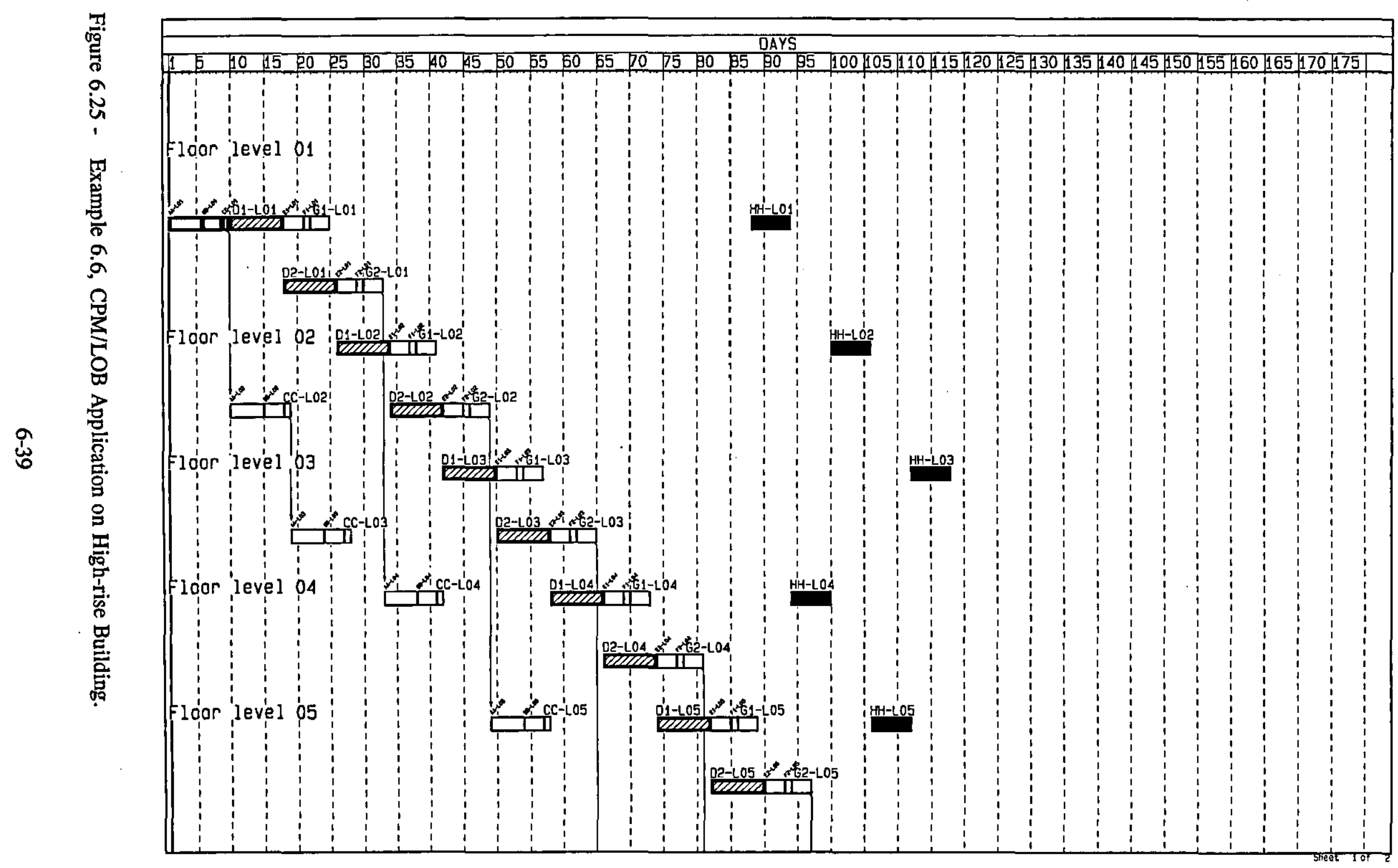




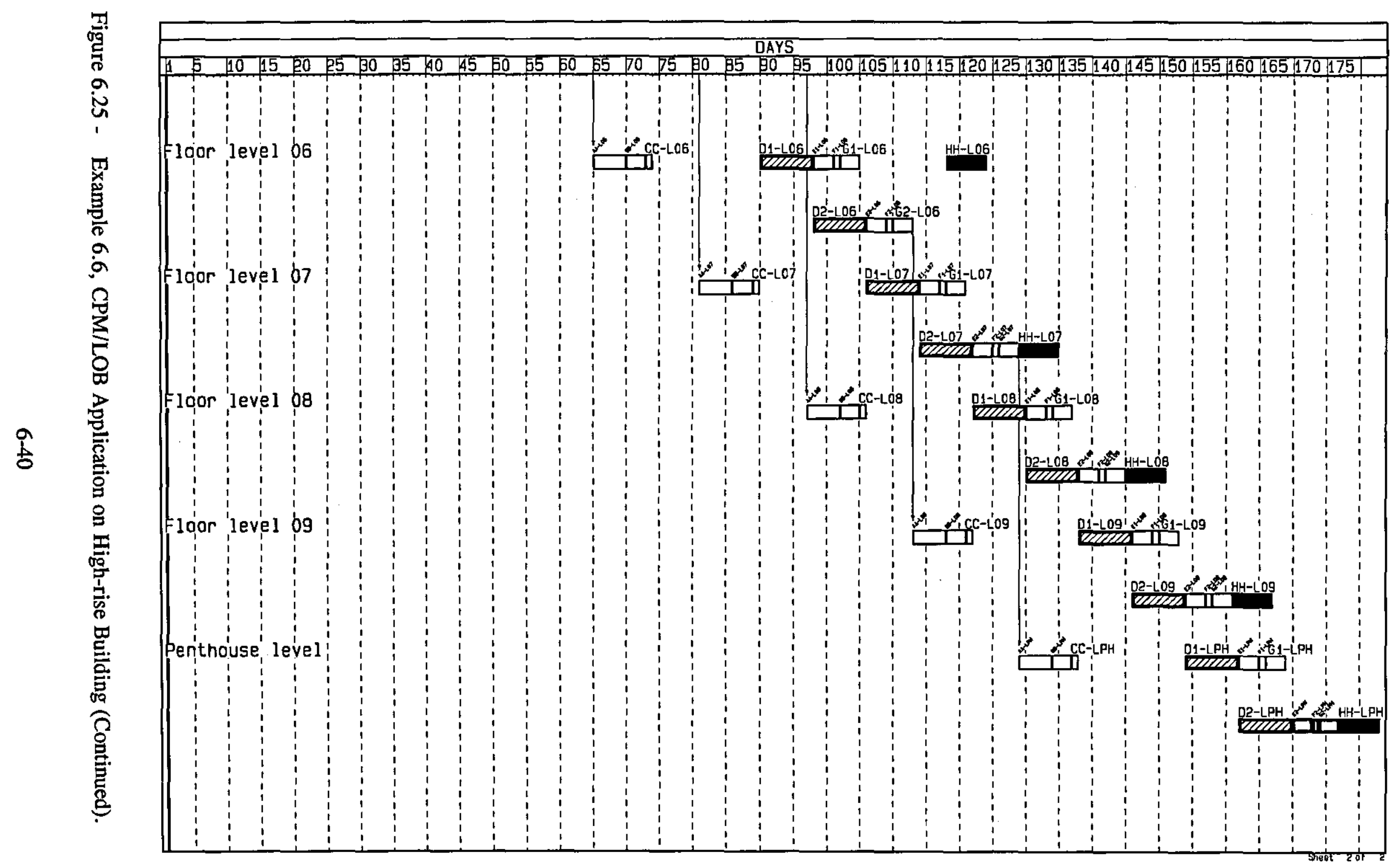


activities on a high-rise building, regardless of cost savings, its progress is independent on previous floors and may follow an unrestricted sequence analogous to that of a housing project activity. Fig. 6.25 shows typical activity $\mathrm{HH}$ following that unrestricted sequence which was driven exclusively by an arbitrary priority code and resource leveling in CPM/LOB. Surely, logic relationships of activity $\mathrm{HH}$ within each subnetwork were not contradicted.

It is vital to emphasize the essential difference between the repetition on a high-rise building and on a housing project. On a high-rise the start of a typical floor is dependent on one or more activities in the preceding floor, whereas on a housing project the start of a typical unit is usually independent on the preceding typical unit. As demonstrated on the complex sequences of the concreting activities, this poses no impracticability to utilize CPM/LOB on a high-rise. Through a limited usage of interface logic relationships this can be achieved. On the other hand, finishing activities can be scheduled with pure resource leveling with no interface logic relationships in a completely unrestricted sequence.

Continuity of a typical activity on a high-rise can be interrupted because of a cyclic activity. A cyclic activity is a repetitive activity that is part of a periodic string of repetitive activities which may start on each subnetwork only after the completion of the whole string on the previous subnetwork. Activities AA, BB and $\mathrm{CC}$ are cyclic. Activity AA can not be continuous because of its cyclic successors BB and $\mathrm{CC}$. On the other hand, although typical activities D1 and D2 are cyclic activities, they maintained alternate continuity because their single durations are more than the remaining total durations of their respective periodic string and they are parallel (D1 > E1 +F1+G1). D1 and D2 (form slab zone 1 and 2) were worked on by one uninterrupted forming squad.

\subsection{Practical Implications}

With the introduction of float in the LOB, there can be many lines of balance between the early and late dates of the activities. Fewer squads may be adequate for typical activities that have float without delaying the project completion.

The rounding up of the quantity of squads before inputting the CPM software along with the nongradual starts of these squads (step flow lines) will result in slightly varying rates of completions, therefore minor squad waiting times for available work are encountered. However, 
this can be overcome by manipulating the schedule with some constraints, or dummy activities representing time buffers that make a squad start later to have no waiting time. Naturally this may push the completion date of the project to a later date. The planner will then weigh the benefits of continuous uninterrupted work for the squads against the savings of an early completion. In most practical cases the latter outweighs.

When monitoring the progress of a project by the valuation curve, projected value calculations based on the traditional schedule of completions (e.g. Table 5.1) produced by the LOB would reduce the overall value by the value of the activities in progress (uncompleted). This is so, because the figures of the schedule of completions do not account for activities in progress and not completed. CPM/LOB corrects this because it is based on CPM and not limited to completions, therefore it accounts properly for the values of activities in progress.

\subsection{Feasibility of CPM/LOB}

CPM/LOB is based on resource-driven rates of completions that can be promptly revised and can produce enhanced LOB information incorporating float times by utilizing the resource management capacity of the CPM software. It eliminates the troubles associated with the change of sequence of operations with a minimal input and indicates their impact as a reality on the completion date. While respecting the branching logic relationships of typical unit networks, CPM/LOB maintains a practical work continuity for the squads by using priority codes for leveling.

In contrast with the many published methods about planning repetitive construction projects, it was demonstrated that CPM/LOB can be applied conveniently not only to develop the initial (or baseline) schedule but to update and accelerate that schedule subsequently. Varying rates of completions for a typical activity is a further sophistication of CPM/LOB that can be undertaken by changing the availability of squads across the duration of execution of that typical activity.

CPM/LOB does not call for training on additional software which the operation personnel are not expected to welcome. And since LOB is now CPM based, time impact analyses can be performed as well as resource-driven impacts on a project in a faster and more accurate fashion. Impacts of delays, undermanning and unbalanced quantities of squads or resources can be directly 
and promptly assessed on the project completion date. The user enjoys a considerable advantage in assessing these impacts, which should help him prove delays and make a more sensible utilization of float.

$\mathrm{CPM} / \mathrm{LOB}$ users have access to the many features available in contemporary software for project planning: multiple calendars, resource aggregation, constraints, summary of activities and graphics and reports. Moreover, a repetitive project network can be as big as a planning software package and a computer can handle and still be manageable without having to reduce the number of activities in fear of the problems of out-of-sequence progress between the typical units. However, out-of-logic progress, which is progress that contradicts the logical relationships between activities within a typical subnetwork will be more distinguishable. No detail will be lost to the detriment of site personnel, yet all parties involved will still be able to judge the balance of the progress through the useful LOB chart which is now called CPM/LOB chart. Typical activities in delay along different branches of the typical unit network can be identified from the CPM/LOB chart as bottlenecks, while the CPM traditional output will indicate the delay of the project completion accurately.

Since the method harnesses the powerful features of contemporary CPM software it is expected that LOB will benefit from the future development of the CPM.

As will be demonstrated in chapter $9, \mathrm{CPM} / \mathrm{LOB}$ was successfully applied by the author on real life projects. 


\section{CHAPTER 7}

\section{COMMON ACTIVITIES IN LOB AND OTHER ISSUES}

\subsection{Introduction}

Most of the repetitive construction projects in real life are, by definition, large and complex. Their planning and scheduling need particular expertise. CPM/LOB has dealt with simplifying and overcoming the problems of work sequence changes and continuity. The flexibility of CPM/LOB puts the process of planning and scheduling repetitive projects in a clear perspective which facilitates additional enhancements. This chapter deals with the definitions and solutions of some essential and practical issues which evolved during the day-to-day management of a large housing project. As of this writing, some of these issues have not been mentioned or identified in the listed literature surveyed by the author.

\subsection{The Effect of Common Activities}

A common activity is one shared by many repetitive units as a result of the method of construction. Technological as well as economical demands may give birth to these common activities. A frequent example is backfilling the foundations of a cluster of attached houses. On a cluster of attached houses the foundations of each house may be independent but their backfilling has to be done for the cluster as a whole and can not be broken down into individual and independent backfillings for each house. Consequently, there could be multiple squads working on the foundations of a cluster of attached houses but only one squad working on the backfilling of the whole cluster after all the foundations of the individual houses have been completed.

Fig 7.1 shows an example of ten units, each has a typical network of four activities. Typical activity $\mathrm{C}$ has float in the typical unit network, however, it is a common activity in the overall network of the ten units. Units one to three share one common activity, C1-3. Units four to eight and nine and ten share common activities $\mathrm{C4-8}$ and C9\&10 respectively. It is noted that the type of work of activity $\mathrm{C}$ on the first, second and third units can not start until after the completion of activity $\mathrm{A}$ on the third unit. This is because typical activity $\mathrm{C}$ for units one, two and three are combined in one common activity: C1-3 which succeeds activities A1, A2 and A3. A delay in activity $\mathrm{C}$ may impact its successor activity $\mathrm{F}$ after consuming the float. In Fig. 7.1 


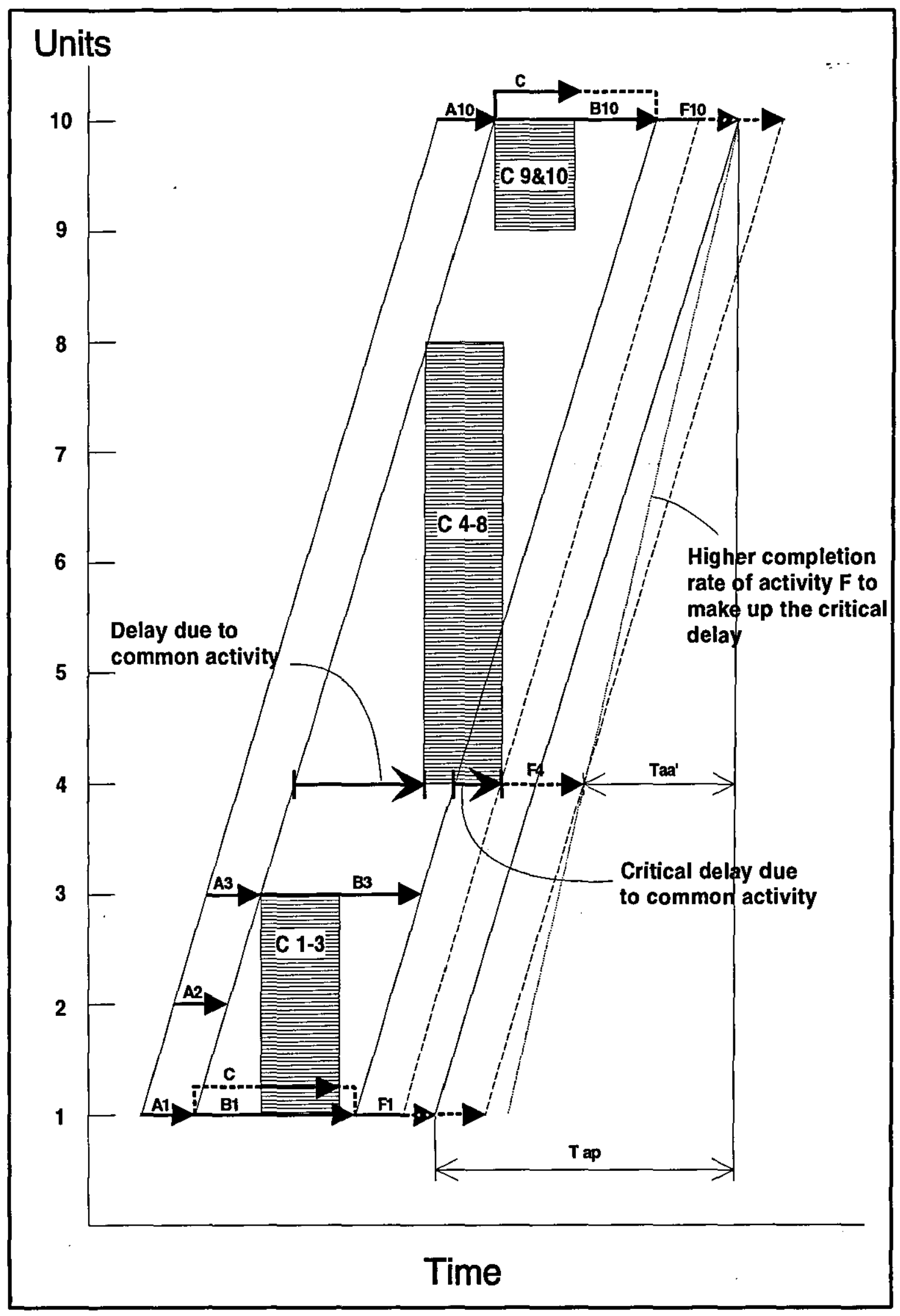

Figure 7.1 - Effect of Common Activities in Repetitive Projects. 
the two solid flow lines connecting activities F1 and F10 represent the starts and completions of this activity when no common activity effect exists. However, when activity $C$ should be worked on in clusters or groups, its impact has to be faced as a reality. Based on the rate of completions shown in the Fig. 7.1, it is clear that the largest cluster of common activity $\mathrm{C}$ has the biggest possible impact on activity $F$, the only successor. This impact is a delay solely attributed to the effect of a common activity. In this case, the impact of the common activity first affected activity F4. This delay of common activity $\mathrm{C}$ is offset by the amount of float available for typical activity $\mathrm{C}$ in the typical network.

The continuity of typical activity B is unaffected, it can run continuously. However, the flow of typical activity $F$ shall be interrupted on unit four. Alternatively, the start of typical activity $\mathrm{F}$ on the first unit has to commence at a latter time to maintain its continuity throughout the project. The two dashed flow lines on the right represent starts and completions of impacted activity F. In either case, continuous or interrupted flow of activity $F$, the whole project completion will be delayed by a duration equal to the critical delay induced by common activity C. It should be noted that typical activity B will have float equal to the delay in the start of its successor, activity F. Typical activity B may therefore follow a lower rate of completions due to the float generated by the effect of the common activity.

In most practical cases the project completion date is imposed. Therefore, the rate of completions of activity $\mathrm{F}$ in this case, and all the impacted activities by the common activity effect in general, have to be increased to make up the delay and finish the project by the imposed finish date. The delay can be calculated as

$$
D=\frac{\text { biggest number of units of a common activity - one }}{\text { rate of completions of project }}
$$

in which $\mathrm{D}$ is the delay due to the common activity.

If a common activity has float, then the critical delay $D_{c r t}$ can be calculated as

$$
D_{c r}=D-T F
$$

where TF is the total float of the typical activity in the unit network before becoming a common activity, and,

$$
D_{c r t} \geq z e r o
$$

Therefore, from equations (7.1) and (7.2) 


$$
D_{c t}=\frac{\text { biggest number of units of a common activity }- \text { one }}{\text { rate of completions of project }}-T F
$$

Consequently $R_{i c}$, the rate of completions of the impacted activities, in this case activity $F$, to finish the project on the imposed completion date, is

$$
R_{i c}=\frac{10-4}{T_{a a}{ }^{\prime}}
$$

where

$$
T_{a a}^{\prime}=\text { project duration }-\frac{4-1}{\text { rate of completion }}-A-D-C-F
$$

This aforementioned approach to the effect of common activities adopts a solution where the delay of the common activity is calculated from an initially determined rate of project completions before the effect of the common activity. Then, only the rates of the affected activities would be increased to make up the delay leaving the completion rates of the other activities unchanged.

Conceivably, a more practical approach would take the effect of the common activity into consideration as a base and starting point in the calculation of the rate of completions of the project. This can be accomplished by solving equation (7.4) and the following equation simultaneously

$$
R_{i p}=\frac{\text { total number of units - one }}{T_{a p}-D_{c n} \text { of common activity }}
$$

where $R_{i p}$ is the rate of completions of the impacted project. $D_{c r}$ is found in terms of the rate of completions from equation (7.4) then substituted in equation (7.7) to find the impacted rate of completions of the project.

\subsubsection{Example 7.1, Effect of a Common Activity}

Consider the first unit network of Fig. 7.2 where there are 10 units to be completed in 28 days. The following situations shall be examined:

1. Calculating the schedule where no common activities exist. 


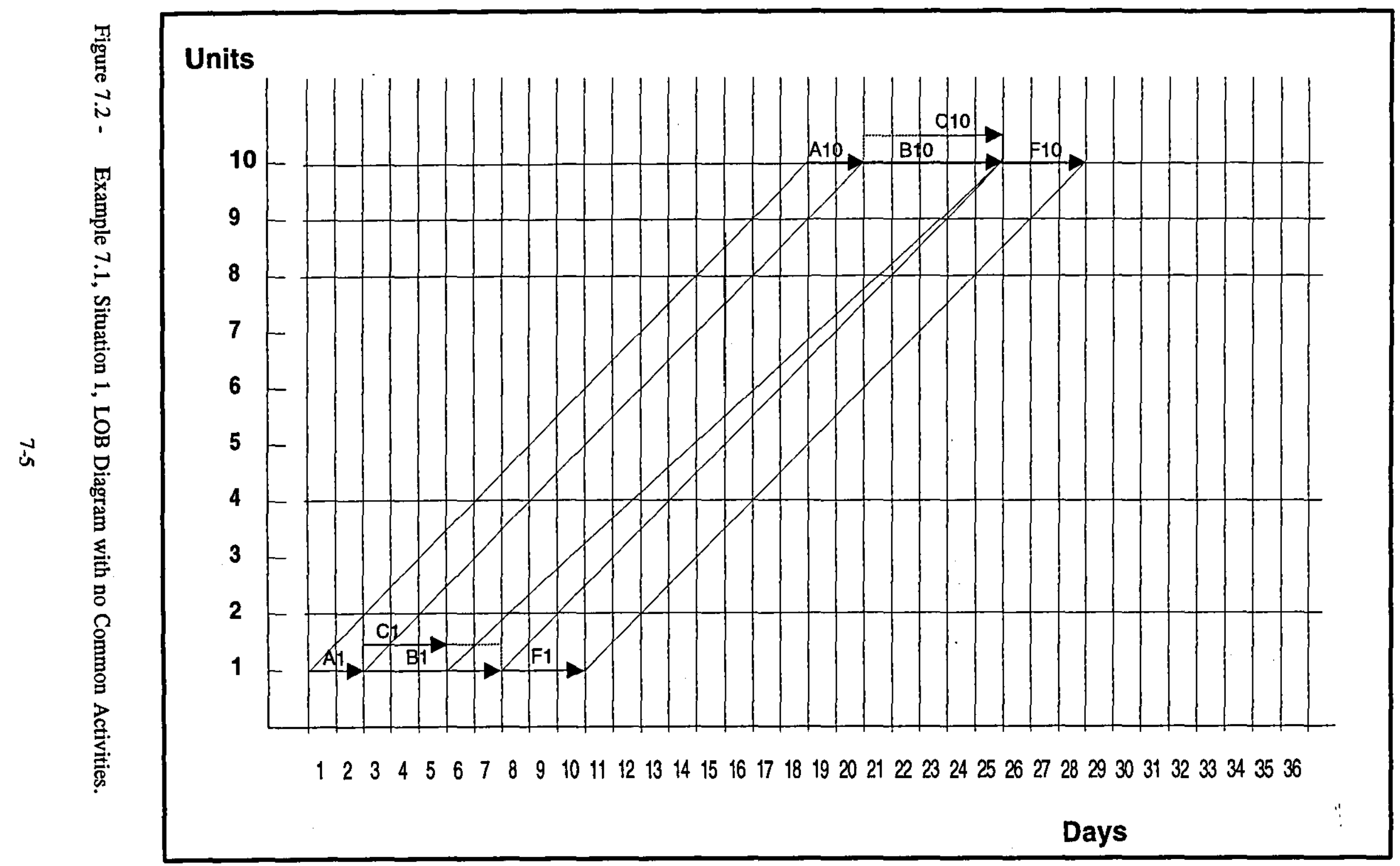


2. Combining typical activity $\mathrm{C}$ in 3 common activities: $\mathrm{C} 1-3, \mathrm{C} 4-8$ and $\mathrm{C} 9-10$ and accelerating only the affected activities.

3. Calculating the schedule based on the effect of common activities to start with.

\section{Situation 1, example 7.1}

$$
T_{\text {ap }}=28-10=18 \text { days }
$$

Fig. 7.2 shows the flow of the typical activities and a completion on day 28 . The quantities of squads are calculated in table 7.1.

\begin{tabular}{|c|c|c|c|c|c|c|}
\hline $\begin{array}{l}\text { Typical } \\
\text { activity }\end{array}$ & $\begin{array}{c}\begin{array}{c}\text { Resource } \\
\text { duration }\end{array} \\
\text { D } \\
\\
\text { (2) } \\
\end{array}$ & $\begin{array}{l}\text { Total } \\
\text { float } \\
\text { TF } \\
\text { (3) }\end{array}$ & $\begin{array}{c}\text { Available time for } \\
\text { completions } \\
T_{\mathrm{a}}= \\
\left\{\mathrm{T}_{\mathrm{ap}}+\mathrm{TF}\right\} \\
\text { (4) }\end{array}$ & $\begin{array}{c}\begin{array}{c}\text { Minimum rate of } \\
\text { completions }\end{array} \\
\mathbf{R}_{\mathrm{ma}}= \\
\left\{(10-1) \div \mathrm{T}_{\mathrm{u}}\right\} \\
\text { (5) }\end{array}$ & $\begin{array}{c}\text { Minimum } \\
\text { quantity of } \\
\text { squads } \\
S_{\mathrm{ma}}= \\
\left\{D \times R_{\mathrm{mad}}\right\} \\
(6) \\
\end{array}$ & $\begin{array}{l}\text { Rounded } \\
\qquad \mathrm{S}_{\mathrm{ma}} \\
(7)\end{array}$ \\
\hline A & 2 & 0 & 18 & 0.50 & 1.00 & 1 \\
\hline B & 5 & 0 & 18 & 0.50 & 2.50 & 3 \\
\hline $\mathrm{C}$ & 3 & 2 & 20 & 0.45 & 1.35 & 2 \\
\hline$F$ & 3 & 0 & 18 & 0.50 & 1.50 & 2 \\
\hline
\end{tabular}

Table 7.1 - Example 7.1, Situation 1, Calculation of the Quantity of Squads.

$S_{\mathrm{ma}}$ is entered into the CPM software then time scheduling and resource leveling is performed. The result is obtained in a bar chart from the CPM software which indicates completion on day 28 . Fig. 7.3 shows this bar chart. 


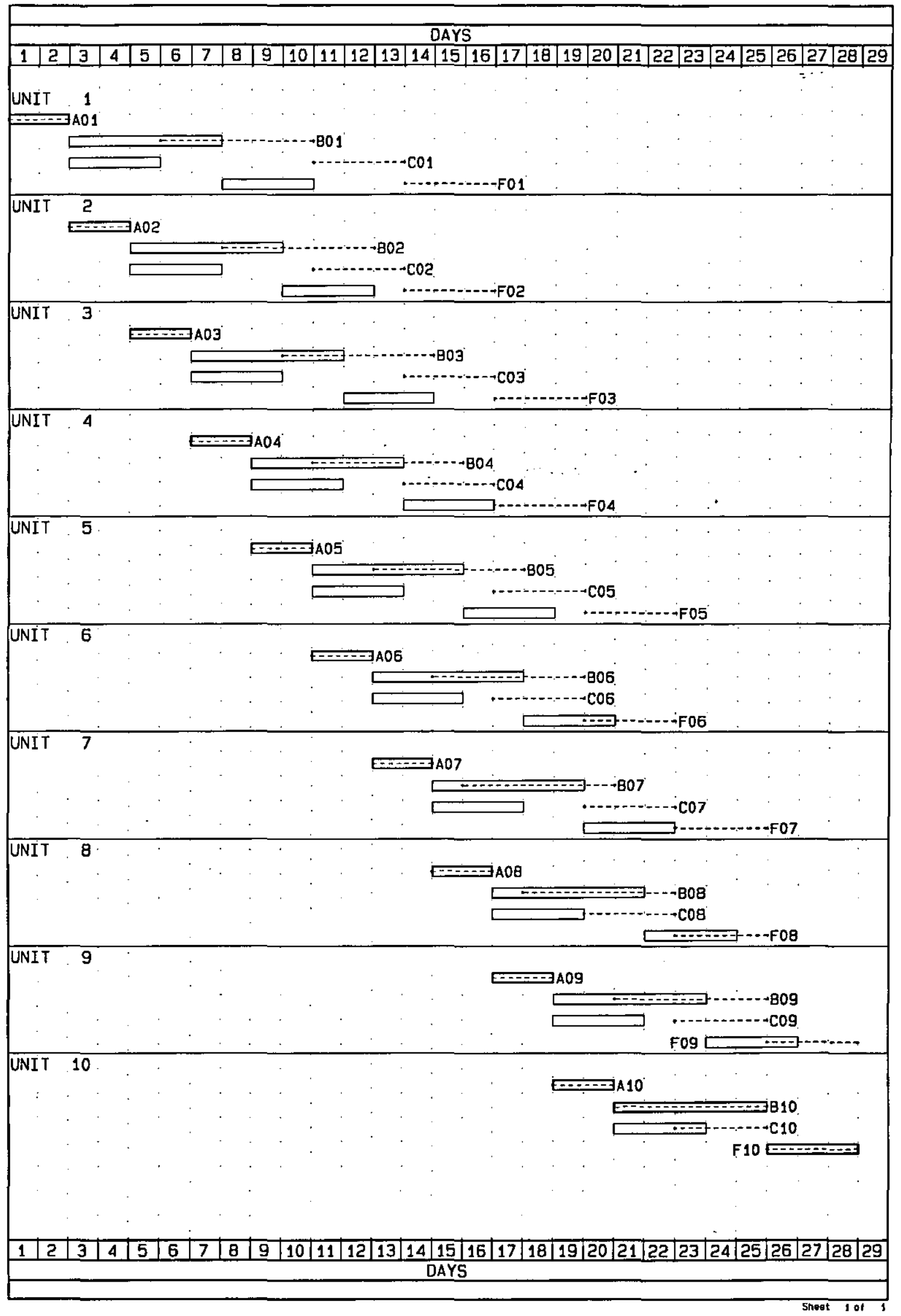

Figure 7.3 - Example 7.1, Situation 1, Bar Chart before Common Activity Effect. 


\section{Situation 2, example 7.1}

In formulating common activities from the network of situation 1, the new common activity C1-3 will take all the predecessors and successors of the deleted activities $\mathrm{C} 01, \mathrm{C} 02$ and C03. The same thing happens with C4-8 and C9-10 respectively. Fig. 7.4 schematically shows the arrangement of the activities of situation 2 after formulating the common activities.

The critical delay can be calculated using equation (7.4):

$\{(5-1) \div 0.5\}-2=6$ days

Fig. 7.5 shows the CPM software output. It is noted that the CPM output indicates a completion on day 31 , which means a delay of only 3 days instead of the 6 days shown in Fig. 7.4. The reasons for this gain are: activities follow a step line flow in the CPM according to the availability of the squads; and the rounding up of the quantity of squads. The step flow lines are closer to reality than the straight flow lines of the LOB diagram of Fig. 7.4.

The following step is accelerating affected activity $\mathrm{F}$ while maintaining its continuity. Fig. 7.6 shows the acceleration of typical activity F on a LOB diagram.

To accelerate typical activity $F$ in the CPM software an increase in its resource availability of squads must be calculated as follows:

from equation (7.6)

$\mathrm{T}_{2 \mathrm{a}}{ }^{\prime}=28-\{(4-1) \div 0.5\}-2-8-3-3$

$\mathrm{T}_{\mathrm{aa}}{ }^{\prime}=6$

and from equation (7.5)

$R_{\mathrm{ic}}=(10-4) \div 6$

$\mathrm{R}_{\mathrm{ic}}=1$

therefore, from equation (6.2) 


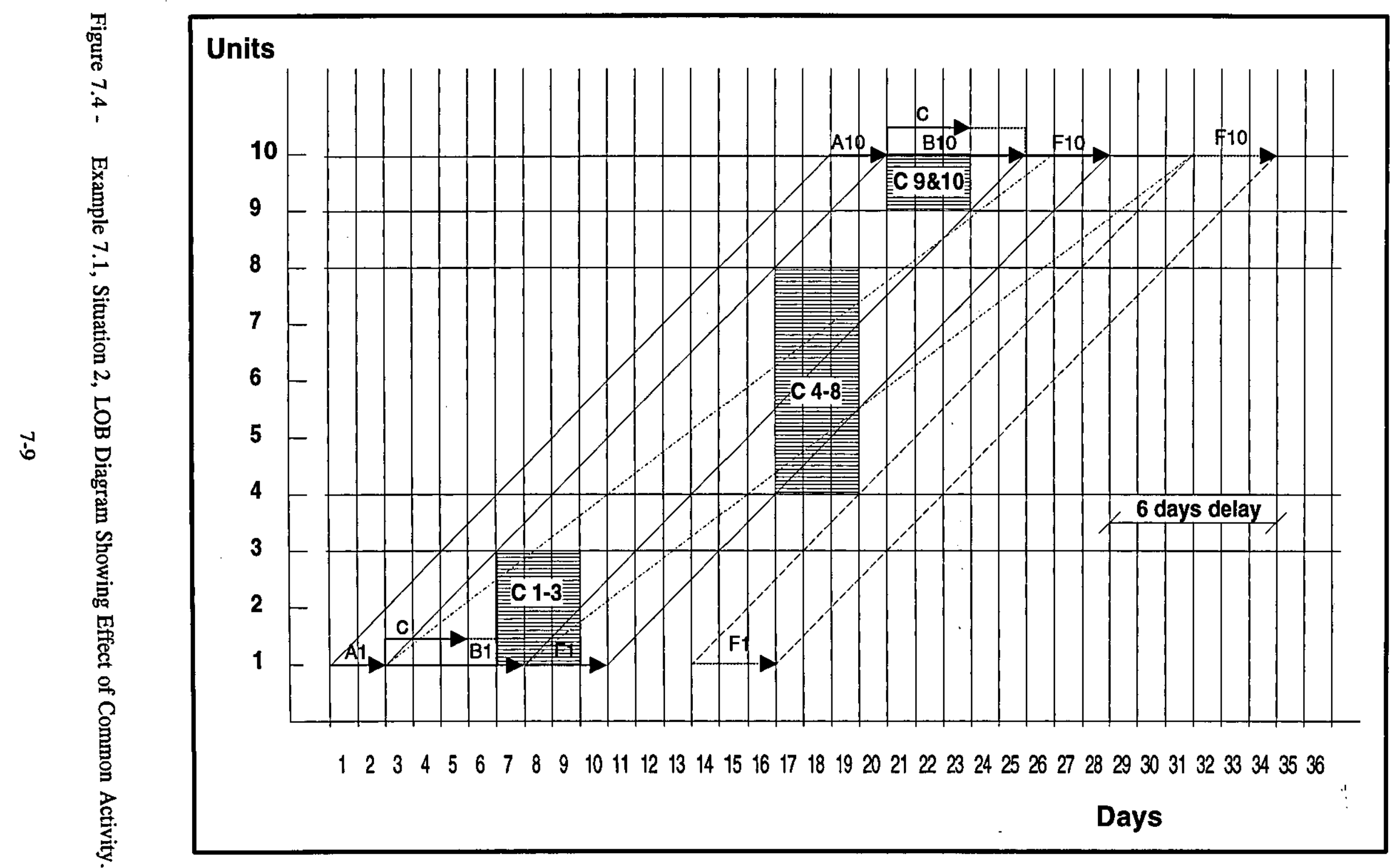


$S_{\mathrm{ma}}=R_{\mathrm{ic}} \times$ duration of resource on activity

$S_{\mathrm{ma}}=1 \times 3$

$S_{\mathrm{ma}}=3$

The availability of the squads for activity $\mathrm{F}$ in the CPM software is therefore increased from 2 to 3, then resource leveling is performed to get the results in Fig. 7.7. It is noted that the rate of typical activity $F$ in Fig. 7.7 is higher than those of the other activities to make up the delay. Typical activity $F$ was also prevented from starting before day 17 to maintain a practical continuity. 


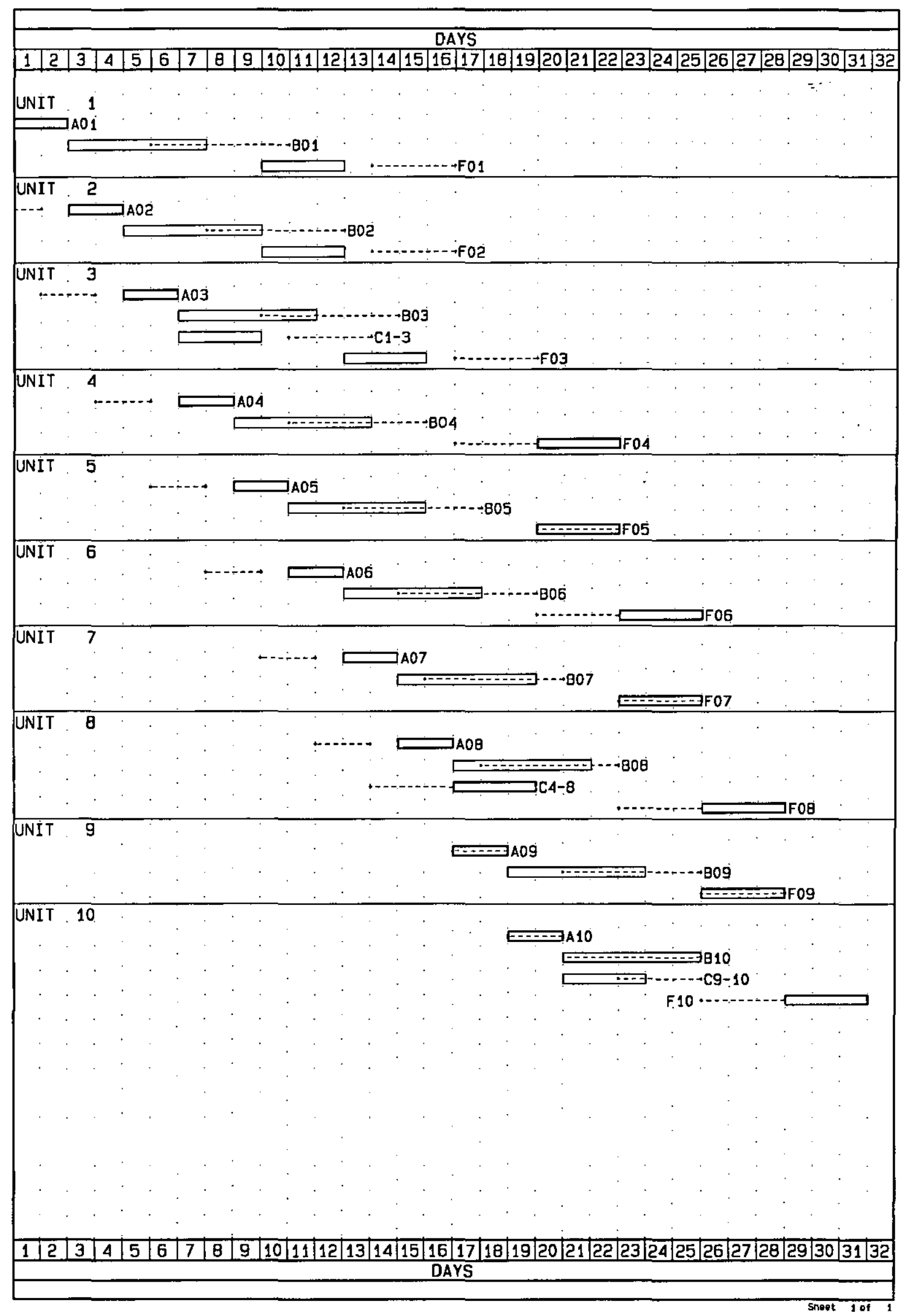

Figure 7.5 - Example 7.1, Situation 2, Bar Chart Showing Effect of Common Activity. 


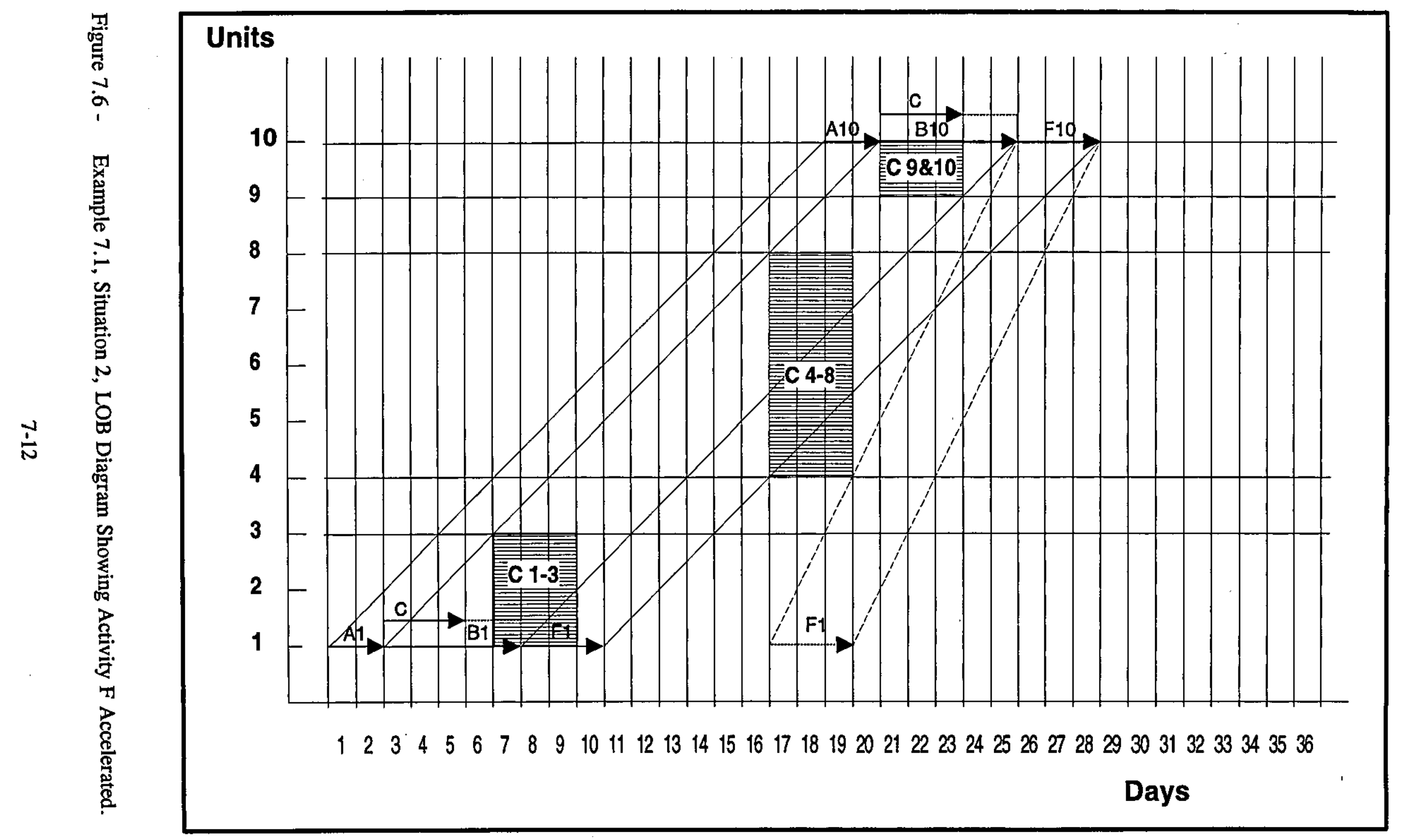




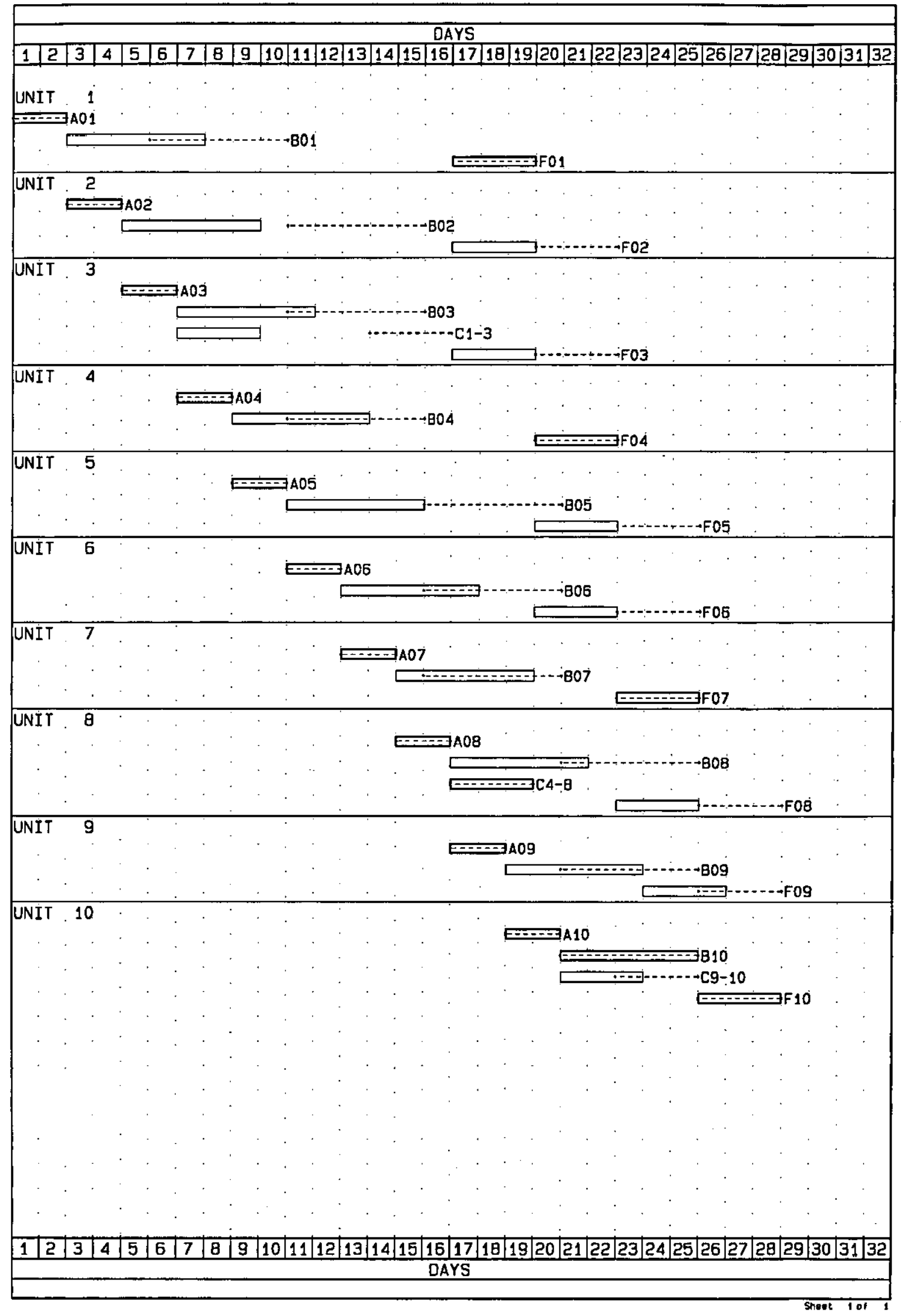

Figure 7.7 - Example 7.1, Situation 2, Bar Chart Showing Typical Activity F Accelerated. 


\section{Situation 3, example 7.1}

Through applying equation (7.4)

$D_{\text {crt }}=\left\{(5-1) \div R_{\text {ip }}\right\}-2$

and from applying equation (7.7)

$R_{\text {ip }}=(10-1) \div\left(18-D_{\text {crr }}\right)$

By solving these two equations we get

$R_{i p}=0.65$ units/day and,

$\mathrm{D}_{\mathrm{crt}}=4.15$ days

Fig. 7.8 shows situation 3 having all the project activities proceeding with one rate of completions (except for the common activities). Notice that because of the higher rate of completions, $D_{c r}$ is reduced from 6 to 4.15 days, and that the rates of completions are insensitive to the sequence of progress on the common activities. In effect, this approach of taking account of the common activity delay along with the rate of project completions is like assuming that the biggest group of common activities is started on first.

\begin{tabular}{|c|c|c|c|c|c|c|}
\hline $\begin{array}{l}\text { Typical } \\
\text { activity }\end{array}$ & $\begin{array}{c}\begin{array}{c}\text { Resource } \\
\text { duration }\end{array} \\
\text { D } \\
\text { (2) } \\
\end{array}$ & $\begin{array}{l}\text { Total } \\
\text { float } \\
\text { TF } \\
\\
\text { (3) } \\
\end{array}$ & $\begin{array}{c}\text { Available time for } \\
\text { completions } \\
T_{w}= \\
\left\{T_{u p}+T F\right\} \\
\text { (4) }\end{array}$ & 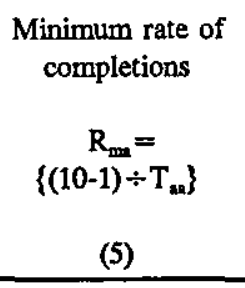 & $\begin{array}{c}\text { Minimum } \\
\text { quantity of } \\
\text { squads } \\
S_{\mathrm{m}}= \\
\left\{D \times R_{\mathrm{max}}\right\} \\
(6) \\
\end{array}$ & $\begin{array}{c}\text { Rounded } \\
\mathbf{S}_{\mathbf{m a}} \\
(7) \\
\end{array}$ \\
\hline A & 2 & 0 & 13.85 & 0.65 & 1.30 & 2 \\
\hline B & 5 & 4.15 & 18.00 & 0.50 & 2.50 & 3 \\
\hline $\mathrm{F}$ & 3 & 0 & 13.85 & 0.65 & 1.95 & 2 \\
\hline
\end{tabular}

Table 7.2 - Example 7.1, Situation 3, Calculation of the Quantity of Squads. 


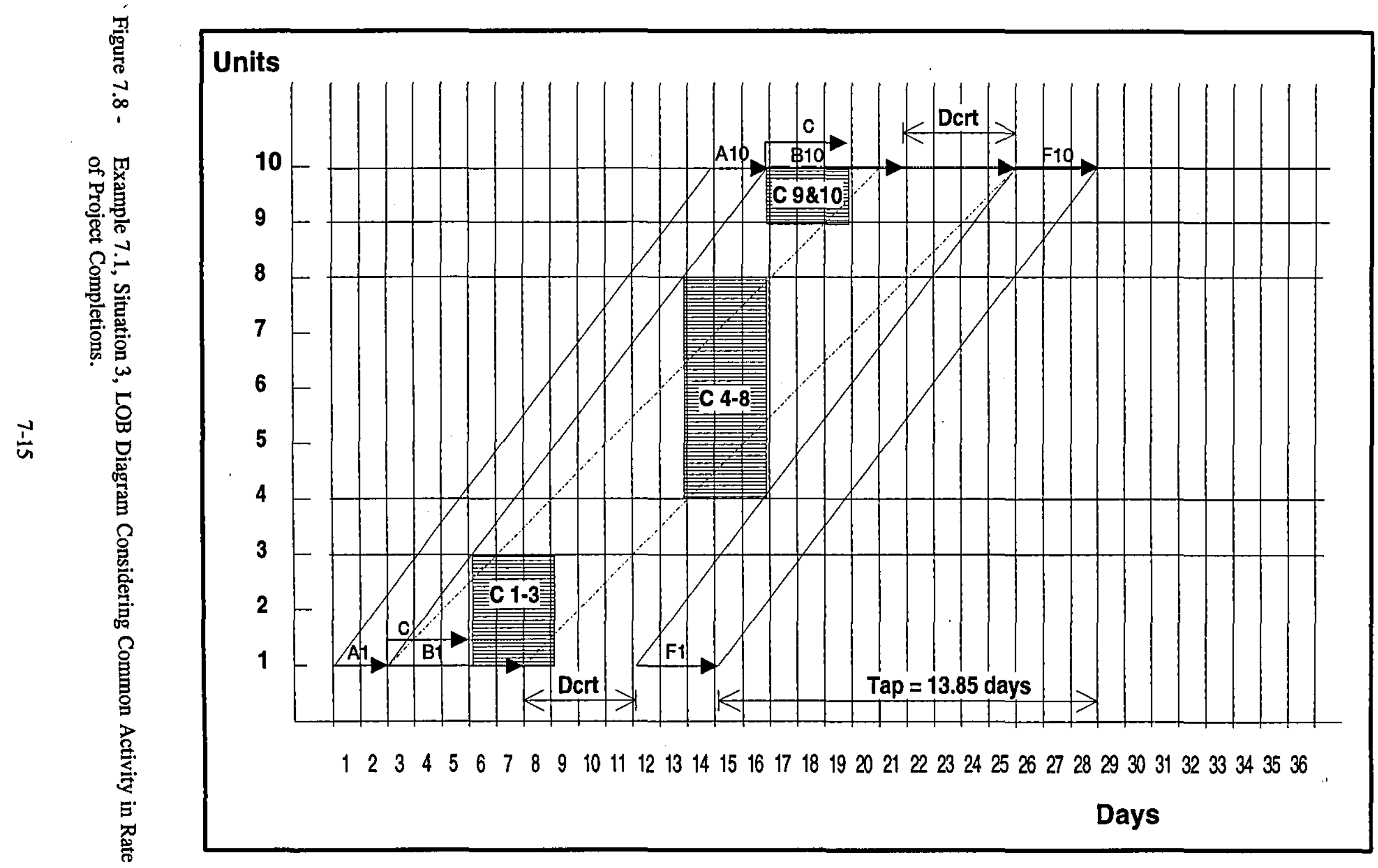


Table 7.2 shows the calculation of the quantity of squads that will be entered into the CPM software as resource availability.

It is noted that the critical delay generated float for typical activity B which may proceed on a lower rate of completions without affecting the project completion.

Fig. 7.9 is the product of the CPM software after resource leveling the quantity of squads calculated in Table 7.2. Again an earlier completion by 3 days was realized on day 25 due to the rounding up of the quantity of squads and the step flow line of completions as opposed to the theoretical straight flow lines of Fig. 7.8.

The previous description and example illustrated how common activities impact the schedule of repetitive projects. By and large, it is therefore understood that if two repetitive projects have everything equal but the existence of common activities, the project with common activities is expected to require a higher rate of project completions thus more squads. The same notion is valid if both projects have common activities but the biggest cluster of common activities in one of them is smaller than the biggest cluster of common activities in the other one. The project having the largest cluster of common activities will have the bigger delay from the effect of common activities.

A favored situation, has been grouping some activities for execution in one pass in anticipation of cost savings. This creates somewhat artificial common activities that may affect the whole project time progress. The author recommends a what-if analysis to compare the cost outcome of both situations. As demonstrated, speedy what-if analyses are possible with CPM/LOB.

Site supervision personnel sometimes, for various reasons, create common activity effects when they prefer or insist on grouping the inspection and approval of some otherwise isolated activities. It is therefore the author's opinion that construction parties should be aware of the consequences of common activities to deal with it in a fair way. 


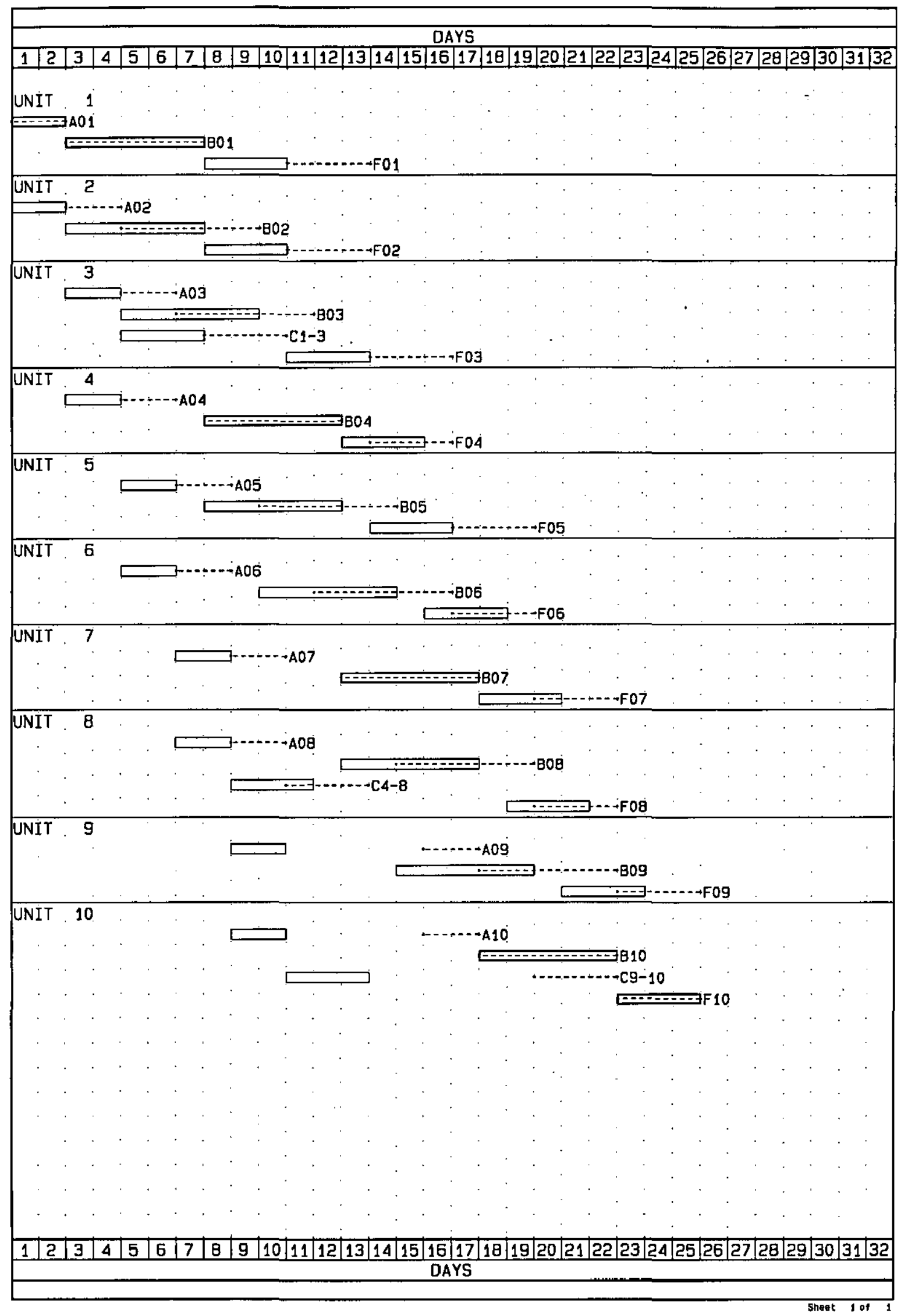

Figure 7.9 - Example 7.1, Situation 3, Bar Chart with a Revised Rate of Project Completions due to Common Activity. 
Inadequate or unbalanced quantities of resources can lead to delays and unnecessary costs. Balancing the quantity of resources available to the project activities is only one side of the issue. There are activities that require a combination of resources; e.g., a concrete roof. This activity needs a squad of Iron workers, carpenters, electricians, plumbers, and a shutter. Balancing the quantity of resources available within such an activity is vital for its economical execution and continuity in addition to the effect on the whole project schedule.

To illustrate this issue, take a housing project with the repetitive activity "Concrete roof slab" that has a duration of 22 days from start of setting up the shutter to the completion of stripping the shutter. The work duration of the carpenters squad is 11 days and concreting takes a day thereafter. The shutter must be available on the first day and must remain 10 days after concreting, that is a total of 22 days. The issue of calendar and workdays is ignored for clarity of illustration. Assume the total quantity of independent concrete roof slabs is 10 and the minimum rate of completions of the activity is 0.182 roof/day. The minimum required quantities of shutters and carpenter squads are calculated using equation (6.2)

$$
\begin{aligned}
& S_{\mathrm{ma}} \text { for shutters }=0.182 \times 22 \\
& \mathrm{~S}_{\mathrm{ma}} \text { for shutters }=4 \text { sets } \\
& \mathrm{S}_{\mathrm{ma}} \text { for carpenters }=0.182 \times 11 \\
& \mathrm{~S}_{\mathrm{ma}} \text { for carpenters }=2 \text { squads }
\end{aligned}
$$

The activity now has balanced resources and should progress at 0.182 roof per day. Fig. 7.10 shows a bar chart and a stack histogram for the usage of these two resources. The last activity "Roof10" finishes on day 66.

The project manager may want to accelerate the rate of completions of the roof slabs by adding another squad of carpenters. Fig. 7.11 shows the effect of this increase and that no earlier completion is possible when increasing the carpenter squads from two to three. This is because the unchanged four shutter sets limit the rate to 0.182 roof/day. The theoretical rate of the carpenter squads is 


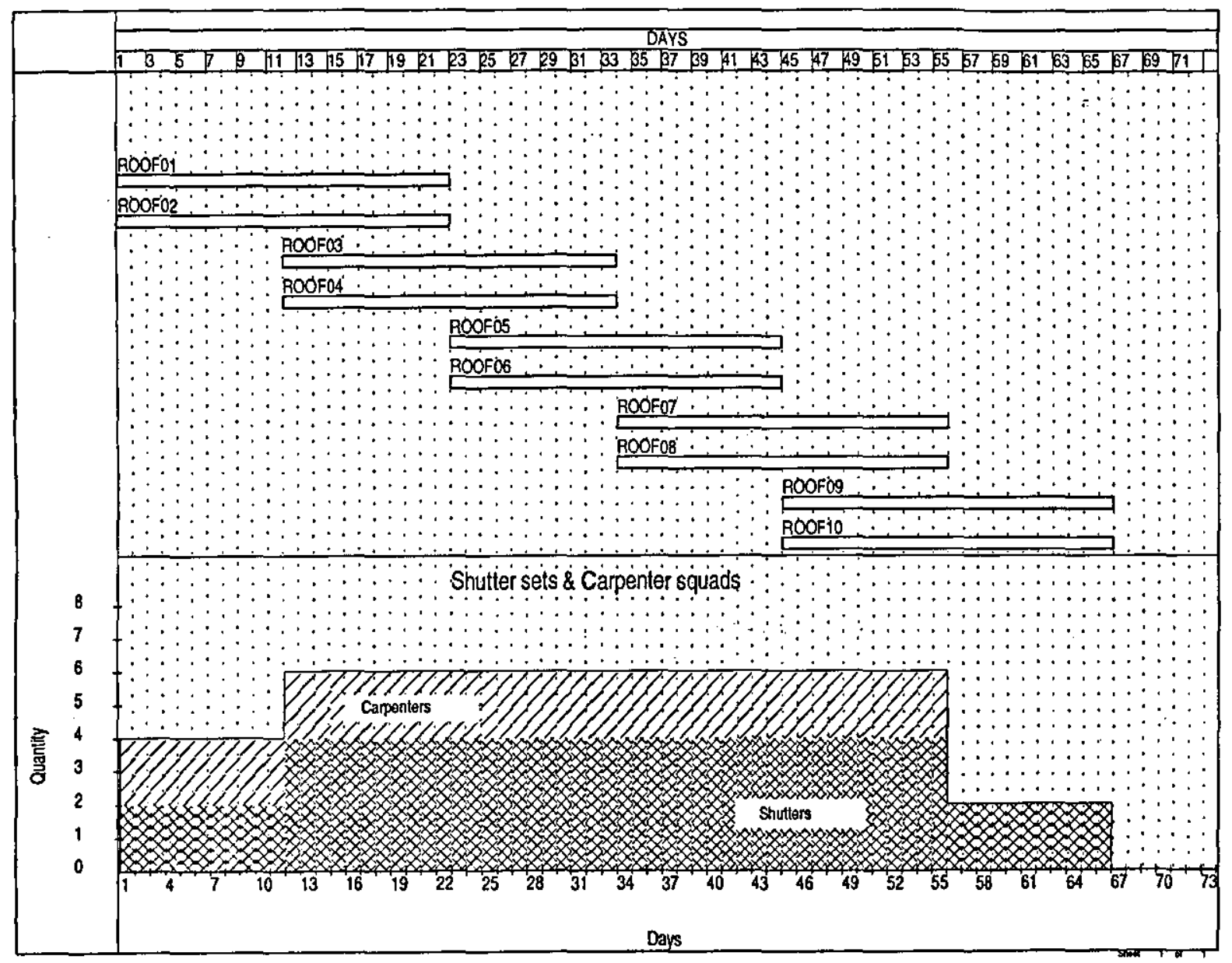

Figure 7.10 - Four Shutter Sets and Two Carpenter Squads Performing a Roof Activity at 0.182 Roof/Day.

$\mathrm{R}$ carpenters $=3 \div 11$

$\mathrm{R}$ carpenters $=0.273$ roof $/ \mathrm{day}$

Obviously the combination of four shutter sets and three carpenter squads is unbalanced because the third squad of carpenters is redundant.

A proportional increase of shutter sets and carpenter squads shall maintain the balance and improve the utilization of these resources. Fig. 7.12 shows the outcome of increasing the shutter sets from four to six thereby matching the potential rate of three carpenter squads

$R_{m}$ shutters $=6 \div 22$

$R_{\mathrm{m}}$ shutters $=0.273$ roof $/$ day 


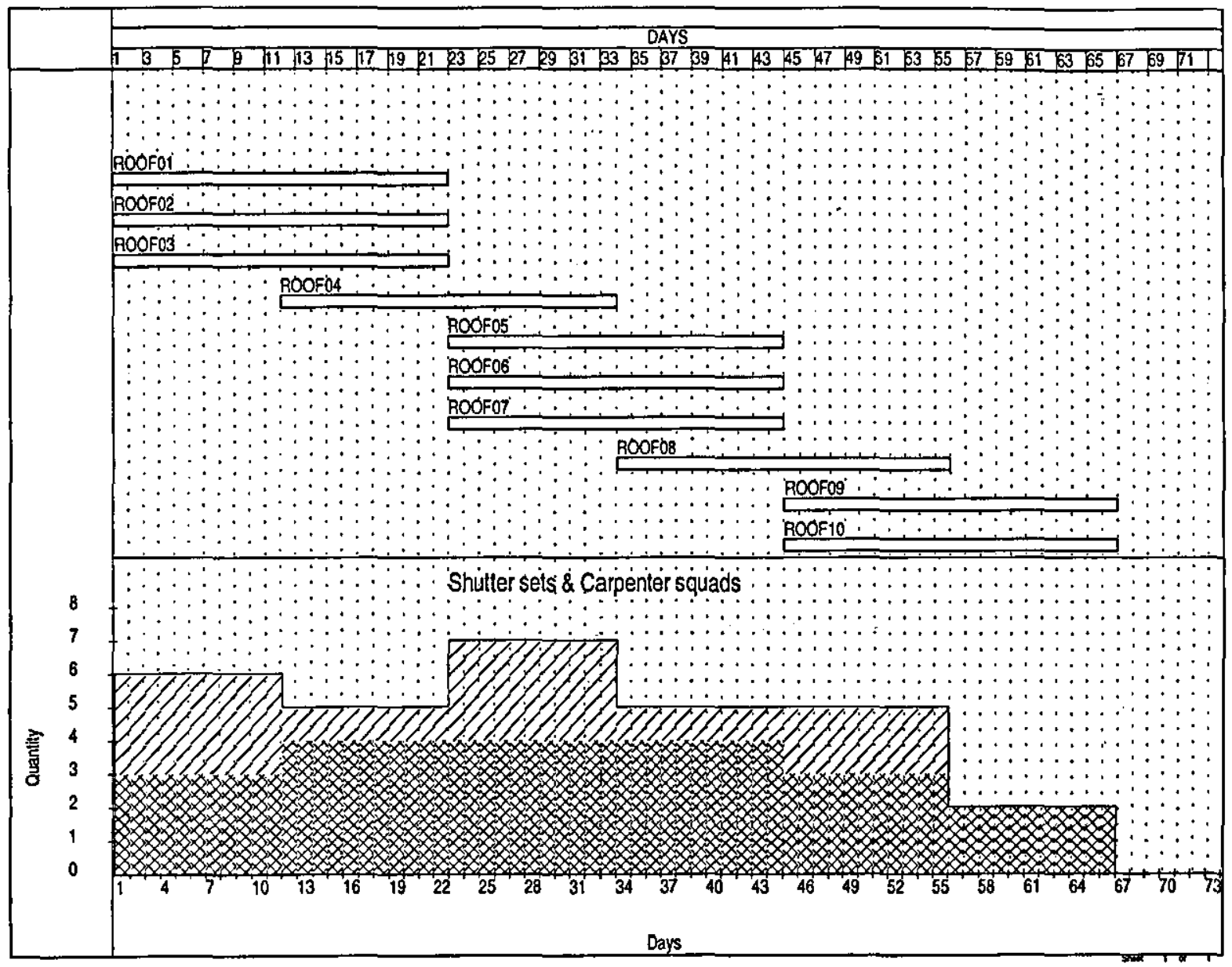

Figure 7.11 - Roof Activity with Unbalanced Resources, Four Shutter Sets and Three Carpenter Squads.

An earlier completion on day 55 is realized at a rate of 0.273 roof/day by increasing both resources $50 \%$.

A peculiar situation may exist here that deserves stopping at and examining. Keeping three carpenter squads but reducing the shutter sets from six to five still achieves the same early completion, day 55. So is the sixth shutter set redundant? Fig. 7.13 shows that, and shows that although the completion occurred on day 55 with only five shutter sets, the overall rate of completion of 0.273 roof/day was not maintained. Roofs 06 and 09 were late and therefore violated the required minimum rate of completions of 0.273 roof/day. This may have a negative impact on the following activities on units 06 and 09 . This impact is definite if the successor activity utilizes one resource thereby following a most vulnerable straight flow line as opposed to a step flow line of an activity performed by more than one squad. 


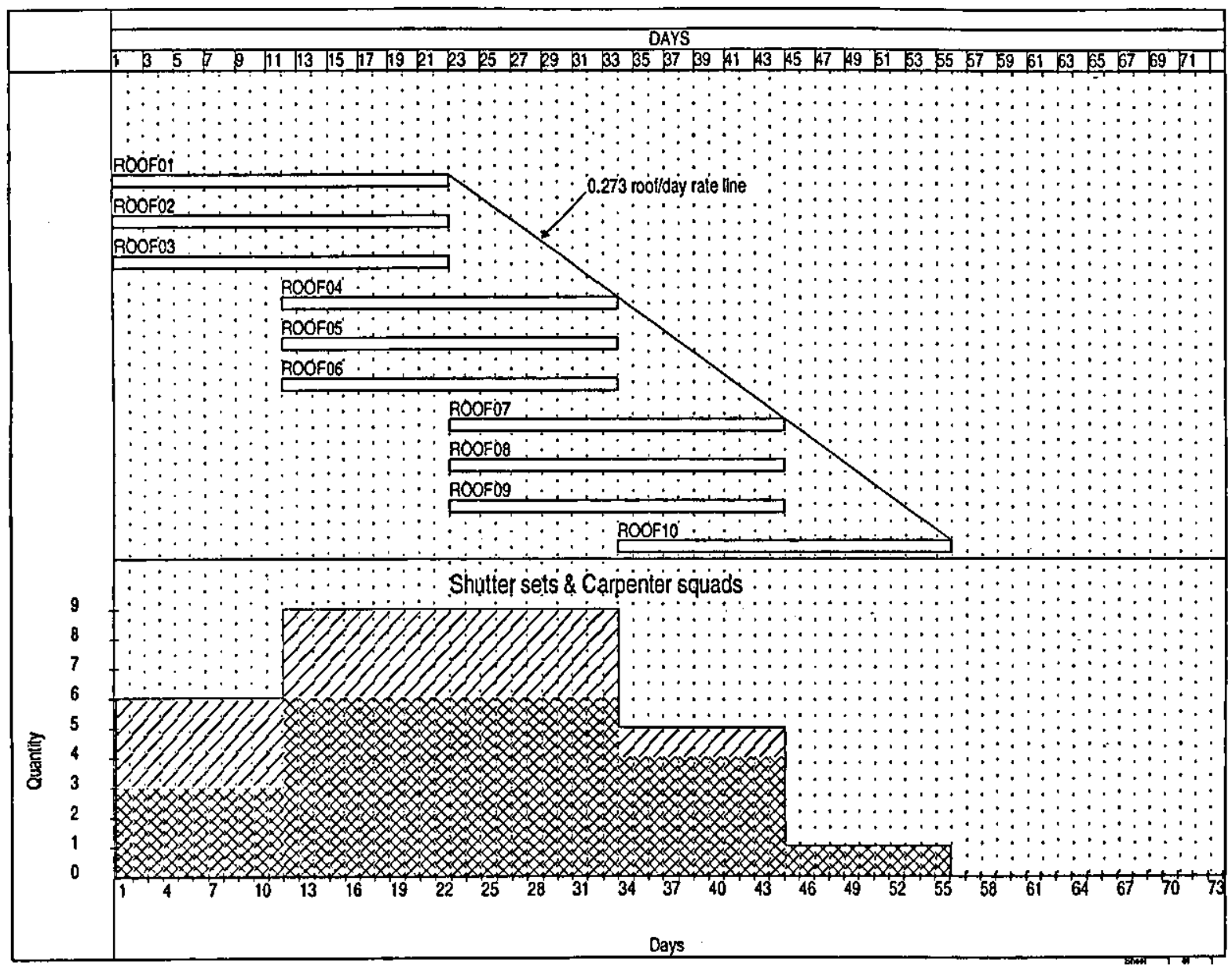

Figure 7.12 - Balanced Six Shutter Sets and Three Carpenter Squads at a Higher Rate of 0.273 Roof/Day.

\subsection{Identifying and Quantifying Out-of-Logic Progress}

The actual progress of activities in CPM often contradicts the predecessor/successor relationships between these activities due to either imperfections in the original plan or subsequently preferred alternatives to circumvent real life complications. Logcher (1989) mentioned that in practice, work was invariably done out-of-sequence and that determining new logic with out-of-sequence progress is a difficult issue.

Out-of-logic progress, although a major potential source of problems in construction, did not attract professionals to confront it in a formalized approach because of its ostensible complexity. Baram (1994), recommended that this issue be taken very seriously in the context of construction delays and disputes. Historically, identification has been accomplished by visual comparison with varying qualities of results. 


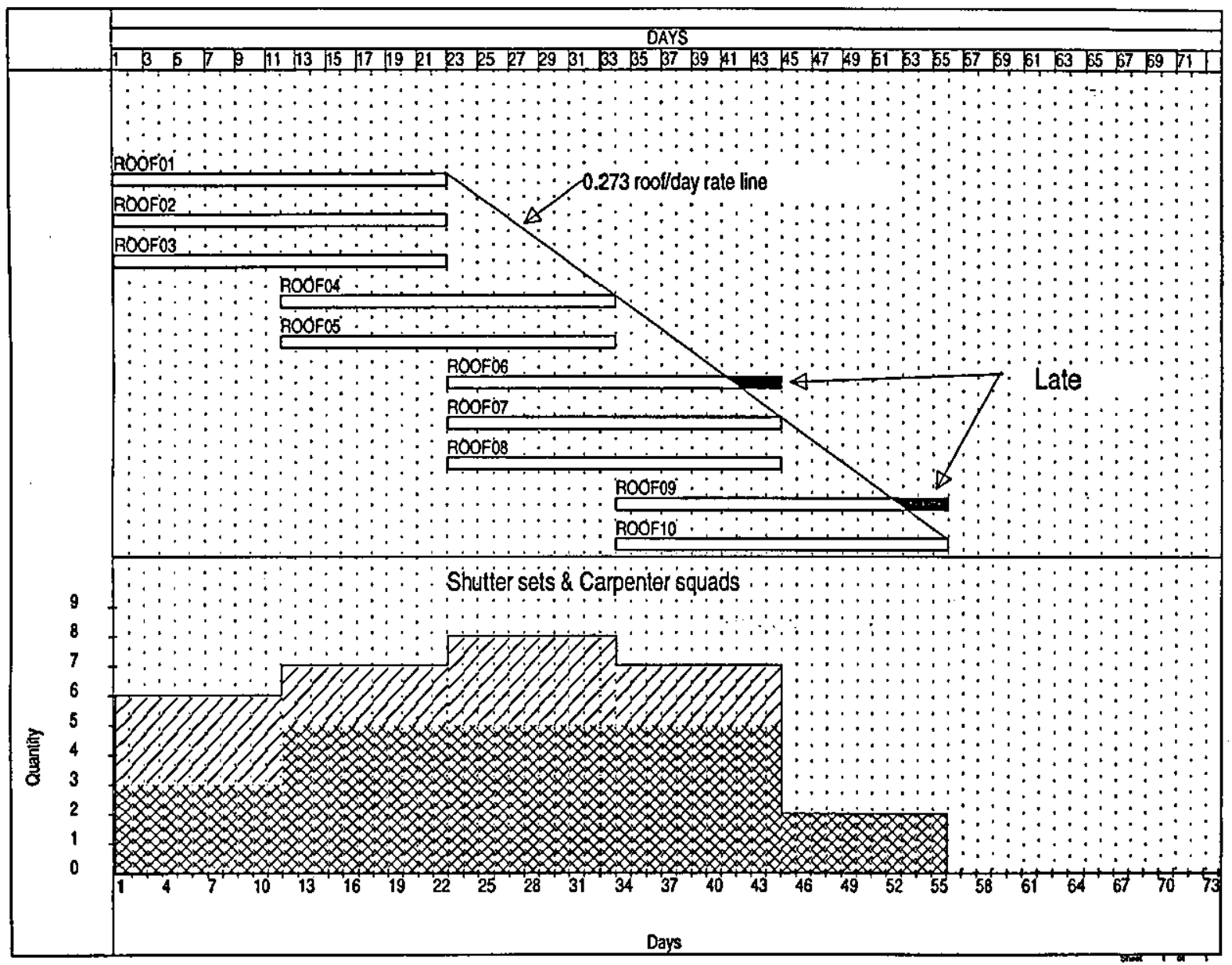

Figure 7.13 - Unbalanced Resources Violating the Minimum Rate Despite Completing on Day 55.

Since the relationships between the CPM activities represent an agreement between the parties involved on the method and logic of the work to be carried out, their violations need identification and quantification in a reasonably fast and easy way. One of the known related works to this issue is offered by some CPM computer packages where only limited exposure to the out-of-logic progress is enabled.

This section presents a simple method for identifying and quantifying out-of-logic progress. The method centers around comparing the as-built dates of the activities with the original predecessor and successor relationships for compliance. The data is generated from the CPM software then taken into a spreadsheet for the eventual comparison and tabular presentation of results. The details of the method can be best explained by the following example. 


\subsubsection{Example 7.2, Out-of-Logic Progress}

The example chosen to demonstrate the method is on constructing a typical wooden house with some external brick walls (see Fig. 7.14). Precedence Diagram was chosen, to show more than the traditional finish to start (FS) relationships the Arrow Diagram would have limited this example to. A seven workday week is assumed for simplicity and clarity of calculations.

It is assumed that the owner of the house has prepared this initial schedule after consultation with all the contractors. The initial schedule is approved and reflects the intention of each party on how and when to do his work in view of the work requirements of the others. For example, the drywall contractor made it clear from the beginning that he needs to deliver and stack the interior drywall inside the building using mechanized equipment before the external walls are closed by the sheeting contractor of activity $\mathrm{K}$. Otherwise he would be forced to unbundle the drywall and dismantle some of the internal walls in order to place the drywall in the appropriate locations on a piece-by-piece basis. Clearly this would take more time and incur more costs. According to this understanding the relationship between activity D DELIVERY OF DRYWALL and activity K EXTERNAL SHEETING AND COMPLETE WALLS is finish to finish (FF) with 3 days lag.

Due to the high possibility of rain damaging the insulation, the owner agreed with the insulation contractor that he would only start his insulation works after the waterproofing of the roof is completed, which is determined to be after seven days from starting the work on the roof, activity $\mathrm{N}$. Hence, in the initial schedule activity $\mathrm{N}$ precedes activity $\mathrm{G}$ by a start to start (SS) relationship with a seven day lag period. This is like saying that waterproofing will take seven days and shingles will take three days making the total duration of activity $\mathrm{N}$ ten days.

The same concept, avoiding rain damage, was applied when activity H DRYWALL, was planned to start after the completion of activity L WINDOWS \& EXTERNAL DOORS. In other words the relationship between them is finish to start (FS).

Activity O SIDEWALKS, was initially planned to start after the completion of the brick walls, activity $\mathrm{M}$, because the sidewalks surround the building and abut and cover the lower portion of the brick walls. 


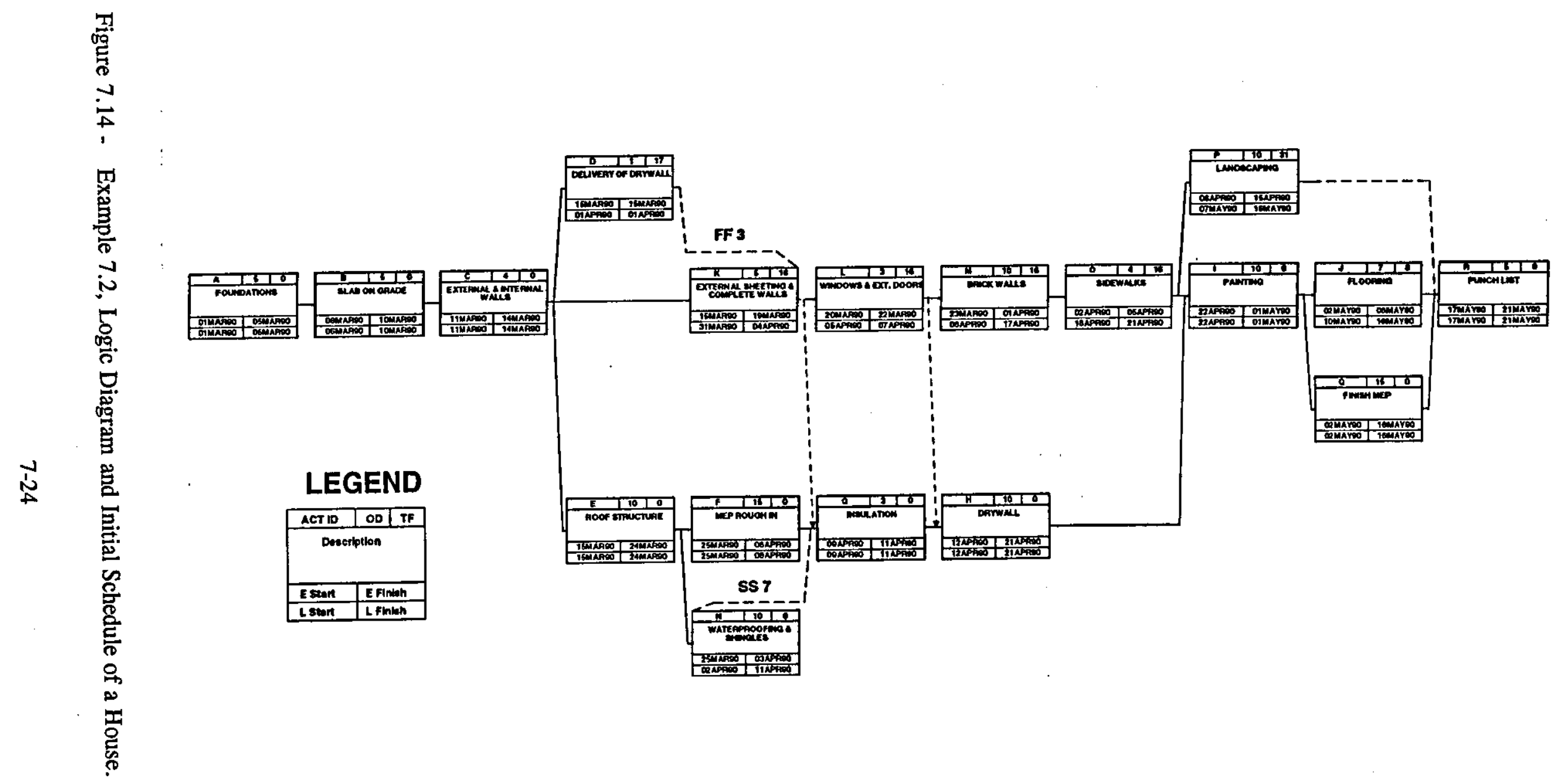


The owner, painting contractor and flooring contractor all agreed that flooring work was to start after the completion of the painting in order not to impede the movement of the painters and to protect the high quality flooring from the paint. The relationship between activity I PAINTING, and J FLOORING is finish to start (FS) with zero lag in line with the agreement. All other relationships in the initial plan are straightforward.

Construction started on March 2, 1990 and completed on May 31, 1990. Fig. 7.15 is an as-built bar chart of the house project that can be used along with the initial logic diagram of Fig. 7.14 to visually compare the actual progress of each activity to the actual progress of its predecessor in order to identify out-of-logic progress. Certainly, if this comparison is not difficult on this example it will be difficult on a large and troubled project. Alternatively the following method can be applied:

\begin{tabular}{|c|c|c|c|c|c|c|c|c|c|c|}
\hline \multicolumn{4}{|c|}{ ACTIVIT } & \multicolumn{6}{|c|}{ SUCCESSOR } & \multirow[b]{2}{*}{$\begin{array}{l}\text { DAYS OUT } \\
\text { OF LOGIC }\end{array}$} \\
\hline 10 & DESCAIPTION & $\begin{array}{l}\text { ACTUAL } \\
\text { START }\end{array}$ & $\begin{array}{l}\text { ACTUAL } \\
\text { FINISH }\end{array}$ & 10 & DESCAIPTION & $\begin{array}{l}\text { ACTUAL } \\
\text { STAAT }\end{array}$ & $\begin{array}{l}\text { ACTUAL } \\
\text { FINISH } \\
\end{array}$ & $\begin{array}{l}\text { LAO } \\
\text { OAYS }\end{array}$ & REL & \\
\hline A & FOUNDATIONS & 2-Mer & 6-Mar & $\mathbf{B}$ & SLAB ON GRADE & 7-Mur & $12+\mathrm{Mr}$ & 0 & F9 & 0 \\
\hline$\hat{B}$ & SLAB ON GRADE & 7-Mar & 12+Mer & c & EXTERNAL \& NTEANAL WALLS & 14Am & $17+\mathrm{Mer}$ & 0 & Fs & -1 \\
\hline C & EXTEFNAL \& NTEPNAL WAUS & 14ther & 17+har & D & DELNEAY OF ORYWALL & $2 a+M$ & 2entrer & 0 & FS & -4 \\
\hline c & EXTERNAL \& NTERNAL WALLS & 14 Mar & $17+\mathrm{Mur}$ & $\mathbf{E}$ & ROOF STRUCTUAE & 19-Mar & $28+$ Mer & 0 & fs & -1 \\
\hline c & EXTERNAL \& NTEFNAL WALS & $14+\mathrm{Mar}$ & 17+Mar & $\mathbf{K}$ & EXT. SHEETING \& COMPLETE WALLS & 18 Mer & 21+Mer & 0 & FS & $\mathbf{0}$ \\
\hline $\mathbf{E}$ & ROOF STRUCTURE & towher & 26-Mar & $\mathbf{F}$ & MEP ROUGH IN & 27 Mar & 16-Apr & 0 & FS & 0 \\
\hline E & ROOF STRUCTUAE & 19+Mar & 26-Mer & $\mathbf{N}$ & WATERPROOFING \& SHINOLES & $12-A \times x$ & 19-Ap & 0 & FS & .18 \\
\hline $\mathbf{K}$ & EXT. SHEETINO \& COMPLETE WALLS & 18-Mar & $21+$ er & a & INSLCATION & 14 Apr & 18-Apr & 0 & FS & 23 \\
\hline $\mathbf{k}$ & EXT. SHEETING \& COMPLETE WALLS & 18-Mer & 21 ther & 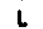 & WINDOWS \& EXT. DOORS & 22+Mer & $25+10$ & 0 & Fs & 0 \\
\hline$D$ & DEUVERY OF OANWHA & $22-1+4$ & $2 a-w$ ir & x. & OXT SHEEMNO 1 CONPLATE WOLS & $10 \mathrm{mo}$ & 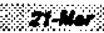 & 3. & H & \% \\
\hline$L$ & WNNOOWS \& EXT. 00ORS & 20 Mar & $25+1 \mathrm{er}$ & H & DRYWALL & 10Apr & 28 Apr & 0 & $\mathbf{F 8}$ & 24 \\
\hline $\mathrm{t}$ & WINDOWS \& EXT. DOORS & $\overline{2}-\mathrm{Mar}$ & 25-Mar & $M$ & BRICK WALS & $1-A p p$ & 14 Apr & 0 & FS & 6 \\
\hline in & BRICK WALs & lapo & $14 \mathrm{Apr}$ & o. & SIOEWALKS & 10 Hor & 16 npr & 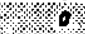 & FS & 8. \\
\hline F & MEP AOUOH WW & $27-$ mar & 16-Apr & a) & MSULATON & 14.4pr & roxprs & 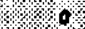 & Fs & 9 \\
\hline$N$ & WATERPROONNO 1 SHINGLES & $12-1 p$ & 1-Apr & a. & nSULATHOH & 1410 & $10 \times p \%$ & 8 & ss. & 6 \\
\hline 0 & SIDEWALKS & 10.Apr & 15-Apr & 1 & PAINTING & 29-Apr & 12-May & 0 & FS & -13 \\
\hline 0 & SIDEWALKS & 10-Apr & 15-Apr & $p$ & LANDSCAPING & 16-Apr & 24Apr & 0 & FS & 0 \\
\hline $\mathbf{p}$ & LANDSCAPINO & 16-Apr & 24Apr & ค & PUNCH LIST & 26-May & 31-May & 0 & FS & 21 \\
\hline 0 & INSLYLATION & 14Apr & 18-Apr & H & DRYWALL & $19-A p x$ & 28.Apr & 0 & Fs & 0 \\
\hline $\mathbf{H}$ & DAYWALL & 12-Apr & 28-Apr & 1 & PAINTING & 29.Apr & $12 \cdot M a y$ & 0 & FS & 0 \\
\hline o & PANTNO: & $26 A_{p o}$ & \%onor & 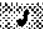 & FLOORNG & ons & How & 8. & rs. & s. \\
\hline 1 & PAINTING & 29-Apr & 12-May & 0 & FINISH MEP & 13-Moy & 25-May & 0 & Fs & 0 \\
\hline a & FINISH MEP & 13-May & 25-May & ค & PUNCHEIST & 26-May & 31- May & 0 & FS & 0 \\
\hline$j$ & FLOOAING & B-May & 15-May & A & PUNCHLIST & 26-Mey & 31-May & 0 & FS & -10 \\
\hline
\end{tabular}

Table 7.3 - Example 7.2, Out-of-Logic Analysis.

Table 7.3 lists all the network activities with their actual start and finish dates. Also in this table are the successors of each activity and the type of relationship and lag between them according to the initial schedule, so that if an activity has more than one successor it will be listed on more than one line, each line describing it with one successor and the relationship between them. All the aforementioned data can be easily produced by the Export facility of the computer program used, Primavera Project Planner ${ }^{\oplus}$ to a spreadsheet where further calculations can be 


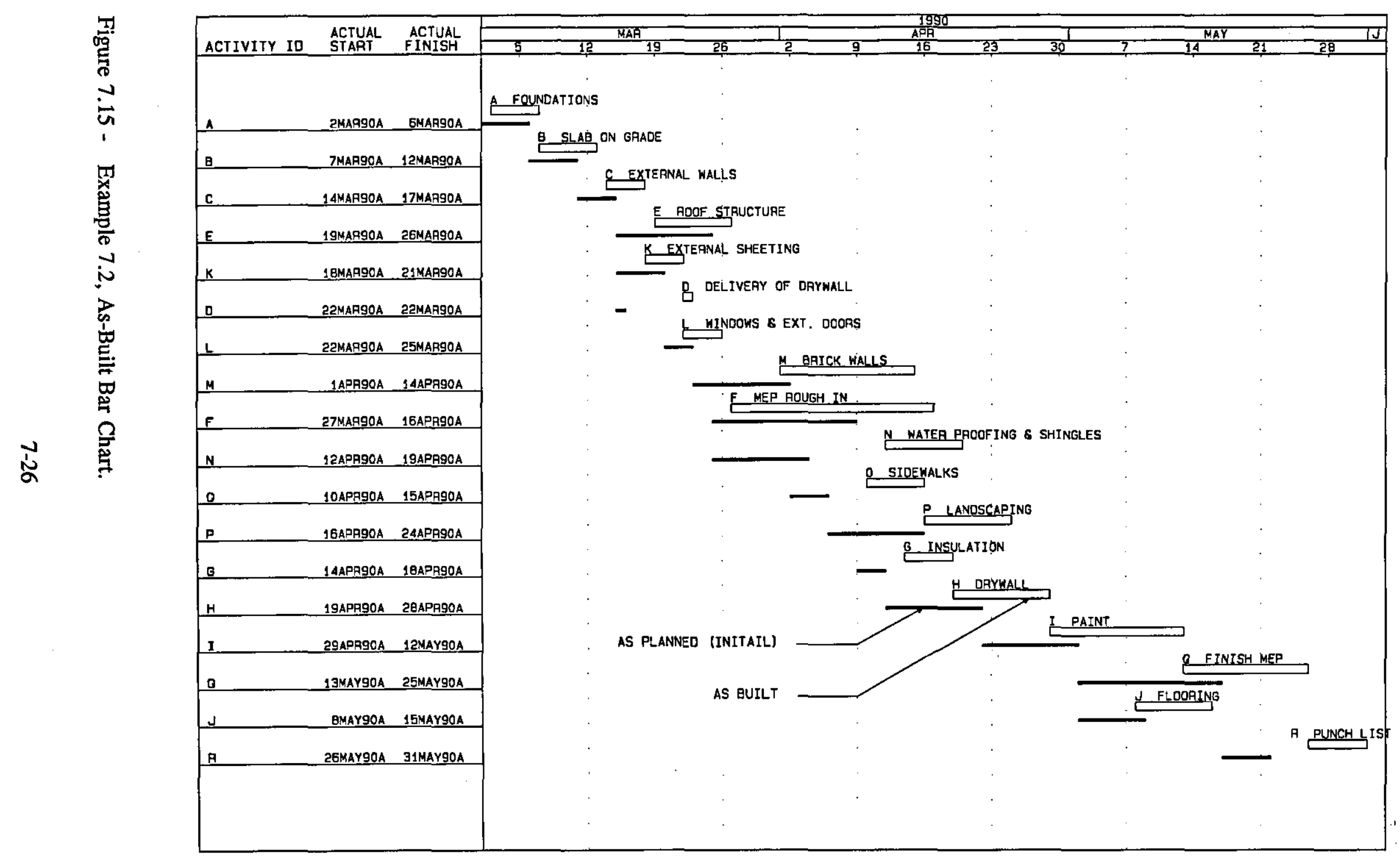


applied conveniently.

The right most column in Table 7.3 shows the days out-of-logic. These were calculated by a formula containing "IF" statements copied to all the cells of the column in the spreadsheet.

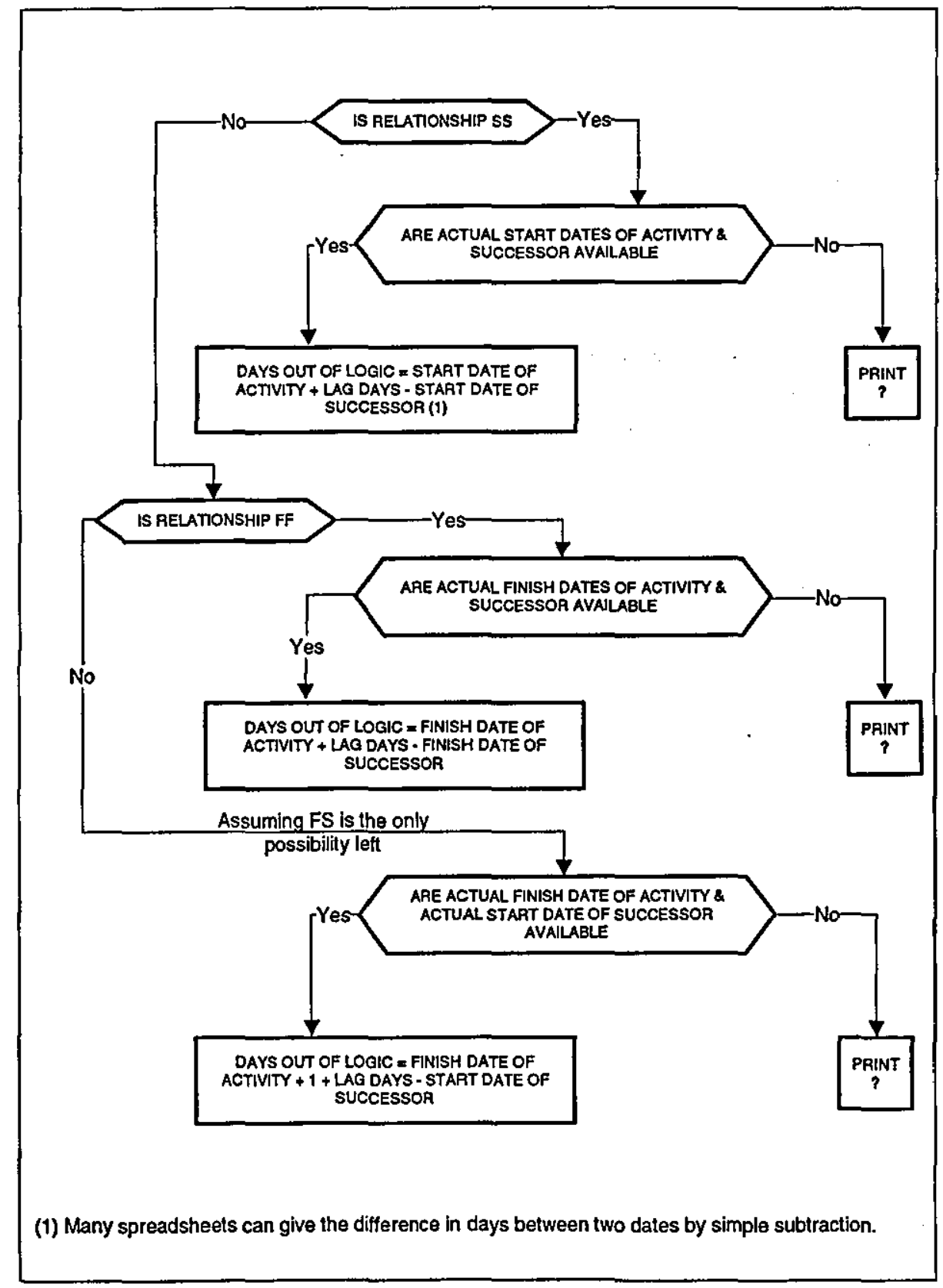

Figure 7.16 - Out-of-Logic Calculation.

Fig. 7.16 is a flow chart showing how these "IF" statements were arranged. They basically identify the type of the relationship then compare the relevant actual start/finish dates for compliance. 
The calculation is simple and straightforward. However, when a date is given as a start date it means the beginning of that particular day, and when a date is given as a finish date it means the end of that day. So for activity D DELIVERY OF DRYWALL, March 22, means the beginning of the day under the actual start column and the end of the day under the actual finish column. For the same reason a correction of one day was necessary to make the calculation for the "FS" relationships consistent since start and finish dates are not comparable.

In Table 7.3, out-of-logic progress happened where positive values were the result of the calculation. The corresponding rows were shaded for more clarity; however, for a large project a sort by descending order of out-of-logic days is recommended. This type of sort can be easily done in a spreadsheet. The scheduler can easily zero in on the identified violations towards exploring the consequences and rectifying the situation.

Clearly, identifying and quantifying out-of-logic progress with this computerized method is fast, simple and less vulnerable to human error than a traditional visual comparison. The method can be applied even after agreed upon changes to the logic take place. This can be performed by comparing the current schedule update with the previous schedule update which incorporated the most recent and agreed upon logic changes.

On a repetitive project in general and particularly on housing projects out-of-logic progress points to serious problems. However, out-of-sequence progress, which does not violate the logic but contradicts the priorities of work order is frequently a strategy matter. CPM/LOB methodology and the method described herein can be employed together to effectively differentiate between the two types of progress and their consequences.

\subsection{The Problem of too many Uncompleted Repetitive Activities}

When a LOB chart shows that a certain quantity of an activity should be completed by a particular cut-off date it also means that there must be an additional quantity of the same activity that is underway at varying stages. This quantity of activities underway is necessary for keeping the progress on schedule, otherwise there will not be enough completions by the next cut-off date. The normal or planned quantity of activities underway is obtained from the difference between the line of balance of completions and the line of balance of starts at a given point in time. The quantity of too many uncompleted activities discussed in this section is above and beyond that 
necessary for the normal progress of work which is calculated from the difference between the quantities of line of balance of completions and the line of balance of starts.

Turban (1968) was the only person according to this author's knowledge that touched on this problem, he stated:

"Measurement of progress is especially difficult in development projects and in partly completed operations. Pure guesses are often the only approach."

A frequently encountered situation in repetitive projects is having too many repetitive activities started-on but not completed for various reasons. An example that was experienced by this author was with a subcontractor who had one unit rate price in his contract for all the concrete foundation works of many houses. Foundation works were bases and column necks, and were represented by one CPM activity called "Foundations". Foreseeing financial problems and possible termination of his contract, this subcontractor over progressed on the bases that had the bulk quantities of concrete, and obviously the money, and ignored the column necks. He was improving his cash flow however, the whole project was suffering. Later, on the same project and while repetitive activity "Brick cladding of external walls" was progressing, a crises happened on a previously completed and major project for the same owner: bricks of cladding walls which were resting on ground beams and going up two stories $(7 \mathrm{~m})$ were collapsing due to inadequate anchorage. The owner stopped the work on activity "Bricks cladding of external walls" beyond a three mete level pending correction of the design, which was to introduce additional anchorage between the cladding bricks and the concrete block unit walls being clad at that level and above. The main contractor expecting a speedy solution by the owner/designer did not stop his brick layers but simply moved them on to start on new houses after partially completing the cladding of each house (after reaching the $3 \mathrm{~m}$ level). Due to the owner inaction, two months later there was a severe problem with scaffold sets, brick layer squads and the succeeding activities.

The planner who periodically depicts the progress of the project on a LOB chart to detect bottle necks and imbalances, is challenged. How is he going to count and present these too many partially completed activities? should he ignore them, work out an equivalent quantity by adding up partially completed activities on top of each other or what?

Not counting them is certainly an understatement of the actual progress. On the other hand, adding partially completed activities towards an equivalent quantity of completed activities 
is definitely not correct, it is a misrepresentation. The answer is going back to these repetitive activities in the CPM and breaking them down into more activities. So for the above examples, activity "Foundations" should be split into "Bases" and "Column necks". Likewise "Brick cladding of external walls" should be two activities one to the $3 \mathrm{~m}$ level and the other above the $3 \mathrm{~m}$ level. A revised line of balance is then calculated and therefore a more representative LOB chart can be produced. Consequently, impacts would be more clear and corrective measures, like acceleration, would be more identifiable for implementation by the project management. 


\section{CHAPTER 8}

\section{MANAGING MULTIPLE CONTRACTS}

\subsection{Introduction}

Huge housing projects have undeniable political importance because of their impacts on people. Sometimes these projects are started by public owners to stimulate the economy. It follows, therefore, that these projects become high-profile and draw considerable public attention. Owners and construction managers face a big responsibility when monitoring housing projects, especially when there are many simultaneous main contracts. The need to generate comparative information about the actual progress of different contracts becomes paramount. Main contractors in turn become under unusual attention, and have to monitor and compare their subcontractors' progress diligently and creatively to stay informed and in control.

This chapter focuses on comparing the progress regularity of multiple housing contracts and developing a new measure of it. The objective is to reduce the opportunity for subjective assessments of various but similar contract progress. This chapter also covers strategic planning issues that enhance early handovers through improved geographical allocation of units and merger.

\subsection{Comparing the Progress Status of Multiple Contractors}

Usually owners and construction managers compare the progress of main contractors based on the earned value as a percentage of the contract sum for each main contractor. The calculation is reported periodically in a table or a graph that is titled "Progress Variance". The essence is the difference between the actual earned value and the planned earned value expressed in percentage of the contract sum. If the variances are presented in a graph, the trends will become clearer than a table presentation. Fig. 8.1 shows an example of the progress variance graph for three contracts taken from a real life project. The horizontal axis represents time and the vertical axis may represent percentage value, or time units, e.g., days or weeks ahead or behind the baseline schedule. Particularly in multiple repetitive projects, these types of reports do not manifest the comparative regularity of the main contractors' progress. On a contract, progress regularity, is the degree of progress uniformity measured against an established line of balance. Information about regularity is obtained from the traditional LOB chart by comparing 


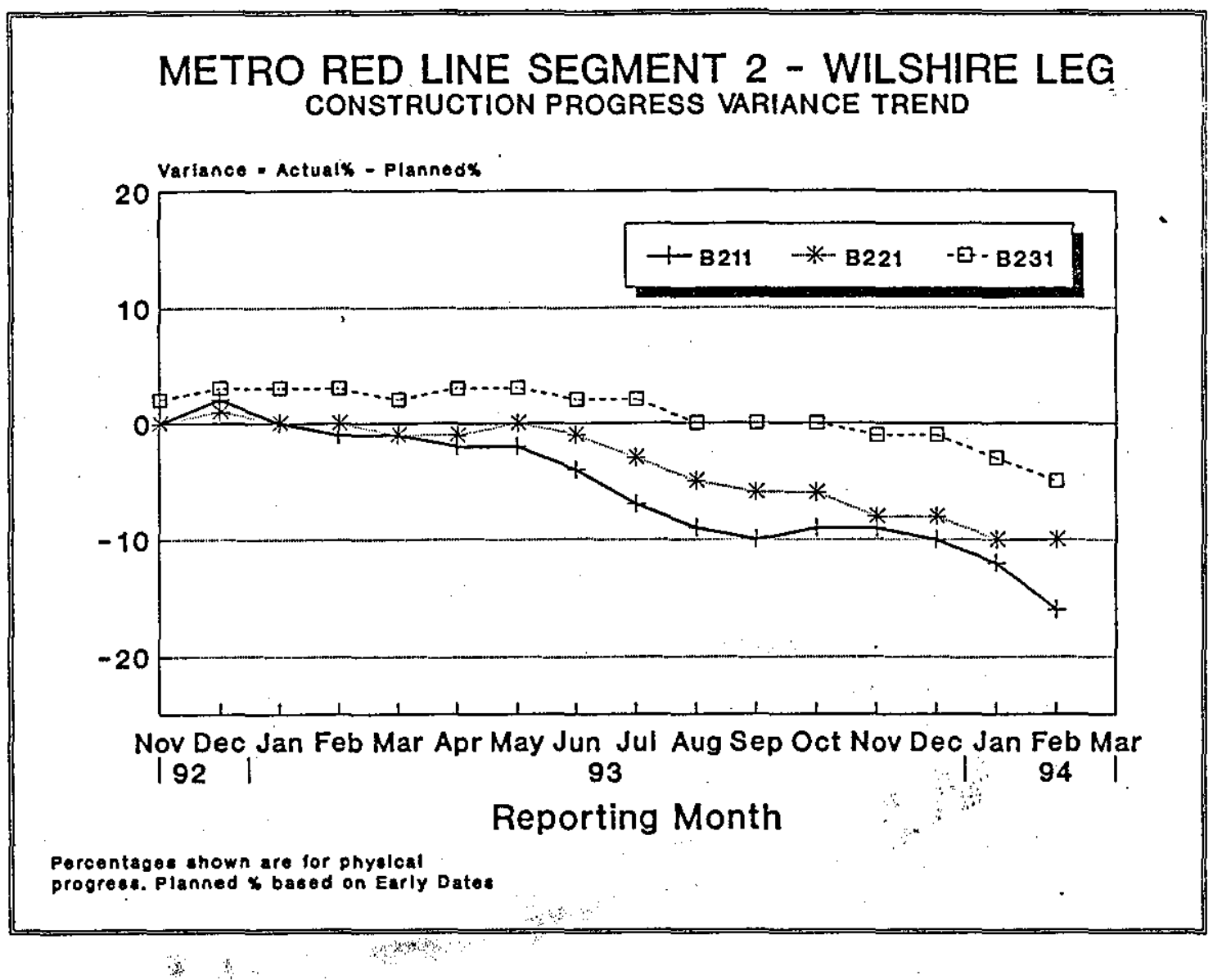

Figure 8.1 - Variance Trend Analysis.

the actual completions with the planned completions, however, it would be more vivid to quantify this regularity. Progress regularity measurement is important because it quantifies the most important aspect of the LOB technique: the balance of the progress. Comparisons of regularity on a multiple prime contract project are usually done in a narrative format not in a numerical format that is expected to enhance the objectivity of the evaluation.

\subsection{Measuring the Progress Regularity of a Repetitive Project}

LOB charts show the balance of the project actual progress, and visually portrays the deviation of each activity completions from its planned completions. However, when these repetitive activities have different rates of completions, e.g., due to float, the mere deviation which is based on completions, does not reflect that. This is important, because showing that a repetitive activity is behind by say 10 unit completions should only be an intermediate qunatification towards determining that it is late by that many days or weeks. For example, due 


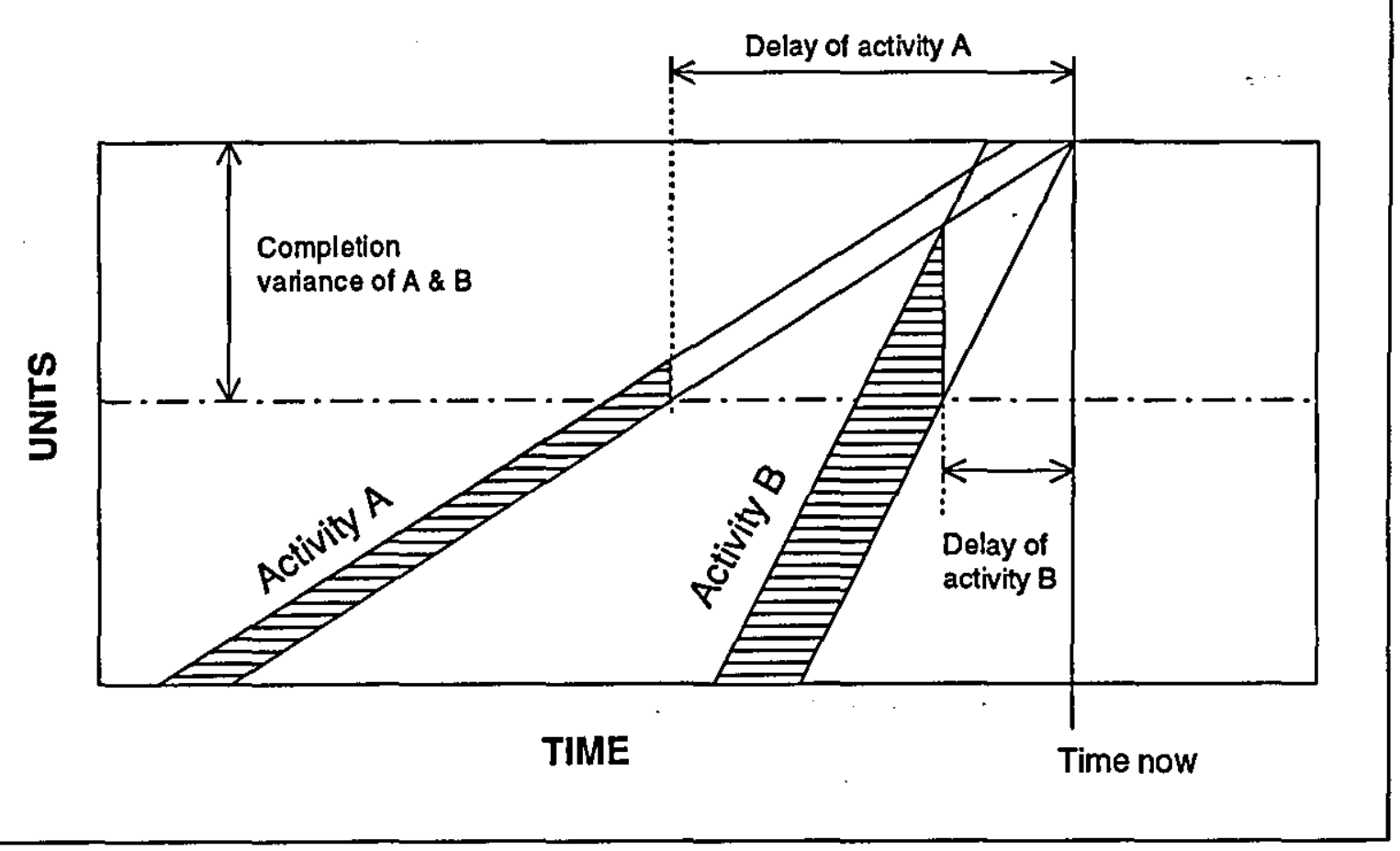

Figure 8.2 - Equal Completion Variances but Different Time Progress.

to varying completion rates, two activities that are behind by the same magnitude of unit completions should not be equally behind the time schedule (see Fig. 8.2). Conversely, if two activities with different completion rates are each behind schedule by say 15 days, they will not have the same deviation from the line of balance on the LOB chart. As far as time progress is concerned, the variance on the LOB chart is not a comparable number for activities with different rates of completion.

Based on the above, it makes more sense to convert the completions variance of an activity on the LOB chart, to time units variance based on its particular rate of completion before comparing it to other activities.

Fig. 8.3, shows an irregular progress of a project. Activities $\mathrm{A}$ and $\mathrm{C}$ completions are within the planned early and late completions, $B$ and $\mathrm{E}$ are behind late completions and D is ahead of early completions. For the purpose of measuring the degree of regularity, the progress of activities $\mathrm{A}$ and $\mathrm{C}$ are considered regular because they are within the bounds of early and late completions, therefore their completions variance is considered zero. However, for the late activities the variance will be measured against the late completions LOB. Conversely, for the early activity, the variance will be measured against the early completions LOB. The completion variances measured above can be converted to time variances by dividing them by the relevant 


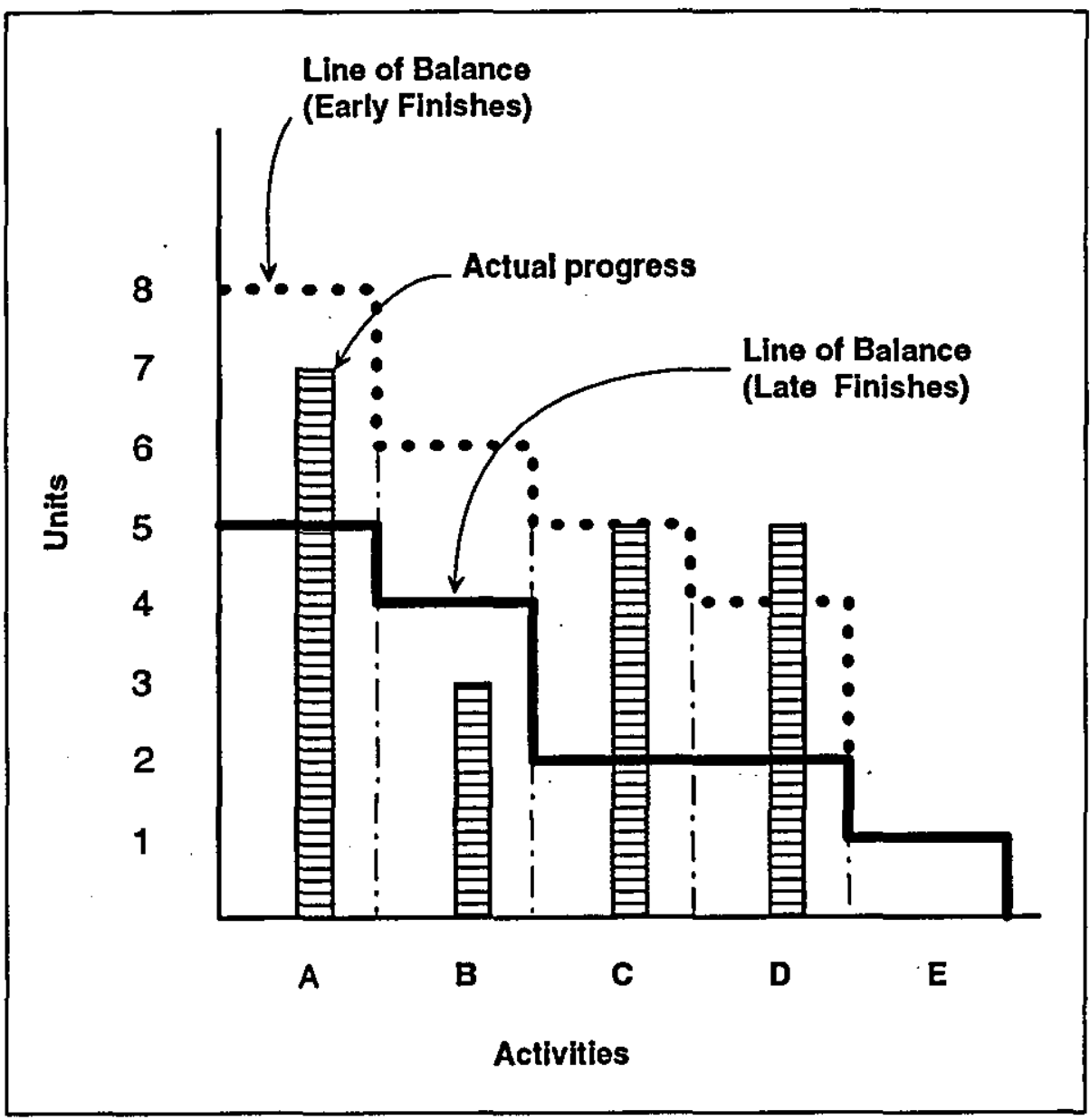

Figure 8.3 - CPM/LOB Chart Showing Irregular Progress.

rates of activity completions. Then the time variances of the activities can be looked at as a set of statistical data or a population for which the standard deviation is a measure of dispersion hence progress regularity. The more regular the progress the lower the variation and the standard deviation.

\begin{tabular}{l|c|c|c|c|c|c}
\hline \hline Activity & A & B & C & D & E & \multicolumn{2}{c}{$\begin{array}{c}\text { Regularity } \\
\sigma_{\mathrm{v}}\end{array}$} \\
\hline Completions variance & 0 & -1 & 0 & +1 & -1 & \\
\hline Rate of completion & 0.2 & 0.1 & 0.2 & 0.3 & 0.4 & 4.48 \\
\hline $\begin{array}{l}\text { Time variance } \\
\text { (in calendar days) }\end{array}$ & 0 & -10 & 0 & +3.3 & -2.5 & \\
\hline \hline
\end{tabular}

Table 8.1 - Calculation of Regularity.

Table 8.1, presents the calculation of the regularity of the progress in Fig. 8.3 assuming arbitrary rates of completion and a single work calendar. 
The time variance should be measured in calendar days, otherwise there maybe an inconsistency. When different activities follow different calendars it is more indicative and comparable to convert progress to a common scale, to calendar days.

It is to be noted that the regularity measure is not an indication of how much behind or ahead the actual progress of a project is. A project that has all of its activities ahead of schedule by say 10 days is as regular in its progress as another project with all its activities late by say 25 days. The reason is, both projects have all their activities uniformly ahead or behind the baseline schedule. Of course in these two projects, the standard deviation of the time variances of the activities is zero, and so is the regularity measure. A zero regularity denotes the highest degree of progress uniformity.

There are challenges to the above approach: a typical activity may have more than one planned rate of completion which complicates the calculation, also deriving its time variance from the actual completions is subject to underestimation or overestimation of the time progress due to many factors. Examples of these factors include: too many uncompleted activities, too few activities in progress, nonwork periods, and delays. Due to those, it is also possible to have the actual completions of a typical activity between the levels of early and late lines of balance on the CPM/LOB chart while its projected completion date is behind the baseline. Although the CPM/LOB chart offers a snapshot of the project balance status, it is not strictly linked to time progress quantifications. However, since a CPM/LOB schedule can readily show how much a typical activity is behind or ahead of its baseline schedule in time units, that information can be alternatively used to measure the regularity. The time variance, TV, of a typical activity can be obtained from the projected early finish date of the last unit activity in order in CPM/LOB compared to its baseline early finish date if it is ahead or late finish date if it is behind

$$
\begin{aligned}
& T V_{\text {ahead }}=E F_{\text {baseline }}-E F_{\text {(actual / current projected) }} \\
& T V_{\text {behind }}=L F_{\text {baseline }}-E F_{\text {(actual / current projected) }}
\end{aligned}
$$

If the projected early finish falls between the early and late finishes of the baseline, the TV is zero

$$
T V=\text { Zero when } E F_{\text {baseline }} \leq E F_{\text {actual / current }} \leq L F_{\text {baseline }}
$$


Whereas the standard deviation of the time variances is a numeral measure of regularity, a regularity chart that presents the time variances in a vertical bar chart will show the visual regularity of the project status, and unlike the CPM/LOB chart, will consider the effects of uncompleted activities and delays towards the latter stages of the project. The following example will illustrate that.

\subsubsection{Example 8.1, Progress Regularity}

This example will illustrate the measurement of the progress regularity of example 6.2 at the end of day 10 of the project. The early and late finish dates are read off the CPM software outputs in Fig. 6.10 (for actual and projected) and Fig. 6.6 (for baseline-example 6.1). Table 8.2 shows the calculations of the TV based on equations (8.1) to (8.3).

\begin{tabular}{c|c|c|c|c}
\hline \multirow{2}{*}{$\begin{array}{c}\text { Typical } \\
\text { activity }\end{array}$} & \multicolumn{2}{|c|}{$\begin{array}{c}\text { Baseline (example 6.1 } \\
\text { Fig. 6.6) }\end{array}$} & $\begin{array}{c}\text { Current projected } \\
\text { EF or AF } \\
\text { (example 6.2 } \\
\text { Fig. 6.10) }\end{array}$ & $\begin{array}{c}\text { Time } \\
\text { variance }\end{array}$ \\
\cline { 2 - 3 } A & EF & LF & 10 & 2 \\
\hline B & 12 & 14 & 15 & 2 \\
\hline C & 28 & 19 & 30 & -1 \\
\hline D & 35 & 35 & 37 & -2 \\
\hline E & 38 & 38 & 40 & -2 \\
\hline F & 28 & 28 & 30 & -2 \\
\hline G & 29 & 29 & 31 & -2 \\
\hline H & 30 & 35 & 32 & 0 \\
\hline \hline
\end{tabular}

Table 8.2 - Example 8.1, Calculations of Time Variance for Progress Regularity.

Note that for typical activity $\mathrm{H}$, the time variance is zero because the projected finish will be between its early and late finishes in the baseline schedule. To illustrate how the regularity would have differed had activities $A$ and $B$ been behind schedule, assume that their time variances 
had been -2 instead of 2 . Obviously the project status would have been in a more uniform delay and the regularity (standard deviation) would have been 0.70 which points to a higher uniformity than the previous value of 1.65 .
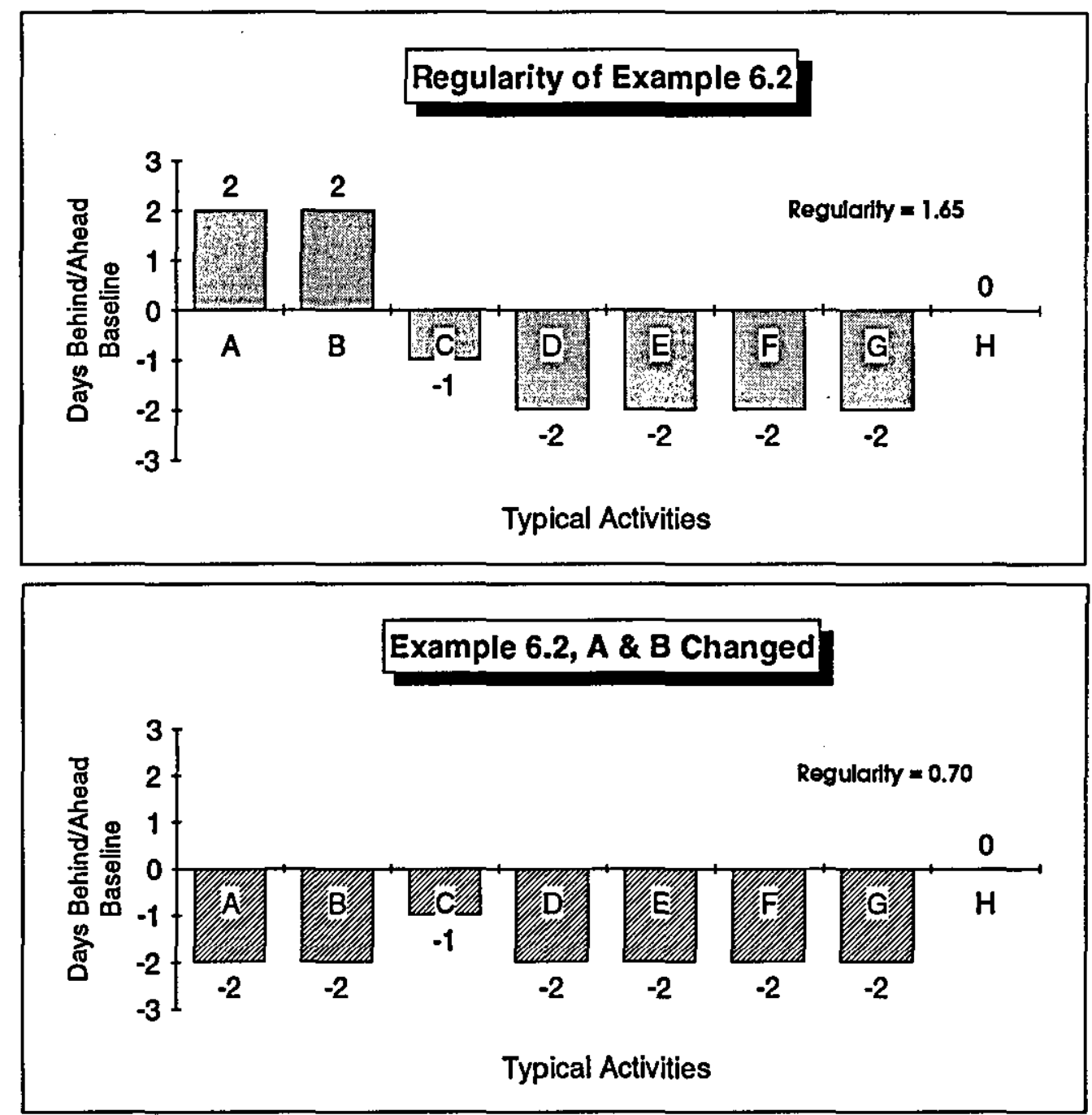

Figure 8.4 - Example 8.1, Regularity Charts.

Fig. 8.4 shows the regularity charts for both cases. This chart portrays the time variances of the typical activities thereby gives an indication of the uniformity as well as the time progress status. So, by merely knowing that typical activity $\mathrm{E}$ is the last typical activity, the chart reader is informed that the overall project progress is 2 days behind schedule. Since the CPM/LOB chart does not show the status of the time progress of the typical activities and sometimes does not agree with it, the regularity chart is recommended to complement the CPM/LOB chart in progress analysis. 
The use of a statistical method to measure the regularity of progress not only compares multiple contracts on a common scale but also facilitates early identification and resolution of imbalance problems.

\subsection{Enhancing Stage Handovers Through Strategic Planning}

On most of housing projects, owners and sometimes contractors prefer to complete and hand over houses in a particular sequence. This sequence is decided at a high level of the strategic planning process and is usually a function of the arrangement of the new houses relative to the existing streets and other amenities of the neighborhood. For example, common sense encourages a planner to choose to complete and hand over new houses that are adjacent to an existing community before those far away from it. By doing so, occupants of newly completed houses shall not have to pass through the areas of uncompleted houses to get to their homes.

The same issue is faced when allocating units to multiple prime contractors on a large housing project. How to allocate in such a way that maximizes the number of units in early stage handovers and therefore makes the handover process less vulnerable to future troubles in progress, given that occupancy should not hinder accessibility.

Fig. 8.5 shows a project jobsite bound by an existing road and community. The feasible direction of progress would be to start from the west end and proceed east bound. This direction of progress always maintains a number of houses occupiable by the owners at any point in time further down the construction progress more than any other direction of progress.

If there are multiple prime contractors on the project, the objective should be securing a unified rate of physical displacement from the western side of the jobsite towards the eastern side. Of course this means considering the rates of completions of these prime contractors when allocating the housing units to them. The objective of this strategy is to avoid a pattern of completions that is unwieldy or awkward to hand over to the owners. Fig 8.6 delineates such an unfortunate possibility where the project participants did not plan ahead for an early partial occupancy. As shown, only those houses completed by contractor $\mathrm{C}$ so far could be realistically considered for handover to the new owners. Allocating houses to contractors and directing progress similar to that shown in Fig. 8.6 for reasons such as, reduction of interference between the workers or preventing thefts of tools are not unheard of in real life construction projects. 


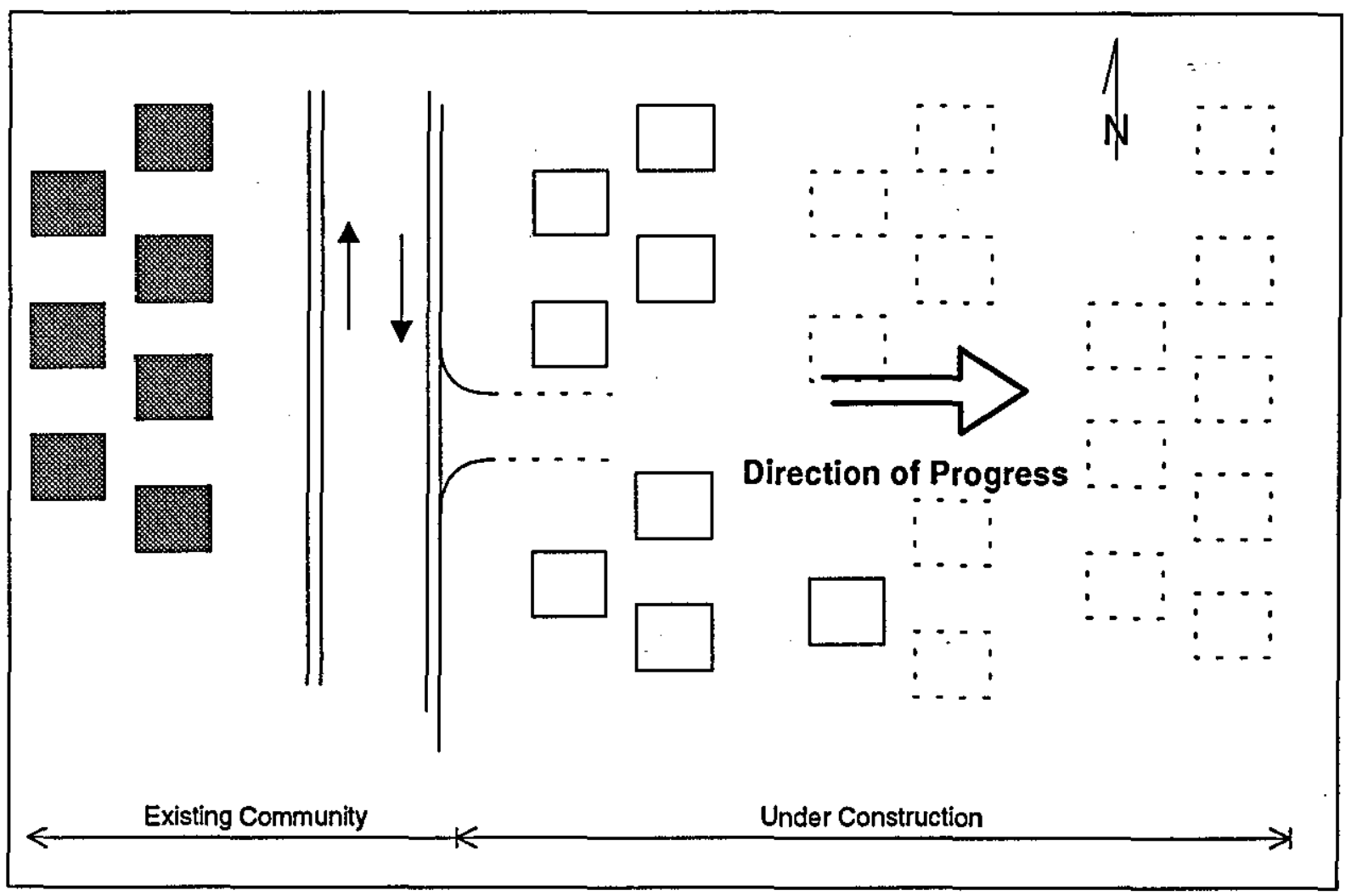

Figure 8.5 - Progress Enhancing Stage Handovers.

\subsubsection{Example 8.2, Enhancing Stage Handovers}

The objective in this example is to improve, in hindsight, a decision on allocating housing units to multiple subcontractors on a completed project. Fig. 8.7 shows a plan of a real life project for the construction of 260 houses in Kuwait. The west side of the site was bound by existing streets and utilities. Consequently the decision was made to start the progress from this west side and go east facilitating stage handovers to the owner in the same direction as houses are completed. The main contractor for these 260 houses had three building subcontractors and another subcontractor for the roads and utilities serving these houses. Due to delays in contracting with the smallest two of the building subcontractors, the main contractor eventually allocated the houses to the three building subcontractors as shown in Fig. 8.7: 185 houses to building subcontractor $\mathrm{L}, 24$ houses to $\mathrm{X}$ and 51 houses to $\mathrm{O}$. The notice to proceed dates and completion dates were as follows: 


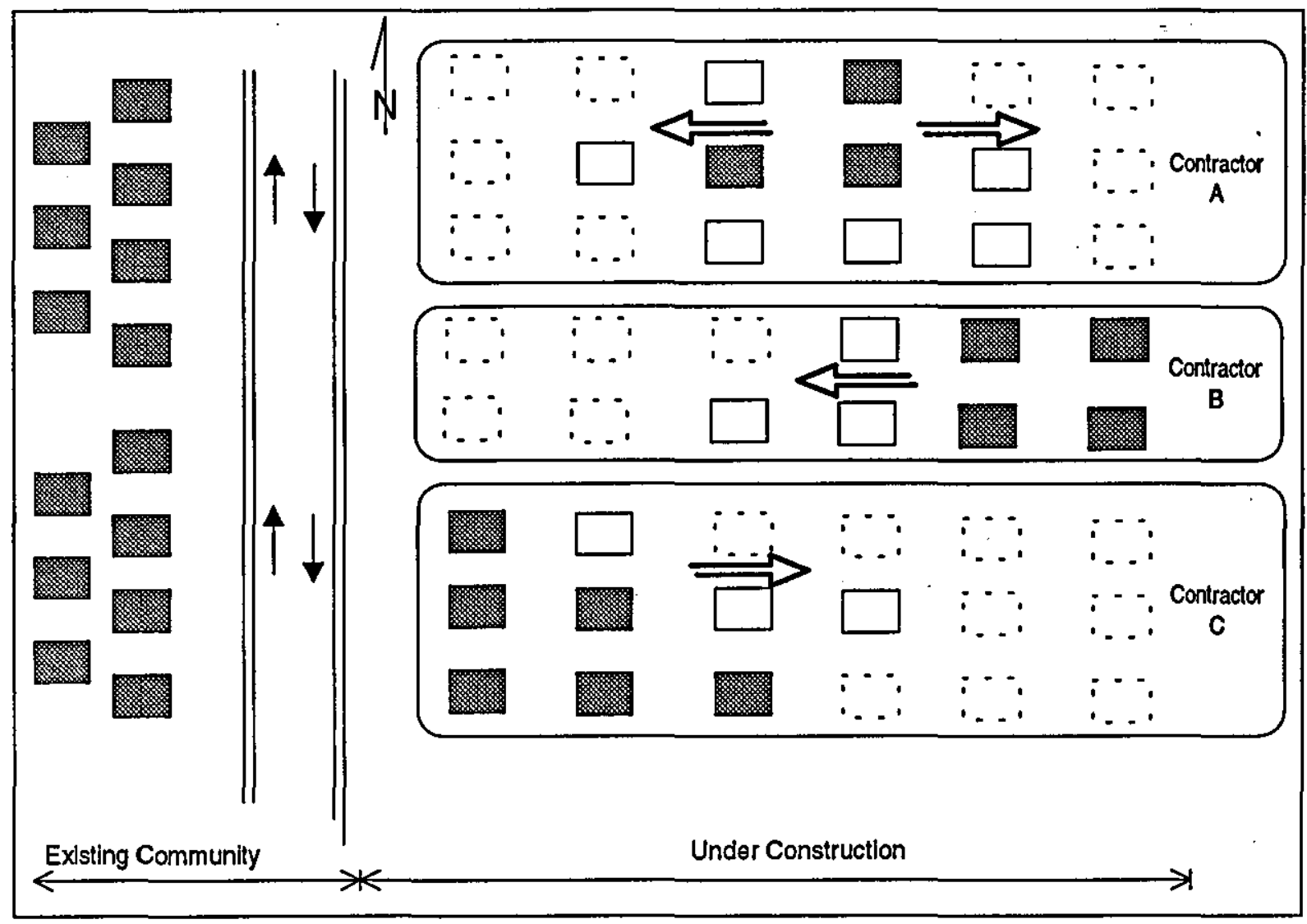

Figure 8.6 - Progress not Enhancing Stage Handovers.

\begin{tabular}{|c|c|c|c|c|}
\hline Subcontr. & $\begin{array}{l}\text { Number of } \\
\text { houses }\end{array}$ & Start date & $\begin{array}{l}\text { Completion } \\
\text { date }\end{array}$ & $\begin{array}{l}\text { Duration } \\
\text { (days) }\end{array}$ \\
\hline $\mathbf{L}$ & 185 & $\begin{array}{l}9 \text { Oct } 86 \\
(\text { day } 1)\end{array}$ & $\begin{array}{l}23 \text { Jul } 88 \\
\text { (day 654) }\end{array}$ & 654 \\
\hline $\mathrm{X}$ & 24 & $\begin{array}{l}10 \text { Jan } 87 \\
\text { (day } 94 \text { ) }\end{array}$ & $\begin{array}{l}17 \text { Apr } 88 \\
\text { (day 557) }\end{array}$ & 464 \\
\hline 0 & 51 & $\begin{array}{l}1 \text { Apr } 87 \\
\text { (day } 175 \text { ) }\end{array}$ & $\begin{array}{l}17 \text { Aug } 88 \\
\text { (day 679) }\end{array}$ & 505 \\
\hline
\end{tabular}




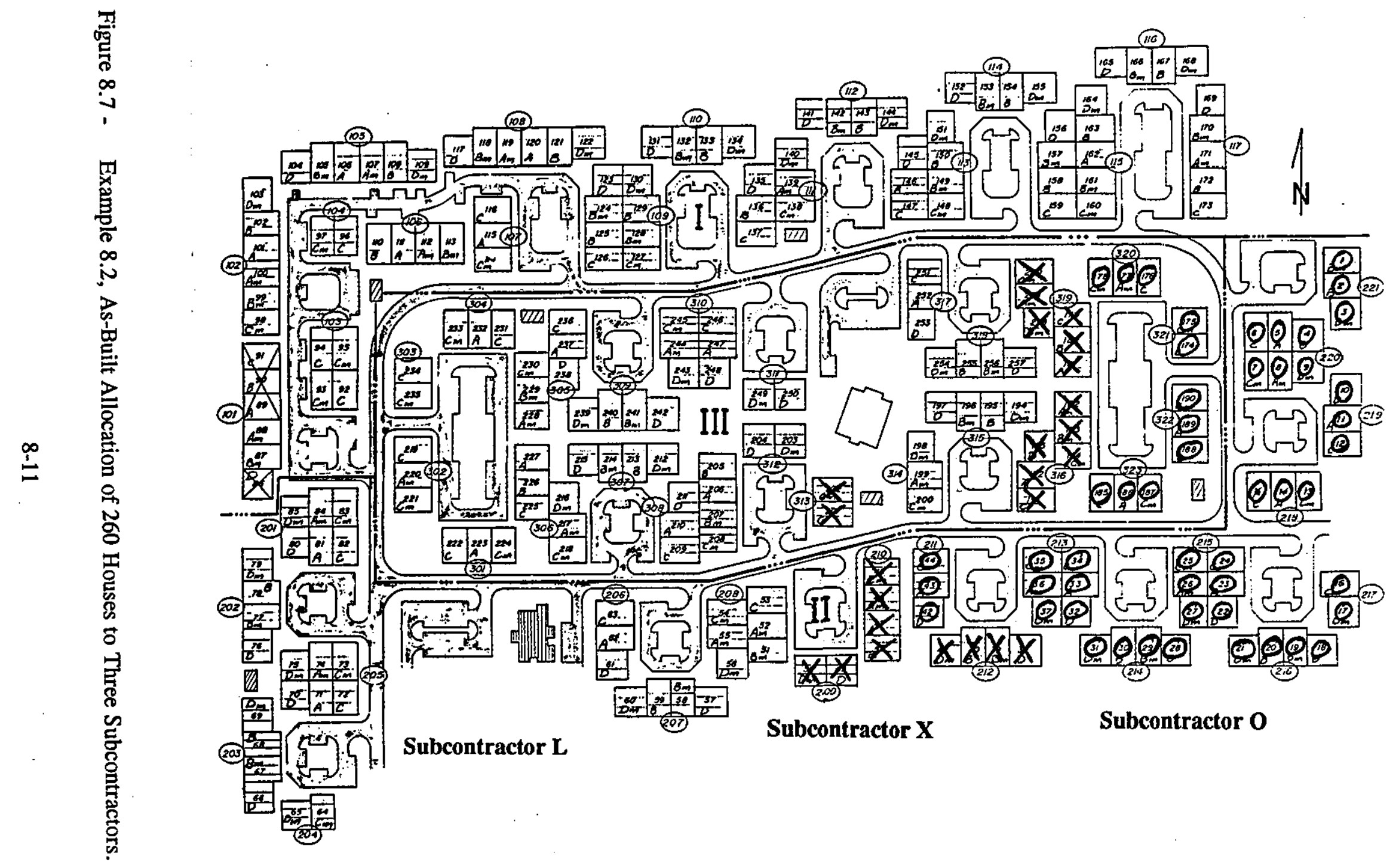


Assuming that the construction of the first house of each subcontractor takes 300 days to complete, the rates of completions can be calculated using equation (6.1):

$\begin{array}{lll}\text { Subcontr } & \begin{array}{l}\text { Rate of completion } \\ \text { (house per month) }\end{array} & \begin{array}{l}\text { Rate relative to } \\ \text { subcontractor X }\end{array} \\ \text { L } & \{(185-1) \times 30\} \div\{654-300\}=15.59 & 3.7 \\ \text { X } & \{(24-1) \times 30\} \div\{464-300\}=4.21 & 1.0 \\ \text { O } & \{(51-1) \times 30\} \div\{505-300\}=7.32 & 1.74\end{array}$

It is clear that if a single physical displacement rate, starting from the west side of the project is desired to enhance stage handovers to the owner, the rates of the subcontractors will have to be considered. So if the main contractor decides to hand over 100 houses at the first stage, that first stage should have its houses allocated to subcontractors $L, X$ and $O$ in the ratio 3.7:1.0:1.74 respectively. If progress goes as planned, this will guarantee that each subcontractor will complete his houses in the first stage at the same time with the other two, therefore enabling a consistent and complete group of houses for handover to the owner. However, since the start and completion dates of the three subcontractors are not the same the above should not be applied throughout the jobsite. Fig. 8.8 shows a linear program derived from the above information which should put the issue in a clear perspective. For each subcontractor the horizontal thick line of three hundred days for the first house is followed by an inclined delivery line.

Subcontractor $\mathrm{L}$ is planned to deliver his first house on day 300 and continue to deliver up to 49 houses alone until before day 394 . Therefore the first 49 houses closer to the existing street and utilities should be allocated to subcontractor $\mathbf{L}$. This will be called the first allocation batch.

For the period between days 394 and 475 , both subcontractors $\mathrm{L}$ and $\mathrm{X}$ will be completing and delivering houses. The planned delivery ratio will be $3.7: 1$ based on the relative 


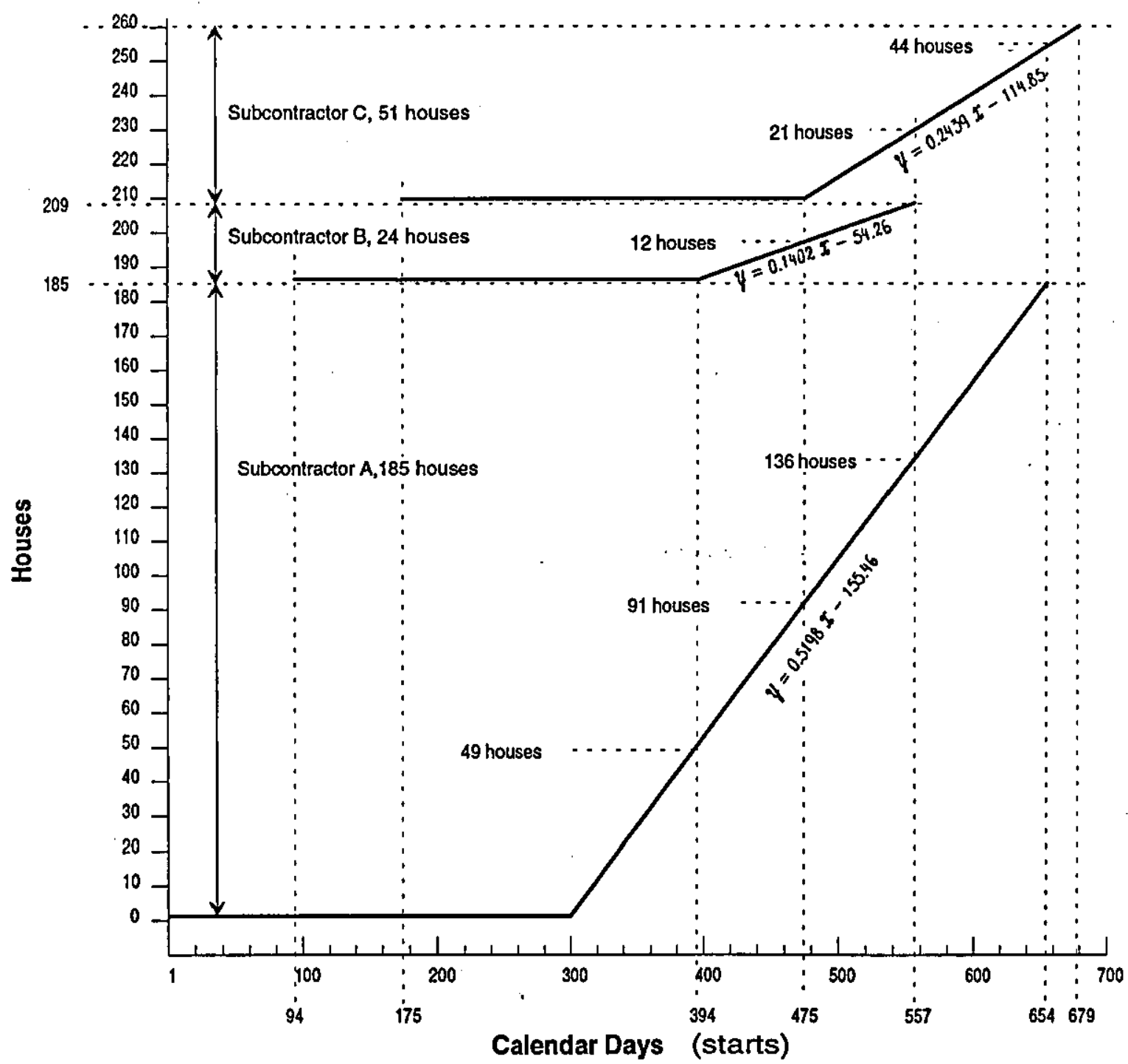

Figure 8.8 - Example 8.2, Linear Program for Three Subcontractors.

rates of the two subcontractors. It follows that the second allocation batch will comprise 12 houses for subcontractor $X$ and 42 houses ( 91 minus 49 ) for subcontractor $L$. These should be allocated to subcontractors $\mathrm{L}$ and $\mathrm{X}$ as close as possible to the existing street and utilities and alternatively in the ratio $3.7: 1$.

The third allocation batch shall be shared by the three subcontractors. Forty five houses for subcontractor L (136 minus 91), 12 houses for subcontractor X (24 minus 12) and 21 houses for subcontractor $\mathrm{O}$. 
The fourth allocation batch will be allocated between subcontractor $\mathrm{L}$ and $\mathrm{O}$ since $\mathrm{X}$ will have completed all his 24 houses. Forty nine houses go to subcontractor L (185 minus 136) and 23 house to subcontractor $O$ (44 minus 21 ).

The last allocation batch goes all to subcontractor $O$ who is the last to complete his

\begin{tabular}{c|c|c|c|c}
\hline \hline \multirow{2}{*}{$\begin{array}{c}\text { Allocation } \\
\text { batch }\end{array}$} & \multicolumn{3}{|c|}{ Subcontractor } & \multirow{2}{*}{$\begin{array}{c}\text { Batch } \\
\text { totals }\end{array}$} \\
\cline { 2 - 5 } & $\mathrm{L}$ & $\mathrm{X}$ & $\mathrm{O}$ & \\
\hline 1 & 49 & 0 & 0 & 49 \\
\hline 2 & 42 & 12 & 0 & 54 \\
\hline 3 & 45 & 12 & 21 & 78 \\
\hline 4 & 49 & 0 & 23 & 72 \\
\hline 5 & 0 & 0 & 7 & 7 \\
\hline \hline Totals & 185 & 24 & 51 & 260 \\
\hline \hline
\end{tabular}

Table 8.3 - Example 8.2, Allocation of Houses to Multiple Subcontractors.

remaining 7 houses. Table 8.3 summarizes the order of allocating the houses to the three subcontractors in a fashion that enhances the chances of delivering houses beginning from the west side of the jobsite and towards the east side. It should be noted that any particular stage of handover may comprise any feasible number of houses regardless of the allocation batch sizes.

Fig. 8.9 shows the alternative geographical allocation of the houses to the three subcontractors based on the above method. Obviously the difference between it and the main contractor's choice (see Fig. 8.7) is massive, and so are the expected implications.

It should be mentioned here that there are other factors that may be taken into consideration when allocating the houses, such as, avoiding having two subcontractors share one cluster of houses if possible, and aiming at completing clusters that form a complete loop before others. A loop (sometimes called a court), is a formation of clusters that are serviced by a common internal road. Other allocation alternatives that maintain the same objective of enhancing stage handovers are possible, but none should be close to the as-built allocation of Fig: 8.7. The as-built allocation leads to a scattered formation of completed houses and reduces the number of 


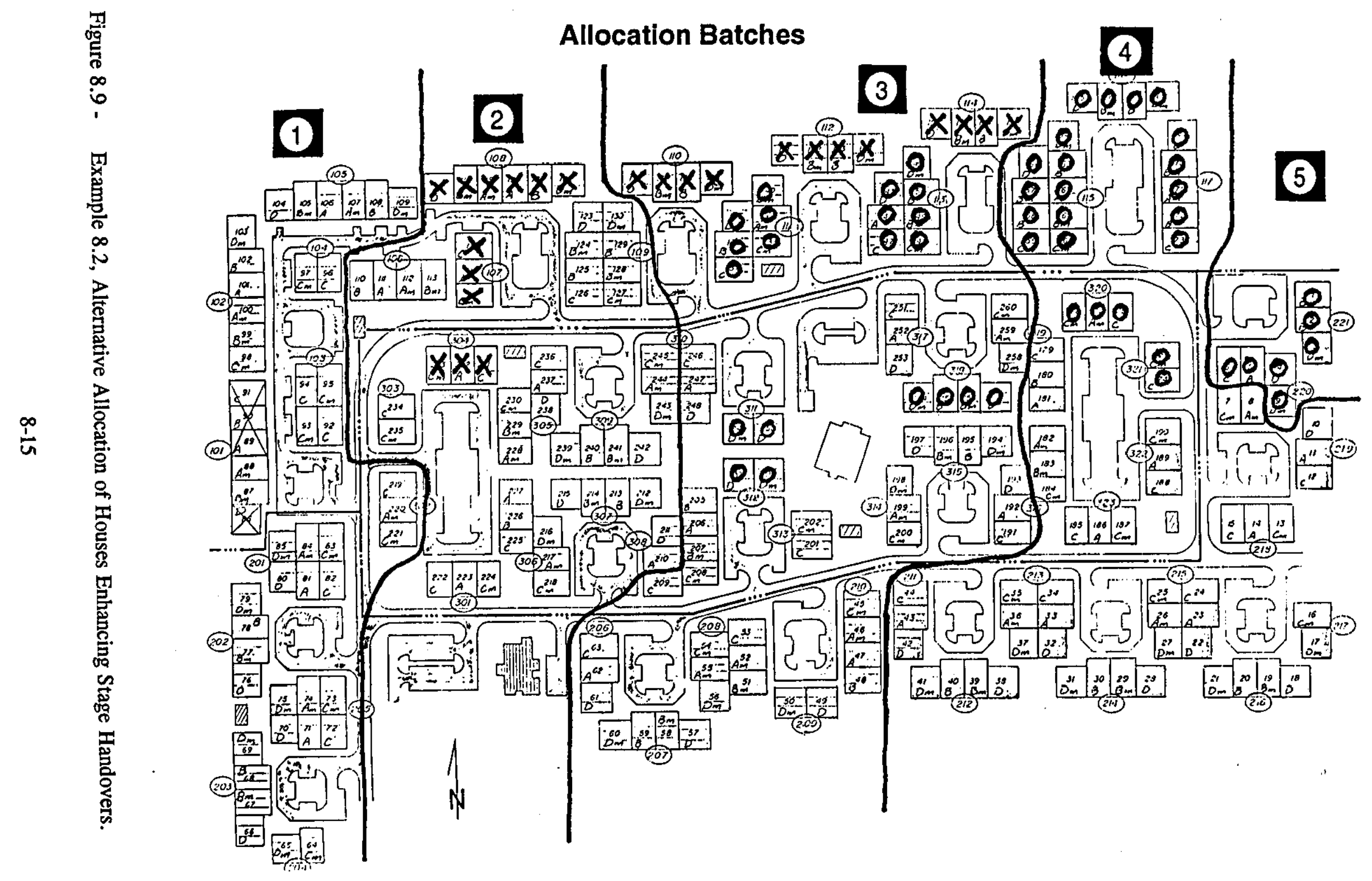


houses the main contractor may hand over at an early stage in a somewhat confined geographical location. Of course this means unnecessary delays in handing over completed but isolated houses of contractors $\mathrm{X}$ and $\mathrm{O}$ and to liquidated damages if stipulated in the contract.

Planning the phases of handover is one of the many reasons project managers change the sequence of work on the houses before and during construction. As the work progresses the project participants rapidly loose their opportunities to change the allocations and the sequence. CPM/LOB offers a responsive tool for the what-if analysis on this issue as planners shuffle the different alternatives of stage handovers and thereby the sequence of operation.

\subsection{Improved uniformity and Earlier Completion through Merger}

Certain events on jobsites may hinder the progress on particular early activities due to various reasons. Examples are: unexpected subsurface conditions, excessive change orders, inclement weather, etc. These may affect only part of a large jobsite and push its progress out of balance. On the other hand, other reasons may affect different activities on other parts of the jobsite, therefore tilting the balance in an opposite fashion to the first part of the jobsite. Examples of those could be excessive change orders on finishing activities, mismanagement of resources, etc.

If the progress of each part of the large project is measured against an independent CPM/LOB chart, it is expected that the two parts will be disparately out of balance. One part late on the somewhat initial activities while the other part ahead on these activities but late on subsequent activities. Both should be behind their time schedules due to the imbalanced statuses.

The posed question is, can these two parts of the project be combined in one part to even out their irregular progress? And if this is possible, will the integrated project gain some time?

The biggest challenge to such an approach, is the contractual relations. These two parts of the project may be assigned to two different contractors or subcontractors, and merging them can be a contractual nightmare. However, if both parts were executed by a single organization, the problem will be reduced to temporary logistical inconveniences. 
Example 8.3 will illustrate the technical feasibility of the merger process towards a more balanced status and an earlier completion.

\subsubsection{Example 8.3, Merger}

The example project comprises twenty units divided equally between two sectors, north and south. Both started together and were planned to finish on the same day. Each sector is a replica of example 6.1 and should be completed by day 38 . Therefore the bar chart of example 6.1 in Fig. 6.6 is an applicable baseline to both sectors' progress. The north sector has houses 1 through 10, and the south sector has houses 11 through 20 .

At the end of day 19 the two sectors progress were at a contrasting imbalance. While the south sector was ahead on some activities, the north sector was behind on the same activities. Figs. 8.10 to 8.13 provide a picture of the status of both sectors. The projected early finish of the northern sector is day 44 , six days behind schedule, while that of the southern sector is day 48 , ten days behind schedule. The regularity for the north and south are 3.59 and 5.67 respectively.

The two sectors shall be combined in one schedule adding their available resources to each other and keeping all other variables fixed. Units 1 and 11, has a priority of 1 , units 2 and 12 has a priority of 2 , and so on for the rest. Fig. 8.14 shows the bar chart of the combined schedule with a projected completion on day 41 , which is earlier than both sectors completion days. Fig. 8.15 shows that the regularity of the new merger is 0.78 , far better than the previous independent sectors.

It is interesting to note that while the level of actual completions of typical activity $G$ in both sectors were between the early and late levels of the lines of balance, its time variance was negative in the southern sector and positive in the northern sector. The same observation is evident on activity $\mathrm{C}$ after merger where the CPM/LOB chart shows it ahead on completions while the table indicates it is 2 days late. There is a similar disparity on activity $G$. This corroborates the notion in section 8.3 that although the CPM/LOB chart depicts activity completions, it gives a temporary indication of the balance status of the project at a certain point in time with no substantial consideration to what is planned soon after. 


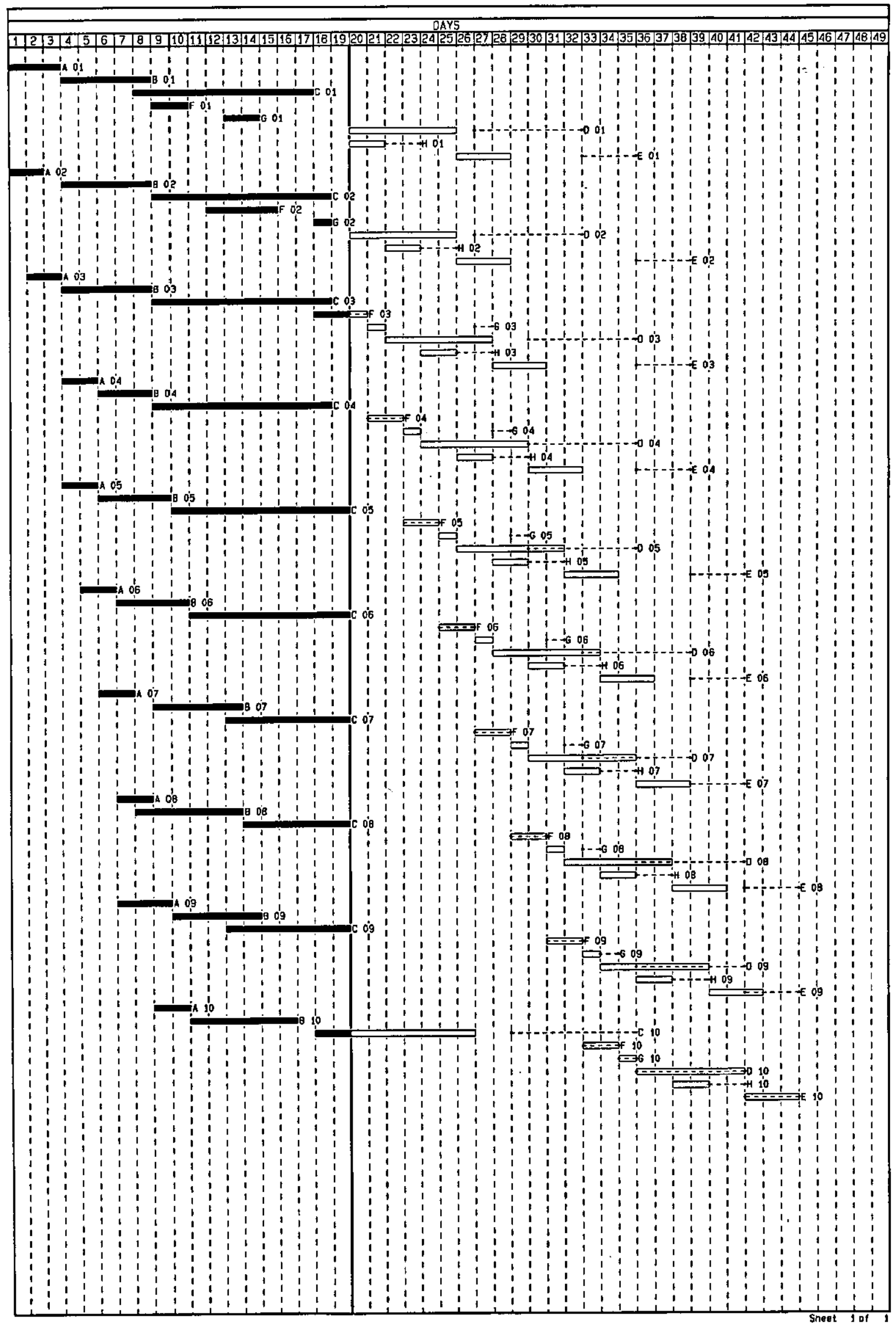

Figure 8.10 - Example 8.3, Bar Chart of North Sector. 


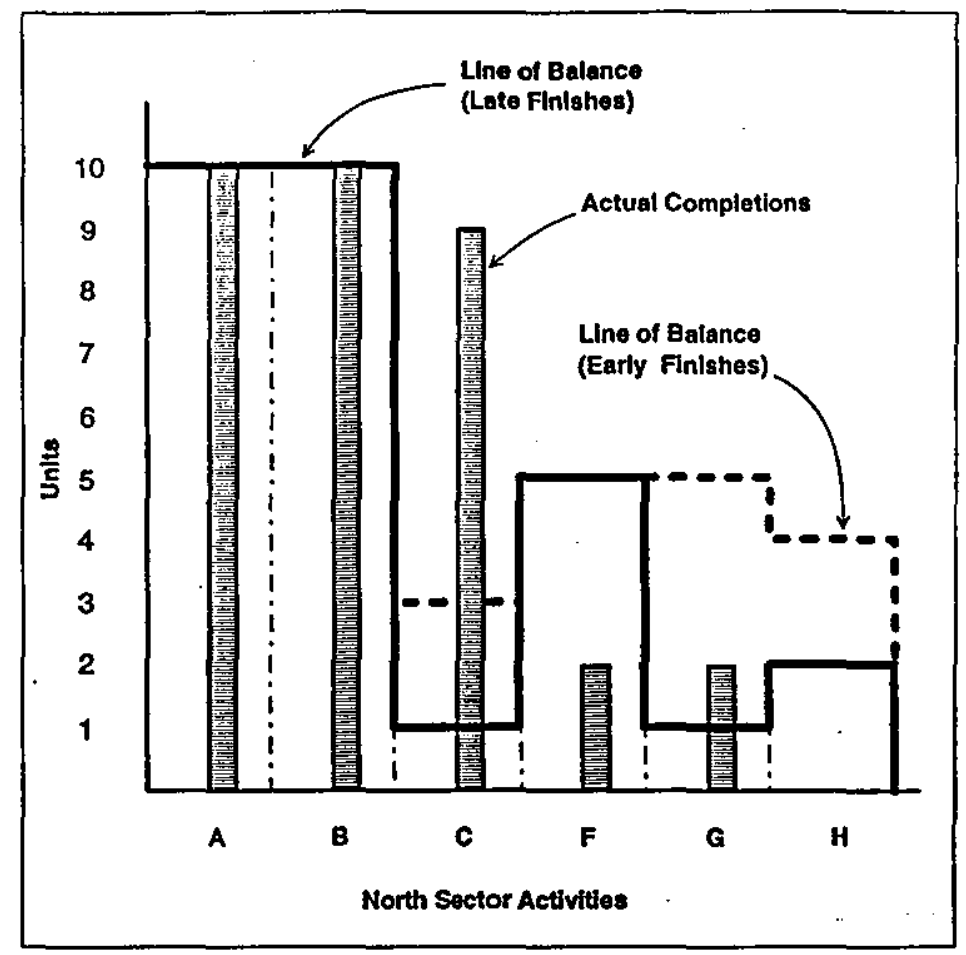

\begin{tabular}{c|c|c|c|c}
\hline \multirow{2}{*}{$\begin{array}{c}\text { Typical } \\
\text { activity } \\
\text { (north sector) }\end{array}$} & \multicolumn{2}{|c|}{$\begin{array}{c}\text { Baseline (example 6.1 } \\
\text { Fig. 6.6) }\end{array}$} & $\begin{array}{c}\text { Current projected } \\
\text { EF or AF } \\
\text { (Fig. 8.10) }\end{array}$ & $\begin{array}{c}\text { Time } \\
\text { variance } \\
\text { TV }\end{array}$ \\
\cline { 2 - 3 } A & 12 & 14 & 10 & 2 \\
\hline B & 17 & 19 & 16 & 1 \\
\hline C & 28 & 29 & 26 & 2 \\
\hline D & 35 & 35 & 41 & -6 \\
\hline E & 38 & 38 & 44 & -6 \\
\hline F & 28 & 28 & 34 & -6 \\
\hline G & 29 & 29 & 35 & -6 \\
\hline H & 30 & 35 & 39 & -4 \\
\hline \hline
\end{tabular}

Figure 8.11 - Example 8.3, CPM/LOB Chart and Regularity Measure of North Sector at End of Day 19. 


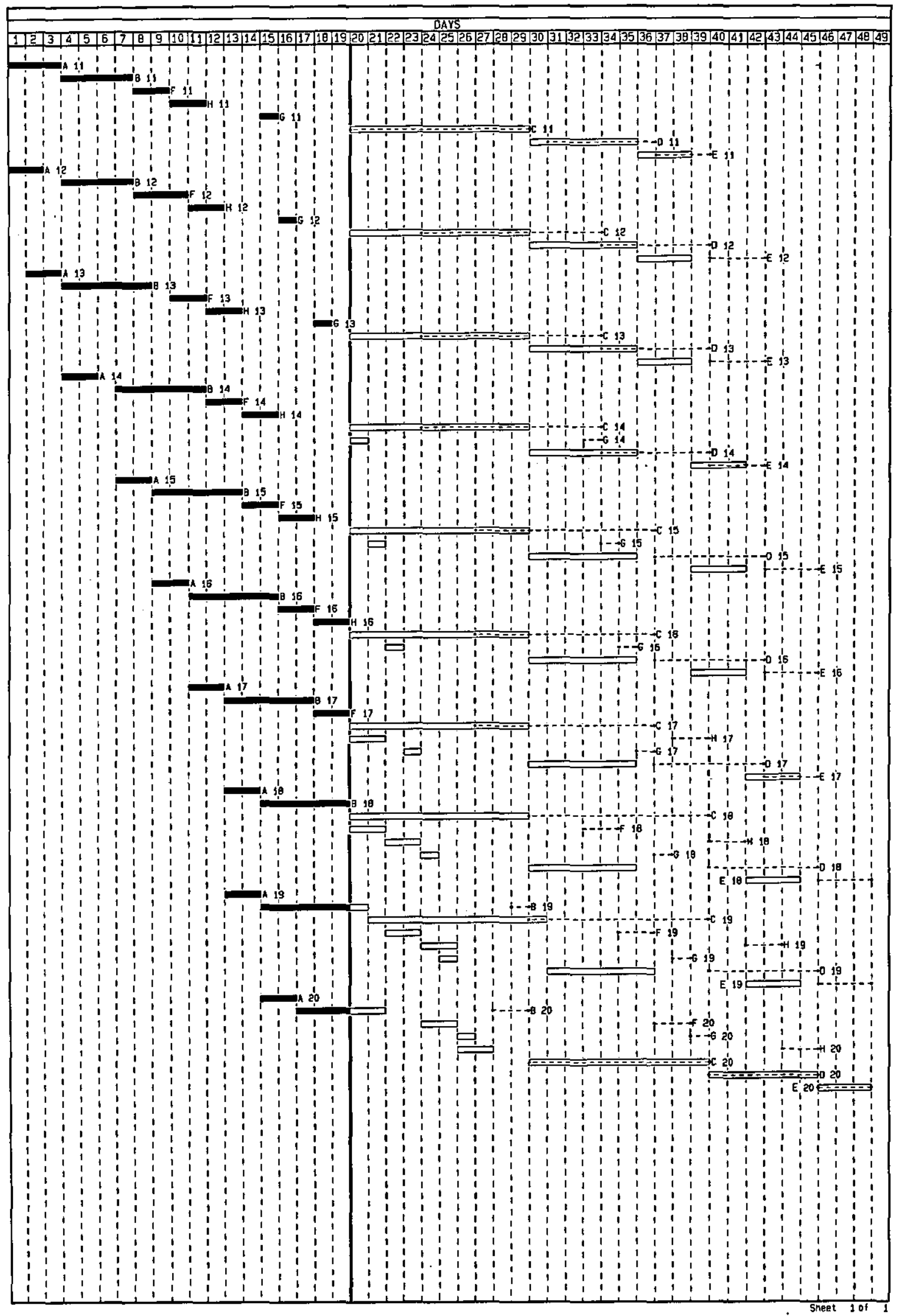

Figure 8.12 - Example 8.3, Bar Chart of South Sector. 


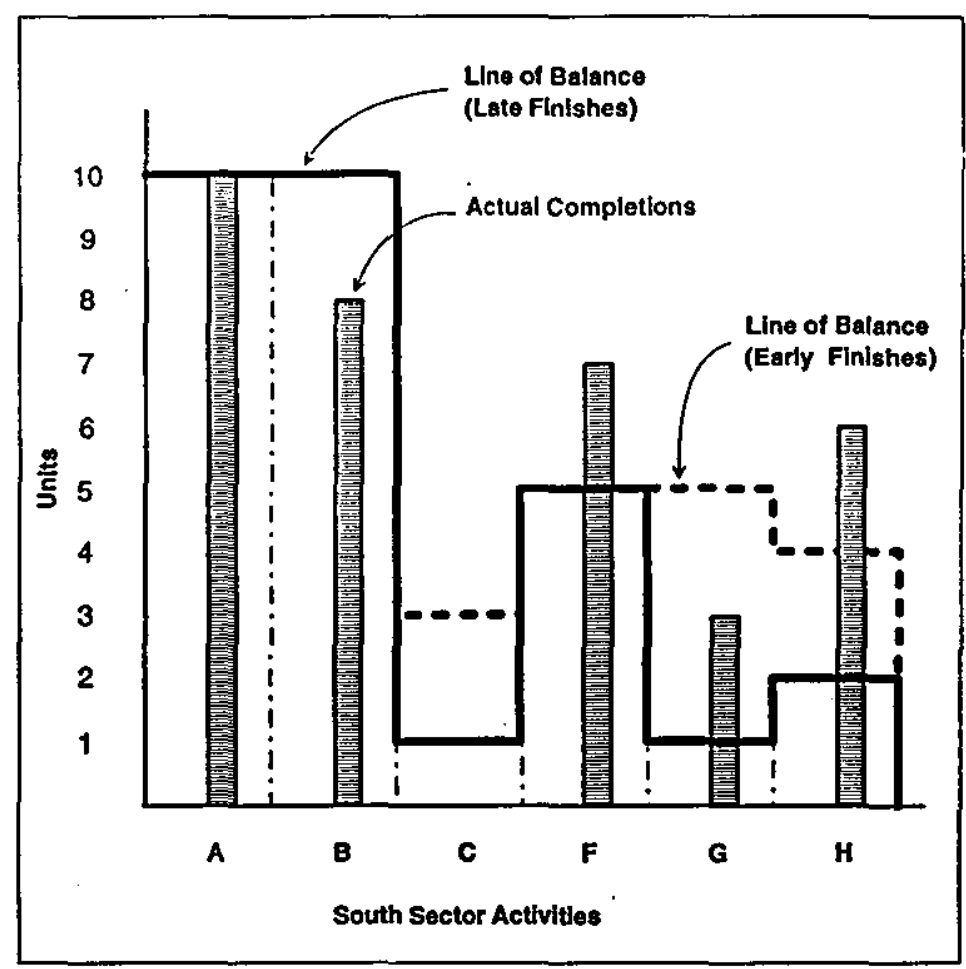

\begin{tabular}{c|c|c|c|c}
\hline \multirow{2}{*}{$\begin{array}{c}\text { Typical } \\
\text { activity } \\
\text { (south sector) }\end{array}$} & \multicolumn{2}{|c|}{$\begin{array}{c}\text { Baseline (example 6.1 } \\
\text { Fig. 6.6) }\end{array}$} & $\begin{array}{c}\text { Current projected } \\
\text { EF or AF } \\
\text { (Fig. 8.12) }\end{array}$ & $\begin{array}{c}\text { Time } \\
\text { variance } \\
\text { TV }\end{array}$ \\
\cline { 2 - 5 } A & EF & LF & 16 & -2 \\
\hline B & 12 & 14 & 21 & -2 \\
\hline C & 28 & 19 & 39 & -10 \\
\hline D & 35 & 35 & 45 & -10 \\
\hline E & 38 & 38 & 48 & -10 \\
\hline F & 28 & 28 & 25 & 3 \\
\hline G & 29 & 29 & 26 & 3 \\
\hline H & 30 & 35 & 27 & 3 \\
\hline \hline
\end{tabular}

Figure 8.13 - Example 8.3, CPM/LOB Chart and Regularity Measure of South Sector at End of Day 19. 


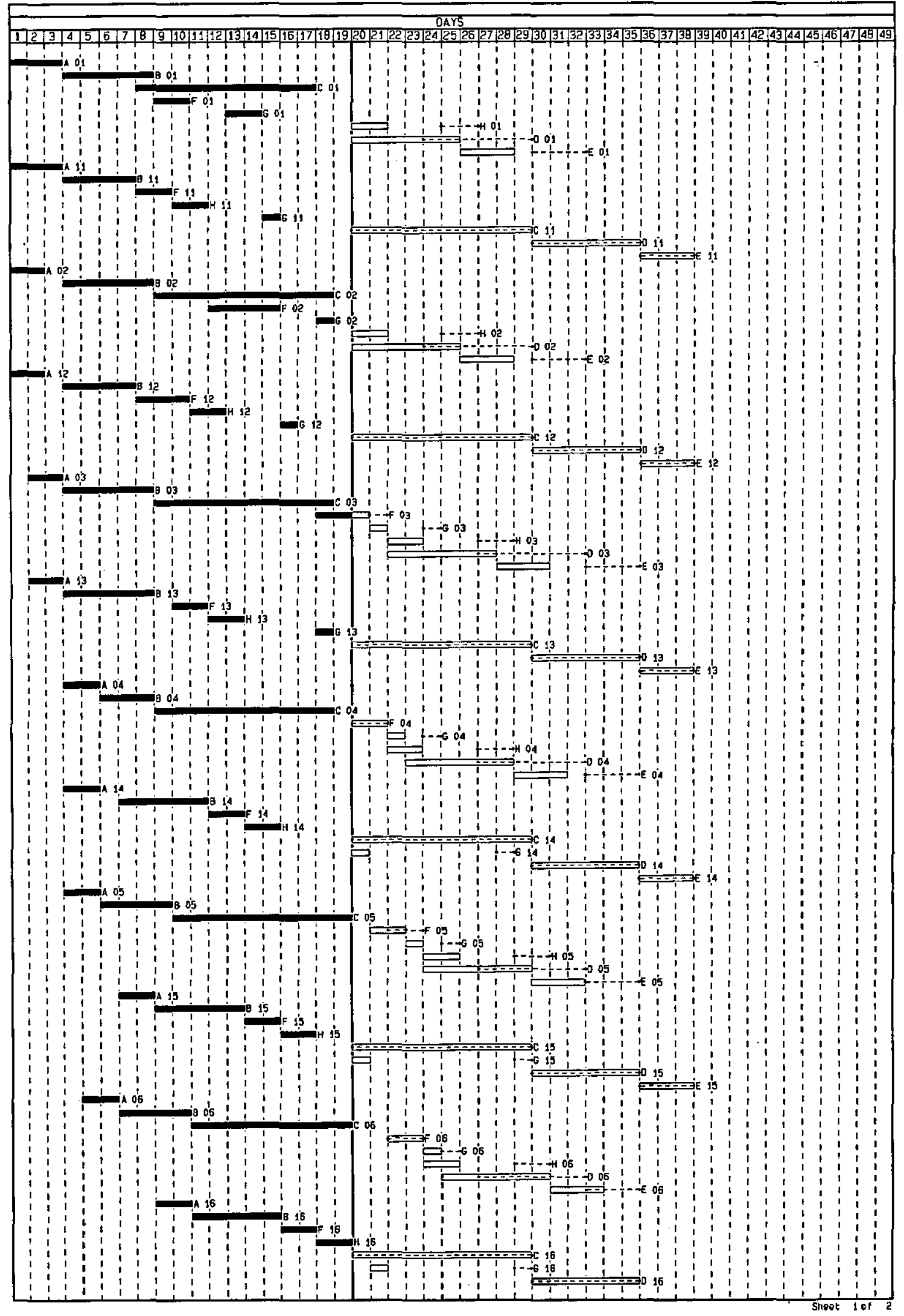

Figure 8.14 - Example 8.3, Bar Chart of Merged Sectors. 


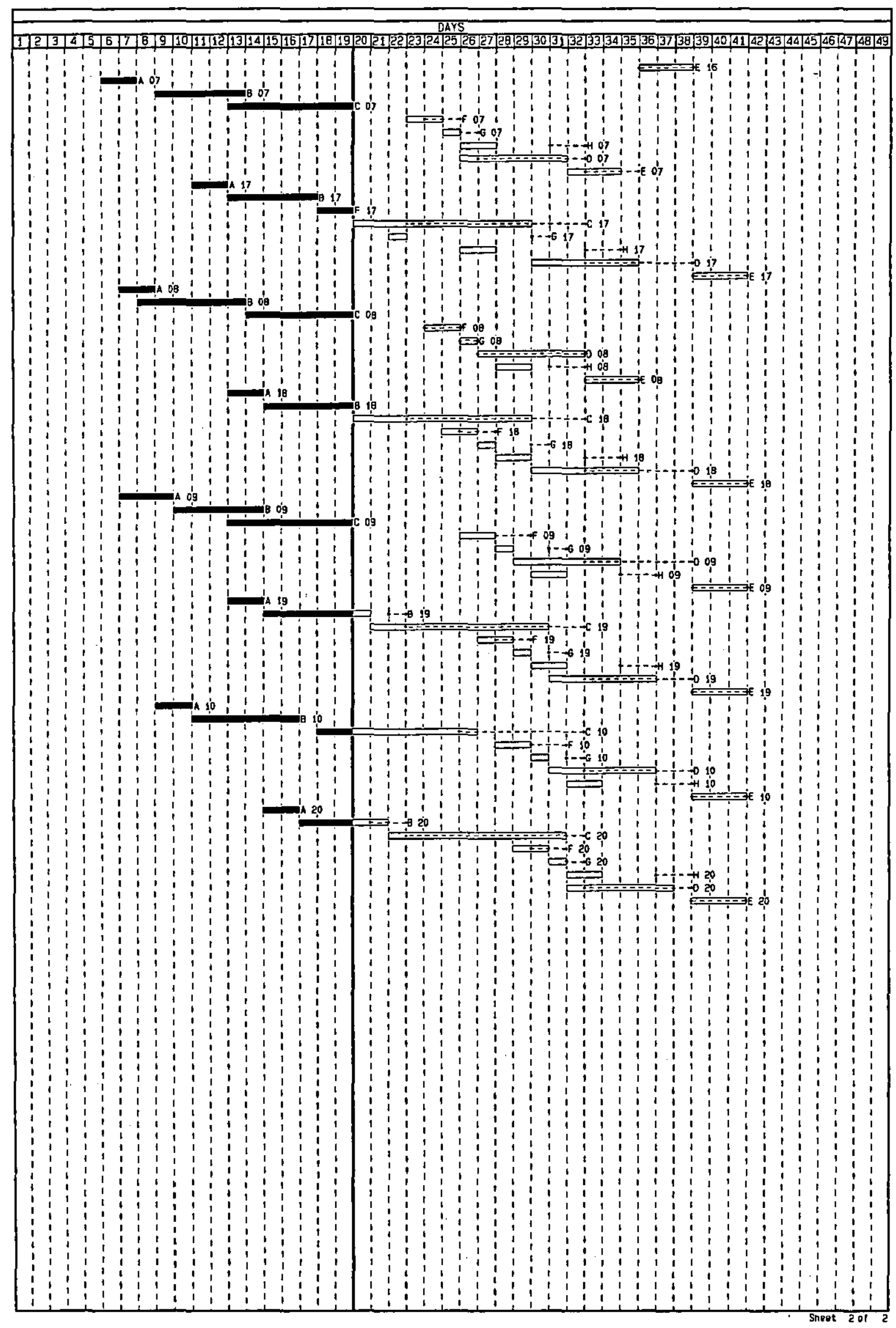

Figure 8.14 - Example 8.3, Bar Chart of Merged Sectors (Continued). 


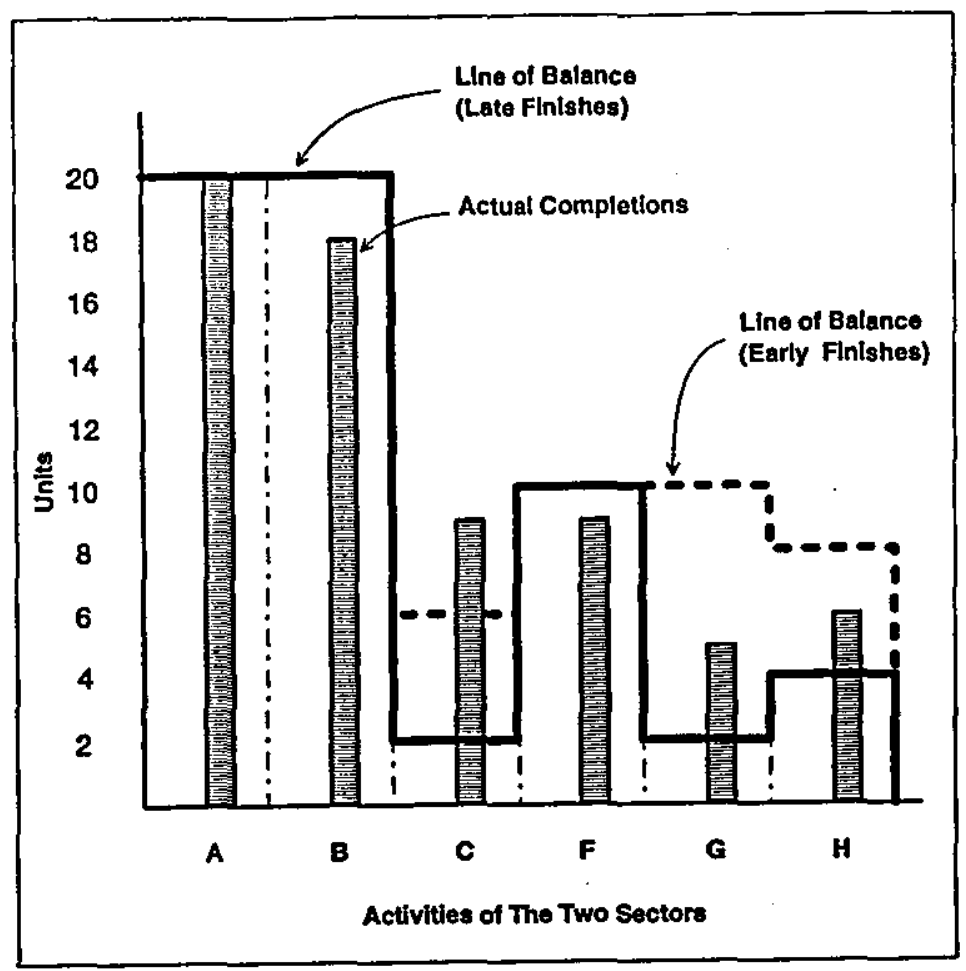

\begin{tabular}{c|c|c|c|c}
\hline \multirow{2}{*}{$\begin{array}{c}\text { Typical } \\
\text { activity } \\
\text { (merged } \\
\text { sectors) }\end{array}$} & EF & LF & $\begin{array}{c}\text { Current projected } \\
\text { EF or AF } \\
\text { (Fig. 8.14) }\end{array}$ & $\begin{array}{c}\text { Time } \\
\text { variance } \\
\text { TV }\end{array}$ \\
\cline { 2 - 3 } A & 12 & 14 & 16 & -2 \\
\hline B & 17 & 19 & 21 & -2 \\
\hline C & 28 & 29 & 31 & -2 \\
\hline D & 35 & 35 & 37 & -2 \\
\hline E & 38 & 38 & 41 & -3 \\
\hline F & 28 & 28 & 30 & -2 \\
\hline G & 29 & 29 & 31 & -2 \\
\hline H & 30 & 35 & 33 & 0 \\
\hline \hline
\end{tabular}

Figure 8.15 - Example 8.3, CPM/LOB Chart and Regularity Measure of Merged Sectors at End of Day 19. 

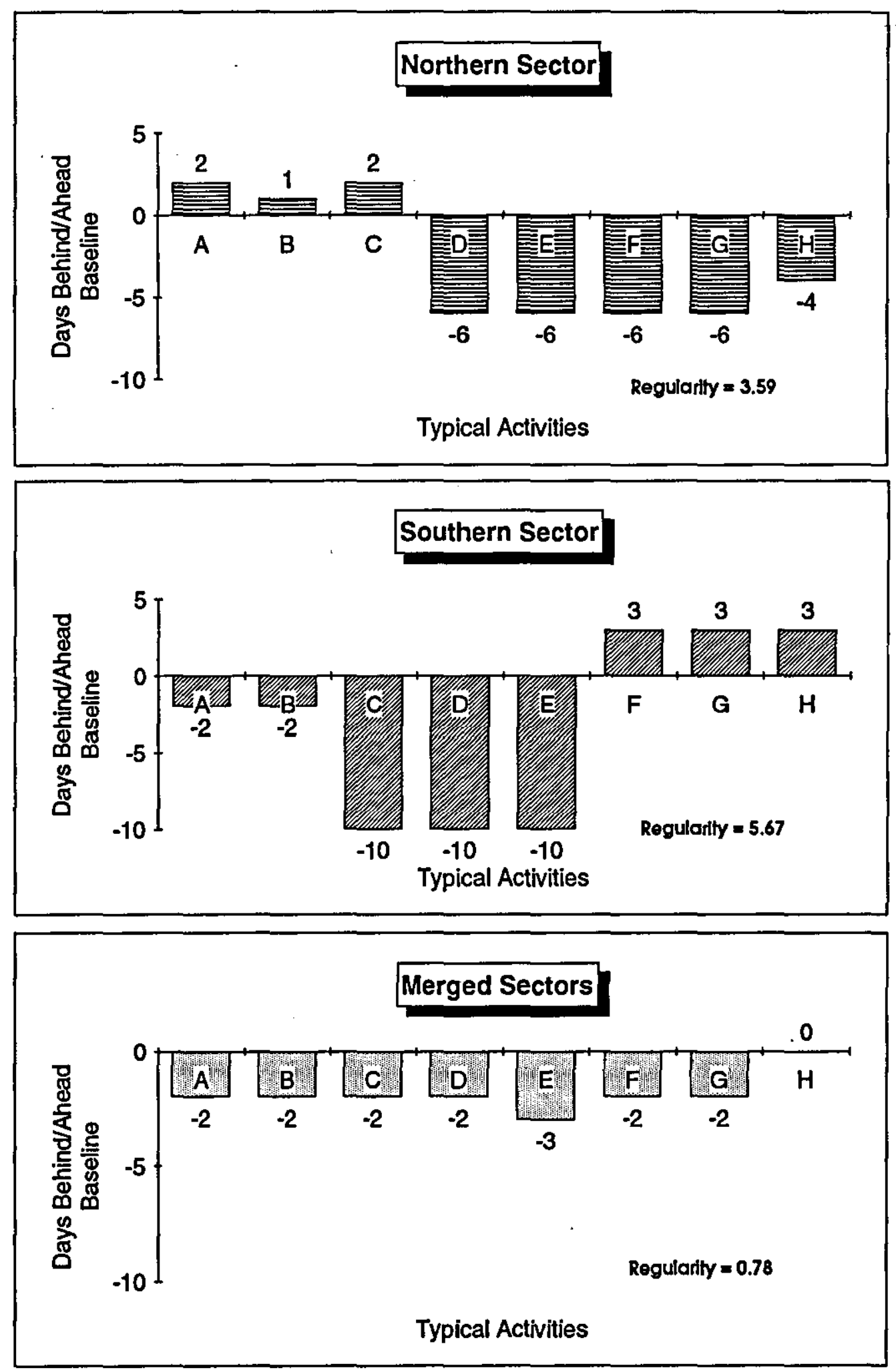

Figure 8.16 - Example 8.3, Regularity Charts at End of Day 19. 
A regularity chart, in addition to the CPM/LOB chart, is suggested and used herein based on the time variance of each typical activity. Fig. 8.16 shows how the regularity chart provides a vivid picture of the progress balance along with a time progress indication. A quick glance reveals the difference between the three statuses of balance by the magnitude of their deviations from the baselines. The visual comparison will be remarkably lucid if the scale of the vertical axis is fixed. 


\section{CHAPTER 9}

\section{VALIDATION THROUGH CASE STUDIES}

\subsection{Introduction}

This chapter shows the application of CPM/LOB on real life construction projects where this author had worked either for the contractor or the construction manager organization. Three applications are provided: The first two are on a housing construction project in Kuwait, and the third is on part of a prison project in California, USA.

Each case study starts with calculating the baseline schedule before the project begins. Then, a point in time well into the project duration is selected (after considerable actual progress and changes have occurred) to update the schedule by entering the real progress data on the activities and measuring the status. This was called the updated schedule which was based on the same quantities of resources in the baseline schedule. Following that and depending on whether the project is ahead or behind schedule, a third schedule, called the accelerated or decelerated schedule, is prepared by setting a new target completion date and calculating revised quantities of team resources to achieve it. Finally each case study concludes with a list of the particular features of CPM/LOB it was used to validate.

The figures and tables are grouped at the end of each case study in the order they were mentioned in the text.

\subsection{Seven Electrical Substations}

These were two transformer substations which would supply electric power to a 260 house development. They were part of a 260 house construction contract between the National Housing Authority of Kuwait (NHA) and a general contracting company called Saud \& Ebrahim Al Abdulrazzak Trading and Contracting Establishment (Al Abdulrazzak) for whom the author had worked. Al Abdulrazzak subcontracted the construction of these electrical substations to Jassim Al Nisif Contracting Company (Al Nisif). The substations were identical and spread over the site. They were numbered $25,26,27,28,29,30$, and 31 and the construction of each one was technically independent of the others. Each substation was about 15 by $20 \mathrm{~m}$ and $4 \mathrm{~m}$ high. Transformers installation and cables laying were not in the scope of the subcontract. 


\subsubsection{Electrical Substations, the Baseline Schedule}

The baseline schedule was prepared by the author to Al Nisif utilizing CPM/LOB on Primavera which, at the time, was not able to level more than six resources in a single run. Fig. 9.1 shows the subcontract calendar, which is a six workday week calendar (weekends on Friday) and includes some religious and national holidays. Construction was to start on December 1, 1987 and complete on August 31, 1988. Fig. 9.2 shows the logic diagram of a typical substation comprising 42 activities. The agreed upon sequence of operation on the substations was: 27,25 , $29,31,30,28$, and 26 . Fig. 9.3 is a tabular schedule report of a typical substation starting on day one (December 12, 1987) and required to complete no later than the last day (August 31, 1988). The available time for completions for each typical activity, $T_{a \mathrm{a}}$, is calculated in a procedure identical to that used in example 6.3 by simply running the schedule of this typical network and obtaining the total floats which are themselves the available times for completions for each typical activity, $\mathrm{T}_{\mathrm{aa}}$. Since total floats are measured by workdays not calendar days, weekends and holidays are accounted for.

Table 9.1 displays the calculations toward finding the minimum resource quantities required to complete seven substations no later than August 31,1988 . Only nineteen typical activities were considered very important and had resources allocated to them. This decision was influenced by Primavera's then limited resource leveling ability as mentioned above. Resources are either: squads or crews, and referred to as CR; or shutters, and referred to as SH. Both are associated with a typical activity number. So CR04, refers to the squad of typical activity 04 LEVELING \& SETTING OUT. The minimum quantities of resources, $S_{\mathrm{ma}}$ Rounded, in column 8 , is entered into the CPM software, Primavera, as resource availability, then time scheduling and resource leveling is performed in line with the above sequence of operation.

Fig. 9.4 is a tabular schedule report of the activities of the seven substations sorted by typical activities then by their early start dates. Under the column titled ACTIVITY ID the first two numbers before the hyphen refer to the substation number while the other two numbers after the hyphen refer to the typical activity number. So 27-04 is typical activity four of substation 27 . The numbers under the column titled CODE are the leveling priorities. It is noted that the projected completion date is August 16, 1988 instead of August 31, 1988. This is due to the rounding up of the number of minimum resources to the next higher integer before entering them onto the CPM software. 
The analysis of the schedule of Fig. 9.4 indicates that the critical path flows through a series of time-critical sequential activities on the first substation (number 27). It then goes through the resource-critical typical activity 60 SANDLIME BRICKS OF WALLS TO FIXTURES \& LINTELS LEVEL on all the substations before making its successors critical to the end and completion of the last substation, number 26 . It is obvious that typical activity 60 was the lowestrate activity and therefore controlled the pace of the project. It was a bottleneck. It pushed its successors and reduced their floats rendering most of them critical because of its comparatively slow rate of progress.

Further examination of Table 9.1 explains why this was the case. Column 9 calculates the rate of each typical activity based on the rounded number of resources as if each typical activity was flowing independently of any predecessor. The lowest rate should point to the typical activity that controls the pace of the project had there been no floats in the typical substation network. But since there was more than one path, and some activities had float in the typical substation network, the rates of the activities having float should be normalized to yield comparable figures and enable identifying the slowest typical activity, hence the first, most resource-critical in the overall project network. Column 10 of Table 9.1 indicates that typical activities 60 and 84 should have been expected to be resource-critical and controlling the pace of the project since they shared the slowest normalized completion rate: 0.100 substation per day. The above LOB calculation puts the analysis in a better perspective: both typical activities 60 and 84 were the slowest and controlled the pace of the project and typical activities $64,66,68,72$, and 78 remained tightly bound between 60 and 84 hence critical. Typical activity 76, since trapped between resource-critical activities on the overall project network, had only its two day float from the typical unit network shown on all substations in Fig. 9.4.

To go back and confirm the effect of rounding up the number of minimum resources, $S_{\operatorname{ma}}$, a separate schedule run was performed on the typical substation network like that of Fig. 9.3 but with August 16, 1988 as the finish-no-later-than date. The available times for completion for all the activities, $T_{2 a}$, were 12 days fewer and the $S_{\mathrm{ma}}$ for typical activities 60 and 84 was exactly equal to one. $S_{\mathrm{ma}}$ for the other activities increased, but after rounding them up they were not different from their original quantities as listed on column 8 of Table 9.1. Hence the resource availability of the overall project schedule was unchanged and August 16, 1988 remained the projected completion date. This proves that when the resource requirement of the dominating (lowest-rate) activity or activities is an integer (in this case, one), the methodology makes the schedule finish exactly on target. 
In chapter six it was stated that the continuity of a working squad could be enhanced by delaying its start to avoid interference with slower predecessor activities through using start constraints in the commercially available CPM software. Figs. 9.5 and 9.6 demonstrate how the continuity of typical activity 26 (which is between typical activities 25 and 32) was raised to full continuity through utilizing some of its float by constraining its start to no earlier than January 17, 1988. Neither its predecessor, typical activity 25 , nor its successor, typical activity 32 were affected.

\subsubsection{Electrical Substations, the Updated Schedule}

The status of the project as of February 2, 1988 is shown in Fig. 9.7, it indicates that there was no change of sequence and that the projected completion date is August 3,1988, 13 calendar days (10 workdays) ahead of the baseline completion date, August 16, 1988. This was obtained by simply entering the percent completes of the activities and running time scheduling and resource leveling using the same resource availabilities of the baseline schedule. It should be noted that the typical activities which did not have resources allocated to them (those not listed on Table 9.1) were not levelled, hence not staggered for proper execution. This reveals the need for allocating team resources for all the typical activities in the baseline schedule to avoid having to address this issue at a subsequent schedule update. Of course this particular situation can be corrected by simply allocating one team resource to each uncompleted activity and running resource leveling.

Fig. 9.8 shows the progress regularity as of February 2, 1988. While all of the typical activities to the left of typical activity 50 are within the early and late completion dates, the others are ahead of the early baseline schedule dates.

Fig. 9.9 isolates the current critical activities for more clarity. It shows that the critical path now flows through the time-critical activities of substation 31; the resource-critical typical activity 60 , starting at substation 31 and to the last substation, number 26; typical activities 64 , $66,68,72,78$, and 84 from substation 31 to 26 ; and finally through the last time-critical activity of substation 26 , activity $26-88$.

Typical activity 60 is the first bottleneck and controls the pace of the progress. The regularity chart shows it 12 calendar days ahead of the baseline schedule which coincides with 
10 workdays per the schedule and the work calendar. Since typical activity 50 is not late and is the only activity that has more than one resource allocated to it (three shutters), it is worth it to do a what-if analysis to see what happens if the shutters were to be reduced to two. It took less than a minute to calculate that on a 486DX2 $66 \mathrm{MHz}$ computer and P3 release 5.1 to realize that the project completion date would be moved fourteen days, from August 3 to August 17, 1988 due to the elimination of one shutter set.

\subsubsection{Electrical Substations, Features Validated}

In this case study the following features of CPM/LOB have been emphasized and validated as superior characteristics over those provided by the current applications on planning and scheduling repetitive projects:

1. Resource-based relationships among repetitive activities which are tedious to create then to update and vulnerable to human error, are replaced with an easier and more realistic scheduling that depends on the availability of team resources and leveling thereof. So for example, the individual activities of typical activity. 50 which required three squads, should have been linked with resource-based relationships as follows: $27-50$ to $31-50,25-50$ to $30-50,29-50$ to $28-50$, and 3150 to 26-50. These have to be revised each time the sequence or the quantity of squads is changed.

2. Repetitive activities can maintain a practical continuity that do not impact the completion date within the CPM context. This continuity can be further enhanced by manipulating the available float. This was demonstrated above on squad CR26 and shown in Figs. 9.5 and 9.6.

3. Through the application of the LOB concept to calculate resources at the outset of the planning process, a considerably better overview of the project is gained. This was demonstrated by an early identification of the required quantities of resources to complete on target and a lucid perception of the critical path flow even before running the schedule on the CPM software.

4. The capability of readily assessing alternative resource-driven strategies on the completion date, which could be inconspicuous though potentially big. 
5. The ability to quantify the state of balance of a project progress through regularity charts that are superior to LOB charts in being rate sensitive, and associated with a single quantification figure. 


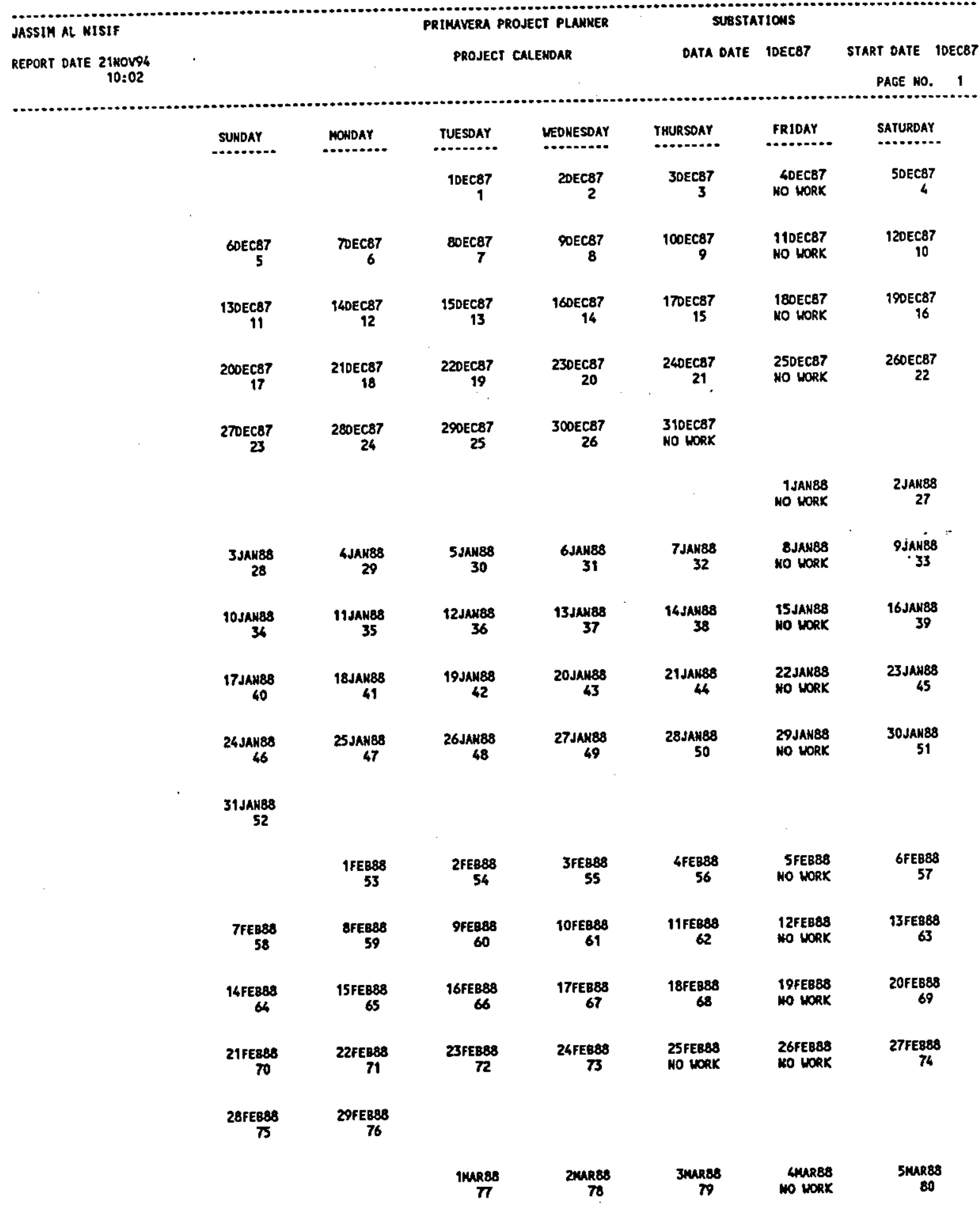

Figure 9.1 - Case 1, Work Calendar. 


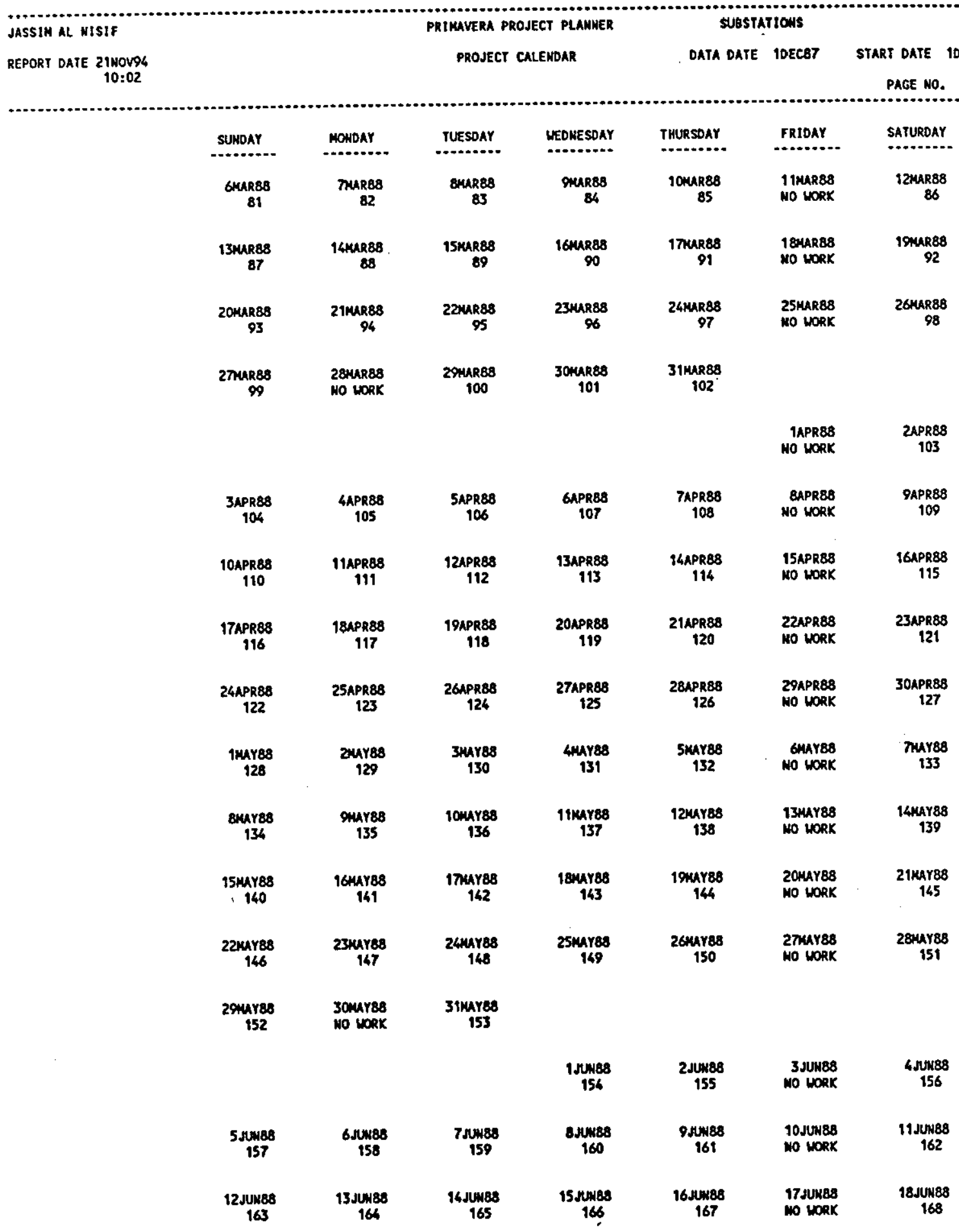

Figure 9.1 - Case 1, Work Calendar (Continued). 


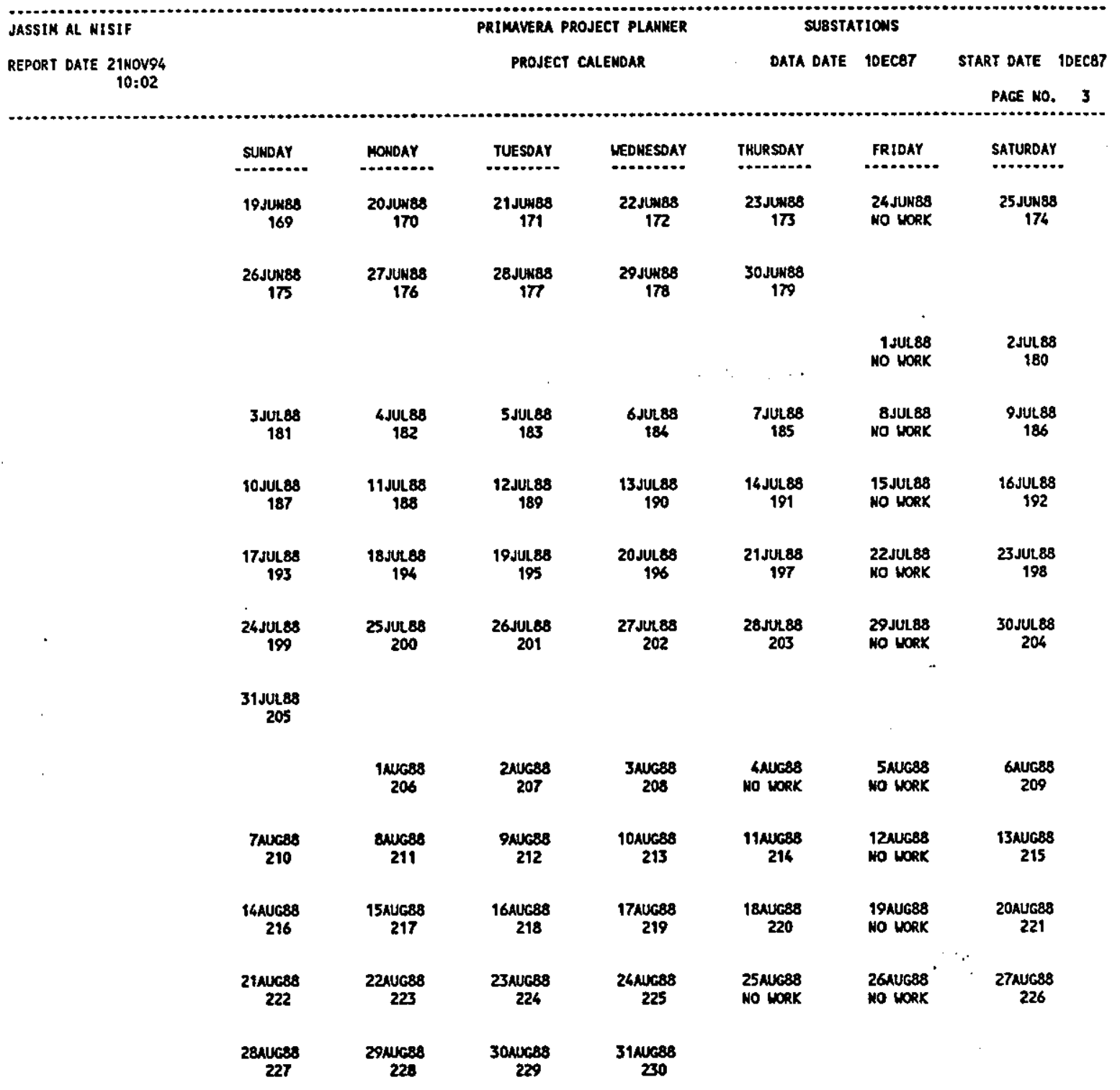

Figure 9.1 - Case 1, Work Calendar (Continued). 


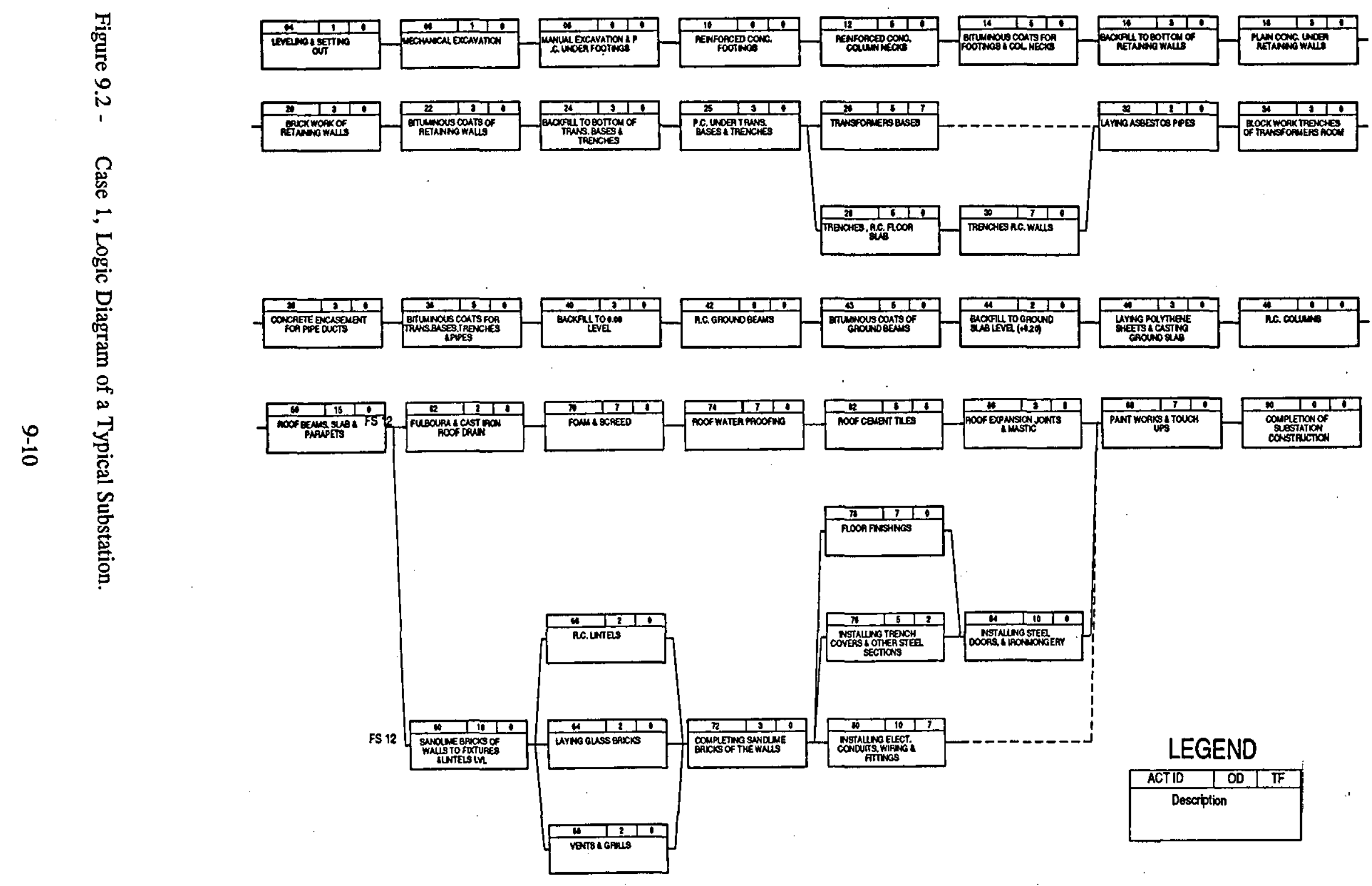




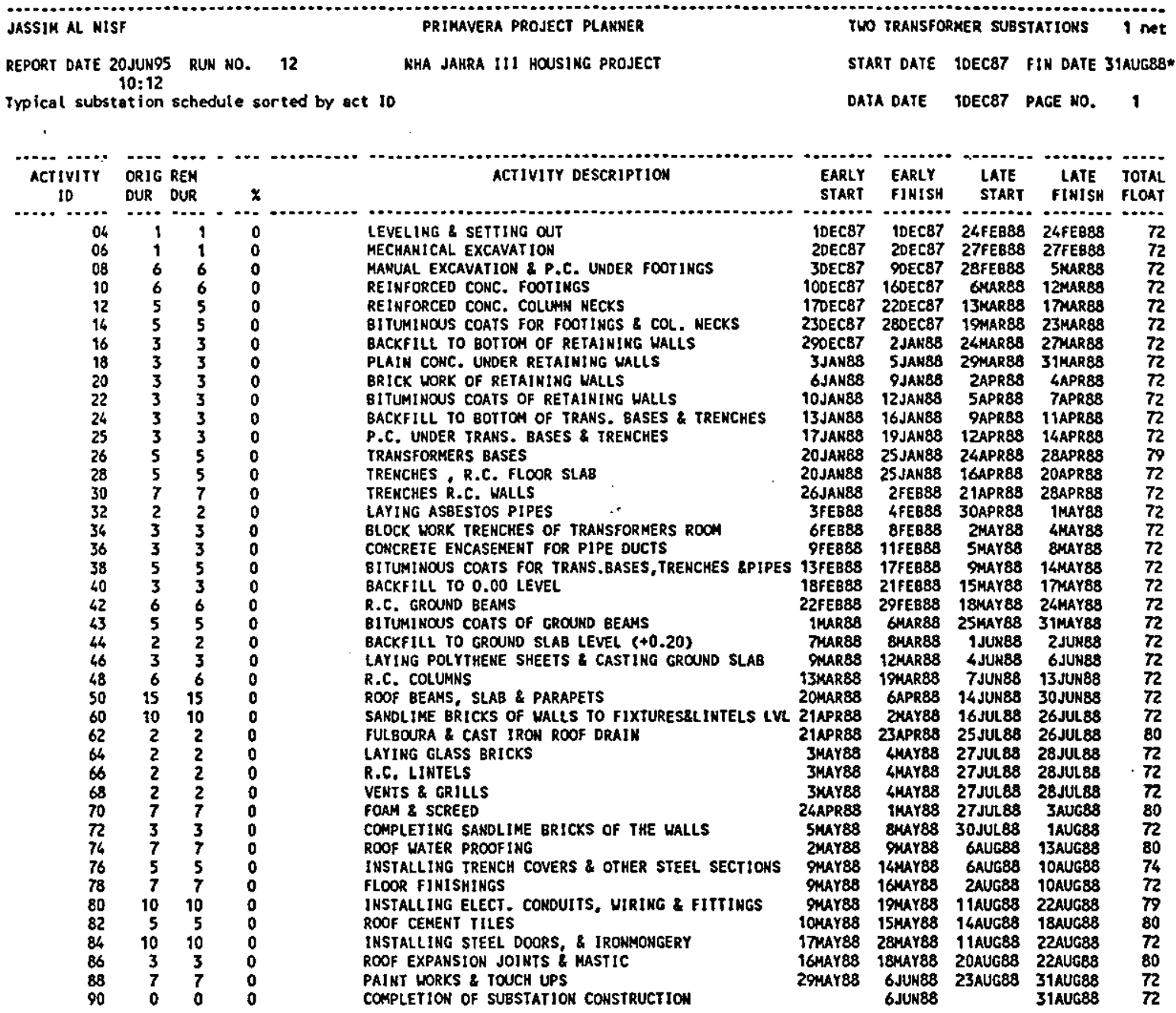

Figure 9.3 - Case 1, Schedule of a Typical Substation Network Showing $\mathbf{T}_{2 \mathrm{a}}$ under the "Total Float" Column. 


\begin{tabular}{|c|c|c|c|c|c|c|c|c|c|}
\hline & & & & & & & & & Normallzed \\
\hline & & & & & & & & Independent & independent \\
\hline & & & Resource & Taa & Rma & Sma & Sma & rate & rate \\
\hline ACT & Activity title & Resource & duration & TF & $\{7-1\}+(5)$ & (6) $\times(4)$ & Rounded & (8) $\div$ (4) & $(9) \times .083 \div(6)$ \\
\hline (1) & (2) & (3) & (4) & (5) & (6) & (7) & (8) & (9) & (10) \\
\hline 04 & EEVELING \& SETTING OUT & CR04 & 1 & 72 & 0.083 & 0.083 & 1 & 1.000 & 1.000 \\
\hline 08 & MANUAL EXCAVATION \& P.C. UNDER FOOTINGS & CR08 & 6 & 72 & 0.083 & 0.500 & 1 & 0.167 & 0.167 \\
\hline 10 & REINFORCED CONC. POOTINGS & SH10 & 6 & 72 & 0.083 & 0.500 & 1 & 0.167 & 0.167 \\
\hline 12 & KEINFORCED CONC. COLUMN NECRS & SE12 & 5 & 72 & 0.083 & 0.417 & 1 & 0.200 & 0.200 \\
\hline 26 & TRANSFORMERS BASES & CR26 & 5 & 79 & 0.076 & 0.380 & 1 & 0.200 & 0.219 \\
\hline 28 & ITRENCHES , R.C. FLOOR SLAB & CR28 & 5 & 72 & 0.083 & 0.417 & 1 & 0.200 & 0.200 \\
\hline 30 & TRENCHES R.C. WALLS & SE3O & 7 & 72 & 0.083 & 0.583 & 1 & 0.143 & 0.143 \\
\hline 38 & BITUMINOUS COATS POR TRANS.BASES, TRENCHES \&PIPES & CR38 & 5 & 72 & 0.083 & 0.417 & 1 & 0.200 & 0.200 \\
\hline 42 & k.c. GROUND BEAMS & SH42 & 6 & 72 & 0.083 & 0.500 & 1 & 0.167 & 0.167 \\
\hline 48 & k.c. COLUMNS & SE4B & 6 & 72 & 0.083 & 0.500 & 1 & 0.167 & 0.167 \\
\hline 50 & ROOP BEAMS, SLAB \& PARAPETS & SH50 & 27 & 72 & 0.083 & 2.250 & 3 & 0.111 & 0.111 \\
\hline 60 & SANDLIME BRICRS OF WALLS TO FIXTURESELINTELS LVL & CR60 & 10 & 72 & 0.083 & 0.833 & 1 & 0.100 & 0.100 \\
\hline 70 & FOAM \& SCREED & CR70 & 7 & 80 & 0.075 & 0.525 & 1 & 0.143 & 0.159 \\
\hline 74 & KOOF WATER PROOPING & CR74 & 7 & $80^{\circ}$ & 0.075 & 0.525 & 1 & 0.143 & 0.159 \\
\hline 78 & FLOOR FINISEINGS & CR78 & 7 & 72 & 0.083 & 0.583 & 1 & 0.143 & 0.143 \\
\hline 80 & INSTALLING ELECT. CONDUITS, WIRING \& PITTINGS & CR80 & 10 & 79 & 0.076 & 0.759 & 1 & 0.100 & 0.110 \\
\hline 82 & ROOP CEMENT TILES & CRB2 & 5 & 80 & 0.075 & 0.375 & 1 & 0.200 & 0.222 \\
\hline 84 & ENSTALLING STEEL DOORS, \& IRONMONGERY & CR84 & 10 & 72 & 0.083 & 0.833 & 1 & 0.100 & 0.100 \\
\hline 88 & PAINT WORRS \& TOUCE UPS & CR88 & 7 & 72 & 0.083 & 0.583 & 1 & 0.143 & 0.143 \\
\hline
\end{tabular}



14:13

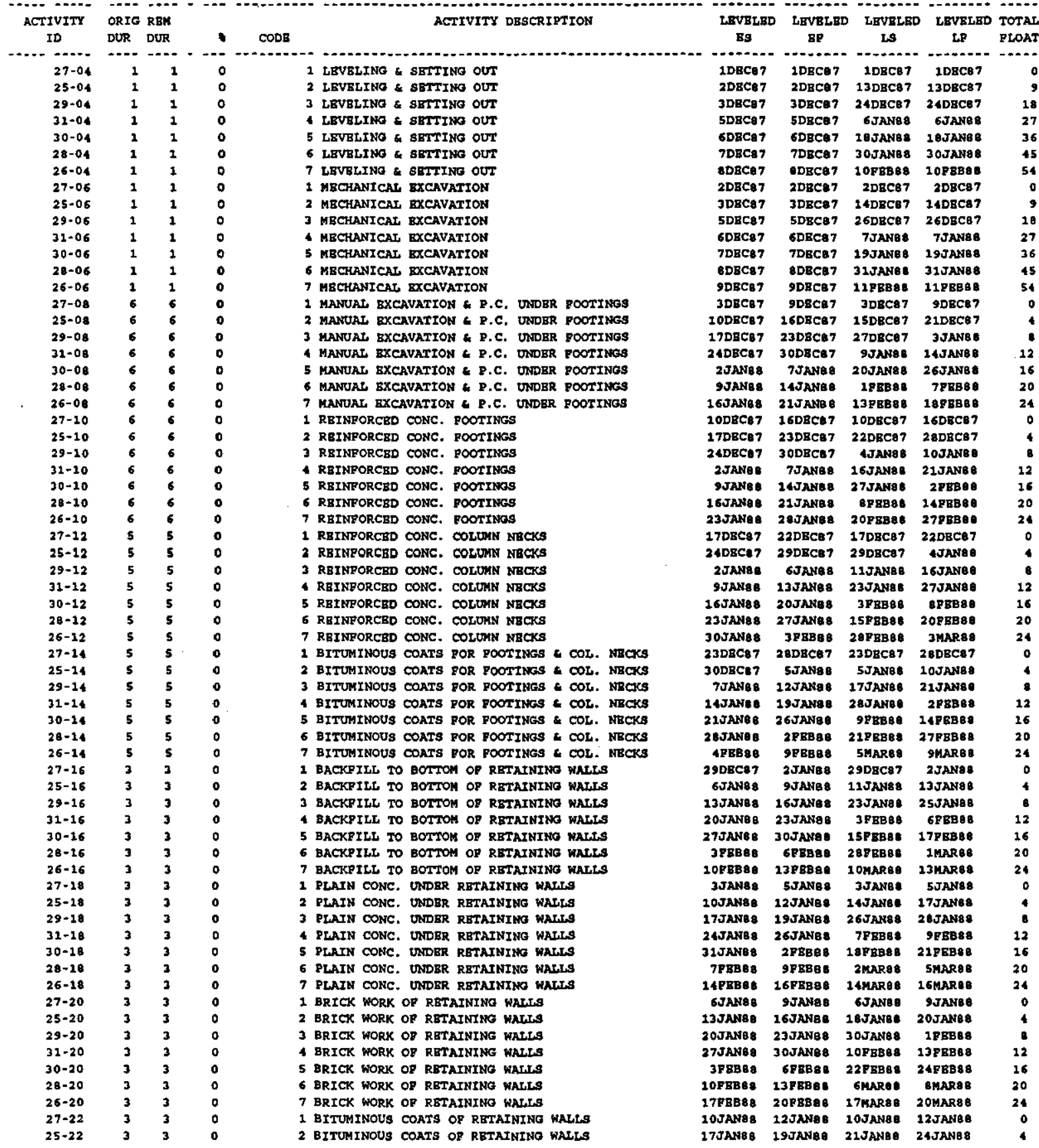

Figure 9.4 - Case 1, Baseline Schedule. 


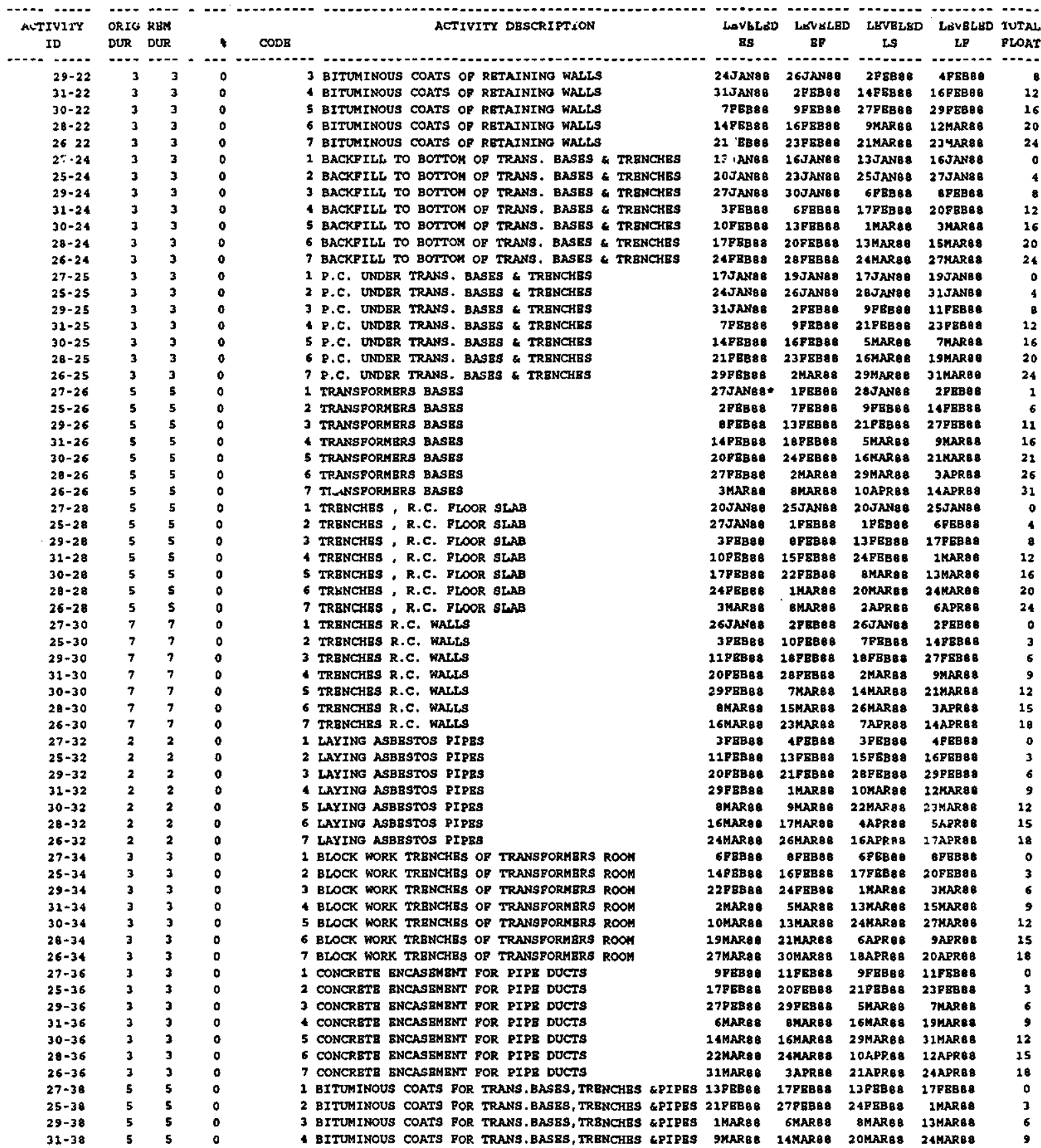

Figure 9.4 - Case 1, Baseline Schedule (Continued). 


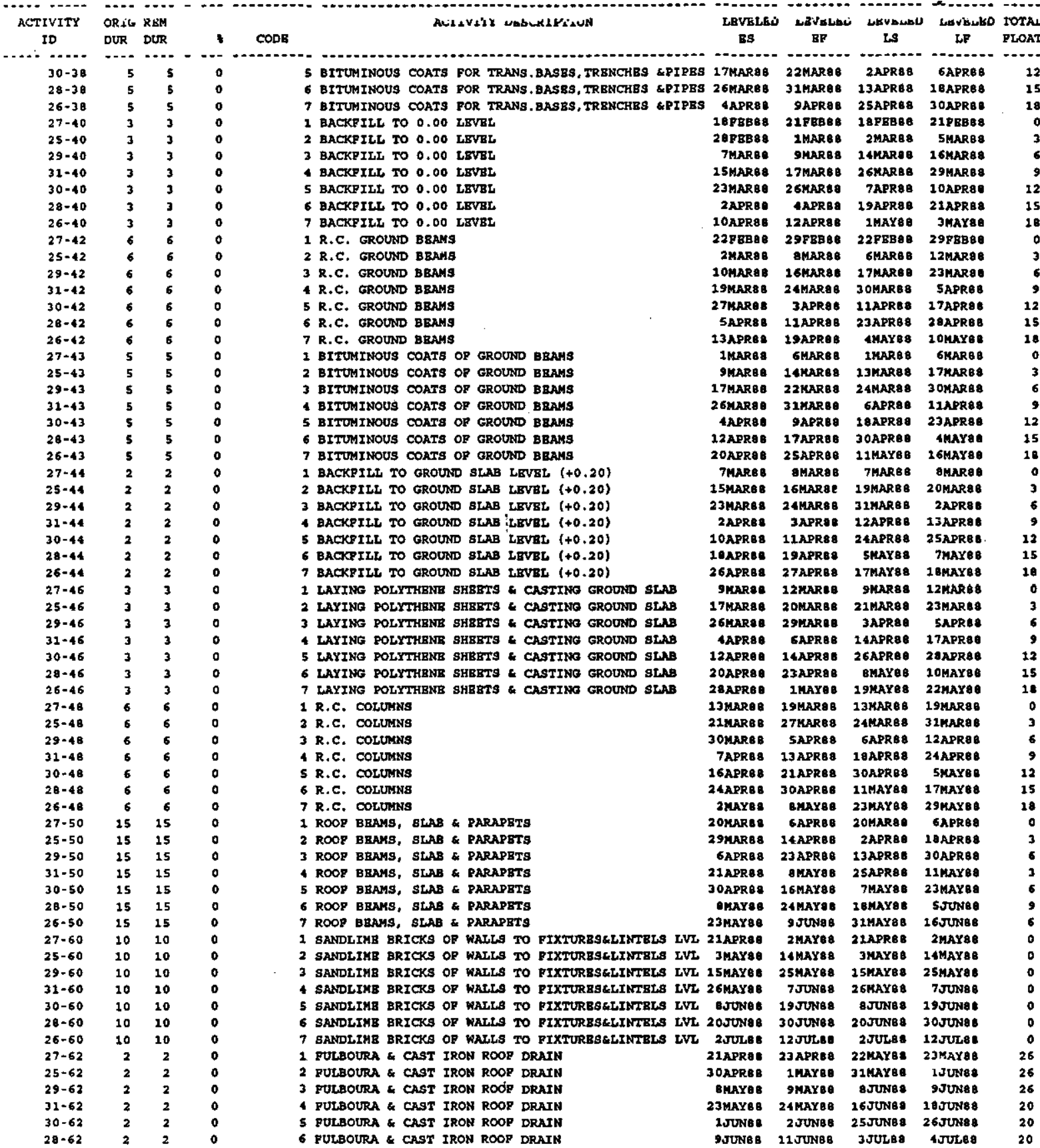

Figure 9.4 - Case 1, Baseline Schedule (Continued). 

14:13

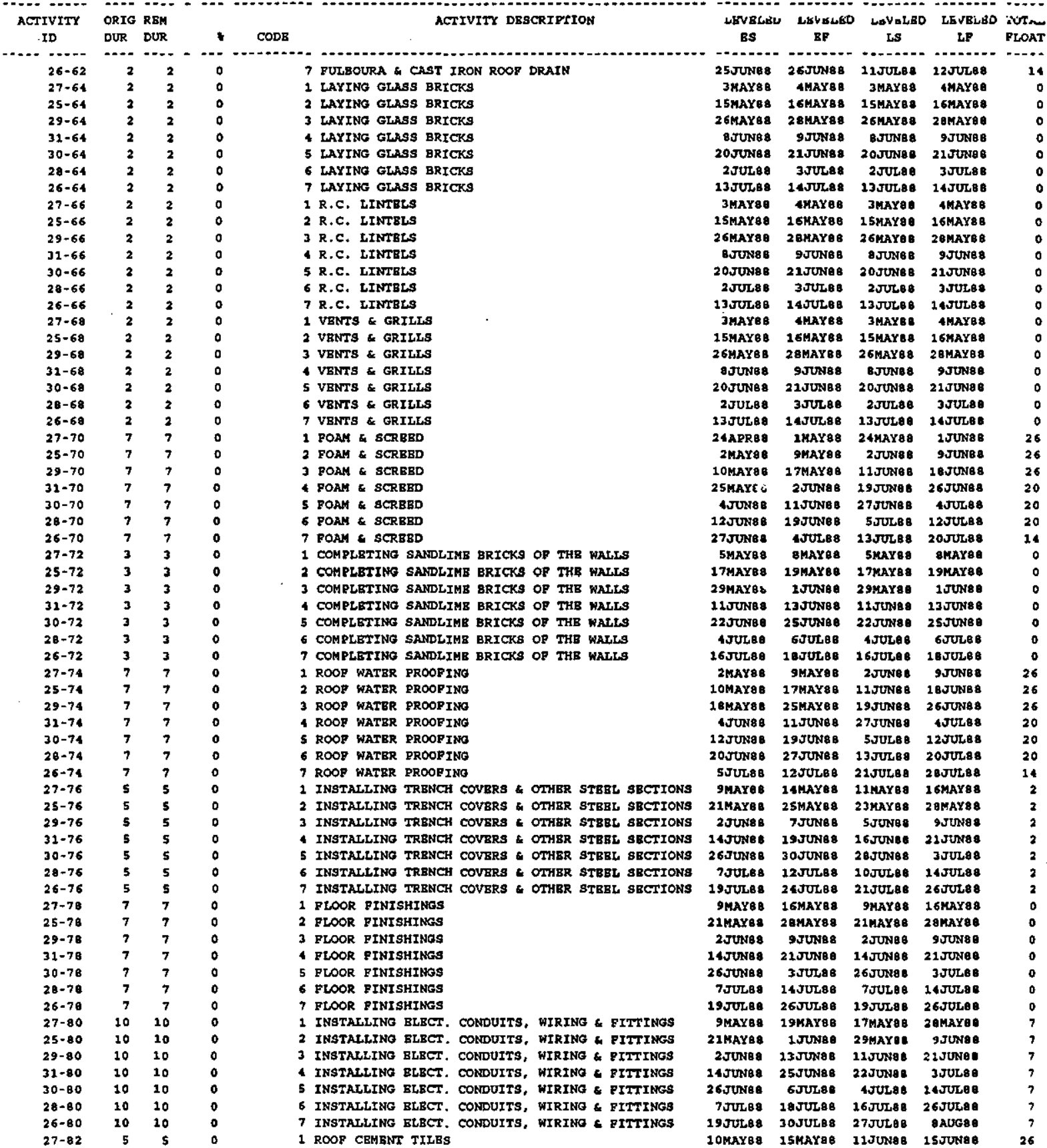

Figure 9.4 - Case 1, Baseline Schedule (Continued). 


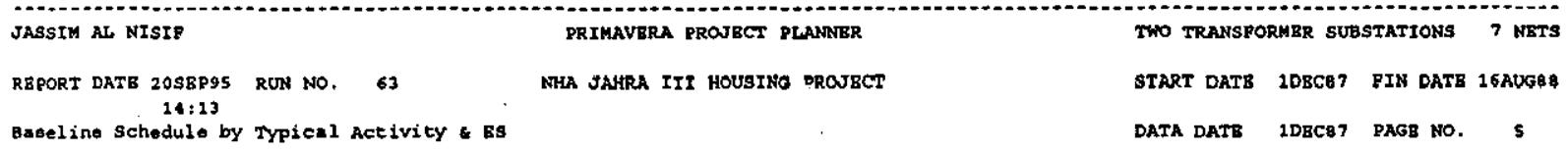

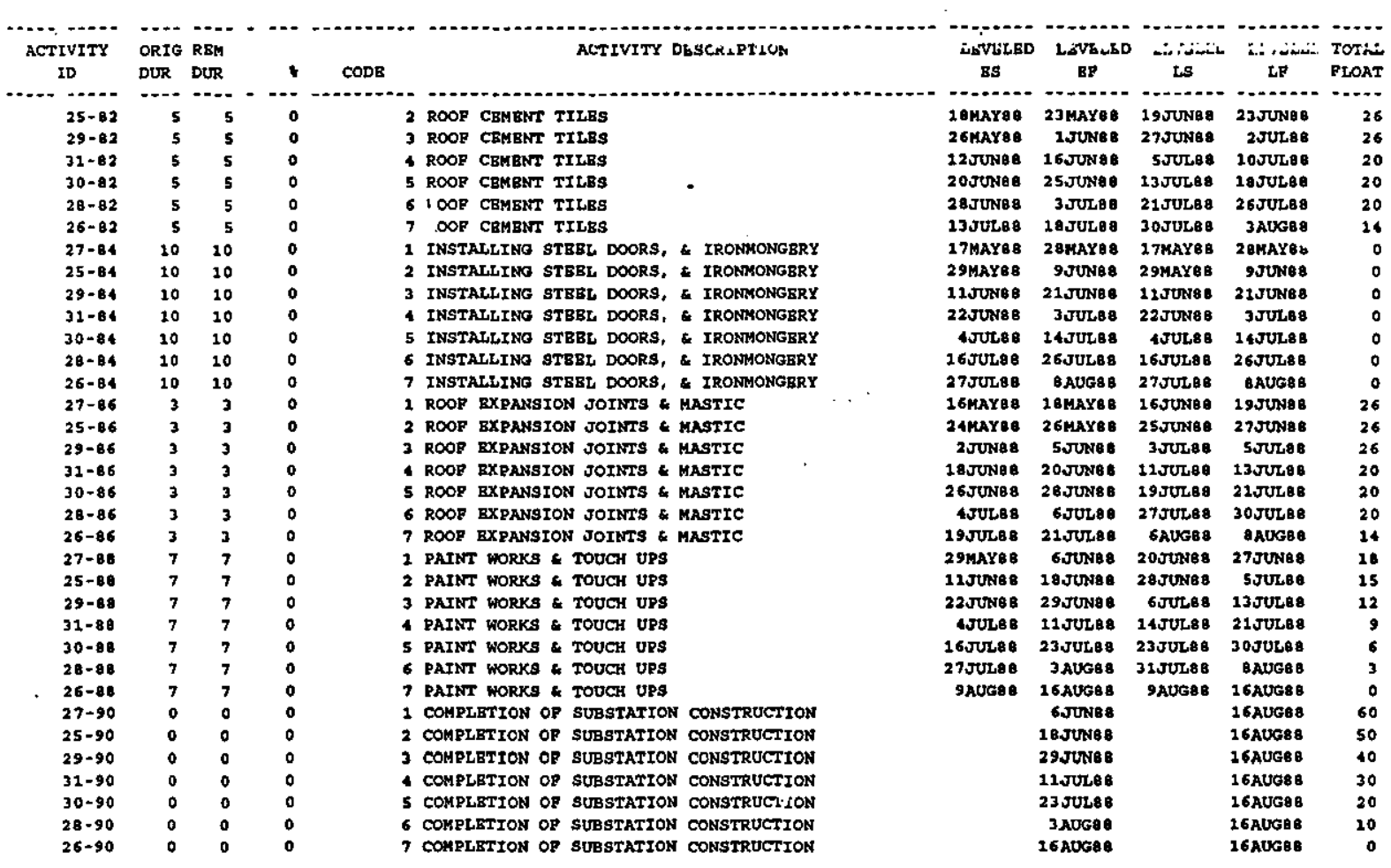

Figure 9.4 - Case 1, Baseline Schedule (Continued). 


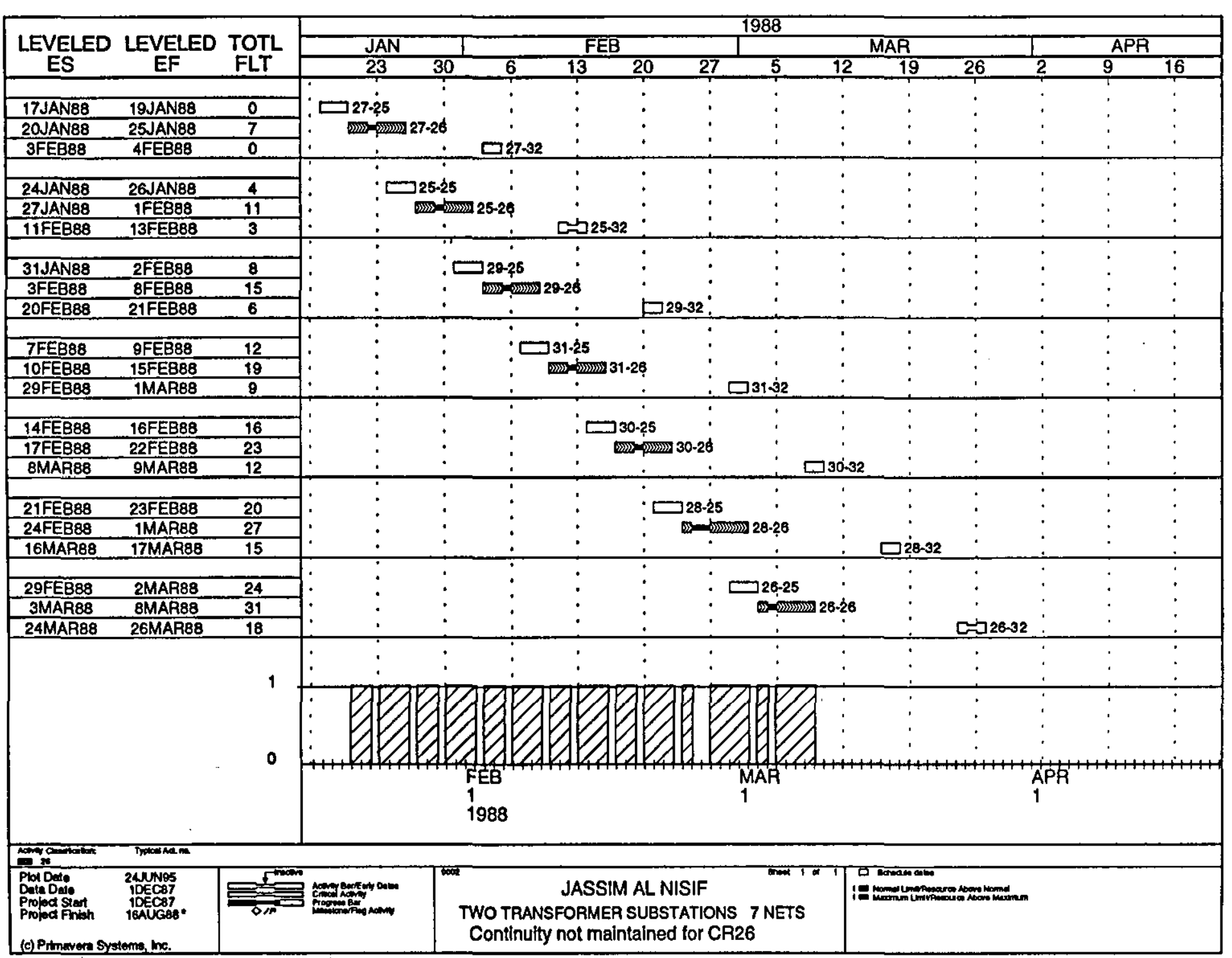




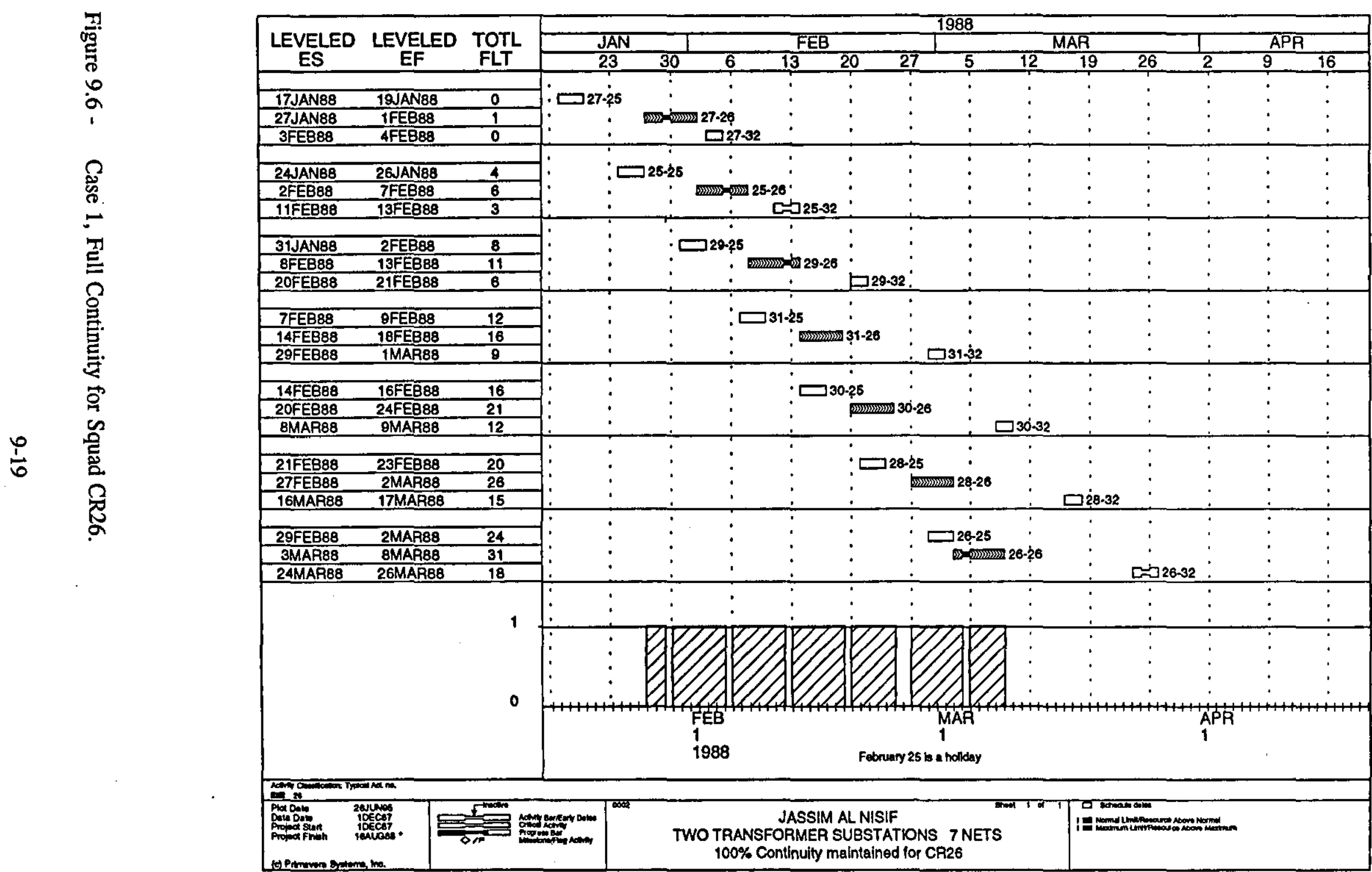




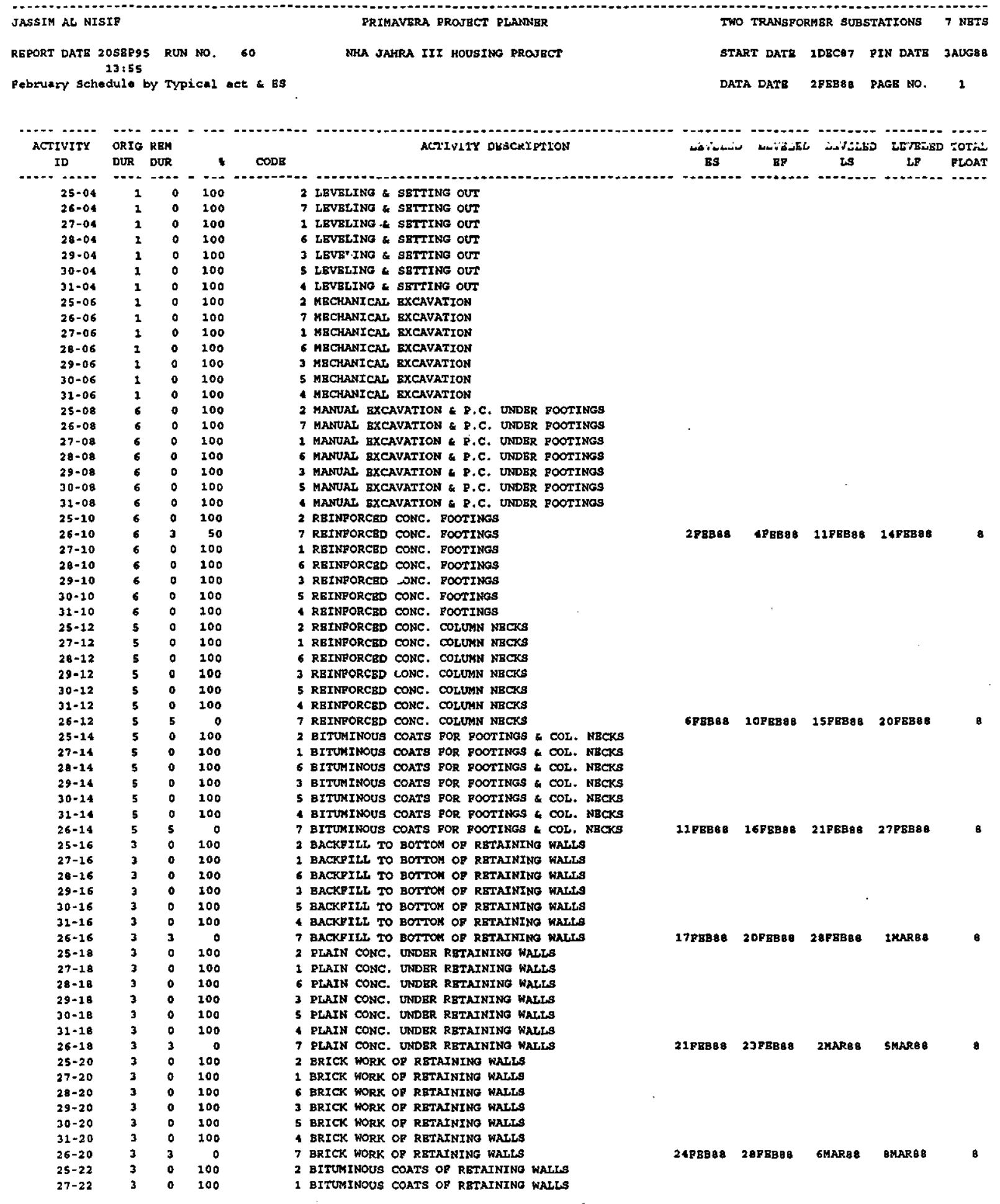

Figure 9.7 - Case 1, Project Status and Schedule Update as of February 2, 1988. 


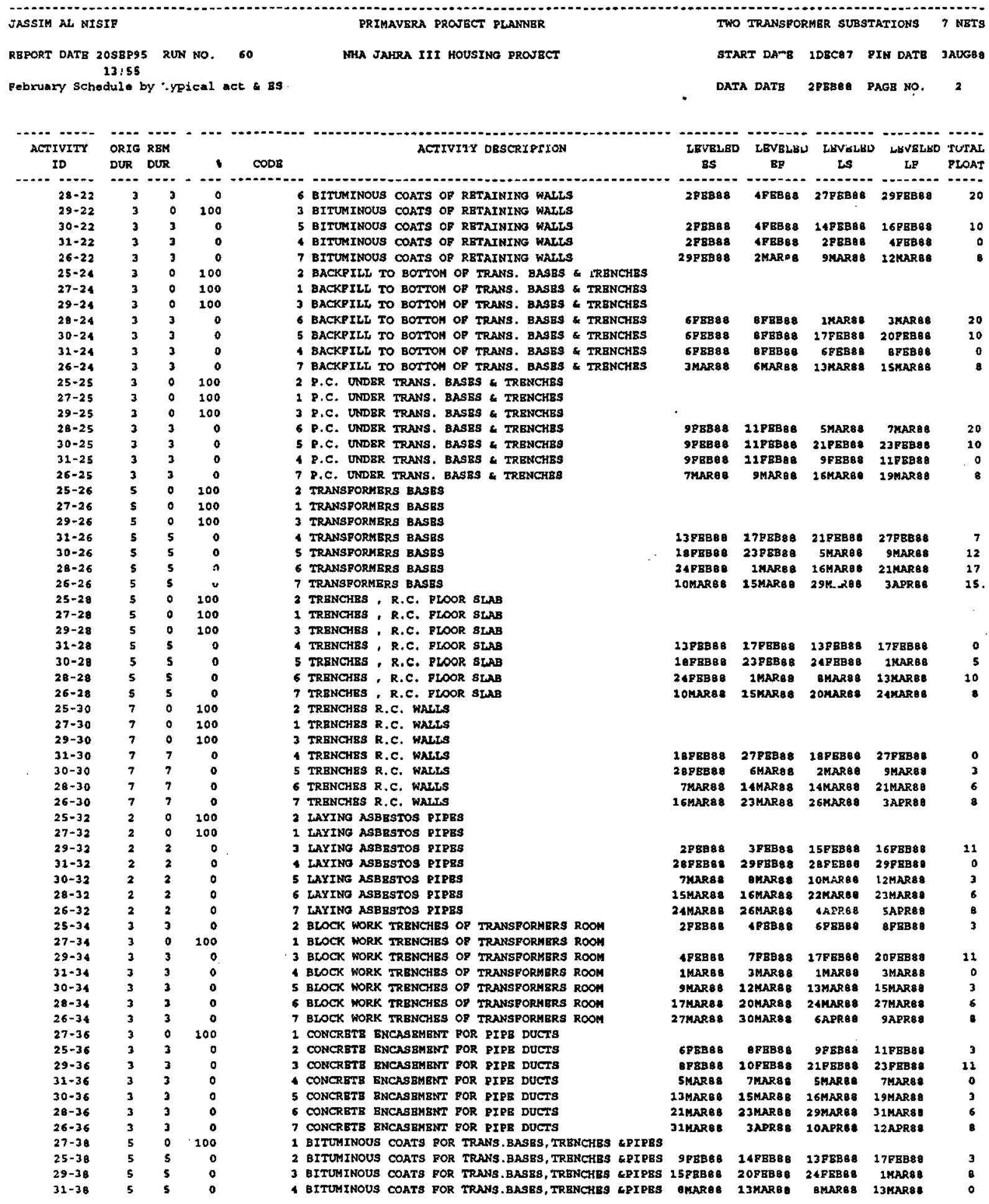

Figure 9.7 - Case 1, Project Status and Schedule Update as of February 2, 1988 (Continued). 
JASSIM AL NISIP

:BPORT DATE 20SBP9S RUN No. 60

$13: 55$

P.bruary Schodule by Typical act \& BS
PRIMAVERA PROTBCT PLANNBR

NHA JAHRA III HOUSING PROTBCT
TWO TRANSFORMBR SUBSTATIONS 7 NETS

START DATE IDBCBY PIN DATB 3AUGB DATA DATE 28BBga pagB nO. 3

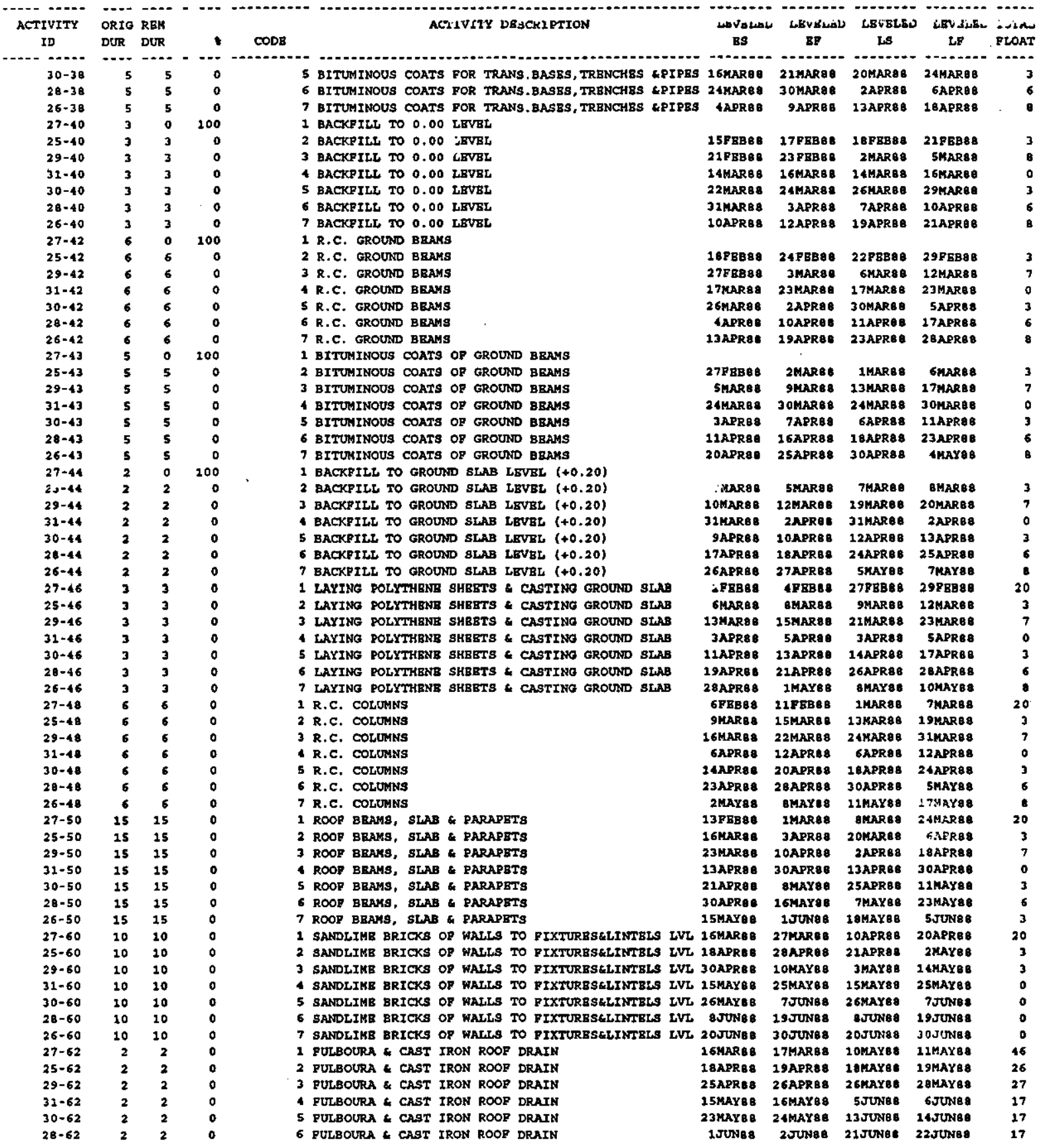

Figure 9.7 - Case 1, Project Status and Schedule Update as of February 2, 1988 (Continued). 


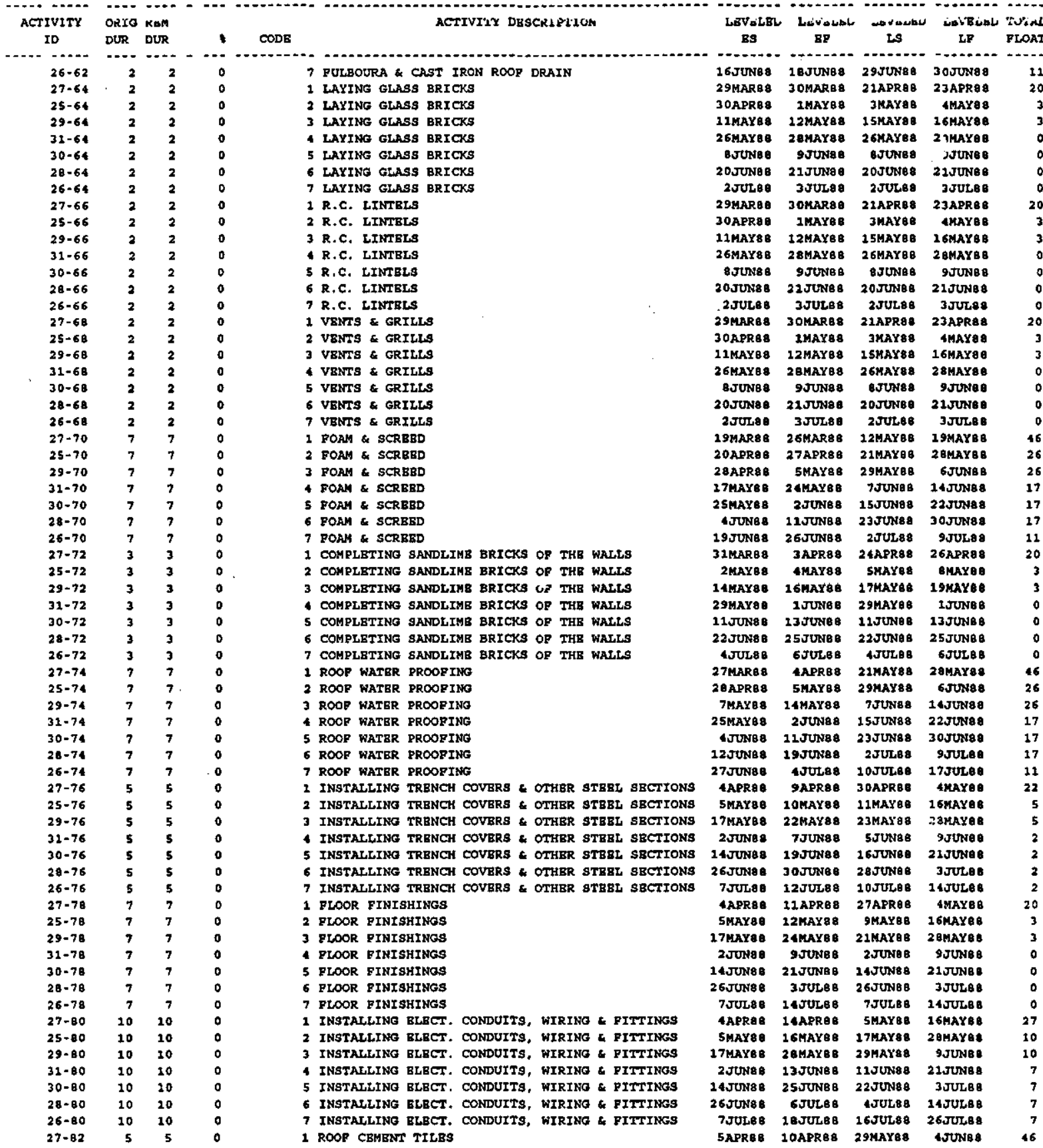

Figure 9.7 - Case 1, Project Status and Schedule Update as of February 2, 1988 (Continued). 


\section{JASSIM AL NISIP}

PRIMAVBRA PROTBCT PLANNER

NHA JAHRA III HOUSING PROTBCT

REPORT DATE 20 SEP95 RUN NO. 60

$13: 55$

Fobruary schedule by Typical act $B$ Bs
TWO TRANSPORMER SUBSTATIONS 7 NETS

START DATE IDECB 7 PIN DATB 3AUGB

DATA DATE 2PBBEB PAGB 0 .

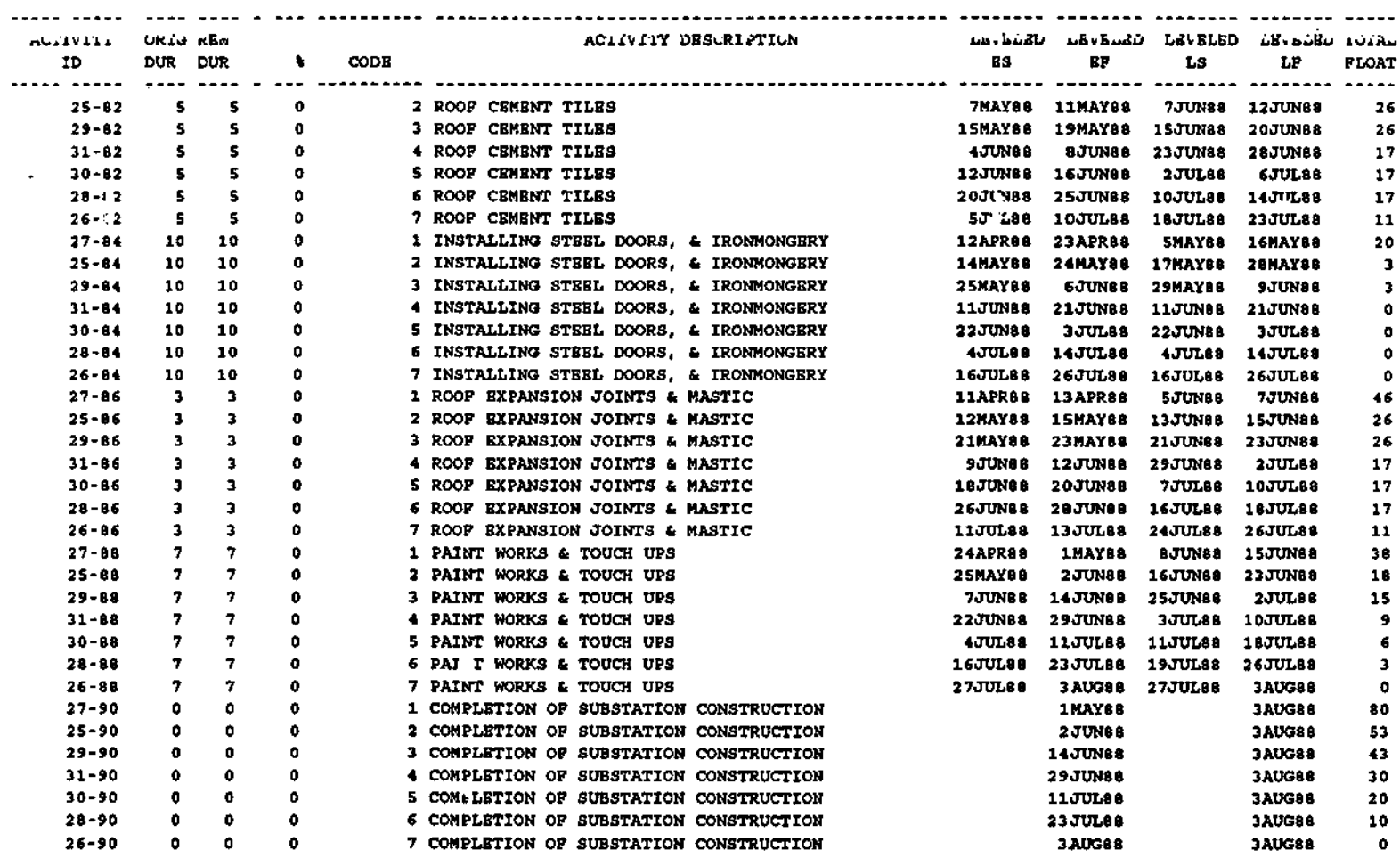

Figure 9.7 - Case 1, Project Status and Schedule Update as of February 2, 1988 (Continued). 


\section{Seven Electrical Substations}

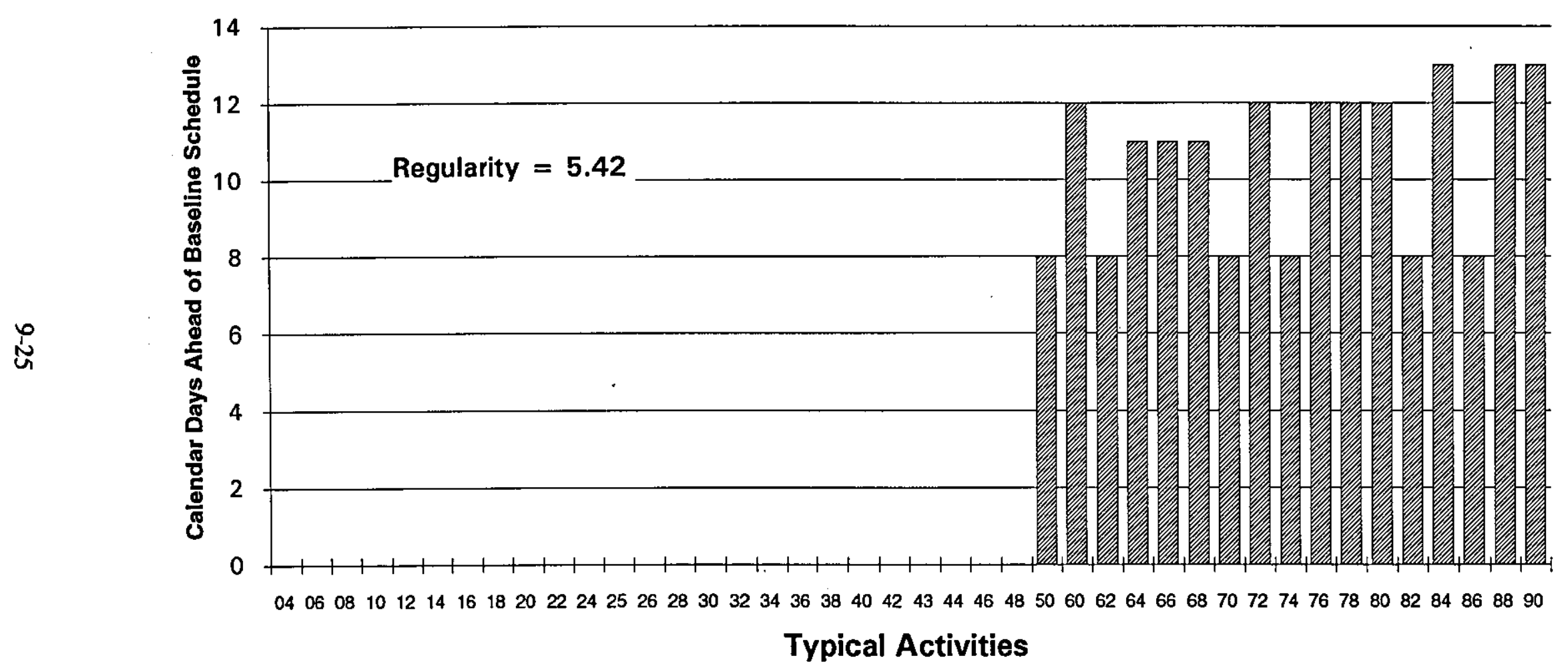

Figure 9.8 - Case 1, Progress Regularity as of February 2, 1988. 


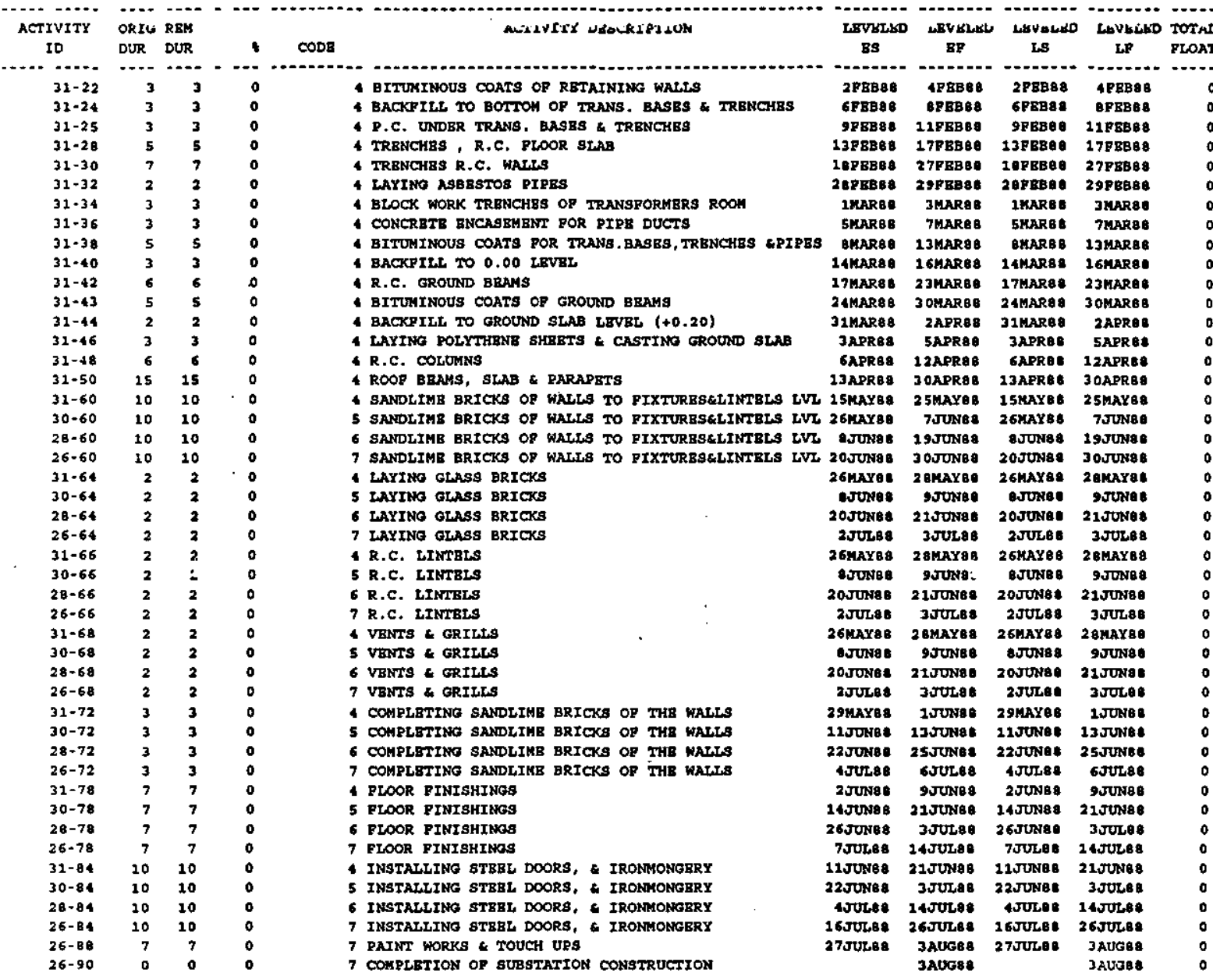

Figure 9.9 - Case 1, Critical Activities as of February 2, 1988. 


\subsection{A Housing Project in Kuwait}

As mentioned above, this was a 260 house construction contract between NHA and $\mathrm{Al}$ Abdulrazzak. There were four types of two story houses, very similar in design and work content: A, B, C, and D. Each floor area was about $300 \mathrm{sq} \mathrm{m}$ and the lot area was about $400 \mathrm{sq} \mathrm{m}$. These houses were somewhat sophisticated and the construction value was equivalent to about $£ 100 \mathrm{~K}$ per house. Fig. 9.10 is an overview of the project layout showing houses on abutting lots called clusters with each cluster containing from two to nine houses. House numbers and types are inside the rectangles and cluster numbers are inside the ovals.

Al Abdulrazzak subcontracted the labor part of the construction to three subcontractors. Seven Seas, one of the three subcontractors, got 51 houses. Due to space limitations this case study was limited to Seven Seas' houses. The subject 51 houses are in 14 clusters: 211, 213-221, and 320-323. Seven Seas' contract duration was from April 1, 1987 to August 17, 1988. Fig. 9.11 shows the project calendar. The project schedule was Al Abdulrazzak's responsibility and was maintained by the author. The CPM network of the project started with activity A, NOTICE TO PROCEED, then B, MOBILIZATION before the 51 subnetworks of the houses began. Activities $\mathrm{Y}$ and $\mathrm{Z}$ succeeded the subnetworks of the houses and were CONTRACTOR'S CONTINGENCY and HANDOVERS \& DEMOBILIZATION respectively. Each house subnetwork comprised 86 activities numbered from 01 to 86 . However, there was only one typical activity, 06 CLUSTER BACKFILLING TO GROUND BEAMS BOTTOM LEVEL for each cluster. This was because backfilling was best done in layers for the whole cluster and was not convenient to do separately for each house. Fig. 9.12 shows a representative typical house subnetwork with activities A, B, Y and Z. The description of each typical activity is in Table 9.2.

\subsubsection{Houses, the Baseline Schedule}

Activity B MOBILIZATION is the predecessor to typical activity 02 PLOT CLEARANCE for all the houses. Likewise, activity Y CONTRACTOR'S CONTINGENCY succeeds typical activity 85 HOUSE COMPLETION for all the houses. In each cluster, common typical activity 06 succeeds typical activity 05 COLUMN NECKS and precedes typical activity 07 PLAIN CONCRETE UNDER GROUND BEAMS for all the houses of that cluster. Common typical activity 06 had a constant duration for all the clusters despite the varying work content 
because the dominating factor affecting its duration was the laboratory results of the compaction test.

After imposing August 17, 1988 as the completion date, time scheduling is run to obtain the available time for activity completions, $\mathrm{T}_{\mathrm{aa}}$. This is readily obtainable from the CPM software as it is equal to the total float figure. The EF of a typical house is workday 276 (February 17, 1988 ) and its LF before activity $Y$ is workday 380 (June 21, 1988), therefore TF, and obviously $\mathrm{T}_{\mathrm{ap}}$, the available time for project completions are 104 workdays $(380-276)$.

Not all the 104 days will be available for completions. The existence of common activity 06 reduces them as demonstrated earlier in section 7.2. The biggest cluster of Seven Seas' houses has six houses, hence the application of equation (7.4) would be as follows:

$D_{\text {crt }}=\left\{(6-1) \div R_{\text {ip }}\right\}-T F$

and since common activity 06 is critical in the typical subnetwork, its TF is equal to zero

$D_{\text {crt }}=\left(5 \div R_{i p}\right)-0$

also, per equation (7.7)

$R_{\text {ip }}=(51-1) \div\left(104-D_{\text {crr }}\right)$

by solving the above two equations simultaneously

$R_{\text {ip }}=0.5288$ house per day, and

$\mathrm{D}_{\mathrm{crt}}=9.45$ days

Table 9.2 shows the calculation of the minimum quantity of resources required to complete the project by August 17, 1988 after considering the effect of common activity 06 . Note that the calculation against typical activity 06 , the common activity, is based on 14 clusters not 51 houses. Also, its $\mathrm{T}_{\mathrm{az}}$ was reduced by $(3-1) \div 0.5288=3.78$ days to allow for the gradual start of its successor typical activity 07 on the last cluster (C221) which has 3 houses. Column 11 of Table 9.2 shows the minimum quantities of 90 resources that should be entered into the CPM software as resource availability. 
The original sequence of operations was as follows:

Cluster 211, Houses: 44, 43, 42;

Cluster 213, Houses: 35, 36, 37, 32, 33, 34;

Cluster 323, Houses: 185, 186, 187;

Cluster 322, Houses: 188, 189, 190;

Cluster 321, Houses: 174, 175;

Cluster 320, Houses: 176, 177, 178;

Cluster 214, Houses: 31, 30, 29, 28;

Cluster 215, Houses: 27, 26, 25, 24, 23, 22;

Cluster 216, Houses: 21, 20, 19, 18;

Cluster 217, Houses: 17, 16;

Cluster 218, Houses: 15, 14, 13;

Cluster 219, Houses: 12, 11, 10;

Cluster 220, Houses: 9, 8, 7, 6, 5, 4; and

Cluster 221, Houses: 3, 2, 1.

After resource leveling per the above sequence, the baseline schedule, comprising 4302 activities, is obtained as shown in Fig. 9.13. It should be mentioned that due to the length of the baseline schedule, Fig. 9.13 was considered for inclusion in an appendix, however, the decision was made to keep it within the body of chapter 9 because it was the only way to demonstrate an adequate exposure of the issue of sequence as well as a thorough validation. In Fig. 9.13, for each repetitive activity, the first three digits of the activity ID refer to the cluster number, the following four characters refer to the house number and the last three characters are the typical activity number. The house number characters are replaced with asterisks on typical activity A06 since it is a common activity on each cluster. The priority code for the sequence of work which was used for leveling is shown under the column titled CODE. August 13, 1988 is the early completion date and is ahead of the target completion date of August 17, 1988. This earlier than required projected completion is attributed to rounding up the quantity of squads. With the effect of a common activity it is not easy to identify the critical path based on the LOB calculations of Table 9.2 before running the CPM schedule. This is because the grouping of the activities in clusters disturbs an otherwise readily identifiable and dominating typical activity or activities.

An alternative calculation for this baseline was done by excluding the effect of the common activity to investigate the effect on the quantity of resources. It was found that the 
necessary increase in the quantity of team resources for each typical activity to make up for the effect of the common activity averaged 8.63 percent. In terms of working capital and cost to a contractor, this could very well be the difference between making a profit and a loss.

\subsubsection{Houses, the Updated Schedule}

The status of the project on January 31,1988 indicated that there were many changes in the sequence of operations. These changes of sequence were identified by comparing the projected completion dates of each house based on a time schedule run before leveling. Fig. 9.14 clearly shows that by listing the final milestone activities, 86 HOUSE COMPLETION, for each house sorted by their EF dates. The numbers under the column titled CODE are the original priorities and are not in order. Therefore the progress was out-of-sequence or did not follow the original priorities. The current sequence of houses is: $43,42,174$ and so on to house 22 per the first column of Fig. 9.14.

The priority codes were therefore changed from the original sequence mentioned above to the current sequence, then resource leveling was prioritized accordingly. Fig. 9.15 shows the project schedule (excluding, due to space limitations, completed and noncritical activities) with a projected completion date on February 21, 1989. This was based on the same quantities of resources calculated for the baseline schedule. Comparison of this date with the initial target date of August 17, 1988, indicates that the project has lost more than six months in the ten month period between April 1, 1987 and January 31, 1988.

Despite the change of sequence in actual progress, another schedule run was performed keeping everything constant except the priority codes, which were changed back to the original sequence. The intention was to expose the effect of not revising the sequence of leveling and adamantly steering the work back to the original sequence regardless of the actual out-of-sequence progress. Fig. 9.16 shows a comparison of the last milestone activity 86 HOUSE COMPLETIONS in both runs. House completions per the current sequence were earlier than those per the original sequence for all the houses except for the first three houses which were identical. This strongly supports the necessity of revising the priority of leveling in the schedule according to the actual sequence in every schedule update. Identifying and correcting out-ofsequence priorities also means avoiding considerable liquidated damages. 
Fig. 9.17 shows the progress regularity of the project. It is based on the comparison of the early finish dates of the activities of house 22 (last house per the current sequence) with the corresponding ES and EF dates of the last house in the baseline schedule (house 1). Actual finish dates for the completed activities were not available, therefore these activities were not shown on the regularity chart. All typical activities are behind schedule and it is clear that the first significant drop occurred on typical activity 11 GF LOFT WALLS \& SLABS. This should indicate that typical activity 11 was a bottleneck. Previous site observations by the author confirm that. Loft slabs were not a profitable item of work and they were purposely bypassed in an effort to get to the following bulk concrete slab items as soon as possible to improve the cash flow. Bypassing the loft slabs on the ground floor and the first floor was physically possible but it contradicted the logic and created out-of-logic progress. This in turn dilutes the accuracy of the regularity chart and indicates that typical activity 12 GF ROOF was the bottleneck since it was more late than its predecessors even when it physically could and did progress regardless of the relationship with its predecessor, activity 11 GF LOFT WALLS \& SLABS.

\subsubsection{Houses, the Revised and Decelerated Schedule}

The starting point here is respecting the repeated occurrence of the out-of-logic progress and revising the logic to produce a schedule that reflects the intended method of construction. As indicated earlier in sections 2.10 and 7.3, some CPM software produce a report about current outof-logic progress. This report is a standard output of Primavera, the software used on this work. The current logic contradictions occurred repeatedly on the relationships between 11 GF LOFT WALLS \& SLABS and 12 GF ROOFS; 14 FF LOFT WALLS \& SLABS and 15 FF ROOFS; and 18 PRECAST WINDOWS ERECTION and 20 GF \& FF CEMENT BLOCK WORKS. Past out-of-logic progress among completed activities could not be reported by Primavera. But it could have been identified and quantified by the method described in section 7.3 had actual dates been available. That would have completed the exposure of out-of-logic progress wherever it happened towards revising the logic between future activities.

The following changes were made to the relationships of the above activities on each house: relationships between typical activities 11 GF LOFT WALLS \& SLABS and 12 GF ROOF were deleted; relationships between typical activities 14 FF LOFT WALLS \& SLABS and 15 FF ROOFS were deleted; typical activities 11 and 12 were made predecessors with FS relationships to typical activities 26 CAST IRON SANITARY PIPES and 27 PLASTERING PREPARATION; 
the relationship of typical activity 18 PRECAST WINDOWS ERECTION with typical activity 20 GF \& FF CEMENT BLOCK WORK was deleted and 18 was made predecessor to 25 WALLEMBEDDED ELECTRICAL INSTALLATIONS with a FS relationship.

Activity 20 GF \& FF CEMENT BLOCK WORK (duration 21 days) on each house was split into two new activities, representing the way it was constructed on site. The two new activities are: 91 GF CEMENT BLOCK WORK (duration 9 days), succeeding 12 GF ROOF with a FS 12 lag relationship; and 92 FF CEMENT BLOCK WORK (duration 12 days), succeeding 15 FF ROOF with a FS 12 lag relationship. Both 91 and 92 inherited all the successors of the deleted 20 GF \& FF CEMENT BLOCK WORK and had squads CR91 and CR92 accordingly. The same status of typical activity 20 was kept by keeping the same remaining durations of it on each house. It should also be noted that the duration of activity Y CONTRACTOR'S CONTINGENCY, was kept unchanged.

After the above revisions of relationships, the schedule run resulted in no out-of-logic progress but the current sequence of completion of the houses changed solely due to the above changes of relationships. Consequently a second revision of the priority codes was necessary. Fig. 9.18 lists activity A86 per the new sequence while showing the current sequence under the column titled CODE.

It was realized that Seven Seas would not be able to mobilized additional resources. So picking a date even after the current projection of February 21, 1989 did not seem unrealistic. This meant deceleration. The author chose March 30,1989 as a new target completion date and calculated the minimum required quantities of resources for each typical activity to achieve it.

Table 9.3 shows the calculation of the minimum quantity of squads required to achieve the new target. Based on the independent normalized rate, typical activities 12 and 17 are expected to be critical activities and control the pace of the progress. Again it should be noted that $T_{2 a}$ for typical activity 06 has been reduced by $(6-1) \div 0.0842=3.78$ days to account for the gradual starts of 6 of its successors on the last cluster (C215). The rounded quantities of squads in column 13 of Table 9.3 are entered into the CPM software and time scheduling then resource leveling are performed per the new sequence to get the decelerated schedule.

Fig. 9.19 shows the decelerated schedule projected completion date exactly on March 30, 1988. The critical path starts on typical activity $17 \mathrm{R} \mathrm{C}$ COLUMNS OF PARAPETS from house 
43 to the last house number 22. It then flows to time-critical activities on house 22 . Only one of the two squads of typical activity 17 was critical due to the nongradual start of work on this activity by the two squads. It is also noted that while typical activity 17 was resource-critical as expected, typical activity 12 GF ROOF was not. The author attributes this to the fact that on January 31,1988 typical activity 17 had no partly completed activities whereas typical activity 12 had six partly completed activities which gave it some leeway and rendered it not as critical as 17 .

The values of $T_{a \mathrm{a}}$ in Table 9.3 can be conveniently manipulated in the spreadsheet to calculate alternative resource requirements for more than one target date. By simply adding or subtracting a certain amount of days an immediate quantity for team resources can be obtained for a later or sooner target completion date to be achieved.

\subsubsection{Houses, Features Validated}

In this case study the following additional features of CPM/LOB have been emphasized and validated as superior characteristics over those provided by the current applications on planning and scheduling repetitive projects:

1. The capability of quantifying the effect of common activities and incorporating that in the schedule.

2. The integration of $\mathrm{LOB}$ with $\mathrm{CPM}$ reduced the combinations of the minimum quantities of required team resources to complete this project on target to one combination. This means avoiding many trials and errors on large schedules like this one.

3. When out-of-sequence progress happens it becomes imperative to identify the new sequence in order to revise the schedule accordingly and make it workable again. While this can be obtained by visual evaluation and comparison of the repetitive units under construction on site, nonobvious differences in time progress can be easily missed. A quick alternative in CPM/LOB is running the schedule containing the actual progress data without leveling then sorting the last typical repetitive activity by early finish dates to identify the current sequence. In traditional applications this is not possible, because resource-based relationships offset the subnetworks of the repetitive units thereby prevent a fair start alignment 
for the purpose of comparing the time progress of the repetitive units, hence the current sequence.

4. Distinguishing out-of-sequence progress easily and changing the priority codes to redirect the work accordingly. Also correcting out-of-logic progress. Alternative resource-based relationship methods are not capable of differentiating between out-of-logic progress and out-of-sequence progress.

5. Through implementing the LOB control on the balance of the project along with the CPM's explicit logic between the activities, the user acquires a better overview of the project. This helps the user to focus and overcome the overwhelming of the large number of the CPM activities inherent with representing repetitive construction projects.

6. The capability of accelerating or decelerating a schedule to meet a target date is quite easy through adjusting $\mathrm{T}_{2 \mathrm{a}}$ values in the spreadsheet and changing the availabilities of team resources in the CPM software. It takes about 15 minutes to manually input the quantities of resources into Primavera release 5.1 and 25 minutes of computer time $(486 \mathrm{DX} 266 \mathrm{MHz})$ to schedule and level this 4300 activity, 90 resource schedule. This experimentation, though possible in other methods, is very tedious because many resource-based relationships have to be revised. 


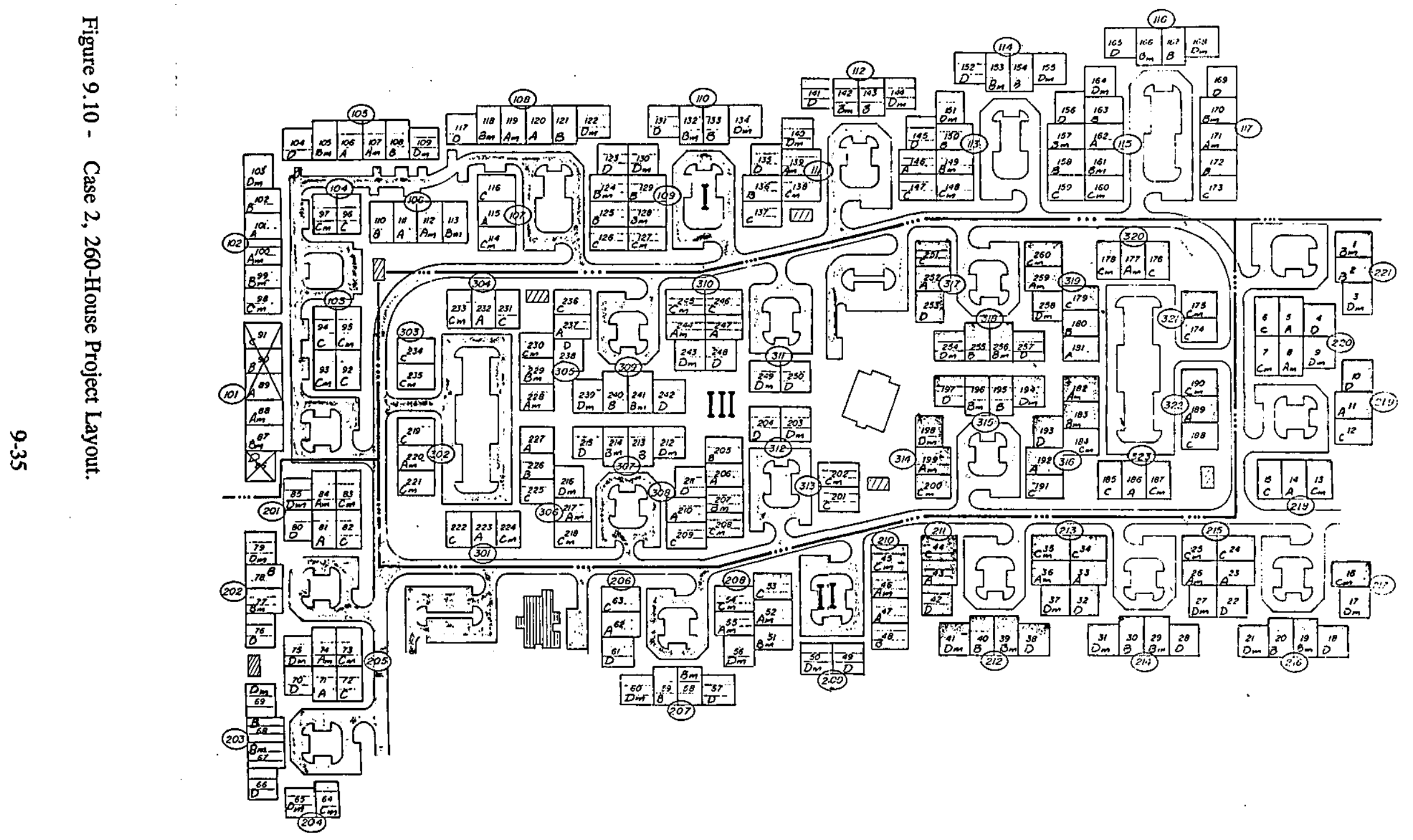




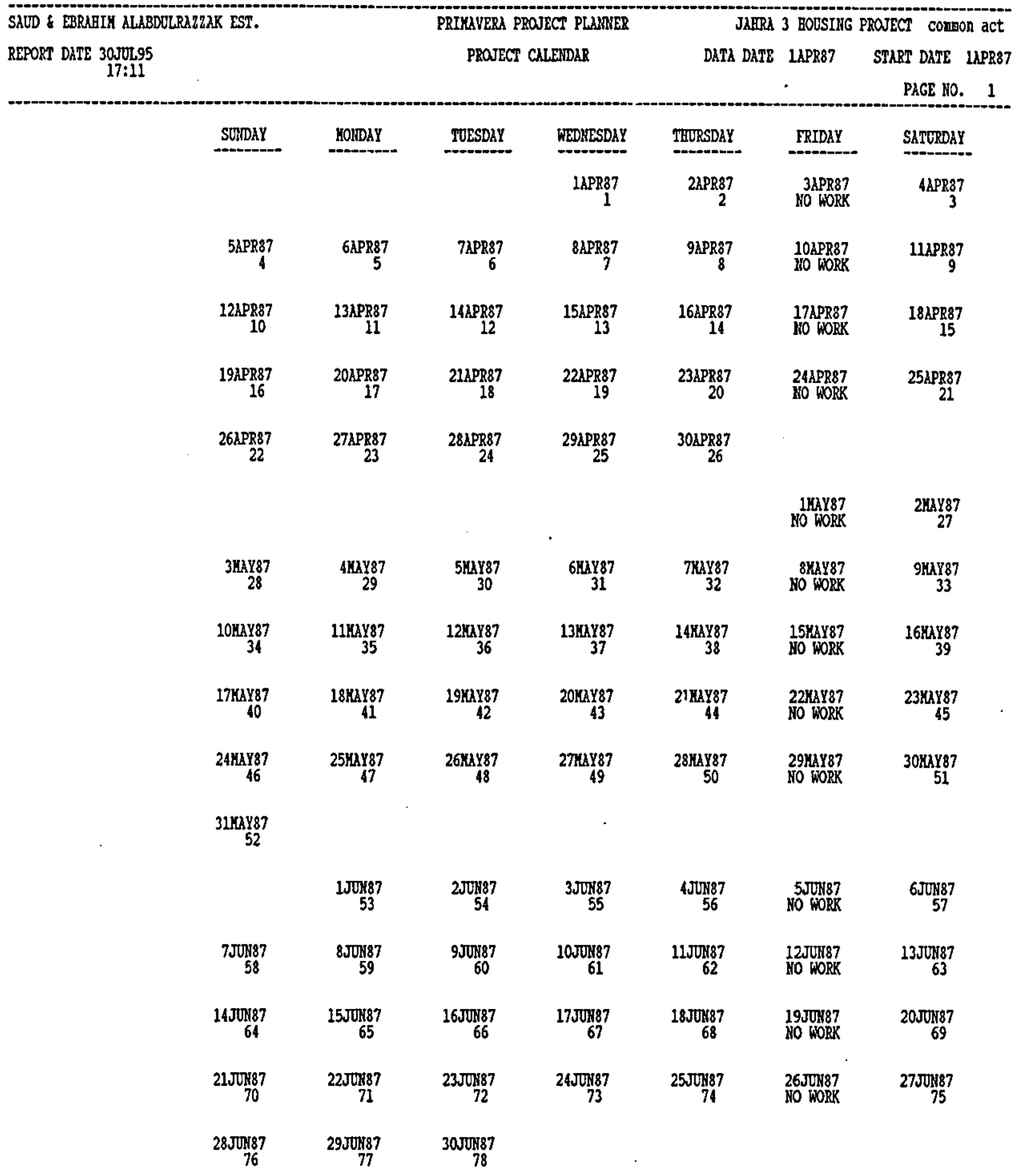

Figure 9.11 - Case 2, Project Calendar. 


\begin{tabular}{|c|c|c|c|c|c|c|}
\hline \multirow{2}{*}{$\begin{array}{l}\text { SAUD \& EBRABIH ALABDULRAL2AK EST. } \\
\text { REPORT DATE 30J0L95 } \\
17: 11\end{array}$} & \multirow{2}{*}{\multicolumn{3}{|c|}{$\begin{array}{l}\text { PRIYAVERA PROJECT PLAWNER } \\
\text { :ROJECT CALEEDDAR }\end{array}$}} & \multicolumn{3}{|c|}{ JAHRA 3 BOUSING PROJECT common act } \\
\hline & & & & DATA DATE & 1APR87 & START DATE 1APR87 \\
\hline amonsv & MNThYY & THESDXY & WEDNESDAY & THURSDAY & FRIDAY & SATURDAY \\
\hline & & & $\begin{array}{c}1 \mathrm{JUL87} \\
79\end{array}$ & $\underset{80}{2 \pi 0487}$ & $\begin{array}{r}3 J 0 L 87 \\
\text { NO WORK }\end{array}$ & $\begin{array}{c}4 \pi 0 L 87 \\
81\end{array}$ \\
\hline $\begin{array}{c}5 J 0 L 87 \\
82\end{array}$ & $\underset{83}{6 \pi U 487}$ & $\underset{34}{7 \pi 087}$ & $\begin{array}{c}8 \mathrm{JOL} 87 \\
85\end{array}$ & $\begin{array}{c}9 \pi 0 L 87 \\
86\end{array}$ & $\begin{array}{l}10 J 0 L 87 \\
\text { NO WORK }\end{array}$ & $\begin{array}{c}11 \pi 0487 \\
87\end{array}$ \\
\hline$\underset{88}{12 \mathrm{JOL} 87}$ & $\begin{array}{c}\text { 13JUL87 } \\
89\end{array}$ & $\begin{array}{c}\text { 14JUL87 } \\
90\end{array}$ & $\begin{array}{c}15 J 0 L 87 \\
91\end{array}$ & $\begin{array}{c}\text { 16. } \\
92\end{array}$ & $\begin{array}{l}\text { 17JUL87 } \\
\text { NO KORK }\end{array}$ & $\underset{93}{\text { 18JUL87 }}$ \\
\hline $\begin{array}{c}19 \mathrm{JUL} 87 \\
94\end{array}$ & $\begin{array}{c}20 J 0 L 87 \\
95\end{array}$ & $\begin{array}{c}21 \mathrm{JuL} 87 \\
96\end{array}$ & $\begin{array}{c}22 \mathrm{JUL87} \\
97\end{array}$ & $\begin{array}{c}23 \mathrm{JUL} 87 \\
98\end{array}$ & $\begin{array}{l}24 \mathrm{JUL} 87 \\
\text { HO WORK }\end{array}$ & $\begin{array}{c}25 \mathrm{JUL} 87 \\
99\end{array}$ \\
\hline $\begin{array}{c}26.0 \mathrm{UL} 87 \\
100\end{array}$ & $\begin{array}{c}27 J u 487 \\
101\end{array}$ & $\begin{array}{c}28 \mathrm{JUL} .87 \\
102\end{array}$ & $\begin{array}{c}2950 t 887 \\
103\end{array}$ & $\begin{array}{c}3050687 \\
104\end{array}$ & $\begin{array}{l}\text { 31JOL87 } \\
\text { NO WORK }\end{array}$ & \\
\hline & & & & & & $\begin{array}{c}1 \mathrm{AJG} 87 \\
105\end{array}$ \\
\hline $\begin{array}{c}240 G 87 \\
106\end{array}$ & $\begin{array}{c}310687 \\
107\end{array}$ & $\begin{array}{c}410687 \\
108\end{array}$ & $\begin{array}{c}510 G 87 \\
109\end{array}$ & $\begin{array}{c}\text { 6.0G87 } \\
110\end{array}$ & $\begin{array}{r}7 \mathrm{AOGB7} \\
\text { NO WORK }\end{array}$ & $\begin{array}{c}810 G 87 \\
111\end{array}$ \\
\hline $\begin{array}{c}920687 \\
112\end{array}$ & $\begin{array}{c}\text { 10AUG87 } \\
113\end{array}$ & $\underset{114}{1140 G 87}$ & $\begin{array}{c}1280687 \\
115\end{array}$ & $\begin{array}{c}13 \lambda 0687 \\
116\end{array}$ & $\begin{array}{l}14 \lambda 0 G 87 \\
\text { HO NORK }\end{array}$ & $\begin{array}{c}\text { 15ADG87 } \\
117\end{array}$ \\
\hline $\begin{array}{c}16 \lambda 0 C 87 \\
118\end{array}$ & $\begin{array}{c}17 \lambda 0 G 87 \\
119\end{array}$ & $\begin{array}{c}1810 G 87 \\
120\end{array}$ & $\begin{array}{c}1910687 \\
121\end{array}$ & $\begin{array}{c}2010 G 87 \\
122\end{array}$ & $\begin{array}{l}\text { 21AUG87 } \\
\text { NO WORK }\end{array}$ & $\begin{array}{c}2210 G 87 \\
123\end{array}$ \\
\hline $\begin{array}{c}23 \times 0 G 87 \\
124\end{array}$ & $\begin{array}{c}24 \lambda \cup G 87 \\
125\end{array}$ & $\begin{array}{c}25.0 G 87 \\
126\end{array}$ & $\begin{array}{c}2640 G 87 \\
127\end{array}$ & $\begin{array}{c}2710687 \\
128\end{array}$ & $\begin{array}{l}2810 G 87 \\
\text { HO WORK }\end{array}$ & $\begin{array}{c}29 A 0 G 87 \\
129\end{array}$ \\
\hline $\begin{array}{c}30 \times 0687 \\
130\end{array}$ & $\begin{array}{c}31 \lambda 0 \mathrm{CB} 87 \\
131\end{array}$ & & & & & \\
\hline & & $\begin{array}{c}\text { 1SEP87 } \\
132\end{array}$ & $\begin{array}{c}2 \text { SEP87 } \\
133\end{array}$ & $\begin{array}{c}\text { 3SEP87 } \\
134\end{array}$ & $\begin{array}{r}\text { 4SEP87 } \\
\text { HO WORK }\end{array}$ & $\underset{135}{\text { 5SEP87 }}$ \\
\hline $\begin{array}{c}\text { 6SEP87 } \\
136\end{array}$ & $\begin{array}{c}\text { 7SEP87 } \\
137\end{array}$ & $\begin{array}{c}\text { 8SEP87 } \\
138\end{array}$ & $\begin{array}{c}\text { 9SEP87 } \\
139\end{array}$ & $\begin{array}{c}\text { 10SEP87 } \\
140\end{array}$ & $\begin{array}{l}\text { 11SEP87 } \\
\text { NO WORK }\end{array}$ & 12SEP87 \\
\hline $\begin{array}{c}135 \mathrm{EP} 87 \\
142\end{array}$ & $\begin{array}{c}\text { 14SEP87 } \\
143\end{array}$ & $\begin{array}{c}\text { 15SEP87 } \\
144\end{array}$ & $\begin{array}{c}16 \text { SEP87 } \\
145\end{array}$ & $\begin{array}{c}17 \mathrm{SEP} 87 \\
146\end{array}$ & $\begin{array}{l}\text { 18SEP87 } \\
\text { KO WORK }\end{array}$ & $\begin{array}{c}\text { I9SERP87 } \\
147\end{array}$ \\
\hline $\begin{array}{c}\text { 20SEP87 } \\
148\end{array}$ & $\begin{array}{c}\text { 21SEP87 } \\
149\end{array}$ & $\begin{array}{c}22 S E P 87 \\
150\end{array}$ & $\begin{array}{c}\text { 23SEP87 } \\
151\end{array}$ & $\begin{array}{c}\text { 24SEP8 } 7 \\
152\end{array}$ & $\begin{array}{l}255 \text { EPP87 } \\
\text { HO WORK }\end{array}$ & 26SEP8 7 \\
\hline $\begin{array}{c}27 S E P 87 \\
154\end{array}$ & $\begin{array}{c}28 \text { SEP87 } \\
155\end{array}$ & $\begin{array}{c}\text { 29SEP87 } \\
156\end{array}$ & $\begin{array}{c}30 \text { SRP } 87 \\
157\end{array}$ & & & \\
\hline
\end{tabular}

Figure 9.11 - Case 2, Project Calendar (Continued). 
SALD \& EBRAHII ALABOULRAZZAK EST. REPORT DATE 30 JOL95 $17: 11$
PRIJUVVERA PROJECT PLANNER PROJECT CALERDAR
JARRA 3 HOUSING PROJECT CON⿴囗十 act DATK DATE LAPR87 START DR.:E 1APR87

PAGE .0. 3

\begin{tabular}{|c|c|c|c|c|c|c|}
\hline SONDAY & HONDAY & TUESDAY & WED) NESAN & TEIDPSDAY & FRIDAY & SAIURDAY \\
\hline & & & & $\begin{array}{c}10 C 787 \\
158\end{array}$ & $\begin{array}{r}200 \mathrm{TP} 87 \\
\text { HO WORK }\end{array}$ & $\begin{array}{r}30 \mathrm{CT} 787 \\
159\end{array}$ \\
\hline 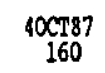 & $\begin{array}{c}50 \text { CT87 } \\
161\end{array}$ & $\begin{array}{c}60 C 187 \\
162\end{array}$ & $\begin{array}{c}70 \mathrm{Cr} 87 \\
163\end{array}$ & $\begin{array}{c}800187 \\
164\end{array}$ & $\begin{array}{r}90 \mathrm{CT} 87 \\
\text { NO WORK }\end{array}$ & $\begin{array}{c}1000787 \\
155\end{array}$ \\
\hline $\begin{array}{c}1100787 \\
166\end{array}$ & $\underset{167}{1200187}$ & $\underset{168}{130 \mathrm{TT} 87}$ & $\begin{array}{c}140 \mathrm{CT} 87 \\
169\end{array}$ & $\begin{array}{c}1500787 \\
170\end{array}$ & $\begin{array}{l}1600787 \\
\text { 10 WORK }\end{array}$ & $\begin{array}{c}1700787 \\
171\end{array}$ \\
\hline $\begin{array}{c}1800787 \\
172\end{array}$ & $\begin{array}{c}190 \mathrm{Cr} 187 \\
173\end{array}$ & $\begin{array}{c}2000187 \\
174\end{array}$ & $\begin{array}{c}210 \mathrm{CT} 87 \\
175\end{array}$ & $\begin{array}{c}220 \mathrm{CT} 87 \\
176\end{array}$ & $\begin{array}{l}230 \mathrm{CT} 87 \\
\text { NO WORK }\end{array}$ & $\begin{array}{c}240 C 187 \\
177\end{array}$ \\
\hline $\begin{array}{c}250 \mathrm{CT} 787 \\
178\end{array}$ & $\begin{array}{c}2600187 \\
179\end{array}$ & $\begin{array}{c}2700 \mathrm{~T} 87 \\
180\end{array}$ & $\begin{array}{c}2800 \mathrm{CT} 87 \\
181\end{array}$ & $\begin{array}{c}2900787 \\
182\end{array}$ & $\begin{array}{l}3000787 \\
110 \text { WORK }\end{array}$ & $\begin{array}{c}310 C 787 \\
183\end{array}$ \\
\hline $\begin{array}{c}1100887 \\
184\end{array}$ & $\begin{array}{c}\text { 2NOV887 } \\
185\end{array}$ & $\begin{array}{c}3 \times 0 V 87 \\
186\end{array}$ & $\begin{array}{l}\text { 48:0187 } \\
187\end{array}$ & $\begin{array}{c}5 \mathrm{H} \text { 10187 } \\
188\end{array}$ & $\begin{array}{r}\text { 6NOVY87 } \\
\text { HO SORK }\end{array}$ & $\begin{array}{c}7 \text { Hov } 87 \\
189\end{array}$ \\
\hline $\begin{array}{c}8 \mathrm{BOV} 87 \\
190\end{array}$ & $\begin{array}{l}920187 \\
191\end{array}$ & $\begin{array}{c}\text { 10NOV87 } \\
192\end{array}$ & $\begin{array}{c}\text { 11NOV87 } \\
193\end{array}$ & $\begin{array}{c}12120087 \\
194\end{array}$ & $\begin{array}{l}13 \text { NOV87 } \\
\text { HO WORK }\end{array}$ & $\begin{array}{c}14500087 \\
195\end{array}$ \\
\hline $\begin{array}{c}1540 v 87 \\
196\end{array}$ & $\begin{array}{c}\text { 16 HOV87 } \\
197\end{array}$ & $\begin{array}{c}17 \text { trov8 } \\
198\end{array}$ & $\begin{array}{c}\text { 1810V87 } \\
199\end{array}$ & $\begin{array}{c}19190187 \\
200\end{array}$ & $\begin{array}{l}20 \text { NOV } 87 \\
\text { NO WORK }\end{array}$ & $\begin{array}{c}21 \text { Now8 } \\
201\end{array}$ \\
\hline $\begin{array}{l}2250487 \\
202\end{array}$ & $\begin{array}{c}23 \text { HOV } 87 \\
203\end{array}$ & $\begin{array}{c}24150187 \\
204\end{array}$ & $\begin{array}{c}25 \text { Nov8 } 7 \\
205\end{array}$ & $\begin{array}{c}26 \text { Nov8 } \\
206\end{array}$ & $\begin{array}{l}27 \text { NOV } 87 \\
\text { HO WORK }\end{array}$ & $\begin{array}{l}\text { 281NOV87 } \\
207\end{array}$ \\
\hline \multirow[t]{2}{*}{$\begin{array}{l}29 \mathrm{NOVy} 87 \\
208\end{array}$} & $\begin{array}{c}30 \mathrm{NOV} 87 \\
209\end{array}$ & & & & & \\
\hline & & $\begin{array}{c}1 \mathrm{DECC87} \\
210\end{array}$ & $\begin{array}{l}2 \mathrm{DEC} 877 \\
211\end{array}$ & $\begin{array}{l}\text { 30EC87 } \\
212\end{array}$ & $\begin{array}{l}\text { 4DEC87 } \\
\text { No WORK }\end{array}$ & $\begin{array}{c}\text { 5DRC87? } \\
213\end{array}$ \\
\hline $\begin{array}{l}\text { 6DEC87 } \\
214\end{array}$ & $\begin{array}{c}715 E C 87 \\
\text { 7DE }\end{array}$ & $\begin{array}{c}8 D E C 87 \\
216\end{array}$ & $\begin{array}{l}9 \mathrm{DEC} 877 \\
217\end{array}$ & $\begin{array}{c}10 D E C 87 \\
218\end{array}$ & $\begin{array}{l}11 D E C 87 \\
110608 K\end{array}$ & $\begin{array}{c}120 E C 87 \\
219\end{array}$ \\
\hline $\begin{array}{r}\text { 13DEC } 227 \\
220\end{array}$ & $\begin{array}{c}\text { 14DEC87 } \\
221\end{array}$ & $\begin{array}{c}15 \mathrm{DEC} C \mathrm{22} \\
222\end{array}$ & $\underset{223}{160 E C 87}$ & $\begin{array}{c}17 D R C 87 \\
224\end{array}$ & $\begin{array}{l}\text { 18DEC87 } \\
\text { YO WORK }\end{array}$ & $\frac{19 \mathrm{DECB} 7}{225}$ \\
\hline $\begin{array}{c}200 E C 87 \\
226\end{array}$ & $\begin{array}{c}21 D E C 87 \\
227\end{array}$ & $\begin{array}{c}22 \mathrm{DEC} 87 \\
228\end{array}$ & $\begin{array}{c}23 \mathrm{DEC} 87 \\
229\end{array}$ & $\begin{array}{c}24 D E C 87 \\
230\end{array}$ & $\begin{array}{l}25 \mathrm{DECB7} \\
\text { HO SORK }\end{array}$ & $\begin{array}{c}26 \mathrm{DECB7} \\
231\end{array}$ \\
\hline \multirow[t]{2}{*}{$\begin{array}{c}\text { 27DEC } 237 \\
232\end{array}$} & $\begin{array}{c}28 D E C 87 \\
233\end{array}$ & $\begin{array}{c}29 \mathrm{DEC} 877 \\
234\end{array}$ & $\begin{array}{c}300 \mathrm{DC} 87 \\
235\end{array}$ & $\begin{array}{l}310 \mathrm{DC} 87 \\
150 \text { WORK }\end{array}$ & & \\
\hline & & & & & $\begin{array}{l}\text { IJNM88 } \\
\text { NO WOORK }\end{array}$ & $\begin{array}{l}\text { 2JAM88 } \\
236\end{array}$ \\
\hline
\end{tabular}

Figure 9.11 - Case 2, Project Calendar (Continued). 
SAUD \& ERRAHIX ALABDULLAZZAK EST.

REPORT DATE 30.0L,95
PRIYAVERA PROJECT PLANWER

PROJECT CALERDAR
JAHRA 3 BOUSING PROJECT common act DATA D'IR 1APR87 START DATE 1APR87

PAGE NO. 4

\begin{tabular}{|c|c|c|c|c|c|c|}
\hline SDIDAY & KORDAY & TIESAYY & WRDNYSSDAY & THURSDAY & PRIDAY & SATURDAY \\
\hline $\begin{array}{c}\text { 3JAH } \\
237\end{array}$ & $\begin{array}{c}\text { 4JAN88 } \\
238\end{array}$ & $\begin{array}{c}\text { 5JhH88 } \\
239\end{array}$ & $\begin{array}{c}6.5 \mathrm{JN} 88 \\
240\end{array}$ & $\begin{array}{c}7 \mathrm{J \lambda} \text { K } 88 \\
241\end{array}$ & $\begin{array}{c}\text { 8JAHS88 } \\
\text { NO WORK }\end{array}$ & $\begin{array}{c}9 \mathrm{JAN} 88 \\
242\end{array}$ \\
\hline $\begin{array}{c}10 \mathrm{JAN} \\
243\end{array}$ & $\underset{244}{11 \mathrm{JJN} 88}$ & $\underset{245}{12 J 4 N 88}$ & $\underset{246}{\text { 13JJN88 }}$ & $\begin{array}{c}14 J \text { AN88 } \\
247\end{array}$ & $\begin{array}{l}\text { 15JAN88 } \\
\text { HO WORR }\end{array}$ & $\begin{array}{c}\text { 16JA4P88 } \\
248\end{array}$ \\
\hline $\begin{array}{c}17 J \text { JN188 } \\
249\end{array}$ & $\begin{array}{c}\text { 18JhN88 } \\
250\end{array}$ & $\begin{array}{c}\text { 19JNH88 } \\
251\end{array}$ & $\begin{array}{c}20 \mathrm{JH} H 88 \\
252\end{array}$ & $\begin{array}{c}21 J 4 N 88 \\
253\end{array}$ & $\begin{array}{l}\text { 22JNAS88 } \\
\text { NO WORK }\end{array}$ & $\begin{array}{c}\text { 23JसN88 } \\
254\end{array}$ \\
\hline $\begin{array}{c}24 J \text { JN188 } \\
255\end{array}$ & $\begin{array}{c}25 \mathrm{JAN} 88 \\
256\end{array}$ & $\begin{array}{c}\text { 26JAhI8 } \\
257\end{array}$ & $\begin{array}{c}27 J A H 88 \\
258\end{array}$ & $\begin{array}{c}28 J \text { JAN8 } 8 \\
259\end{array}$ & $\begin{array}{l}29 J \mathrm{JH} 88 \\
\text { NO KORK }\end{array}$ & $\begin{array}{c}30 \mathrm{JAH} 88 \\
260\end{array}$ \\
\hline
\end{tabular}

$\underset{261}{31 \mathrm{JAH}}$

\begin{tabular}{|c|c|c|c|c|c|c|}
\hline & 1FEB88 & $\frac{2 \text { 2PEB88 }}{263}$ & $\begin{array}{c}\text { 3FEB88 } \\
264\end{array}$ & $\begin{array}{c}\text { 4FEB88 } \\
265\end{array}$ & $\begin{array}{r}5 \text { FEBB88 } \\
\text { NO WORK }\end{array}$ & $\begin{array}{c}\text { 6FEB888 } \\
266\end{array}$ \\
\hline $\begin{array}{c}7 \mathrm{FEB88} \\
267\end{array}$ & $\begin{array}{c}8 \mathrm{FEBB8} \\
268\end{array}$ & $\begin{array}{c}\text { 9PERBS8 } \\
269\end{array}$ & 10PER88 $\frac{270}{270}$ & $\begin{array}{c}\text { 11FER88 } \\
271\end{array}$ & $\begin{array}{l}\text { 12FEBS8 } \\
\text { HO WORK }\end{array}$ & $\frac{13 F E B 88}{272}$ \\
\hline $\begin{array}{c}\text { 14FEB88 } \\
273\end{array}$ & $\begin{array}{c}\text { 15FEB } 88 \\
274\end{array}$ & $\underset{275}{16 \text { FEB88 }}$ & $\frac{\text { 17FEB888 }}{276}$ & 18FEB88 & $\begin{array}{l}\text { 19FEB88 } \\
\text { NO WORK }\end{array}$ & 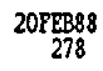 \\
\hline $\begin{array}{c}21 \mathrm{PEB} 88 \\
279\end{array}$ & $\begin{array}{c}22 \mathrm{PEB} 88 \\
280\end{array}$ & $\begin{array}{c}23 \text { PEB88 } \\
281\end{array}$ & $\begin{array}{c}24 \mathrm{PEB} 88 \\
282\end{array}$ & $\begin{array}{l}25 \text { FEB88 } \\
\text { HO WORK }\end{array}$ & $\begin{array}{l}26 \mathrm{~F} \text { HB88 } \\
\text { NO WORK }\end{array}$ & $\underset{27 \mathrm{FEB8} 8}{283}$ \\
\hline \multirow[t]{2}{*}{$\begin{array}{c}28 \mathrm{FEB} 88 \\
284\end{array}$} & $\begin{array}{c}29 \mathrm{PEB} 88 \\
285\end{array}$ & & & & & \\
\hline & & $\begin{array}{c}1101888 \\
286\end{array}$ & $\begin{array}{c}2 \text { 2KAR88 } \\
287\end{array}$ & $\underset{288}{3 \text { MIRP88 }}$ & $\begin{array}{l}\text { 4hLRR88 } \\
\text { HO WORK }\end{array}$ & $\begin{array}{c}\text { 5:LARR88 } \\
289\end{array}$ \\
\hline $\begin{array}{c}\text { 6.LAR88 } \\
290\end{array}$ & $\begin{array}{c}\text { THAR88 } \\
291\end{array}$ & $\begin{array}{c}\text { 8HAR88 } \\
292\end{array}$ & $\begin{array}{c}\text { 9HAR88 } \\
293\end{array}$ & $\begin{array}{c}\text { 10,HAR88 } \\
294\end{array}$ & $\begin{array}{l}11 \text { MR88 } \\
\text { NO WORK }\end{array}$ & $\underset{295}{12 \operatorname{MR} 88}$ \\
\hline $\begin{array}{c}\text { 13) } \\
296\end{array}$ & $\begin{array}{c}14 \mathrm{KAR} 88 \\
297\end{array}$ & $\underset{298}{15 \mathrm{KAR} 88}$ & $\underset{299}{16 \mathrm{KAR} 88}$ & $\underset{300}{17 \text { ThR88 }}$ & $\begin{array}{l}18 \text { KAR8 } \\
\text { NO WORK }\end{array}$ & $\begin{array}{c}\text { 19HAR8 } 8 \\
301\end{array}$ \\
\hline$\underset{302}{20 \mathrm{KAR} 88}$ & $\begin{array}{c}21 \text { KAR } 88 \\
303\end{array}$ & $\frac{22 \text { NAR88 }}{304}$ & $\begin{array}{c}23 \text { IAR8 } \\
305\end{array}$ & $\frac{24 h \text { RR8 }}{306}$ & $\begin{array}{l}\text { 25HAR88 } \\
\text { NO WORK }\end{array}$ & 26IUR88 \\
\hline \multirow[t]{2}{*}{$\begin{array}{c}27 \text { ILR88 } \\
308\end{array}$} & $\begin{array}{l}\text { 281LAR88 } \\
\text { NO WORK }\end{array}$ & $\begin{array}{c}\text { 29:1AR88 } \\
309\end{array}$ & $\begin{array}{c}\text { 30IAAR88 } \\
310\end{array}$ & $\begin{array}{c}31 \mathrm{WhR} 88 \\
311\end{array}$ & & \\
\hline & . & & & & $\begin{array}{l}\text { 1APR88 } \\
\text { NO WORK }\end{array}$ & $\begin{array}{c}2 \mathrm{APRB8} \\
312\end{array}$ \\
\hline
\end{tabular}

Figure 9.11 - Case 2, Project Calendar (Continued). 
SAUD \& BBRAHIM ALABDULRAZ2MK EST. REPORT DATE 30JUL95

\section{PRIMUVERA PROJECT PLANNER}

PROJECT CALER AR
JAHRA 3 HOUSING PROJECT common act DATA DATE LAPR87 START DATE 1APR87

PAGE HO. 5

\begin{tabular}{|c|c|c|c|c|c|c|}
\hline STpinav & yoNnaY & MTIFSDAY & WEDNRSDAY & THORSDDYY & FRIDAY & SATURDAI \\
\hline $\begin{array}{c}3 A P R 88 \\
313\end{array}$ & $\begin{array}{c}4 \mathrm{APR} 88 \\
314\end{array}$ & $\begin{array}{c}5 \text { APRP8 } \\
315\end{array}$ & $\begin{array}{c}\text { 6APR88 } \\
316\end{array}$ & $\begin{array}{c}\text { 7APR88 } \\
317\end{array}$ & $\begin{array}{r}\text { 8APR88 } \\
\text { NO WORR }\end{array}$ & $\begin{array}{c}\text { 9APR88 } \\
318\end{array}$ \\
\hline $\begin{array}{c}\text { 10APR88 } \\
319\end{array}$ & $\begin{array}{c}\text { 114PR88 } \\
3 ? !\end{array}$ & $\begin{array}{c}\text { 12APR88 } \\
321\end{array}$ & $\begin{array}{c}\text { 13APR88 } \\
322\end{array}$ & $\underset{323}{14 \lambda P R 88}$ & $\begin{array}{l}\text { 15APR88 } \\
\text { HO WORK }\end{array}$ & $\begin{array}{c}16 \alpha P R 88 \\
324\end{array}$ \\
\hline $\begin{array}{c}17 \text { APR88 } \\
325\end{array}$ & $\begin{array}{c}\text { 18APR88 } \\
326\end{array}$ & $\begin{array}{c}\text { 19APR88 } \\
327\end{array}$ & $\begin{array}{c}\text { 20APR88 } \\
328\end{array}$ & $\begin{array}{c}21 \text { 11PR88 } \\
329\end{array}$ & $\begin{array}{l}\text { 22APR88 } \\
\text { KO WORK }\end{array}$ & $\begin{array}{c}23 \mathrm{APR} 88 \\
330\end{array}$ \\
\hline $\begin{array}{c}24 \text { APR88 } \\
331\end{array}$ & $\begin{array}{c}25 \text { APR88 } \\
332\end{array}$ & $\begin{array}{c}\text { 26APR88 } \\
333\end{array}$ & $\frac{27 \mathrm{APR} 88}{334}$ & $\frac{28 \text { APR88 }}{335}$ & $\begin{array}{l}\text { 29.9PR88 } \\
\text { NO WORK }\end{array}$ & $\begin{array}{c}30 \text { APR88 } \\
336\end{array}$ \\
\hline$\underset{337}{1144 y 88}$ & $\begin{array}{c}26 \text { KAY } 88 \\
338\end{array}$ & $\begin{array}{c}\text { 3)גूY88 } \\
339\end{array}$ & $\begin{array}{c}\text { 4HAYY88 } \\
340\end{array}$ & $\begin{array}{c}5 \mathrm{k} \text { KYy88 } \\
341\end{array}$ & $\begin{array}{l}\text { 6LAY88 } \\
110 \text { WORK }\end{array}$ & $\begin{array}{c}7 \text { ThY } \\
342\end{array}$ \\
\hline $\begin{array}{c}\text { 8HAY88 } \\
343\end{array}$ & $\begin{array}{c}\text { 9rAY88 } \\
344\end{array}$ & $\begin{array}{c}1010 \mathrm{MAY} 88 \\
345\end{array}$ & $\begin{array}{c}\text { I1LRY88 } \\
346\end{array}$ & $\begin{array}{c}\text { 12HAY88 } \\
347\end{array}$ & $\begin{array}{l}1364 Y 88 \\
\text { HO WORR }\end{array}$ & $\underset{348}{14 M A Y 88}$ \\
\hline $\begin{array}{c}15 \text { KuY } 88 \\
349\end{array}$ & $\begin{array}{c}16 \mathrm{KAYY} 88 \\
350\end{array}$ & $\begin{array}{c}17 \text { Way } 88 \\
351\end{array}$ & $\frac{18 M \text { MY }}{352}$ & $\begin{array}{c}1912 \text { KYY } 88 \\
353\end{array}$ & $\begin{array}{l}2014 \text { Y } 88 \\
\text { NO WORK }\end{array}$ & $\underset{354}{21 \text { rays8 }}$ \\
\hline$\frac{224 \text { AYY8 }}{355}$ & $\begin{array}{c}23 \text { 23AY88 } \\
356\end{array}$ & $\begin{array}{c}\text { 24IMAY88 } \\
357\end{array}$ & $\frac{25 \text { HAY88 }}{358}$ & $\begin{array}{c}26 \text { !hy } 88 \\
359\end{array}$ & $\begin{array}{l}27 \mathrm{H} \text { HY8 } \\
\text { NO WORR }\end{array}$ & $\begin{array}{c}28 \mathrm{HAY88} \\
360\end{array}$ \\
\hline \multirow[t]{2}{*}{$\underset{361}{294 k y 88}$} & $\begin{array}{l}\text { 301KYY88 } \\
\text { HO WORK }\end{array}$ & $\begin{array}{c}31 \text { 119Y88 } \\
362\end{array}$ & & & & \\
\hline & & & $\begin{array}{c}1.01188 \\
363\end{array}$ & 250788 & $\begin{array}{r}35 \mathrm{~W} / 88 \\
\text { NO WORK }\end{array}$ & $\begin{array}{c}40 \mathrm{H} / 88 \\
365\end{array}$ \\
\hline $\begin{array}{c}5 \sqrt{0488} \\
366\end{array}$ & $\begin{array}{c}6 \mathrm{JON} 88 \\
367\end{array}$ & $\begin{array}{c}750188 \\
368\end{array}$ & $\begin{array}{c}\text { 8JuN88 } \\
369\end{array}$ & $\begin{array}{c}900188 \\
370\end{array}$ & $\begin{array}{l}\text { 10JW188 } \\
\text { HO WORK }\end{array}$ & $\begin{array}{c}11 \pi 0788 \\
371\end{array}$ \\
\hline$\underset{372}{12 \sqrt{J} 188}$ & $\underset{373}{13 \mathrm{~J} / 188}$ & $\underset{374}{14 \pi 0788}$ & $\begin{array}{c}15 \sqrt{0188} \\
375\end{array}$ & $\begin{array}{c}\text { 16JUY88 } \\
376\end{array}$ & $\begin{array}{l}\text { 17JU188 } \\
\text { KO WORK }\end{array}$ & $\begin{array}{l}\text { 18JJWN88 } \\
377\end{array}$ \\
\hline $\begin{array}{c}1900 \text { N88 } \\
378\end{array}$ & $\begin{array}{c}20 \sqrt{3} 188 \\
379\end{array}$ & $\begin{array}{c}21 \text { JWW88 } \\
380\end{array}$ & $\begin{array}{c}22 \text { Jow } 88 \\
381\end{array}$ & $\begin{array}{c}23 \mathrm{JWN} / 88 \\
382\end{array}$ & $\begin{array}{l}\text { 24JUN88 } \\
\text { HO WORK }\end{array}$ & $\begin{array}{c}25 \text { JuN } 88 \\
383\end{array}$ \\
\hline \multirow[t]{2}{*}{$\begin{array}{c}26 \sqrt{0+888} \\
384\end{array}$} & $\begin{array}{c}27 \text { Jण188 } \\
385\end{array}$ & $\begin{array}{c}28 \mathrm{JUH} \\
386\end{array}$ & $\begin{array}{c}29 \sqrt{01388} \\
387\end{array}$ & $\begin{array}{c}3000488 \\
388\end{array}$ & & \\
\hline & & & & & $\begin{array}{r}1 J U L 88 \\
\text { NO WORK }\end{array}$ & $\begin{array}{c}250188 \\
389\end{array}$ \\
\hline $\begin{array}{l}300188 \\
390\end{array}$ & 450L88 & $\begin{array}{c}500488 \\
392\end{array}$ & $\begin{array}{c}6 \mathrm{JUL} 88 \\
393\end{array}$ & $\underset{394}{70 L 88}$ & $\begin{array}{l}8 J 0288 \\
110 \text { WORK }\end{array}$ & $\underset{395}{9 J U L 88}$ \\
\hline
\end{tabular}

Figure 9.11 - Case 2, Project Calendar (Continued). 


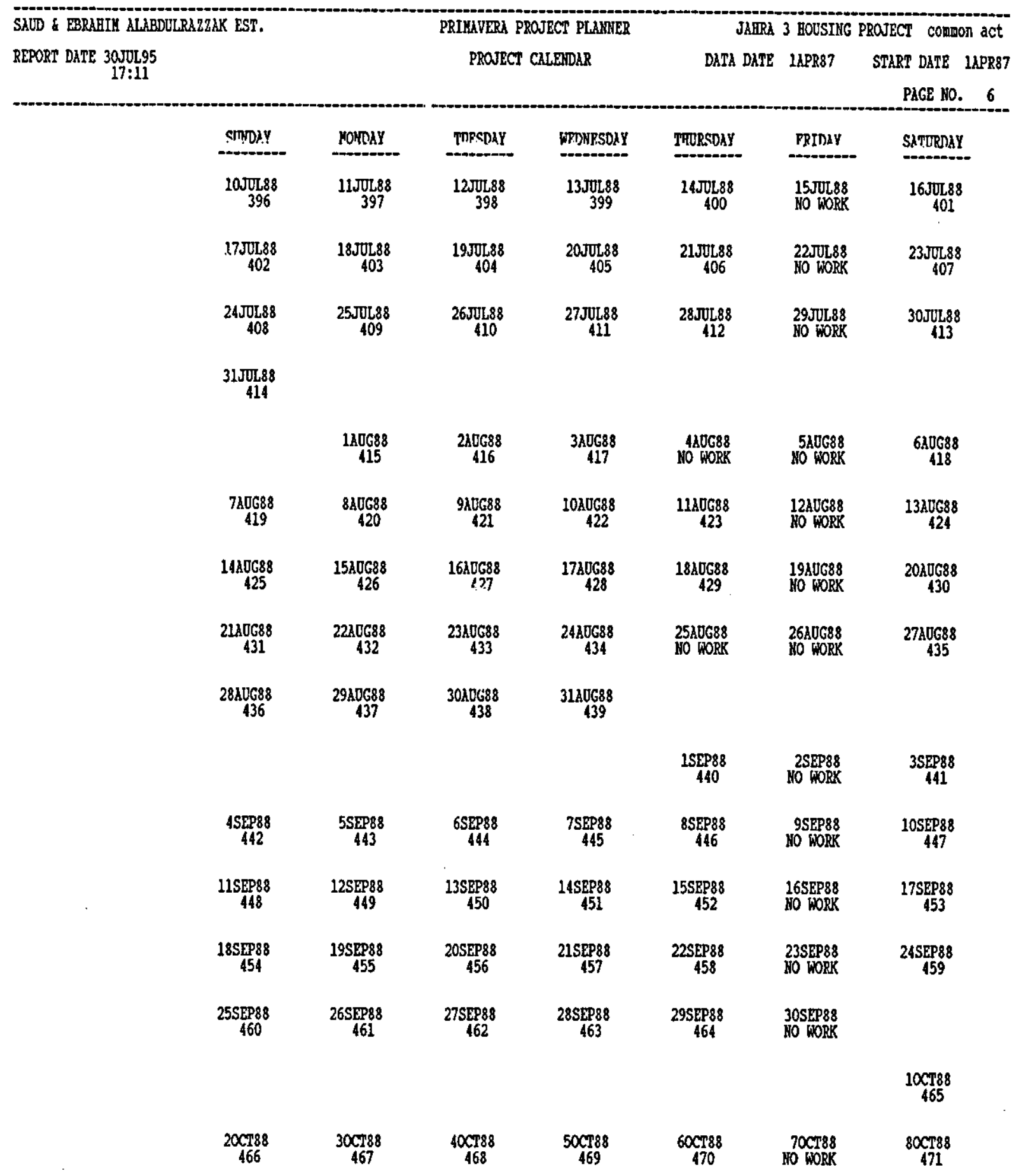

Figure 9.11 - Case 2, Project Calendar (Continued). 


\begin{tabular}{|c|c|c|c|c|c|c|}
\hline \multirow{2}{*}{$\begin{array}{l}\text { SAUD \& EBRAHIH ALABDULRAZ2AK EST. } \\
\text { REPORT DATE 30JUL95 } \\
17: 11\end{array}$} & & \multirow{2}{*}{\multicolumn{2}{|c|}{$\begin{array}{l}\text { PRIMAVERA PROJECT PLLNNER } \\
\text { PROJECT CALEIDAR }\end{array}$}} & \multicolumn{3}{|c|}{ JAHR 3 HOUSING PROJECT comnon act } \\
\hline & & & & DATA DATE & LAPR87 & 3TART DATE LAPR87 \\
\hline SDNDAY & WONI)AY & TUESDAY & WEDHESTAY & THORSDAY & FRIDAY & SATURDAY \\
\hline$\underset{472}{90 \mathrm{TT} 88}$ & $\begin{array}{c}1000 \mathrm{~T} 88 \\
473\end{array}$ & $\underset{474}{1100788}$ & $\begin{array}{c}120 \mathrm{CT} 88 \\
475\end{array}$ & $\begin{array}{c}130 \mathrm{CT} 88 \\
476\end{array}$ & $\begin{array}{l}140 \mathrm{CT} 88 \\
\text { NO WORK }\end{array}$ & $\underset{477}{1500788}$ \\
\hline $\begin{array}{c}160 \mathrm{CT} 88 \\
478\end{array}$ & $\begin{array}{c}170 \mathrm{CT} 88 \\
479\end{array}$ & $\begin{array}{c}180 \mathrm{CT} 88 \\
480\end{array}$ & $\begin{array}{c}1900188 \\
481\end{array}$ & $\begin{array}{c}200 C T 88 \\
482\end{array}$ & $\begin{array}{l}210 \mathrm{CT} 88 \\
\text { NO WORK }\end{array}$ & $\begin{array}{c}220 \mathrm{CT} 88 \\
483\end{array}$ \\
\hline $\begin{array}{c}230 \mathrm{CT} 88 \\
484\end{array}$ & $\begin{array}{c}2400 \mathrm{~T} 88 \\
485\end{array}$ & $\begin{array}{c}250 \mathrm{CT} 88 \\
486\end{array}$ & $\begin{array}{c}2600188 \\
487\end{array}$ & $\begin{array}{c}270 \mathrm{CT} 88 \\
488\end{array}$ & $\begin{array}{l}280 \mathrm{TT} 88 \\
\text { NO WORK }\end{array}$ & $\begin{array}{c}290 \mathrm{CT} 88 \\
489\end{array}$ \\
\hline $\begin{array}{c}300 \mathrm{CT} 88 \\
490\end{array}$ & $\begin{array}{c}310 \mathrm{CP8} 88 \\
491\end{array}$ & & & & & \\
\hline & & $\underset{492}{1110 \text { V88 }}$ & $\begin{array}{c}2150 v 88 \\
493\end{array}$ & $\begin{array}{c}3 \text { NOV88 } \\
494\end{array}$ & $\begin{array}{r}410088 \\
\text { NO WORK }\end{array}$ & $\begin{array}{c}5 \text { NOW } 88 \\
495\end{array}$ \\
\hline $\begin{array}{c}\text { 6NOV88 } \\
496\end{array}$ & $\begin{array}{c}\text { 7Nov88 } \\
497\end{array}$ & $\begin{array}{c}8110 v 88 \\
498\end{array}$ & $\begin{array}{c}9 \text { NOV88 } \\
499\end{array}$ & $\begin{array}{c}\text { 1010V88 } \\
500\end{array}$ & $\begin{array}{l}11110 \text { W8 } \\
\text { HO } \text { WORK }\end{array}$ & $\begin{array}{c}\text { 12NOV88 } \\
501\end{array}$ \\
\hline $\begin{array}{c}\text { 13NOV88 } \\
502\end{array}$ & $\begin{array}{c}1410088 \\
503\end{array}$ & $\begin{array}{c}15 y 0 v 88 \\
504\end{array}$ & $\begin{array}{c}16 \text { NOV88 } \\
505\end{array}$ & $\begin{array}{c}17 \text { Hov88 } \\
506\end{array}$ & $\begin{array}{l}18 \text { NOV88 } \\
\text { HO WORK }\end{array}$ & $\begin{array}{c}1910 V 88 \\
507\end{array}$ \\
\hline $\begin{array}{c}20 \text { Nov88 } \\
508\end{array}$ & $\begin{array}{c}21110 v 88 \\
509\end{array}$ & $\begin{array}{c}2210 v 88 \\
510\end{array}$ & $\begin{array}{c}\text { 23NOV88 } \\
511\end{array}$ & $\begin{array}{c}2410088 \\
512\end{array}$ & $\begin{array}{l}25 \text { NON88 } \\
\text { HO HORK }\end{array}$ & $\begin{array}{c}26 \text { Nov88 } \\
513\end{array}$ \\
\hline $\begin{array}{c}2710788 \\
514\end{array}$ & $\begin{array}{c}2810 V 88 \\
515\end{array}$ & $\begin{array}{c}2910 \mathrm{~V} 88 \\
516\end{array}$ & $\begin{array}{c}\text { 30N0v88 } \\
517\end{array}$ & & & \\
\hline & & & & $\begin{array}{c}10 E C 88 \\
518\end{array}$ & $\begin{array}{r}2 D E C 88 \\
\text { NO WORK }\end{array}$ & $\begin{array}{c}30 \text { 3DC88 } \\
519\end{array}$ \\
\hline $\begin{array}{c}4 \mathrm{DEC} 88 \\
520\end{array}$ & $\begin{array}{c}50 \mathrm{DCC} 88 \\
521\end{array}$ & $\begin{array}{c}60 \mathrm{DCB8} \\
522\end{array}$ & $\begin{array}{c}7 D C_{528} \\
523\end{array}$ & $\begin{array}{c}80 E C 88 \\
524\end{array}$ & $\begin{array}{l}\text { 9DEC88 } \\
\text { NO WORK }\end{array}$ & $\begin{array}{c}10 D E C 88 \\
525\end{array}$ \\
\hline$\underset{526}{11 D E C 88}$ & $\underset{527}{120 \mathrm{DC} 88}$ & $\begin{array}{c}\text { 13DECS8 } \\
528\end{array}$ & $\begin{array}{c}14 D E C 88 \\
529\end{array}$ & $\underset{530}{250 R C 88}$ & $\begin{array}{l}\text { 16DEC88 } \\
110 \text { WORK }\end{array}$ & $\begin{array}{c}17 D R C 88 \\
531\end{array}$ \\
\hline $\begin{array}{c}18 D E C 88 \\
532\end{array}$ & $\underset{533}{19 D E C 88}$ & $\begin{array}{c}2002 c 88 \\
534\end{array}$ & $\underset{535}{21 D E C 88}$ & $\begin{array}{c}220 \mathrm{DEC} 88 \\
536\end{array}$ & $\begin{array}{l}\text { 23DEC88 } \\
\text { HO WORK }\end{array}$ & $\begin{array}{c}240 \mathrm{DC} 88 \\
537\end{array}$ \\
\hline $\begin{array}{c}250 \mathrm{DC} 88 \\
538\end{array}$ & $\begin{array}{c}26 \text { DEC88 } \\
539\end{array}$ & $\underset{540}{270 E C 88}$ & $\begin{array}{c}28 D E C 88 \\
541\end{array}$ & $\begin{array}{c}29 D E C 88 \\
542\end{array}$ & $\begin{array}{l}\text { 30DEC88 } \\
\text { NO WORK }\end{array}$ & $\begin{array}{c}310 E C 88 \\
543\end{array}$ \\
\hline $\begin{array}{l}\text { 1JAH89 } \\
\text { NO WORK }\end{array}$ & $\begin{array}{c}2 \mathrm{JAK} 89 \\
544\end{array}$ & $\begin{array}{c}\text { 3Jג1899 } \\
545\end{array}$ & $\begin{array}{c}4 J \lambda H 89 \\
546\end{array}$ & $\begin{array}{c}\text { 5JAN89 } \\
547\end{array}$ & $\begin{array}{r}6 \mathrm{JAN89} \\
\text { NO WORK }\end{array}$ & $\begin{array}{c}7 \mathrm{JAN} 89 \\
548\end{array}$ \\
\hline $\begin{array}{c}\text { 8JAN89 } \\
549\end{array}$ & $\begin{array}{c}9 \text { ग)मू89 } \\
550\end{array}$ & $\begin{array}{c}\text { 10JA1489 } \\
551\end{array}$ & $\begin{array}{c}\text { 11JKN89 } \\
552\end{array}$ & $\begin{array}{c}12 J \lambda N 89 \\
553\end{array}$ & $\begin{array}{l}\text { 13JגH189 } \\
\text { NO WORR }\end{array}$ & $\underset{554}{14 J \lambda N 89}$ \\
\hline
\end{tabular}

Figure 9.11 - Case 2, Project Calendar (Continued). 


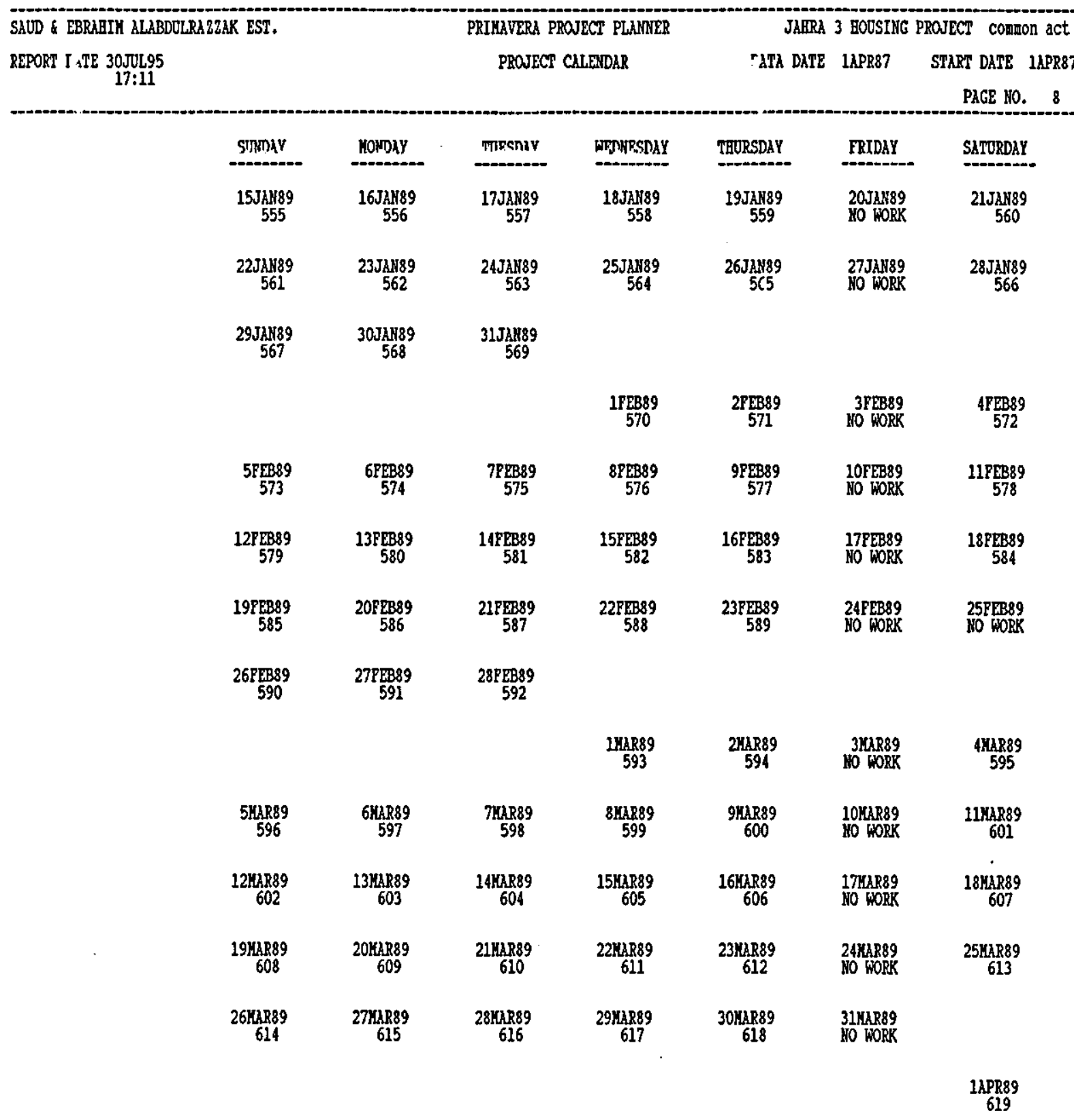

Figure 9.11 - Case 2, Project Calendar (Continued). 


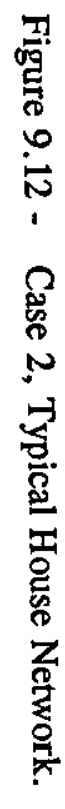

\section{LEGEND}

\begin{tabular}{|l|l|}
\hline ACT ID & TF \\
\hline
\end{tabular}




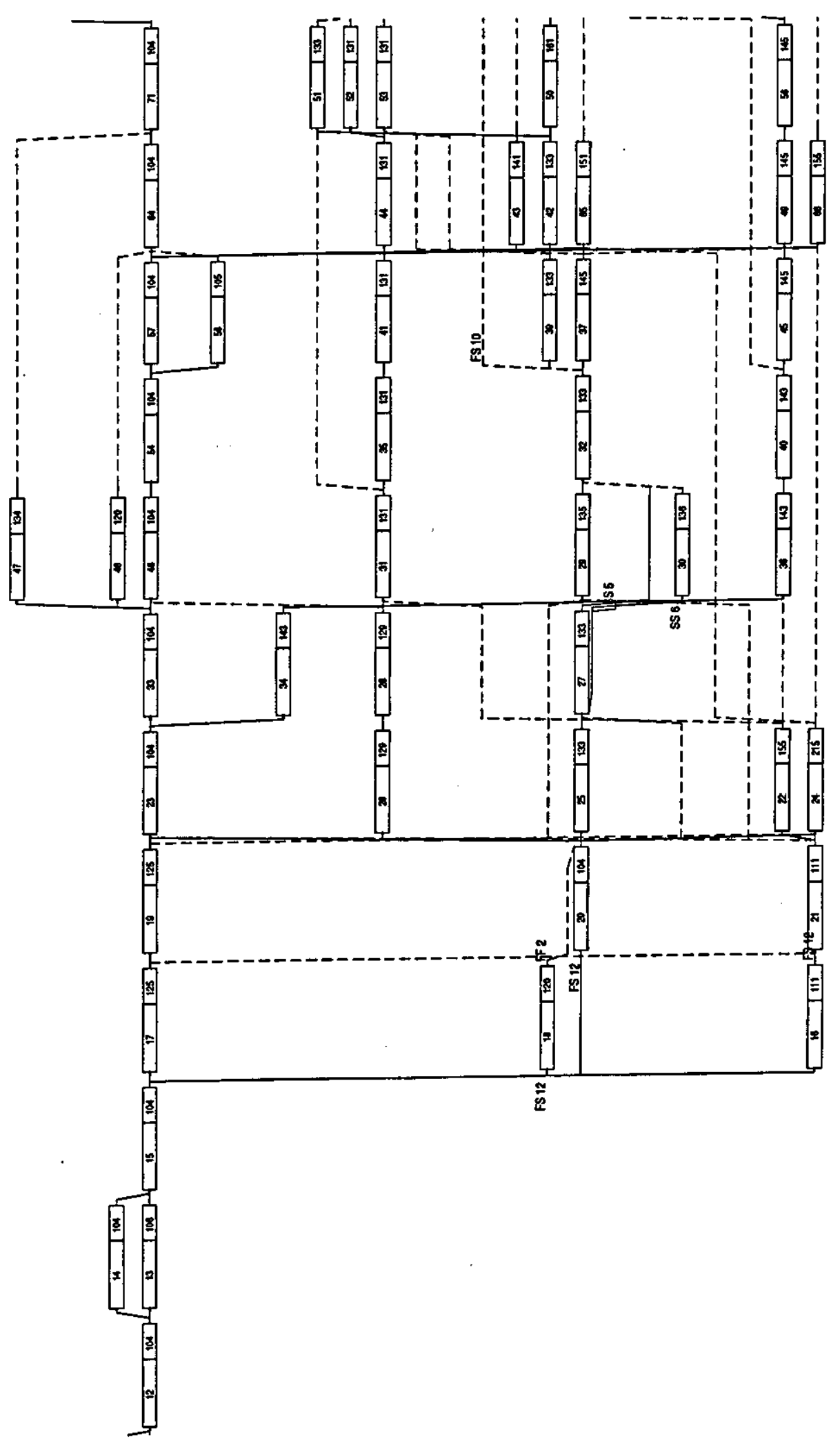

Figure 9.12 - Case 2, Typical House Network (Continued). 


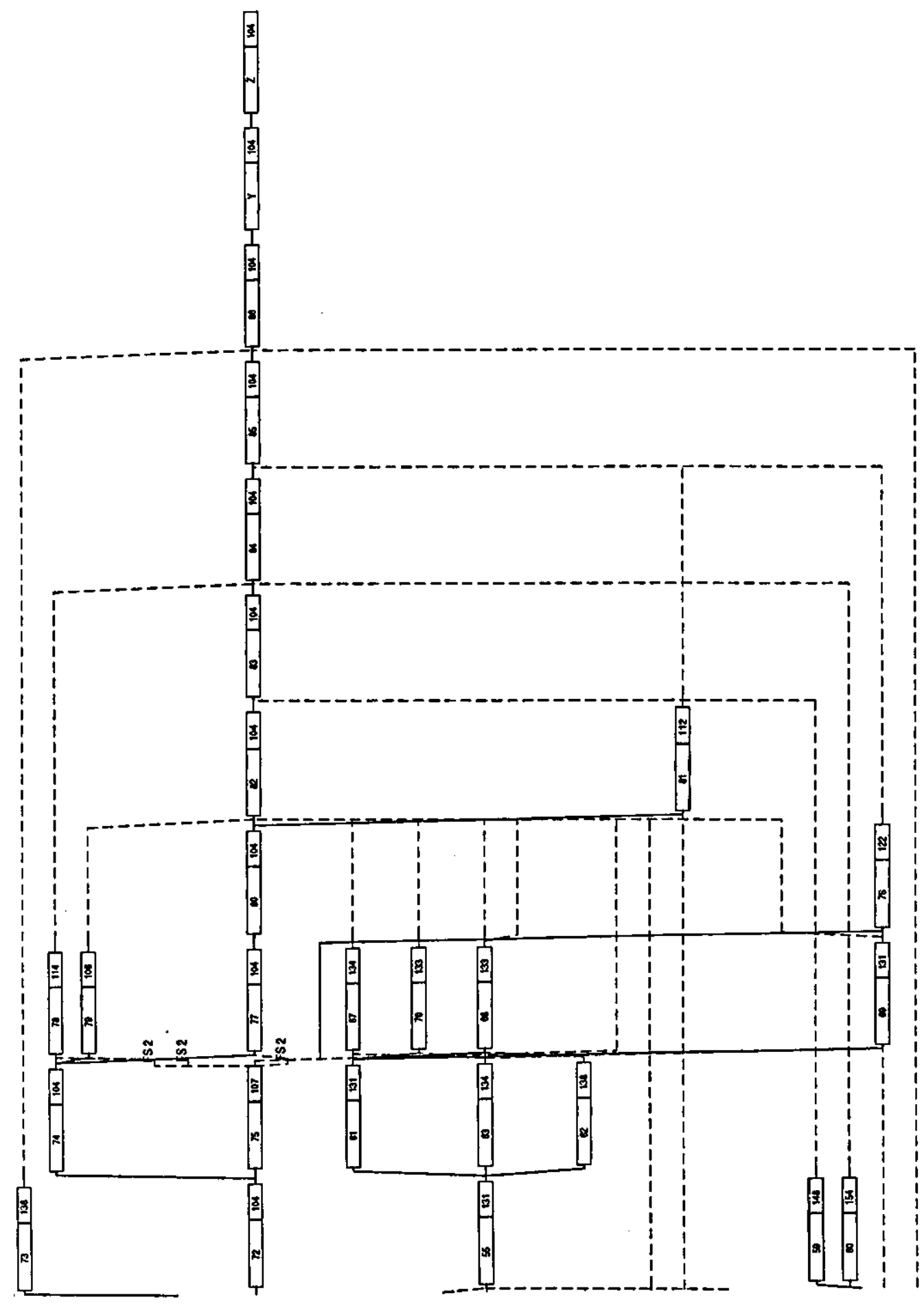

Figure 9.12 - Case 2, Typical House Network (Continued). 


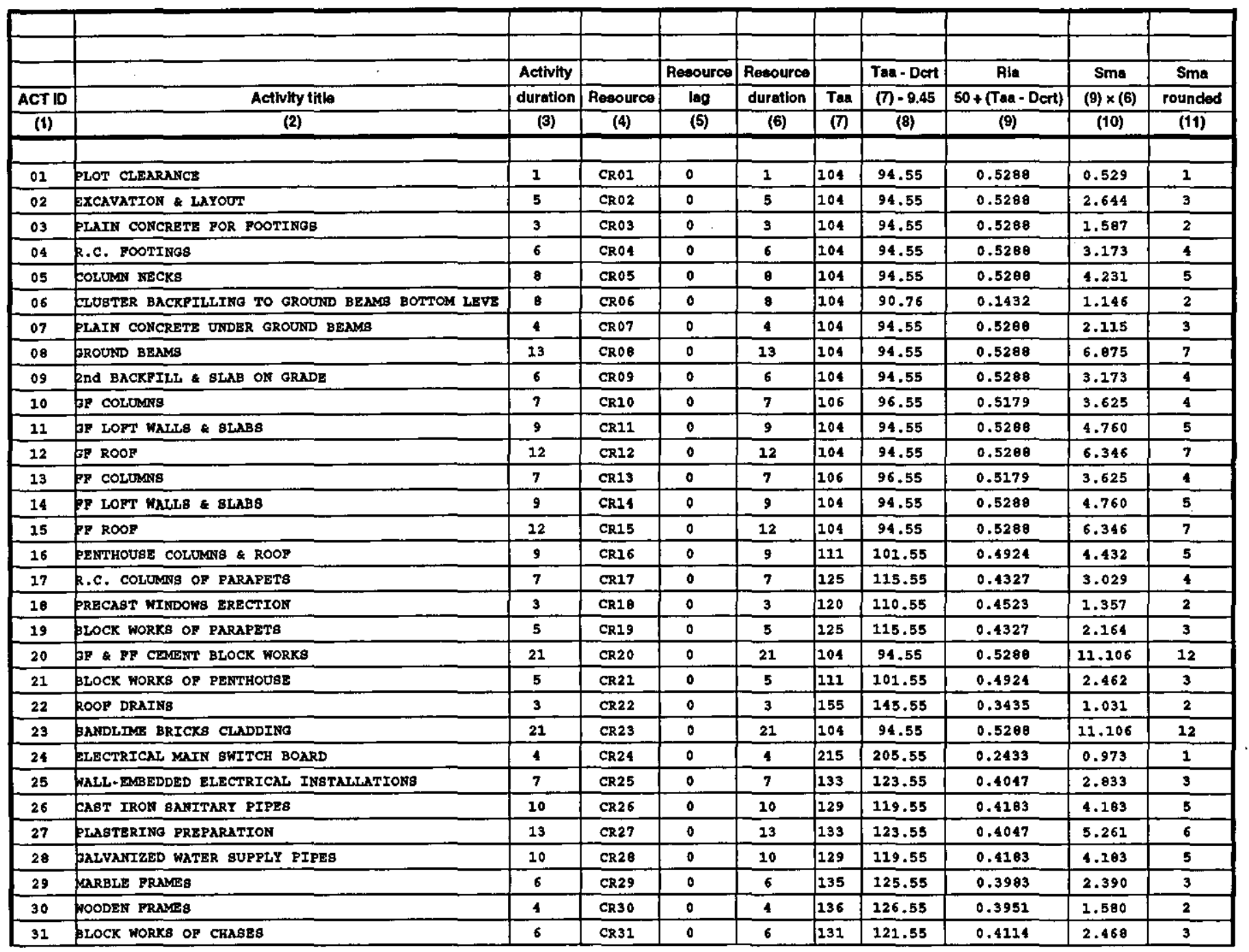




\begin{tabular}{|c|c|c|c|c|c|c|c|c|c|c|}
\hline & & Anthithy & & Domenureal & Lanirmal & & Tan-Dat & & & \\
\hline ACT ID & Activity title & duration & Resource & lag & duration & Taa & $(7) \cdot 9.45$ & $50+($ Taa $-D e r t)$ & $(9) \times(6)$ & rounded \\
\hline (1) & (2) & (3) & (4) & (5) & (6) & $(7)$ & (8) & (9) & (10) & (11) \\
\hline 32 & FLABTERINO & $\boldsymbol{\theta}$ & CR32 & 0 & 8 & 133 & 123.55 & 0.4047 & 3.238 & 4 \\
\hline 33 & BOUNDARY WALL BUB-STRUCTURE (PIain conc. to GB. & 24 & CR33 & 0 & 24 & 204 & 94.55 & 0.5288 & 12.692 & 13 \\
\hline 34 & FARAPETS COPING \& TANK SUPPORTS & 6 & CR34 & o & 6 & 243 & 133.55 & 0.3744 & 2.246 & 3 \\
\hline 35 & PORCELAIN WALI TILES & 12 & CR33 & 0 & 12 & 131 & 121.55 & 0.4114 & 4.936 & 5 \\
\hline 36 & ROOF FOMM \& SCREED & 10 & CR36 & o & 10 & 143 & 133.55 & 0.3744 & 3.744 & 4 \\
\hline 37 & ELECTRICAI \& TELEPHONE BOARDS, PANELS \& WIRING & 6 & CR37 & 0 & 6 & 145 & 135.55 & 0.3689 & 2.213 & 3 \\
\hline 39 & TERRAZZO FLOOR TILES \& AXIRTING & 13 & CR39 & 0 & 13 & 133 & 123.55 & 0.4047 & 5.261 & 6 \\
\hline 40 & ROOP WATER PROOPING LAYERS & 6 & CR40 & 0 & 6 & 143 & 133.55 & 0.3744 & 2.246 & 3 \\
\hline 41. & BANITARY PITTINGS (P10OT Embodded) & 4 & CR41 & o & 4 & 131 & 121.55 & 0.4114 & 1.645 & 2 \\
\hline 42 & MARBLE STAIRB E BKIRTING & 6 & CR42 & 0 & 6 & 133 & 123.55 & 0.4047 & 2.428 & 3 \\
\hline 43 & JOINARY WORKS & 4 & CR43 & $\circ$ & 4 & 141 & 131.55 & 0.3803 & 1.520 & 2 \\
\hline 44 & WTTERPROOPINO IN WET PLOOR AREAS & 6 & CR44 & 0 & 6 & 131 & 121.55 & 0.4114 & $2.46 \theta$ & 3 \\
\hline 45 & KOOF EMBEDDED DRAINAGE PIPES & 2 & CR45 & 0 & 2 & 145 & 135.55 & 0.3689 & 0.738 & 1 \\
\hline 46 & BOUNDARY WALL REIN. CONC. COLUMASB & 7 & CR46 & 0 & 7 & 120 & 110.55 & 0.4523 & 3.166 & 4 \\
\hline 47 & FRECAST UNITS OF BODKDARY WALL & 3 & CR47 & 0 & 3 & 134 & 124.55 & 0.4015 & 1.204 & 2 \\
\hline 48 & FARD PLOMBING PRECABT MANHOLE & 6 & CR4B & 0 & 6 & 104 & 94.55 & 0.5288 & 3.173 & 4 \\
\hline 49 & ROOP THERMAL INGULATION \& TILING & 10 & CR49 & 0 & 10 & 145 & 135.55 & 0.3689 & 3.689 & 4 \\
\hline 50 & BTAIRB HAKDRAILB & 5 & CR50 & 0 & 5 & 161 & 151.55 & 0.3299 & 1.650 & 2 \\
\hline 51 & PLASTERING OP CHASES, SXIRTINO EMBEDDED PLUMB & 6 & CR51 & o & 6 & 133 & 123.55 & 0.4047 & 2.428 & 3 \\
\hline 52 & CERAMIC TILES & 7 & CR52 & 0 & 7 & 231 & 121.55 & 0.4114 & 2.880 & 3 \\
\hline 53 & GYPSOM BOARDS FALBE CEILING & 7 & CR53 & 0 & 7 & 131 & 121.55 & 0.4114 & 2.880 & 3 \\
\hline 54 & YARD PLTMBING PIPES & 10 & CR54 & 0 & 10 & 104 & 94.55 & 0.5288 & 5.280 & 6 \\
\hline 55 & EAIFI PREPARATION (Primer a Putty Coate) & 12 & CR55 & 0 & 12 & 132 & 121.55 & 0.4314 & 4.936 & 5 \\
\hline 56 & WATER TANX $E$ EXPOSED ROOP PIPINO & 7 & CR56 & O & 7 & 145 & 135.55 & 0.3689 & 2.582 & 3 \\
\hline 57 & YARD ELECTRICAL PIPEB \& HANDHOLES & $?$ & CR57 & 0 & 7 & 104 & 94.55 & 0.5288 & 3.702 & 4 \\
\hline 58 & BANITARY HOOBE CONNBCTION & 6 & CR5O & 0 & 6 & 105 & 95.55 & 0.5233 & 3.140 & 4 \\
\hline 59 & KOOF MASTIC \& TOUCH-UPS & 6 & CR59 & 0 & 6 & 148 & 138.55 & 0.3609 & 2.165 & 3 \\
\hline 60 & MATER TANK SHED & 4 & CR60 & 0 & 4 & 154 & 144.55 & 0.3459 & 1.384 & 2 \\
\hline 61 & ALUMANOM PRAMES, WINDOWS \& DOORS & 9 & CR61 & 0 & 9 & 131 & 121.55 & 0.4114 & 3.702 & 1 \\
\hline 62 & ELECTRICAI BOARDS CONNECTION & 4 & CR62 & 0 & 4 & 138 & 128.55 & 0.3890 & 1.556 & 2 \\
\hline 63 & PAINT, FIRBT AECOND COATS & 6 & CR63 & 0 & 6 & 134 & 124.55 & 0.4015 & 2.409 & 3 \\
\hline
\end{tabular}




\begin{tabular}{|c|c|c|c|c|c|c|c|c|c|c|}
\hline & & & & & & & & & & \\
\hline & & & & & & & & & & \\
\hline & & Activity & & Resource & Resource & & TaA - Dort & Aia & Sma & Sma \\
\hline ACT ID & Activity thite & duration & \begin{tabular}{|l|} 
Resources \\
\end{tabular} & \begin{tabular}{|l|l|} 
lag \\
\end{tabular} & \begin{tabular}{|l|} 
duration \\
\end{tabular} & Taa & $(7) \cdot 9.45$ & $50+(T a A \cdot D c t)$ & $(9) \times(6)$ & rounded \\
\hline (1) & (2) & (3) & (4) & (5) & (6) & (7) & (8) & (9) & $(10)$ & (11) \\
\hline & & & & & & & & & & \\
\hline 64 & YARD EARTH WORKS \& PLAIN CONCRETS & 10 & CR64 & 0 & 10 & 104 & 94.55 & $0.528 \theta$ & 5.288 & 6 \\
\hline 65 & ENTERNAI MAIN CABLES & 2 & CR65 & 0 & 2 & 151 & 141.55 & 0.3532 & 0.706 & 1 \\
\hline 66 & ELECTRICAL HOUGE CONNECTION & 3 & CR66 & 0 & 3 & 155 & 145.55 & 0.3435 & 1.031 & 2 \\
\hline 67 & IRONMONGERY WORKB YOR YOODEN DOORS & 3 & CR67 & 0 & 3 & 134 & 124.55 & 0.4015 & 1.204 & 2 \\
\hline 68 & INTERNAI ELBCTRICAI PITTINGS & 4 & CR69 & 0 & 4 & 133 & 223.55 & 0.4047 & 1.619 & 2 \\
\hline 69 & BANITARY PIXTURES \& ACCESSORIES & 6 & CR69 & 0 & 6 & 131 & 121.55 & 0.4114 & 2.468 & 3 \\
\hline 70 & AIUAMINOM ACCEBSORIES & 4 & CR70 & 0 & 4 & 133 & 223.55 & 0.4047 & 1.619 & 2 \\
\hline 72 & BRICX WORKS OF BOTKDARY WALL & 9 & CR71 & 0 & 9 & 104 & 94.55 & $0.528 \theta$ & 4.760 & 5 \\
\hline 72 & BOUNDARY WALL CAST IN BITU COPING & 6 & CR72 & 0 & 6 & 104 & 94.55 & $0.528 \theta$ & 3.173 & 4 \\
\hline 73 & WMTER BUPPLY HOUQB CONNECTION & 1 & CR73 & 0 & 1 & 138 & 128.55 & 0.3890 & 0.389 & 2 \\
\hline 74 & YXRD TERRAZZO TILES \& MASTIC WORKB & 10 & CR74 & 0 & 10 & 104 & 94.55 & 0.5288 & 5.200 & 6 \\
\hline 75 & FRAMES E PIRISHES OF YARD HAND/MANBOLES & 5 & CR75 & 0 & 5 & 207 & 97.55 & 0.5126 & 2.563 & 3 \\
\hline 76 & ELUMAING NETWORKS TEBTINO & 2 & CR76 & 0 & 2 & 122 & 212.55 & 0.4443 & 0.809 & 1 \\
\hline 77 & ALUMIXOM EXTERYAL PALAE CEILING & 5 & CR77 & 0 & 5 & 104 & 94.55 & $0.52 \theta \theta$ & 2.644 & 3 \\
\hline 78 & HGRICULTURAI BOIL & 3 & CR78 & Q & 3 & 114 & 104.55 & 0.4783 & 1.435 & 2 \\
\hline 79 & FTEEL GATES INSTALLATTON \& PATNT UNDERCOATB & 4 & $\overline{C R 79}$ & 0 & 4 & 106 & 96.53 & 0.5179 & 2.072 & 3 \\
\hline Bo & EXTERKAL ELECTRICAL PITTINGS & 1 & CR8O & 0 & 1 & 104 & 94.55 & $0.528 \mathrm{~B}$ & 0.529 & 1 \\
\hline 81 & ELECTRICAL \& TELEPHONE NETWORKB TEGTTNG & $\overrightarrow{1}$ & CRE1 & 0 & 1 & 212 & 102.55 & 0.4976 & 0.488 & 1 \\
\hline 82 & GABT COAT OF PAINT & 3 & CR82 & 0 & 3 & 104 & 94.55 & 0.5288 & 1.587 & 2 \\
\hline 83 & ENT \& EXT. TRRRAZZO TILES GRINDING \& POLISHING & 4 & CR 83 & 0 & 4 & 104 & 94.55 & 0.5288 & 2.115 & 3 \\
\hline 84 & Fougs PTNAL CLENMING & 2 & CR84 & 0 & 2 & 104 & 94.55 & 0.5288 & 1.058 & 2 \\
\hline 85 & PINAL PAINT \& TOUCH-UPB & 4 & CRES & 0 & 4 & 104 & 94.55 & 0.5288 & 2.115 & 3 \\
\hline 23 & BANDLIME BRICKS CLADDING & 21 & SCAPPOLD & 10 & 11 & 104 & 94.55 & 0.5288 & 5.017 & 6 \\
\hline 10 & SP coltowass & 7 & $8 \mathrm{H} 10$ & 0 & 9 & 106 & 96.55 & 0.5179 & 4.661 & 5 \\
\hline 12 & BP ROOP & 12 & $8 \mathrm{H12}$ & 0 & 24 & 104 & 94.55 & 0.5288 & 12.692 & 13 \\
\hline 13 & COLUTANB & 7 & 8H13 & 0 & 9 & 106 & 96.55 & 0.5179 & 4.661 & 5 \\
\hline 15 & ProOP & 12 & BH15 & 0 & 24 & 104 & 94.55 & 0.5288 & 12.692 & 13 \\
\hline 16 & FETTHOUSE COLOWNS \& ROOP & 9 & $8 \mathrm{sH}$ & 0 & 21 & 111 & 201.55 & 0.4924 & 10.340 & 11 \\
\hline 33 & FOUNDARY MALL SUB-ATRUCTURE (Plain Conc. to GB. & 24 & BH33 & 18 & 7 & 104 & 94.55 & 0.5288 & 3.702 & 4 \\
\hline
\end{tabular}


SAUD \& EBRAHIM ALRABDULPAZZAK EST.

FEPORT DATE 21SEP9S RUN NO. 121
PRTMAIREA PFJTETT PLiNRE?

SEVEN SEAS, COHGTRUTTZUN OF 51 HOUSES
H HOUS GTART DATE 1APRG 7 FIN DATE 13AUGRB DATA DATE LAPRg? PAGE NO.

\begin{tabular}{|c|c|}
\hline$\underset{\text { ID }}{\text { ACTIVI }}$ & $\begin{array}{l}\text { ORIO } \\
\text { PUR }\end{array}$ \\
\hline$\cdots$ & i \\
\hline$\hat{B}$ & 24 \\
\hline $\bar{x}$ & 18 \\
\hline$z$ & 30 \\
\hline $211 \mathrm{HO44AO1}$ & 1 \\
\hline $211 \mathrm{HO} 3 \mathrm{AOOL}$ & I \\
\hline $211 \mathrm{H} 042 \mathrm{AO1}$ & 1 \\
\hline $213 \mathrm{HO} 035 \mathrm{AO} 1$ & 1 \\
\hline $213 \mathrm{H} 036 \mathrm{AO} 1$ & 1 \\
\hline $223 \mathrm{HO37AOL}$ & 2 \\
\hline $213 \mathrm{H} 032 \mathrm{AOI}$ & 1 \\
\hline $223 \mathrm{HO} 33 \mathrm{AOO}$ & $i$ \\
\hline $213 \mathrm{H} 034 \mathrm{~A} 01$ & 1 \\
\hline $323 \mathrm{H} 185 \mathrm{AO}$ & 1 \\
\hline $323 \mathrm{H} 106 \mathrm{AOO}$ & 1 \\
\hline $323 \mathrm{H1O7A01}$ & 1 \\
\hline $322 H 188 A 01$ & $\vec{\jmath}$ \\
\hline $322 \mathrm{H1B9A01}$ & 1 \\
\hline $322 \mathrm{H} 190 \mathrm{AO} O$ & 1 \\
\hline $321 \mathrm{H174A01}$ & $i$ \\
\hline $321 \mathrm{H} 175 \mathrm{AOO} 2$ & 1 \\
\hline $320 \mathrm{H176A01}$ & 1 \\
\hline $320 \mathrm{H177 \textrm {AO } 1}$ & 1 \\
\hline $320 \mathrm{H278AO1}$ & 1 \\
\hline $214 \mathrm{HO31 \textrm {AOI }}$ & 1 \\
\hline $214 \mathrm{HO} 30 \mathrm{AOO}$ & 1 \\
\hline $214 \mathrm{HO2} 9 \mathrm{AO} 2$ & 1 \\
\hline $214 \mathrm{HO} \mathrm{OAO1}$ & 1 \\
\hline $215 H 027 \mathrm{AO} 1$ & 1 \\
\hline $215 \mathrm{H026A01}$ & $i$ \\
\hline $215 \mathrm{HO25AOL}$ & $i$ \\
\hline $215 H 024 A 01$ & 1 \\
\hline $225 \mathrm{HO} 023 \mathrm{AOL}$ & 1 \\
\hline $215 \mathrm{HO22A01}$ & 1 \\
\hline $216 \mathrm{HO2} 2 \mathrm{AOI}$ & 1 \\
\hline $216 \mathrm{HO2OAOL}$ & 1 \\
\hline 216HO19AO1 & 1 \\
\hline $216 \mathrm{HO1BAOL}$ & 1 \\
\hline $217 \mathrm{H} 017 \mathrm{AO1}$ & 1 \\
\hline 217K016AO1 & 2 \\
\hline 218HO15A02 & 1 \\
\hline $218 H 014 \mathrm{AO}$ & 1 \\
\hline $21 \mathrm{BH} 013 \mathrm{AO}$ & 1 \\
\hline $219 \mathrm{HO} 12 \mathrm{AO}$ & 1 \\
\hline $219 H O 11 A 01$ & 1 \\
\hline $219 \mathrm{HO10A01}$ & 1 \\
\hline $220 \mathrm{HOOSAOI}$ & 1 \\
\hline $220 \mathrm{HOOBAO1}$ & 1 \\
\hline $220 \mathrm{HOOTAO1}$ & 1 \\
\hline $220 \mathrm{HOOGAOI}$ & 1 \\
\hline $220 \mathrm{HOOSAOI}$ & $i$ \\
\hline $220 \mathrm{HOO4AOI}$ & 1 \\
\hline $221 \mathrm{HOO3AO1}$ & 1 \\
\hline $221 \mathrm{HOO} 2 \mathrm{AOL}$ & 1 \\
\hline $221 \mathrm{HOOLAOI}$ & 1 \\
\hline $211 H 044 A 02$ & 5 \\
\hline $211 H 043 A 02$ & 5 \\
\hline $211 H 042 A 02$ & 5 \\
\hline $213 H 035 A 02$ & 5 \\
\hline $213 \mathrm{HO} 036 \mathrm{AO2}$ & 5 \\
\hline $233 \mathrm{HO37A02}$ & 5 \\
\hline $223 \mathrm{HO} 2 \mathrm{AO}$ & 5 \\
\hline $213 \mathrm{HO} 33 \mathrm{AO2}$ & 5 \\
\hline $213 H 034 A 02$ & 5 \\
\hline $323 K 185 A 02$ & 5 \\
\hline $323 \mathrm{H186A02}$ & 5 \\
\hline $323 \% 187 \mathrm{AO2}$ & 5 \\
\hline $322 \mathrm{HIBOAO2}$ & 5 \\
\hline $322 \mathrm{HIB9A02}$ & 5 \\
\hline 322H190A02 & 5 \\
\hline $321 \mathrm{H} 174 \mathrm{AO2}$ & 5 \\
\hline $3214175 A 02$ & 5 \\
\hline $320 \mathrm{H} 176 \mathrm{AO2}$ & 5 \\
\hline $320 H 177 \mathrm{AO2}$ & 5 \\
\hline $320 \mathrm{H178A02}$ & 5 \\
\hline $214 H 031 \mathrm{AO2}$ & 5 \\
\hline $214 \mathrm{HO} 030 \mathrm{AO} 2$ & 5 \\
\hline $214 \mathrm{HOZ} 9 \mathrm{AOZ}$ & 5 \\
\hline $214 \mathrm{HO2}$ OA02 & 5 \\
\hline $215 \mathrm{H027A02}$ & 5 \\
\hline $215 \mathrm{H} 026 \mathrm{AOZ}$ & s \\
\hline $215 \mathrm{HO2SAO2}$ & $\mathbf{s}$ \\
\hline $215 \mathrm{HO24A02}$ & 5 \\
\hline $215 K 023 \mathrm{AOZ}$ & 5 \\
\hline $215 \mathrm{H} 022 \mathrm{AOZ}$ & 5 \\
\hline $216 \mathrm{HO21A02}$ & 5 \\
\hline $216 \mathrm{HO2OAO2}$ & 5 \\
\hline $216 \mathrm{HO} 19 \mathrm{AOO}$ & 5 \\
\hline $216 \mathrm{HO1BAO2}$ & 5 \\
\hline 217H017A02 & 5 \\
\hline $217 \mathrm{HO16AO2}$ & 5 \\
\hline 218H015A02 & 5 \\
\hline $21 \mathrm{QHO14A02}$ & 5 \\
\hline 21 BHO13AO2 & 5 \\
\hline $219 \mathrm{H} 012 \mathrm{AO} 2$ & 5 \\
\hline 219HO1LAO2 & 5 \\
\hline
\end{tabular}

Figure 9.13 - Case 2, Baseline Schedule. 
JAUD \& EBRAHIM ALABDULPAZZAK SST

REPORT DATE $215 E P G S$ RUN HO 121

Bageline Schedule by Typical Act 1V1EY E ES
PRIMAVEAA PRCUECT PLANNER

SEVEN SEAS, SONSTRUCTION OF 51 HOUSES
JAHRA 3 HOUSING PROJECT COMMOR ACE

START DATE 1APR87 FIN DATE 23AUG9S

DATA DATE LAPRO7 PAGE NO. 2

\begin{tabular}{|c|}
\hline $\begin{array}{c}\text { ACTIVITY } \\
\text { ID }\end{array}$ \\
\hline I19HO1OADZ \\
\hline $220 \mathrm{HOOSAOZ}$ \\
\hline $220400 B A 02$ \\
\hline $220 \mathrm{HOO} 7 \mathrm{AO2}$ \\
\hline $220 \mathrm{HOOOGAOZ}$ \\
\hline $220 \mathrm{HOO5AO2}$ \\
\hline $22 \mathrm{OH} 004 \mathrm{AO} 2$ \\
\hline $22 \mathrm{LHOOJAO2}$ \\
\hline $221 \mathrm{H} 002 \mathrm{~A} 02$ \\
\hline $221 \mathrm{HOO} 1 \mathrm{A02}$ \\
\hline $211 \mathrm{H} 044 \mathrm{AO}$ \\
\hline $211 \mathrm{H} 043 \mathrm{A0} 3$ \\
\hline 211H042A03 \\
\hline $213 \mathrm{HO} 35 \mathrm{AO} 3$ \\
\hline $213 \mathrm{HO} 63 \mathrm{GA03}$ \\
\hline $213 \mathrm{HO} 37 \mathrm{AO} 3$ \\
\hline $213 \mathrm{HO} 32 \mathrm{AO} 3$ \\
\hline $213 \mathrm{HO} 33 \mathrm{AOO}$ \\
\hline $213 \mathrm{HO3} 4 \mathrm{AO} 3$ \\
\hline 323H1BSA03 \\
\hline $323 \mathrm{H} 1 \mathrm{~B} 6 \mathrm{~A} 03$ \\
\hline $323 \mathrm{H1 \theta 7A03}$ \\
\hline $322 \mathrm{HLOQAO} 3$ \\
\hline $322 \mathrm{B1O9A03}$ \\
\hline $322 \mathrm{H190A03}$ \\
\hline $321 \mathrm{H174A03}$ \\
\hline $321 \mathrm{H} 175 \mathrm{AO03}$ \\
\hline $320 \% 176 A 03$ \\
\hline $320 \mathrm{H177A03}$ \\
\hline $3204178 \times 03$ \\
\hline $214 \mathrm{HO} 32 \mathrm{AO}$ \\
\hline $214 \mathrm{HO30A03}$ \\
\hline $214 \mathrm{HO} 2903$ \\
\hline $214 \mathrm{HO} 02 \mathrm{BAO}$ \\
\hline $215 \mathrm{HO27A03}$ \\
\hline $225 \mathrm{HO} 26 \mathrm{AO}$ \\
\hline $215 \mathrm{H} 025 \mathrm{~A} 03$ \\
\hline $215 \mathrm{HO} 24 \mathrm{A03}$ \\
\hline 215H023AO3 \\
\hline $215 \mathrm{HO22A03}$ \\
\hline $216 \mathrm{HO21A03}$ \\
\hline $216 \mathrm{HO20A03}$ \\
\hline $216 \mathrm{H019A03}$ \\
\hline $216 \mathrm{HO} 10 \mathrm{AO} 03$ \\
\hline $217 \mathrm{HO17A03}$ \\
\hline $217 \mathrm{HO} 16 \mathrm{A03}$ \\
\hline 210HO15AO3 \\
\hline $218 \mathrm{HO} 14 \mathrm{AO} 3$ \\
\hline 218HO13A03 \\
\hline $219 H 012 \mathrm{AO} 3$ \\
\hline $219 \mathrm{HO} 21 \mathrm{AO} 03$ \\
\hline $219 \mathrm{HO} 20 \mathrm{A0} 03$ \\
\hline $220 \mathrm{HOO9A03}$ \\
\hline $220 \mathrm{HOOBAO3}$ \\
\hline $220 \mathrm{HO07A03}$ \\
\hline $220 \mathrm{HOO} 6 \mathrm{AO} 3$ \\
\hline $220 \mathrm{HOOSAO3}$ \\
\hline $22 \mathrm{OHOO4A03}$ \\
\hline $221 \mathrm{HO} 003 \mathrm{A0} 3$ \\
\hline $221 \mathrm{HOO2A03}$ \\
\hline $221 \mathrm{H} 001 \mathrm{A03}$ \\
\hline 211 HO44AO4 \\
\hline 211H043AO4 \\
\hline 211H042A04 \\
\hline 213HO35AO4 \\
\hline $213 H 036 \mathrm{AO} 4$ \\
\hline $213 \mathrm{HO} 037 \mathrm{AO4}$ \\
\hline $213 \mathrm{HO} 032 \mathrm{AOA}$ \\
\hline \\
\hline $213 \mathrm{HO} 34 \mathrm{AO4}$ \\
\hline $323 \mathrm{H} 185 \mathrm{AO} 4$ \\
\hline $323 \mathrm{H} 186 \mathrm{AO} 04$ \\
\hline $323 \mathrm{H} 187 \mathrm{AO} 4$ \\
\hline $322 \mathrm{H} 108 \mathrm{AOS}$ \\
\hline $322 \mathrm{H} 1 \mathrm{BAA04}$ \\
\hline $322 \mathrm{H190A04}$ \\
\hline $321 \mathrm{HLT4A04}$ \\
\hline $321 \mathrm{H} 175 \mathrm{AOA}$ \\
\hline $320 \mathrm{H176A04}$ \\
\hline $320 \mathrm{HL77AO4}$ \\
\hline $320 \mathrm{H178A04}$ \\
\hline $214 \mathrm{H} 031 \mathrm{AO}$ \\
\hline $214 \mathrm{HO} 30 \mathrm{AOA}$ \\
\hline $214 \mathrm{HO29A04}$ \\
\hline 214H028AO4 \\
\hline $\begin{array}{l}215 \mathrm{HO27AOA} \\
215 \mathrm{HO2} 6 \mathrm{AOA}\end{array}$ \\
\hline $215 \mathrm{H} 026 \mathrm{AOA}$ \\
\hline $215 \mathrm{HO25AO4}$ \\
\hline 215HO24AO4 \\
\hline $215 \mathrm{HO}^{23 \mathrm{AO}}$ \\
\hline $215 \mathrm{HO22AO4}$ \\
\hline $216 \mathrm{HO} 21 \mathrm{AO} 04$ \\
\hline $216 \mathrm{HO2OAO4}$ \\
\hline $216 \mathrm{HO19A04}$ \\
\hline $216 \mathrm{HO} 28 \mathrm{AOA}$ \\
\hline 217H017A04 \\
\hline
\end{tabular}

Figure 9.13 - Case 2, Baseline Schedule (Continued). 
3AUD S EBrAHIM ALABDULRAZZAK EST

REPORT DATE 21SEPSS RUN NO. 121 $25: 46$
PP.IMATERA PRCEECT PLANNE

SEVEN SEAS, CONSTRUCTION OE 51 HOUSES
JAHPA 3 HOUSING PPOJECT EOMmEN 26

START DATE HAPRE? FIN DATE L3AUGga

DATA DATE LAPRE7 PAGE NO.

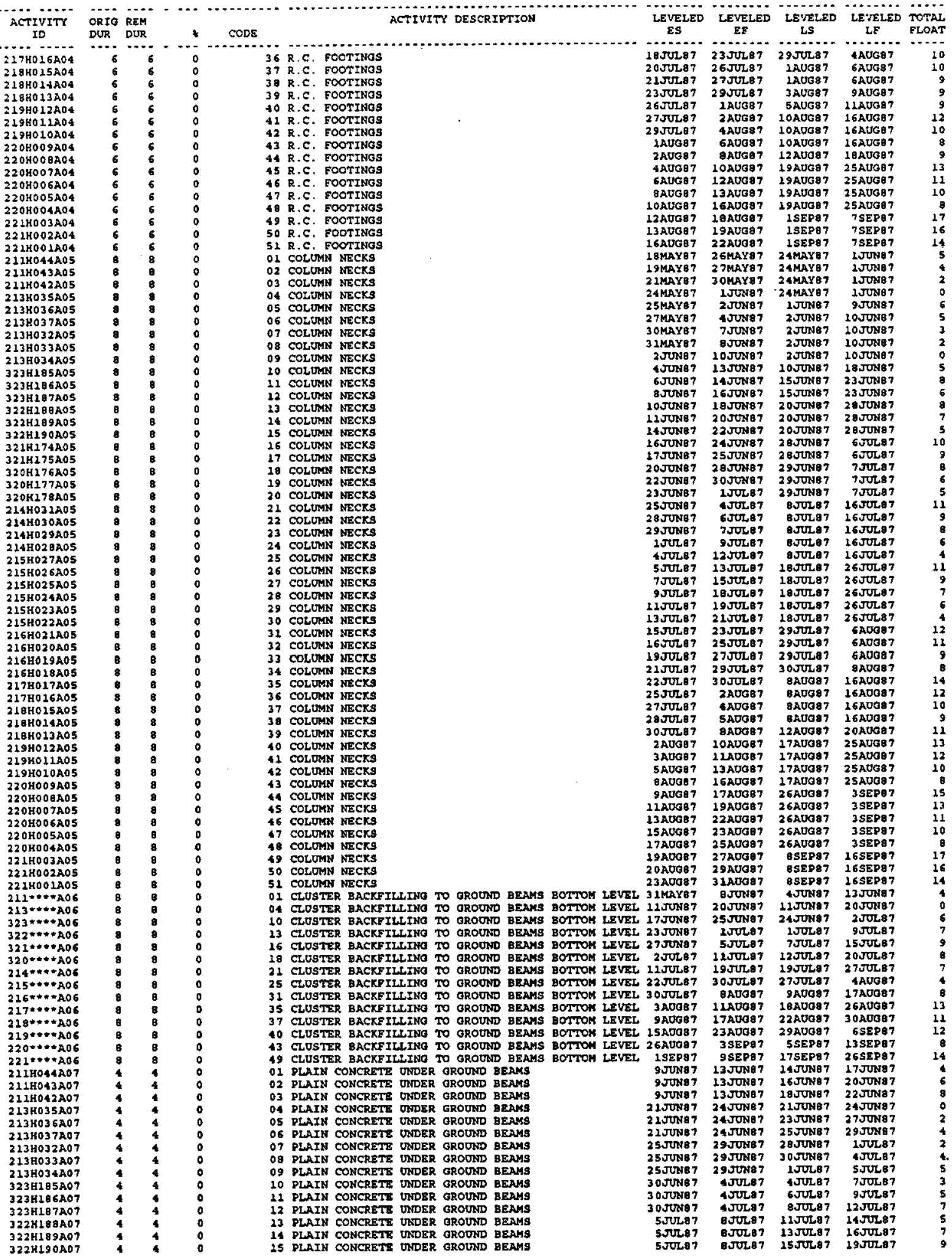

Figure 9.13 - Case 2, Baseline Schedule (Continued). 


\begin{tabular}{|c|c|}
\hline ACTIVITY & $\begin{array}{l}\text { ORIG } \\
\text { DUR }\end{array}$ \\
\hline $321 H 194407$ & 4 \\
\hline $321 \mathrm{M} 175 \mathrm{AO} 7$ & 4 \\
\hline $320 \mathrm{H} 176 \mathrm{AO} 7$ & 4 \\
\hline $320 \mathrm{~K} 177 \mathrm{AO} 7$ & 4 \\
\hline $320 \mathrm{H} 178 \mathrm{AO0} 7$ & 4 \\
\hline $214 \mathrm{H} 031 \mathrm{AO} 7$ & 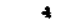 \\
\hline $214 \mathrm{HO} 030 \mathrm{AO} ?$ & 4 \\
\hline $214 \mathrm{HO} 29 \mathrm{AO} 7$ & 4 \\
\hline $214 \mathrm{HO} 2 \mathrm{BAO} 7$ & 4 \\
\hline $215 \mathrm{H027A07}$ & 4 \\
\hline $215 \mathrm{HO} 26 \mathrm{AO} 7$ & 4 \\
\hline $225 \mathrm{HO} 25 \mathrm{AO} 7$ & 4 \\
\hline $215 \mathrm{HO2} 4 A 07$ & 4 \\
\hline $215 H 023 \mathrm{AO} 7$ & 4 \\
\hline $215 \mathrm{HO22A07}$ & 4 \\
\hline $216 \mathrm{HO2} 1 \mathrm{AO} 07$ & 4 \\
\hline $2164020 \mathrm{A07}$ & 4 \\
\hline $216 \mathrm{HO} 19 \mathrm{AO} 7$ & 4 \\
\hline $216 \mathrm{HO} 01 \mathrm{AAO} 7$ & 4 \\
\hline $217 \mathrm{H} 027 \mathrm{AO} 7$ & 4 \\
\hline $217 \mathrm{HO} 16 \mathrm{AO} 7$ & 4 \\
\hline $2194015 A 07$ & 4 \\
\hline $219 H 014 \mathrm{AO} 7$ & 4 \\
\hline $219 \mathrm{H} 023 \mathrm{A0} 07$ & 4 \\
\hline $219 \mathrm{HO} 012 \mathrm{AO} 7$ & 4 \\
\hline $219 H 011 A 07$ & 4 \\
\hline $219 H 010 A 07$ & 4 \\
\hline $2201009 A 07$ & 4 \\
\hline $220 \mathrm{HOOBAO} 7$ & 4 \\
\hline $2204007 A 07$ & 4 \\
\hline $220 \mathrm{H} 006 \mathrm{AO} 7$ & 4 \\
\hline $220 \mathrm{HOO} 5 \mathrm{AOC}$ & 4 \\
\hline $220 \mathrm{HOO} \triangle A 07$ & 4 \\
\hline $221 \mathrm{HO} 003 \mathrm{A07}$ & 4 \\
\hline $221 \mathrm{H} 002 \mathrm{AO} 7$ & 4 \\
\hline $221 \mathrm{HOO} 1 \mathrm{AO} 7$ & 4 \\
\hline $211 \mathrm{HO44AOQ}$ & 13 \\
\hline $211 H 043 A 08$ & 13 \\
\hline $211 \mathrm{H} 042 \mathrm{~A} 0 \mathrm{~B}$ & 13 \\
\hline $213 \mathrm{HO} 3 \mathrm{SAOB}$ & 13 \\
\hline $213 \mathrm{HO} 6 \mathrm{AOOB}$ & 13 \\
\hline $213 \mathrm{H} 037 \mathrm{AOO}$ & 13 \\
\hline $213 \mathrm{HO} 2 \mathrm{AOQ}$ & 13 \\
\hline $213 \mathrm{HO} 33 \mathrm{AOO}$ & 13 \\
\hline $213 \mathrm{HO} 34 \mathrm{AOO}$ & 13 \\
\hline $323 \mathrm{H} 185 \mathrm{AO}$ & 13 \\
\hline $323 \mathrm{H} 186 \mathrm{~A} 0 \mathrm{~g}$ & 13 \\
\hline $323 \mathrm{H} 187 \mathrm{AOO}$ & 13 \\
\hline $322 \mathrm{H} 18 \mathrm{8A0} 0 \mathrm{a}$ & 13 \\
\hline $322 \mathrm{H} 189 \times 0 \mathrm{O}$ & 13 \\
\hline $322 H 190 A 08$ & 13 \\
\hline $321 \% 174 A 08$ & 13 \\
\hline $321 \mathrm{H} 175 \mathrm{AOB}$ & 13 \\
\hline $320 \mathrm{H176A08}$ & 13 \\
\hline $320 \mathrm{HL} 77208$ & 13 \\
\hline $320 \mathrm{H17} 7 \mathrm{AOO}$ & .13 \\
\hline $214 \mathrm{HO} 1 \mathrm{AOO}$ & 23 \\
\hline $214 \mathrm{HO} 0 \mathrm{OAOS}$ & 13 \\
\hline $2141029 A 09$ & 13 \\
\hline $214 \mathrm{KO2BA08}$ & 13 \\
\hline $215 \mathrm{HO27AOO}$ & 13 \\
\hline $215 H 026 \mathrm{AOO}$ & 13 \\
\hline $215 \mathrm{H} 025 \mathrm{AOB}$ & 13 \\
\hline $215 \% 024 \mathrm{~A} 0 \mathrm{~B}$ & 13 \\
\hline $215 H 023 A 08$ & 13 \\
\hline $215 \mathrm{HO22AOB}$ & 13 \\
\hline $216 \mathrm{HO21A0B}$ & 13 \\
\hline $216 \mathrm{HO} 020 \mathrm{AOO}$ & 13 \\
\hline $216 \mathrm{HO} 19 \mathrm{AOO}$ & 13 \\
\hline $216 \mathrm{HO} 1 \mathrm{BAOS}$ & 13 \\
\hline 217H017A08 & 13 \\
\hline $217 \mathrm{HO} 26 \mathrm{AOS}$ & 13 \\
\hline $218 H 015 A 08$ & 13 \\
\hline $218 \mathrm{HO14 \lambda 09}$ & 13 \\
\hline 21日HO13AOO & 13 \\
\hline $219 K 012 A 08$ & 13 \\
\hline $219 \mathrm{HO11A08}$ & 13 \\
\hline $219 \mathrm{H} 010 \mathrm{AO}$ & 13 \\
\hline 220HOO9AOB & 13 \\
\hline $2204009 A 0 B$ & 13 \\
\hline $22010007 \mathrm{~A} 08$ & 13 \\
\hline $220 H 006 \mathrm{AOB}$ & 13 \\
\hline $220 H 005 A O B$ & 13 \\
\hline $2204004 A 0 B$ & 13 \\
\hline $221 \mathrm{HOO} \mathrm{AOB}$ & 13 \\
\hline $221 \mathrm{HOO2AOB}$ & 13 \\
\hline $221 \mathrm{HOO1AOB}$ & 13 \\
\hline $211 H 044 A 09$ & 6 \\
\hline $2114043 \mathrm{AO} 9$ & 6 \\
\hline $211 \mathrm{HO} 2 \mathrm{AOO}$ & 6 \\
\hline $213 \mathrm{H} 035 \mathrm{~A} 09$ & 6 \\
\hline $213 \mathrm{HO} 036 \mathrm{AOO}$ & 6 \\
\hline $213 H 037 A 09$ & 6 \\
\hline 213 H032A09 & 6 \\
\hline $213 \mathrm{HO} 3 \mathrm{AO9}$ & 6 \\
\hline 213 H034A09 & 6 \\
\hline
\end{tabular}

Figure 9.13 - Case 2, Baseline Schedule (Continued). 


\begin{tabular}{|c|c|c|}
\hline \begin{tabular}{c} 
Actrvith \\
yo \\
\hdashline
\end{tabular} & $\begin{array}{l}\text { ORIG } \\
\text { DUR } \\
\cdots\end{array}$ & $\begin{array}{l}\text { REM } \\
\text { DUR } \\
\cdots \ldots\end{array}$ \\
\hline $323 \mathrm{H} 1 \mathrm{BSA09}$ & 6 & 6 \\
\hline $323 \mathrm{H}: B 6 \mathrm{AO} 9$ & 6 & 6 \\
\hline $323 H 187 A 09$ & 6 & 6 \\
\hline $322 \mathrm{H} 1 \mathrm{BgA09}$ & 6 & 6 \\
\hline 322H189A09 & 6 & 6 \\
\hline $322 \mathrm{H} 190 \mathrm{~A} 09$ & 6 & 6 \\
\hline $321 \mathrm{H} 174 \mathrm{~A} 09$ & 6 & 6 \\
\hline $321 H 275 A 09$ & 6 & 6 \\
\hline $320 \mathrm{H176A09}$ & 6 & 6 \\
\hline $320 \mathrm{H} 177 \mathrm{~A} 09$ & 6 & 6 \\
\hline $320 H 179 A 09$ & 6 & 6 \\
\hline $214 \mathrm{H} 031 \mathrm{A09}$ & 6 & 6 \\
\hline $214 \mathrm{H} 030 \mathrm{~A} 09$ & 6 & 6 \\
\hline $214 \mathrm{HO29AOS}$ & 6 & 6 \\
\hline $2144028 A 09$ & 6 & 6 \\
\hline $215 \mathrm{H} 027 \mathrm{A09}$ & 6 & 6 \\
\hline $215 \mathrm{H} 026 \mathrm{AOO}$ & 6 & 6 \\
\hline $215 \mathrm{H} 025 \mathrm{AOO}$ & 6 & 6 \\
\hline $215 \mathrm{HO} 024 \mathrm{AOS}$ & 6 & 6 \\
\hline $215 \mathrm{H} 023 \mathrm{~A} 09$ & 6 & 6 \\
\hline $215 \mathrm{H} 022 \mathrm{AOO}$ & 6 & 6 \\
\hline $216 \mathrm{H} 021 \mathrm{~A} 09$ & 6 & 6 \\
\hline $216 \mathrm{HO} 020 \mathrm{AOS}$ & 6 & 6 \\
\hline $216 \mathrm{HO} 19 \mathrm{AOO}$ & 6 & 6 \\
\hline $216 \mathrm{HO18A09}$ & 6 & 6 \\
\hline $217 H 017 A 09$ & 6 & 6 \\
\hline $217 \mathrm{HO16AOS}$ & 6 & 6 \\
\hline $219 \mathrm{H} 015 \mathrm{~A} 09$ & 6 & 6 \\
\hline $218 \mathrm{HO} 014 \mathrm{AO} 09$ & 6 & 6 \\
\hline $219 \mathrm{H} 013 \mathrm{~A} 09$ & 6 & 6 \\
\hline 219H012A09 & 6 & 6 \\
\hline $2194013 \mathrm{AOO}$ & 6 & 6 \\
\hline $219 H 010 A 09$ & 6 & 6 \\
\hline $220 H 009 A 09$ & 6 & 6 \\
\hline $2204008 A 09$ & 6 & 6 \\
\hline $2204007 A 09$ & 6 & 6 \\
\hline $2204006 A 09$ & 6 & 6 \\
\hline 220H005A09 & $\begin{array}{l}6 \\
6\end{array}$ & 6 \\
\hline $\begin{array}{l}220 \mathrm{H} 004 \mathrm{AOS} \\
221 \mathrm{H} 003 \mathrm{AOO}\end{array}$ & 6 & 6 \\
\hline $221 \% 002 \mathrm{AOS}$ & 6 & 6 \\
\hline $221 H 001 A 09$ & 6 & 6 \\
\hline $211 \mathrm{H} 044 \mathrm{~A} 20$ & 7 & 7 \\
\hline $211 \mathrm{H} 043 \mathrm{AlO}$ & 7 & 7 \\
\hline $211 \mathrm{HO42A10}$ & 7 & $?$ \\
\hline $213 \mathrm{H} 035 \mathrm{~A} 2 \mathrm{O}$ & 7 & 7 \\
\hline $213 \mathrm{H} 036 \mathrm{A1O}$ & 7 & 7 \\
\hline $2238037 A 20$ & 7 & 7 \\
\hline 213H032A10 & $?$ & 7 \\
\hline $213 \mathrm{HO} 33 \mathrm{~A}=0$ & 7 & 7 \\
\hline $213 H 034210$ & 7 & 7 \\
\hline $323 \mathrm{H} 105 \mathrm{AO} 10$ & 7 & 7 \\
\hline $323 \mathrm{HLB6A10}$ & 7 & 7 \\
\hline $323 H 187210$ & 7 & 7 \\
\hline $322 \mathrm{H280A10}$ & 7 & 7 \\
\hline $322 \mathrm{~K} 289 \mathrm{AlO}$ & 7 & 7 \\
\hline $322 \mathrm{H190A10}$ & 7 & 7 \\
\hline $321 \mathrm{H} 74 \mathrm{AlO}$ & 7 & 7 \\
\hline $3214175 A 10$ & 7 & $?$ \\
\hline $320 H 276 A 10$ & 7 & 7 \\
\hline $320 \mathrm{HL} 77 \mathrm{ALO}$ & 7 & 7 \\
\hline $320 H 178 A 10$ & 7 & 7 \\
\hline 214 HO31A10 & 7 & 7 \\
\hline $214 \mathrm{H} 030 \mathrm{AlO}$ & 7 & 7 \\
\hline $214 H 029 A 10$ & 7 & 7 \\
\hline 214 HO2 OA1O & 7 & 7 \\
\hline $215 \mathrm{H} 027 \mathrm{AlO}$ & 7 & 7 \\
\hline $215 H 026 A 10$ & 7 & 7 \\
\hline $215 \mathrm{HO25A10}$ & 7 & 7 \\
\hline $225 \mathrm{HO24A10}$ & 7 & 7 \\
\hline 215H023A10 & ? & 7 \\
\hline $215 H 022 \mathrm{AlO}$ & 7 & 7 \\
\hline $226 \mathrm{HO21 \textrm {A } 1 0}$ & 7 & 7 \\
\hline $216 \mathrm{HO} 0 \mathrm{OA} 1 \mathrm{O}$ & 7 & $?$ \\
\hline $216 \mathrm{HO} 29 \mathrm{AlO}$ & 7 & 7 \\
\hline $216 \mathrm{HO} 20 \mathrm{~A} 10$ & 7 & 7 \\
\hline $217 H 017 A 10$ & 7 & 7 \\
\hline $217 \mathrm{H} 016 \mathrm{~A} 10$ & 7 & 7 \\
\hline $218 \mathrm{H} 015 \mathrm{AlO}$ & 7 & 7 \\
\hline $218 \mathrm{H} 014 \mathrm{AlO}$ & 7 & 7 \\
\hline $218 H 013 \mathrm{AlO}$ & 7 & 7 \\
\hline 219H012A10 & 7 & 7 \\
\hline $219 \mathrm{HO} 11 \mathrm{ALO}$ & 7 & 7 \\
\hline $219 H 010 A 10$ & $?$ & $?$ \\
\hline 22OHOO9A10 & 7 & 7 \\
\hline 22 OHOOBALO & 7 & 7 \\
\hline $220 \mathrm{HOO7A10}$ & 7 & 7 \\
\hline $220 \mathrm{H} 006 \mathrm{~A} 10$ & 7 & 7 \\
\hline $220 H 005 A 10$ & 7 & 7 \\
\hline $220 \mathrm{HOO4A.10}$ & 7 & $?$ \\
\hline $221 H 003 A 10$ & 7 & 7 \\
\hline $221 \mathrm{H} 002 \mathrm{~A} 10$ & 7 & 7 \\
\hline $221 \mathrm{H} 001 \mathrm{ALO}$ & 7 & 7 \\
\hline 211H044A11 & 9 & 9 \\
\hline $231 \mathrm{H} 043 \mathrm{Al1}$ & 9 & 9 \\
\hline $211 H 042 A 11$ & 9 & 9 \\
\hline
\end{tabular}

Figure 9.13 - Case 2, Baseline Schedule (Continued). 
GAUO SA EBRAH IM ALABDULRAZZAX EST.

REPORT JATE IISEPYS PUN NO. 121

Baseline schedul $15 ;$ by Typical Activity \& ES
PRIMAVERA PROTECT PLANNER

SEVEN SEAS, CONSTRUCTION OF 51 HOUSES

\begin{tabular}{|c|c|}
\hline $\begin{array}{c}\text { ACTIVITY } \\
\text { ID }\end{array}$ & $\begin{array}{l}\text { ORIG } \\
\text { DUR } \\
-. .\end{array}$ \\
\hline $213 \mathrm{H} 035 \mathrm{~A} 11$ & \\
\hline$=13 \mathrm{HO} 36 \mathrm{AL}$ & 9 \\
\hline$=13 \mathrm{HO} 37 \mathrm{AL1}$ & 9 \\
\hline $213 H 032 A 11$ & 9 \\
\hline $213 \mathrm{HO} 33 \mathrm{~A} .11$ & 9 \\
\hline $2134034 A_{111}$ & 9 \\
\hline $323 \mathrm{H} 185 \mathrm{A11}$ & 9 \\
\hline $3234186 A 11$ & 9 \\
\hline $323 \mathrm{H} 187 \mathrm{All}$ & 9 \\
\hline $322 \mathrm{H18BAIL}$ & 9 \\
\hline $322 \mathrm{H} 1 \mathrm{BgA} 1 \mathrm{I}$ & 9 \\
\hline $322 \mathrm{H} 190 \mathrm{~A}, 1$ & 9 \\
\hline $321 \mathrm{H} 174 \mathrm{Al1}$ & 9 \\
\hline $321 \mathrm{H} 175 \mathrm{~A} 11$ & 9 \\
\hline $320 \mathrm{H} 176 \mathrm{Al} 1$ & 9 \\
\hline $320 \mathrm{H} 177 \mathrm{ALI}$ & 9 \\
\hline $3201178 \mathrm{Al1}$ & 9 \\
\hline $234 K 031 \mathrm{Al1}$ & 9 \\
\hline $214 \mathrm{H} 030 \mathrm{~A} 11$ & 9 \\
\hline $2144029 A 11$ & 9 \\
\hline $21+K 028 A 11$ & 9 \\
\hline 215 H027A11 & 9 \\
\hline $215 \mathrm{HO}^{2} 6 \mathrm{AL} 1$ & 9 \\
\hline $215 \mathrm{HO} 2 \mathrm{SAII}$ & 9 \\
\hline 215 HO24A11 & 9 \\
\hline $215 \mathrm{HO} 23 \mathrm{Al1}$ & 9 \\
\hline $215 \mathrm{H} 022 \mathrm{~A} 11$ & 9 \\
\hline $216 \mathrm{HO21A21}$ & 9 \\
\hline 2164020211 & 9 \\
\hline $216 \% 019 A 12$ & 9 \\
\hline $2164018 A 11$ & 9 \\
\hline 217H017A11 & 9 \\
\hline 217 H016A11 & 9 \\
\hline $218 \mathrm{H} 025 \mathrm{~A} 11$ & 9 \\
\hline $219 H O 14211$ & 9 \\
\hline 218KO13A11 & 9 \\
\hline $219 H 012 A 11$ & 9 \\
\hline 219 HO11A11 & 9 \\
\hline $219 H 010 A 11$ & 9 \\
\hline $220 \times 009 A 11$ & 9 \\
\hline $2204008 A 11$ & 9 \\
\hline $2204007 A 11$ & 9 \\
\hline $220 \mathrm{HOOSA11}$ & 9 \\
\hline $220 \mathrm{HOO} 5 \mathrm{AII}$ & 9 \\
\hline $220 \mathrm{HOO4A11}$ & 9 \\
\hline $222 \mathrm{H} 003 \mathrm{~A} 11$ & 9 \\
\hline $221 \mathrm{HOO2AI1}$ & 9 \\
\hline $221 \mathrm{HOOLA11}$ & 9 \\
\hline $211 H 044 A 22$ & 12 \\
\hline $2111043 A 12$ & 12 \\
\hline $211 \mathrm{HO42A12}$ & 22 \\
\hline $213 \mathrm{H} 035 \mathrm{~A} 12$ & 12 \\
\hline 2134036212 & 12 \\
\hline $213 \mathrm{H} 037 \mathrm{~A} 12$ & 12 \\
\hline $2134032 A 12$ & 12 \\
\hline $213 H 033 A 12$ & 12 \\
\hline 213 H034A12 & 12 \\
\hline $323 \mathrm{H} 1 \mathrm{OSA} 12$ & 12 \\
\hline $323 \mathrm{H186A12}$ & 12 \\
\hline $323 \mathrm{H} 187 \mathrm{~A} 22$ & 12 \\
\hline $322 \mathrm{H} 188 \mathrm{~A} 12$ & 12 \\
\hline $322 \mathrm{H} 189 \mathrm{AL2}$ & 12 \\
\hline $322 \mathrm{H} 190 \mathrm{~A} 12$ & 12 \\
\hline $321 K 174 A 12$ & 12 \\
\hline $321 H 275 A 12$ & 12 \\
\hline $3204176 A 12$ & 12 \\
\hline $320 H 177 A 12$ & 12 \\
\hline $320 H_{279 A 12}$ & 12 \\
\hline $214 H 031 \mathrm{L12}$ & 12 \\
\hline $214 \mathrm{HO} 0 \mathrm{OA12}$ & 12 \\
\hline $2144029 A 12$ & 12 \\
\hline $214 \mathrm{HO2BA12}$ & 12 \\
\hline $215 \mathrm{H} 027 \mathrm{A1Z}$ & 12 \\
\hline $215 \mathrm{HO} 26 \mathrm{A12}$ & 12 \\
\hline $215 \mathrm{HO25A12}$ & 12 \\
\hline $215 \mathrm{H} 024 \mathrm{Al} 2$ & 12 \\
\hline $215 H 023 \mathrm{A12}$ & 12 \\
\hline $215 H 022 A 12$ & 12 \\
\hline $216 \mathrm{HO2} 1 \mathrm{AL2}$ & 12 \\
\hline $216 H 020212$ & 12 \\
\hline $216 H 019 A 12$ & 12 \\
\hline $2164018 A 12$ & 12 \\
\hline $217 H 017 A 12$ & 12 \\
\hline $217 \mathrm{H} 016 \mathrm{Al2}$ & 12 \\
\hline $2184015 A 12$ & 12 \\
\hline $219 H 014 A 12$ & 12 \\
\hline $218 H 013 \mathrm{A12}$ & 12 \\
\hline $219 H 012 A 12$ & 12 \\
\hline $219 H 011 A 12$ & 12 \\
\hline 2194010212 & 12 \\
\hline $220 \mathrm{H} 009 \mathrm{A12}$ & 12 \\
\hline $220 \mathrm{HOOBA12}$ & 12 \\
\hline $220 \mathrm{HOO} 7 \mathrm{Al2} 2$ & 12 \\
\hline $220 \% 006 A 12$ & 12 \\
\hline $220 \mathrm{HOOSA12}$ & 32 \\
\hline $220 \mathrm{HOO4A12}$ & 12 \\
\hline
\end{tabular}

JAHRA 3 HOUSING PROEET COMmON $2 C \mathrm{C}$ START DATE 1APRB 7 FIN DATE 13AUG89 DATA DATE 1APRE? PAGE NO. 6 LEVELED LEVELED LEVELED LEVELED TOTAL ......- -...... -.................

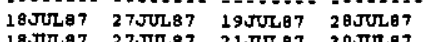

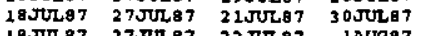

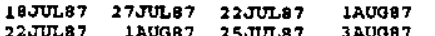

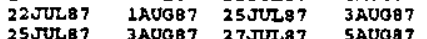

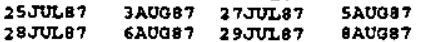

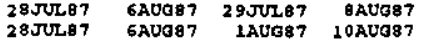
$\begin{array}{rrrr}2850287 & 6 A U 087 & 1 A U G 87 & 10 A U 087 \\ 2 A U 097 & 11 A 0 G 87 & 3 A U G B 7 & 12 A U G B 7\end{array}$ 2AUSB7 11AUGG97 4 AUUGB 7 13AUGG7 4AUG87 13AUG97 GAUGE7 16AUGO7 BAUGB7 17AUGB? GAUGB? 18AUGG? 9AUGB 7 1AAUOB? 11AUOB 7 20AUG97 12AUGB7 22AUGB7 13AUOS7 23AUOA7 12AUG87 22AUG87 26AUG87 25AUG87 17AUGS7 26AUGa? 18AUO87 27AUOS? 18AUOS7 27AUGS7 19AUGB7 29AUOB7 $\begin{array}{llll}19 A U G 87 & 29 A U G 87 & 22 A U G 87 & 31 \text { AUGB }\end{array}$ $\begin{array}{llll}23 A U G 87 & 1 S E P B 7 & 24 A U G 87 & 2 S E P 17 \\ 24 A U G 87 & 2 S E P B 7 & 26 A U G 97 & 5 S E P 97\end{array}$ $\begin{array}{llll}24 A U G 87 & 2 S E P B 7 & 26 A U G 97 & 5 S E P 97 \\ 27 A U G B 7 & 6 S E P B 7 & 29 A U Q 97 & 7 S E P 87\end{array}$ $\begin{array}{llll}27 A U G B 7 & 6 S E P B 7 & 29 A U Q 87 & 75 E P 87 \\ 29 A U B 87 & 7 S E P 87 & 31 A 0097 & \text { 9SEPB7 }\end{array}$

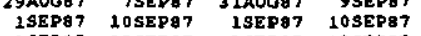
2SEPE7 12SEP 7 3SEP87 13SEP87 3SEP97 13SEPQ7 6SEPB7 15SEPB7 7SEPB7 16SEPE7 ASEPB7 17SEPE7 OSEP87 17SEP97 10SEPE7 20SEPE7 12SEPB7 21SEPB 73 13SEPB7 22SEPB7

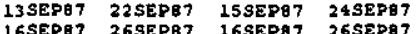
$\begin{array}{llll}16 S E P 87 & 26 S E P B 7 & 16 S E P 87 & 26 S 2 P 87 \\ 17 S E P 97 & 27 S E P 97 & 19 S E P 97 & 28 S E P 97\end{array}$ $\begin{array}{llll}17 S E P 97 & \text { 27SEP87 } & 19 \text { SEP97 } & \text { 28SEP97 } \\ \text { 19SEPA7 } & 2 \text { BSEP97 } & 21 \text { SEPB7 } & 30 \text { SEP87 }\end{array}$ $\begin{array}{rrrr}\text { 19SEPA7 } & 2 \text { 8SEP97 } & 21 \text { SEPB7 } & 30 \text { SEP87 } \\ \text { 22SEPB7 } & \text { 1OCTA7 } & 23 \text { SEPB7 } & 300 T B 7\end{array}$ $\begin{array}{llll}\text { 22SEPB7 } & \text { 1OCTA7 } & 23 S E P B 7 & 30 C T B 7 \\ 23 S E P B 7 & \text { उOCTB7 } & 26 S E P B 7 & 50 C T B 7\end{array}$ $\begin{array}{llll}23 S E P B 7 & 30 C T 87 & 26 S E P B 7 & 50 C T B 7 \\ 27 S E P B 7 & 60 C T B 7 & 28 S E P B 7 & 70 C T 87\end{array}$ 2ASEPB7 7OCTB7 29SEPA7 BOCT87 10CT87 120CT87 10CT87 110CT87 30сT87 120CT87 40CTr87 130CT87 10CT87 130CTE7 60CT87 150CT87 70CT87 170CTa7 80CT87 280CT87

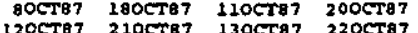

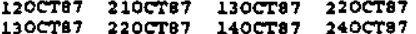

$\begin{array}{llll}130 C T 87 & 220 C T 87 & 140 C T 87 & 240 C T 87 \\ 170 C T 87 & 260 C T 87 & 170 C T 87 & 260 C T B 7\end{array}$

$\begin{array}{llll}170 C T 87 & 260 C T 87 & 170 C T \theta 7 & 260 C T 87 \\ 180 C T 87 & 270 C T 97 & 190 C T 87 & 280 C T \theta 7\end{array}$

190CTA7 28OCTA7 210CTB7 310CTA7 220CT87 1NOVA7 240CTE7 2NOVB7 240CTB7 2NOVB7 260CTB7 4NOV87 270CTB? 5NOVB7 270CTE7 5NOV97 16JUL87 29JUL87 210TL297 3AU087 $16 \pi 0 L 872900 L 872500 L 67$ 6AVG87 16J0L87 29J0L97 27JUL87 9ADGB? $\begin{array}{llll}2 \text { G JUL87 } & \text { 10AUGB7 } 29 \text { JUL87 } & \text { 11AUG87 }\end{array}$

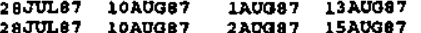

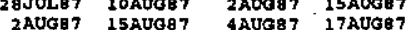
$\begin{array}{llll}\text { 4AUGQ7 17AUGS7 GAUGS7 } & 19 A 0 G B 7\end{array}$ 8AUOS7 20AUOQ9 9AJGB7 22AUOB7 8AUOB 7 20AUG97 11AUGB? 24AUGG7 12AUCO7 2SAUG87 13AUOB? 26AUOB7 12AUG87 25AUGB7 15AUG97 27AUOB7 $15 A U G 87$ 27AUGB7 17AUGG9 30AUG87 18AUGB 7 31AUGB7 19AUG87 1SEPB7 19AUGE7 19EPB7 22AUGB7 3SEPB7

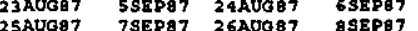
$\begin{array}{rrrr}25 A U G 97 & \text { 7SEP87 } & \text { 26AUG87 } & \text { SSEPB7 } \\ 27 A U G 97 & \text { 9SEPS7 } & 29 A U G 87 & 20 S E P B 7\end{array}$ $\begin{array}{rrrr}27 A U Q 97 & 9 S E P 97 & \text { 29AUGB 7 } & \text { 20SEPQ7 } \\ 29 A 0087 & 10 S E P Q 7 & 30 A 0087 & 12 S E P 97\end{array}$ $30 A \cup 097$ 12SEPE7 1SEP97 14SEP87 2SEPE7 15SEPB? 3SEPQ7 16SEPA7 5SEPB7 17SEPQ7 6SEPQ7 19SEP87 7SEPB7 20SEPB 7 ASEPB7 21 SEPPB 7 9SEPB7 22SEPA7 10SEPB7 23SEPB7 12SEP87 24SEP97 12SEPA7 24SEP87 13SEP87 26SEP87 14SEPA7 27SEP97

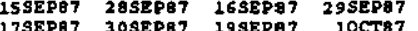

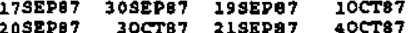
$\begin{array}{llll}20 S E P B 7 & 30 C T 87 & 21 \text { SEPA7 } & \text { 40CT87 } \\ 22 S E P 97 & \text { 5OCT87 } & 235 E P 97 & \text { 60CT87 }\end{array}$ 24SEPB7 70CT87 26SEPB7 BOCTB 7 27SEP97 100CT87 27SEPB7 100CT87 28SEP87 110CT87 29SEPB7 120CT87 30SEP87 130CT87 10CTB7 140CT87 30CT87 150CT97 40CT87 170CT97 50CTE7 180CT87 60CT87 190CT87 70CT87 200CT87 80CT97 210CT87 $100 C T 97$ 220CTB7 100CT87 220CT97 120CT17 250CT87 120CT87 250CT87 $\begin{array}{llll}130 C T 87 & 260 C T 87 & 140 C T 87 & 270 C T B 7 \\ 150 C T 87 & 2 \text { BOCT87 } & 170<T 87 & 290 C T 87\end{array}$ $\begin{array}{rrrr}150 C T 87 & 280 C T 87 & 170 C T 87 & 290 C T B 7 \\ 280 C T 87 & 310 C T 87 & 190 C T B 7 & \text { INOVE }\end{array}$ 200CT87 2NOV87 210CTB7 3NOV87 $\begin{array}{llll}220 C T 87 & \text { 4NOV87 240CTE? 5NOVB7 }\end{array}$ 250 Cr87 7NOV87 250CT日7 7NOV87 $270 \mathrm{CTB} 7$ 9NOV97 $270 \mathrm{CTE7}$ $280 C T$ T 70 NOV87 $290 \mathrm{CTE}$ ? 11NOV87

Figure 9.13 - Case 2, Baseline Schedule (Continued). 


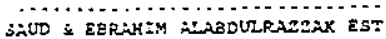

PEPCRT DATE :1SEPYS RUN NO. 121

Baseline Sehodule by Typical Activaty \& ES
PRIMAVERA PROTECT PLANNER

SEVEN SEAS, CONSTRUCTION OF 51 HOUSES
JAHPA 3 HOUSING PROJECT ECMmCn ACL

START DATE 1APRG 7 FIN DATE L3AUGBG

DATA DATE IAPRE? PAGE NO. ?

\begin{tabular}{|c|c|}
\hline $\begin{array}{l}\text { ACTIVITT } \\
\text { ID }\end{array}$ & $\begin{array}{l}\text { ORIO } \\
\text { DUR }\end{array}$ \\
\hline $2214003 \mathrm{AL2}$ & 12 \\
\hline $221 \mathrm{H} 002 \mathrm{~A} 12$ & 12 \\
\hline $221 H 001 A 12$ & 12 \\
\hline $211 \mathrm{HO44A13}$ & 7 \\
\hline $211 \mathrm{HO4}_{3 \mathrm{Al} 3}$ & 7 \\
\hline $211 \% 042 \mathrm{~A} 13$ & 7 \\
\hline $2134035 \mathrm{Al} 3$ & 7 \\
\hline $213 \mathrm{H} 036 \mathrm{~A} 13$ & 7 \\
\hline 213 HO37AJ3 & 7 \\
\hline $213 \mathrm{H} 032 \mathrm{A13}$ & 7 \\
\hline 233 HO33A13 & 7 \\
\hline & 7 \\
\hline $323 \mathrm{~K} 285 \mathrm{Al} 3$ & 7 \\
\hline $3234286 \mathrm{~A} 13$ & 7 \\
\hline $3234197 A 13$ & 7 \\
\hline $322 \mathrm{KIOBA} 3$ & 7 \\
\hline $322 \mathrm{H} 109 \mathrm{Al} 3$ & 7 \\
\hline $322 \mathrm{H} 190 \mathrm{AL} 3$ & $\rightarrow$ \\
\hline $32111744 A 13$ & 7 \\
\hline $321 k 175 A 13$ & 7 \\
\hline $3204176 \mathrm{Al3}$ & 7 \\
\hline $320 H 177 A 23$ & 7 \\
\hline $320 \mathrm{H} 178 \mathrm{~A} / 3$ & 7 \\
\hline $214 \mathrm{H} 03$ LA13 & 7 \\
\hline 214 H03OA13 & 7 \\
\hline $214 \mathrm{HO29A13}$ & 7 \\
\hline $214 H 02 B A 13$ & 7 \\
\hline $215 \mathrm{HO2}_{\mathrm{AA} 13}$ & 7 \\
\hline $215 \mathrm{HO26A13}$ & 7 \\
\hline $2154025 \mathrm{Al} 3$ & 7 \\
\hline $215 H 024 A 13$ & 7 \\
\hline $215 H 023 A 13$ & 7 \\
\hline $215 H 022 A 13$ & 7 \\
\hline $216 \mathrm{HO2} 1 \mathrm{Al} 3$ & 7 \\
\hline $216 \mathrm{HO2OA13}$ & 7 \\
\hline $216 \mathrm{HO} 19 \mathrm{~A} 3$ & 7 \\
\hline $216 \mathrm{HO29A13}$ & 7 \\
\hline $217 \mathrm{HO17A13}$ & 7 \\
\hline $217 \mathrm{HO16A13}$ & 7 \\
\hline $218 \mathrm{HO} 15 \mathrm{Al} 3$ & 7 \\
\hline $218 H 014 A 13$ & 7 \\
\hline $219 \mathrm{HO} 13 \mathrm{AL} 3$ & 7 \\
\hline $219 \mathrm{HO} 12 \mathrm{Al} 3$ & 7 \\
\hline $2194011 \mathrm{~A} 13$ & 7 \\
\hline $219 \mathrm{HOLOA13}$ & 7 \\
\hline $220 \mathrm{HOOO9A13}$ & 7 \\
\hline $220 H 00$ BA13 & 7 \\
\hline $220 \mathrm{HOO} 7 \mathrm{Al} 3$ & $\rightarrow$ \\
\hline $220 \mathrm{HOOGA13}$ & 7 \\
\hline $2204005 A 13$ & 7 \\
\hline $220 H 004 A 13$ & 7 \\
\hline $221 \mathrm{H} 003 \mathrm{~A} 13$ & 7 \\
\hline $221 H 002 \mathrm{A13}$ & 7 \\
\hline & 7 \\
\hline $211 H 044 A 14$ & 9 \\
\hline $211 H 043 A 14$ & 9 \\
\hline $211 \mathrm{HO42A14}$ & 9 \\
\hline $2134035 A 14$ & 9 \\
\hline $213 \mathrm{H} 036 \mathrm{Al} 4$ & 9 \\
\hline $213 \mathrm{HO} 7 \mathrm{ALA}$ & 9 \\
\hline 213 H032A14 & 9 \\
\hline $213 \mathrm{H} 033 \mathrm{Al} 4$ & 9 \\
\hline $213 \mathrm{H} 034 \mathrm{AL}$ & 9 \\
\hline $323 \mathrm{H} 185 \mathrm{SA} 14$ & 9 \\
\hline $323 \mathrm{H} 186 \mathrm{Al4}$ & 9 \\
\hline $323 \mathrm{H1B7A21}$ & 9 \\
\hline $322 \mathrm{H1B8A14}$ & 9 \\
\hline $322 \mathrm{H} 189 \mathrm{~A} 14$ & 9 \\
\hline $322 \mathrm{H} 190 \mathrm{AL}$ & 9 \\
\hline 3216174414 & 9 \\
\hline $321 \mathrm{H} 175 \mathrm{Al} 4$ & 9 \\
\hline $320 H 176214$ & 9 \\
\hline $320 \mathrm{H} 177 \mathrm{A14}$ & 9 \\
\hline $320 H_{278 A 14}$ & 9 \\
\hline $\begin{array}{l}2144031 \mathrm{Al} 4 \\
214 \mathrm{H} 030 \mathrm{~A}\end{array}$ & 9 \\
\hline 214 OO30A14 & ? \\
\hline $214 H 029 A 14$ & $?$ \\
\hline $214 \mathrm{HO} 2 \mathrm{BA} 14$ & 9 \\
\hline $225 \mathrm{H} 027 \mathrm{A14}$ & 9 \\
\hline $21513026 \mathrm{Al}$ & 9 \\
\hline $215 \% 025 A 14$ & 9 \\
\hline $215 \mathrm{H} 024 \mathrm{Al}$ & 9 \\
\hline $215 H_{023 A 14}$ & ; \\
\hline $215 H 022 A 14$ & 9 \\
\hline $216 \mathrm{HO}_{2} \mathrm{LAL} 4$ & 9 \\
\hline $216 \mathrm{HO20A14}$ & 9 \\
\hline $216 H 019 A 14$ & 9 \\
\hline $216 \mathrm{HOLAA14}$ & 9 \\
\hline 217 HO17A14 & 9 \\
\hline $217 \mathrm{HO} 6 \mathrm{AA14}$ & 9 \\
\hline $2194015 A 14$ & 9 \\
\hline $219 H 014 A 14$ & 9 \\
\hline $218 H O 13 A 14$ & 9 \\
\hline $219 \mathrm{HO} 12 \mathrm{Al} 4$ & 9 \\
\hline 219 HO1LA14 & 9 \\
\hline $219 \mathrm{HO} 20 \mathrm{A14}$ & 9 \\
\hline
\end{tabular}

Figure 9.13 - Case 2, Baseline Schedule (Continued). 


\begin{tabular}{|c|c|}
\hline $\begin{array}{l}\text { ACTIVITY } \\
\text { ID }\end{array}$ & $\begin{array}{l}\text { ORIO } \\
\text { DUR }\end{array}$ \\
\hline 220 OMOOALI4 & 9 \\
\hline $220 \mathrm{HOOSA14}$ & 9 \\
\hline $220 \mathrm{H} 007 \mathrm{~A} 14$ & 9 \\
\hline 220 H006A14 & 9 \\
\hline $220 \mathrm{H005A14}$ & 9 \\
\hline $220 \mathrm{H} 004 \mathrm{A14}$ & 9 \\
\hline $221 \% 003 \mathrm{ALA}$ & 9 \\
\hline $221 H 002 \mathrm{Al4}$ & 9 \\
\hline $221 \mathrm{H} 001 \mathrm{~A} 14$ & 9 \\
\hline $2114044 A 15$ & 12 \\
\hline $2114043 A 15$ & 12 \\
\hline 211 HO42A15 & 12 \\
\hline 213 4035A15 & 12 \\
\hline 213 H036A15 & 12 \\
\hline 213 H037A15 & 12 \\
\hline $213 \mathrm{H} 032 \mathrm{A15}$ & 12 \\
\hline $213 \mathrm{BO} 3 \mathrm{~A} \mathrm{AlS}$ & 12 \\
\hline $213 B 034 A 15$ & 12 \\
\hline $323 \mathrm{~B} 185 \mathrm{~A} 15$ & 12 \\
\hline $323 \mathrm{H} 186 \mathrm{Al} 5$ & 12 \\
\hline $323 K 187 A 15$ & 12 \\
\hline $322 \mathrm{H} 18 \mathrm{BA} 5$ & 12 \\
\hline $322 \mathrm{~B} 289 A 15$ & 12 \\
\hline $322 \mathrm{H190A15}$ & 12 \\
\hline $321 H 174 A 15$ & 12 \\
\hline $321 K 175 A 15$ & 12 \\
\hline $320 \mathrm{H276A1S}$ & 12 \\
\hline $320 \mathrm{H177A1S}$ & 12 \\
\hline $320 H 178 A 15$ & 12 \\
\hline $214 \% 031 \mathrm{A15}$ & 12 \\
\hline $214 \mathrm{H} 030 \mathrm{~A} 15$ & 12 \\
\hline $214 \mathrm{H} 029 \mathrm{~A} 25$ & 12 \\
\hline $214 \mathrm{H} 02 \mathrm{gALS}$ & 12 \\
\hline 215 H027A1S & 12 \\
\hline $215 \mathrm{HO26A25}$ & 12 \\
\hline $215 H 025 A 15$ & 12 \\
\hline $215 \mathrm{HO24A15}$ & 12 \\
\hline $215 \mathrm{H} 023 \mathrm{~A} 15$ & 12 \\
\hline $215 \mathrm{H} 022 \mathrm{~A} 15$ & 12 \\
\hline $216 \mathrm{H} 021 \mathrm{~A} 15$ & 12 \\
\hline $216 \mathrm{H} 020 \mathrm{~A} 15$ & 12 \\
\hline $216 \mathrm{HO} 19 \mathrm{A15}$ & 12 \\
\hline $216 \mathrm{H} 018 \mathrm{~A} 15$ & 12 \\
\hline $217 \mathrm{H} 017 \mathrm{ALS}$ & 12 \\
\hline $227 \mathrm{HO} 6 \mathrm{AA15}$ & 12 \\
\hline $218 H 015 A 15$ & 12 \\
\hline $218 H 014 A 15$ & 12 \\
\hline $228 \mathrm{HO} 13 \mathrm{~A} 15$ & 12 \\
\hline $219 \mathrm{KO} 012 \mathrm{~A} 15$ & 12 \\
\hline $219 \mathrm{HO} 11 \mathrm{~A} 15$ & 12 \\
\hline 219 HOLOA15 & 12 \\
\hline $220 \mathrm{H} 009 \mathrm{~A} 15$ & 12 \\
\hline $220 \mathrm{OH} 008 \mathrm{AL} 5$ & 12 \\
\hline $220 \mathrm{HOO} 7 \mathrm{A15}$ & 12 \\
\hline $220 \mathrm{HOOGA15}$ & 12 \\
\hline $220 \mathrm{HOO5A15}$ & 12 \\
\hline 220 HOO4A15 & 12 \\
\hline 22 IHO03A15 & 12 \\
\hline $222 \mathrm{HOO} 2 \mathrm{A1S}$ & 12 \\
\hline $221 \mathrm{HOO} 1 \mathrm{A15}$ & 12 \\
\hline $211 H 044 A 16$ & 9 \\
\hline $211 \mathrm{HO4} 3 \mathrm{Al6}$ & 9 \\
\hline $211 \mathrm{HO42A16}$ & 9 \\
\hline $233 \mathrm{~K} 035 \mathrm{~A} 16$ & 9 \\
\hline $213 \% 036 A 16$ & 9 \\
\hline $213 H 037 A 16$ & 9 \\
\hline $2134032 \mathrm{~A} 16$ & 9 \\
\hline $213 \mathrm{~K}_{03} 3 \mathrm{A2} 6$ & 9 \\
\hline $2134034 A 16$ & 9 \\
\hline 323 H195A16 & 9 \\
\hline $323 \mathrm{H1O6 \textrm {ALC }}$ & 9 \\
\hline $323 \mathrm{~K} 187 \mathrm{~A} 16$ & 9 \\
\hline $322 \mathrm{HIaBA16}$ & 9 \\
\hline $322 \mathrm{H} 199 \mathrm{~A} 26$ & 9 \\
\hline $322 \mathrm{~K} 190 \mathrm{~A} 16$ & 9 \\
\hline $321 \% 174 \mathrm{AL} 6$ & 9 \\
\hline $321 K 175 \mathrm{~A}] 6$ & 2 \\
\hline $320 \mathrm{H} 176 \mathrm{~A} 16$ & 9 \\
\hline $320 \mathrm{H} 177 \mathrm{~A} 16$ & 9 \\
\hline $320 \mathrm{H178A16}$ & 9 \\
\hline $214 H 031 \mathrm{L16}$ & 9 \\
\hline $214 H_{030 A 16}$ & 9 \\
\hline $214 H 029 A 16$ & 9 \\
\hline $214 K 028 A 16$ & 9 \\
\hline 215 H027A16 & 9 \\
\hline $215 \mathrm{H} 026 \mathrm{~A} 16$ & 9 \\
\hline $215 \mathrm{H} 025 \mathrm{~A} 16$ & 9 \\
\hline $215 \mathrm{H} 024 \mathrm{~A} .16$ & 9 \\
\hline $215 \mathrm{H} 023 \mathrm{~A} 16$ & 9 \\
\hline $215 H 022 A 16$ & 9 \\
\hline $216 \mathrm{HO} 21 \mathrm{~A} 16$ & 9 \\
\hline $216 \mathrm{HO2OA16}$ & 9 \\
\hline 216HO19A16 & 9 \\
\hline $216 \% 010 A 16$ & 9 \\
\hline $217 \mathrm{H} 017 \mathrm{~A} 16$ & 9 \\
\hline $217 \mathrm{HO16A16}$ & 9 \\
\hline
\end{tabular}

Figure 9.13 - Case 2, Baseline Schedule (Continued). 


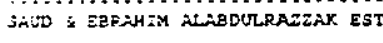

PRIMAVERA PROTECT PLWWNER

PEPOPT IATE I1SEP95 RUN NO. 121

SEVEN SEAS, CONSTRUCTION OF 52 HOUSES
JAHRA 3 HOUSING PROJECT COMMON aCt

START DATE 1APRB? FIN DATE 13AUG88 DATA DATE LAPRE? PAGE NO.

\begin{tabular}{|c|c|}
\hline $\begin{array}{c}\text { ActIVITY } \\
\text { ID }\end{array}$ & $\begin{array}{l}\text { ORIG } \\
\text { DUR }\end{array}$ \\
\hline 218HO15A16 & \\
\hline $219 H 014 \mathrm{Al} 6$ & 9 \\
\hline $219 H 013 \mathrm{AL6}$ & 9 \\
\hline $219 \mathrm{HO12A16}$ & 9 \\
\hline $2194011 \mathrm{~A} 16$ & 9 \\
\hline 219 HO1OAL5 & 9 \\
\hline $220 \mathrm{H009A16}$ & 9 \\
\hline $220 \mathrm{H} 008 \mathrm{A16}$ & 9 \\
\hline $2209007 A 26$ & 9 \\
\hline $220 \mathrm{OH} 006 \mathrm{Al} 6$ & 9 \\
\hline $2204005 A 16$ & 9 \\
\hline $220 \mathrm{HOO4A36}$ & 9 \\
\hline $221 \mathrm{H} 003 \mathrm{~A} 16$ & 9 \\
\hline $221+9002 A 16$ & 9 \\
\hline $221 \mathrm{HOOLA16}$ & 9 \\
\hline $2116044 A 17$ & 7 \\
\hline $211 \mathrm{H} 043 \mathrm{~A} 17$ & 7 \\
\hline 211H042A17 & 7 \\
\hline $213 \mathrm{H} 035 \mathrm{~A} 17$ & 7 \\
\hline $213 \mathrm{H} 036 \mathrm{AL} 7$ & 7 \\
\hline $213 B 037 A 17$ & 7 \\
\hline $213 \mathrm{H} 032 \mathrm{AL} 7$ & 7 \\
\hline 213H033A17 & 7 \\
\hline $213 H 034 A 17$ & 7 \\
\hline $323 \mathrm{H} 185 \mathrm{~A} 27$ & 7 \\
\hline 323H286A17 & 7 \\
\hline $323 \mathrm{H} 1 \mathrm{E} 7 \mathrm{~A} 27$ & 7 \\
\hline $322 \mathrm{H189A17}$ & 7 \\
\hline $322 \mathrm{HLB9A27}$ & 7 \\
\hline $322 \mathrm{H} 190 \mathrm{~A} 27$ & $?$ \\
\hline $321 H 174 A 17$ & 7 \\
\hline $3211175 A 17$ & 7 \\
\hline $320 \mathrm{H} 276 \mathrm{AL} 7$ & 7 \\
\hline $320 \mathrm{H} 177 \mathrm{AL} 7$ & 7 \\
\hline $320 \mathrm{H} 178 \mathrm{~A} 17$ & 7 \\
\hline $214 \mathrm{HO} 2 \mathrm{AA17}$ & 7 \\
\hline $2143030 A 17$ & 7 \\
\hline 214H029A17 & 7 \\
\hline $214 \mathrm{HO} 28 \mathrm{A17}$ & 7 \\
\hline $215 \mathrm{HO} 27 \mathrm{~A} 17$ & 7 \\
\hline $215 \mathrm{KO} 26 \mathrm{Al} 7$ & 7 \\
\hline 215 HO25A17 & 7 \\
\hline $215 H 024 A 17$ & 7 \\
\hline $215 \mathrm{HO23A17}$ & 7 \\
\hline $225 \mathrm{H} 022 \mathrm{~A} 17$ & 7 \\
\hline $216 \mathrm{HO21A17}$ & 7 \\
\hline $216 \mathrm{HO20A17}$ & 7 \\
\hline $216 \mathrm{HO} 19 \mathrm{~A} 17$ & 7 \\
\hline $216 \mathrm{HO1BA17}$ & 7 \\
\hline $217 \mathrm{H} 017 \mathrm{AL} 7$ & 7 \\
\hline $217 \mathrm{HO} 6 \mathrm{GA17}$ & 7 \\
\hline $218 H 015 A 17$ & 7 \\
\hline 218 HO14A17 & 7 \\
\hline $21 \mathrm{BHO} 33 \mathrm{Al} 7$ & 7 \\
\hline 219 H012A17 & 7 \\
\hline $219 H 011 A 17$ & 7 \\
\hline $219 \mathrm{HO} 10 \mathrm{Al7}$ & 7 \\
\hline $220 \mathrm{HOO9A27}$ & 7 \\
\hline $220 \mathrm{OHOOBA17}$ & 7 \\
\hline $220 \mathrm{H} 007 \mathrm{A17}$ & 7 \\
\hline $220 \mathrm{HO06A17}$ & 7 \\
\hline $220 \mathrm{H} 005 \mathrm{Al} 7$ & 7 \\
\hline 220 HOO4A.17 & 7 \\
\hline $221 \mathrm{H} 003 \mathrm{Al} 7$ & 7 \\
\hline $221 \mathrm{H} 002 \mathrm{Al} 7$ & 3 \\
\hline $22111001 \mathrm{A17}$ & 7 \\
\hline $211 \% 044 A 18$ & 3 \\
\hline $211 H 043 A 18$ & 3 \\
\hline 211K042A18 & 3 \\
\hline $213 \mathrm{H} 035 \mathrm{~A} 18$ & 3 \\
\hline $213 \mathrm{H} 036 \mathrm{A18}$ & 3 \\
\hline $213 K 037 \mathrm{Al} \theta$ & 3 \\
\hline $213 \mathrm{HO}_{3} 2 \mathrm{~A} 18$ & 3 \\
\hline $213 \mathrm{H} 033 \mathrm{~A} 18$ & 3 \\
\hline 213 H034AI8 & 3 \\
\hline $323 \mathrm{HLESAl8}$ & 3 \\
\hline $323 \mathrm{H} 186 \mathrm{AL} 2$ & 3 \\
\hline 323 H107A18 & 3 \\
\hline $322 \mathrm{H198AI \theta}$ & 3 \\
\hline $322 \mathrm{H} 189 \mathrm{A1} 8$ & 3 \\
\hline $322 \mathrm{H190A18}$ & 3 \\
\hline $321 \mathrm{H174A28}$ & 3 \\
\hline $321 \mathrm{H} 175 \mathrm{~A} 18$ & 3 \\
\hline $320 \mathrm{H} 176 \mathrm{A2} 8$ & 3 \\
\hline $320 \mathrm{H} 177 \mathrm{ALO}$ & 3 \\
\hline $320 \mathrm{HL78A1 \textrm {B }}$ & 3 \\
\hline $214 \mathrm{H} 031 \mathrm{ALO} \theta$ & 3 \\
\hline $214 \mathrm{H} 030 \mathrm{~A} 18$ & 3 \\
\hline 214H029A19 & 3 \\
\hline $2144028 \mathrm{Al} \theta$ & 3 \\
\hline $215 \mathrm{HO27A18}$ & 3 \\
\hline $215 \mathrm{H} 026 \mathrm{~A} 18$ & 3 \\
\hline $215 \mathrm{HO2SA}^{2}$ & 3 \\
\hline $215 \mathrm{H} 024 \mathrm{~A} 18$ & 3 \\
\hline $215 \mathrm{H} 023 \mathrm{ALO}$ & 3 \\
\hline & \\
\hline
\end{tabular}

Figure 9.13 - Case 2, Baseline Schedule (Continued). 


\begin{tabular}{|c|c|}
\hline $\begin{array}{c}\text { ACrIVITY } \\
\text { :D }\end{array}$ & $\begin{array}{l}\text { ORIG } \\
\text { DUR } \\
\cdots\end{array}$ \\
\hline $2164021 A 18$ & 3 \\
\hline $216 H 020 A 28$ & 3 \\
\hline $216 \mathrm{H} 019 \mathrm{~A} 1 \mathrm{~B}$ & 3 \\
\hline $\begin{array}{l}216 \mathrm{B018A1 \theta} \\
217 \mathrm{H027A18}\end{array}$ & 3 \\
\hline $\begin{array}{l}217 \mathrm{H} 027 \mathrm{A18} \\
217 \mathrm{HO} 6 \mathrm{AlB}\end{array}$ & 3 \\
\hline $\begin{array}{l}217 \mathrm{HO16A18} \\
218 \mathrm{HO} 15 \mathrm{AlB}\end{array}$ & 3 \\
\hline $\begin{array}{l}218 \mathrm{H} 015 \mathrm{Al} 8 \\
218 \mathrm{H} 014 \mathrm{Al} 18\end{array}$ & 3 \\
\hline $\begin{array}{l}218 H 014 A 18 \\
2184013 A 18\end{array}$ & 3 \\
\hline $\begin{array}{l}2184013 \mathrm{~A} 18 \\
219 \mathrm{H} 012 \mathrm{A1g}\end{array}$ & 3 \\
\hline 219H012A19 & 3 \\
\hline $219 \mathrm{HO11A28}$ & 3 \\
\hline $\begin{array}{l}219 H 010 \mathrm{A18} \\
220 \mathrm{O} 009 \mathrm{A2B}\end{array}$ & 3 \\
\hline $\begin{array}{l}220 \mathrm{HOO9A28} \\
220 \mathrm{HOOBA18}\end{array}$ & 3 \\
\hline $\begin{array}{l}220 \mathrm{HOO} 8 \mathrm{~A} 18 \\
220 \mathrm{H} 007 \mathrm{~A}\end{array}$ & 3 \\
\hline $220 \mathrm{H} 007 \mathrm{Al}$ & 3 \\
\hline $220 \mathrm{H006R18}$ & 3 \\
\hline $220 \mathrm{HOOSAL} 8$ & 3 \\
\hline $220 \mathrm{H} 004 \mathrm{~A} 1 \mathrm{~B}$ & 3 \\
\hline $221 \mathrm{H} 003 \mathrm{AlB}$ & 3 \\
\hline $221 \mathrm{HOO} 2 \mathrm{~A} 18$ & 3 \\
\hline $221 \mathrm{~B} 001 \mathrm{~A} 18$ & 3 \\
\hline 211 H044A19 & 5 \\
\hline $2118043 A 19$ & 5 \\
\hline $2114042 \mathrm{~A} 19$ & 5 \\
\hline 2139035A19 & 5 \\
\hline $213 \mathrm{HO} 36 \mathrm{AL}$ & 5 \\
\hline $213 \mathrm{H} 037 \mathrm{~A} 19$ & 5 \\
\hline $213 \mathrm{H} 032 \mathrm{Alg}$ & 5 \\
\hline $213 \mathrm{H} 033 \mathrm{~A} 19$ & 5 \\
\hline $213 \mathrm{HO34A19}$ & 5 \\
\hline 3233185A19 & 5 \\
\hline $323 \mathrm{H186A19}$ & 5 \\
\hline 323 H187A19 & 5 \\
\hline $322 \mathrm{H} 188 \mathrm{Al} 9$ & 5 \\
\hline $322 \mathrm{H} 189 \mathrm{Al} 9$ & 5 \\
\hline $322 \mathrm{H} 190 \mathrm{~A} 19$ & 5 \\
\hline $321 H 174 A 19$ & 5 \\
\hline $321 \mathrm{H175A19}$ & 5 \\
\hline $320 \mathrm{HI76A19}$ & 5 \\
\hline $3201177 \mathrm{~A} 19$ & 5 \\
\hline $320 \mathrm{H} 17 \mathrm{BAl} 9$ & 5 \\
\hline $214 \mathrm{HO} 31 \mathrm{Alg}$ & 5 \\
\hline 214 H030A19 & 5 \\
\hline 214H029A19 & $\mathbf{5}$ \\
\hline 214H028A29 & 5 \\
\hline 215H027A19 & 5 \\
\hline $215 H 026 \mathrm{AL} 9$ & 5 \\
\hline $215 \mathrm{HO25A19}$ & 5 \\
\hline $215 \% 024 A 29$ & 5 \\
\hline $215 \mathrm{HO}^{23 \mathrm{Al}}$ & 5 \\
\hline 215H022A19 & 5 \\
\hline $216 \mathrm{H} 02 \mathrm{AA19}$ & 5 \\
\hline $2163020 \mathrm{~A} 19$ & 5 \\
\hline 216H019A19 & 5 \\
\hline $2164010 \mathrm{A19}$ & 5 \\
\hline $2174017 A 19$ & 5 \\
\hline 217 1616A19 & 5 \\
\hline 218 HO15A19 & 5 \\
\hline 218H014A19 & 5 \\
\hline 219H013A29 & 5 \\
\hline $219 \mathrm{HO} 2 \mathrm{AL} 9$ & 5 \\
\hline 219HO1LA19 & 5 \\
\hline 219H010A19 & 5 \\
\hline $220 \mathrm{HOOSA19}$ & 5 \\
\hline $220 \mathrm{HOOBAI} 9$ & 5 \\
\hline $220 H 007 \mathrm{Al} 9$ & 5 \\
\hline $220 \mathrm{HOOGA19}$ & 5 \\
\hline $220 \mathrm{H} 005 \mathrm{Al} 9$ & 5 \\
\hline $220 H 004 A 19$ & 5 \\
\hline 221Н003A19 & 5 \\
\hline 221HOO2A19 & 5 \\
\hline $221 \mathrm{HOO}$ ANIS & $\mathbf{5}$ \\
\hline $211 \mathrm{HO44A2O}$ & 21 \\
\hline $21 \mathrm{H} 043 \mathrm{~A} 2 \mathrm{O}$ & 21 \\
\hline $211 \mathrm{HO42A20}$ & 21 \\
\hline $213 \mathrm{H} 035 \mathrm{A2} 0$ & 21 \\
\hline $213 \% 036 A 20$ & 21 \\
\hline $213 \mathrm{H}_{03} 7 \mathrm{A20}$ & 21 \\
\hline $213 H 032 A 20$ & 21 \\
\hline $213 \mathrm{H} 033 \mathrm{~A} 2 \mathrm{O}$ & 21 \\
\hline $213 \mathrm{H} 034 \mathrm{~A} 2 \mathrm{O}$ & 21 \\
\hline $323 \mathrm{H} 185 \mathrm{~A} 2 \mathrm{O}$ & 21 \\
\hline $323 \mathrm{H} 186 \mathrm{~A} 20$ & 21 \\
\hline $323 \mathrm{H197A2O}$ & 21 \\
\hline $322 K 188 A 20$ & 21 \\
\hline $322 \mathrm{H} 189 \mathrm{A2O}$ & 21 \\
\hline $322 \mathrm{H} 190 \mathrm{~A} 20$ & 21 \\
\hline $321 \mathrm{HL} 74 \mathrm{~A} 2 \mathrm{O}$ & 21 \\
\hline $321 \mathrm{H} 175 \mathrm{~A} 2 \mathrm{O}$ & 21 \\
\hline $320 \mathrm{HL} 76200$ & 21 \\
\hline $320 \mathrm{H} 177 \mathrm{AO} O$ & 21 \\
\hline $320 \mathrm{H17} 7 \mathrm{BA2O}$ & 21 \\
\hline $214 \mathrm{HO} 31 \mathrm{A2O}$ & 21 \\
\hline $214 \mathrm{HOOSOA2O}$ & 21 \\
\hline $214 \mathrm{HO29A2O}$ & 21 \\
\hline $214 H 028 A 20$ & 21 \\
\hline
\end{tabular}

Figure 9.13 - Case 2, Baseline Schedule (Continued). 
zATD \& EBRAHTS ALABDLLRAJZAK EST.

PEPORT OATE $215 E P 95$ RUN NO 121

gasel zne Schedule by T/Pical Activity \& ES
PRIMAVERA PROTECT PLANNER

SEVEN SEAS, CONSTRUCTION OF 51 HOUSES
JAKPA 3 HOUSING PROTECT COMmON ACE

START DATE LAPRa? FIN DATE 13AUOB8

DATA DATE LAPR87 PAGE NO.

\begin{tabular}{|c|c|c|c|c|}
\hline $\begin{array}{c}\text { ACTIVITV } \\
\text { ID }\end{array}$ & $\begin{array}{l}\text { ORIG } \\
\text { DUR } \\
\cdots . .\end{array}$ & $\begin{array}{l}\text { REM } \\
\text { DUR } \\
\cdots \cdots\end{array}$ & $\ldots$ & $\begin{array}{c}\text { CODE } \\
\end{array}$ \\
\hline $215 \mathrm{HO} 27 \mathrm{~A} 20$ & 21 & 21 & 0 & 25 \\
\hline$=15 H 026 A 20$ & 21 & 21 & 0 & 26 \\
\hline $215 \mathrm{H} 025 \mathrm{~A} 20$ & 21 & 21 & 0 & 27 \\
\hline $215 \mathrm{H} 024 \mathrm{~A} 20$ & 21 & 22 & 0 & 28 \\
\hline $215 \mathrm{HO2} 3 \mathrm{~A} 20$ & 21 & 21 & 0 & 29 \\
\hline $215 H 022 A 2 O$ & 21 & 21 & 0 & 30 \\
\hline $216 \mathrm{HO21A2O}$ & 21 & 21 & $\therefore$ & 31 \\
\hline $\begin{array}{l}216 \mathrm{HO} 20 \mathrm{~A} 20 \\
216 \mathrm{HO} 19 \mathrm{A2}\end{array}$ & 22 & 21 & 0 & 32 \\
\hline $\begin{array}{l}216 \mathrm{HO} 19 \mathrm{A2} 0 \\
216 \mathrm{HO} 19 \mathrm{~A} 2 \mathrm{O}\end{array}$ & 21 & 21 & 0 & $\begin{array}{l}33 \\
34\end{array}$ \\
\hline $216 \mathrm{HO} 18 \mathrm{A2O}$ & 21 & 21 & 0 & $\begin{array}{l}34 \\
35\end{array}$ \\
\hline $217 \mathrm{HO} 17 \mathrm{A2O}$ & 21 & 21 & $\therefore$ & $\begin{array}{l}35 \\
35\end{array}$ \\
\hline $\begin{array}{l}217 \mathrm{HO16A20} \\
218 \mathrm{BO} 015 \mathrm{~A} 20\end{array}$ & $\begin{array}{l}21 \\
21\end{array}$ & $\begin{array}{l}21 \\
21\end{array}$ & $\begin{array}{l}0 \\
0\end{array}$ & $\begin{array}{l}36 \\
37\end{array}$ \\
\hline $218 K 014 A 20$ & 21 & 21 & 0 & 38 \\
\hline $218 \mathrm{HO} 13220$ & 21 & 21 & 0 & 39 \\
\hline $219 \mathrm{HO} 12 \mathrm{A2} O$ & 21 & 21 & : & 40 \\
\hline $219 \mathrm{HO} 11 \mathrm{A20}$ & 21 & 21 & 0 & 11 \\
\hline $219 \mathrm{HO} 10 \mathrm{A2O}$ & 21 & 21 & 0 & $\begin{array}{l}12 \\
43\end{array}$ \\
\hline $\begin{array}{l}220 \mathrm{HOOOA20} \\
220 \mathrm{HOOBA20}\end{array}$ & $\begin{array}{l}21 \\
21\end{array}$ & $\begin{array}{l}21 \\
21\end{array}$ & 0 & $\begin{array}{l}43 \\
44\end{array}$ \\
\hline $220 \mathrm{OHOO} 7 \mathrm{A2O}$ & 21 & 21 & 0 & 45 \\
\hline $220 \mathrm{OHOO6A2O}$ & 21 & 21 & 0 & 46 \\
\hline $220 \mathrm{HOOSA} 20$ & 21 & 21 & 0 & 47 \\
\hline $220 \mathrm{HOO} 4 \mathrm{A2} O$ & 21 & 21 & 0 & 48 \\
\hline $2218003 A 20$ & 21 & 21 & 0 & 49 \\
\hline $221 \mathrm{HOOZA20}$ & 21 & 21 & $0^{0}$ & 50 \\
\hline $\begin{array}{l}221 \mathrm{HOO} 1 \mathrm{A2} \\
211 \mathrm{HO44A21}\end{array}$ & $\begin{array}{r}21 \\
5\end{array}$ & 215 & $\begin{array}{l}0 \\
0\end{array}$ & $\begin{array}{l}51 \\
01\end{array}$ \\
\hline $21210043 A 21$ & 3 & 5 & 0 & 02 \\
\hline $211 \mathrm{HO42A21}$ & 5 & 5 & 0 & 03 \\
\hline $213 \mathrm{HO} 35 \mathrm{~A} 21$ & 5 & 5 & 0 & 04 \\
\hline $213 \mathrm{H} 036 \mathrm{A21}$ & 5 & 5 & 0 & 05 \\
\hline 2134037221 & 5 & 5 & 0 & 06 \\
\hline $213 \mathrm{H} 032 \mathrm{A21}$ & 5 & s & 0 & 07 \\
\hline $213 \mathrm{HO33A21}$ & 5 & $\mathbf{5}$ & 0 & $O B$ \\
\hline $213 H 034 A 21$ & $\mathbf{s}$ & 5 & 0 & 09 \\
\hline $323 \mathrm{HIOSA21}$ & $\mathbf{s}$ & 5 & $\therefore$ & 10 \\
\hline $323 \mathrm{H} 186 \mathrm{A2} 1$ & 5 & 5 & 0 & 11 \\
\hline $323 \mathrm{H} 187 \mathrm{~B} 2 \mathrm{I}$ & 5 & 5 & 0 & 22 \\
\hline $322 H 180221$ & 5 & $\mathbf{5}$ & 0 & 13 \\
\hline $322 \mathrm{H} 189 \mathrm{A2} 1$ & 5 & 5 & 0 & 14 \\
\hline $322 \mathrm{H} 190 \mathrm{A2} 2$ & 5 & 5 & 0 & 15 \\
\hline $321 H 174 A 21$ & 5 & 5 & $\therefore$ & 16 \\
\hline $\begin{array}{l}3214175 A 21 \\
320 H 176 A 21\end{array}$ & 5 & 3 & : & 17 \\
\hline $\begin{array}{l}320 \mathrm{H} 176 \mathrm{A2} 1 \\
320 \mathrm{H} 177 \mathrm{A2} 1\end{array}$ & 5 & 5 & 0 & 18 \\
\hline $\begin{array}{l}320 \mathrm{H} 177 \mathrm{7A2} 1 \\
320 \mathrm{H} 178 \mathrm{AA2} 2\end{array}$ & 5 & 5 & 0 & 19 \\
\hline $320 \mathrm{H} 178 \mathrm{~A} 22$ & 5 & s & 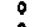 & 20 \\
\hline $214 \mathrm{H} 031 \mathrm{A2} 2$ & $\mathbf{s}$ & 5 & 0 & 21 \\
\hline 2144030821 & $\mathbf{5}$ & 5 & 0 & 22 \\
\hline 2144029421 & 5 & $\mathbf{s}$ & 0 & 23 \\
\hline $214 \mathrm{HO} 28 \mathrm{A2} 1$ & s & 5 & 0 & 24 \\
\hline $2154027 \mathrm{A21}$ & $\mathbf{s}$ & $\mathbf{s}$ & 0 & 25 \\
\hline $215 \mathrm{H} 026 \mathrm{A2} 1$ & 5 & $\mathbf{5}$ & 0 & 26 \\
\hline $215 H 025 A 21$ & 5 & 5 & 0 & 27 \\
\hline $215 H 024 A 21$ & 5 & 5 & 0 & 28 \\
\hline $215 \mathrm{H} 023 \mathrm{A2} 1$ & $\mathbf{5}$ & 5 & 0 & 29 \\
\hline $2154022 A 21$ & $\mathbf{s}$ & 5 & : & 30 \\
\hline $216 \mathrm{H} 021 \mathrm{~A} 21$ & $\mathrm{~s}$ & 5 & 0 & 31 \\
\hline $216 \mathrm{HO20A21}$ & $\mathbf{s}$ & 5 & 0 & 32 \\
\hline $216 \mathrm{HO} 39 \mathrm{~A} 21$ & $\mathbf{5}$ & 5 & 0 & 33 \\
\hline $216 \mathrm{HO218A21}$ & 5 & 5 & 0 & 34 \\
\hline $2174027 \mathrm{A2} 1$ & 5 & 5 & 0 & 35 \\
\hline $217 \mathrm{H} 016 \mathrm{A21}$ & 5 & 5 & 0 & 36 \\
\hline $2104015 A 21$ & 5 & 5 & 0 & 37 \\
\hline $218 K 014 A 21$ & 5 & 5 & 0 & 38 \\
\hline $218 \mathrm{HO} 13 \mathrm{A21}$ & 5 & 5 & 0 & 39 \\
\hline $219 \mathrm{HO} 22 \mathrm{A2} 1$ & 5 & 5 & 0 & 40 \\
\hline $219 \mathrm{H} 011 \mathrm{A2} 1$ & 5 & 5 & 0 & $\$ 1$ \\
\hline $219 \mathrm{HO} 10 \mathrm{A2} 1$ & 5 & 5 & 0 & 42 \\
\hline $220 \mathrm{HOOON221}$ & 5 & $\mathbf{s}$ & 0 & 43 \\
\hline 2201000821 & 5 & 5 & 0 & 44 \\
\hline $220 \mathrm{H} 007 \mathrm{A2} 1$ & 5 & 5 & 0 & 45 \\
\hline $220 \mathrm{H} 006 \mathrm{A2} 1$ & 5 & 5 & 0 & 46 \\
\hline $220 \mathrm{HOOSA21}$ & 5 & 5 & 0 & 47 \\
\hline $220 \mathrm{HOO4A2I}$ & $s$ & 5 & 0 & 48 \\
\hline $221 \mathrm{H} 003 \mathrm{A2} 1$ & 5 & 5 & 0 & 49 \\
\hline $221 \mathrm{HOO02 \textrm {A } 2 1}$ & 5 & 5 & 0 & 50 \\
\hline $221 \mathrm{HOOLA22}$ & 5 & 5 & 0 & 51 \\
\hline $212 \mathrm{HO} 04 \mathrm{A22}$ & 3 & 3 & 0 & 01 \\
\hline $211 \mathrm{HO} 43 \mathrm{AZ2}$ & 3 & 3 & 0 & 02 \\
\hline $2118042 A 22$ & 3 & 3 & 0 & 03 \\
\hline $213 \mathrm{H} 035 \mathrm{A22}$ & 3 & 3 & 0 & 04 \\
\hline $213 \mathrm{H} 036 \mathrm{~A} 22$ & 3 & 3 & 0 & 05 \\
\hline $213 \mathrm{H} 037 \mathrm{A22}$ & 3 & 3 & 0 & 06 \\
\hline $213 \mathrm{HO} 32 \mathrm{A2Z}$ & 3 & 3 & 0 & 07 \\
\hline $213 \mathrm{HO} 33 \mathrm{A22}$ & 3 & 3 & 0 & $O B$ \\
\hline $213 \mathrm{H} 034 \mathrm{A2Z}$ & 3 & 3 & 0 & 09 \\
\hline $323 \mathrm{H} 185 \mathrm{~A} 22$ & 3 & 3 & 0 & 10 \\
\hline $323 \mathrm{H} 186 \mathrm{~A} 22$ & 3 & 3 & 0 & 11 \\
\hline $323 \mathrm{H} 187 \mathrm{A22}$ & 3 & 3 & 0 & 12 \\
\hline $322 \mathrm{H} 198 \mathrm{A2} 2$ & 3 & 3 & 0 & 13 \\
\hline $322 \mathrm{H} 189 \mathrm{~A} 22$ & 3 & 3 & 0 & 14 \\
\hline $322 \mathrm{HL} 90 \mathrm{A2} 2$ & 3 & 3 & 0 & 15 \\
\hline $321 \mathrm{H} 174 \mathrm{A22}$ & 3 & 3 & 0 & 16 \\
\hline $32 \mathrm{LH} 175 \mathrm{A22}$ & 3 & 3 & 0 & 17 \\
\hline $32 \mathrm{OH} 176 \mathrm{A2} 2$ & 3 & 3 & 0 & 18 \\
\hline
\end{tabular}

Figure 9.13 - Case 2, Baseline Schedule (Continued). 


\begin{tabular}{|c|c|}
\hline $\begin{array}{c}\text { ACTIVITY } \\
\text { ID }\end{array}$ & $\begin{array}{l}\text { ORIG } \\
\text { OUR }\end{array}$ \\
\hline $320 \mathrm{~K} 277222$ & 3 \\
\hline $320 H 178 A 22$ & 3 \\
\hline $224 H 03 L A 22$ & 3 \\
\hline $214 \mathrm{HO30A22}$ & 3 \\
\hline $214 \mathrm{HO29A22}$ & 3 \\
\hline $224 H 028 A 22$ & 3 \\
\hline $215 H 027 A 22$ & 3 \\
\hline $215 H 026 A 22$ & 3 \\
\hline $215 H 025 A 22$ & 3 \\
\hline $215 H 024 A 222$ & 3 \\
\hline $215 \mathrm{HO23A22}$ & 3 \\
\hline $215 \mathrm{HO22A22}$ & 3 \\
\hline $216 H 022822$ & 3 \\
\hline $216 \mathrm{HO20A22}$ & 3 \\
\hline $216 \mathrm{HO} 19 \mathrm{A2} 2$ & 3 \\
\hline $216 \mathrm{HOLAA22}$ & 3 \\
\hline 2271017222 & 3 \\
\hline $2174016 A 22$ & 3 \\
\hline $2164015 A 22$ & 3 \\
\hline $219 H 014 \Delta 22$ & 3 \\
\hline $2104013 A 22$ & 3 \\
\hline $2194012 A 22$ & 3 \\
\hline 21940111222 & 3 \\
\hline 2194010222 & 3 \\
\hline $2204009 A 22$ & 3 \\
\hline $220 \mathrm{HOOOA22}$ & 3 \\
\hline $2204007 A 22$ & 3 \\
\hline $220 H 006 \mathrm{A2} 2$ & 3 \\
\hline $220 \mathrm{HOOSA22}$ & 3 \\
\hline $22 O H O O 4 A 22$ & 3 \\
\hline $221 \mathrm{H} 003 \mathrm{A2} 2$ & 3 \\
\hline $221 \mathrm{H} 002 \mathrm{A22}$ & 3 \\
\hline $22114001 A 22$ & 3 \\
\hline $211 H 044 A 23$ & $2 \overrightarrow{1}$ \\
\hline $211 H 043 A 23$ & 21 \\
\hline $211 \mathrm{H} 042 \mathrm{A23}$ & 21 \\
\hline $213 \mathrm{H} 03 \mathrm{SA2} 23$ & 21 \\
\hline $213 \mathrm{H} 036 \mathrm{A23}$ & 21 \\
\hline $213 H 037 A .23$ & 21 \\
\hline $213 \mathrm{HO} 2 \mathrm{~A} 23$ & 21 \\
\hline $213 \mathrm{H} 033 \mathrm{~A} 23$ & 21 \\
\hline $213 H 034 A 23$ & 21 \\
\hline $323 \mathrm{H} 185 \mathrm{A23}$ & 21 \\
\hline $323 \mathrm{H186A23}$ & 21 \\
\hline $323 \mathrm{H} 187 \times 23$ & 21 \\
\hline $322 \mathrm{H} 188 \mathrm{~A} 23$ & 21 \\
\hline $322 \mathrm{H189A23}$ & 21 \\
\hline $322 \mathrm{H} 190 \mathrm{A23}$ & 21 \\
\hline $321 H 174 A 23$ & 21 \\
\hline $321 H 175 A 23$ & 21 \\
\hline $320 \mathrm{H} 276 \mathrm{A23}$ & 21 \\
\hline $320 \mathrm{H1} 77223$ & 21 \\
\hline $320 \mathrm{H178A23}$ & 21 \\
\hline $214 \mathrm{H} 031 \mathrm{~A} 23$ & 21 \\
\hline $224 \mathrm{H}_{030 \mathrm{ON23}}$ & 21 \\
\hline $214 \mathrm{HO2} 9 \mathrm{A2} 3$ & 21 \\
\hline $214 \mathrm{HO} 2 \mathrm{BA2} 3$ & 21 \\
\hline $215 \mathrm{HO27A23}$ & 21 \\
\hline $215 H_{026 A 23}$ & 21 \\
\hline $215 \mathrm{HO2SA23}$ & 21 \\
\hline $215 \mathrm{H} 024 \mathrm{A2} 3$ & 21 \\
\hline $215 \mathrm{HO} 23 \mathrm{A2} 23$ & 21 \\
\hline $2154022 A 23$ & 21 \\
\hline $216 \mathrm{H} 021 \mathrm{A23}$ & 21 \\
\hline $216 \mathrm{H} 020 \mathrm{A23}$ & 21 \\
\hline $216 \mathrm{HO} 29 \mathrm{A23}$ & 21 \\
\hline $216 \mathrm{HO1BA23}$ & 21 \\
\hline 2174017423 & 21 \\
\hline 2174016223 & 21 \\
\hline $218 \mathrm{HO15A23}$ & 21 \\
\hline $219 \mathrm{HO} 14 \mathrm{A23}$ & 21 \\
\hline $218 \mathrm{HO} 13 \mathrm{A23}$ & 21 \\
\hline $219 H O 12 \mathrm{A23}$ & 21 \\
\hline $219 \mathrm{HO1LA23}$ & 21 \\
\hline $2194010 A 23$ & 21 \\
\hline 220 HOO9A23 & 21 \\
\hline $220 \mathrm{HOOBA23}$ & 21 \\
\hline 2204007223 & 21 \\
\hline $220 \mathrm{HOOGA23}$ & 21 \\
\hline $220 \mathrm{HOOS223}$ & 21 \\
\hline $22 \mathrm{OHOO4A23}$ & 21 \\
\hline $221 \mathrm{H} 003 \mathrm{A23}$ & 21 \\
\hline $2214002 A 23$ & 21 \\
\hline $221 \mathrm{H} 001223$ & 21 \\
\hline $211 H 044 A 24$ & 4 \\
\hline $211 \mathrm{H} 043 \times 24$ & $i$ \\
\hline $211 H 042 A 24$ & 4 \\
\hline $213 \mathrm{HO35A24}$ & 4 \\
\hline $213 \mathrm{HO} 6 \mathrm{6A24}$ & 4 \\
\hline $213 \mathrm{H} 037 \mathrm{A24}$ & 4 \\
\hline $213 \mathrm{HO} 2 \mathrm{A24}$ & 4 \\
\hline $213 \mathrm{H} 033 \mathrm{A24}$ & 4 \\
\hline $2134034 A 24$ & 4 \\
\hline $323 \mathrm{H} 185 \mathrm{A24}$ & 4 \\
\hline $323 \mathrm{H1B6A24}$ & 4 \\
\hline $323 H 187 A 34$ & 4 \\
\hline
\end{tabular}

Figure 9.13 - Case 2, Baseline Schedule (Continued). 


\begin{tabular}{|c|c|c|c|c|c|}
\hline $\begin{array}{l}\text { ACTIVITY } \\
\text { ID }\end{array}$ & $\begin{array}{l}\text { ORIG } \\
\text { DUR } \\
\ldots . .\end{array}$ & $\begin{array}{l}\text { REM } \\
\text { DUR }\end{array}$ & $*$ & CODE & \\
\hline $322 \mathrm{H} 18 \mathrm{BA} 24$ & 4 & 4 & 0 & 13 & ELECTR ICAL \\
\hline $322 \mathrm{H} 189 \mathrm{A24}$ & 4 & 4 & 0 & 14 & ELECTR ICAL \\
\hline $322 \mathrm{H190A24}$ & & 4 & 0 & 15 & ELECTR ICAI \\
\hline $321 \mathrm{H174 \lambda 24}$ & & 4 & 0 & 16 & ELECTR ICAL \\
\hline $\begin{array}{l}321 H 175 A 24 \\
3201176 A 24\end{array}$ & 4 & 4 & $\begin{array}{l}0 \\
0\end{array}$ & $\begin{array}{l}17 \\
19\end{array}$ & ELECTR ICAL \\
\hline $320 H 177 A 24$ & 4 & 4 & 0 & 19 & $\begin{array}{l}\text { ELECTRICAL } \\
\text { ELECTRICAL }\end{array}$ \\
\hline 3201178224 & 4 & 4 & 0 & 20 & ELECTR ICAL \\
\hline $214 \mathrm{HO}^{2} 3 \mathrm{A2} 4$ & 4 & 4 & 0 & 21 & ELECTRICAS \\
\hline $214 \mathrm{H} 030 \mathrm{A24}$ & 4 & 4 & 0 & 22 & ELECTR ICAL \\
\hline $214 \mathrm{HO} 29 \mathrm{~A} 24$ & 4 & 4 & 0 & 23 & ELECTR ICAL \\
\hline $214 \mathrm{HO} 028 \mathrm{~A} 24$ & 4 & 4 & 0 & 24 & ELECTR ICAL \\
\hline $215 \mathrm{H} 027 \mathrm{A24}$ & 4 & 4 & 0 & 25 & ELECTR ICAL \\
\hline $215 \% 026 \mathrm{A24}$ & & 4 & 0 & 26 & ELECTR ICAL \\
\hline $\begin{array}{l}215 \mathrm{H}_{025 \mathrm{~A} 4} \\
215 \mathrm{HO2} 4 \mathrm{~A} 24\end{array}$ & 4 & 4 & $:$ & 27 & ELECTRICAL \\
\hline 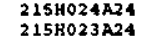 & 4 & 4 & $\begin{array}{l}0 \\
0\end{array}$ & $\begin{array}{l}29 \\
29\end{array}$ & $\begin{array}{l}\text { ELECTR ICAL } \\
\text { ELECTR ICAL }\end{array}$ \\
\hline $215 H 022 A 24$ & 4 & 4 & 0 & 30 & ELECTRICAE \\
\hline $216 \mathrm{HO} 21 \mathrm{A24}$ & 4 & 4 & 0 & 31 & ELECTR ICAL \\
\hline $216 \mathrm{H020A24}$ & 4 & 4 & 0 & 32 & ELECTRICAL \\
\hline $216 \mathrm{H019A24}$ & 4 & 4 & 0 & 33 & ELECTR ICAL \\
\hline $216 \mathrm{H} 028 \mathrm{A24}$ & 4 & 4 & 0 & 34 & ELECTR ICAL \\
\hline $217 \mathrm{HO17A24}$ & 4 & 4 & 0 & 35 & ELECTRICAC \\
\hline $\begin{array}{l}217 \mathrm{H} 016 \mathrm{A24} \\
218 \mathrm{HO} 25 \mathrm{A24}\end{array}$ & 4 & 4 & $:$ & $\begin{array}{l}36 \\
37\end{array}$ & $\begin{array}{l}\text { ELECTRICAL } \\
\text { ELECTRICAL }\end{array}$ \\
\hline $218 H 014 A 24$ & 4 & 4 & 0 & 30 & \\
\hline $218 \mathrm{HO} 13 \mathrm{~A} 24$ & 4 & 4 & 0 & 39 & ELECTRICAL \\
\hline $219 \mathrm{HO} 012 \mathrm{A24}$ & 4 & 4 & 0 & 40 & ELECTRICAL \\
\hline $219 \mathrm{H} 011 \mathrm{~A} 24$ & 4 & 4 & 0 & 41 & ELECTRICAL \\
\hline $2194010 A 24$ & 4 & 4 & 0 & 42 & ELECTRICAL \\
\hline $220 \mathrm{HOO} 9 \mathrm{A24}$ & 4 & 4 & 0 & 43 & ELECTRICAL \\
\hline $220 \mathrm{H} 00 \mathrm{OA24}$ & 4 & 4 & 0 & 44 & ELECTR ICAL \\
\hline 2204007224 & & 4 & : & 45 & ELECTRICAL \\
\hline $\begin{array}{l}220 \mathrm{H} 006 \mathrm{AP24} \\
220 \mathrm{H} 005 \mathrm{A24}\end{array}$ & 4 & 4 & $\begin{array}{l}0 \\
0\end{array}$ & $\begin{array}{l}16 \\
47\end{array}$ & $\begin{array}{l}\text { ELECTRICAL } \\
\text { ELECTRICAL }\end{array}$ \\
\hline $220 \mathrm{HOO4A24}$ & 4 & 4 & 0 & 48 & ELECTRICAL, \\
\hline $221 \mathrm{H} 003 \mathrm{A24}$ & 4 & 4 & 0 & 49 & ELECTRICAL \\
\hline $221 \mathrm{H} 002 \mathrm{~A} 24$ & 4 & 4 & 0 & 50 & ELECTRICAL \\
\hline $221 \mathrm{H} 001224$ & 4 & 4 & 0 & 51 & ELECTRICAL \\
\hline $211 H 044 A 25$ & 7 & 7 & 0 & 01 & WALL-EMBED \\
\hline $211 \mathrm{H} 043 \mathrm{~A} 25$ & 7 & 7 & 0 & 02 & WALL-EMBED \\
\hline 211H042A25 & 7 & 7 & 0 & 03 & WALL-EMBED \\
\hline 213H035A2S & ? & 7 & 0 & 04 & WALL-EMBED \\
\hline $2134036 \mathrm{A25}$ & 7 & 7 & 0 & 05 & WALC-EMBED \\
\hline $213 H 037 \not 25$ & 7 & 7 & 0 & 06 & WALL-EMBED \\
\hline 213H032A25 & 7 & 7 & 0 & 07 & WALL-EMBED \\
\hline $213 \mathrm{H} 033 \mathrm{~A} 25$ & 7 & 7 & 0 & 08 & WALE-EMBED \\
\hline $223 \mathrm{H} 034 \mathrm{A25}$ & 7 & 7 & 0 & 09 & WALL-EMBED \\
\hline $323 \mathrm{HI} 55 \mathrm{A25}$ & 7 & 7 & 0 & 10 & WALE-EMBED \\
\hline $323 \mathrm{H} 196 \mathrm{~A} 25$ & 7 & $?$ & 0 & 11 & HALL-EMBED \\
\hline $323 \mathrm{H187A25}$ & 7 & 7 & 0 & 12 & WALL-EMBED \\
\hline $322 \mathrm{K18 \theta A25}$ & 7 & 7 & 0 & 13 & WALL-EMBED \\
\hline $322 \mathrm{H} 189 \mathrm{~A} 25$ & 7 & 7 & 0 & 14 & WALLL-EMBED \\
\hline $322 \mathrm{H} 190 \mathrm{A25}$ & 7 & 7 & 0 & 15 & WALL-EMBED \\
\hline $321 H 174225$ & 7 & 7 & 0 & 16 & WALLL-EMEED \\
\hline $321 \% 175 A 25$ & 7 & 7 & 0 & 17 & WALL-EMBED \\
\hline $320 \mathrm{H} 176 \mathrm{~A} 25$ & 7 & 7 & 0 & 18 & WALL-EMBED \\
\hline $320 \mathrm{H} 177 \mathrm{~A} 25$ & 7 & $?$ & 0 & 19 & HALL-EMBED \\
\hline $320 \mathrm{H} 178 \mathrm{~A} 25$ & 7 & $?$ & 0 & 20 & WALL-EMBED \\
\hline $214 \mathrm{H} 031 \mathrm{A2S}$ & 7 & 7 & 0 & 21 & WALL-EMBED \\
\hline 214H030A25 & 7 & 7 & 0 & 22 & WALL-EMBED \\
\hline $214 \mathrm{HO29A2S}$ & 7 & 7 & 0 & 23 & WALL-EMBED \\
\hline $214 \mathrm{HO} 028 \mathrm{A2S}$ & 7 & 7 & 0 & 24 & WALL-EMBED \\
\hline $215 H 027 A 25$ & 7 & 7 & 0 & 25 & WALL-EMBED \\
\hline $215 \mathrm{H} 026 \mathrm{A25}$ & $?$ & 7 & 0 & 26 & WALL-EMBED \\
\hline $215 \mathrm{H} 025 \mathrm{~A} 25$ & 7 & 7 & 0 & 27 & WALL-EMBED \\
\hline $215 H 024 A 25$ & 7 & 7 & 0 & 28 & WALL-EMBED \\
\hline $215 H 023 A 25$ & 7 & 7 & 0 & 29 & WALL-EMBED \\
\hline $215 \mathrm{H} 022 \mathrm{~A} 25$ & 7 & 7 & 0 & 30 & WALS-EMBED \\
\hline $216 \mathrm{HO} 21 \mathrm{A25}$ & 7 & $?$ & 0 & 31 & WALL-EMBED \\
\hline $216 \mathrm{HO2OA25}$ & 7 & 7 & 0 & 32 & WALL-EMBED \\
\hline $216 \mathrm{H} 019 \mathrm{A25}$ & 7 & 7 & 0 & 33 & WALL-EMBED \\
\hline $216 \mathrm{HO} 18 \mathrm{A25}$ & 7 & 7 & 0 & 34 & WALL-EMBED \\
\hline 2174027A25 & 7 & 7 & 0 & 35 & WALZ, - EMBEO \\
\hline $2174016 \mathrm{A25}$ & 7 & 7 & 0 & 36 & WALL-EMBED \\
\hline $218 \mathrm{BO} 015 \mathrm{~A} 2 \mathrm{~S}$ & 7 & 7 & 0 & 37 & WALL-EMEED \\
\hline 218 HO14A25 & 7 & 7 & 0 & 38 & WALI-EMBED \\
\hline $219 H 023 A 25$ & 7 & 7 & 0 & 39 & WALL-EMBED \\
\hline $219 \mathrm{HO} 12 \mathrm{A25}$ & 7 & 7 & 0 & 40 & WALL-EMEED \\
\hline $219 \mathrm{HO} 11 \mathrm{A25}$ & 7 & 7 & 0 & 41 & WALL-EMBED \\
\hline $219 \mathrm{H} 010225$ & 7 & 7 & 0 & 42 & WALL-EMBED \\
\hline $2204009 A 25$ & 7 & 7 & 0 & 43 & WALL-EMBED \\
\hline $220 \mathrm{HOOBA25}$ & 7 & 7 & 0 & 44 & WALL-EMBED \\
\hline 2201007 A25 & 7 & 7 & 0 & 45 & WAV.L-EMBED \\
\hline $220 \mathrm{H} 006 \mathrm{~A} 25$ & 7 & 7 & 0 & 46 & WALL-EMBED \\
\hline $22011005 A 25$ & 7 & 7 & 0 & 47 & WALL-EMBED \\
\hline $22 \mathrm{OH} 004 \mathrm{~A} 25$ & 7 & 7 & 0 & 48 & WALL -EMBED \\
\hline $221 \mathrm{H} 003 \mathrm{A25}$ & 7 & 7 & 0 & 49 & WALL-EMBED \\
\hline $2214002 \mathrm{A25}$ & $?$ & 7 & 0 & 50 & WALL-EMBED \\
\hline $221 \mathrm{H} 001 \mathrm{A25}$ & 7 & 7 & 0 & 51 & WALL-EMBED \\
\hline 2114044226 & 10 & 10 & 0 & 01 & CAST IRON \\
\hline $211 \mathrm{HO} 33 \mathrm{A26}$ & 10 & 10 & 0 & 02 & CAST IRON \\
\hline $1042 A 26$ & 10 & 10 & 0 & 03 & CAST IRON \\
\hline $2134035 \mathrm{~A} 26$ & 10 & 10 & 0 & 04 & CAST IRON \\
\hline $213 H 036 A 26$ & 10 & 10 & 0 & 05 & CAST IRON \\
\hline & & & & & \\
\hline
\end{tabular}

Figure 9.13 - Case 2, Baseline Schedule (Continued). 
EALD S EBRAHIM ALABDULPJتEAK EST

PEPORT CATE IISEP9S RUN NO :21 $5:+6$
PRIMAVERA PROTECT PLLNNAER

SEVEN SEAS, CONSTRUCTION OF S1 HOUSES
JAHRA 3 HOUSING PROETCT COMmON aCt START DATE LAPRB7 FIN DATE 13AUGG

DATA DATE IAPRg? SAGE NO. 14

\begin{tabular}{|c|c|}
\hline $\begin{array}{c}\text { ACTIVITY } \\
\text { ID } \\
\ldots\end{array}$ & $\begin{array}{l}\text { ORYO } \\
\text { DUR }\end{array}$ \\
\hline $213 \mathrm{H}_{0} 32 \mathrm{A2} 6$ & 10 \\
\hline 2138033326 & 10 \\
\hline $\begin{array}{l}2134034 A 26 \\
3234195026\end{array}$ & 10 \\
\hline $\begin{array}{l}3234195 A 26 \\
3234186226\end{array}$ & 10 \\
\hline $\begin{array}{l}3234186 A 26 \\
323 H 187 A 26\end{array}$ & 10 \\
\hline $\begin{array}{l}323 \mathrm{H} 187 \mathrm{A2} 6 \\
322 \mathrm{H1BOA26}\end{array}$ & 10 \\
\hline $\begin{array}{l}322 \mathrm{H} 1 \mathrm{BQA26} 6 \\
322 \mathrm{H} 1 \mathrm{BgA2} 6\end{array}$ & 10 \\
\hline & 10 \\
\hline $\begin{array}{l}322 H 190 A 26 \\
321 \mathrm{H} 74 A 26\end{array}$ & 10 \\
\hline $\begin{array}{l}321 H 174 A 26 \\
3221175 A 26\end{array}$ & 10 \\
\hline 3214175226 & 10 \\
\hline $\begin{array}{l}320 \mathrm{H} 176 \mathrm{A26} \\
320 \mathrm{1} 77 \mathrm{A2} 6\end{array}$ & 10 \\
\hline $\begin{array}{l}320 \% 177 A 26 \\
320 H 179 A 26\end{array}$ & 10 \\
\hline $\begin{array}{l}3201179 A 26 \\
2144031426\end{array}$ & 10 \\
\hline $\begin{array}{l}214 H 031226 \\
214 H 030 A 26\end{array}$ & 10 \\
\hline 2144030026 & 10 \\
\hline $214 \mathrm{HO} 29 \mathrm{A2} 6$ & 10 \\
\hline $224 H 028 A 26$ & 10 \\
\hline $2154027 A 26$ & 10 \\
\hline $215 H 026 A 26$ & 20 \\
\hline $225 H 025 A 26$ & 10 \\
\hline $215 \mathrm{H} 024 \mathrm{A2} 6$ & 10 \\
\hline $215 \mathrm{H} 023 \mathrm{A2} 6$ & 10 \\
\hline $215 \mathrm{HO222026}$ & 10 \\
\hline $216 \mathrm{H} 021 \mathrm{A2} 6$ & 10 \\
\hline 2164020426 & 10 \\
\hline $216 \mathrm{HO19A26}$ & 10 \\
\hline $216 \mathrm{HO1} 1 \mathrm{AA26}$ & 10 \\
\hline $217 \mathrm{HO17A26}$ & 10 \\
\hline $217 \mathrm{HO16A26}$ & 10 \\
\hline $218 \mathrm{HO15A26}$ & 10 \\
\hline $219 H 014 A 26$ & 10 \\
\hline $218 \mathrm{HO} 13 \mathrm{A2} 6$ & 10 \\
\hline $219 \mathrm{HO} 012 \mathrm{2} 26$ & 10 \\
\hline $219 \mathrm{HO} 13 \mathrm{A2} 6$ & 10 \\
\hline $219 H 010 A 26$ & 10 \\
\hline $220 \mathrm{H} 009 \mathrm{~A} 26$ & 20 \\
\hline $220 \mathrm{HOOSA26}$ & 10 \\
\hline $220 H 007 A 26$ & 10 \\
\hline $220 \mathrm{OHO06A26}$ & 10 \\
\hline $220 \mathrm{HOOSA26}$ & 10 \\
\hline $220 H_{004 A 26}$ & 10 \\
\hline 2214003 A26 & 10 \\
\hline $221 \mathrm{H} 003 \mathrm{A26}$ & 10 \\
\hline $221 \% 001 A 26$ & 10 \\
\hline $2114044 A 27$ & 13 \\
\hline $2318043 A 27$ & 13 \\
\hline $211 \mathrm{H} 042 \mathrm{A2} 7$ & 13 \\
\hline $213 \mathrm{H} 035 \mathrm{A2} 7$ & 13 \\
\hline $213 \mathrm{H} 036227$ & 13 \\
\hline $213 \mathrm{H} 037 \mathrm{A27}$ & 13 \\
\hline 2134032827 & 13 \\
\hline $213 \mathrm{H} 033 \mathrm{A2} 7$ & 13 \\
\hline $213 \mathrm{H} 034 \mathrm{A27}$ & 23 \\
\hline $323 \mathrm{HLSSA27}$ & 13 \\
\hline $323 \mathrm{H} 186 \mathrm{A27}$ & 13 \\
\hline $323 \mathrm{H187A27}$ & 13 \\
\hline $322 \mathrm{H} 188 \mathrm{A27}$ & 13 \\
\hline $322 \mathrm{KIO9A27}$ & 23 \\
\hline $322 \mathrm{H} 190 \mathrm{A2} 7$ & 13 \\
\hline $321 \mathrm{~K} 174 \mathrm{A2} 7$ & 13 \\
\hline 32114175227 & 13 \\
\hline $320 \mathrm{H176A27}$ & 13 \\
\hline $320 \mathrm{H} 177 \mathrm{A27}$ & 13 \\
\hline $320 \mathrm{H} 178 \mathrm{A27}$ & 13 \\
\hline $214 \mathrm{~K} 031 \mathrm{A27}$ & 13 \\
\hline $214 \mathrm{HO} 030 \mathrm{A2} 7$ & 13 \\
\hline $214 \mathrm{H029A27}$ & 13 \\
\hline $214 \mathrm{HO2}$ AA27 & 13 \\
\hline $215 \mathrm{H} 027 \mathrm{A27}$ & 13 \\
\hline $215 \mathrm{H} 026227$ & 13 \\
\hline 2159025227 & 23 \\
\hline $215 H 024 A 27$ & 13 \\
\hline $215 K 023 \times 27$ & 13 \\
\hline $2154022 \times 27$ & 13 \\
\hline $216 \mathrm{HO2} 1 \mathrm{A27}$ & 13 \\
\hline $216 \mathrm{HO20A27}$ & 13 \\
\hline $216 \mathrm{HO} 29 \mathrm{A2} 7$ & 13 \\
\hline $216 \mathrm{HO} 10227$ & 13 \\
\hline $2174017 A 27$ & 13 \\
\hline $217 \mathrm{HO16A27}$ & 13 \\
\hline $2184015 A 27$ & 13 \\
\hline $218 \mathrm{HO} 014 \mathrm{A2} 7$ & 13 \\
\hline $21 \mathrm{BHO} 013 \mathrm{A2} 7$ & 23 \\
\hline $219 \mathrm{H} 012 \mathrm{A2} 7$ & 23 \\
\hline $219 \mathrm{HO} 11 \mathrm{A27}$ & 13 \\
\hline $219 \mathrm{HO} 20 \mathrm{A2} 7$ & 13 \\
\hline $220 H 009 \times 27$ & 13 \\
\hline $220 \mathrm{HOOBA27}$ & 13 \\
\hline $2201007 A 27$ & 13 \\
\hline $220 \mathrm{HO00627}$ & 13 \\
\hline $220 \% 005 A 27$ & 13 \\
\hline $2204004 \mathrm{A2} 7$ & 13 \\
\hline $2218003 \mathrm{A2} 7$ & 13 \\
\hline $221 \% 002 A 27$ & 13 \\
\hline & \\
\hline
\end{tabular}

Figure 9.13 - Case 2, Baseline Schedule (Continued). 


\begin{tabular}{|c|c|}
\hline $\begin{array}{l}\text { ACTIVITY } \\
\text { ID }\end{array}$ & $\begin{array}{l}\text { ORIG } \\
\text { DUR }\end{array}$ \\
\hline $211 \% 044 A 28$ & 10 \\
\hline $211 \mathrm{HO} 43 \mathrm{~A} 2 \mathrm{O}$ & 10 \\
\hline $2119042 A 28$ & 10 \\
\hline $213 H 035 A 28$ & 10 \\
\hline $2139036 \mathrm{~A} 2 \mathrm{\theta}$ & 10 \\
\hline $213 \mathrm{HO} 37 \mathrm{A28}$ & 10 \\
\hline $2131032 A 28$ & 10 \\
\hline $213 H 033 A 28$ & 10 \\
\hline $2134034 A 2 B$ & 10 \\
\hline $3234185 A 28$ & 10 \\
\hline $323 \mathrm{H186A28}$ & 10 \\
\hline $323 H 187 A 28$ & 10 \\
\hline $322 \mathrm{H18 \theta A28}$ & 20 \\
\hline $322 \mathrm{H} 189 \mathrm{~A} 28$ & 10 \\
\hline $322 H 190228$ & 10 \\
\hline $321 K 174 A 28$ & 10 \\
\hline $321 \% 175 A 28$ & 10 \\
\hline $320 \mathrm{H} 176 \mathrm{A28}$ & 10 \\
\hline $320 \mathrm{H177A28}$ & 10 \\
\hline $320 \mathrm{H178A28}$ & 10 \\
\hline 2144031228 & 10 \\
\hline $214 H 030288$ & 20 \\
\hline $2144029 A 28$ & 10 \\
\hline 2144028228 & 10 \\
\hline $215 \mathrm{H} 027 \mathrm{A28}$ & 10 \\
\hline $215 H 026 A 28$ & 10 \\
\hline $225 H 025 A 28$ & 10 \\
\hline $215 H 024 A 28$ & 10 \\
\hline $215 H_{023 A 28}$ & 10 \\
\hline $215 H 022 A 28$ & 10 \\
\hline $2164021 \mathrm{A2} 8$ & 10 \\
\hline 2164020228 & 10 \\
\hline $216 \mathrm{HO19A2B}$ & 10 \\
\hline 216401628 & 10 \\
\hline $217 \mathrm{HO17A28}$ & 10 \\
\hline 2174016228 & 10 \\
\hline $21 \mathrm{OHO} 15 \mathrm{~A} 28$ & 10 \\
\hline $218 K 014 A 28$ & 10 \\
\hline $218 \mathrm{BH} 013 \mathrm{A2B}$ & 10 \\
\hline $219 H 012 \mathrm{A2} 8$ & 10 \\
\hline $219 \mathrm{HO} 11 \mathrm{A28}$ & 10 \\
\hline $229 K 010 A 28$ & 10 \\
\hline $220 H 009 A 28$ & 10 \\
\hline $220 \mathrm{HOOBA2B}$ & 10 \\
\hline $22 \mathrm{OH} 007228$ & 10 \\
\hline $220 \mathrm{HHOOGA28}$ & 10 \\
\hline $220 \mathrm{H} 005 \mathrm{A2} 8$ & 10 \\
\hline $220 \mathrm{H} 004 A 28$ & 20 \\
\hline 2214003228 & 10 \\
\hline $221 \mathrm{H0O2A2 \theta}$ & 10 \\
\hline $2214001 \times 2 \theta$ & 10 \\
\hline $2114044 A 29$ & 6 \\
\hline $211 \% 043 A 29$ & 6 \\
\hline $211 \% 042 A 29$ & 6 \\
\hline $2134035 A 29$ & 6 \\
\hline $213 \mathrm{H} 036 \mathrm{A2} 9$ & 6 \\
\hline $213 H 037429$ & 6 \\
\hline $213 \mathrm{H}_{032 \mathrm{~A} 2 \mathrm{~S}}$ & 6 \\
\hline $213 \mathrm{H}_{03} 3 \mathrm{~A} 29$ & 6 \\
\hline 2134034429 & 6 \\
\hline $323 \mathrm{H} 185 \mathrm{AN2}$ & 6 \\
\hline 32341862029 & 6 \\
\hline $323 \mathrm{H} 1877239$ & 6 \\
\hline $322 \mathrm{H} 188 \mathrm{~A} 29$ & 6 \\
\hline 3224189229 & 6 \\
\hline $322 \mathrm{H} 190 \mathrm{~A} 2 \mathrm{O}$ & 6 \\
\hline $321 \mathrm{H174A29}$ & 6 \\
\hline $321 \mathrm{H175A29}$ & 6 \\
\hline $320 \mathrm{H} 176 \mathrm{A2} 9$ & 6 \\
\hline $320 \mathrm{H} 177229$ & 6 \\
\hline 320H178A29 & 6 \\
\hline $214 \mathrm{H} 031 \mathrm{A2g}$ & 6 \\
\hline $214 \mathrm{HO} 030 \mathrm{A29}$ & 6 \\
\hline $214 \mathrm{HO} 29 \mathrm{A2}$ & 6 \\
\hline $214 \mathrm{HO}_{2}$ BA29 & 6 \\
\hline $215 \mathrm{HO}^{2} 7 \not 29$ & 6 \\
\hline $215 \mathrm{HO} 26 \mathrm{A2} 9$ & 6 \\
\hline $215 H_{025 A 29}$ & 6 \\
\hline $215 H_{024 A 29}$ & 6 \\
\hline 215H023A29 & 6 \\
\hline $215 \mathrm{HO} 22 \mathrm{A29}$ & 6 \\
\hline $216 \mathrm{HO22A29}$ & \\
\hline 2164020029 & 6 \\
\hline $216 \mathrm{H} 019 \mathrm{A29}$ & 6 \\
\hline 2164018 A29 & 6 \\
\hline $217 \mathrm{H} 017 \mathrm{A29}$ & 6 \\
\hline $217 \mathrm{HO16A29}$ & 6 \\
\hline $218 H 015 A 29$ & 6 \\
\hline $210 \mathrm{HO} 14 \mathrm{A2} 9$ & 6 \\
\hline 218HO13A29 & 6 \\
\hline $219 \mathrm{HO} 2 \mathrm{AA29}$ & 6 \\
\hline 219HO11A29 & 6 \\
\hline $229 \mathrm{HO} 10 \mathrm{A2}$ & 6 \\
\hline $22 \mathrm{OH} 009 \mathrm{AZS}$ & 6 \\
\hline $220 \mathrm{HOOOA29}$ & 6 \\
\hline $22 \mathrm{OH} 007 \mathrm{A2} 9$ & 6 \\
\hline
\end{tabular}

Figure 9.13 - Case 2, Baseline Schedule (Continued). 


\begin{tabular}{|c|c|c|c|c|}
\hline $\begin{array}{l}\text { ACTIVITY } \\
\text { ID }\end{array}$ & $\begin{array}{l}\text { ORIG } \\
\text { DUR }\end{array}$ & $\begin{array}{l}\text { REM } \\
\text { DUR }\end{array}$ & * & CODE \\
\hline $220 \mathrm{H} 006 \mathrm{~A} 29$ & 6 & 6 & 0 & 46 \\
\hline $2201005 A 29$ & 6 & 6 & 0 & 47 \\
\hline $2204004 A 29$ & 6 & 6 & 0 & 49 \\
\hline $221 \mathrm{H} 003 \mathrm{A2} 9$ & 6 & 6 & 0 & 49 \\
\hline $22111002 A 29$ & 6 & 6 & 0 & so \\
\hline $221 \mathrm{H} 001 \mathrm{A2} 9$ & 6 & 6 & 0 & 51 \\
\hline $21111044 A 30$ & 4 & 4 & 0 & 01 \\
\hline $21114043 \mathrm{A30}$ & 4 & 4 & 0 & 02 \\
\hline $211 \mathrm{HO42A30}$ & 4 & 4 & 0 & 03 \\
\hline $2134035 A 30$ & 4 & 4 & 0 & 04 \\
\hline $213 \mathrm{HO} 36 \mathrm{A3O}$ & & 4 & 0 & 05 \\
\hline $2134037 \mathrm{A3} 0$ & 4 & 4 & 0 & 06 \\
\hline $2134032 A 30$ & 4 & 4 & 0 & 07 \\
\hline $213 \mathrm{HO} 33 \mathrm{A3O}$ & 4 & 4 & 0 & 08 \\
\hline $213 \mathrm{HO} 44 \mathrm{A3O}$ & 4 & 4 & 0 & 09 \\
\hline $323 \mathrm{H} 195 \mathrm{~A} 3 \mathrm{O}$ & 4 & 4 & 0 & 10 \\
\hline $323 H 186 \mathrm{A3O} O$ & 4 & 4 & 0 & 11 \\
\hline $323 \mathrm{H} 187 \mathrm{A3} 30$ & 4 & 4 & 0 & 12 \\
\hline $322 \mathrm{H1O8A30}$ & 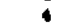 & 4 & 0 & 23 \\
\hline $322 \mathrm{H} 189 \mathrm{A3} \mathrm{O}$ & 4 & 4 & 0 & 14 \\
\hline $322 \mathrm{H} 190 \mathrm{~A} 3 \mathrm{O}$ & 4 & 4 & 0 & 15 \\
\hline $321 \mathrm{H174A30}$ & 4 & 4 & 0 & 16 \\
\hline $321 \mathrm{H} 175 \mathrm{A3O}$ & 4 & 4 & 0 & 17 \\
\hline $3204176 A 30$ & 4 & 4 & 0 & 18 \\
\hline $3201177 A 30$ & 4 & 4 & 0 & 19 \\
\hline 320H278A3O & 4 & 4 & 0 & 20 \\
\hline $214 \mathrm{HO} 1 \mathrm{A3} \mathrm{O}$ & 4 & 4 & 0 & 21 \\
\hline $214 \mathrm{HHO30A30}$ & 4 & 4 & 0 & 22 \\
\hline $224 \mathrm{H} 029 \mathrm{A3O}$ & 4 & 4 & 0 & 23 \\
\hline $214 \mathrm{HO28030}$ & 4 & 4 & 0 & 24 \\
\hline $215 \mathrm{HO}_{02} 7 \mathrm{A3} 0$ & 4 & 4 & 0 & 25 \\
\hline $215 H 026 \AA 30$ & 1 & 4 & 0 & 26 \\
\hline $215 \mathrm{H} 025 \mathrm{~A} 3 \mathrm{O}$ & 4 & 4 & 0 & 27 \\
\hline $215 \mathrm{HO24A30}$ & 4 & 4 & 0 & 28 \\
\hline $215 H_{023 A 3 O}$ & 4 & 4 & 0 & 29 \\
\hline $215 \mathrm{HO}^{2} 2 \mathrm{~A} 30$ & 4 & 4 & 0 & 30 \\
\hline $\begin{array}{l}2164021030 \\
2164020030\end{array}$ & 4 & 4 & 0 & 31 \\
\hline $\begin{array}{l}216 \mathrm{HO} 020230 \\
216 \mathrm{HO} 19 \mathrm{A3} 0\end{array}$ & 4 & 4 & 0 & $\begin{array}{r}32 \\
33\end{array}$ \\
\hline $216 \mathrm{HOLSA3O}$ & 4 & 4 & 0 & $\begin{array}{l}33 \\
34\end{array}$ \\
\hline $2174017 \times 30$ & $i$ & 4 & 0 & $\begin{array}{l}34 \\
35\end{array}$ \\
\hline 2174016230 & 4 & 4 & 0 & 36 \\
\hline $218 H 015 A 30$ & 4 & 4 & 0 & 37 \\
\hline $218 H 014430$ & 4 & 4 & 0 & 38 \\
\hline $218 H 013 A 30$ & 4 & 4 & 0 & 39 \\
\hline $219 H 012 A 30$ & 4 & 1 & 0 & 40 \\
\hline $219 H 011$ A3O & 4 & 4 & 0 & 41 \\
\hline $219 H 010030$ & 4 & 4 & 0 & 42 \\
\hline $220 \mathrm{HOOSA30}$ & 4 & 4 & 0 & 43 \\
\hline $220 \mathrm{HOOBA3O}$ & 4 & 4 & 0 & 44 \\
\hline $220 \mathrm{H0O7A30}$ & 4 & 4 & 0 & 45 \\
\hline $22 \mathrm{OHOO6A3O}$ & 4 & 4 & 0 & 46 \\
\hline $220 \mathrm{HOOSA30}$ & 4 & 4 & 0 & 17 \\
\hline $2204004 A 30$ & 4 & 4 & 0 & 48 \\
\hline $\begin{array}{l}221 \% 003 \mathrm{A3O} \\
221 \mathrm{H} 002 \mathrm{A3} 0\end{array}$ & 4 & 4 & 0 & 49 \\
\hline $\begin{array}{l}221 \mathrm{H} 002 \mathrm{A3} 0 \\
221 \mathrm{H} 001 \mathrm{A3} 0\end{array}$ & 4 & 4 & 0 & 50 \\
\hline $\begin{array}{l}221 \mathrm{H} 001 \mathrm{A3} 0 \\
211 \mathrm{HO} 4 \mathrm{~A} 3 \mathrm{~B}\end{array}$ & 6 & 6 & 0 & 51 \\
\hline & 6 & 6 & 0 & $\begin{array}{l}01 \\
02\end{array}$ \\
\hline $211 \mathrm{H042 \textrm {A3 } 1}$ & 6 & 6 & 0 & 03 \\
\hline $2234035 A 31$ & 6 & 6 & 0 & 04 \\
\hline & 6 & 6 & 0 & 05 \\
\hline & 6 & 6 & 0 & 08 \\
\hline 2134034231 & 6 & 6 & : & 09 \\
\hline $323 \mathrm{H} 105 \mathrm{~A} 31$ & 6 & 6 & 0 & 10 \\
\hline $\begin{array}{l}323 \mathrm{H187A31} \\
322 \mathrm{H} 188 \mathrm{AB} 1\end{array}$ & 6 & 6 & 0 & 12 \\
\hline $\begin{array}{l}322 \mathrm{H} 189 \mathrm{AB} 1 \\
322 \mathrm{H} 189 \mathrm{~A} 3 \mathrm{l}\end{array}$ & 6 & 6 & : & 13 \\
\hline $322 \mathrm{H} 189 \mathrm{A3}$ & 6 & 6 & $\circ$ & 14 \\
\hline $322 \mathrm{HI} 190231$ & 6 & 6 & 0 & $\begin{array}{l}15 \\
16\end{array}$ \\
\hline $\begin{array}{l}321 \mathrm{H175A31} \\
320 \mathrm{H176A33}\end{array}$ & 6 & 6 & 0 & $\begin{array}{l}17 \\
18\end{array}$ \\
\hline $\begin{array}{l}320 \mathrm{H176A31} \\
320 \mathrm{H177A3}\end{array}$ & 6 & 6 & $\begin{array}{l}0 \\
0\end{array}$ & 18 \\
\hline $320 \mathrm{H} 177 \mathrm{~A} 31$ & 6 & 6 & : & $\begin{array}{l}19 \\
20\end{array}$ \\
\hline $320 \mathrm{H} 178 \mathrm{A3} 1$ & 6 & 6 & 0 & $\begin{array}{l}20 \\
21\end{array}$ \\
\hline 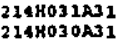 & $\begin{array}{l}6 \\
6\end{array}$ & 5 & $: 0$ & $\begin{array}{l}21 \\
22\end{array}$ \\
\hline $\begin{array}{l}2148030 A 31 \\
214 H 029 A 31\end{array}$ & 6 & 6 & 0 & $\begin{array}{l}22 \\
23\end{array}$ \\
\hline $214 \mathrm{H} 028 \mathrm{~A} 31$ & 6 & 6 & 0 & 24 \\
\hline $215 \mathrm{HO27A31}$ & 6 & 6 & 0 & 25 \\
\hline $215 \mathrm{HO} 26 \mathrm{A3} 1$ & 6 & 6 & 0 & 26 \\
\hline $215 \mathrm{HO} 25 \mathrm{SA} 1$ & 6 & 6 & 0 & 27 \\
\hline $2154024 \times 31$ & 6 & 6 & 0 & 28 \\
\hline $215 K 023 \mathrm{A31}$ & 6 & 6 & 0 & 29 \\
\hline $215 \mathrm{HO}_{02 \mathrm{~A} 3 \mathrm{I}}$ & 6 & 6 & 0 & 30 \\
\hline $216 \mathrm{HO} 1 \mathrm{A3} 1$ & 6 & 6 & 0 & 31 \\
\hline $216 H 020 A 31$ & 6 & 6 & 0 & 32 \\
\hline $216 \mathrm{HO19A31}$ & 6 & 6 & 0 & 33 \\
\hline $216 \mathrm{HO} 18 \mathrm{BA} 1$ & 6 & 6 & 0 & 34 \\
\hline $2174017 \times 31$ & 6 & 6 & 0 & 35 \\
\hline $217 \mathrm{H016 \textrm {A } 3 1}$ & 6 & 6 & 0 & 36 \\
\hline 218 O15A31 & 6 & 6 & 0 & 37 \\
\hline 218 HO14A31 & 6 & 6 & 0 & 38 \\
\hline $2184013 \mathrm{A31}$ & 6 & 6 & 0 & 39 \\
\hline
\end{tabular}

Figure 9.13 - Case 2, Baseline Schedule (Continued). 
jaUO \& EBRAHIM ALABDULRAZZAK EST

PEPORT DATE 21SEP95 RUN NO. 121

$15: 46$
PRTMATEPA PROJECT PLANNE

SEVEN SEAS, CONSTRUCTION OF 5 h hOUSES
JAKRA 3 HOUSTNG PROTECT COMmON aC:

START DATE LAPRB7 FIN DATE 13AUGg8 DATA DATE LAPRg7 PAGE NO.

\begin{tabular}{|c|c|}
\hline \begin{tabular}{c} 
ACTIVITY \\
ID \\
\hdashline
\end{tabular} & $\begin{array}{l}\text { ORIG } \\
\text { DUR }\end{array}$ \\
\hline $2191012 \mathrm{~A} 31$ & 6 \\
\hline $219 H 011 \mathrm{~A} 31$ & 6 \\
\hline $2195010 \mathrm{AJ} 1$ & 6 \\
\hline $220 H 009 A 31$ & 6 \\
\hline $220 \mathrm{OHOOSAJ1}$ & 6 \\
\hline $2204007 \mathrm{A3} 1$ & 6 \\
\hline $220 \mathrm{HOO6A31}$ & 6 \\
\hline $220 \mathrm{H} 005 \mathrm{A3} 1$ & 6 \\
\hline $2204004 A 31$ & 6 \\
\hline 2214003231 & 6 \\
\hline $22111002 A 31$ & 6 \\
\hline $221 \mathrm{HOO} 2 \mathrm{A31}$ & 6 \\
\hline $22114044 A 32$ & 日 \\
\hline $21111043 \mathrm{A32}$ & B \\
\hline $211 \% 042 A 32$ & 8 \\
\hline $213 H 035 \mathrm{A32}$ & 8 \\
\hline $213 \mathrm{HO} 636 \mathrm{A3} 2$ & a \\
\hline $213 \mathrm{HO} 77332$ & 8 \\
\hline $213 \mathrm{H} 032 \mathrm{A32}$ & B \\
\hline 2134033032 & $B$ \\
\hline $213 \mathrm{H} 034 \mathrm{A32}$ & B \\
\hline $323 \mathrm{H} 1 \mathrm{BSA32}$ & 8 \\
\hline $323 \mathrm{H} 186 \mathrm{GA32}$ & 8 \\
\hline $323 \mathrm{H} 187 \mathrm{A32}$ & 8 \\
\hline $322 \mathrm{H} 18 \mathrm{BAB} 32$ & g \\
\hline $322 \mathrm{HLQSA32}$ & 8 \\
\hline $322 \mathrm{H} 190 \mathrm{OA} 2$ & 8 \\
\hline $32 \mathrm{LH} 174 \mathrm{A3} 2$ & 8 \\
\hline $321 \mathrm{H} 175 \mathrm{~A} 32$ & a \\
\hline $320 \mathrm{H176A32}$ & a \\
\hline $320 \mathrm{H} 177 \mathrm{A32}$ & g \\
\hline $320 \mathrm{H} 178 \mathrm{AA} 32$ & 8 \\
\hline $214 \mathrm{H} 031 \mathrm{~A} 32$ & 8 \\
\hline $214 \mathrm{HO} 30 \mathrm{A32}$ & a \\
\hline 2144029232 & $\theta$ \\
\hline $214 \mathrm{HO} 2 \mathrm{BA} 32$ & a \\
\hline $215 \mathrm{H} 027 \mathrm{A32}$ & g \\
\hline $215 \mathrm{H} 026 \mathrm{A32}$ & a \\
\hline $2154025 A 32$ & 9 \\
\hline $2154024 A 32$ & g \\
\hline $215 \mathrm{H} 023 \mathrm{A32}$ & g \\
\hline $215 \mathrm{H} 022 \mathrm{A32}$ & 8 \\
\hline $2164021 A 32$ & 8 \\
\hline $2164020 A 32$ & g \\
\hline $\begin{array}{l}2164019 A 32 \\
216 \mathrm{HO19A32}\end{array}$ & 8 \\
\hline $216 \mathrm{HO} 18 \mathrm{A32}$ & 8 \\
\hline $2174017 \mathrm{A32}$ & 8 \\
\hline $\begin{array}{l}217 \mathrm{HO} 016 \mathrm{A32} \\
210 \mathrm{H} 01 \mathrm{SA32}\end{array}$ & 8 \\
\hline $\begin{array}{l}219 \mathrm{H} 015 \mathrm{SA} 2 \\
218 \mathrm{HO14A32}\end{array}$ & 8 \\
\hline $\begin{array}{l}218 \mathrm{HO} 14 \mathrm{A32} \\
218 \mathrm{HO}=13 \mathrm{A32}\end{array}$ & 8 \\
\hline $210 \mathrm{HO} 013 \mathrm{A32}$ & 8 \\
\hline 219H012A32 & B \\
\hline $219 \mathrm{HO} 11 \mathrm{A32}$ & 8 \\
\hline $219 \mathrm{HO} 10 \mathrm{A3} 2$ & 8 \\
\hline $22 \mathrm{OHOO9A32}$ & $\theta$ \\
\hline $22 \mathrm{OHOOBA32}$ & $\theta$ \\
\hline $220 \mathrm{H} 007 \mathrm{A32}$ & 8 \\
\hline $220 \mathrm{HOOGA32}$ & $\mathbf{g}$ \\
\hline $220 \mathrm{HOOSA32}$ & 9 \\
\hline $220 H 004232$ & 8 \\
\hline $221 \mathrm{HO0} 3 \mathrm{A32}$ & 8 \\
\hline & 8 \\
\hline $2218001 \mathrm{A32}$ & 8 \\
\hline $21113044 A 33$ & 24 \\
\hline $211 \mathrm{H} 043 \mathrm{A3} 3$ & 24 \\
\hline $211 \mathrm{H042A33}$ & 24 \\
\hline $213 \mathrm{H} 035 \mathrm{~A} 33$ & 24 \\
\hline $213 \mathrm{HO} 36 \mathrm{A3} 3$ & 24 \\
\hline $213 \mathrm{HO} 3 \mathrm{7A3} 3$ & 24 \\
\hline $223 H 032 A 33$ & 24 \\
\hline $213 \mathrm{H} 033 \mathrm{A33}$ & 24 \\
\hline $213 \mathrm{HO} 034 \mathrm{A3} 3$ & 24 \\
\hline $323 \mathrm{H} 185 \mathrm{~A} 33$ & 24 \\
\hline $323 \mathrm{H} 1862 \lambda 33$ & 24 \\
\hline $323 \mathrm{H} 187 \mathrm{A33}$ & 24 \\
\hline $322 \mathrm{H} 18 \mathrm{BA} 33$ & 24 \\
\hline $322 \mathrm{H} 189233$ & 24 \\
\hline $3224190 \mathrm{A3} 3$ & 24 \\
\hline $321 \mathrm{H} 174 \mathrm{~A} 33$ & 24 \\
\hline $32 \mathrm{LH} 175 \mathrm{~A} 33$ & 24 \\
\hline $320 \mathrm{H} 176 \mathrm{~A} 33$ & 24 \\
\hline $320 \mathrm{H} 277 \mathrm{A3} 3$ & 24 \\
\hline $320 \mathrm{H17}$ 8A33 & 24 \\
\hline $214 \mathrm{HO} 32 \mathrm{A3} 3$ & 24 \\
\hline $214 \mathrm{HO}_{030 \mathrm{A3} 3}$ & 24 \\
\hline $214 K 029 A 33$ & 24 \\
\hline $214 \mathrm{HO} 8 \mathrm{~A} 33$ & 24 \\
\hline $215 \mathrm{HO27A33}$ & 24 \\
\hline $215 \mathrm{SHO} 26 \mathrm{A3} 3$ & 24 \\
\hline $215 \mathrm{H} 025 \mathrm{~A} 33$ & 24 \\
\hline $215 \mathrm{HO}^{24 \mathrm{~A} 33}$ & 24 \\
\hline $215 \mathrm{H} 023 \mathrm{A3} 3$ & 24 \\
\hline $215 \mathrm{H}_{022 \mathrm{~A}} 33$ & 24 \\
\hline $216 \mathrm{H} 021 \mathrm{~A} 33$ & 24 \\
\hline $216 \mathrm{KO} 020 \mathrm{A33}$ & 24 \\
\hline $216 \mathrm{HO} 19 \mathrm{~A}_{3} 3$ & 24 \\
\hline
\end{tabular}

Figure 9.13 - Case 2, Baseline Schedule (Continued). 
SAUD \& EBRAHTM ALABDULRAZZAK EST REPORT DATE $215 E$ P95 RUN NO. 121 REPORT DATE $215: 46$
PRTMATERA PRCJECT PLWNAER

SEVEN SEAS, CONSTRUCTION OF 51 HOUSES
JAKRA 3 KOUSING PROJECT COMmEN A:?

START DATE LAPR87 FIN DATE LDAFTuS

dATA DATE lapre? PAGE No. Ha

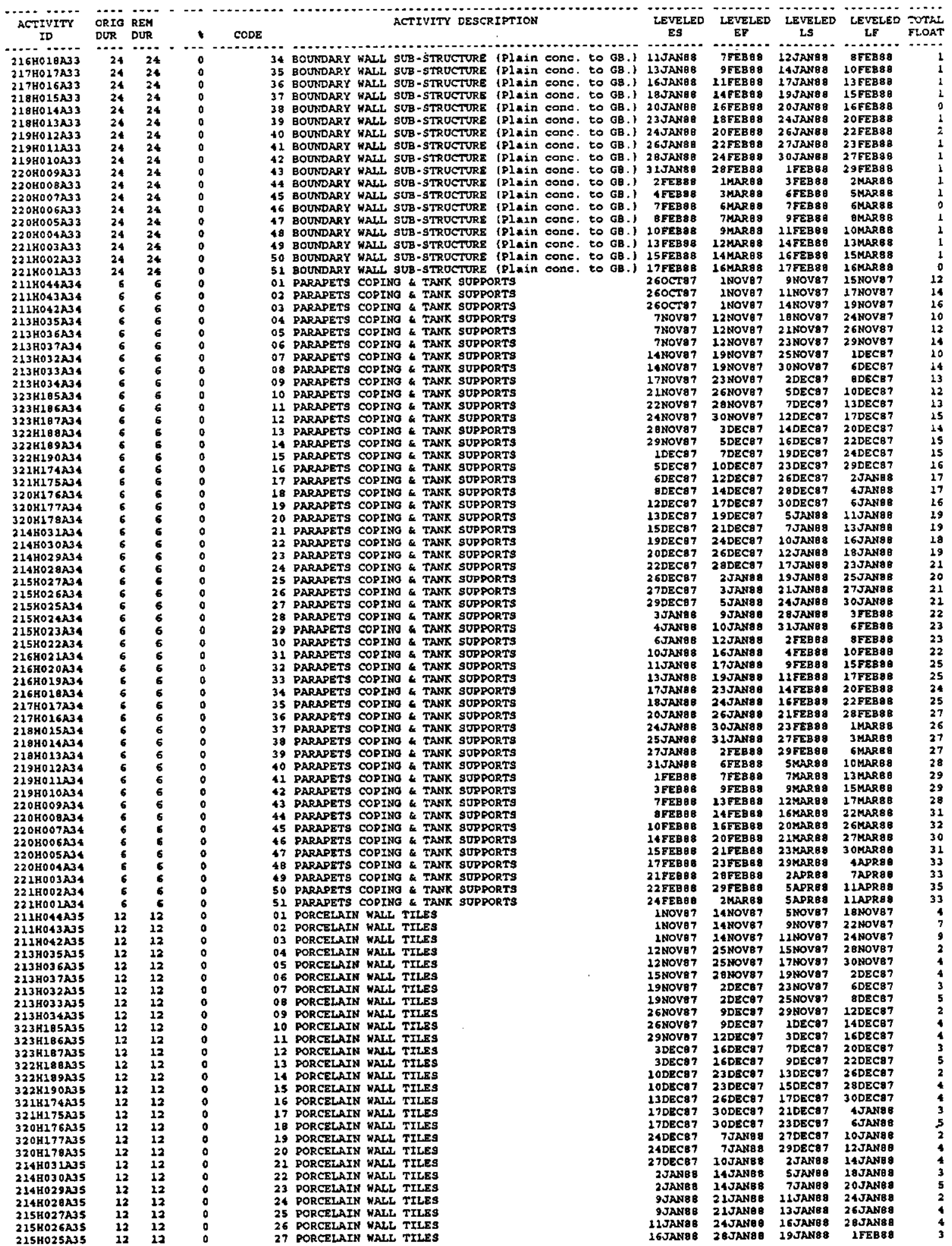

Figure 9.13 - Case 2, Baseline Schedule (Continued). 


\begin{tabular}{|c|c|}
\hline $\begin{array}{l}\text { ACTIVITY } \\
\text { ID }\end{array}$ & $\begin{array}{l}\text { ORIO } \\
\text { DUR }\end{array}$ \\
\hline 215 HO24A35 & 12 \\
\hline $215 \mathrm{H} 023 \mathrm{A35}$ & 12 \\
\hline $215 \mathrm{H} 022 \mathrm{~A} 35$ & 12 \\
\hline $216 \mathrm{HO21A35}$ & 12 \\
\hline $216 \mathrm{HO202035}$ & 12 \\
\hline $216 \mathrm{HO} 19 \mathrm{A3} 5$ & 12 \\
\hline $216 \mathrm{HO1BA35}$ & 12 \\
\hline $217 H 017235$ & 12 \\
\hline $217 \mathrm{HO} 16 \mathrm{~A} 35$ & 12 \\
\hline $218 B 015 A 35$ & 12 \\
\hline $210 \mathrm{HO14A35}$ & 12 \\
\hline $218 \mathrm{HO} 13235$ & 12 \\
\hline $219 \mathrm{HO} 12 \mathrm{A35}$ & 12 \\
\hline $219 H 0211235$ & 12 \\
\hline $219 \mathrm{HO} 10 \mathrm{A3} 5$ & 12 \\
\hline $220 \mathrm{HOOOAA35}$ & 12 \\
\hline $220 \mathrm{HOOBA35}$ & 12 \\
\hline $220 \mathrm{H} 007 \mathrm{A35}$ & 12 \\
\hline $220 \mathrm{HOOOSA35}$ & 12 \\
\hline $220 \mathrm{HOOSA35}$ & 12 \\
\hline $220 \mathrm{HOO4A35}$ & 12 \\
\hline $221 \mathrm{HOO} 3 \mathrm{A3} 5$ & 12 \\
\hline $2211002 \mathrm{A3S}$ & 12 \\
\hline $2214001 \mathrm{A35}$ & 12 \\
\hline $211 \mathrm{H} 044236$ & 10 \\
\hline $21111043 \mathrm{A3} 6$ & 10 \\
\hline $211 \mathrm{H} 042 \mathrm{~A}_{36}$ & 10 \\
\hline $213 \mathrm{H} 035 \mathrm{~S} 36$ & 10 \\
\hline $2134036 A 36$ & 10 \\
\hline $213 \mathrm{HO37A36}$ & 10 \\
\hline $213 \mathrm{HO} 32 \mathrm{A36}$ & 10 \\
\hline $213 \mathrm{H} 033 \mathrm{A3} 6$ & 10 \\
\hline 2134034436 & 10 \\
\hline $323 \mathrm{H} 185 \mathrm{A3} 6$ & 10 \\
\hline $323 \mathrm{H} 286 \mathrm{A36}$ & 10 \\
\hline $323 \mathrm{H} 187 \mathrm{A3} 6$ & 10 \\
\hline $322 \mathrm{H} 18 \mathrm{OA} 36$ & 10 \\
\hline $322 \mathrm{H} 189 \mathrm{~A} 36$ & 10 \\
\hline $322 H 190 A 36$ & 10 \\
\hline $3228174 A 36$ & 10 \\
\hline $3214175 A 36$ & 10 \\
\hline $320 H 176 \mathrm{A3} 6$ & 10 \\
\hline $320 M 1777 A^{3} 6$ & 10 \\
\hline $32042778 \mathrm{A3} 6$ & 10 \\
\hline $214 \mathrm{H} 03$ LA36 & 10 \\
\hline $214 \mathrm{H} 030 \mathrm{A3} 6$ & 10 \\
\hline $214 \mathrm{HO29A36}$ & 10 \\
\hline $214 \mathrm{H} 02 \mathrm{BA} 36$ & 10 \\
\hline $215 K 027 \times 36$ & 10 \\
\hline $215 H 026 A 36$ & 10 \\
\hline $215 H 025 A 36$ & 10 \\
\hline $2154024 A 36$ & 10 \\
\hline $215 H 023 \Omega 36$ & 20 \\
\hline $215 h 0222136$ & 10 \\
\hline $2164021 A 36$ & 10 \\
\hline 2164020236 & 10 \\
\hline $216 \mathrm{HO} 19 \mathrm{A36}$ & 10 \\
\hline $2168018 \times 36$ & 10 \\
\hline 2174017236 & 10 \\
\hline $2174016 A 36$ & 20 \\
\hline $218 B 015 A 36$ & 10 \\
\hline 218 H014A36 & 10 \\
\hline $218 \mathrm{HO} 13 \mathrm{A3} 6$ & 10 \\
\hline $219 \mathrm{HO} 12 \mathrm{A3} 6$ & 10 \\
\hline $219 \mathrm{H} 011236$ & 10 \\
\hline $219 \mathrm{H} 010 \mathrm{A3} 6$ & 10 \\
\hline $220 \mathrm{H} 009 \mathrm{~A} 36$ & 10 \\
\hline $220 \mathrm{HOOSA36}$ & 10 \\
\hline $220 \mathrm{H} 007 \mathrm{~A} 36$ & 10 \\
\hline $220 \mathrm{H} 006 \mathrm{A3} 6$ & 10. \\
\hline $220 \mathrm{HOOSA36}$ & 10 \\
\hline $220 \mathrm{HOOQ4A36}$ & 10 \\
\hline $221 H 003 A 36$ & 10 \\
\hline $2216002 A 36$ & 10 \\
\hline $221 H 001 A 36$ & 10 \\
\hline 211 H044A37 & 6 \\
\hline 2116043037 & 6 \\
\hline $211 \mathrm{H} 042 \mathrm{A3} 7$ & 6 \\
\hline $213 \mathrm{H} 035 \mathrm{A3} 7$ & 6 \\
\hline 2134036237 & 6 \\
\hline $213 \mathrm{H} 037 \mathrm{A37}$ & 6 \\
\hline $2134032 \times 37$ & 6 \\
\hline 2134033317 & 6 \\
\hline $213 \mathrm{H} 034 \mathrm{A3} 7$ & 6 \\
\hline $323 \mathrm{H} 185 \mathrm{~A} 37$ & 6 \\
\hline $323 \mathrm{H} 186 \mathrm{~A} 37$ & 6 \\
\hline $323 \mathrm{H} 187 \mathrm{A3} 7$ & 6 \\
\hline $322 \mathrm{H} 188 \mathrm{BA3} 7$ & 6 \\
\hline $322 \mathrm{H} 189 \mathrm{~A} 37$ & 6 \\
\hline $3224190 A 37$ & 6 \\
\hline $321 \% 174237$ & 6 \\
\hline $321 \mathrm{H175A37}$ & 6 \\
\hline $3201176 A 37$ & 6 \\
\hline $320 K 1777237$ & 6 \\
\hline $320 \mathrm{H} 278237$ & 6 \\
\hline $214 \mathrm{HO} 31 \mathrm{A3} 7$ & 6 \\
\hline
\end{tabular}

Figure 9.13 - Case 2, Baseline Schedule (Continued). 


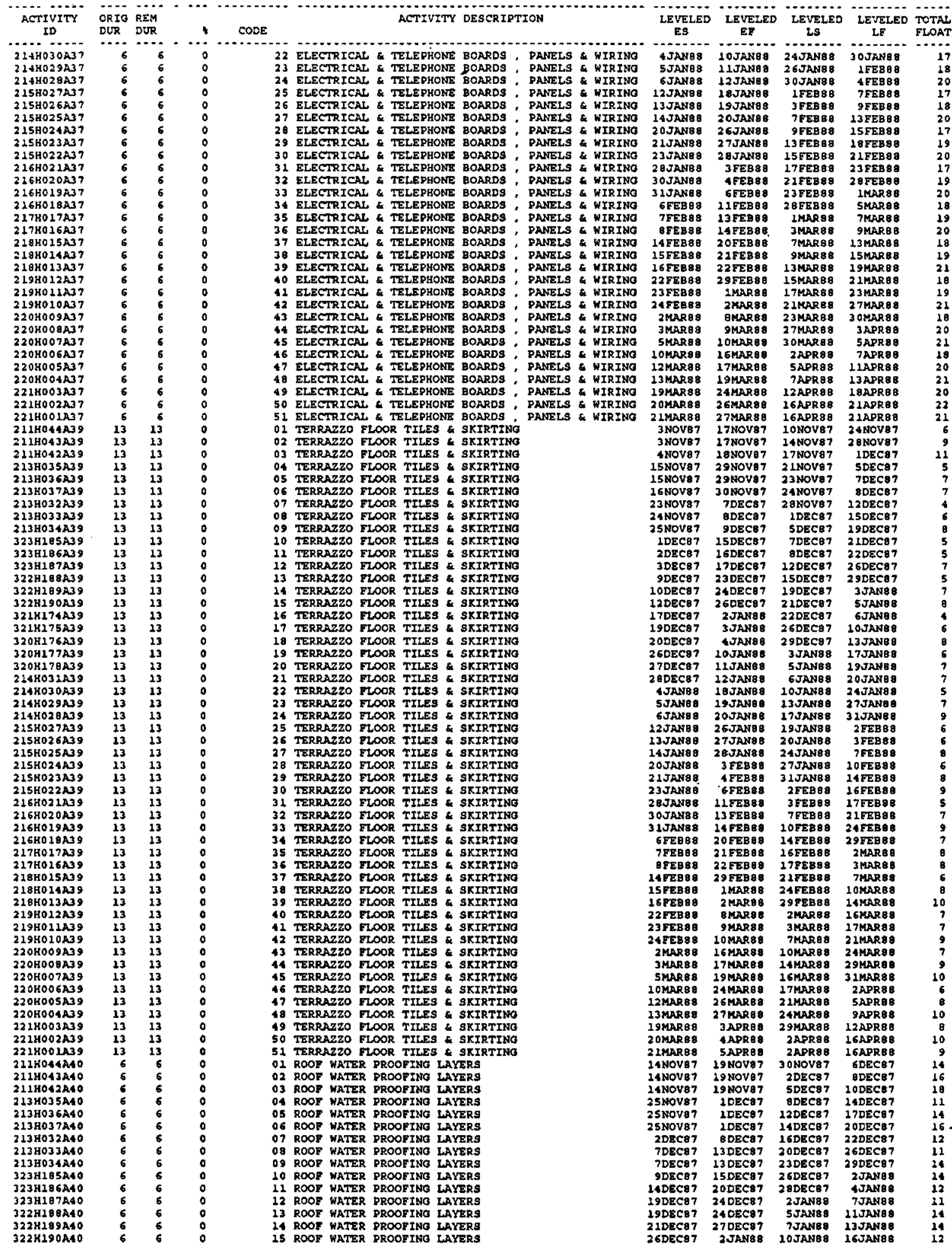

Figure 9.13 - Case 2, Baseline Schedule (Continued). 


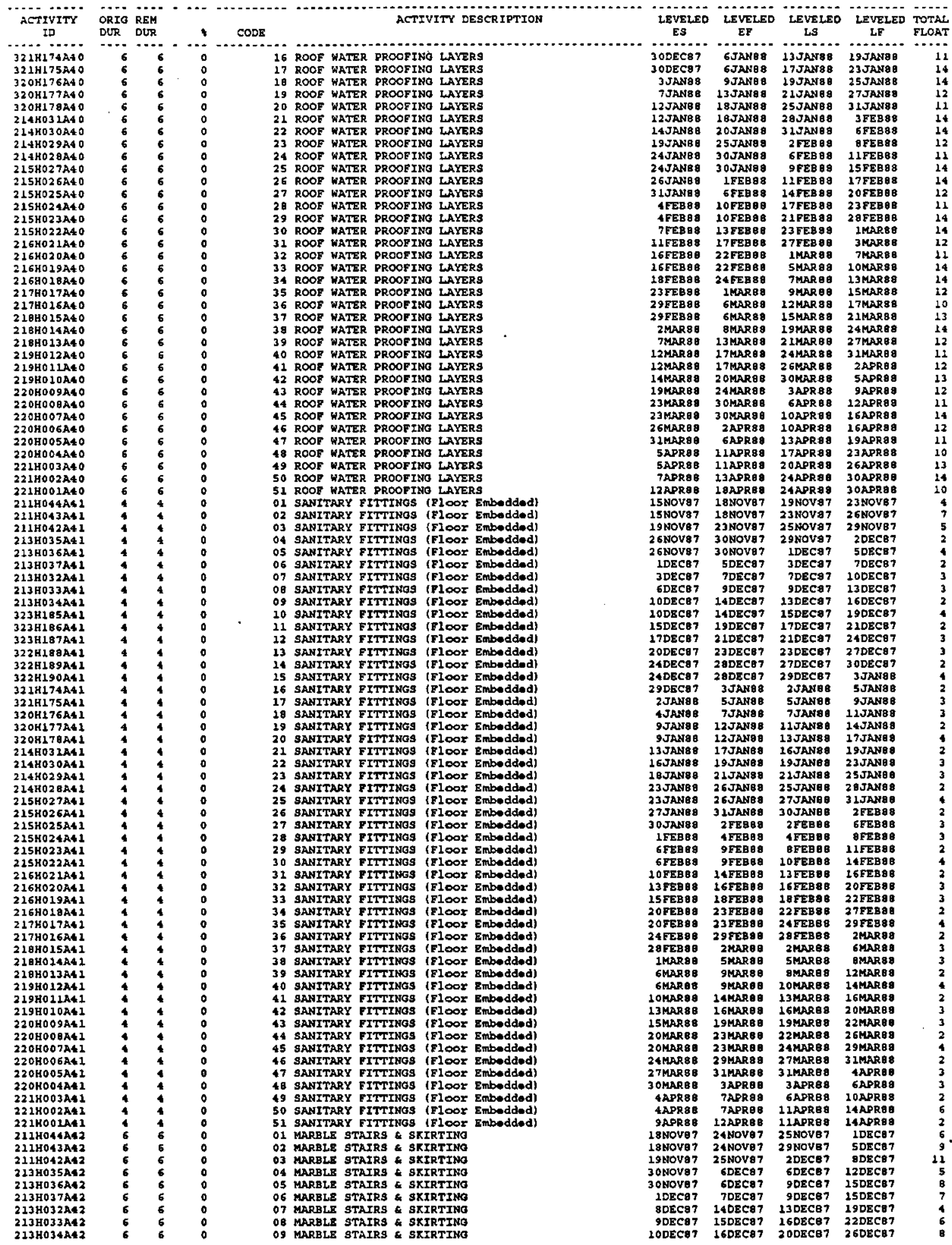

Figure 9.13 - Case 2, Baseline Schedule (Continued). 


\begin{tabular}{|c|}
\hline ACTIVITY \\
\hline \\
\hline 323 HLASA42 \\
\hline $323 \mathrm{H186A42}$ \\
\hline $3234187 \mathrm{A42}$ \\
\hline $322 \mathrm{H} 1 \mathrm{BOA42}$ \\
\hline $322 \mathrm{H189A42}$ \\
\hline $322 \mathrm{H} 190 \mathrm{~A} 42$ \\
\hline $321 H 174 A 42$ \\
\hline $32 \mathrm{LH} 175 \mathrm{~A} 42$ \\
\hline $320 \mathrm{H} 176 \mathrm{A42}$ \\
\hline $3204177 A 42$ \\
\hline $320 \mathrm{H} 170 \mathrm{~A} 42$ \\
\hline $214+4031 A 42$ \\
\hline $2144030 A \$ 2$ \\
\hline $214 \mathrm{HO} 29 \mathrm{A4} 2$ \\
\hline $214 \mathrm{HO} 2 \mathrm{BA} 42$ \\
\hline $215 \mathrm{H} 027 \mathrm{A42}$ \\
\hline $215 H 026 A 42$ \\
\hline $215 H 025 A 42$ \\
\hline $2154024 A 42$ \\
\hline 215 H023A42 \\
\hline $215 H 022 A 42$ \\
\hline $216 \mathrm{HO} 21 \mathrm{A42}$ \\
\hline $216 \mathrm{HO2OA42}$ \\
\hline 216H019A42 \\
\hline $216 \mathrm{HO} 19 \mathrm{~A}+2$ \\
\hline $217 \mathrm{HO} 17 \mathrm{A42}$ \\
\hline $2174016 \mathrm{AE2}$ \\
\hline $21 \mathrm{BH} 015 \mathrm{~A} 42$ \\
\hline $218 \mathrm{HO} 14 \mathrm{A42}$ \\
\hline $21 B H O 13 A 42$ \\
\hline $219 \mathrm{HO} 12 \mathrm{A42}$ \\
\hline $219 H 011 \mathrm{A42}$ \\
\hline $219 \mathrm{HO1OA42}$ \\
\hline 22 OHOO9A42 \\
\hline $220 \mathrm{HOOOBA42}$ \\
\hline $220 \mathrm{HOO} 7 \mathrm{A42}$ \\
\hline $230 \mathrm{HOOOAA42}$ \\
\hline 22 OHOO5A42 \\
\hline $220 \mathrm{HOO} 4 \mathrm{A42}$ \\
\hline $221 H 003 A 42$ \\
\hline $221 \mathrm{HOO2A42}$ \\
\hline 221 HOOLAt2 \\
\hline $2114044 A 43$ \\
\hline $211 K 043 A 43$ \\
\hline $211{ }_{0} 042 A 43$ \\
\hline $213 H 035 A 43$ \\
\hline $213 H 036 \mathrm{A43}$ \\
\hline $213 \mathrm{H} 037 \mathrm{A4} 3$ \\
\hline $213 \mathrm{HO}_{2} 2 \mathrm{A43}$ \\
\hline $213 H_{03} 3 \mathrm{~A} 43$ \\
\hline $213 H 034 A 43$ \\
\hline 323 H185A43 \\
\hline $323 \mathrm{H1}_{18 \mathrm{A4} 3}$ \\
\hline $323 \mathrm{H} 187 \mathrm{A6} 3$ \\
\hline $322 H 188 A 43$ \\
\hline 322 K1E9A43 \\
\hline $322 \mathrm{H} 190 \mathrm{AS3}$ \\
\hline $32 \mathrm{LH} 174 \mathrm{~A} 43$ \\
\hline $321 \mathrm{H} 175 \mathrm{~A} 43$ \\
\hline $320 \mathrm{H} 176 \mathrm{A4} 3$ \\
\hline $320 \mathrm{H} 177 \mathrm{A43}$ \\
\hline $320 \mathrm{HI} 78 \mathrm{A4} 3$ \\
\hline $214 \mathrm{HO} 032 \mathrm{A4} 3$ \\
\hline $214 \mathrm{HO30A43}$ \\
\hline $214 \mathrm{HO29A43}$ \\
\hline $2144028 A 43$ \\
\hline $215 \mathrm{HO27A43}$ \\
\hline $215 \mathrm{HO26A43}$ \\
\hline $215 \mathrm{HO2SA43}$ \\
\hline $215 \mathrm{HO} 24 \mathrm{~A} 43$ \\
\hline $215 H 023 \AA 43$ \\
\hline $215 H 022 A 43$ \\
\hline $226 \mathrm{KO} 21 \mathrm{~A} 43$ \\
\hline $216 \mathrm{HO} 20 \mathrm{A4} 3$ \\
\hline $216 \mathrm{HO} 19 \mathrm{A43}$ \\
\hline $2164018 A 63$ \\
\hline $217 \mathrm{HO17A43}$ \\
\hline $217 \mathrm{HO16A43}$ \\
\hline $21 \mathrm{BH} 015 \mathrm{~A} 43$ \\
\hline $210 \mathrm{HO} 14 \mathrm{A43}$ \\
\hline $21 \mathrm{BHO} 023 \mathrm{A43}$ \\
\hline $219 H 012 A 43$ \\
\hline $219 \mathrm{HOL1A43}$ \\
\hline $219 \mathrm{HO} 0 \mathrm{OA43}$ \\
\hline $220 \mathrm{HOOOAA43}$ \\
\hline $220 \mathrm{HOOOA43}$ \\
\hline 220 HOO7A43 \\
\hline $220 \mathrm{HOOSA43}$ \\
\hline 22 HOOOSA43 \\
\hline $220 \mathrm{HOO} 4 \mathrm{A43}$ \\
\hline $221 \mathrm{H} 003 \mathrm{A4} 3$ \\
\hline $221 \mathrm{H} 002 \mathrm{~A} 43$ \\
\hline $221 \mathrm{HOOLA43}$ \\
\hline 211 H0S4A44 \\
\hline $221 \mathrm{HO} 043 \mathrm{A44}$ \\
\hline $211 H 042 A 44$ \\
\hline
\end{tabular}

Figure 9.13 - Case 2, Baseline Schedule (Continued). 
SAUD \& EBRAHTM ALABDULRAZZAK EST

PEPORT DATE 21 SEP9S RUN NO. 121

Baseline Schedule by

PRTMAVERA PROJECT PLANNER

SEVEN SEAS, CONSTRUCTION OF 51 hOUSES
JAHRA 3 HOUSING PROJECT cOmmON aAE

START DATE IAPR8? FIN DATE 13AUG3:

DATA DATE LAPRB7 PAGE NO.

23

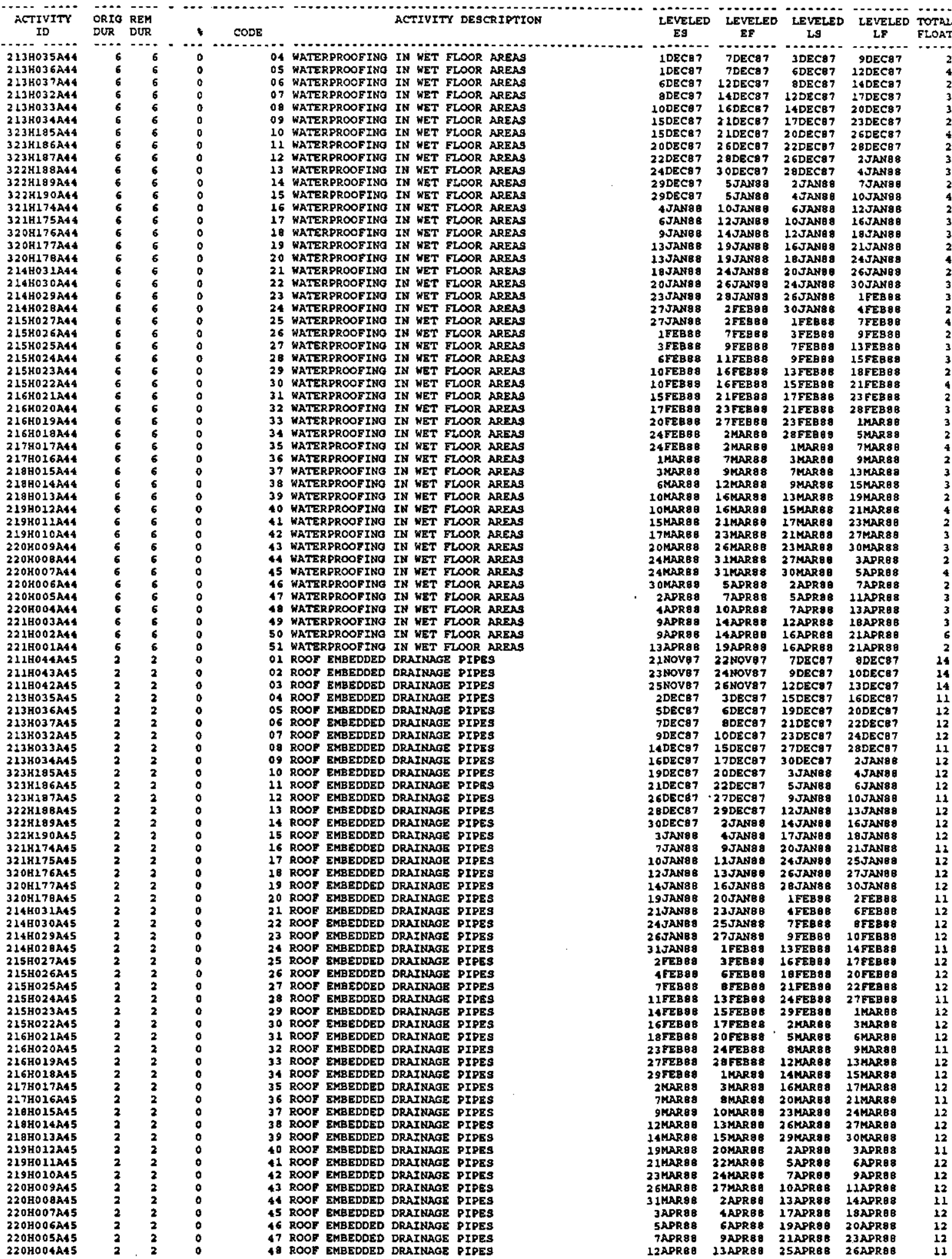

Figure 9.13 - Case 2, Baseline Schedule (Continued). 
SAUD \& EBrahtM ALABDULRAZZAX EST.

REPORT DATE 2 1SEP95 RUN NO. 121 $15: 46$
PRIMAVERA PROJECT PLANNER

SEVEN SEAS, CONSTRUCTION OF 51 HOUSES
JAHRA 3 HOUSING PROJEET common ace

START DATE IAPRE7 FTN DATE IJAUGA

DATA DATE IAPR8? PAGE NO. 24

\begin{tabular}{|c|c|}
\hline $\begin{array}{l}\text { ACTIVITT } \\
\text { ID }\end{array}$ & $\begin{array}{l}\text { ORIO } \\
\text { DUR }\end{array}$ \\
\hline $221 K 003 A 45$ & 2 \\
\hline $221 H 002 A 45$ & 2 \\
\hline $221 \mathrm{H} 001 \mathrm{A4} 5$ & 2 \\
\hline $211 \mathrm{H044A46}$ & 7 \\
\hline $211 H 043 A 46$ & 7 \\
\hline $2114042 A 46$ & 7 \\
\hline $213 H 035 A 46$ & 7 \\
\hline $213 \mathrm{H036A46}$ & 7 \\
\hline $213 H 037 A 46$ & 7 \\
\hline $213 H 032 A 46$ & 7 \\
\hline $213 H 033 A 46$ & 7 \\
\hline 2134034246 & 7 \\
\hline $323 \mathrm{H} 185 \mathrm{~A} 46$ & 7 \\
\hline $323 \mathrm{H196A46}$ & 7 \\
\hline $323 H 187 A 46$ & 7 \\
\hline $322 \mathrm{H18} 8 \mathrm{~A}+6$ & 7 \\
\hline $322 \mathrm{HLO} 9 \mathrm{A4} 6$ & 7 \\
\hline $322 H 190 A 46$ & 7 \\
\hline $32111274 A 46$ & 7 \\
\hline $321 H 175 A 46$ & 7 \\
\hline $320 H 176 A 46$ & 7 \\
\hline $320 \mathrm{H} 177 \mathrm{A46}$ & $?$ \\
\hline $320 H 178 A 46$ & 7 \\
\hline $214 H 031 A 46$ & 7 \\
\hline 2144030246 & 7 \\
\hline $214 H 029 A 46$ & 7 \\
\hline $314 \mathrm{HO2BA46}$ & 7 \\
\hline $215 H 027 A 46$ & 7 \\
\hline $215 \mathrm{HO2} 6 \mathrm{A4} 6$ & 7 \\
\hline $215 \mathrm{HO} 25 \mathrm{A4} 6$ & 7 \\
\hline $215 H 024 A 46$ & 7 \\
\hline $215 H 023 \mathrm{A4} 6$ & 7 \\
\hline $215 \mathrm{H} 022 \mathrm{At} 6$ & $?$ \\
\hline $216 \mathrm{H} 021 \mathrm{A4} 6$ & 7 \\
\hline $216 H 020 A 46$ & 7 \\
\hline 2164019246 & 7 \\
\hline $216 \% 018 A 46$ & 7 \\
\hline $2174017 \mathrm{A4} 6$ & 7 \\
\hline $217 \% 016 A 46$ & 7 \\
\hline $218 H 015 A 46$ & 7 \\
\hline $21 B H 014 A 46$ & 7 \\
\hline $2184013 A 46$ & 7 \\
\hline $219 H 012 A 46$ & 7 \\
\hline $219 H_{0} 11 \mathrm{A4} 6$ & 7 \\
\hline $219 H 010 A 46$ & 7 \\
\hline 220 HOO9A46 & 7 \\
\hline $220 \mathrm{HOOBA46}$ & 7 \\
\hline $22010007 A 46$ & 7 \\
\hline 220H006A46 & 7 \\
\hline $220 H 005 A 46$ & 7 \\
\hline $220 \mathrm{H} 004 \mathrm{~A} 46$ & 7 \\
\hline 221H0O3A46 & 7 \\
\hline $221 \mathrm{H} 002 \mathrm{~A} 46$ & 7 \\
\hline $221 \mathrm{H} 001 \mathrm{A4} 6$ & 7 \\
\hline $211 \mathrm{HO44A47}$ & 3 \\
\hline $213 H 043447$ & 3 \\
\hline $211 H 042 A 47$ & 3 \\
\hline 213H035A47 & 3 \\
\hline $223 \times 036 \mathrm{A4}$ ? & 3 \\
\hline $213 H 037 A 47$ & 3 \\
\hline $213 \mathrm{H} 032 \mathrm{A4} 7$ & 3 \\
\hline $213 \mathrm{H} 033 \mathrm{~A} 47$ & 3 \\
\hline 213 Ho34A47 & 3 \\
\hline $323 \mathrm{H} 185 \mathrm{A4} 7$ & 3 \\
\hline $323 \mathrm{H1B6A4} 7$ & 3 \\
\hline $323 H 187 \mathrm{A4} 7$ & 3 \\
\hline $322 \mathrm{H} 188 \mathrm{AA}$ ? & 3 \\
\hline $322 \mathrm{H} 189 \mathrm{A4} 7$ & 3 \\
\hline $322 \mathrm{H} 190 \mathrm{~A} 47$ & 3 \\
\hline $321 \mathrm{H174A4}$ ? & 3 \\
\hline $321 \mathrm{H} 175 \mathrm{A4} 7$ & $\mathbf{3}$ \\
\hline $320 \mathrm{H176A47}$ & 3 \\
\hline $320 H 177 A 47$ & 3 \\
\hline $320 \mathrm{H} 178 \mathrm{A4} 7$ & 3 \\
\hline $214 H 031 A 47$ & 3 \\
\hline 214H030A47 & 3 \\
\hline $214 H 029447$ & 3 \\
\hline $2141028 A 47$ & 3 \\
\hline $235 \mathrm{HO27A47}$ & 3 \\
\hline $215 H_{026 \mathrm{A4}} 7$ & 3 \\
\hline $215 H 025 A 47$ & 3 \\
\hline 215 H024A4? & 3 \\
\hline $215 H 023 A 47$ & 3 \\
\hline $225 \mathrm{HO22A47}$ & 3 \\
\hline $216 \mathrm{HO2} 1 \mathrm{A47}$ & 3 \\
\hline $216 H 020 A 47$ & 3 \\
\hline $216 H 019 A 47$ & 3 \\
\hline $216 \mathrm{HO18A4}$ ? & 3 \\
\hline $217 \mathrm{HO17A47}$ & 3 \\
\hline 217 H016A47 & 3 \\
\hline 218H015A47 & 3 \\
\hline $218 H 014 A 47$ & 3 \\
\hline $218 \mathrm{H} 013 \mathrm{~A} 47$ & 3 \\
\hline 219H012A47 & 3 \\
\hline $219 H 011 \mathrm{A4} 7$ & 3 \\
\hline $219 H 010 A 47$ & 3 \\
\hline
\end{tabular}

Figure 9.13 - Case 2, Baseline Schedule (Continued). 
SAUD \& EBRAHIM ALABDULRAZZAK EST

REPORT DATE 21 SEPSS RUN NO. 121

Baceline sehodile by Typical Aetivity 15 ES
PRIMAVERA PROJECT PLANNER

SEVEN SEAS, CONSTRUCTION OF 51 HOUSES
JAHRA 3 HOUSING PROAECT COMMON ACE

START DATE LAPRE? FIN DATE L3AUG9B

DATA DATE LAPRB? PAGE NO. 25

\begin{tabular}{|c|c|c|c|c|}
\hline $\begin{array}{c}\text { ACTIVITY } \\
\text { ID }\end{array}$ & $\begin{array}{l}\text { ORIO } \\
\text { DUR }\end{array}$ & $\begin{array}{l}\text { REM } \\
\text { DUR }\end{array}$ & 1 & CODE \\
\hline $220 \mathrm{H} 009 \mathrm{A4} 7$ & 3 & 3 & 0 & 43 \\
\hline $220 \mathrm{HOOBA4}$ ? & 3 & 3 & 0 & 44 \\
\hline $220 \mathrm{H} 007 \mathrm{A4} 7$ & 3 & 3 & 0 & 45 \\
\hline $220 \mathrm{HOO} 0 \mathrm{AA4}$ ? & 3 & 3 & 0 & 46 \\
\hline $220 \mathrm{HOOSA47}$ & 3 & 3 & 0 & 47 \\
\hline $220 \mathrm{HOO4A47}$ & 3 & 3 & 0 & 48 \\
\hline $221 \mathrm{H} 003 \mathrm{A4} 7$ & 3 & 3 & 0 & 49 \\
\hline $221 \mathrm{H} 002 \mathrm{A4} 7$ & 3 & 3 & 0 & 50 \\
\hline 221H001A47 & 3 & 3 & 0 & 51 \\
\hline 211 HO44A48 & 6 & 6 & 0 & 01 \\
\hline $2121 \mathrm{HO} 33 \mathrm{A48}$ & 6 & 6 & 0 & 02 \\
\hline $21140042 A 40$ & 6 & 6 & 0 & 03 \\
\hline $213 \mathrm{HO} 35 \mathrm{~A} 4 \mathrm{~B}$ & 6 & 6 & 0 & 04 \\
\hline $213 \mathrm{HO} 36 \mathrm{A4O}$ & 6 & 6 & 0 & os \\
\hline $223 \mathrm{H037A48}$ & 6 & 6 & 0 & 06 \\
\hline $2134032 \mathrm{~A} 4 \mathrm{~B}$ & 6 & 6 & 0 & 07 \\
\hline $213 \mathrm{HO} 33 \mathrm{A48}$ & 6 & 6 & 0 & OB \\
\hline $213 H 034 A 4 B$ & 6 & 6 & 0 & 09 \\
\hline 323 HIOSA48 & 6 & 6 & 0 & 20 \\
\hline $323 \mathrm{H1} 66 \mathrm{AAB}$ & 6 & 6 & 0 & 11 \\
\hline 323H1日7A4O & 6 & 6 & 0 & 12 \\
\hline $322 \mathrm{H} 18 \mathrm{AA} 4 \mathrm{O}$ & 6 & 5 & 0 & 13 \\
\hline $322 \mathrm{H} 1 \mathrm{BAA} 4$ & 6 & 6 & 0 & 14 \\
\hline $322 \mathrm{H} 190 \mathrm{~A} 4 \mathrm{~B}$ & 6 & 6 & 0 & 15 \\
\hline $321 \mathrm{H} 274 \mathrm{A48}$ & 6 & 6 & 0 & 16 \\
\hline $321 \mathrm{H175A4O}$ & 6 & 6 & 0 & 17 \\
\hline $320 \mathrm{H} 176 \mathrm{A4} 8$ & 6 & 6 & 0 & 18 \\
\hline 3201777 A48 & 6 & 6 & 0 & 19 \\
\hline $320 \mathrm{OH} 78 \mathrm{~A} 4 \mathrm{~B}$ & 6 & 6 & 0 & 20 \\
\hline $214 \mathrm{HO} 32 \mathrm{A4} \mathrm{O}$ & 6 & 6 & 0 & 21 \\
\hline $214 \mathrm{HO} 30 \mathrm{A48}$ & $\begin{array}{l}6 \\
6\end{array}$ & $\begin{array}{l}6 \\
6\end{array}$ & 0 & $\begin{array}{l}22 \\
23\end{array}$ \\
\hline $\begin{array}{l}214 \mathrm{HO29A48} \\
214 \mathrm{HO2SA4B}\end{array}$ & $\begin{array}{l}6 \\
6\end{array}$ & 6 & 0 & $\begin{array}{l}23 \\
24\end{array}$ \\
\hline $215 H 027 A 49$ & 6 & 6 & 0 & 25 \\
\hline $215 \mathrm{H} 026 \mathrm{~A} 4 \mathrm{~g}$ & 6 & 6 & 0 & 26 \\
\hline $215 \mathrm{HO25A4 \theta}$ & 6 & 6 & 0 & 27 \\
\hline 215 HO24A48 & 6 & 6 & 0 & 29 \\
\hline $215 \mathrm{HO2} 3 \mathrm{A48}$ & 6 & 6 & 0 & 29 \\
\hline $215 \mathrm{HO} 22 \mathrm{A4O}$ & 6 & 6 & 0 & 30 \\
\hline $216 \mathrm{HO2} 1 \mathrm{~A} 4 \mathrm{~B}$ & 6 & 6 & 0 & 31 \\
\hline $216 \mathrm{HO2OA48}$ & 6 & 6 & 0 & 32 \\
\hline 236HO19A4B & 6 & 6 & 0 & 33 \\
\hline $216 \mathrm{HO}$ QAA48 & 6 & 6 & 0 & 34 \\
\hline 217HO17A48 & 6 & 6 & 0 & 35 \\
\hline $217 \mathrm{HO16A48}$ & 6 & 6 & 0 & 36 \\
\hline $228 \mathrm{HO} 15 \mathrm{A4} \mathrm{B}$ & 6 & 6 & 0 & 37 \\
\hline & $\begin{array}{l}6 \\
6\end{array}$ & 6 & $:$ & $\begin{array}{l}38 \\
39\end{array}$ \\
\hline $\begin{array}{l}218 H 013 A 48 \\
219 H 022 A 48\end{array}$ & 6 & 6 & 0 & 40 \\
\hline $219 H O 11 A 4 B$ & 6 & 6 & 0 & 41 \\
\hline 219 HO10A48 & 6 & 6 & 0 & 42 \\
\hline $220 \mathrm{HOO} A 48$ & 6 & 6 & 0 & 43 \\
\hline $220 \mathrm{HOOOA4}$ & 6 & 6 & 0 & 44 \\
\hline $220 \mathrm{HOO}$ A48 & 6 & 6 & 0 & 45 \\
\hline $2201006 \mathrm{A4} 8$ & 6 & 6 & 0 & 46 \\
\hline $\begin{array}{l}220 \mathrm{HOOSA4B} \\
220 \mathrm{H} 004 \mathrm{~A} 4 \mathrm{~B}\end{array}$ & 6 & 6 & $:$ & $\begin{array}{l}47 \\
48\end{array}$ \\
\hline $\begin{array}{l}220 \mathrm{H} 004 \mathrm{A4} 8 \\
221 \mathrm{H} 003 \mathrm{A4g}\end{array}$ & 6 & 6 & 0 & $\begin{array}{l}48 \\
49\end{array}$ \\
\hline $2211 \% 002 \mathrm{A48}$ & 6 & 6 & 0 & 50 \\
\hline $221 \mathrm{H} 001 \mathrm{A48}$ & 6 & 6 & 0 & 51 \\
\hline $212 H 044 A 49$ & 10 & 10 & 0 & 01 \\
\hline $211 \mathrm{HO43A49}$ & 10 & 10 & 0 & 02 \\
\hline $21111042 \mathrm{A4} 9$ & 10 & 10 & 0 & 03 \\
\hline $2134035 A 49$ & 10 & 10 & 0 & 04 \\
\hline $213 \mathrm{HO} 36 \mathrm{A49}$ & 10 & 10 & 0 & os \\
\hline $213 \mathrm{HO} 37 \mathrm{A4} 9$ & 10 & 10 & 0 & 06 \\
\hline $213 \mathrm{HO} 32 \mathrm{A4} 9$ & 10 & 10 & 0 & 07 \\
\hline $213 \mathrm{HO} 33 \mathrm{A49}$ & 10 & 10 & 0 & 08 \\
\hline 213HO34A49 & 10 & 10 & 0 & 09 \\
\hline $323 \mathrm{HL} 125 \mathrm{A4} 9$ & 10 & 10 & 0 & 10 \\
\hline $323 \mathrm{H} 186 \mathrm{A4} 9$ & 10 & 10 & 0 & 11 \\
\hline $323 \mathrm{H} 197 \mathrm{~A} 49$ & 10 & 10 & 0 & 12 \\
\hline $322 \mathrm{H} 18 \mathrm{BA} 49$ & 10 & 10 & 0 & 23 \\
\hline 322H189A49 & 10 & 10 & 0 & 14 \\
\hline $322 \mathrm{H190A49}$ & 10 & 20 & 0 & 15 \\
\hline $322 \mathrm{H} 174 \mathrm{A49}$ & 10 & 10 & 0. & 16 \\
\hline $321 \mathrm{H275A49}$ & 10 & 10 & 0 & 17 \\
\hline $320 \mathrm{H176A49}$ & 10 & 10 & 0 & 18 \\
\hline $320 \mathrm{H177A49}$ & 10 & 10 & 0 & 19 \\
\hline $320 \mathrm{H} 278 \mathrm{~A} 49$ & 10 & 10 & 0 & 20 \\
\hline 214H031A49 & 10 & 10 & 0 & 21 \\
\hline 214 H030A49 & 10 & 10 & 0 & 22 \\
\hline $214 \mathrm{HO29A49}$ & 10 & 10 & 0 & 23 \\
\hline $214 K 028 A 49$ & 10 & 10 & 0 & 24 \\
\hline $215 H 027 A 49$ & 10 & 10 & 0 & 25 \\
\hline $215 H 026 A 49$ & 10 & 10 & 0 & 26 \\
\hline $215 H 025 A 49$ & 10 & 10 & 0 & 27 \\
\hline $215 H 024 A 49$ & 10 & 10 & 0 & 28 \\
\hline $215 \mathrm{HO} 23 \mathrm{A4} 9$ & 10 & 10 & 0 & 29 \\
\hline $215 H 022 A 49$ & 20 & 10 & 0 & 30 \\
\hline $216 \mathrm{HO2} 1 \mathrm{A49}$ & 10 & 10 & 0 & 31 \\
\hline $216 H 020 A 49$ & 10 & 10 & 0 & 32 \\
\hline $216 \% 029 A 49$ & 10 & 10 & 0 & 33 \\
\hline 216HO18A49 & 10 & 10 & 0 & 34 \\
\hline $2174027 A 49$ & 10 & 10 & 0 & 35 \\
\hline $217 \mathrm{HO16A49}$ & 10 & 10 & 0 & 36 \\
\hline
\end{tabular}

Figure 9.13 - Case 2, Baseline Schedule (Continued). 


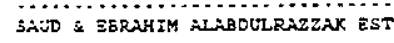

PEPOPT DATE IISEP95 RLN NO 121

Bakoline schedule by Trpical Activity \& ES
PRIMAVERA PROJECT PLANNER

3EVEN SEAS, CONSTRUCTION OF 51 HOUSES
JAHPA 3 hOUSING PROJECT fOMmOn act

START DATE LAPRE? FIN DATE L3AUG98

DATA DATE LAPRS; PAOE NO. 26

\begin{tabular}{|c|c|c|}
\hline $\begin{array}{l}\text { ACTIVITY } \\
\text { ID }\end{array}$ & $\begin{array}{l}\text { ORIO } \\
\text { DUR }\end{array}$ & $\begin{array}{l}\text { REM } \\
\text { DUR }\end{array}$ \\
\hline 2184015449 & 10 & 10 \\
\hline $2194014 A+9$ & 10 & 10 \\
\hline $2184013 \mathrm{~A} 49$ & 10 & 20 \\
\hline 2194012A49 & 10 & 10 \\
\hline 219HO11A49 & 10 & 10 \\
\hline $219 \mathrm{HO} 0 \mathrm{OA4} 9$ & 10 & 10 \\
\hline $220 \mathrm{HOOOA49}$ & 10 & 10 \\
\hline $220 \% 008 A 49$ & 10 & 20 \\
\hline $220 H 007 A 49$ & 10 & 10 \\
\hline $220 \mathrm{HOO6A49}$ & 10 & 20 \\
\hline $2208005 A 49$ & 10 & 10 \\
\hline $2204004 A 49$ & 10 & 20 \\
\hline $2218003 A 49$ & 10 & 10 \\
\hline $221 \mathrm{HOO} 2 \mathrm{A4g}$ & 10 & 10 \\
\hline $221 H 001 A 49$ & 10 & 10 \\
\hline 211 1K044A50 & 5 & 5 \\
\hline $211 \% 043 A 50$ & 5 & 5 \\
\hline $21111042 A 50$ & $\mathbf{5}$ & 5 \\
\hline 213H035ASO & 5 & 5 \\
\hline 213 HO36A5O & $\mathbf{5}$ & 5 \\
\hline $213 \mathrm{HO} 3 \mathrm{ASO} \mathrm{O}$ & $\mathbf{5}$ & 5 \\
\hline $2134032 A 50$ & 5 & 5 \\
\hline 213 H033A50 & $\mathbf{5}$ & 5 \\
\hline $213 \mathrm{HO34A5O}$ & $\mathbf{5}$ & 5 \\
\hline 323 H105ASO & 5 & 5 \\
\hline 323 H106ASO & $\mathbf{s}$ & 5 \\
\hline 323 H187A5O & 5 & 5 \\
\hline $322 \mathrm{H1O8A5O}$ & 5 & 5 \\
\hline $322 \mathrm{H} 1 \mathrm{Q9A5O}$ & $\mathbf{5}$ & 5 \\
\hline $322 \mathrm{H190A50}$ & 5 & 5 \\
\hline 32111744250 & 5 & 5 \\
\hline $32114175 A 50$ & 5 & 5 \\
\hline $320 H 176 A 50$ & $\mathbf{5}$ & 5 \\
\hline $320 H 177 A 50$ & 5 & 5 \\
\hline 32OH178ASO & 5 & 5 \\
\hline 214 HO31ASO & 5 & 5 \\
\hline 21411030050 & 5 & 5 \\
\hline $214 H 029 A 50$ & 5 & 5 \\
\hline 214HO2QASO & 5 & 5 \\
\hline $215 \mathrm{HO} 27 \mathrm{ASO}$ & 5 & $\mathbf{5}$ \\
\hline $215 \mathrm{H} 026 \mathrm{A5O}$ & 5 & 5 \\
\hline $215 \mathrm{H} 025 \mathrm{~A} 5 \mathrm{O}$ & 5 & 5 \\
\hline $215 H 024 A 50$ & 5 & 5 \\
\hline $215 H 023 \mathrm{ASO}$ & 5 & 5 \\
\hline $215 H 022 A 50$ & $\mathbf{5}$ & 5 \\
\hline $216 \mathrm{HO21A50}$ & 5 & 5 \\
\hline $216 \mathrm{HO} 20 \mathrm{ASO}$ & 5 & 5 \\
\hline 216HO19ASO & 5 & 5 \\
\hline 216KO1BASO & 5 & 5 \\
\hline $2174017 A 50$ & 5 & 5 \\
\hline $2174016 A 50$ & 5 & 5 \\
\hline 218 OHO15A50 & 5 & 5 \\
\hline $218 H 014250$ & 5 & 5 \\
\hline 218HO13ASO & 5 & 5 \\
\hline 219HO12ASO & 5 & 5 \\
\hline $219 \mathrm{HO} 11 \mathrm{ASO}$ & $\mathbf{3}$ & 5 \\
\hline $219 \mathrm{HO10A5O}$ & 5 & 5 \\
\hline $220 \mathrm{HOOOAASO}$ & $\mathbf{5}$ & 5 \\
\hline 22 OHOOBASO & 5 & 5 \\
\hline $220 \mathrm{HOO} 7 \mathrm{ASO}$ & $\mathbf{s}$ & 5 \\
\hline $220 \mathrm{HOOO6DSO}$ & 5 & 5 \\
\hline $220 \mathrm{HOOSASO}$ & 5 & 5 \\
\hline $220 H 004 A 50$ & 5 & 5 \\
\hline 22 LHO03ASO & 5 & 5 \\
\hline $22110002 A 50$ & 5 & 5 \\
\hline $221 \mathrm{HOO} 1 \mathrm{ASO}$ & 5 & 5 \\
\hline 211H044AS1 & 6 & 6 \\
\hline 21110043251 & 6 & 6 \\
\hline 211H042AS1 & 6 & 6 \\
\hline 213 H035A51 & 6 & 6 \\
\hline & 6 & $\begin{array}{l}6 \\
6\end{array}$ \\
\hline $2134037 A 51$ & & $\begin{array}{l}6 \\
6\end{array}$ \\
\hline $213 H 032 A 52$ & $\begin{array}{l}6 \\
6\end{array}$ & $\begin{array}{l}6 \\
6\end{array}$ \\
\hline 213H033AS1 & 6 & 6 \\
\hline $2134034 A 51$ & 6 & $\begin{array}{l}6 \\
5\end{array}$ \\
\hline $\begin{array}{l}323 \text { H185AS1 } \\
323 \text { H186ASL1 }\end{array}$ & 6 & 6 \\
\hline & & 6 \\
\hline $322 \mathrm{H1O8AS1}$ & 6 & 6 \\
\hline $322 \mathrm{HLA9AS1}$ & 6 & 6 \\
\hline $322 H 190 A 51$ & 6 & 6 \\
\hline 321H174AS1 & 6 & 6 \\
\hline $321 \mathrm{H175AS1}$ & 6 & 6 \\
\hline $320 \mathrm{H} 176 \mathrm{~A} 51$ & 6 & 6 \\
\hline $320 \mathrm{H} 277 \mathrm{~S} 1$ & 6 & 5 \\
\hline $\begin{array}{l}320 \mathrm{H} 178 \mathrm{AS1} \\
214 \mathrm{HO3} \text { IASL }\end{array}$ & $\begin{array}{l}6 \\
6\end{array}$ & $\begin{array}{l}6 \\
6\end{array}$ \\
\hline $\begin{array}{l}214 \mathrm{H} 031 \mathrm{LS1} \\
214 \mathrm{H} 030 \mathrm{AS} 1\end{array}$ & 6 & 6 \\
\hline $214 \mathrm{H030A51}$ & 6 & 6 \\
\hline $2144029 A 51$ & & \\
\hline $214 \mathrm{HO2BA5} 1$ & 6 & $\begin{array}{l}6 \\
6\end{array}$ \\
\hline $\begin{array}{l}215 H 027 A 51 \\
215 H 026 A 51\end{array}$ & 6 & 6 \\
\hline $215 \mathrm{HO}^{26 \mathrm{AS} 1}$ & 6 & 5 \\
\hline 215H025AS1 & 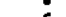 & 0 \\
\hline $215 \mathrm{H} 024 \mathrm{AS} 1$ & 6 & 6 \\
\hline $215 \mathrm{H} 023 \mathrm{AS} 1$ & 6 & 6 \\
\hline $215 \mathrm{H} 022 \mathrm{AS} 2$ & 6 & 6 \\
\hline
\end{tabular}

Figure 9.13 - Case 2, Baseline Schedule (Continued). 
3AUT I EBPAHTM ALLBDULRAZZAK EST.

PEPORT DATE $213 E P 95$ RUN NO. 121

Baseline Schedule by Trpical Activity \& ES
PRIMATERA PRONECT PLANNER

JAHRA 3 HOUSING PROJECT COMmON net

SEVEN SEAS, CONSTPUCTION OF 51 HOUSES
START DATE LAPRE? FIN DATE 13AUGG8

DATA DATE LAPRE? PAGE NO. 27

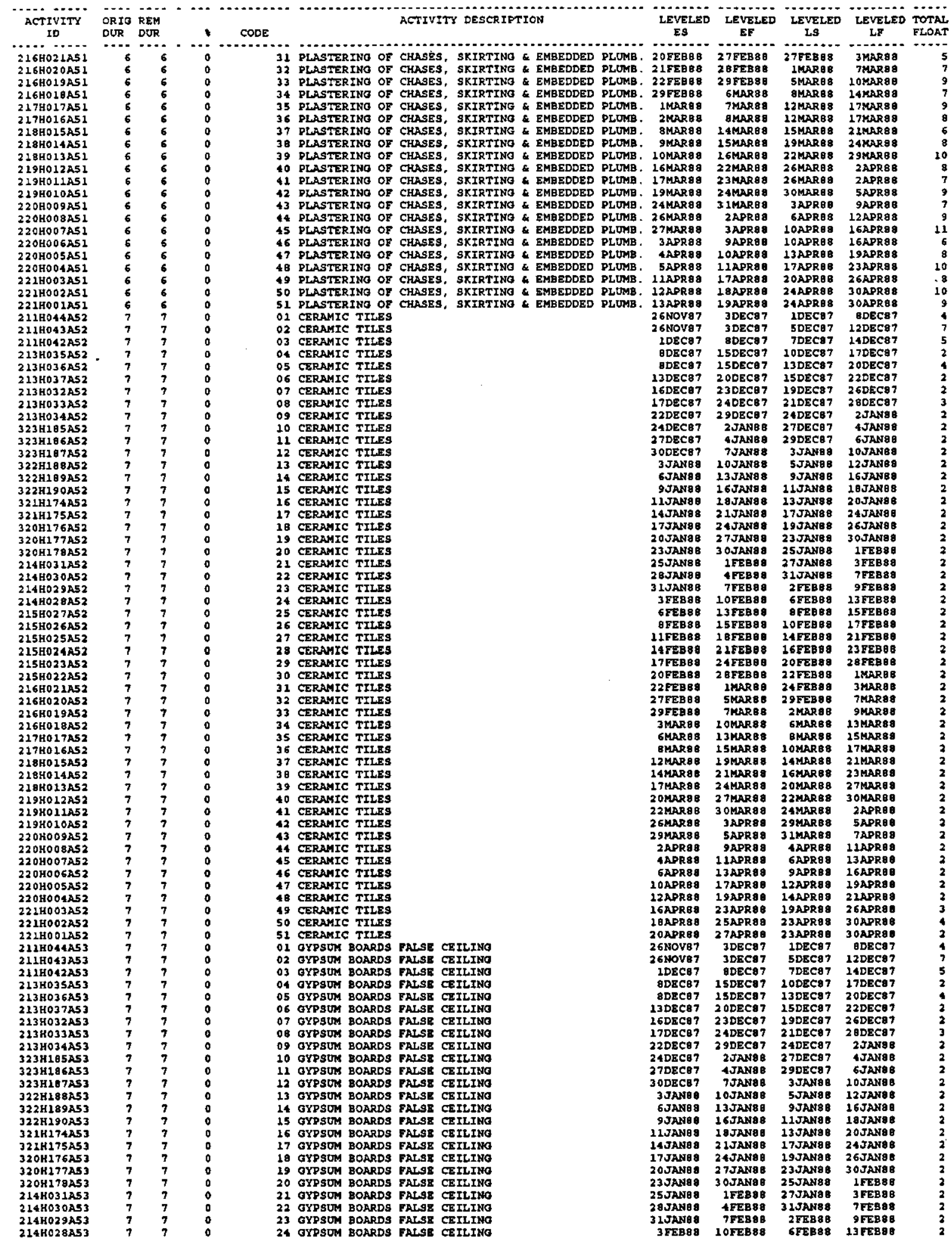

Figure 9.13 - Case 2, Baseline Schedule (Continued). 
SAUD \& EBRAHIM ALABDULRAzZAK EST

REPORT DATE 21 ISEP95 RUN NO. 121 $15: 46$
PRIMAVERA PROJECT PLANAER

SEVEN SEAS, CONSTRUCTION OF 51 HOUSES
JAHRA 3 HOUSING PROJECT COMmon act

START DATE IAPROT FIN DATE 13 AUGg9

DATA DATE LAPRB7 PAGE NO. 29

\begin{tabular}{|c|c|c|}
\hline $\begin{array}{l}\text { ACTIVITY } \\
\text { I0 }\end{array}$ & $\begin{array}{l}\text { ORIO } \\
\text { DUR }\end{array}$ & $\begin{array}{l}\text { REM } \\
\text { DURR }\end{array}$ \\
\hline $215 \mathrm{H} 027 \mathrm{AS} 3$ & 7 & 7 \\
\hline $215 \mathrm{H026253}$ & 7 & 7 \\
\hline $215 \mathrm{HO25AS3}$ & 7 & 7 \\
\hline $215 \mathrm{H} 024 A 53$ & 7 & 7 \\
\hline $215 \mathrm{H} 023 \mathrm{AS} 3$ & $?$ & 7 \\
\hline $215 \mathrm{H} 022 \mathrm{AS} 3$ & 7 & 7 \\
\hline $216 \mathrm{H} 021 \mathrm{AS} 3$ & 7 & 7 \\
\hline $216 \mathrm{H} 020 \mathrm{AS} 3$ & 7 & 7 \\
\hline $216 \mathrm{H} 019 \mathrm{AS} 3$ & 7 & 7 \\
\hline $216 \mathrm{HO} 018 \mathrm{~A} 53$ & 7 & 7 \\
\hline $217 \mathrm{HO} 17 \mathrm{AS} 3$ & ? & 7 \\
\hline $217 \mathrm{HO} 16 \mathrm{AS} 3$ & 7 & 7 \\
\hline $21 \mathrm{aH} 015 \mathrm{AS} 3$ & 7 & 7 \\
\hline $218 \mathrm{H} 014 A 53$ & 7 & 7 \\
\hline $21 \mathrm{BH} 013 \mathrm{AS3}$ & 7 & 7 \\
\hline $219 \mathrm{HO} 012 \mathrm{AS3}$ & 7 & 7 \\
\hline $219 \mathrm{H} 011 \mathrm{AS3}$ & 7 & 7 \\
\hline $219 \mathrm{HO} 10 \mathrm{AS} 3$ & 7 & 7 \\
\hline $220 \mathrm{HOO} 9 \mathrm{~A} 53$ & 7 & 7 \\
\hline $22 \mathrm{OH} 008 \mathrm{~A} 53$ & 7 & 7 \\
\hline $220 \mathrm{HOO}^{2} \mathrm{AS3}$ & $?$ & 7 \\
\hline $220 \mathrm{HOO6AS3}$ & 7 & 7 \\
\hline $220 H 005 A 53$ & 7 & 7 \\
\hline $22 \mathrm{OHOO} 04 \mathrm{AS} 3$ & 7 & 7 \\
\hline $221 \mathrm{H} 003 \mathrm{AS} 3$ & 7 & 7 \\
\hline $221 \mathrm{HOO} 2 \mathrm{AS3}$ & 7 & 7 \\
\hline $222 \mathrm{H} 001 \mathrm{AS} 3$ & 7 & 7 \\
\hline $211 \mathrm{HO44A54}$ & 10 & 10 \\
\hline $211 \mathrm{HO4} 3 \mathrm{AS4}$ & 10 & 10 \\
\hline $211 \mathrm{H042 \textrm {AS4 }}$ & 10 & 10 \\
\hline $2136035 A 54$ & 10 & 20 \\
\hline $213 \mathrm{H036A54}$ & 10 & 10 \\
\hline $213 \mathrm{HO37AS4}$ & 10 & 20 \\
\hline $213 \mathrm{H} 032 \mathrm{AS4}$ & 10 & 10 \\
\hline 2134033254 & 10 & 10 \\
\hline 2134034254 & 10 & 20 \\
\hline $323 \mathrm{H} 185 \mathrm{A54}$ & 10 & 10 \\
\hline $323 \mathrm{H} 866 \mathrm{S4}$ & 10 & 10 \\
\hline $323 \mathrm{H} 187 \mathrm{AS4}$ & 10 & 10 \\
\hline $322 \mathrm{H} 18 \mathrm{BAS4}$ & 10 & 10 \\
\hline $322 \mathrm{H199A54}$ & 10 & 10 \\
\hline $322 \mathrm{H190A54}$ & 10 & 10 \\
\hline 3211417454 & 10 & 10 \\
\hline $321 \mathrm{HL} 75 \mathrm{SA}$ & 10 & 10 \\
\hline $320 \mathrm{H176A54}$ & 10 & 10 \\
\hline $320 \mathrm{H} 177 \mathrm{A5}$ & 10 & 10 \\
\hline $320 \mathrm{HL} 7 \mathrm{BA54}$ & 10 & 10 \\
\hline 2141032054 & 10 & 10 \\
\hline $214 H 030 A 54$ & 10 & 10 \\
\hline $2144029 A 54$ & 10 & 10 \\
\hline 214H028A54 & 10 & 10 \\
\hline $225 H_{027254}$ & 10 & 10 \\
\hline 2154026254 & 10 & 10 \\
\hline $215 \mathrm{H} 025 \mathrm{A54}$ & 10 & 10 \\
\hline 215H024A54 & 10 & 10 \\
\hline $215 \mathrm{H} 023 \mathrm{~A} 54$ & 10 & 10 \\
\hline $215 \mathrm{H} 022254$ & 10 & 10 \\
\hline $216 \mathrm{H021254}$ & 10 & 10 \\
\hline $216 H 020 \triangle 54$ & 20 & 10 \\
\hline $216 \mathrm{H} 019 \mathrm{A54}$ & 10 & 10 \\
\hline 216H018A54 & 10 & 10 \\
\hline $217 \mathrm{H} 017254$ & 10 & 10 \\
\hline $217 \mathrm{H} 016 \mathrm{AS4}$ & 10 & 10 \\
\hline 218H02SAS4 & 10 & 10 \\
\hline $228 \mathrm{H} 014 \mathrm{AS}$ & 10 & 10 \\
\hline $218 \mathrm{H} 013 \mathrm{AS4}$ & 10 & 20 \\
\hline $219 \mathrm{H} 012 \mathrm{AS4}$ & 10 & 20 \\
\hline $\begin{array}{l}\text { 219H011AS4 } \\
219 H 010 A 54\end{array}$ & $\begin{array}{l}10 \\
20\end{array}$ & $\begin{array}{l}10 \\
10\end{array}$ \\
\hline $220 H 009 A 54$ & 20 & 20 \\
\hline $220 \mathrm{HOOBAS4}$ & 10 & 10 \\
\hline $220 \mathrm{H} 007 \mathrm{AS4}$ & 20 & 10 \\
\hline $220 \mathrm{HO} 06 \mathrm{AS4}$ & 10 & 20 \\
\hline $220 H 005 A 54$ & 10 & 10 \\
\hline $2201004 A 54$ & 20 & 10 \\
\hline $221 \mathrm{H} 003 \mathrm{A54}$ & 10 & 20 \\
\hline $221 \mathrm{H} 002 \mathrm{~A} 54$ & 10 & 10 \\
\hline $221 H 001 A 54$ & 20 & 20 \\
\hline $211 \mathrm{H044 \lambda 55}$ & 12 & 12 \\
\hline $211 H 043255$ & 1.2 & 12 \\
\hline $2114042 A 55$ & 12 & 12 \\
\hline $2134035 A 55$ & 12 & 12 \\
\hline $2134036 \mathrm{A5}$ & 12 & 12 \\
\hline $213 \mathrm{H} 037255$ & 12 & 12 \\
\hline $2134032 \mathrm{A5S}$ & 12 & 12 \\
\hline $213 \mathrm{HO} 33 \mathrm{ASS}$ & 12 & 12 \\
\hline $2134034 \times 55$ & 12 & 12 \\
\hline $323 \mathrm{H} 185 \times 55$ & 12 & 12 \\
\hline $323 \mathrm{H} 186 \mathrm{ASS}$ & 12 & 12 \\
\hline $323 \mathrm{H} 187 \times 55$ & 12 & 12 \\
\hline 322H188ASS & 12 & 12 \\
\hline 322H189A5S & 12 & 12 \\
\hline $322 \mathrm{H} 190 \mathrm{~A} 55$ & 12 & 12 \\
\hline $321 \mathrm{H} 174 \mathrm{ASS}$ & 12 & 12 \\
\hline $321 \mathrm{H175A55}$ & 12 & 12 \\
\hline $320 \mathrm{H} 176 \mathrm{AS5}$ & 12 & 12 \\
\hline
\end{tabular}

Figure 9.13 - Case 2, Baseline Schedule (Continued). 


\begin{tabular}{|c|c|c|c|}
\hline \begin{tabular}{c} 
ACrIVITY \\
ID \\
\hdashline
\end{tabular} & $\begin{array}{l}\text { ORIG } \\
\text { DUR } \\
-. .\end{array}$ & $\begin{array}{l}\text { REM } \\
\text { DUR } \\
\ldots . .\end{array}$ & 5 \\
\hline $320 \mathrm{H} 177 \mathrm{ASS}$ & 22 & 12 & 0 \\
\hline $320 \mathrm{H} 17$ 8ASS & 12 & 12 & 0 \\
\hline $22+\mathrm{H} 031 \mathrm{~A} 55$ & 12 & 12 & 0 \\
\hline 214H030AS5 & 12 & 12 & 0 \\
\hline $214 \mathrm{HO29A55}$ & 12 & 12 & 0 \\
\hline 214 HO2 OASS & 12 & 12 & 0 \\
\hline $215 H 027 A 55$ & 12 & 12 & 0 \\
\hline $215 \mathrm{H} 026 \mathrm{A55}$ & 12 & 12 & 0 \\
\hline 215HO2SASS & 12 & 12 & 0 \\
\hline $215 \mathrm{HO24A55}$ & 12 & 12 & 0 \\
\hline $215 \mathrm{HO} 23 \mathrm{A5}$ & 12 & 12 & 0 \\
\hline $215 B 022 A 55$ & 12 & 12 & 0 \\
\hline $216 \mathrm{H021255}$ & 12 & 12 & 0 \\
\hline $216 \mathrm{HO2OA55}$ & 12 & 12 & 0 \\
\hline 216\%019A55 & 12 & 12 & 0 \\
\hline 2164018255 & 12 & 12 & 0 \\
\hline 217 H017AS5 & 12 & 12 & 0 \\
\hline $2174016 \times 55$ & 12 & 22 & 0 \\
\hline 218 HOLSAS5 & 12 & 12 & 0 \\
\hline 238 HO 14AS5 & 12 & 12 & 0 \\
\hline $228 \mathrm{HO} 013 \mathrm{AS5}$ & 12 & 22 & 0 \\
\hline $219 \mathrm{HO} 12 \mathrm{ASS}$ & 12 & 12 & 0 \\
\hline 219 HO11ASS & 12 & 12 & 0 \\
\hline 219H010AS5 & 12 & 12 & 0 \\
\hline $220 \mathrm{HOO}$ AS5 & 12 & 12 & 0 \\
\hline 22OHOOAASS & 12 & 12 & 0 \\
\hline $220 \mathrm{H} 007 \mathrm{ASS}$ & 12 & 12 & 0 \\
\hline 22 OHOOGAS5 & 12 & 12 & 0 \\
\hline $220 \mathrm{HOOSA55}$ & 12 & 12 & 0 \\
\hline 220 BOO4A5S & 12 & 12 & 0 \\
\hline 221B003A5S & 12 & 12 & 0 \\
\hline $221 \mathrm{H} 002 \mathrm{A5} 5$ & 12 & 12 & : \\
\hline $221 H 001 A 55$ & 12 & 12 & 0 \\
\hline 21111044256 & 7 & 7 & 0 \\
\hline $2114043 \times 56$ & 7 & 7 & 0 \\
\hline 2114042256 & 7 & 7 & 0 \\
\hline 213H035A56 & 7 & 7 & 0 \\
\hline $213 K 036 \mathrm{~A} 56$ & $\begin{array}{l}7 \\
7\end{array}$ & $\begin{array}{l}7 \\
7\end{array}$ & 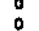 \\
\hline $213 \mathrm{H} 037 \mathrm{A5} 6$ & 7 & 7 & 0 \\
\hline $213 \mathrm{H} 032 \mathrm{~A} 56$ & 7 & 7 & 0 \\
\hline 21310033256 & 7 & 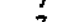 & 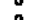 \\
\hline 213H034AS6 & 7 & 7 & o \\
\hline $323 \mathrm{HI} 85 \mathrm{AS} 6$ & 7 & 7 & 0 \\
\hline $323 \mathrm{H} 186 \mathrm{AS} 6$ & 7 & 7 & 0 \\
\hline $323 \mathrm{HI} 197 \mathrm{AS} 6$ & 7 & 7 & 0 \\
\hline $322 \mathrm{H} 18 \mathrm{BAS} 6$ & 7 & 7 & 0 \\
\hline 322H189AS6 & 7 & 7 & 0 \\
\hline $322 \mathrm{H190A56}$ & 7 & 7 & 0 \\
\hline $321 \mathrm{H174A56}$ & 7 & 7 & 0 \\
\hline 321H175AS6 & 7 & 7 & 0 \\
\hline $320 \mathrm{H176A56}$ & 7 & 7 & 0 \\
\hline $320 \mathrm{H177256}$ & 7 & $?$ & 0 \\
\hline 320H278AS 6 & 7 & 7 & 0 \\
\hline 214H031A56 & 7 & 7 & 0 \\
\hline 214 H030AS 6 & 7 & 7 & 0 \\
\hline 214H029AS6 & 7 & 7 & 0 \\
\hline $\begin{array}{l}214 \mathrm{HO2} \text { QA56 } \\
215 \mathrm{HO27ASG}\end{array}$ & $\begin{array}{l}7 \\
7\end{array}$ & 7 & : \\
\hline $\begin{array}{l}\text { 215H027AS6 } \\
215 H 026 A 56\end{array}$ & 7 & 7 & 0 \\
\hline $\begin{array}{l}215 H 026 A 56 \\
215 H 025 A 56\end{array}$ & 7 & 7 & 0 \\
\hline $\begin{array}{l}215 \mathrm{H} 025 A 56 \\
215 H 024 A 56\end{array}$ & 7 & 7 & 0 \\
\hline 215H024A56 & & & \\
\hline $225 \mathrm{H}_{023 A 56}$ & 7 & 7 & 0 \\
\hline $225 H 022 A 56$ & 7 & 7 & 0 \\
\hline $216 \mathrm{HO} 021 \mathrm{A56}$ & 7 & 3 & 0 \\
\hline $216 \mathrm{HO20A56}$ & 7 & 7 & 0 \\
\hline 226H019A56 & 7 & 7 & 0 \\
\hline 216H018A56 & 7 & 7 & 0 \\
\hline $\begin{array}{l}\text { 217H017A56 } \\
217 \mathrm{HO} 16 \mathrm{AS} 6\end{array}$ & $\begin{array}{l}7 \\
7\end{array}$ & $\begin{array}{l}7 \\
7\end{array}$ & : \\
\hline $218 H 014 A 56$ & 7 & 7 & 0 \\
\hline 218HO13A56 & 7 & 7 & 0 \\
\hline 219H012A56 & 7 & 7 & 0 \\
\hline $219 H 011 \mathrm{AS} 6$ & 7 & 7 & 0 \\
\hline 219H010A5 6 & 7 & 7 & 0 \\
\hline $220 H 009 A 56$ & 7 & 7 & 0 \\
\hline $220 \mathrm{HOOBASE}$ & 7 & 7 & 0 \\
\hline $\begin{array}{l}220 \text { 2007AS } \\
220 \text { HOOGAS6 }\end{array}$ & $\begin{array}{l}7 \\
7\end{array}$ & $\begin{array}{l}7 \\
7\end{array}$ & $\begin{array}{l}0 \\
0\end{array}$ \\
\hline $220 \mathrm{H} 005 \mathrm{~A} 56$ & 7 & 7 & 0 \\
\hline 220 HOO4AS 6 & 7 & 7 & 0 \\
\hline 221H003A56 & 7 & 7 & 0 \\
\hline $221 \mathrm{H} 002 \mathrm{~A} 56$ & 7 & 7 & 0 \\
\hline 221HOO1AS6 & 7 & 7 & 0 \\
\hline 211 H044A57 & 7 & 7 & 0 \\
\hline 211 HO43AS7 & 7 & 7 & 0 \\
\hline 211H042A57 & $?$ & $?$ & 0 \\
\hline 213 H035AS7 & $?$ & 7 & 0 \\
\hline 213H036A57 & 7 & 7 & 0 \\
\hline $213 \mathrm{H037A57}$ & 7 & 7 & 0 \\
\hline $213 \mathrm{H} 032 \mathrm{AS} 7$ & 7 & 7 & 0 \\
\hline 213 H033A57 & $?$ & 7 & 0 \\
\hline $213 \mathrm{H} 034 \mathrm{~A} 57$ & 7 & 7 & 0 \\
\hline $323 \mathrm{H105AS7}$ & 7 & 7 & 0 \\
\hline $323 \mathrm{HLBGAS7}$ & 7 & $\mathbf{7}$ & 0 \\
\hline $323 \mathrm{HLO7A5} 7$ & 7 & 7 & 0 \\
\hline
\end{tabular}

Figure 9.13 - Case 2, Baseline Schedule (Continued). 
Jiltc \& zBFjhIM ALABCULRAzZAK EST

PEPORT DATE 21SEP9S RUN NO. 121

PRIMAVERA PROTECT PLANNER

SEVEN SEAS, CONSTRUCTION OF 51 MOUSES
JAHRA 3 HOUSING PROJECT COMmON NCE

START DATE LAPRg7 EIN DATE $13 A$ HGG

Baseline Schecule by Typical Aetivity o ES

DATA DATE LAPR87 PAGE RO. 30

\begin{tabular}{|c|c|c|}
\hline $\begin{array}{l}\text { ACTIVITY } \\
\text { ID }\end{array}$ & $\begin{array}{l}\text { ORIG } \\
\text { DURR }\end{array}$ & $\begin{array}{l}\text { REM } \\
\text { DUR }\end{array}$ \\
\hline 332H188AS7 & 7 & 7 \\
\hline $3224189 \times 57$ & 7 & 7 \\
\hline 322H190AS7 & $?$ & 7 \\
\hline $3221 \mathrm{H} 17+257$ & $?$ & 7 \\
\hline 3211175457 & 7 & 7 \\
\hline $320 H 176 A 57$ & 7 & 7 \\
\hline $3204177 A 57$ & 7 & 7 \\
\hline $320 \mathrm{H} 178 \mathrm{~A} 57$ & 7 & 7 \\
\hline 214 H031AS? & $?$ & 7 \\
\hline $214 \mathrm{H} 030 \mathrm{AS} 7$ & $?$ & 7 \\
\hline $214 \mathrm{HO29AS7}$ & 7 & 7 \\
\hline $214 H 028 A S 7$ & 7 & 7 \\
\hline $215 \mathrm{HO27AS7}$ & $?$ & $\tau$ \\
\hline $215 \mathrm{H} 026 \mathrm{AS} ?$ & 7 & $\tau$ \\
\hline $215 \mathrm{HO} 25 \mathrm{AS} 7$ & 7 & 7 \\
\hline $215 \mathrm{H} 024 A 57$ & 7 & 7 \\
\hline $215 \mathrm{HO23A5} 7$ & 7 & 7 \\
\hline $215 H 022857$ & 7 & $?$ \\
\hline 2164021857 & 7 & 7 \\
\hline 2164020257 & 7 & 7 \\
\hline $2164019 A 57$ & ? & 7 \\
\hline $216 \mathrm{HO19A57}$ & 7 & 7 \\
\hline $217 H 017 A 57$ & 7 & 7 \\
\hline $217 \mathrm{H} 016 \mathrm{AS} 7$ & $?$ & 7 \\
\hline 218HO1SAS? & 7 & 7 \\
\hline $218 H 014257$ & 7 & 7 \\
\hline 218H013AS7 & 7 & 7 \\
\hline $219 \mathrm{HO} 012 \mathrm{AS} 7$ & 7 & 7 \\
\hline 219H011AS? & 7 & 7 \\
\hline $219 H 010 A 57$ & 7 & 7 \\
\hline $2204009 A 57$ & 7 & 7 \\
\hline 220HOO8AS7 & 7 & 7 \\
\hline $220 \mathrm{H} 007257$ & $?$ & 7 \\
\hline 2204006 A57 & 7 & 7 \\
\hline $\begin{array}{l}2204005 A 57 \\
2204004 A 57\end{array}$ & 7 & $\begin{array}{l}7 \\
7\end{array}$ \\
\hline $2214003 A 57$ & $?$ & 7 \\
\hline $2234002 A 57$ & $?$ & 7 \\
\hline $221 \mathrm{H} 001 \mathrm{~A} 57$ & 7 & 7 \\
\hline $211 \mathrm{HO44A58}$ & 6 & 6 \\
\hline $2114043 A 58$ & 6 & 6 \\
\hline $212 \mathrm{H} 042 \mathrm{ASO}$ & 6 & 5 \\
\hline $213 \mathrm{H} 035 \mathrm{ASB}$ & 6 & 6 \\
\hline $223 \mathrm{H} 036$ A58 & 6 & 6 \\
\hline $213 \mathrm{H} 037 \mathrm{AS} 8$ & 6 & 6 \\
\hline $213 \mathrm{HO} 032 \mathrm{A5} \mathrm{g}$ & 6 & 6 \\
\hline $213 \mathrm{H} 033 \mathrm{ASO}$ & 6 & 6 \\
\hline $213 \mathrm{HH} 034 \mathrm{ASB}$ & 6 & 6 \\
\hline 323HLOSAS 8 & 6 & 6 \\
\hline $323 \mathrm{H} 186 \mathrm{AS} 8$ & 6 & 6 \\
\hline $323 \mathrm{H} 1 \mathrm{~B} 7 \mathrm{ASB}$ & 6 & 6 \\
\hline 322H1BAASB & 6 & 6 \\
\hline $322 \mathrm{H} 169 \mathrm{AS} \mathrm{B}$ & 6 & 6 \\
\hline 322H190A5B & 6 & 6 \\
\hline $321 \mathrm{H} 174 A 58$ & 6 & 6 \\
\hline $322 H 175 A 58$ & 6 & 6 \\
\hline $320 \mathrm{H176A59}$ & 6 & 6 \\
\hline $320 \mathrm{H} 177 \mathrm{AS} 8$ & 6 & 6 \\
\hline $320 \mathrm{H178A58}$ & 6 & 6 \\
\hline $214 \mathrm{H} 032 \mathrm{AS} 8$ & 6 & 6 \\
\hline $214 \mathrm{H03OA58}$ & 6 & 6 \\
\hline $214 H 029 A 5 B$ & 6 & 6 \\
\hline 214H028A58 & 6 & 6 \\
\hline $215 H 027 A 58$ & 6 & 6 \\
\hline $215 \mathrm{HO} 26 \mathrm{AS} \theta$ & 6 & 6 \\
\hline $215 H 025 A 5 \theta$ & 6 & 6 \\
\hline $215 \mathrm{HO24ASO}$ & 6 & 6 \\
\hline $215 \mathrm{H} 023 \mathrm{~A} 5 \mathrm{O}$ & 6 & 6 \\
\hline $215 H 022 A 58$ & 6 & 6 \\
\hline $216 \mathrm{H} 021 \mathrm{AS} 8$ & 6 & 6 \\
\hline $216 \mathrm{H020A58}$ & 6 & 6 \\
\hline $216 \mathrm{HO19A5O}$ & 6 & 6 \\
\hline 216 HO1BASB & 6 & 6 \\
\hline 217 H017A58 & 6 & 6 \\
\hline $217 \mathrm{HO16A58}$ & 6 & 6 \\
\hline 218 1815A59 & 6 & 6 \\
\hline 218HO14A58 & 6 & 6 \\
\hline $218 \mathrm{HO} 13 \mathrm{AS} 8$ & 6 & 6 \\
\hline $2194012 A 58$ & 6 & 6 \\
\hline $219 \mathrm{HO11A58}$ & 6 & 6 \\
\hline $219 \mathrm{HO10A58}$ & 6 & 6 \\
\hline $220 \mathrm{HOOSA58}$ & 6 & 6 \\
\hline $220 \mathrm{HOOOADB}$ & 6 & 6 \\
\hline $220 \mathrm{H} 007 \mathrm{AS} \mathrm{B}$ & 6 & 6 \\
\hline $220 H 006 A 58$ & $\begin{array}{l}6 \\
6\end{array}$ & 6 \\
\hline $220 \mathrm{OH} 005258$ & $\begin{array}{l}6 \\
6\end{array}$ & 6 \\
\hline $220 \mathrm{HOOANSB}$ & 6 & 6 \\
\hline $221 \mathrm{H} 003 \mathrm{~A} 5 \mathrm{~B}$ & 6 & 6 \\
\hline 2224002258 & 6 & 6 \\
\hline 221H001AS8 & 6 & 6 \\
\hline 211HO44AS9 & 6 & 6 \\
\hline $2114043 A 59$ & 6 & 6 \\
\hline $211 H 042 A 59$ & 6 & 6 \\
\hline 213H035A59 & 6 & 6 \\
\hline 213H036AS9 & 6 & 6 \\
\hline 2134037259 & 6 & 6 \\
\hline
\end{tabular}

Figure 9.13 - Case 2, Baseline Schedule (Continued). 
SALC S EBPAHIM ILABDULRAZZAK EST.

P.EPORT DATE 21 SEPSS RUN NO. 121

PRIMAVERA PROTECT PLANNER

SEVEN SEAS, CONSTRUCTION OF 51 HOUSES
JAHRA 3 HOUSINO PROJECT cOMmON act

START DATE IAPRA7 FIN DATE 13AUOBS DATA DATE IAPRg7 PAGE NO. 31

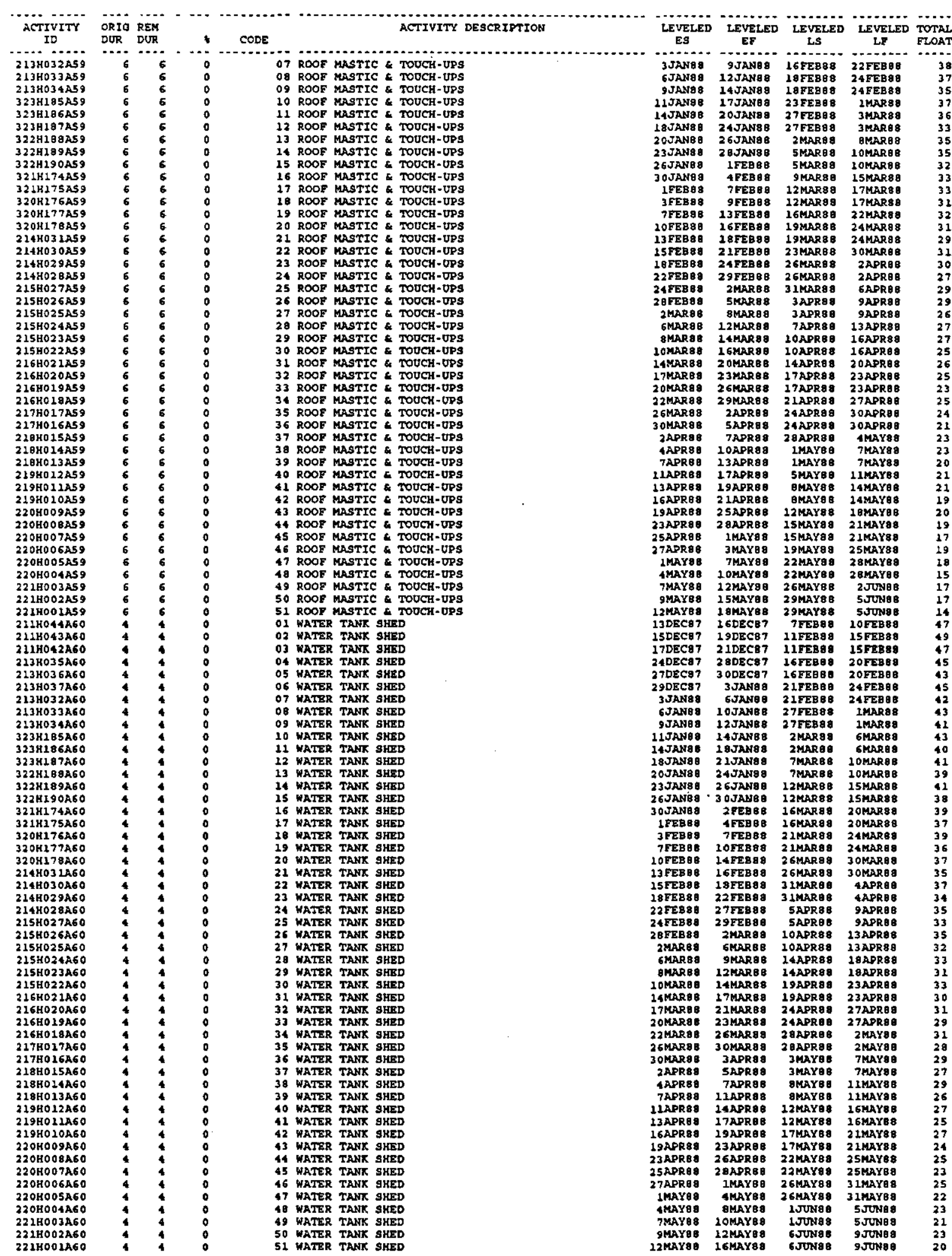

Figure 9.13 - Case 2, Baseline Schedule (Continued). 
BAND \& ESRAHTM ALABOULRAZZAK EST

PEPORT DATE :1SEP95 RUN NO. 12 \&

is: 46
PRTMAVERA PROTECT PEANRER

SEVEN SEAS, CONSTRUCTION OF 51 HOUSES
JAHRA 3 HOUSING DROJECT COMUON ACE

START DATE LAPR97 FIN DATE L3AUG89

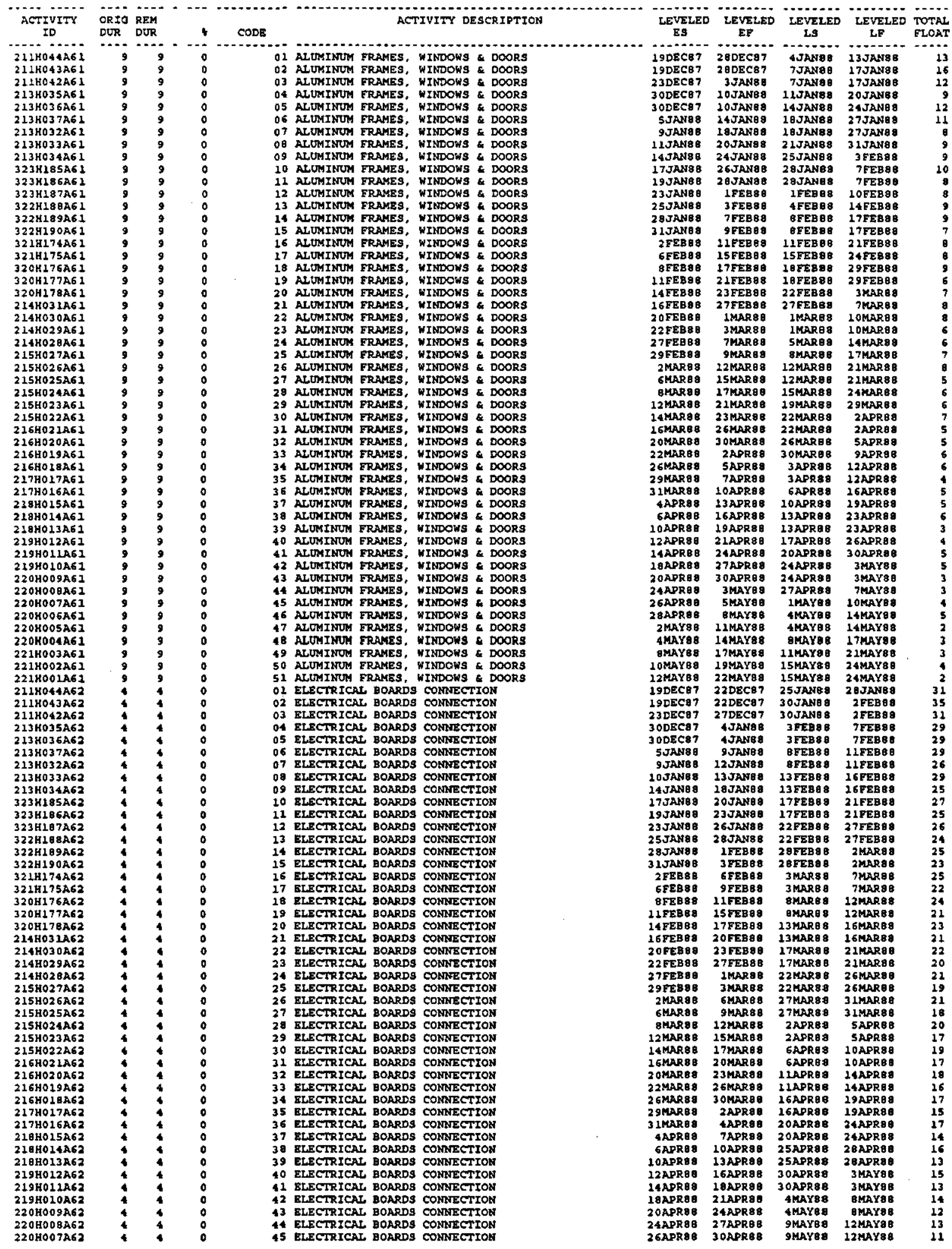

Figure 9.13 - Case 2, Baseline Schedule (Continued). 


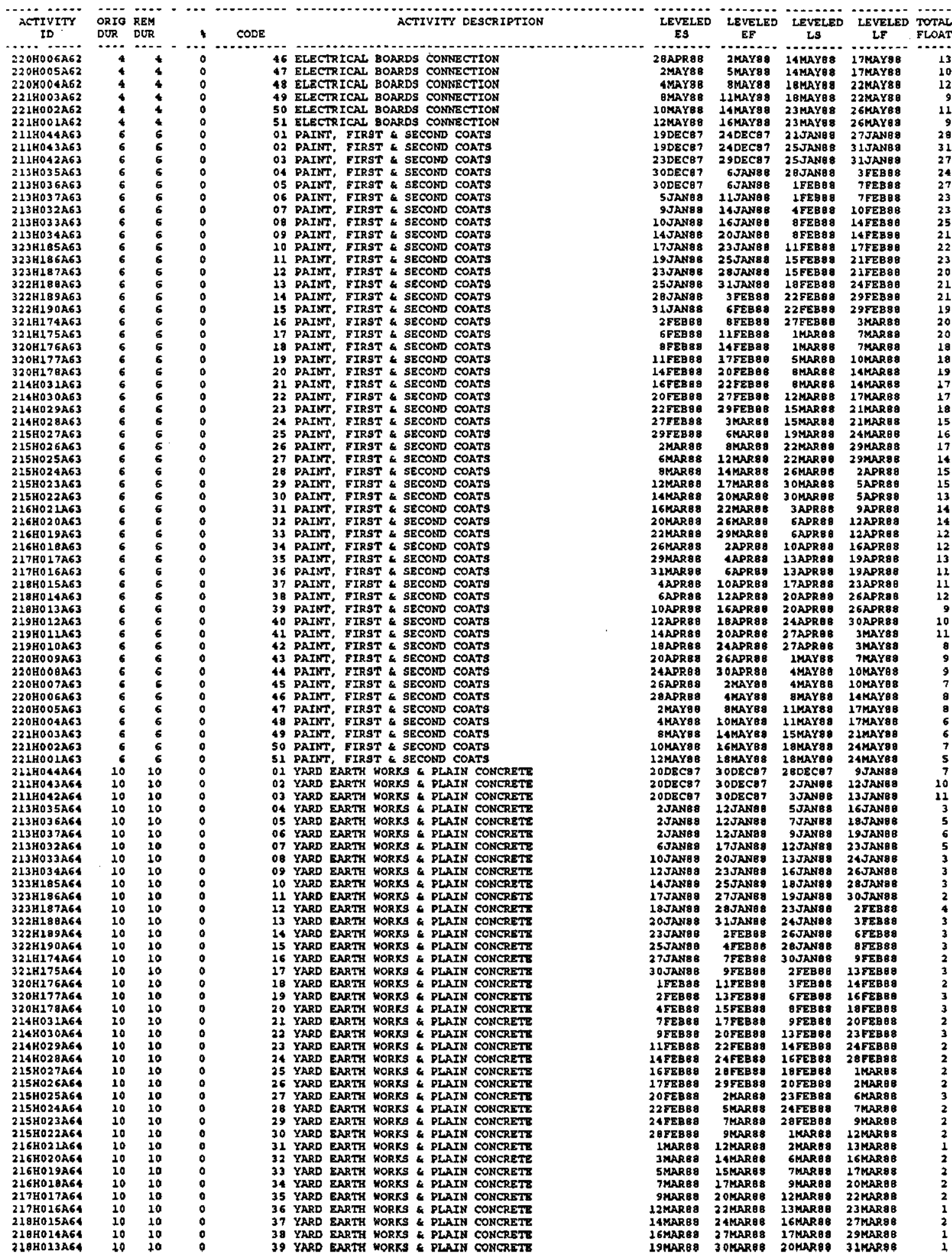

Figure 9.13 - Case 2, Baseline Schedule (Continued). 
JALD \& ESRAHIM ATABDULRAZZAK EST

PEPCRT DATE $21 S E 995$ RUN NO. 121 is: 46
PRIMAVERA PROJECT PLANNER

SEVEN SEAS, CONSTRUCTION OF 51 HOUSES
JAHRA 3 HOUSING PROEECT COMENON ACE

START DATE LAPRB? FIN DATE 13AUG8B DATA DATE LAPR87 PAGE NO. 34

\begin{tabular}{|c|c|}
\hline $\begin{array}{l}\text { ACTIVITY } \\
\text { ID }\end{array}$ & $\begin{array}{l}\text { ORIG } \\
\text { OUR }\end{array}$ \\
\hline $219 M 012 \mathrm{~A} 64$ & 10 \\
\hline $219 H 011 \mathrm{~A} 64$ & 20 \\
\hline $219 H 010 A 64$ & 10 \\
\hline $2201009 A 64$ & 10 \\
\hline $220 \mathrm{HOOBA64}$ & 10 \\
\hline $220 H 007 A 64$ & 10 \\
\hline $220 \mathrm{HOOGAG4}$ & 10 \\
\hline $220 H 005 A 64$ & 10 \\
\hline $220 H 004 A 64$ & 10 \\
\hline 221HO03A64 & 10 \\
\hline $221 \% 002 A 64$ & 10 \\
\hline $221 H 001 A 64$ & 10 \\
\hline $2111 \% 044 A 65$ & 2 \\
\hline $21110043 \AA 65$ & 2 \\
\hline $21110042 A 65$ & 2 \\
\hline 213 HOS5A65 & 2 \\
\hline 213 H 036 A65 & 2 \\
\hline $213 H 037 A 65$ & 2 \\
\hline $213 H 032 A 65$ & 2 \\
\hline $2134033 A 65$ & 2 \\
\hline 213 H034A65 & 2 \\
\hline 323 M185A65 & 2 \\
\hline $323 H 186 A 65$ & 2 \\
\hline 323H187A65 & 2 \\
\hline $322 \mathrm{HzB8A65}$ & 2 \\
\hline $322 \mathrm{H} 8 \mathrm{BA} 65$ & 2 \\
\hline 322 H290A65 & 2 \\
\hline $321 H 174 A 65$ & 2 \\
\hline $321 H 175 A 65$ & 2 \\
\hline $320 \mathrm{H176A65}$ & 2 \\
\hline $320 H 177 \mathrm{A65}$ & 2 \\
\hline $320 H 17$ A65 & 2 \\
\hline $214 \mathrm{HO31A65}$ & 2 \\
\hline $214 \mathrm{H} 030 \mathrm{~A} 65$ & 2 \\
\hline $2144029 A 65$ & 2 \\
\hline $214 \mathrm{HO2}$ BA65 & 2 \\
\hline 215H027A65 & 2 \\
\hline 215 H026A65 & 2 \\
\hline $215 H 025 A 65$ & 2 \\
\hline 21SH024A65 & 2 \\
\hline $215 \mathrm{H} 023 \mathrm{A65}$ & 2 \\
\hline $215 H 022 A 65$ & 2 \\
\hline $216 H 021 A 65$ & 3 \\
\hline 216 H02 OA 65 & $i$ \\
\hline $216 \mathrm{HO} 19 \mathrm{A65}$ & 2 \\
\hline $216 \mathrm{HO} 01 \mathrm{BA} 65$ & 2 \\
\hline 217H017A65 & 2 \\
\hline $217 \mathrm{H} 016 \mathrm{~A} 65$ & 2 \\
\hline 219 H015A65 & 2 \\
\hline 218HO14A65 & 2 \\
\hline $218 H 013 \mathrm{~A} 65$ & 2 \\
\hline 219H012A65 & 2 \\
\hline $219 H 011 A 65$ & 2 \\
\hline 219 H01OA65 & 2 \\
\hline $220 H 009 A 65$ & 2 \\
\hline $220 \mathrm{HOOBA65}$ & 2 \\
\hline $220 H 007 A 65$ & 3 \\
\hline 220 H006A65 & 2 \\
\hline 220HOOSAG5 & 2 \\
\hline $220 \mathrm{H004A65}$ & 2 \\
\hline 221H003A65 & 2 \\
\hline 221H002A65 & 2 \\
\hline 221H001A65 & 2 \\
\hline $212 H 044 A 66$ & 3 \\
\hline $211 H 043 A 66$ & 3 \\
\hline $211 H 042 A 66$ & 3 \\
\hline $213 H 035 A 66$ & 3 \\
\hline 213 H036A66 & 3 \\
\hline $213 \mathrm{H} 037 \mathrm{~A} 66$ & 3 \\
\hline $213 \mathrm{H} 032 \mathrm{~A} 66$ & 3 \\
\hline 213K033A66 & 3 \\
\hline 213H034A66 & 3 \\
\hline $323 H 185 A 66$ & 3 \\
\hline $323 \mathrm{H} 186 \mathrm{~A} 66$ & 3 \\
\hline $323 H 187 A 66$ & 3 \\
\hline $322 \mathrm{H18QA66}$ & 3 \\
\hline $3224189 A 66$ & 3 \\
\hline $322 H 190 A 66$ & 3 \\
\hline З21H174A56 & $\mathbf{3}$ \\
\hline $321 \mathrm{HITSA66}$ & 3 \\
\hline З20H176A66 & 3 \\
\hline $320 H 177 A 66$ & 3 \\
\hline $320 H 178 A 66$ & 3 \\
\hline $214 \mathrm{H} 031 \mathrm{A66}$ & 3 \\
\hline $214 \mathrm{H} 030 \mathrm{~A} 66$ & 3 \\
\hline $214 H 029 A 66$ & 3 \\
\hline $214 \mathrm{H028A66}$ & 3 \\
\hline $215 \mathrm{H027A66}$ & 3 \\
\hline $215 \mathrm{H} 026 \mathrm{~A} 66$ & 3 \\
\hline $215 H 025 A 66$ & 3 \\
\hline $215 H 024 A 66$ & 3 \\
\hline $215 \mathrm{HO23A66}$ & 3 \\
\hline $2154022 \mathrm{A66}$ & 3 \\
\hline $216 \mathrm{H} 021 \mathrm{~A} 66$ & 3 \\
\hline $216 \mathrm{HO2OA66}$ & 3 \\
\hline $216 \mathrm{HO} 19 \mathrm{~A} 66$ & 3 \\
\hline
\end{tabular}

Figure 9.13 - Case 2, Baseline Schedule (Continued). 
SAUD 5 EBRATIM ALABDULRAZZAK EST

REPORT DATE 21SEP95 RUN NO. 121

PRIMAVERA PRQNECT PLANNER

SEVEN SEAS, CONSTRUCTION OF 51 HOUSES
JAHRA 3 HOUSING PROTECT JOmmon ace

START DATE LAPRB 7 FIN DATE 13AUOG9

DATA DATE IAPRE? PAGE NO. 35

\begin{tabular}{|c|c|}
\hline $\begin{array}{l}\text { ACTIVITY } \\
\text { ID }\end{array}$ & $\begin{array}{l}\text { ORIO } \\
\text { DUR }\end{array}$ \\
\hline $216 \mathrm{HO} 19 \mathrm{~A} 6 \mathrm{6}$ & \\
\hline $227 H 017 A 66$ & 3 \\
\hline $2174016 \mathrm{~A} 66$ & 3 \\
\hline 218 HO15A66 & 3 \\
\hline $2194014 A 66$ & 3 \\
\hline $218 \mathrm{HO13266}$ & 3 \\
\hline $219 \mathrm{HO} 2 \mathrm{A6G}$ & 3 \\
\hline $219 \mathrm{H} 011 \mathrm{A66}$ & 3 \\
\hline 219 H010A66 & 3 \\
\hline $220 \mathrm{HOOOAG6}$ & 3 \\
\hline $220 \mathrm{HOOBAG6}$ & 3 \\
\hline $220 \mathrm{H} 007 \mathrm{A6} 6$ & 3 \\
\hline $220 \mathrm{H006A66}$ & 3 \\
\hline $220 \mathrm{HOO5AG6}$ & 3 \\
\hline $220 \mathrm{HO04A66}$ & 3 \\
\hline 22 LHO03A66 & 3 \\
\hline $221 \mathrm{H} 002 \mathrm{A66}$ & 3 \\
\hline $221 \mathrm{H} 001 \mathrm{A66}$ & 3 \\
\hline 211 H044A67 & 3 \\
\hline $21111043 A 67$ & 3 \\
\hline $211 H 042 A 67$ & 3 \\
\hline $213 H 035 A 67$ & 3 \\
\hline $213 H 036 A 67$ & 3 \\
\hline $213 H 037 A 67$ & 3 \\
\hline $2133 \mathrm{HO32A67}$ & 3 \\
\hline $213 H 033 A 67$ & 3 \\
\hline $213 \mathrm{HO34A67}$ & 3 \\
\hline $323 \mathrm{H} 185 \mathrm{~A} 67$ & 3 \\
\hline $323 H 186 A 67$ & 3 \\
\hline $3233187 A 67$ & 3 \\
\hline $322 \% 288 A 67$ & 3 \\
\hline $322 H 189 A 67$ & 3 \\
\hline $322 \mathrm{H190A67}$ & 3 \\
\hline $321 \% 174 A 67$ & 3 \\
\hline $321 H 175 A 67$ & 3 \\
\hline $320 \mathrm{HL76A67}$ & 3 \\
\hline $320 H 277 A 67$ & 3 \\
\hline $320 H 178 A 67$ & 3 \\
\hline $2144031 \mathrm{A67}$ & 3 \\
\hline $214 \mathrm{HO3OA67}$ & 3 \\
\hline $214 H 029 A 67$ & 3 \\
\hline $2144028 A 67$ & 3 \\
\hline $2154027 \mathrm{A67}$ & 3 \\
\hline $215 \mathrm{H} 026 \mathrm{A67}$ & 3 \\
\hline $215 \mathrm{H} 025 \mathrm{~A} 67$ & 3 \\
\hline $215 \mathrm{H} 024 \mathrm{~A} 67$ & 3 \\
\hline $215 H 023 A 67$ & 3 \\
\hline $215 \mathrm{H} 022 \mathrm{~A} 67$ & 3 \\
\hline $216 \mathrm{HO} \angle \mathrm{LA} 67$ & 3 \\
\hline 2164020267 & 3 \\
\hline $216 \mathrm{H} 029 \mathrm{A6} 7$ & 3 \\
\hline $216 \mathrm{HO} 2 \mathrm{BA67}$ & 3 \\
\hline $217 \mathrm{H017A67}$ & 3 \\
\hline 217 H016A67 & 3 \\
\hline 218H015A67 & 3 \\
\hline $218 \mathrm{HO14A67}$ & 3 \\
\hline $218 H 023 A 67$ & 3 \\
\hline 219H012A67 & 3 \\
\hline $219 \mathrm{HO11A67}$ & 3 \\
\hline 219H010A67 & 3 \\
\hline $220 \mathrm{HOO9A67}$ & 3 \\
\hline $220 \mathrm{HOOOA67}$ & 3 \\
\hline $220 \mathrm{HOO} 7 \mathrm{A67}$ & 3 \\
\hline $220 \mathrm{HOO} 6 \mathrm{A67}$ & 3 \\
\hline $220 \mathrm{H} 005 \mathrm{~A} 67$ & 3 \\
\hline $2201004 A 67$ & 3 \\
\hline $221 \mathrm{H} 003 \mathrm{~A} 67$ & 3 \\
\hline $221 \mathrm{H} 002 \mathrm{~A} 67$ & 3 \\
\hline $221 \mathrm{H} 001 \mathrm{A6} 7$ & 3 \\
\hline $211 H 044 A 68$ & 4 \\
\hline $21110043 A 60$ & 1 \\
\hline $211 \mathrm{H} 042 \mathrm{~A} 6 \mathrm{~B}$ & 4 \\
\hline $213 \mathrm{HO} 35 \mathrm{~A} 6 \mathrm{~B}$ & 4 \\
\hline $213 \mathrm{H} 036 \mathrm{A6O}$ & 1 \\
\hline $213 \mathrm{H} 037 \mathrm{A6B}$ & 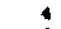 \\
\hline $213 H 032 \mathrm{A6g}$ & 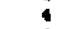 \\
\hline $213 \mathrm{H} 033 \mathrm{~A} 6 \mathrm{\theta}$ & 4 \\
\hline $213 \mathrm{HO34A6 \theta}$ & 4 \\
\hline $323 \mathrm{H} 185 \mathrm{~A} 6 \mathrm{~g}$ & 1 \\
\hline $323 \mathrm{HLQ6A69}$ & \\
\hline 323H187A68 & 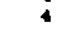 \\
\hline $322 \mathrm{H} 18 \mathrm{BA} 6 \mathrm{~g}$ & 4 \\
\hline $322 \mathrm{H189A68}$ & 4 \\
\hline $322 \mathrm{H190A68}$ & 4 \\
\hline $32114274 A 68$ & 4 \\
\hline $321 H 175 A 68$ & 4 \\
\hline $320 H 176 A 68$ & 4 \\
\hline $320 H 177 A 68$ & 4 \\
\hline $320 \mathrm{H178A68}$ & 4 \\
\hline 214H031A68 & 4 \\
\hline $214 \mathrm{H030A6 \textrm {O }}$ & 4 \\
\hline $214 \mathrm{HO29A68}$ & 4 \\
\hline $2144028 A 68$ & 4 \\
\hline $215 \mathrm{HO27A68}$ & 4 \\
\hline $\begin{array}{r}215 \mathrm{HO26A68} \\
215 \mathrm{HO25A68}\end{array}$ & 4 \\
\hline $215 \mathrm{HO25A6B}$ & \\
\hline
\end{tabular}

Figure 9.13 - Case 2, Baseline Schedule (Continued). 
SAUD \& EBRAHIM ALABDCLRAZZZAK EST.

REPORT DATE 21 SEP95 RUN NO. 12 $15: 46$
PRIMAVERA PROEETT PLUNNER

SEVEN SEAS, CONSTRUCTION OF 51 HOUSES

JAHRA 3 HOUSING PROTECT COMTOD ".CO

START DATE IAPR日7 FIN DATE 13AUG8ם

DATA DATE 1APRB 7 PAGE NO. 36

\begin{tabular}{|c|c|c|}
\hline $\begin{array}{l}\text { ACTIVITY } \\
\text { ID }\end{array}$ & $\begin{array}{l}\text { ORIG } \\
\text { DUR }\end{array}$ & $\begin{array}{l}\text { REM } \\
\text { DUR }\end{array}$ \\
\hline $215 H 024 A 68$ & & 4 \\
\hline $215 B 023 \mathrm{A6B}$ & & \\
\hline $2154022 \mathrm{Abg}$ & 4 & 4 \\
\hline $216 \mathrm{HO} 1 \mathrm{AA69}$ & 4 & 4 \\
\hline $2164020 A 69$ & 4 & 4 \\
\hline $2164019 \mathrm{A68}$ & 4 & 4 \\
\hline $216 \mathrm{HO18A68}$ & 4 & 4 \\
\hline $217 \mathrm{HO} 017 \mathrm{~A} 6 \mathrm{\theta}$ & 4 & 4 \\
\hline $217 H 016 A 68$ & 4 & 4 \\
\hline $219 H 015 \mathrm{~A} 6 \mathrm{~B}$ & 4 & 4 \\
\hline $2194014 A 69$ & 4 & 4 \\
\hline $218 H 013 \mathrm{~A} 6 \mathrm{~B}$ & 4 & 4 \\
\hline $229 \mathrm{HO12A68}$ & 4 & 4 \\
\hline $219 H 011260$ & 4 & 4 \\
\hline $219 \mathrm{HO1OA69}$ & 4 & 4 \\
\hline $220 \mathrm{HOO9A6 \theta}$ & 4 & \\
\hline $220 \mathrm{HOOBA68}$ & 4 & 4 \\
\hline $2204007 A 68$ & 4 & 4 \\
\hline $2204006 A 68$ & 4 & 4 \\
\hline $220 \mathrm{HOO05A68}$ & 4 & 4 \\
\hline $220 \mathrm{H} 004 \mathrm{~A} 6 \mathrm{~B}$ & 1 & 4 \\
\hline $221 \mathrm{H} 003 \mathrm{~A} 68$ & 4 & 4 \\
\hline $221 \mathrm{H} 002 \mathrm{~A} 6 \mathrm{~B}$ & 4 & 4 \\
\hline $2214001 \mathrm{A6B}$ & 4 & 4 \\
\hline $2114044 A 69$ & 6 & 6 \\
\hline $211 \mathrm{H} 043 A 69$ & 6 & 6 \\
\hline $211 H 042 A 69$ & 6 & 6 \\
\hline 213 H035A69 & 6 & 6 \\
\hline $213 \mathrm{H} 036 \mathrm{~A} 69$ & 6 & 6 \\
\hline $2134037 A 69$ & 6 & 6 \\
\hline $2134032 A 69$ & 6 & 6 \\
\hline $2134033 \mathrm{~A} 69$ & 6 & 6 \\
\hline 213 H034A69 & 6 & 6 \\
\hline 323H185A69 & 6 & 6 \\
\hline $323 \mathrm{H} 186 \mathrm{AG9}$ & 6 & 6 \\
\hline $3234187 A 69$ & 6 & 6 \\
\hline $322 \mathrm{KLB8AS9}$ & 6 & 6 \\
\hline 322H199A69 & 6 & 6 \\
\hline $322 \mathrm{H} 190 \mathrm{~A} 69$ & 6 & 6 \\
\hline $321 \mathrm{H174A69}$ & 6 & 6 \\
\hline $32111275 A 69$ & 6 & 6 \\
\hline $320 \mathrm{H} 176 \mathrm{AG} 9$ & 6 & 6 \\
\hline $32041777 A 69$ & 6 & 6 \\
\hline $320 \mathrm{H279A69}$ & 6 & 6 \\
\hline $214 H 031 \mathrm{A69}$ & 6 & 6 \\
\hline $214 H 030 A 69$ & 6 & 6 \\
\hline 214H029A69 & 6 & 6 \\
\hline 214H028A69 & 6 & 6 \\
\hline 215H027A69 & 6 & 6 \\
\hline $2154026 A 69$ & 6 & 6 \\
\hline $215 \% 025 A 69$ & 6 & 6 \\
\hline 215HO24AG9 & 6 & 6 \\
\hline $225 \mathrm{HO23AG9}$ & 6 & 6 \\
\hline 215H022A69 & 6 & 6 \\
\hline $216 \mathrm{H} 0212669$ & 6 & 6 \\
\hline $216 \mathrm{HO2OA69}$ & 6 & 6 \\
\hline 226H019A69 & 6 & 6 \\
\hline $216 \mathrm{HO} 018 \mathrm{A69}$ & 6 & 6 \\
\hline $\begin{array}{l}2174017 A 69 \\
2174015 \mathrm{~A} 69\end{array}$ & 6 & 6 \\
\hline $\begin{array}{l}217 \mathrm{HO16A69} \\
218 \mathrm{HO15A69}\end{array}$ & 6 & 6 \\
\hline $218 \mathrm{H} 014 \mathrm{~A} 69$ & 6 & 6 \\
\hline 21BHO13A69 & 6 & 6 \\
\hline $2194012 A 69$ & 6 & 6 \\
\hline $219 \mathrm{HO} 011269$ & 6 & 6 \\
\hline $219 \mathrm{HO10A69}$ & 6 & 6 \\
\hline $220 \mathrm{HOO} 9 \mathrm{AG9}$ & 6 & 6 \\
\hline $2204008 A 69$ & 6 & 6 \\
\hline $\begin{array}{l}220 \mathrm{HOOTA69} \\
220 \mathrm{0006469}\end{array}$ & & 6 \\
\hline $220 H 006 A 69$ & 6 & 6 \\
\hline 220H005A69 & 6 & 6 \\
\hline $220 \mathrm{OH} 004 \mathrm{~A} 69$ & 6 & 6 \\
\hline $2214003 A 69$ & 6 & 6 \\
\hline $221 \mathrm{H} 002 \mathrm{~A} 69$ & 6 & 6 \\
\hline $2214001 \mathrm{~A} 69$ & 6 & 6 \\
\hline 211 H044A70 & 4 & 4 \\
\hline $2114043 A 70$ & 4 & 4 \\
\hline $2114042 A 70$ & 4 & 4 \\
\hline $213 \mathrm{H} 035 \mathrm{~A} 7 \mathrm{O}$ & & 4 \\
\hline $213 \mathrm{HO} 36 \mathrm{~A} 70$ & & 4 \\
\hline 213 HO37A70 & & 4 \\
\hline $213 H 032 A>0$ & & 4 \\
\hline $2136033 A 70$ & 4 & 4 \\
\hline $213 H 0344 A 70$ & & 4 \\
\hline 323H185A70 & 4 & 4 \\
\hline $323 \mathrm{HL186A70}$ & & \\
\hline $323 \mathrm{H187A70}$ & 4 & 4 \\
\hline $322 \mathrm{HIA8A7O}$ & 4 & 4 \\
\hline $322 \mathrm{H} 189 \mathrm{~A} 70$ & & 4 \\
\hline $32218190 A 70$ & & 4 \\
\hline $321 \mathrm{H174A70}$ & & \\
\hline $321 H 175 A 70$ & & 4 \\
\hline $320 H 176 A 70$ & 4 & 4 \\
\hline $320 \mathrm{~K} 177 \times 70$ & & 4 \\
\hline $320 \mathrm{K178A70}$ & & 4 \\
\hline $214 \mathrm{H} 031 \mathrm{~A} 70$ & & 4 \\
\hline
\end{tabular}

Figure 9.13 - Case 2, Baseline Schedule (Continued). 


\begin{tabular}{|c|c|c|}
\hline $\begin{array}{c}\text { ACTIVITY } \\
\text { ID }\end{array}$ & $\begin{array}{l}\text { ORIO } \\
\text { DUR }\end{array}$ & $\begin{array}{l}\text { REM } \\
\text { DUR }\end{array}$ \\
\hline 214 HO3OA7O & 4 & 4 \\
\hline $2144029 A 70$ & & \\
\hline $214 H 028 A 70$ & 4 & 4 \\
\hline $215 K 027 A 70$ & 4 & 4 \\
\hline $215 H 026 A 70$ & 4 & 4 \\
\hline $215 \% 025 A 70$ & & 4 \\
\hline $215 H 024 A 70$ & 4 & 4 \\
\hline $215 H 023 A 70$ & 4 & 4 \\
\hline $215 H 022 A 70$ & 4 & 4 \\
\hline $216 \mathrm{H} 021 \mathrm{~A} 70$ & 4 & 4 \\
\hline $216 \% 020 A 70$ & 4 & 4 \\
\hline $216 \% 019 A 70$ & 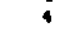 & 4 \\
\hline $236 \mathrm{HO} 18 \mathrm{~A} 70$ & 4 & 4 \\
\hline $2174027 A 70$ & 4 & 4 \\
\hline $217 \mathrm{H016A70}$ & 4 & 4 \\
\hline $228+4035 A 70$ & 4 & 4 \\
\hline 218 HO14A7O & 4 & 4 \\
\hline $218 H 013 \mathrm{~A} 70$ & 4 & 4 \\
\hline $219 \mathrm{HO} 12 \mathrm{~A} 70$ & 4 & 4 \\
\hline $219 \mathrm{HO11A70}$ & 1 & 4 \\
\hline $219 \mathrm{H} 010 \mathrm{~A} 70$ & 4 & 4 \\
\hline $220 H 009 A 70$ & 4 & 4 \\
\hline $220 \mathrm{HOOBA70}$ & 4 & 4 \\
\hline $2204007 A 70$ & 4 & 4 \\
\hline $220 \mathrm{HOOGA70}$ & 4 & 4 \\
\hline $220 \mathrm{HOO} 5 \mathrm{~A} 70$ & 4 & 4 \\
\hline $220 \mathrm{HO04A70}$ & 4 & 4 \\
\hline $221 \% 003 A 70$ & 4 & 4 \\
\hline $221 \mathrm{2HOO2A70}$ & 4 & 4 \\
\hline $\begin{array}{l}221 H 001 A 70 \\
211 H 044 A 71\end{array}$ & 9 & 9 \\
\hline $2114043 A 71$ & 9 & 9 \\
\hline $2119042 A 71$ & 9 & 9 \\
\hline 213H035A71 & 9 & 9 \\
\hline $213 \mathrm{H} 036 \mathrm{AT1}$ & 9 & 9 \\
\hline $213 \mathrm{HO} 37 \mathrm{~A} 71$ & 9 & 9 \\
\hline $213 \mathrm{HO} 32 \mathrm{AT}$ & 9 & 9 \\
\hline $213 \mathrm{H} 033 \mathrm{~A} 71$ & 9 & 9 \\
\hline $213 H 034 A 71$ & 9 & 9 \\
\hline $323 \mathrm{H} 185 A 71$ & 9 & 9 \\
\hline $323 \mathrm{H} 186 \mathrm{~A} 71$ & 9 & 9 \\
\hline $323 H 187 A 71$ & 9 & 9 \\
\hline $322 \mathrm{H} 188 \mathrm{~A} 71$ & 9 & 9 \\
\hline $322 \mathrm{HLE} 9 \mathrm{~A} 71$ & 9 & 9 \\
\hline $322 \mathrm{H} 190 \mathrm{OA} 7 \mathrm{I}$ & 9 & 9 \\
\hline $321 \mathrm{H174A71}$ & 9 & 9 \\
\hline $321 \mathrm{H175A72}$ & ? & 9 \\
\hline $320 \mathrm{H176A71}$ & 9 & 9 \\
\hline $320 \mathrm{OH} 177 \mathrm{~A} 7 \mathrm{I}$ & 9 & 9 \\
\hline $320 \mathrm{H} 178 \mathrm{~A} 71$ & 9 & 9 \\
\hline 214H031A71 & 9 & 9 \\
\hline $214 H 030 A 71$ & 9 & 9 \\
\hline 214H029A71 & s & 9 \\
\hline 214H028A71 & 9 & 9 \\
\hline $215 \mathrm{HO27A71}$ & 9 & 9 \\
\hline $215 \mathrm{HO26A71}$ & 9 & 9 \\
\hline $215 H 025 \mathrm{~A} 71$ & 9 & 9 \\
\hline $215 H 024 A 71$ & 9 & 9 \\
\hline $215 H 023 \mathrm{~A} 71$ & 9 & 9 \\
\hline $215 H 022 A 71$ & 9 & 9 \\
\hline $216 \mathrm{HO21A71}$ & 9 & 9 \\
\hline $216 H 020 A 71$ & 9 & 9 \\
\hline $216 \mathrm{HO29A71}$ & 9 & 9 \\
\hline 216H018A71 & 9 & 9 \\
\hline $217 H 017871$ & 9 & 9 \\
\hline $217 \% 016271$ & 9 & 9 \\
\hline $21 \mathrm{BHO} 25 \mathrm{~T} 2$ & 9 & 9 \\
\hline 210 QHO14A71 & 9 & 9 \\
\hline 21 Q18013A71 & 9 & 9 \\
\hline 219HO12A71 & 9 & 9 \\
\hline $219 H 011 A 71$ & 9 & 9 \\
\hline $219 \mathrm{HO10A71}$ & 9 & 9 \\
\hline $220 \mathrm{H} 009 \mathrm{~A} 71$ & 9 & 9 \\
\hline $220 \mathrm{H008A71}$ & 9 & 9 \\
\hline $220 \mathrm{HOO} 7 \mathrm{A71}$ & 9 & 9 \\
\hline 2201006271 & 9 & 9 \\
\hline $\begin{array}{l}220 \mathrm{HOOSA71} \\
220 \mathrm{HOOOAA71}\end{array}$ & 9 & 9 \\
\hline $220 H 004 A 71$ & 9 & 9 \\
\hline $\begin{array}{l}221 H 003 A 71 \\
221 H 002 A 71\end{array}$ & $?$ & 9 \\
\hline $221 H 002 A 71$ & $?$ & 9 \\
\hline $221 \mathrm{HOO1A71}$ & $\begin{array}{l}9 \\
6\end{array}$ & 9 \\
\hline 211 HO4A72 & 6 & 6 \\
\hline 211 H043A72 & 6 & 6 \\
\hline $211 \mathrm{~K} 042 \mathrm{~A} 72$ & 6 & 6 \\
\hline $213 \mathrm{HO} 35 \mathrm{SA2}$ & 6 & 6 \\
\hline $213 \mathrm{H} 036 \mathrm{~B} 72$ & 6 & 6 \\
\hline $213 \mathrm{HO} 37 \mathrm{A72}$ & 6 & 6 \\
\hline $213 \mathrm{HO32A72}$ & 6 & 6 \\
\hline $2134033 A 72$ & 6 & 6 \\
\hline $223 \mathrm{HO34A72}$ & 6 & 6 \\
\hline $323 \mathrm{H185A72}$ & 6 & 6 \\
\hline 323H186A72 & 6 & 6 \\
\hline 323 H187A72 & 6 & 6 \\
\hline $3224188 A 72$ & 6 & 6 \\
\hline 322H189A72 & $\begin{array}{l}6 \\
6\end{array}$ & $\begin{array}{l}6 \\
6\end{array}$ \\
\hline & & \\
\hline
\end{tabular}

Figure 9.13 - Case 2, Baseline Schedule (Continued). 
SAUD \& EBRAHIM ALABDURRAZZAX EST.

REPORT DATE $215 E P 95$ RUN NO. 121

gasoline Sehodulo by Typical Activity \& Es
PRIMAVERA PROUECT PLANNER

SEVEN SEAS, CONSTRUCTION OF 51 HOUSES
JAHRA 3 HOUSING PPOTECT COMTOR ACE

START DATE IAPR87 EIN DATE L3AUGSB

DATA DATE LAPRg7 PAGE NO. 3 B

\begin{tabular}{|c|c|c|c|c|}
\hline $\begin{array}{c}\text { ACTIVITY } \\
\text { ID }\end{array}$ & $\begin{array}{l}\text { ORTO } \\
\text { DUR } \\
-\end{array}$ & $\begin{array}{l}\text { REM } \\
\text { DUR }\end{array}$ & 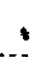 & CODE \\
\hline $321 \mathrm{H} 174 \mathrm{~A} 72$ & 6 & 6 & 0 & 16 \\
\hline $321 H 175 A 72$ & 6 & 6 & 0 & .17 \\
\hline $320 \mathrm{H176A72}$ & 6 & 6 & 0 & 18 \\
\hline $320 H 177 A 72$ & 6 & 6 & 0 & 19 \\
\hline $320 \mathrm{H178A72}$ & 6 & 6 & 0 & 20 \\
\hline $\begin{array}{l}214 \mathrm{H} 031 \mathrm{AA72} \\
214 \mathrm{H} 030 \mathrm{~A} 72\end{array}$ & $\begin{array}{l}6 \\
6\end{array}$ & $\begin{array}{l}6 \\
6\end{array}$ & $:$ & $\begin{array}{l}21 \\
22\end{array}$ \\
\hline $314 \mathrm{H} 029 \mathrm{~A} 72$ & 6 & 6 & 0 & 23 \\
\hline $214 \mathrm{HO28A72}$ & 6 & 6 & 0 & 24 \\
\hline $215 \mathrm{H027A72}$ & 6 & 6 & 0 & 25 \\
\hline $215 \mathrm{H} 026 \mathrm{~A} 72$ & 6 & 6 & 0 & 26 \\
\hline $215 \mathrm{H} 025 \mathrm{A72}$ & 6 & 6 & 0 & 27 \\
\hline $215 \mathrm{H} 024 \mathrm{~A} 72$ & 6 & 6 & 0 & 29 \\
\hline $215 \mathrm{HO} 33 \mathrm{~A} 72$ & 6 & 6 & 0 & 29 \\
\hline $215 \mathrm{H} 022 \mathrm{A72}$ & & 6 & 0 & 30 \\
\hline $\begin{array}{l}2164021 \mathrm{AA72} \\
216 \mathrm{H} 020 \mathrm{~A} 72\end{array}$ & $\begin{array}{l}6 \\
6\end{array}$ & $\begin{array}{l}6 \\
6\end{array}$ & $\stackrel{0}{0}$ & $\begin{array}{l}31 \\
32\end{array}$ \\
\hline $216 \mathrm{H} 019 \mathrm{~A} 72$ & 6 & 6 & 0 & 33 \\
\hline $216 \mathrm{HO} 2 \mathrm{\theta A72}$ & 6 & 6 & 0 & 34 \\
\hline $217 \mathrm{H} 017 \mathrm{~A} 72$ & 6 & 6 & 0 & 35 \\
\hline $217 H 016 A 72$ & 6 & 6 & 0 & 36 \\
\hline $218 \mathrm{HO} 015 \mathrm{~A} 72$ & 6 & 6 & 0 & 37 \\
\hline $218 H 024 A 72$ & 6 & 6 & 0 & 38 \\
\hline $21 \mathrm{HO} 13 \mathrm{~A} 72$ & 6 & 6 & 0 & 39 \\
\hline $219 \mathrm{HO} 012 \mathrm{~A} 72$ & 6 & 6 & 0 & 40 \\
\hline $219 \mathrm{HO} 11 \mathrm{~A} 72$ & 6 & 6 & 0 & 11 \\
\hline $219 \mathrm{H} 010 \mathrm{~A} 72$ & 6 & 6 & 0 & 42 \\
\hline $230 \mathrm{H} 009 \mathrm{~A} 72$ & 6 & 6 & 0 & 43 \\
\hline $220 \mathrm{OH} 008 \mathrm{~A} 72$ & 6 & 6 & 0 & 44 \\
\hline $220 \mathrm{H} 007 \mathrm{A2} 2$ & 6 & 6 & 0 & 45 \\
\hline $220 \mathrm{H} 006 \mathrm{~A} 72$ & 6 & 6 & 0 & 46 \\
\hline $\begin{array}{l}220 \mathrm{H} 005 \mathrm{~A} 72 \\
220 \mathrm{H} 004 \mathrm{72}\end{array}$ & $\begin{array}{l}6 \\
6\end{array}$ & 6 & $: 0$ & $\begin{array}{l}47 \\
18\end{array}$ \\
\hline $\begin{array}{l}220 \mathrm{H} 004 \mathrm{AA} 72 \\
22 \mathrm{H} 003 \mathrm{~A} 72\end{array}$ & 6 & 6 & 0 & $\begin{array}{l}18 \\
49\end{array}$ \\
\hline $221 \mathrm{H} 002 \mathrm{~A} 72$ & 6 & 6 & 0 & so \\
\hline $221 \mathrm{H} 001 \mathrm{~A} 72$ & 6 & 6 & 0 & 51 \\
\hline $211 \mathrm{H} 044 \mathrm{~A} 73$ & 1 & 1 & 0 & 01 \\
\hline $212 \mathrm{HO} 3 \mathrm{~A}$.73 & 1 & 1 & 0 & 02 \\
\hline $211 \mathrm{H042A73}$ & 1 & 1 & 0 & 03 \\
\hline $213 \mathrm{H} 035 \mathrm{AA3}$ & 1 & 1 & 0 & 04 \\
\hline $213 \mathrm{H} 036 \mathrm{A73}$ & 1 & 1 & 0 & os \\
\hline $213 \mathrm{H} 037 \mathrm{A73}$ & 1 & 1 & 0 & 06 \\
\hline $213 \mathrm{HO} 32 \mathrm{~A} 73$ & 2 & 1 & 0 & 07 \\
\hline $2133033 A 73$ & $i$ & $i$ & 0 & 08 \\
\hline $213 \mathrm{H} 034 \mathrm{~A} 73$ & 1 & $\overline{1}$ & 0 & 09 \\
\hline $323 H 185 A 73$ & 1 & 1 & 0 & 10 \\
\hline $323 \mathrm{H} 286 \mathrm{~A} 73$ & 1 & 1 & 0 & 11 \\
\hline 323H187A73 & i & 1 & 0 & 12 \\
\hline $322 \% 180 A 73$ & 1 & 1 & 0 & 13 \\
\hline $322 \mathrm{H}_{189 A 73}$ & 1 & 1 & 0 & 14 \\
\hline $322 \mathrm{H} 190 \mathrm{~A} 73$ & 1 & 1 & 0 & 15 \\
\hline $321 \mathrm{H} 174 \mathrm{~A} 73$ & 1 & 1 & 0 & 16 \\
\hline $321 H 175 A 73$ & 1 & 1 & 0 & 17 \\
\hline $3204176 A 73$ & 1 & 1 & 0 & 18 \\
\hline $320 \mathrm{H177A73}$ & I & $\overline{1}$ & 0 & 19 \\
\hline $320 \mathrm{H178A73}$ & 1 & 1 & 0 & 20 \\
\hline 214H031A73 & 1 & 1 & 0 & 21 \\
\hline $214 \mathrm{H} 030 \mathrm{OA} 73$ & $\overrightarrow{1}$ & $i$ & 0 & 22 \\
\hline $2144029 A 73$ & 1 & 1 & 0 & 23 \\
\hline $214 \mathrm{HO28A73}$ & 1 & 1 & 0 & 24 \\
\hline $215 \mathrm{HO} 27 \mathrm{~A} 73$ & 1 & 1 & 0 & 25 \\
\hline $215 \mathrm{HO} 26 \mathrm{A73}$ & 1 & 1 & 0 & 26 \\
\hline $215 \mathrm{H025A73}$ & 1 & 1 & 0 & 27 \\
\hline $215 H 024 A 73$ & 2 & 1 & 0 & 28 \\
\hline $215 \mathrm{H} 023 \mathrm{A73}$ & $\mathbf{1}$ & 1 & 0 & 29 \\
\hline $215 H 022 A 73$ & $\mathbf{1}$ & 1 & 0 & 30 \\
\hline $216 \mathrm{HO21A73}$ & $\mathbf{1}$ & 1 & 0 & 31 \\
\hline $216 \mathrm{HO2OA73}$ & 1 & 1 & 0 & 32 \\
\hline $216 \mathrm{HO19A73}$ & $i$ & 1 & 0 & 33 \\
\hline $2166018 A 73$ & 1 & 1 & 0 & 34 \\
\hline $217 \mathrm{H} 017 \mathrm{A73}$ & 1 & 2 & 0 & 35 \\
\hline $217 \mathrm{H} 016 \mathrm{AA73}$ & 1 & 1 & 0 & 36 \\
\hline 218 1815A73 & 1 & 1 & 0 & 37 \\
\hline $218 \mathrm{OH} 014 \mathrm{~A} 73$ & 1 & i & 0 & 38 \\
\hline $218 \mathrm{H} 013 \mathrm{~A} 73$ & 1 & 1 & 0 & 39 \\
\hline $2194012 \mathrm{~A} 73$ & 1 & 1 & 0 & 40 \\
\hline $219 \mathrm{HO} 11 \mathrm{~A} 73$ & 1 & 1 & 0 & 41 \\
\hline $2194010 A 73$ & $i$ & 1 & 0 & 42 \\
\hline $220 \mathrm{HOO} A \mathrm{~A} 3$ & 1 & $i$ & 0 & 43 \\
\hline $220 \mathrm{HOOOA73}$ & 1 & 1 & 0 & 44 \\
\hline $2204007 A 73$ & 1 & 1 & 0 & 45 \\
\hline $220 \mathrm{H} 006 \mathrm{AP} 73$ & $\mathbf{1}$ & 1 & 0 & 46 \\
\hline $22 \mathrm{OHOOSA73}$ & 1 & 1 & 0 & \\
\hline $220 \mathrm{HOO4A73}$ & $i$ & 1 & 0 & 49 \\
\hline $221 \% 003 A 73$ & 1 & 1 & 0 & 49 \\
\hline $221 \mathrm{HOO2A73}$ & 1 & 1 & 0 & 50 \\
\hline $2214001 \mathrm{~A} 73$ & 1 & 1 & 0 & 51 \\
\hline $211 H 044 A 74$ & 10 & 10 & 0 & 01 \\
\hline $211 \% 043 A 74$ & 20 & 10 & 0 & 02 \\
\hline $21114012 \mathrm{A74}$ & 10 & 10 & 0 & 03 \\
\hline 213 H035A74 & 10 & 10 & 0 & 04 \\
\hline $213 H 036274$ & 10 & 10 & 0 & 05 \\
\hline $2234037 A 74$ & 10 & 10 & 0 & 06 \\
\hline $213 \mathrm{H} 032 \mathrm{~A} 74$ & 20 & 10 & 0 & 07 \\
\hline $223 \mathrm{H}_{033 \mathrm{~A} 74}$ & 10 & 10 & 0 & 08 \\
\hline $213 \% 034 A 74$ & 10 & 20 & 0 & 09 \\
\hline
\end{tabular}

Figure 9.13 - Case 2, Baseline Schedule (Continued). 


\begin{tabular}{|c|c|}
\hline $\begin{array}{c}\text { ACTIVITY } \\
\text { ID }\end{array}$ & $\begin{array}{l}\text { ORIG } \\
\text { DUR } \\
\cdots . .\end{array}$ \\
\hline $323 \mathrm{~K} 105 \mathrm{~A} 74$ & 10 \\
\hline $323 \mathrm{HLO6A74}$ & 10 \\
\hline $323 \mathrm{H} 187 \mathrm{~A} 74$ & 10 \\
\hline $322 \mathrm{H} 188 \mathrm{~A} 74$ & 10 \\
\hline $322 \mathrm{H} 199 \mathrm{~A} 74$ & 10 \\
\hline $322 \mathrm{H190A74}$ & 10 \\
\hline $321 \mathrm{H} 174 \mathrm{~A} 74$ & 10 \\
\hline $321 \mathrm{H} 175 \mathrm{~A} 7 \mathrm{4}$ & 10 \\
\hline $320 \mathrm{H} 176 \mathrm{~A} 74$ & 10 \\
\hline $320 \mathrm{HL} 77 \mathrm{A74}$ & 10 \\
\hline $320 \mathrm{H178A74}$ & 20 \\
\hline $214 \mathrm{H} 031 \mathrm{~A} 74$ & 10 \\
\hline $214 \mathrm{H} 030 \mathrm{A74}$ & 10 \\
\hline $214 \mathrm{H029A74}$ & 10 \\
\hline $2144026 \mathrm{AA} 74$ & 10 \\
\hline $2154027 A 74$ & 10 \\
\hline $215 H 026 \mathrm{~A} 74$ & 10 \\
\hline $215 \mathrm{HO25A74}$ & 10 \\
\hline $215 \mathrm{H} 024 \mathrm{~A} 74$ & 10 \\
\hline $2154023 \mathrm{~A} 74$ & 10 \\
\hline $215 K 022 \mathrm{~A} 74$ & 10 \\
\hline $216 \mathrm{H02} 2 \mathrm{A74}$ & 10 \\
\hline $216 \% 020 \mathrm{~A} 74$ & 10 \\
\hline $216 \mathrm{H029A74}$ & 10 \\
\hline $216 \mathrm{HO18A74}$ & 10 \\
\hline $217 \mathrm{H027A74}$ & 10 \\
\hline $217 \% 016 A 74$ & 10 \\
\hline $219 H 015 \mathrm{~A} 74$ & 10 \\
\hline 219H014A74 & 10 \\
\hline $21 \mathrm{BHO} 13 \mathrm{~A} 74$ & 10 \\
\hline $219 H 022 A 74$ & 10 \\
\hline & 10 \\
\hline $219 H 010 A 74$ & 10 \\
\hline $220 \mathrm{H} 009 \mathrm{~A} 74$ & 10 \\
\hline $220 \mathrm{HOOBA74}$ & 10 \\
\hline $220 \mathrm{H} 007 \mathrm{A74}$ & 10 \\
\hline $220 H 006 \mathrm{A74}$ & 10 \\
\hline $220 \mathrm{HOOSA74}$ & 10 \\
\hline $220 \mathrm{H004A74}$ & 10 \\
\hline 221H003A74 & 10 \\
\hline 221 H002A74 & 10 \\
\hline 221H001A74 & 10 \\
\hline $2114044 A 75$ & 5 \\
\hline 211H043A75 & 5 \\
\hline 211H042A75 & 5 \\
\hline $2134035 A 75$ & 5 \\
\hline 213 H036A75 & 5 \\
\hline 213H037A75 & 5 \\
\hline $213 H 032 A 75$ & 5 \\
\hline $213 \mathrm{H} 033 \mathrm{~A} 75$ & 5 \\
\hline $213 H 034275$ & 5 \\
\hline $323 \mathrm{H} 185 A 75$ & 5 \\
\hline $323 \mathrm{H} 186 \mathrm{~A} 75$ & 5 \\
\hline $323 \mathrm{H} 187 \mathrm{~A} 75$ & 5 \\
\hline $322 \mathrm{H188A75}$ & 5 \\
\hline $322 \mathrm{H} 189 \mathrm{ASS}$ & 5 \\
\hline $322 \mathrm{H} 190 \mathrm{~A} 75$ & 5 \\
\hline $3224174 A 75$ & 5 \\
\hline $321 \mathrm{H} 175 \mathrm{~A} 75$ & 5 \\
\hline $320 \mathrm{H} 176 \mathrm{~A} 75$ & 5 \\
\hline $320 H 177$ A5 & 5 \\
\hline $320 \mathrm{H} 17 \mathrm{BA} 75$ & 5 \\
\hline $214 \mathrm{H} 031 \mathrm{~A} 75$ & 5 \\
\hline $214 \mathrm{H} 030 \mathrm{~A} 7 \mathrm{~S}$ & 5 \\
\hline $214 \mathrm{H029A75}$ & 5 \\
\hline $214 \mathrm{HO28A75}$ & 5 \\
\hline $215 \mathrm{H027A75}$ & 5 \\
\hline $215 \mathrm{HO26A75}$ & 5 \\
\hline 215H025A75 & 5 \\
\hline $2154024 \mathrm{~A} 75$ & 5 \\
\hline $215 \mathrm{H} 023 \mathrm{~A} 75$ & 5 \\
\hline $215 \mathrm{HO} 22 \mathrm{A75}$ & 5 \\
\hline $216 \mathrm{HO2} 1 \mathrm{~A} 75$ & 5 \\
\hline $216 \mathrm{H} 020 \mathrm{~A} 75$ & 5 \\
\hline $216 \mathrm{HO19A75}$ & 5 \\
\hline $216 \mathrm{HO} 01 \mathrm{BA} 75$ & 5 \\
\hline $2177017 \mathrm{ATS}$ & 5 \\
\hline 217 HO16A75 & \\
\hline $210 H 015 A 75$ & 5 \\
\hline 21BH014A75 & 5 \\
\hline 218H013A75 & $\mathbf{5}$ \\
\hline $219 H 012 \mathrm{~A} 75$ & 5 \\
\hline $219 \mathrm{HO} 11 \mathrm{A75}$ & 5 \\
\hline $219 \mathrm{H} 010 \mathrm{~A} 35$ & 5 \\
\hline $22 \mathrm{OH} 009 \mathrm{~A} 75$ & 5 \\
\hline $22 \mathrm{OH} 00 \mathrm{BA} 7 \mathrm{~S}$ & 5 \\
\hline $220 \mathrm{H} 007 \mathrm{~A} 75$ & 5 \\
\hline $220 \mathrm{H} 006 \mathrm{GA} 5$ & 5 \\
\hline $220 \mathrm{H} 005 \mathrm{~A} 7 \mathrm{~S}$ & 5 \\
\hline $2204004 A 73$ & 5 \\
\hline $221 \% 003 A 75$ & 5 \\
\hline $221 \% 002 \mathrm{~A} 75$ & $\mathbf{5}$ \\
\hline $221 \% 001 A 75$ & 5 \\
\hline $221 H 044 A 76$ & 2 \\
\hline $211 \mathrm{H} 043 \mathrm{~A} 76$ & 2 \\
\hline $211 \mathrm{H} 042 \mathrm{~A} 76$ & 2 \\
\hline
\end{tabular}

Figure 9.13 - Case 2, Baseline Schedule (Continued). 
SAUO ST EBRAHIM ALABDTLRAZZAK EST

P.EPORT DATE 21 SEP95 RIN NO. 121
PRIMAVERA PPONECT PLANRER

SEVEN SEAS. CONSTRUCTION OF 51 HOUSES
JAHRA 3 HOUSING PROJECT COMmON acE

START DATE IAPR87 FIN DATE 13AUG8B

DATA DATE LAPRaT PAGE NO. 40

\begin{tabular}{|c|c|}
\hline $\begin{array}{c}\text { ACTIVITY } \\
\text { ID }\end{array}$ & $\begin{array}{l}\text { ORIO } \\
\text { DUR }\end{array}$ \\
\hline $213 H 035 A 76$ & \\
\hline $213 B 036 A 76$ & 2 \\
\hline $213 \mathrm{HO37A76}$ & 2 \\
\hline $213 H 032 A 76$ & 2 \\
\hline $2134033 A 76$ & 2 \\
\hline $213 H 034 A 76$ & 2 \\
\hline 323H185A76 & 2 \\
\hline $323 H 186 A 76$ & 2 \\
\hline $323 \mathrm{H187A76}$ & 2 \\
\hline $322 H 188 A 76$ & 2 \\
\hline $322 \mathrm{H} 189 \mathrm{~A}>6$ & 2 \\
\hline $3224190 \mathrm{~A} 76$ & 2 \\
\hline $321 H 174 A 76$ & 2 \\
\hline $321 \mathrm{H} 175 \mathrm{~A} 76$ & 2 \\
\hline $320 H 176 A 76$ & 2 \\
\hline $320 H 177 A 76$ & 2 \\
\hline $320 H 178 A 76$ & 2 \\
\hline $214 H 031 A 76$ & 2 \\
\hline $214 H 03$ OAT6 & 2 \\
\hline $214 H 029 A 76$ & 2 \\
\hline $214 H 028 A 76$ & 2 \\
\hline $215 H_{027 A 76}$ & 2 \\
\hline $215 \mathrm{H026A76}$ & 2 \\
\hline $215 H 025 A 76$ & 2 \\
\hline $215 H 024 A 76$ & 2 \\
\hline $215 \mathrm{H} 023 \mathrm{AT} 6$ & 2 \\
\hline $215 H 022 A 76$ & 2 \\
\hline 216H021A76 & 2 \\
\hline $216 \mathrm{H020A76}$ & 2 \\
\hline $216 H 029 A 76$ & 2 \\
\hline $216 \mathrm{HO18A76}$ & 2 \\
\hline $217 H 017 A 76$ & 2 \\
\hline $217 \mathrm{HO16A76}$ & 2 \\
\hline 218HO15A76 & 2 \\
\hline $219 H 014 A 76$ & 2 \\
\hline $218 H 023 A 76$ & 2 \\
\hline 219HO12A76 & 2 \\
\hline 219HO11A76 & 2 \\
\hline $219 \mathrm{HO1OA76}$ & 2 \\
\hline $220 H 009 A 76$ & 2 \\
\hline $2204008 A 76$ & 2 \\
\hline $220 H 007 A 76$ & 2 \\
\hline $220 H 006 A 76$ & 2 \\
\hline $220 \mathrm{HOOSA76}$ & 2 \\
\hline $22010004 A 76$ & 2 \\
\hline $221 H 003 A 76$ & 2 \\
\hline $221 H 002 A 76$ & 2 \\
\hline $221 \mathrm{H} 002 \mathrm{~A} 76$ & 2 \\
\hline $21111044 A 77$ & 5 \\
\hline $2114043 A>7$ & 5 \\
\hline $21118042 A 77$ & 5 \\
\hline $2134035 A 77$ & 5 \\
\hline $213 \mathrm{H} 036 \mathrm{~A} 77$ & 5 \\
\hline $213 \mathrm{H037A77}$ & 5 \\
\hline $213 H 032 A 77$ & 5 \\
\hline $213 \mathrm{HO} 33 \mathrm{A77}$ & 5 \\
\hline $213 \mathrm{HO34A77}$ & 5 \\
\hline 323H185A77 & 5 \\
\hline $323 \mathrm{H} 186 \mathrm{~A} 77$ & 5 \\
\hline $3233187 A 77$ & 5 \\
\hline $322 \mathrm{H188A77}$ & $\mathbf{5}$ \\
\hline $322 \mathrm{H189A77}$ & 5 \\
\hline $322 \mathrm{H} 190 \mathrm{~A} 77$ & 5 \\
\hline $3211 \mathrm{1744A77}$ & 5 \\
\hline $32114175 A 77$ & 5 \\
\hline $3204176 \mathrm{A77}$ & 5 \\
\hline $320 \mathrm{H177A77}$ & $\mathbf{s}$ \\
\hline $320 \mathrm{HL} 7 \mathrm{BA77}$ & 5 \\
\hline $214 \mathrm{HO} 1 \mathrm{~A} 77$ & 5 \\
\hline $214103030 A 77$ & $\mathbf{5}$ \\
\hline $214 \mathrm{H029A77}$ & 5 \\
\hline $214 H 020 A 77$ & 5 \\
\hline $215 \mathrm{H027A77}$ & 5 \\
\hline $215 \mathrm{HO26A77}$ & 5 \\
\hline $215 H 025 A 77$ & 5 \\
\hline $215 H 024 A 77$ & 5 \\
\hline $2154023 A 77$ & 5 \\
\hline $215 \mathrm{HO22A37}$ & 5 \\
\hline 216HO21A77 & 5 \\
\hline $216 \mathrm{HO20A77}$ & 5 \\
\hline $216 \mathrm{HO} 19 \mathrm{~A} 77$ & 5 \\
\hline $216 \mathrm{HO19A77}$ & $\mathbf{s}$ \\
\hline $217 \mathrm{H017A77}$ & 5 \\
\hline 217H016A77 & $\mathbf{5}$ \\
\hline 218 1815A77 & 5 \\
\hline $21 \mathrm{EHO14A77}$ & 5 \\
\hline $218 \mathrm{H} 013 \mathrm{~A} 77$ & 5 \\
\hline $319 H 012277$ & 5 \\
\hline $219 \mathrm{H} 011 \mathrm{~A} 77$ & 5 \\
\hline $219 H 010 \mathrm{A77}$ & 5 \\
\hline $220 \mathrm{H} 009 \mathrm{~A} 77$ & 5 \\
\hline $220 \mathrm{H} 008 \mathrm{~A} 77$ & 5 \\
\hline $220 \mathrm{H} 007 \mathrm{A77}$ & 5 \\
\hline $220 H 006 A 77$ & 5 \\
\hline $220 \mathrm{H005A77}$ & 5 \\
\hline $220 \mathrm{H} 004 \mathrm{~A} 77$ & 5 \\
\hline
\end{tabular}

Figure 9.13 - Case 2, Baseline Schedule (Continued). 
SAUD \& EBraHIM aLABDULRAzzAK EST,

REPORT OATE 21 SEP9S RUN NO. 121 $15: 46$
PRIMAVERA PPOJECT PLANNER

SEVEN SEAS. CONSTRUCTION OF 51 HOUSES
JAHRA 3 HOUSTHG PROJECT EOMmON aCT

START DATE LAPRg? FIN DATE 13AUGBa

DATA DATE LAPRg7 PAGE NO."

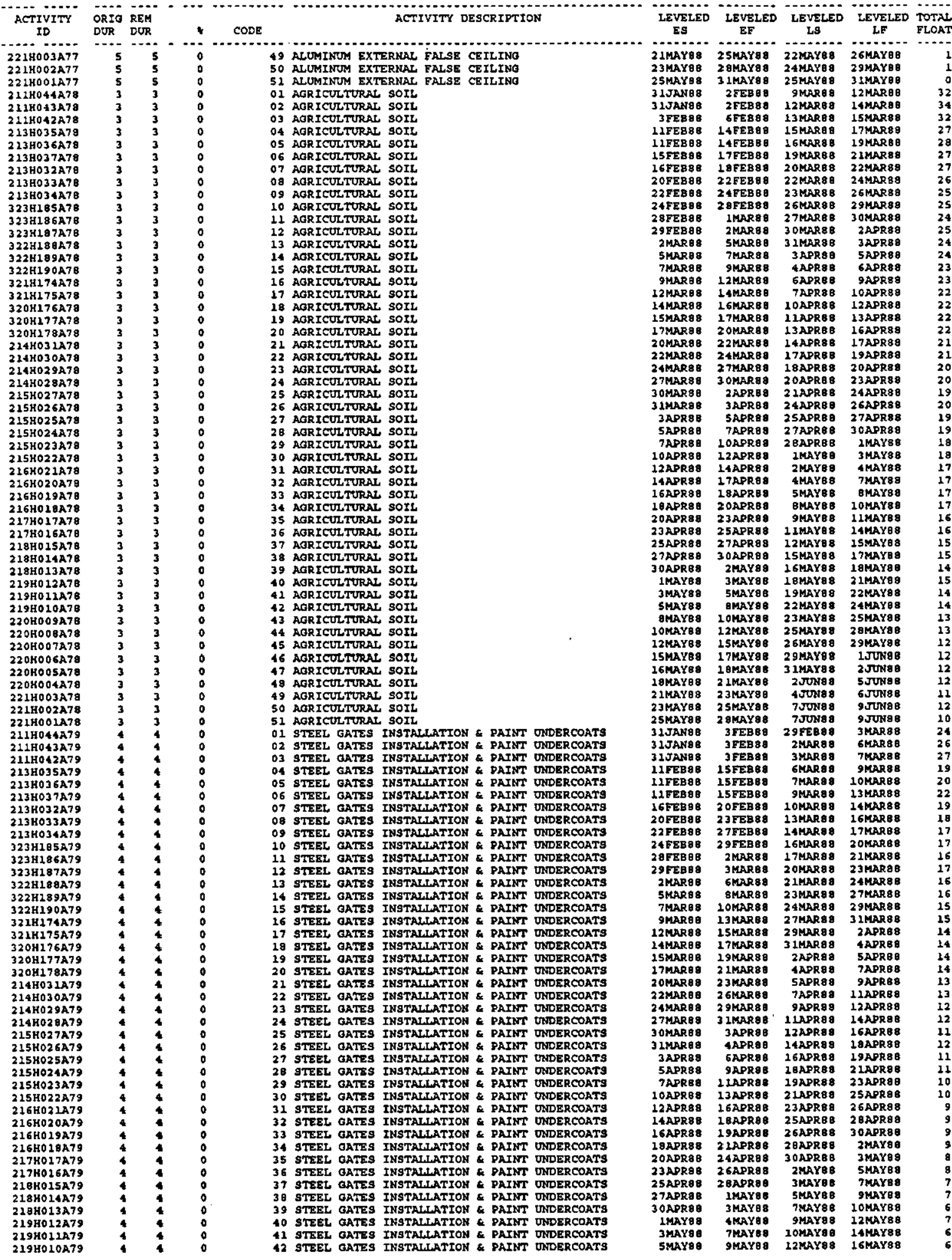

Figure 9.13 - Case 2, Baseline Schedule (Continued). 


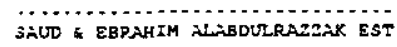

PEPORT DATE 21SEPYS RUN NO. 121

Baseline schedule by Typical Aetivity \& ES
PRIMAVERA PROJECT PLANIER

SEVEN SEAS, CONSTRUCTION OF 51 HOUSES

\begin{tabular}{|c|c|}
\hline$\underset{\text { AD }}{\text { ACTIVIY }}$ & $\begin{array}{l}\text { ORIO } \\
\text { DUR }\end{array}$ \\
\hline 22 OHOO9A79 & \\
\hline $220 H 000279$ & \\
\hline $220 H 007 A 79$ & 4 \\
\hline $2204006 \mathrm{~A} 79$ & 4 \\
\hline $2204005 A 79$ & 4 \\
\hline $220 H 004 A 79$ & 4 \\
\hline $221 \% 003 \mathrm{~A} 79$ & 4 \\
\hline $221 H 002 A 79$ & 4 \\
\hline 2214001279 & 4 \\
\hline $2114044 A 80$ & 1 \\
\hline $2114043 A 90$ & $i$ \\
\hline $2114042 A 90$ & 1 \\
\hline $213 \mathrm{HO} 35 \mathrm{ABO}$ & 1 \\
\hline 213 H036A日O & 1 \\
\hline $213 \mathrm{HO} 37 \mathrm{ABO}$ & 1 \\
\hline $213 \mathrm{HO} 32 \mathrm{ABO}$ & 1 \\
\hline $213 \mathrm{HO33A} 00$ & 1 \\
\hline $213 H 034 A 80$ & 1 \\
\hline $323 \mathrm{H} 185 \mathrm{ABO}$ & 1 \\
\hline $323 \mathrm{H} 286 \mathrm{ABO}$ & 1 \\
\hline $323 H 187$ A9O & 1 \\
\hline 322H1B8A9O & $i$ \\
\hline $322 \mathrm{H1B9ABO}$ & 1 \\
\hline 322H19OA9O & 1 \\
\hline $3211174 A 80$ & 1 \\
\hline $321 H 175 A 80$ & $i$ \\
\hline $320 \mathrm{H} 176 \mathrm{ABO}$ & 1 \\
\hline $320 H 177 A B O$ & 1 \\
\hline $320 \mathrm{H178ABO}$ & 1 \\
\hline 2 14H03 LABO & $\mathbf{1}$ \\
\hline $2114 \mathrm{OO}$ OABO & 1 \\
\hline $214 H 029 A 80$ & i \\
\hline $214 H 028 A B O$ & 1 \\
\hline $215 \mathrm{HO27A80}$ & 1 \\
\hline $2154026 A 80$ & 1 \\
\hline $215 H 025 A 80$ & 1 \\
\hline $215 H 024 A 80$ & 1 \\
\hline $215 H 023 A 80$ & 1 \\
\hline $215 H 022 A 80$ & 2 \\
\hline $216 \mathrm{HO} 21 \mathrm{AQO}$ & 1 \\
\hline $2164020 A 80$ & 1 \\
\hline $216 \mathrm{HO} 19 \mathrm{ABO}$ & $i$ \\
\hline $216 \mathrm{HO} 1 \mathrm{BABO}$ & 1 \\
\hline $217 \mathrm{HO} 27 \mathrm{ABO}$ & 1 \\
\hline $217 \mathrm{HO} 16 \mathrm{ABO}$ & 1 \\
\hline 218HO15A8O & 1 \\
\hline 21BHO14ABO & $i$ \\
\hline $21 \mathrm{BHO13ABO}$ & 1 \\
\hline $219 \mathrm{HO12ABO}$ & 1 \\
\hline 219HO11A8O & 1 \\
\hline $219 \mathrm{HO10A9O}$ & 1 \\
\hline $220 \mathrm{HOO9A8O}$ & 1 \\
\hline $220 \mathrm{NOOOA80}$ & 1 \\
\hline $22014007 A 80$ & 1 \\
\hline $2201006 A 80$ & 1 \\
\hline $220 \mathrm{HOOSA80}$ & 1 \\
\hline $220 \% 004 A 80$ & 1 \\
\hline $221 H 003 \mathrm{ABO}$ & 1 \\
\hline $221 \mathrm{HOO2ABO}$ & 1 \\
\hline $221 \% 002 \mathrm{ABO}$ & 1 \\
\hline 211 O44ABI & 1 \\
\hline $211 H 043 A O 1$ & i \\
\hline $211 \mathrm{HO42AO1}$ & 1 \\
\hline $213 \mathrm{HO3SABD}$ & 1 \\
\hline $213 \mathrm{HO} 36 \mathrm{AO1}$ & 1 \\
\hline $213 \mathrm{HO} 7 \mathrm{Ag1}$ & 1 \\
\hline $213 \mathrm{HO} 2 \mathrm{AQZ}$ & 1 \\
\hline $213 \mathrm{HO} 33 \mathrm{ABI}$ & 1 \\
\hline $213 \mathrm{HO34AB1}$ & 1 \\
\hline 323H185AR2 & 1 \\
\hline $323 \mathrm{H} 186 \mathrm{Ag1}$ & 1 \\
\hline $323 \mathrm{H1B7A91}$ & 1 \\
\hline $322 \mathrm{HIBBAB1}$ & 1 \\
\hline $322 H 189 A 81$ & 1 \\
\hline $322 \mathrm{H} 190 \mathrm{AB} 1$ & 1 \\
\hline $321 \mathrm{H174A81}$ & 1 \\
\hline $321 \mathrm{H} 175 \mathrm{ABI}$ & 1 \\
\hline $320 \mathrm{H} 176 \mathrm{~A} 81$ & 1 \\
\hline $320 \mathrm{HL77A81}$ & 1 \\
\hline $320 \mathrm{H278A81}$ & 1 \\
\hline $214 \mathrm{H} 031 \mathrm{A81}$ & 1 \\
\hline $214 \mathrm{HO} 30 \mathrm{AB} 1$ & 1 \\
\hline $214 \mathrm{HO29A81}$ & 1 \\
\hline $214 \mathrm{HO2} B A 81$ & 1 \\
\hline $215 H 027 A 81$ & 1 \\
\hline $215 \mathrm{HO2} 6 \mathrm{AB} 1$ & 1 \\
\hline $215 H 025 A 81$ & 1 \\
\hline $215 H 024 A 81$ & i \\
\hline $215 \mathrm{HO23AB1}$ & 1 \\
\hline $215 H 022 A 91$ & i \\
\hline $216 \mathrm{HO2} 1 \mathrm{ABI}$ & 1 \\
\hline $216 H 020 A 81$ & 1 \\
\hline $216 \mathrm{HO19AB1}$ & 1 \\
\hline $216 K 018 A B 1$ & 1 \\
\hline 217H017A日1 & 1 \\
\hline $2138026 \mathrm{AB1}$ & 1 \\
\hline
\end{tabular}

ACTIVITY DESCRIPTION

\$3 STEEL GATES INSTALLATION \& PAINT UNDERCOATS

41 STEEL GATES INSTALLATION \& PAINT UNDERCOATS

46 STEEL GATES INSTTALILATION O PAINT UNDERCOATS

17 STEEE GATES INSTALLATION \& PAINT UNDERCOATS

49 STEEL GATES INSTALLATION \& PAINT ONDERCOATS

作

11 STEEL GATES INSTALLATION \& PAINT UNDERCOATS

OI EXTERNAL ELECTRICAL FITTINGS

O3 EXTERNAI ELECTRICAL FITTINOS

O4 EXTERNAL ELECTR ICAL FITTINGS

O5 EXTERNAL ELECTRICAD FITTINGS

O6 EXTERNAL ELECTRICAI FITTINGS

OA EXTERNAL ELECTR ICAL FITTINGS

o9 EXTERNAL ELECTRICAL FITTINGS

EXTRNA ELCTRTCA PITINas

12 EXTERNAL STECTR TCAL ETTTINGS

13 EXTERNAL ELECTR ICAL TITTTHOS

14 EXTERNAL ELECTRICAL EITTTNOS

15 EXTERNAL ELECTRICAL FITTINGS

16 EXTERNAL ELECTRICAL FITTINGS

17 EXTERNAL ELECTRICAL FITTINGS

19 EXTERNAL ELECTRICAL FITTINGS

20 EXTERNAL ELECTRICAL FITTINGS

21 EXTERNAL ELECTRICAL FITTINGS

EXTERAL ELECTRTCAL EITTINOS

25 EXTTERNAL ELECTRICAL FITTINGS

26 EXTERNAL ELECTRICAL FITTINGS

ELECTRICAL FITTINAS

EXTERNAL ELECTRICAL FITTINOS

O EXTERNAL ELECTRICAL FITTINGS

31 EXTTERNAL ELECTRICAL FITTTNOS

32 EXTERNAL ELECTRICAL FITTINOS

3.

NNAL ELECTRICAL FITTINGS

6 EXTERAL ELETRICAE FITTNOS

37 EXTERNAL ELECTRICAL PTTINGS

38 EXTERNAL ELECTRICAL FITTINGS

39 EXTERNAL ELECTRICAL FITTINGS

40 EXTERNAL ELECTRICAL FITTINOS

EXTERNAL ELECTR ICAL FITTINGS

4.

I5 EXTRNAL ELETRICAL FTTINGS

46 EXTERNAL ELECTRICAL FITTINGS

EXTERNAL ELECTRICAL FITTINOS

EXTERNAL ELECTRICAL FITTINOS

9 EXTERANAL ELECTRICN FITTINOS

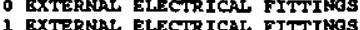

Ol ELECTRICAL E TELEPHONE NETWORKS TESTING

02 ELECTRICAL \& TELEPHONE NETWORKS TESTIN

O3 ELECTKICAL TELEPHONE NETWORKS TESTING

O4 ELECTRICAL \& TELEPHONE NETWORKS TESTIMO

OS ELECTRICAL \& TELEPHONE NETWORKS TESTINO

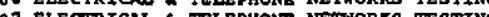

ELECTRICAR a TELEPHONE NETTORYs TEgTTVG

O ELECTRTCAL A TELEPHONE NETWORKS TESTING

10 ELECTRICAL A TELEPHONE NETWORRS TESTING

11 ELECTRICAI \& TELEPHONE NETWORKS TESTINO

12 ELECTRICAT \& TELEPHONE NETWORKS TESTING

13 ELECTRICAL \& TELEPHONE NETWORKS TESTTM

14 ELSCTRICAL \& TELEPHONE NETWORKS TESTING

15 ELECTRICAL \& TELEPHONE NETWORXS TESTIN 16 ELECTRICAL \& TELEPHONE NETWORXS TESTINO 17 ELLCARICNL \& TELEPHONE NETWORKS TESTING

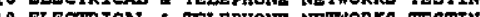
PHONE METWORKS TESTING 21 ELECTTICAL TTLPHON NETTORKS TESTING 22 ELECTRTCAI A TELEPHONE NETWORKS TESTING 23 ELECTRICAI A TELEPHONE NETWORKS TTSTING 24 ELECTRICAL A TELEPHONE NETWORKS TZSTINO 25 ELECTRICAI A TELEPHONE NETHORKS TESTING 26 ELECTRICAL \& TELRPHONE NETWORKS TESTING 27 ELECTRICAL TELEPHONE NETWORKS TESTING 29 ELLCTRICA \& TELEPHONE NETWORKS TESTING TER 31 ELECTRTCAL G TELEPHONE

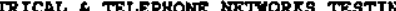
33 ELECTPICAL AELEPHONE NETWORYS TESTING 34 ELECTRICAL A TRLEPHONE NETWORXS TESTING 35 ELECTRICAL TELEPHONE NETWORKS TESTING
EXTERNA ELECTRICAL EITTrNos

44 EXTERNAI ELETR ICAL FITTIMOS
JAHRA 3 HOUSINO PROJECT COMMON ACE

START DATE LAPRE7 FIN DATE L3AUGg

DATA DATE LAPR日7 PAGE NO. 42
LEVELED LEVELED LEVELED LEVELED TOTAT

\begin{tabular}{|c|c|c|c|c|}
\hline & MAYB & & 17МАYВ8 & \\
\hline 10 MAY8B & MAYBB & 16 MAYBO & 19MAYAB & \\
\hline & 16MAY8B & 17MAYQB & 21MAYBB & \\
\hline 15MAY8 & 18MAY 8 & 19MAYG & 23 MAY8 & \\
\hline 16MAY日9 & 19MAY日 & 21 MAY 88 & 24MAY88 & \\
\hline IaMAYGg & 22 MAY8g & 23 MAY 88 & 26 MAY88 & \\
\hline 21 MAY89 & 24 MAY98 & 24MAY98 & 2 BMAY日 8 & \\
\hline 23 MAY98 & 2 SMAY8 8 & 2 OMAYYB & 1 JUNA 8 & \\
\hline 25 MAY8 B & 2 9MAY 88 & 2 SMAY8 & 15ONA8 & \\
\hline GFEBB & 6FEB 8 & JMAR8 & उMAR98 & \\
\hline $7 F E B 88$ & 7FE9 8 & 6MARB日 & 6MARBO & \\
\hline BFEB 8 & 8FEB日8 & 7 MARB & 7MAR8 & \\
\hline $27 \mathrm{FEB} 8 \mathrm{~g}$ & 17FEB98 & 9MAREQ & 9MAR88 & 17 \\
\hline 19FEB日8 & 1AFEB89 & 1OMARE9 & 10MARA9 & 17 \\
\hline 20FEBa8 & $20 F 889$ & 13MARBQ & 13MAR89 & 18 \\
\hline 23 FEB88 & 23 FEBS & 24MAAR 8 & 14MARE9 & \\
\hline 27 FEB8 & 27 FEB 88 & 16MARA & 16MAR日9 & \\
\hline $29 \mathrm{FEBaB}$ & 29 FEBB & 17WAR9B & 17MARE 8 & 15 \\
\hline 2MAR8B & 2MARB B & 20MARE 8 & $\begin{array}{l}20 \text { MARB } 8 \\
2 \text { MARB B }\end{array}$ & $\begin{array}{l}15 \\
14\end{array}$ \\
\hline 5MARB & SMARB B & 21 MAR8 & $\begin{array}{l}2 \text { IMAR日B } \\
2 \text { MAARgB }\end{array}$ & 18 \\
\hline $\begin{array}{l}\text { GMARB B } \\
\text { gMARRBg }\end{array}$ & $\begin{array}{l}\text { 6MARE8 } \\
\text { OKAREg }\end{array}$ & $\begin{array}{l}23 \text { MAABB } \\
24 \text { MARBg }\end{array}$ & $\begin{array}{l}\text { 23MAR88 } \\
24 \text { MAR89 }\end{array}$ & \\
\hline LOMARB & IOKARSA & 27 MAR 19 & 27 MAR89 & 14 \\
\hline 13MARBO & 13MAROB & 29 MARES & 29 MAR89 & 13 \\
\hline 15MARa & 15MAREB & 31 MAR8 8 & 31MARB9 & 13 \\
\hline 17 1 MARaB & 17MARB & 2APR98 & 2APREB & 12 \\
\hline 2 OMARB 8 & 20 MARB 8 & 4APRa8 & 4APRE 8 & \\
\hline 21 MARB 8 & 21MARB & 5APR8B & SAPR日B & \\
\hline 23MAREA & 23\%ARE8 & 7APRBg & 7APR8B & 12 \\
\hline 26 MAREA & 2 GMAR89 & 9APRB & 9APRAB & 11 \\
\hline 29 MAREA & 29 MARg日 & 11APRE9 & 11APRAB & 11 \\
\hline 31 MAR $9 \theta$ & 31 MAR 8B & 12APREA & 12ASR88 & 10 \\
\hline 3APRgB & 3APR日8 & 14APRE9 & 14APRBg & 10 \\
\hline 5APR 88 & SAPRBB & 16APR9B & 16APRE9 & \\
\hline 6APRB B & CAPRB B & 18APRB & 18APR日9 & 10 \\
\hline SAPREB & 9ADRB & 19APR8 & 19APR日8 & 9 \\
\hline LAPREB & 12APREO & 21APRBO & 21APR8 & \\
\hline 3APR日9 & 13APR8 & 23APREB & 23APR98 & \\
\hline 6APRE & 16APR89 & 25APRB & 25APR8B & \\
\hline LAAPR日A & 1OAPR89 & 26APRB & & \\
\hline 20APRB9 & 20 APRAg & 2 BAPR日 8 & 28APR8\% & \\
\hline 21 APRS & 21APR 83 & 30APR8 8 & 3OAPRE9 & \\
\hline 24APR:8 & 24APRBB & 2MAY9E & 2MAY日8 & \\
\hline 2 GAPRB B & 26APRB & 3MAY马Q & 3MAYG B & \\
\hline 2 OAPRB & 28APRE & 5 MAY88 & 5MAY8 & \\
\hline 1MAYBg & 1MAYB & 7MAYB8 & 7MAYBQ & \\
\hline $\begin{array}{l}3 \text { MAYQ8 } \\
5 \text { MAY98 }\end{array}$ & $\begin{array}{l}3 \text { MAY8 } \\
5 \text { MAY8B }\end{array}$ & $\begin{array}{r}\text { 9MAY98 } \\
\text { IOMAYQ8 }\end{array}$ & 9MAY8 & \\
\hline 7 MAY98 & 7 MAYSB & $\begin{array}{l}\text { 10MAYYg } \\
12 \text { MAYGg }\end{array}$ & $\begin{array}{l}\text { 10LAY88 } \\
12 \text { MAY8B }\end{array}$ & 5 \\
\hline 9MAY98 & 9MAY8B & 16MAYG & & 4 \\
\hline 11 MAYaв & 21MAYBB & 36 MAY98 & 16 MAY9 8 & 4 \\
\hline 14 MAY88 & 14 MAY 88 & 27MAY98 & 17 MaYeB & 3 \\
\hline LMAYB 8 & 16 MAY88 & 19MAY88 & 29MAYBB & 3 \\
\hline L8KAY89 & 18 MAY 89 & 21 MAYG & & 2 \\
\hline 21 MAY 89 & 21 MAY90 & 23MAY99 & 23 MAY89 & 2 \\
\hline 2 MAY 98 & 22MAY日e & 24MAYBO & 24 MAYB & \\
\hline 24 MAY8B & 24MAYB & 25 MAY9 8 & 2 GMAYBg & \\
\hline 26 MAY8E & 26MAYB8 & 2 gMAY98 & 2 BMAYAs & \\
\hline 9 MAY9B & 29 MAY 88 & 31 MAYBo & 31 MAYgB & \\
\hline 1JUN88 & 1)TNA & $150 N 88$ & 1 J & \\
\hline 7FEB 8 & $7 F 888$ & 24MARBa & 24MAR98 & 39 \\
\hline 8FEB 8 & 8FEB 90 & 26MARA8 & 26 MARBa & 39 \\
\hline 9EEg & 9FEB & 27 TMAREB & 27MARE : & \\
\hline 18FEB 8 & 19FEB98 & 30MAREA & 30MARES & 33 \\
\hline OFEB8 & 20FEBB B & 3 1MAR8B & $32 \mathrm{MAR}$ & 33 \\
\hline 1 FEg9日 & 21 FEB 8 & 2APROB & 2AP & 33 \\
\hline 4FEBa 8 & 24FEG8 8 & 4APR8B & 1APR日B & 32 \\
\hline $8 F 8888$ & 2 9FEB 8 & SAPREB & SAPR8B & \\
\hline IMAR日B & IMAR日8 & 6APRE8 & GAPRAB & 30 \\
\hline & 3MAR日8 & 9APRE\& & 9APR & 30 \\
\hline & 6MARA9 & 10APREA & $10 A 0$ & 29 \\
\hline 7MAR89 & 7MAR9日 & 11APR日9 & $11 \mathrm{AP}$ & 29 \\
\hline 9MARE8 & 9MAR9O & & & 29 \\
\hline 12MAR日8 & 12MAAR8 & 14APREB & 14APR 88 & 28 \\
\hline & 14MAR & & & 27 \\
\hline $16 \mathrm{MAP}$ & 16MAR88 & IBA & $18 \mathrm{~A}$ & 27 \\
\hline IMAR & 19 MARE 8 & 19APR9B & 19APRB & 26 \\
\hline $1 \mathrm{M}$ & & & & 25 \\
\hline $2 \mathrm{M}$ & 22 MARE8 & 23APR8B & 23APRAB & 26 \\
\hline & 26MARE8 & $24 A$ & 242 & 25 \\
\hline & $27 \mathrm{M}$ & 25A & & \\
\hline OMAR & 30MARB & $27 \mathrm{~A}$ & 27APRA 8 & 24 \\
\hline 2APR8B & 2APRBQ & 28APREA & 28APR8B & 23 \\
\hline 4APR8B & 4APRag & 30APRE9 & 30APR9 & 22 \\
\hline & 6APR98 & 2MAY & 2MAY8 & 22 \\
\hline & & & & 22 \\
\hline 108 & 10ADI & & & 21 \\
\hline PR & 12APR日O & 7MAY 88 & 7MAYB8 & 21 \\
\hline APR & 14APR9B & gKAY80 & BMAY8: & 20 \\
\hline & 17APRB 6 & 9 9АУ8в & 9MAYBg & 19 \\
\hline 197 & 19AP & 11MAY98 & $11 \mathrm{M}$ & 19 \\
\hline & $21 \mathrm{APR}$ & 12MAY9B & $12 \mathrm{MAY}$ & 28 \\
\hline & & 14MAY8S & 24MAYOS & 18 \\
\hline & & 16MAY88 & 16MAY98 & 28 \\
\hline & & $7 M$ & $17 \mathrm{M}$ & \\
\hline & & & & \\
\hline
\end{tabular}

Figure 9.13 - Case 2, Baseline Schedule (Continued). 
SAUD \& EBRAHIM ALABDULRAZZAX EST

P.EPORT DATE 21 SEP95 RUN NO 12 $15: 46$
PRIMAVERA PRQTECT PLANNER

SEVEN SEAS, CONSTRUCTION OF 51 HOUSES
JAHRA 3 YOUSING PROJECT GOMmOO

START DATE LAPR日? FIN DATE L3AUGBB

DATA DATE IAPRG? PAGE NO. 43

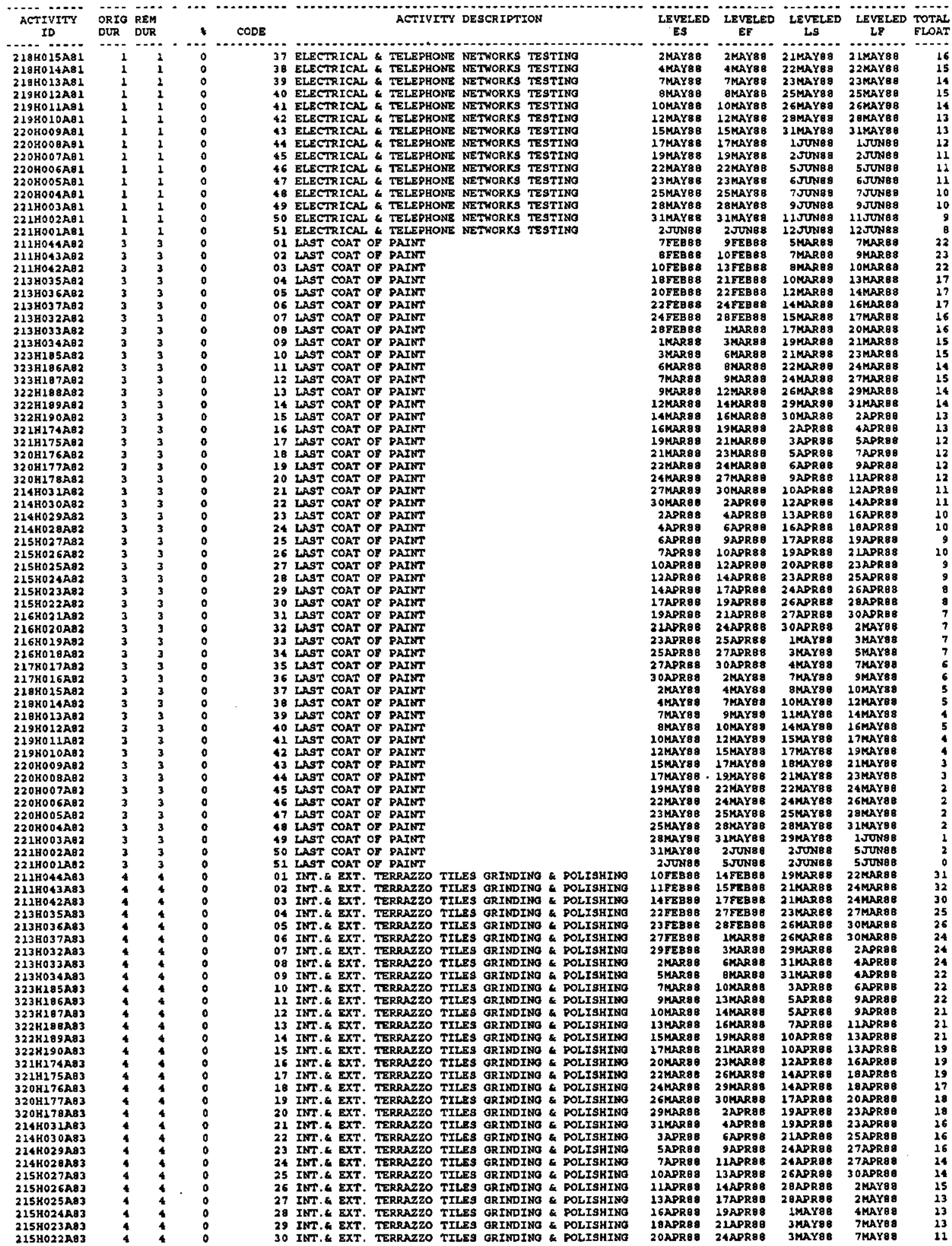

Figure 9.13 - Case 2, Baseline Schedule (Continued). 


\begin{tabular}{|c|c|}
\hline $\begin{array}{c}\text { ACTIVITY } \\
\text { ID }\end{array}$ & $\begin{array}{l}\text { ORIO } \\
\text { DUR }\end{array}$ \\
\hline $216 \mathrm{H} 021 \mathrm{AB} 3$ & \\
\hline $216 \mathrm{H} 020 \mathrm{AOB}$ & 4 \\
\hline $216 \mathrm{HO19AB3}$ & \\
\hline $216 \mathrm{HO18AB3}$ & 4 \\
\hline $2174017 \mathrm{ABO}$ & 4 \\
\hline $217 \mathrm{HO16AB3}$ & 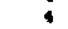 \\
\hline 218H015A日3 & \\
\hline $21 B H 024 A B 3$ & \\
\hline 21BH013A日3 & 4 \\
\hline $219 \mathrm{HOL12AB3}$ & 4 \\
\hline $219 \mathrm{HO} 01 \mathrm{AAB} 3$ & 4 \\
\hline $219 H 020 A 83$ & 4 \\
\hline 220H009AB3 & 4 \\
\hline $220 \mathrm{HOOBAB} 3$ & 4 \\
\hline $220 \mathrm{H} 007 \mathrm{Ag} 3$ & 4 \\
\hline $220 \mathrm{HO06 \textrm {ABO }}$ & 4 \\
\hline $220 \mathrm{HOOSA93}$ & 4 \\
\hline $220 \mathrm{HOOAA93}^{2}$ & 4 \\
\hline $221 \mathrm{H} 003 \mathrm{AB3}$ & 4 \\
\hline $221 \% 002 \mathrm{ABO}$ & 4 \\
\hline 221H001A93 & 4 \\
\hline $211 \mathrm{H} 044 \mathrm{~A} \theta 4$ & 2 \\
\hline $211 H_{043 A 84}$ & 2 \\
\hline 211H042A日4 & 2 \\
\hline 213H03SAE4 & 2 \\
\hline $213 \mathrm{H} 036 \mathrm{ABA}$ & 2 \\
\hline $223 \mathrm{HO37A94}$ & 2 \\
\hline 213H032A84 & 2 \\
\hline $213 \mathrm{H} 033 \mathrm{~A} 84$ & 2 \\
\hline $213 H_{034 A 94}$ & 2 \\
\hline $323 \mathrm{H} 185 \mathrm{~A} 94$ & 2 \\
\hline $323 \mathrm{H} 186 \mathrm{ABA}$ & 2 \\
\hline 323H187A.84 & 2 \\
\hline $322 \mathrm{H18BAQ4}$ & 2 \\
\hline $322 \mathrm{H} 189 \mathrm{Ag} 4$ & 2 \\
\hline $322 \mathrm{H} 190 \mathrm{OAB} 4$ & 2 \\
\hline $321 \mathrm{H} 174 \mathrm{~A} 94$ & 2 \\
\hline 321H175A84 & 2 \\
\hline $320 \mathrm{H} 176 \mathrm{~A} 84$ & 2 \\
\hline $320 \mathrm{H177A84}$ & 2 \\
\hline $320 \mathrm{H} 17 \mathrm{aAB4}$ & 2 \\
\hline 214H031A84 & 2 \\
\hline $214 \mathrm{H} 030 \mathrm{~A} 84$ & 2 \\
\hline $224 \mathrm{HO29A84}$ & 2 \\
\hline 214HO28A84 & 2 \\
\hline $215 \mathrm{HO27A84}$ & 2 \\
\hline $215 \mathrm{H026A84}$ & 2 \\
\hline 225H025AO4 & 2 \\
\hline $215 \mathrm{HO24AS4}$ & 2 \\
\hline $215 \mathrm{HO23A84}$ & 2 \\
\hline $215 \mathrm{HO} 022 \mathrm{ABA}$ & 2 \\
\hline 226H02 LAA4 & 2 \\
\hline 216HO2OAB4 & 2 \\
\hline 216H019A94 & 2 \\
\hline 216HO18A94 & 2 \\
\hline 217H027AB4 & 2 \\
\hline 217H026A84 & 2 \\
\hline 218H015A94 & 2 \\
\hline 21BHO14AB4 & 2 \\
\hline 21BH013A84 & 2 \\
\hline $219 \mathrm{HO12AB4}$ & 2 \\
\hline 219HO11AO4 & 2 \\
\hline & 2 \\
\hline $220 \mathrm{HOO9AB4}$ & 2 \\
\hline 220H008A94 & 2 \\
\hline $2204007 \mathrm{AB4}$ & 2 \\
\hline $220 H 006 \mathrm{AO4}$ & 2 \\
\hline $220 \mathrm{HOOSA84}$ & 2 \\
\hline $\begin{array}{l}220 \mathrm{HOO4AB4} \\
221 \mathrm{H} 003 \mathrm{ABS}\end{array}$ & 2 \\
\hline $\begin{array}{l}221 H_{003 A B A} \\
221 H 002 \mathrm{ABA}\end{array}$ & 2 \\
\hline & 2 \\
\hline 221H0O1AG4 & 2 \\
\hline 211H044ABS & 4 \\
\hline 211H043A85 & \\
\hline 211HO42ABS & \\
\hline 213H035A85 & \\
\hline 2234036485 & 4 \\
\hline 213H037A9S & 4 \\
\hline $213 \mathrm{HO} 032 \mathrm{ABS}$ & \\
\hline $213 H 033 A 85$ & \\
\hline 213H034A95 & 4 \\
\hline 323 H1OSA95 & 4 \\
\hline $3234186 \mathrm{ABS}$ & 4 \\
\hline 323H187AB5 & 4 \\
\hline 322H188ABS & \\
\hline $322 \mathrm{K189ABS}$ & 4 \\
\hline $322 \mathrm{H} 190 \mathrm{ABS}$ & \\
\hline $321 \mathrm{H174A85}$ & 4 \\
\hline $32214275 A 85$ & 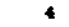 \\
\hline 320H276A95 & 4 \\
\hline $320 H 277 A 85$ & \\
\hline $320 H 278 A 85$ & \\
\hline $214 H 031 A 85$ & 4 \\
\hline 214 HO3OAA5 & 4 \\
\hline $214 \mathrm{HO29A85}$ & 4 \\
\hline $214 \mathrm{HO2}$ AAB 5 & 4 \\
\hline
\end{tabular}

Figure 9.13 - Case 2, Baseline Schedule (Continued). 


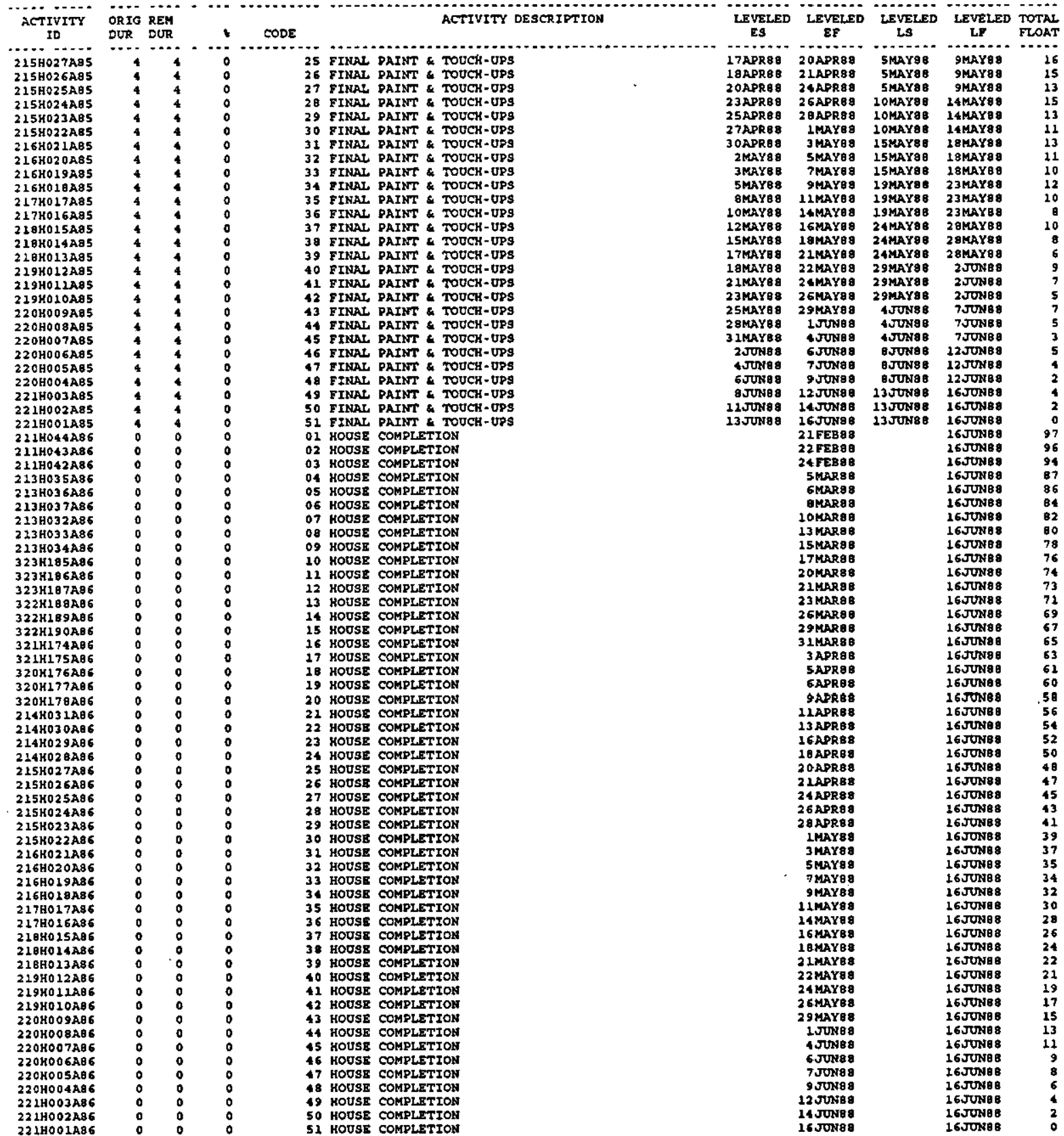

Figure 9.13 - Case 2, Baseline Schedule (Continued). 


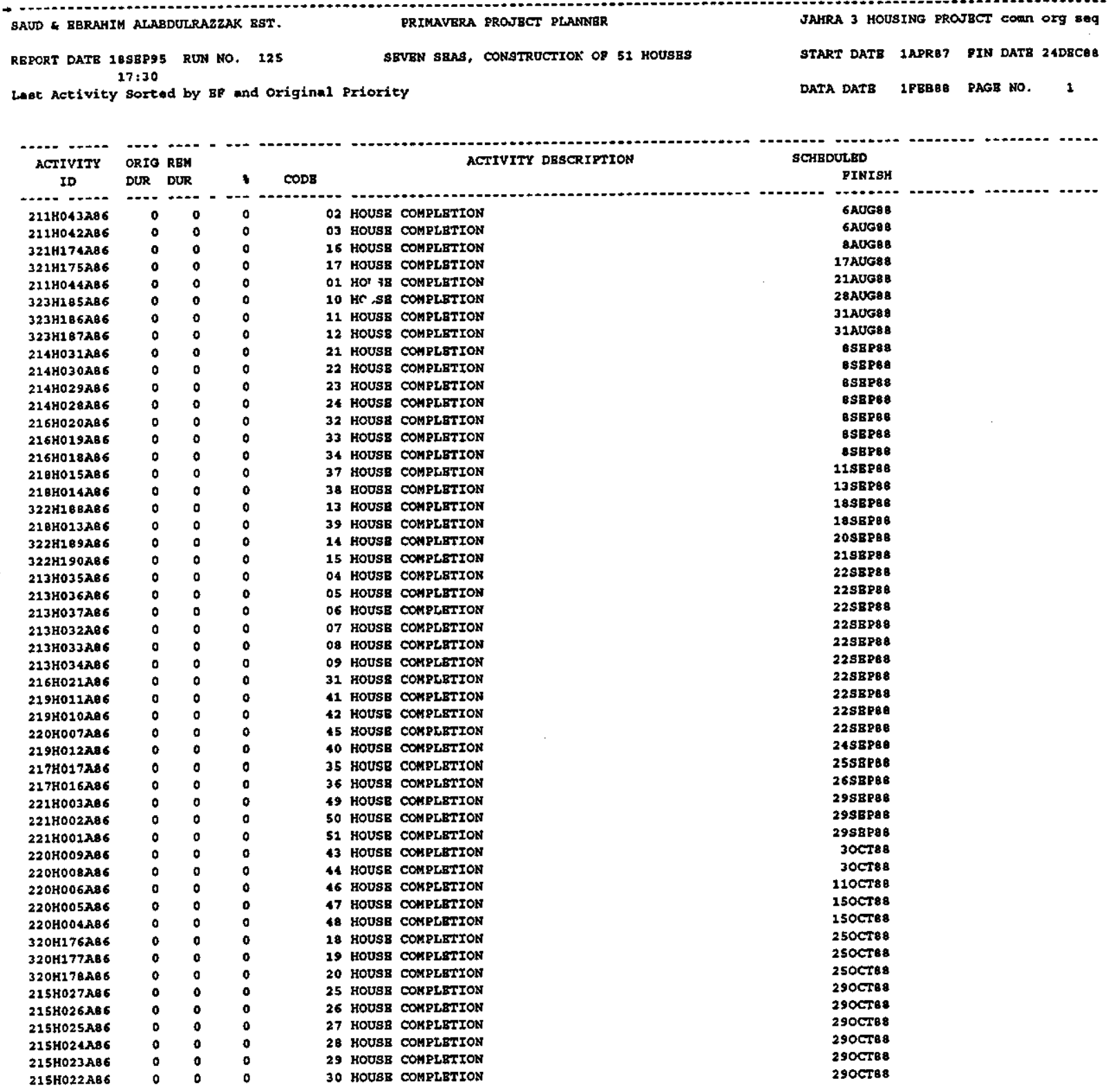

Figure 9.14 - Case 2, Current Sequence of Operation, as Indicated by House Projected Completions. 


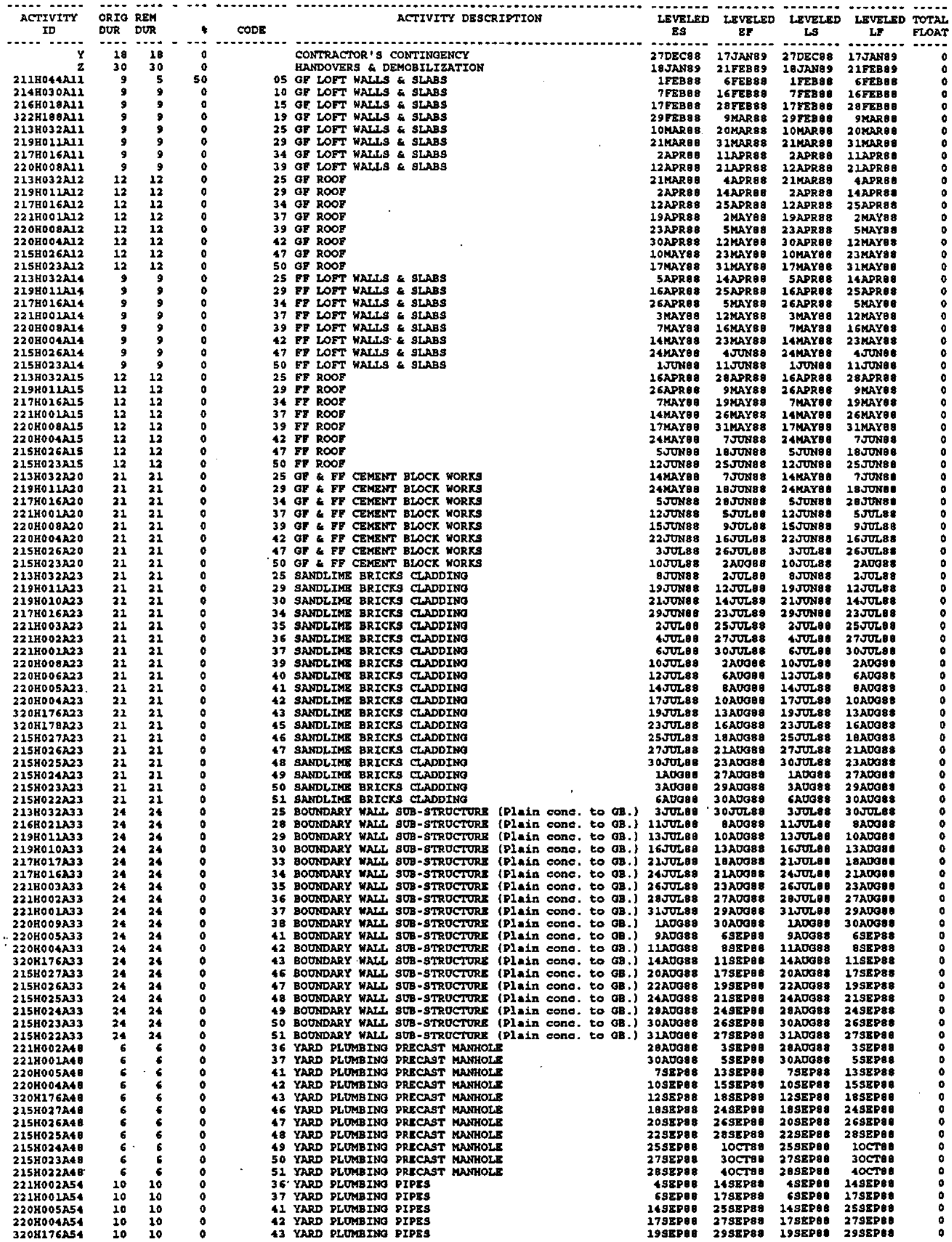

Figure 9.15 - Case 2, Status and Schedule Update as of January 31, 1988. 


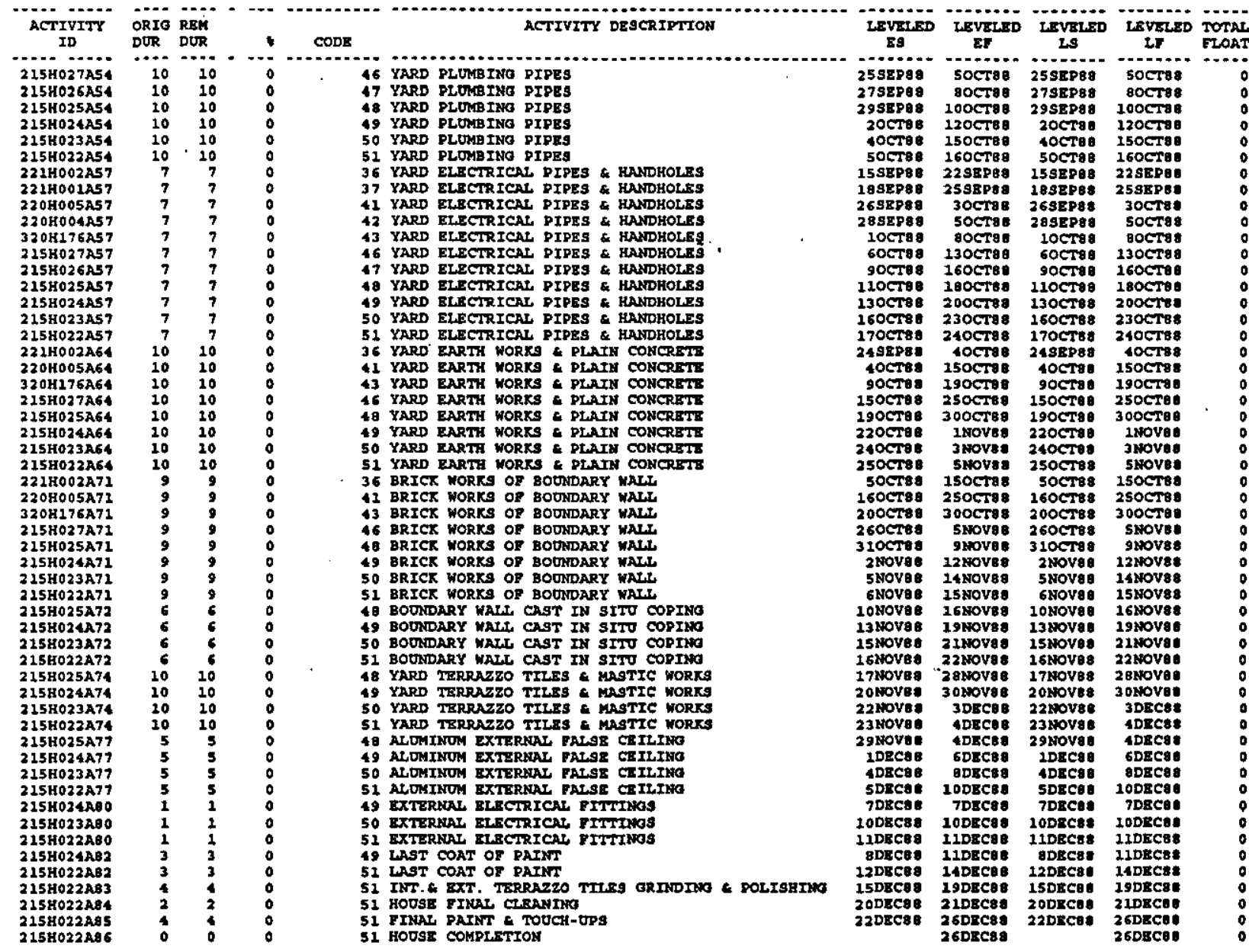

Figure 9.15 - Case 2, Status and Schedule Update as of January 31, 1988 (Continued). 


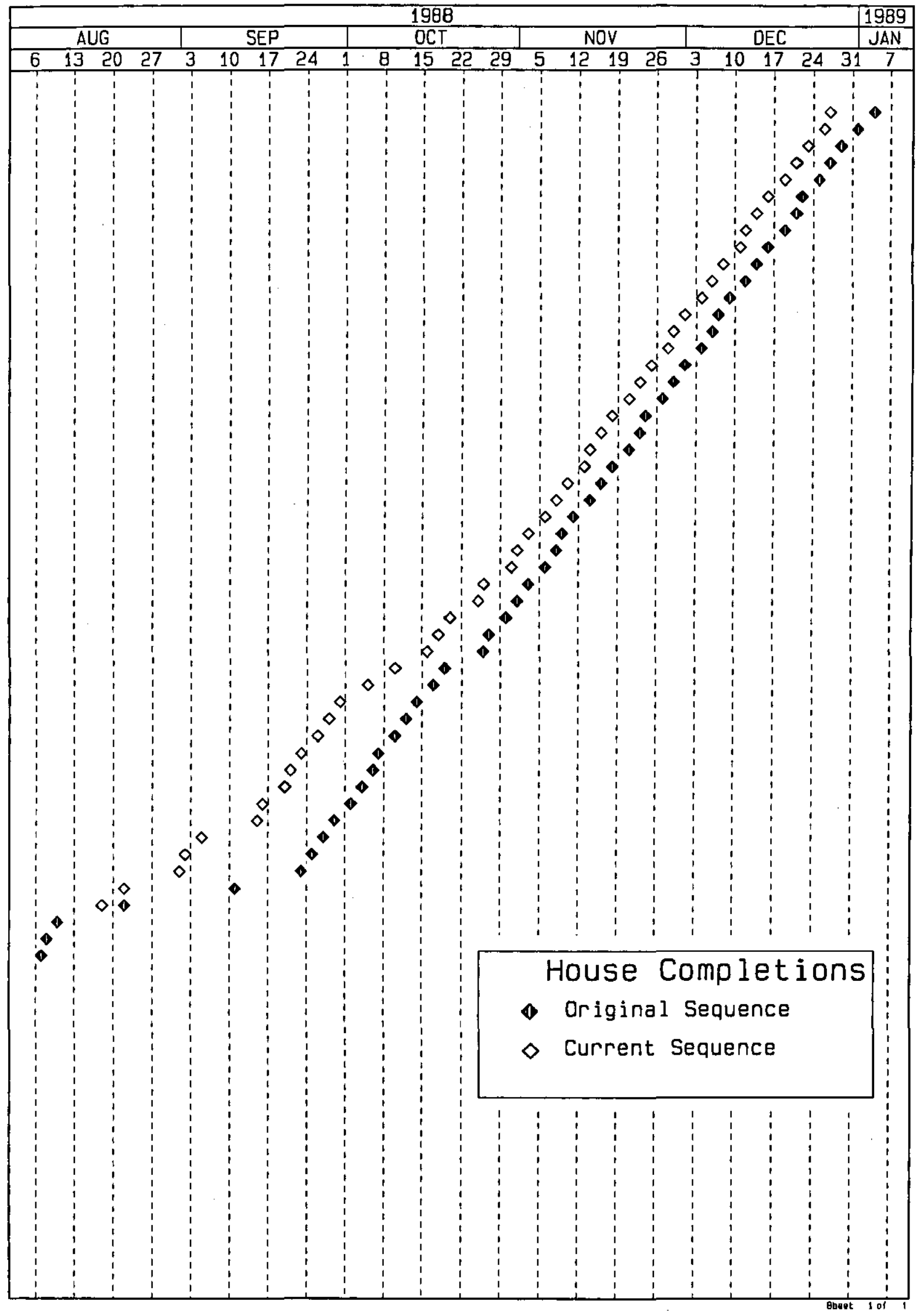

Figure 9.16 - Case 2, Correcting Out-of-Sequence Progress Versus Ignoring it in Leveling Resources. 


\section{Typical Activities}

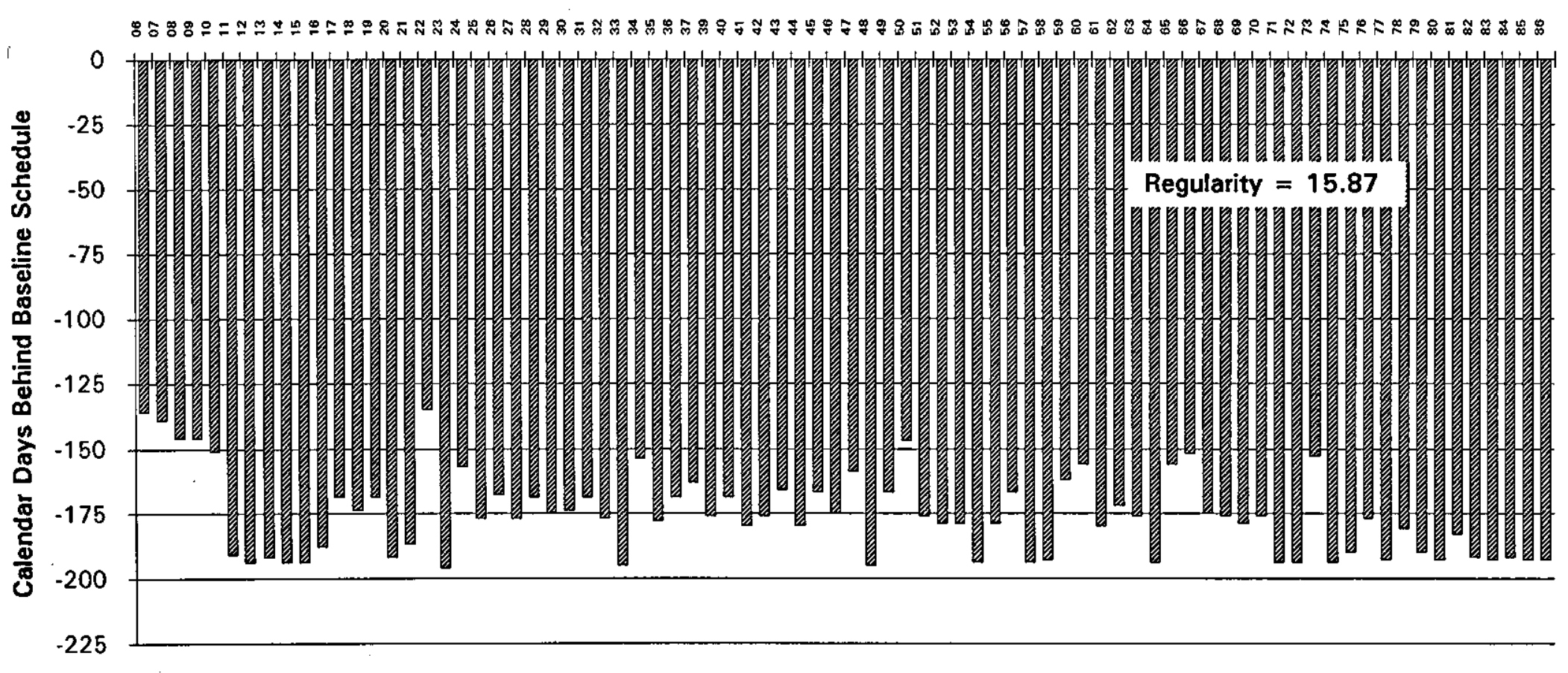

\section{Houses}

Figure 9.17 - Case 2, Progress Regularity as of January 31, 1988. 


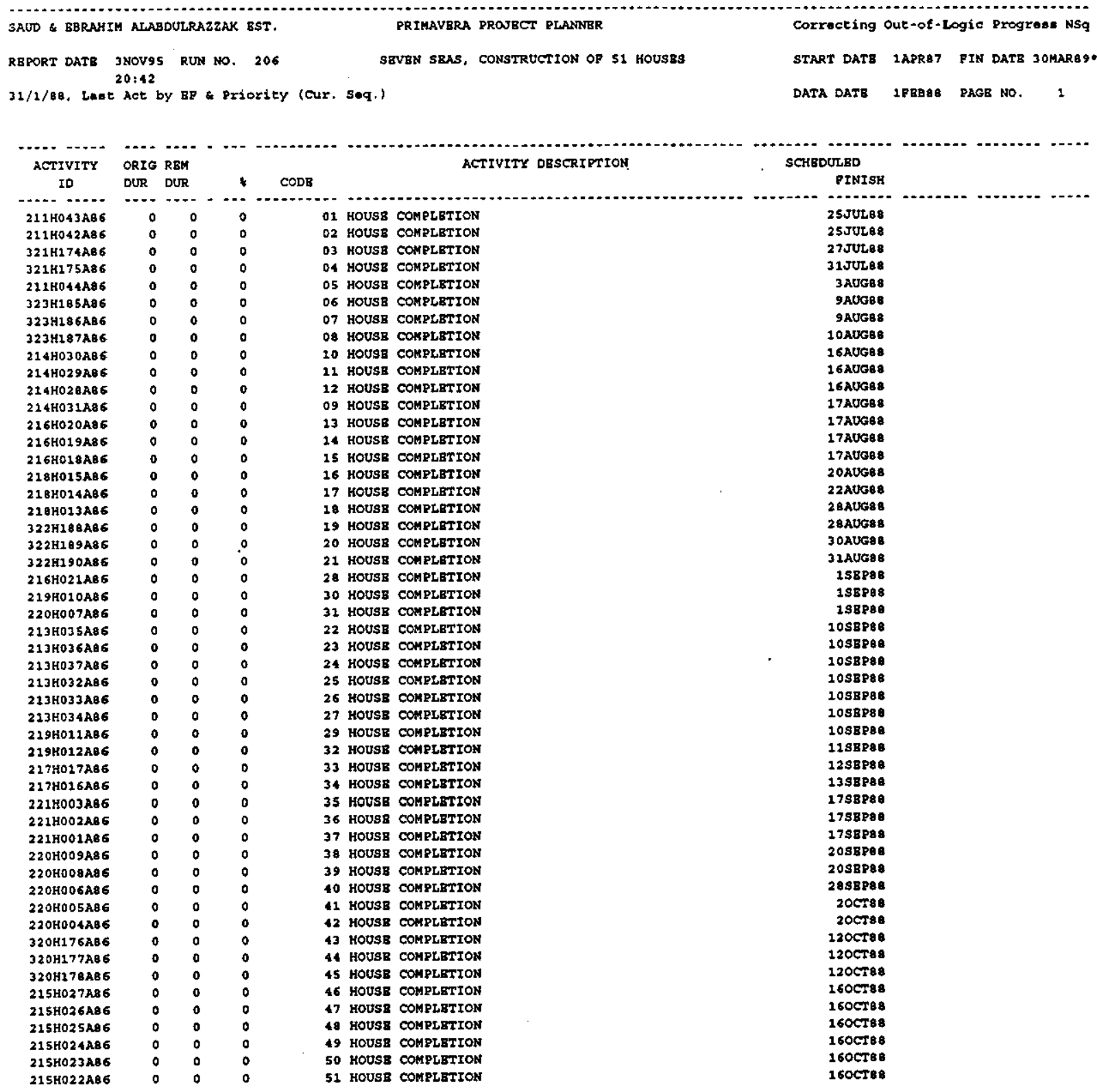

Figure 9.18 - Case 2, New Sequence after Correcting Out-of-Logic Progress. 


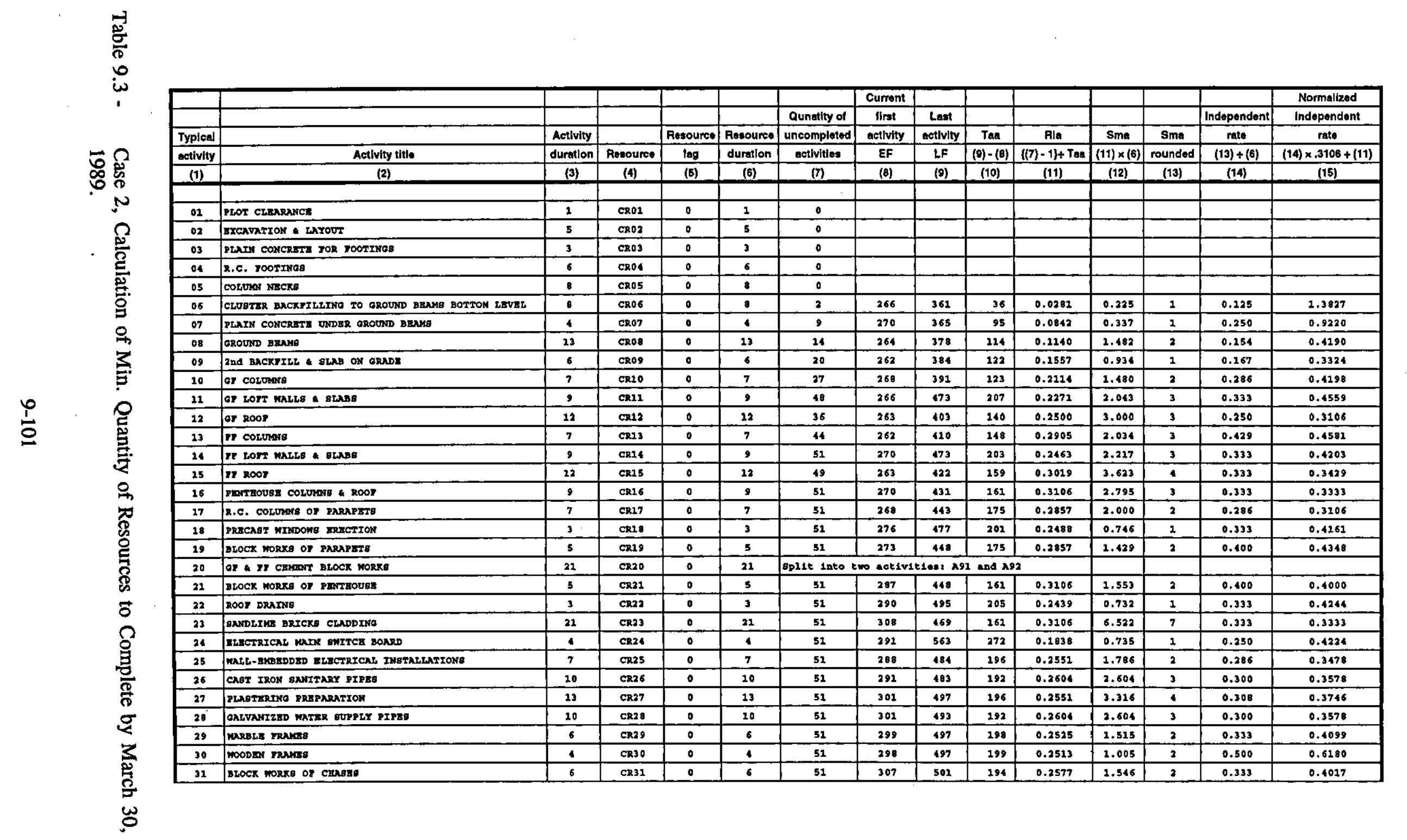




\begin{tabular}{|c|c|c|c|c|c|c|c|c|c|c|c|c|c|c|}
\hline & & & & & & & Current & & & & & & & Normallzed \\
\hline & & & & & & Qunnatity of & flrst & Last & & & & & 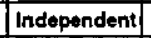 & independent \\
\hline Typical & & Aetivity & & Resouree & Pesource & uncomplotod & activity & ectivity & Tas & Ris & Sma & Sma & rate & rate \\
\hline settivity & Aetivity titlo & duration & \begin{tabular}{|l|l|} 
Regource \\
\end{tabular} & \begin{tabular}{|l|}
$\operatorname{lag}$ \\
\end{tabular} & duration & sectvititios & EF & LF & $(9) \cdot(8)$ & $\{(7)-1\}+$ Ta & $(11) \times(6)$ & Founded & $(13)+(8)$ & $(14) \times .3106+(11)$ \\
\hline (1) & (2) & (3) & (4) & (5) & (8) & (7) & (8) & (9) & (10) & (11) & (12) & (13) & (14) & (15) \\
\hline$\sigma^{n}$ & 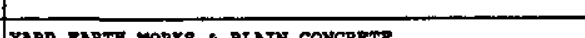 & 30 & 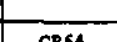 & 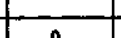 & 10 & 5 & 3.5 & 536 & 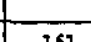 & 0.0010 & 3.108 & 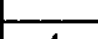 & 0.000 & 0.909 \\
\hline 65 & 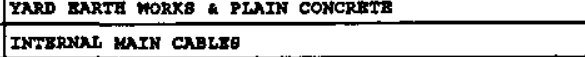 & $\frac{1}{2}$ & CR65 & 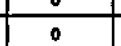 & $\frac{20}{2}$ & $\frac{52}{52}$ & $\frac{305}{357}$ & 565 & $\frac{208}{208}$ & 0.02404 & 0.481 & 1 & $\begin{array}{l}0.400 \\
0.500\end{array}$ & 0.0000 \\
\hline 66 & BLBCTRICAL HOUQR COMNBCTYON & 3 & C2866 & 0 & 3 & 51 & 358 & 570 & 212 & 0.2358 & 0.708 & 2 & 0.333 & 0.4389 \\
\hline 67 & IROMMONOGRY MORKA YOR HOODEN DOORA & 3 & C2.67 & $\circ$ & 3 & 32 & 360 & 557 & 197 & 0.2538 & 0.761 & 1 & 0.333 & 0.4079 \\
\hline 68 & 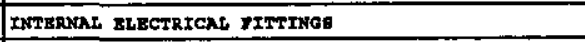 & 4 & CR68 & 0 & 4 & 51 & 361 & 557 & 196 & 0.2551 & 1.020 & 2 & 0.500 & 0.6087 \\
\hline 69 & 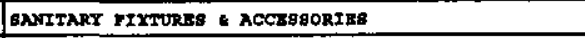 & 6 & CR.69 & 0 & 6 & 51 & 363 & 557 & 194 & 0.2577 & 1.546 & 2 & 0.333 & 0.4017 \\
\hline 70 & 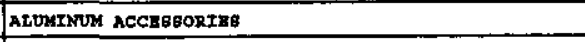 & 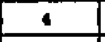 & CR70 & 0 & 4 & 51 & 361 & 557 & 196 & 0.2551 & 1.020 & 2 & 0.500 & 0.6087 \\
\hline 71 & BRICX MORRA OF BOONDARR MAIL & 9 & CR71 & 0 & 9 & 52 & 374 & 533 & 162 & 0.3206 & 2.795 & 3 & 0.333 & 0.3333 \\
\hline 72 & BOCNDARY MALL CAST IN BITU COPING & 6 & $\mathrm{CR72}$ & 0 & 6 & 51 & 380 & 541 & 161 & 0.3106 & 1.863 & 2 & 0.333 & 0.3333 \\
\hline 73 & 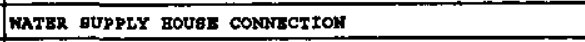 & 1 & $\mathrm{CR73}$ & 0 & 1 & 51 & 373 & 570 & 195 & 0.2564 & 0.256 & 1 & 2.000 & 2.2112 \\
\hline 74 & YYARD TZRRAZZZO TILISB \& MABTTC NORRB & 10 & CR74 & 0 & 10 & 51 & 390 & 551 & 161 & 0.3106 & 3.106 & 4 & 0.400 & 0.4000 \\
\hline 75 & 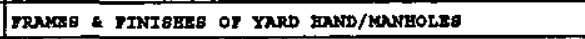 & 5 & CX75 & 0 & 5 & 51 & 385 & 549 & 164 & 0.3049 & 2.524 & 2 & 0.400 & 0.4075 \\
\hline 76 & 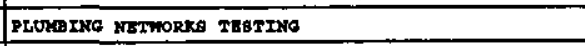 & 2 & CR76 & 0 & 2 & 51 & 387 & 566 & 179 & 0.2793 & 0.559 & 1 & 0.500 & 0.5559 \\
\hline 77 & 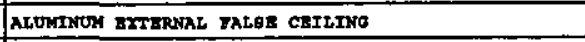 & 5 & CR77 & 0 & 5 & 51 & 395 & 556 & 161 & 0.3106 & 1.553 & 2 & 0.400 & 0.4000 \\
\hline 78 & AGRICULTURAL BOIL & 3 & CR78 & 0 & 3 & 51 & 393 & 564 & 171 & 0.2934 & 0.877 & 1 & 0.333 & 0.3540 \\
\hline 79 & 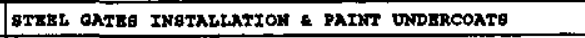 & 4 & CR79 & 0 & 4 & 51 & 394 & 557 & 163 & 0.3067 & 3.227 & 2 & 0.500 & 0.5062 \\
\hline 80 & 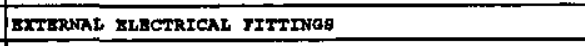 & 1 & CR80 & 0 & 1 & 51 & 396 & 557 & 363 & 0.3106 & 0.321 & 1 & 1.000 & 1.0000 \\
\hline 82 & 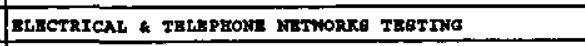 & 1 & CR81 & 0 & 2 & 51 & 397 & 566 & 169 & 0.2959 & 0.296 & 1 & 1.000 & 3.0497 \\
\hline 82 & \begin{tabular}{|l|l|} 
LAOT COAT OP PANRT \\
\end{tabular} & 3 & CR82 & $\circ$ & 3 & 51 & 399 & 560 & 162 & 0.3100 & 0.932 & 1 & 0.333 & 0.3333 \\
\hline 83 & 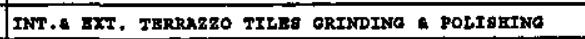 & 4 & CR83 & 0 & 4 & 51 & 403 & 564 & 161 & 0.3106 & 1.242 & 2 & 0.500 & 0.5000 \\
\hline 84 & MOUBB YTMAX CLENATNO & 2 & CR84 & 0 & 2 & 51 & 405 & 566 & 161 & 0.3108 & 0.621 & 1 & 0.500 & 0.5000 \\
\hline 85 & TrMAL PATNT \& TOVCR-UPB & 4 & CRBS & 0 & 4 & 51 & 409 & 570 & 161 & 0.3206 & 1.242 & 2 & 0.500 & 0.5000 \\
\hline 91 & Or CRHENT BLOCK MORRA & 9 & CR91 & 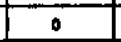 & 9 & 44 & 274 & 448 & 274 & 0.2471 & 2.224 & 3 & 0.333 & 0.4189 \\
\hline 92 & II CBMENT BLOCK MORKG & 12 & CRQ92 & 0 & 12 & 51 & 281 & 448 & 167 & 0.2994 & 3.593 & 4 & 0.333 & 0.3458 \\
\hline 23 & OANDLITAS BRICKB CLADDXNG & 21 & SCARYOLD & 20 & 21 & 32 & 308 & 469 & 361 & 0.3106 & 3.416 & 4 & 0.364 & 0.3636 \\
\hline 30 & ar cozurest & 7 & 8H10 & 0 & 9 & 27 & 268 & 391 & 123 & 0.2114 & 1.902 & 2 & 0.222 & 0.3265 \\
\hline 12 & or 8000 & 12 & 8812 & 0 & 24 & 36 & 263 & 403 & 140 & 0.2500 & 6.000 & 6 & 0.250 & 0.3106 \\
\hline 13 & or cotonors & 7 & ons 3 & 0 & 9 & 44 & 262 & 410 & 248 & 0.2905 & 2.615 & 3 & 0.333 & 0.3563 \\
\hline 15 & $n$ Roor & 12 & $8 \mathrm{BR2}$ & 0 & 24 & 49 & 263 & 422 & 159 & 0.3019 & 7.245 & 8 & 0.333 & 0.3429 \\
\hline 16 & 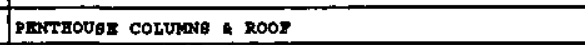 & 9 & 8816 & 0 & 21 & 52 & 270 & 431 & 161 & 0.3106 & 6.522 & 7 & 0.333 & 0.3333 \\
\hline 33 & 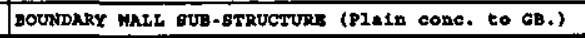 & 24 & $8 \mathrm{B33}$ & 28 & 7 & 51 & 332 & 493 & 261 & 0.3106 & 2.174 & 3 & 0.429 & 0.4286 \\
\hline
\end{tabular}




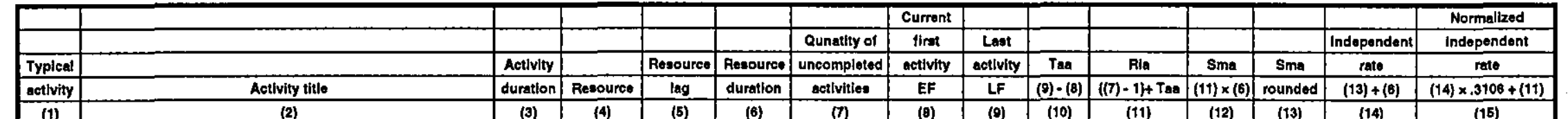

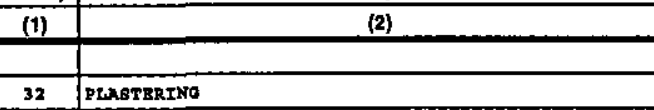

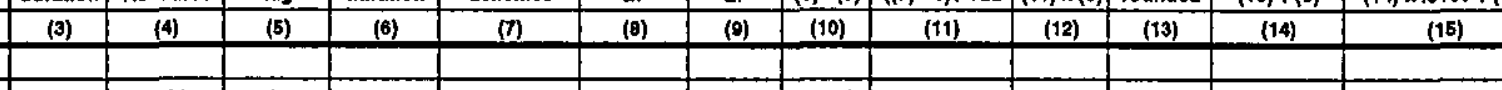

34 BOUNDARY MALL GUB-ETRUCTURR (919ID

35 PORCELATM MALI IILEB

36 ROOI JOAM \& BCREASD

39 TERrazZZ MLOK TILES A BKIRTIMo

40 ROOF NATRR PROOYTNG LAYRRB

41 BANTTARY RITTINGB (7loor Emboddod)

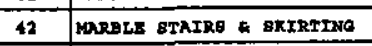

43 JoINary morke

14 matsRPROOFING IN WLT TLOOR NREAS

45 ROOY IMBSRDDED DRAINAGS PIPBS

46 BOUNDNRY WaLL RYIN. CONC. COLUnerg

47 PRECABT UNITS OI BODNDARY MaIt.

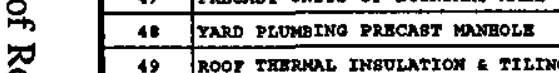

49 ROOI TREsRaL INGO

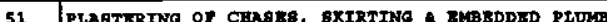
52 CERMMTe TILEs

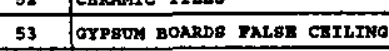

54 YNRD PLTMBSTMG PIPEA

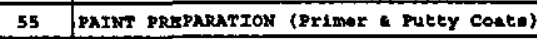

56 MATER TNNK \& EXPOBSD ROOI PIPINO

57 YARD BLSCTRICAI PTPEB \& EANDEOLIE

58 BNMTTARY hOUgE COBNBCTION

59 roOY MatTIC E TOUCH-UPB

60 MATER TANR Bgaso

61 ALEMINUM MRAMRE, MTMDOMS \& DOORE

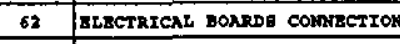

63 PAINT, IIRET a BBCOND COAIB \begin{tabular}{|c|c|c|c|c|}
\hline 8 & $C 832$ & 0 & $B$ & 51 \\
\hline 24 & $C \times 33$ & 0 & 24 & 51 \\
\hline
\end{tabular}

\begin{tabular}{|l|l|l|l|l|l|l|l|l|}
\hline 51 & 309 & 505 & 196 & 0.2531 & 2.041 & 3 & 0.375 & 0.4565 \\
\hline & 332 & 493 & 161 & 0.3106 & 7.453 & 8 & 0.333 & 0.333 \\
\hline
\end{tabular}

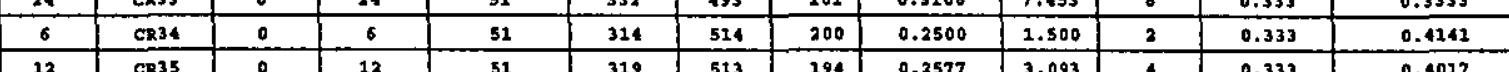

\begin{tabular}{|c|c|c|c|c|c|c|c|c|c|c|c|c|}
\hline 12 & CR 35 & 0 & 12 & 51 & 319 & 513 & 194 & 0.2577 & 3.093 & 4 & 0.333 & 0.4017 \\
\hline 10 & CR36 & 0 & 10 & 51 & 324 & 524 & 200 & 0.2500 & 2.500 & 3 & 0.300 & 0.3727 \\
\hline
\end{tabular}

\begin{tabular}{|c|c|c|c|c|c|c|c|c|c|c|c|c|}
\hline 6 & CR37 & 0 & 6 & 52 & 315 & 523 & 208 & 0.2404 & 1.442 & 2 & 0.333 & 0.4306 \\
\hline 13 & CR39 & 0 & 13 & 52 & 322 & 518 & 196 & 0.2551 & 3.316 & 1 & 0.308 & 0.3746 \\
\hline
\end{tabular}

\begin{tabular}{|c|c|c|c|c|c|c|c|c|c|c|c|c|}
\hline 13 & CR39 & 0 & 13 & 52 & 322 & 518 & 196 & 0.2551 & 3.316 & 1 & 0.308 & 0.3746 \\
\hline 6 & CR40 & 0 & 6 & 51 & 330 & 530 & 200 & 0.2500 & 1.500 & 2 & 0.333 & 0.4141 \\
\hline
\end{tabular}

\begin{tabular}{|c|c|c|c|}
\hline & 6 & CR41 & 0 \\
\hline 6 & CR42 & 0 \\
\hline 1 & CR43 & 0
\end{tabular}

\begin{tabular}{l|l|l|l|l|l|l|l|l|l}
1 & 51 & 323 & 537 & 194 & 0.2577 & 1.031 & 2 & 0.500 & 0.6025 \\
\hline
\end{tabular}

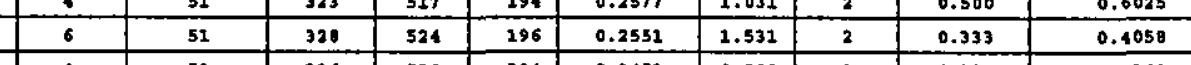

\begin{tabular}{|c|c|c|c|c|c|c|c|c|c|c|c|c|}
\hline 1 & CR43 & 0 & 4 & 53 & 326 & 530 & 304 & 0.2451 & 0.980 & 1 & 0.250 & 0.3168 \\
\hline 6 & CR44 & 0 & 6 & 52 & 329 & 323 & 194 & 0.2577 & 1.546 & 2 & 0.333 & 0.4017 \\
\hline
\end{tabular}

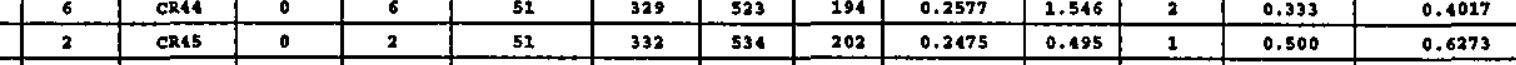

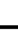

\begin{tabular}{|c|c|c|c|}
\hline 3 & $\mathrm{CR} 46$ & 0 \\
\hline $\mathrm{CR47}$ & 0 \\
\hline
\end{tabular}

\begin{tabular}{|c|c|c|c|c}
\hline 3 & CR47 & 0 & 3 & \\
\hline 6 & CR48 & 0 & 6 & \\
\hline 10 & CR49 & 0 & 10 & \\
\hline 5 & CR50 & 0 & 5 & \\
\hline 6 & CR51 & 0 & 6 &
\end{tabular}

\begin{tabular}{l|l|l|l|l|l|l|l|l}
52 & 335 & 516 & 177 & 0.2825 & 1.977 & 2 & 0.286 & 0.3141 \\
\hline & 336 & 191 & 0.2618 & 0.785 & 2 & 0.333 & 0.3954
\end{tabular}

\begin{tabular}{l|c|c|c|c|c|c|c|c}
52 & 335 & 326 & 191 & 0.2618 & 0.785 & 2 & 0.333 & 0.3954 \\
\hline 51 & 338 & 199 & 161 & 0.3206 & 1.863 & 2 & 0.333 & 0.3333
\end{tabular}

\begin{tabular}{l|l|l|l|l|l|l|l|l}
51 & 336 & 199 & 161 & 0.3206 & 1.863 & 2 & 0.333 & 0.3333 \\
\hline 51 & 342 & 544 & 202 & 0.2475 & 2.475 & 3 & 0.300 & 0.3764
\end{tabular}

\begin{tabular}{|c|c|c|c|c|c|c|c|c|c|c|c|c|}
\hline & & & & & & & & & & & & \\
\hline 6 & CR51 & 0 & 6 & 51 & 336 & 530 & 196 & 0.2551 & 1.531 & 2 & 0.333 & 0.4058 \\
\hline 7 & CR52 & 0 & 7 & 51 & 336 & 530 & 394 & 0.2577 & 1.804 & 2 & 0.286 & 0.3443 \\
\hline 7 & $\mathrm{CR} 53$ & 0 & 7 & 51 & 336 & 530 & 194 & 0.2577 & 1.804 & 2 & 0.286 & 0.3443 \\
\hline 10 & c254 & 0 & 10 & 51 & 348 & 509 & 262 & 0.3106 & 3.106 & 4 & 0.400 & 0.4000 \\
\hline 12 & CR55 & 0 & 12 & 51 & 349 & 542 & 194 & 0.2577 & 3.093 & 4 & 0.333 & 0.4017 \\
\hline 7 & CR56 & 0 & 7 & 52 & 349 & 551 & 202 & 0.2475 & 1.733 & 2 & 0.286 & 0.358 \\
\hline 7 & CR57 & 0 & 7 & 51 & 355 & 516 & 161 & 0.3106 & 2.174 & 3 & 0.429 & 0.428 \\
\hline 6 & CR5B & 0 & 5 & 51 & 354 & 516 & 162 & 0.3006 & 2.852 & 2 & 0.333 & 0.335 \\
\hline 6 & CR59 & 0 & 6 & 52 & 355 & 560 & 205 & 0.2439 & 1.463 & 3 & 0.333 & 0.424 \\
\hline 4 & CR60 & 0 & 4 & 31 & 353 & 364 & 211 & 0.2370 & 0.948 & 1 & 0.250 & 0.3276 \\
\hline 9 & CR61 & 0 & 9 & 51 & 357 & 551 & 294 & 0.2577 & 2.320 & 3 & 0.333 & 0.4017 \\
\hline 4 & CR62 & 0 & 4 & 51 & 352 & 553 & 201 & 0.2488 & 0.995 & 1 & 0.250 & 0.3121 \\
\hline 6 & CR63 & 0 & 6 & 52 & 354 & 552 & 297 & 0.2538 & 1.523 & 2 & 0.333 & 0.4079 \\
\hline
\end{tabular}

\begin{tabular}{l|l|l|l|l|l|l|l|l}
51 & 342 & 544 & 202 & 0.2475 & 2.475 & 3 & 0.300 & 0.3764 \\
\hline 51 & 333 & 557 & 224 & 0.2232 & 1.216 & 2 & 0.400 & 0.5565 \\
\hline
\end{tabular}




\begin{tabular}{|c|c|c|}
\hline$\underset{\text { ID }}{\text { ACTIVIT }}$ & $\begin{array}{l}\text { ORIG } \\
\text { OUR }\end{array}$ & $\begin{array}{l}\text { REM } \\
\text { DUR }\end{array}$ \\
\hline$\gamma$ & 10 & 18 \\
\hline & 30 & 30 \\
\hline $320 * \cdots+* A 06$ & 8 & 5 \\
\hline $215 * * * \# 06$ & 8 & 8 \\
\hline $320 \mathrm{H} 176 \mathrm{~A} 07$ & 4 & 1 \\
\hline $320 \mathrm{H} 177 \mathrm{A07}$ & 4 & 4 \\
\hline $320 \mathrm{HL} 7 \mathrm{BAO} 7$ & 4 & 4 \\
\hline $215 \mathrm{H} 027 \mathrm{AO} 7$ & 4 & 4 \\
\hline $215 \mathrm{HO} 026 \mathrm{AO} 7$ & 4 & 1 \\
\hline $\begin{array}{l}215 \mathrm{H} 025 \mathrm{~A} 07 \\
215 \mathrm{H024A07}\end{array}$ & 4 & 4 \\
\hline $215 \mathrm{H} 023 \mathrm{A0} 7$ & 4 & 4 \\
\hline $215 \mathrm{HO22A07}$ & 4 & 4 \\
\hline $220 \mathrm{HOO9AOB}$ & 13 & 3 \\
\hline $220 \mathrm{HOOOAOB}$ & 13 & 3 \\
\hline $220 \mathrm{HOOGAOB}$ & 13 & 10 \\
\hline $220 \mathrm{HOOSAOB}$ & 13 & 23 \\
\hline $220 \mathrm{HOOAAOB}$ & 13 & 13 \\
\hline $320 \mathrm{H} 176 \mathrm{AOO}$ & 13 & 13 \\
\hline $320 \mathrm{H} 177 \mathrm{AOOB}$ & 13 & 13 \\
\hline $320 \mathrm{H} 178 \mathrm{AOB}$ & 13 & 13 \\
\hline $215 \mathrm{HO} 27 \mathrm{AOB}$ & 13 & 13 \\
\hline $215 K 026 A 08$ & 13 & 13 \\
\hline $2154025 A 08$ & 13 & 13 \\
\hline 215 H024AC8 & 13 & 13 \\
\hline $215 H 023 \mathrm{AO} 8$ & 13 & 13 \\
\hline $215 \mathrm{HO22A08}$ & 13 & 13 \\
\hline $219 \mathrm{H} 012 \mathrm{AOS}$ & 6 & 1 \\
\hline 2174017A09 & 6 & 2 \\
\hline $217 \mathrm{HO} 16 \mathrm{AO} 9$ & 6 & 3 \\
\hline 221 HOO3A09 & 6 & 6 \\
\hline $221 \mathrm{HOO2AOS}$ & 6 & 6 \\
\hline $221 \mathrm{HOOLAOS}$ & 6 & 6 \\
\hline $2204009 A 09$ & 6 & 6 \\
\hline $220 \mathrm{H} 008 \mathrm{BAO}$ & 6 & 6 \\
\hline $220 \% 006 A 09$ & 5 & 6 \\
\hline $2201005 A 09$ & 6 & 6 \\
\hline $220 \mathrm{H} 004 \mathrm{AOO}$ & 6 & 6 \\
\hline $320 \mathrm{H} 176 \mathrm{AOS}$ & 6 & 6 \\
\hline $320 \mathrm{H} 177 \mathrm{AOS}$ & 6 & 6 \\
\hline $320 \mathrm{H} 178 \mathrm{AO} 9$ & 6 & 6 \\
\hline 215H027A09 & 6 & 6 \\
\hline $215 H 026 A 09$ & 6 & 6 \\
\hline 215H025A09 & 6 & 6 \\
\hline 215H024A09 & 6 & 6 \\
\hline 215 H023A09 & 6 & 6 \\
\hline 215 H022A09 & 6 & 6 \\
\hline 213 H035A10 & 7 & 7 \\
\hline $2134036 A 10$ & 7 & 7 \\
\hline 213 HO37ALO & 7 & 7 \\
\hline 213 H032ALO & 7 & 7 \\
\hline 213 H033ALO & 7 & 3 \\
\hline $213 \mathrm{HO} 03 \& \mathrm{~A} 1 \mathrm{O}$ & 7 & $?$ \\
\hline $219 \mathrm{HO} 1 \mathrm{AlO}$ & 7 & 7 \\
\hline $219 \mathrm{HO} 02 \mathrm{AlO}$ & 7 & 7 \\
\hline $217 \mathrm{HO} 77 \mathrm{~A} 10$ & 7 & 7 \\
\hline $217 \mathrm{H} 026 \mathrm{~A} 10$ & 7 & 7 \\
\hline $221 \mathrm{H} 003 \mathrm{AlO}$ & 7 & 7 \\
\hline $221 \mathrm{H} 002 \mathrm{~A} 10$ & 7 & 7 \\
\hline $221 \mathrm{HOOLA10}$ & 7 & 7 \\
\hline $220 \mathrm{HOO} 9 \mathrm{A1O}$ & 7 & 7 \\
\hline $220 \mathrm{HOOBA10}$ & 7 & 7 \\
\hline $220{ }^{2} 006 A 10$ & 7 & 7 \\
\hline $220 H 005 A 10$ & 7 & 7 \\
\hline $220 \% 004 A 10$ & $?$ & 7 \\
\hline $320 \mathrm{H} 176 \mathrm{AlO}$ & 7 & 7 \\
\hline $320 \mathrm{H} 177 \mathrm{A2O}$ & 7 & 7 \\
\hline $320 \mathrm{H} 178 \mathrm{ALO}$ & 7 & 7 \\
\hline $215 H_{027 A 10}$ & 7 & 7 \\
\hline $215 K 026210$ & 7 & 7 \\
\hline & 7 & 7 \\
\hline $\begin{array}{l}215 \mathrm{HO24AAO} \\
215 \mathrm{H} 023 \mathrm{ALO}\end{array}$ & 7 & $\begin{array}{l}7 \\
7\end{array}$ \\
\hline & 7 & 7 \\
\hline 215H022A10 & 9 & 5 \\
\hline 321H175A11 & 9 & 5 \\
\hline 211H044A11 & 9 & 5 \\
\hline $323 \mathrm{H} 285 \mathrm{~A} 11$ & 9 & 6 \\
\hline $323 \mathrm{H} 286 \mathrm{Al1}$ & 9 & 9 \\
\hline $323 \mathrm{H} 187 \mathrm{A11}$ & 9 & 9 \\
\hline $2144030 \mathrm{A11}$ & 9 & 9 \\
\hline $214 \mathrm{KO29A13}$ & 9 & ? \\
\hline 214 21428A13 & 9 & 9 \\
\hline $214 \mathrm{H} 031 \mathrm{A11}$ & 9 & 9 \\
\hline $216 \mathrm{HO} 020 \mathrm{Al1}$ & 9 & 9 \\
\hline $216 \mathrm{HO} 29 \mathrm{Al1}$ & 9 & 9 \\
\hline 216H018A11 & 9 & 2 \\
\hline 218HO1SA11 & 9 & 9 \\
\hline 218H014A1] & 9 & 9 \\
\hline 218KO13A11 & 9 & 9 \\
\hline $322 \mathrm{H} 18 \mathrm{BAN1}$ & 9 & 9 \\
\hline $322 \mathrm{H189A12}$ & 9 & 9 \\
\hline $322 \mathrm{H} 190 \mathrm{Al1}$ & 9 & 9 \\
\hline $216 \mathrm{HO} 21 \mathrm{~A} 11$ & 9 & 9 \\
\hline $219 \mathrm{HO} 010 \mathrm{Al} 1$ & 9 & 9 \\
\hline $220 \mathrm{H} 007 \mathrm{A1}$ & 9 & 9 \\
\hline 213H035A11 & 9 & 9 \\
\hline
\end{tabular}

Figure 9.19 - Case 2, Revised and Decelerated Schedule. 
3AUD \& EBPAHIM ALABDULRAzZAX EST

REPORT DATE 21SEP9S RUN NO. 20S

Rovd \& Decol Sehl by Typseal Activity a Es
PRIMAVERA PRCTECT PLWNIER

SEVEN SEAS, CONSTRUCTTON OF 51 HOUSES
Correcting Out-of-Logie Progrobs NSq

START DATE IAPRE7 FIN DATE 3OMARB9*

DATA DATE IFEBQS PAGE NO. 2

\begin{tabular}{|c|c|}
\hline $\begin{array}{c}\text { ACTIVITY } \\
\text { ID }\end{array}$ & $\begin{array}{l}\text { ORIO } \\
\text { DUR }\end{array}$ \\
\hline $2134036 \mathrm{~A} 11$ & 9 \\
\hline $213 H 037 \mathrm{Al1}$ & 9 \\
\hline $213 \mathrm{HO} 32 \mathrm{A12}$ & 9 \\
\hline $213 \mathrm{HO} 3 \mathrm{Al1}$ & 9 \\
\hline 213 HO34A11 & 9 \\
\hline $219 H 011 \mathrm{AL1}$ & 9 \\
\hline $219 H 012 A 11$ & 9 \\
\hline 217 HOLTAII & 9 \\
\hline $217 \mathrm{HO16A11}$ & 9 \\
\hline 221HO03A11 & 9 \\
\hline $22111002 A 11$ & ? \\
\hline $221 H 001 A 11$ & 9 \\
\hline $220 \mathrm{HOOSAII}$ & 9 \\
\hline $220 \mathrm{HOOBA11}$ & 9 \\
\hline $220 \mathrm{HOOGA11}$ & 9 \\
\hline $220 \mathrm{HOOSA11}$ & 9 \\
\hline $220 \mathrm{HOO4A11}$ & 9 \\
\hline $320 \mathrm{H} 176 \mathrm{AII}$ & 9 \\
\hline 3201177211 & 9 \\
\hline $320 \mathrm{H} 178 \mathrm{1} 1$ & 9 \\
\hline $215 \mathrm{H} 027 \mathrm{Al1}$ & 9 \\
\hline $215 \mathrm{HO26A11}$ & 9 \\
\hline $215 H 025 A 11$ & 9 \\
\hline $215 \mathrm{HO24A11}$ & 9 \\
\hline $215 \mathrm{HO23A12}$ & 9 \\
\hline $215 \mathrm{HO} 2 \mathrm{ALI}$ & 9 \\
\hline $219 \mathrm{HO} 015 \mathrm{~A} 12$ & 12 \\
\hline $219 H 014 A 12$ & 12 \\
\hline $2194013 A 12$ & 12 \\
\hline $322 \mathrm{H1OQALZ}$ & 12 \\
\hline $322 \mathrm{H} 1 \mathrm{GAAL2}$ & 12 \\
\hline 3224190212 & 12 \\
\hline $216 \mathrm{HO2LA12}$ & 12 \\
\hline 219 HOIOA12 & 12 \\
\hline $220 \mathrm{H007A12}$ & 12 \\
\hline $213 \mathrm{HO} 35 \mathrm{AL} 2$ & 12 \\
\hline $213 H 036 \mathrm{BA12}$ & 12 \\
\hline $213 \mathrm{HO37A12}$ & 12 \\
\hline 213 H032A12 & 12 \\
\hline 213 H033A12 & 12 \\
\hline $213 \mathrm{HO34A12}$ & 12 \\
\hline $219 \mathrm{HOL11A12}$ & 12 \\
\hline $219 \mathrm{HO12A12}$ & 12 \\
\hline $217 \mathrm{HO} 17 \mathrm{Al2}$ & 12 \\
\hline $217 \mathrm{HO16A12}$ & 12 \\
\hline $221 H 003 A 12$ & 12 \\
\hline $221 \mathrm{HOO2A12}$ & 12 \\
\hline $221 \mathrm{HOOLA12}$ & 12 \\
\hline $220 \mathrm{HOO} A \mathrm{~L} 2$ & 12 \\
\hline $220 \mathrm{HOOBA22}$ & 12 \\
\hline $220 \mathrm{HOOGA12}$ & 12 \\
\hline $220 \mathrm{HOOSA12}$ & 12 \\
\hline $220 \mathrm{HOO4A12}$ & 12 \\
\hline $320 \mathrm{H} 176 \mathrm{LL2}$ & 12 \\
\hline $320 H 277 A 12$ & 12 \\
\hline $320 \mathrm{H} 17 \mathrm{OA} 12$ & 12 \\
\hline $215 \% 027 A 12$ & 12 \\
\hline 215 SO2GALZ & 12 \\
\hline $215 H 025 A 12$ & 12 \\
\hline $215 \mathrm{H} 024 A 12$ & 12 \\
\hline 215 H023AI2 & 12 \\
\hline $215 \mathrm{H} 022 \mathrm{Al} 2$ & 12 \\
\hline $323 \mathrm{H187A23}$ & 7 \\
\hline $214 \mathrm{HO3OA13}$ & 7 \\
\hline $214 H 029 A 13$ & 7 \\
\hline $214 \mathrm{HO2BA13}$ & 7 \\
\hline $214 \mathrm{HO} 11 \mathrm{A13}$ & 7 \\
\hline $216 \mathrm{HO20A13}$ & 7 \\
\hline $216 \mathrm{HO} 019 \mathrm{~A} 13$ & 7 \\
\hline $216 H 018 A 13$ & 7 \\
\hline $218 H 015 A 13$ & 7 \\
\hline $218 \mathrm{HO} 14 \mathrm{~A} 23$ & 7 \\
\hline $218 \mathrm{HO} 13 \mathrm{~A} 13$ & 7 \\
\hline $322 \mathrm{HLOBA13}$ & 7 \\
\hline 3224189213 & 7 \\
\hline $322 \mathrm{H} 190 \mathrm{Al} 3$ & 7 \\
\hline $216 H 021 A 13$ & 7 \\
\hline $219 H 020 A 13$ & 7 \\
\hline $220 \mathrm{HOO} 7 \mathrm{Al3}$ & 7 \\
\hline $223 \mathrm{HO} 55 \mathrm{Al} 3$ & 7 \\
\hline $2134036 \mathrm{~A} 13$ & 7 \\
\hline $213 H 037 A 13$ & 7 \\
\hline $213 \mathrm{H} 032 \mathrm{Al3}$ & 7 \\
\hline $213 \mathrm{HO}_{03} \mathrm{~A} 13$ & 7 \\
\hline $213 H 034 A 13$ & 7 \\
\hline $219 H 011 \mathrm{AL3}$ & 7 \\
\hline $219 H 012 A \times 3$ & 7 \\
\hline 217 HO17A13 & 7 \\
\hline $217 \mathrm{HO16A13}$ & $?$ \\
\hline 2216003213 & 7 \\
\hline $221 \% 002 A 13$ & 7 \\
\hline $22111001 A 13$ & $?$ \\
\hline $2204009 \mathrm{Al} 3$ & 7 \\
\hline $220 \mathrm{H} 009 \mathrm{AL} 3$ & 7 \\
\hline $220 \mathrm{H} 006 \mathrm{AL} 3$ & 7 \\
\hline 220 HOOSA13 & 7 \\
\hline
\end{tabular}

Figure 9.19 - Case 2, Revised and Decelerated Schedule (Continued). 
SAUD \& EBRAHIM ALABDULRAEZAK EST

REPORT DATE 2ISEPYS RUN NO. 205

Ravd Decel SehI by TYPienl Activity E ES
PRIMAVERA PROEECT PLANNER

SEVEN SEAS, CONSTRUCTION OF 51 HOUSES
Correcting Out-of-Log 26 Progrese NSq START DATE 1APRE7 FIN DATE 3OMAR99* DATA DATE LFEB日 PAGE NO, 3

\begin{tabular}{|c|c|}
\hline $\begin{array}{c}\text { ACTIVITY } \\
\text { ID }\end{array}$ & $\begin{array}{l}\text { ORIO } \\
\text { DUR }\end{array}$ \\
\hline $220 \mathrm{H} 004 \mathrm{~A} 13$ & 7 \\
\hline $3204176 \mathrm{A13}$ & 7 \\
\hline $320 \mathrm{H} 177 A 13$ & 7 \\
\hline $320 H 178 A 13$ & 7 \\
\hline $215 \% 027 A 13$ & 7 \\
\hline $215 \mathrm{HO} 2 \mathrm{EA} 13$ & 7 \\
\hline $215 \mathrm{H} 025 \mathrm{~A} 13$ & 7 \\
\hline $215 \mathrm{H} 024 \mathrm{~A} 13$ & 7 \\
\hline $215 \mathrm{H} 023 \mathrm{Al} 3$ & 7 \\
\hline $215 \mathrm{HO}_{022 \mathrm{~A} 33}$ & 7 \\
\hline 211 H043A14 & 9 \\
\hline $211 \mathrm{HO42A14}$ & 9 \\
\hline $321 \% 174 A 14$ & 9 \\
\hline 3211175214 & 9 \\
\hline 211 H044A14 & 9 \\
\hline 323 HLOSALA & 9 \\
\hline $323 \mathrm{HLB} 6 \mathrm{Al}$ & 9 \\
\hline 323 H107Al4 & 9 \\
\hline $214 \mathrm{H} 030 \mathrm{~A} 14$ & 9 \\
\hline $214 H 029 A 14$ & $?$ \\
\hline $214 \mathrm{HO} 2 \mathrm{AA} 14$ & 9 \\
\hline $214 K 031 \mathrm{Al} 4$ & 9 \\
\hline 2164020814 & ? \\
\hline $216 \mathrm{HO} 19 \mathrm{A14}$ & 9 \\
\hline 216 HO18A14 & 9 \\
\hline 21 GHO15AL4 & 9 \\
\hline $218 \mathrm{HO} 14 \mathrm{Al} 4$ & 9 \\
\hline 218H013A14 & 9 \\
\hline $322 \mathrm{HLOBAI4}$ & 9 \\
\hline $322 \mathrm{HLO9A14}$ & 9 \\
\hline $322 \mathrm{H} 190 \mathrm{OA} 4$ & 9 \\
\hline $216 \mathrm{H} 021 \mathrm{N14}$ & 9 \\
\hline $239 \mathrm{HO} 0 \mathrm{AD} 14$ & 9 \\
\hline $220 \mathrm{H} 007 \mathrm{Al} 4$ & 9 \\
\hline 213 H035A14 & 9 \\
\hline $213 H 036 A 14$ & 9 \\
\hline $213 \mathrm{HO} 7 \mathrm{A24}$ & 9 \\
\hline $213 \mathrm{H}_{032 \mathrm{Al}}$ & 9 \\
\hline 213 H033A14 & 9 \\
\hline 213 H034A14 & 9 \\
\hline $219 \mathrm{HO} 12 \mathrm{A14}$ & 9 \\
\hline 219H012A14 & 9 \\
\hline 217H017A14 & 9 \\
\hline $2174016 \mathrm{Al} 4$ & 9 \\
\hline $221 \mathrm{HOOO3A14}$ & 9 \\
\hline $222 \mathrm{HOOO2A14}$ & 9 \\
\hline $221 \mathrm{H} 00 \mathrm{LA14}$ & 9 \\
\hline $220 \mathrm{HOOSA14}$ & 9 \\
\hline $2204008 A 14$ & 9 \\
\hline $220 \mathrm{HOOSA14}$ & 9 \\
\hline $220 H 005 A 14$ & 9 \\
\hline $220 \mathrm{HOOAAL4}$ & 9 \\
\hline $320 \mathrm{H} 176 \mathrm{~A} 14$ & 9 \\
\hline $320 \mathrm{H} 177 \mathrm{Al} 4$ & 9 \\
\hline $320 \mathrm{H} 17 \mathrm{AAL} 4$ & 9 \\
\hline $215 \mathrm{HO2} 7 \mathrm{A14}$ & 9 \\
\hline $215 \mathrm{H} 026 \mathrm{Al} 4$ & 9 \\
\hline 215H025A14 & 9 \\
\hline $2154024 A 14$ & 9 \\
\hline $215 H 023 A 14$ & 9 \\
\hline $215 H_{022 A 14}$ & 9 \\
\hline $321 \mathrm{H} 174 A 15$ & 12 \\
\hline $321 K 175 \mathrm{AlS}$ & 12 \\
\hline 211H044A15 & 12 \\
\hline $323 \mathrm{H} 185 \mathrm{SASS}$ & 12 \\
\hline $323 \mathrm{H} 186 \mathrm{AL}$ & 12 \\
\hline $323 \mathrm{HLO7A15}$ & 12 \\
\hline 214 H03 OA15 & 12 \\
\hline $214 \mathrm{H029A15}$ & 22 \\
\hline $214 \mathrm{HO28A15}$ & 12 \\
\hline $214 \mathrm{~K} 031 \mathrm{~A} 15$ & 12 \\
\hline $2161020 A 15$ & 12 \\
\hline $216 \mathrm{HO19A15}$ & 12 \\
\hline 216H018A15 & 12 \\
\hline 219H015A15 & 12 \\
\hline $218 \mathrm{H} 014 \mathrm{Al} 5$ & 12 \\
\hline $2184013 A 15$ & 12 \\
\hline $322 \mathrm{H} 188 \mathrm{~A} 15$ & 12 \\
\hline 322K189ALS & 12 \\
\hline 322 M190A15 & 22 \\
\hline $216 \mathrm{HOZ} 1 \mathrm{AlS}$ & 12 \\
\hline 219 KO10A15 & 12 \\
\hline $220 \mathrm{HOO} 7 \mathrm{A1S}$ & 12 \\
\hline 213 H035A15 & 12 \\
\hline $213 \mathrm{H} 036 \mathrm{Al} 15$ & 12 \\
\hline $223 H 037 A 15$ & 12 \\
\hline $213 \mathrm{H} 032 \mathrm{Al5}$ & 12 \\
\hline 2134033315 & 12 \\
\hline $213 \mathrm{H} 034 \mathrm{ALS}$ & 12 \\
\hline $219 \mathrm{H} 011 \mathrm{~A} 1 \mathrm{~S}$ & 12 \\
\hline 219HO12A1S & 12 \\
\hline $217 \mathrm{HO} 17 \mathrm{ALS}$ & 12 \\
\hline $217 \mathrm{HO} 16 \mathrm{A15}$ & 12 \\
\hline $221 \mathrm{H} 003 \mathrm{AlS}$ & 12 \\
\hline $221 \mathrm{H} 002 \mathrm{R15}$ & 12 \\
\hline $221 \mathrm{H} 001 \mathrm{~A} 15$ & 12 \\
\hline
\end{tabular}

Figure 9.19 - Case 2, Revised and Decelerated Schedule (Continued). 


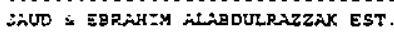

P.EPORT DATE 215EPSS RUN NO. 205

PRIMAVERA PROFECT PLANNER

SEVEN SEAS, CONSTRUCTION OF 51 MOUSES

Reyd \& oecel Sehl by Tpical Activity \& ES
Correcting Out-of-Logie Progres: NSq START DATE 1APRE? EIN DATE 3OMAR99* DATA DATE LFEBBa PAGE NO.

\begin{tabular}{|c|c|}
\hline $\begin{array}{l}\text { ACTIVITY } \\
\text { ID }\end{array}$ & $\begin{array}{l}\text { ORIO } \\
\text { DUR }\end{array}$ \\
\hline 220 Hоogals & 12 \\
\hline $220 H 008 A_{15}$ & 12 \\
\hline $220 \mathrm{H} 006 \mathrm{A1S}$ & 12 \\
\hline $2204005 \mathrm{~A} 15$ & 12 \\
\hline $220 \mathrm{HOO4ALS}$ & 12 \\
\hline $320 H 176 \mathrm{~A} 15$ & 12 \\
\hline $320 H 277 \mathrm{~A} 15$ & 12 \\
\hline $320 \mathrm{H} 270 \mathrm{~A} 15$ & 12 \\
\hline $2154027 A 15$ & 12 \\
\hline $2154026 \mathrm{ALS}$ & 12 \\
\hline 215 HO25A1S & 12 \\
\hline $2154024 A 15$ & 12 \\
\hline 215 H023A1S & 12 \\
\hline $215 H 022 \mathrm{AlS}$ & 12 \\
\hline $211 \mathrm{H} 043 \mathrm{Al} 6$ & 9 \\
\hline $211 H 042 A 26$ & 9 \\
\hline $321 H 174 A 16$ & 9 \\
\hline $321 \mathrm{H} 175 \mathrm{~A} 16$ & 9 \\
\hline 211 H044A16 & 9 \\
\hline 323 H185A16 & 9 \\
\hline $323 \mathrm{H} 186 \mathrm{~A} 16$ & 9 \\
\hline 323 H187A16 & $?$ \\
\hline $214 \mathrm{H} 030 \mathrm{~A} 16$ & $?$ \\
\hline $214 \mathrm{HO} 29 \mathrm{Al} 6$ & 9 \\
\hline $214 \mathrm{HO28A16}$ & 9 \\
\hline $214 \mathrm{H} 031 \mathrm{~A} 16$ & $?$ \\
\hline 216 HO20A16 & $?$ \\
\hline 216H019A16 & 9 \\
\hline $216 \mathrm{HO} 18 \mathrm{~A} 16$ & 9 \\
\hline $218 \mathrm{HO15A16}$ & 9 \\
\hline $2184014 A 16$ & 9 \\
\hline 218 O13A16 & $?$ \\
\hline $322 H 188 A 16$ & 9 \\
\hline 322 H189A16 & 9 \\
\hline $322 \mathrm{~K} 190 \mathrm{A16}$ & 9 \\
\hline $216 H 021 A 16$ & 9 \\
\hline $2194010 A 16$ & 9 \\
\hline $220 \mathrm{H} 007 \mathrm{A16}$ & 9 \\
\hline $213 H 035 A 16$ & 9 \\
\hline 213 H036A16 & 9 \\
\hline 213 H037A.16 & 9 \\
\hline $2134032 A 26$ & 9 \\
\hline $2134033 A 16$ & 9 \\
\hline $213 \mathrm{H} 034 \mathrm{~A} 16$ & 9 \\
\hline $2194011 \mathrm{AN} 16$ & 9 \\
\hline $219 \mathrm{HO} 12 \mathrm{A16}$ & 9 \\
\hline 217H017A16 & 9 \\
\hline 217 016A16 & 9 \\
\hline $221 \mathrm{HOO} \mathrm{AD16}$ & 9 \\
\hline $221 \mathrm{H} 002 \mathrm{~A} 16$ & 9 \\
\hline 221HOO1A16 & 9 \\
\hline $220 \mathrm{HOOQA.16}$ & 9 \\
\hline $220 \mathrm{HOOBA16}$ & 9 \\
\hline $220 \mathrm{HOO} 0 \mathrm{AL} 6$ & 9 \\
\hline $220 \mathrm{HOOSA16}$ & 9 \\
\hline $220 \mathrm{HOO4A16}$ & 9 \\
\hline $320 H_{176 A 16}$ & 9 \\
\hline $320 \mathrm{H} 177 \mathrm{~A} 16$ & 9 \\
\hline $320 \mathrm{H178A16}$ & 9 \\
\hline $215 \mathrm{H} 027 \mathrm{~A} 16$ & 9 \\
\hline $215 \mathrm{H} 026 \mathrm{~A} 16$ & 9 \\
\hline 215 H025A16 & 9 \\
\hline 215 HO24A.16 & 9 \\
\hline $215 H 023 A 16$ & 9 \\
\hline $215 \mathrm{HO22A16}$ & $?$ \\
\hline $211 \mathrm{HO} 43 \mathrm{~A} 17$ & 7 \\
\hline $211 \mathrm{HO} 2 \mathrm{A17}$ & $?$ \\
\hline $321 H_{174 A 17}$ & 7 \\
\hline $321 K 175 A 17$ & 7 \\
\hline 211 HO44A17 & 7 \\
\hline $323 \mathrm{H} 105 \mathrm{AL} 7$ & 7 \\
\hline $323 \mathrm{H} 186 \mathrm{~A} 17$ & 7 \\
\hline $323 K 187 A 17$ & 7 \\
\hline 214 HO3 OAL7 & 7 \\
\hline $2144029 A 17$ & 7 \\
\hline $214 \mathrm{HO2BA17}$ & 7 \\
\hline $214 H 0312017$ & 7 \\
\hline $2164020 A 17$ & 7 \\
\hline $216 H 019 A 17$ & 7 \\
\hline $216 H 018 A 17$ & 7 \\
\hline $219 H 015 A 17$ & 7 \\
\hline $218 H 014 A 17$ & 7 \\
\hline $218 H 013 A 17$ & 7 \\
\hline $322 \mathrm{K1B8A17}$ & 7 \\
\hline $322 \mathrm{H} 199 \mathrm{~A} 17$ & 7 \\
\hline $322 \mathrm{H} 190 \mathrm{A1} 7$ & 7 \\
\hline $216 \mathrm{HO}_{2} 1 \mathrm{A1} 7$ & 7 \\
\hline 219 HO1OA17 & 7 \\
\hline $220 \mathrm{H}_{007 \mathrm{~A}} 7$ & 7 \\
\hline 213 H035A.17 & 7 \\
\hline $213 \mathrm{H036A17}$ & 7 \\
\hline $213 \mathrm{H} 037 \mathrm{~A}=7$ & 7 \\
\hline $213 H 032 \mathrm{~A} 17$ & 7 \\
\hline $213 \mathrm{H} 033 \mathrm{~A} 17$ & 7 \\
\hline $213 H 034 A 17$ & 7 \\
\hline $219 H 011 \mathrm{~A} 17$ & 7 \\
\hline
\end{tabular}

Figure 9.19 - Case 2, Revised and Decelerated Schedule (Continued). 


\begin{tabular}{|c|c|}
\hline $\begin{array}{l}\text { ACTIVITY } \\
\text { ID }\end{array}$ & $\begin{array}{l}\text { ORIG } \\
\text { DUR }\end{array}$ \\
\hline $219 \mathrm{H} 012 \mathrm{Al} 3$ & 7 \\
\hline $217 \mathrm{HO17A17}$ & 7 \\
\hline 217 HO16A17 & $?$ \\
\hline $22 \mathrm{JHO03A17}$ & 7 \\
\hline $221 \mathrm{HOO} 2 \mathrm{~A} 17$ & 7 \\
\hline 22 LHOOLA17 & 7 \\
\hline $220 H 009 A 17$ & 7 \\
\hline $220 \mathrm{H} 000 \mathrm{~A} 17$ & 7 \\
\hline $220 H 006 A 17$ & 7 \\
\hline $220 \mathrm{H} 005 \mathrm{Al} 7$ & 7 \\
\hline $220 \mathrm{H} 004 A 17$ & 7 \\
\hline $320 H_{176 A 17}$ & 7 \\
\hline $320 \mathrm{H} 277 \mathrm{AL} 7$ & 7 \\
\hline $320 \mathrm{H278A17}$ & 7 \\
\hline $215 H 027 A 17$ & 7 \\
\hline $2154026 \mathrm{Al7}$ & 7 \\
\hline $215 H 025 A 17$ & 7 \\
\hline $215 \mathrm{HO24AI7}$ & 7 \\
\hline $215 \mathrm{HO23A17}$ & 7 \\
\hline $2154022 A 17$ & 7 \\
\hline $211 H 043 A 18$ & 3 \\
\hline $21111042 A 18$ & 3 \\
\hline $321 \% 174 A 19$ & 3 \\
\hline $321 \mathrm{H} 275 \mathrm{AlB}$ & 3 \\
\hline $211 H 044 A 18$ & 3 \\
\hline 323 HIO5A18 & 3 \\
\hline $323 H 286 A 18$ & 3 \\
\hline 323HIO7AL8 & 3 \\
\hline 214H030A19 & 3 \\
\hline $214 H 029 A 18$ & 3 \\
\hline $214 H 02$ BNAg & 3 \\
\hline 214 HO3 IA18 & 3 \\
\hline $216 \mathrm{HO2OA19}$ & 3 \\
\hline $216 \mathrm{HO19A18}$ & 3 \\
\hline $216 \mathrm{HO1BA18}$ & 3 \\
\hline 218H015A18 & 3 \\
\hline 218HO14A18 & 3 \\
\hline $218 H 013 A 18$ & 3 \\
\hline $3224268 A 19$ & 3 \\
\hline $322 \mathrm{H1 \theta 9A18}$ & 3 \\
\hline $322 \mathrm{HI} 9 \mathrm{AAl}$ & 3 \\
\hline $226 H 021228$ & 3 \\
\hline 219 HO10A18 & 3 \\
\hline $220 \mathrm{HOO7A28}$ & 3 \\
\hline 213 HO35A18 & 3 \\
\hline $213 H 036 A 28$ & 3 \\
\hline $213 H_{037 A 18}$ & 3 \\
\hline $213 H 032 A 18$ & 3 \\
\hline $213 \mathrm{HO} 33 \mathrm{ALO}$ & 3 \\
\hline $213 H 034 A 18$ & 3 \\
\hline $219 \mathrm{HOL1A1B}$ & 3 \\
\hline $219 H 012 A 1 B$ & 3 \\
\hline $217 \mathrm{HO} 27 \mathrm{A18}$ & 3 \\
\hline $217 \mathrm{H} 016 \mathrm{AB}$ & 3 \\
\hline $221 \mathrm{H} 003 \mathrm{AlB}$ & 3 \\
\hline $221 H 002 A 1 B$ & 3 \\
\hline $221 \mathrm{HOO1A1B}$ & 3 \\
\hline $220 \mathrm{HOO} A \mathrm{Al} 8$ & $\mathbf{3}$ \\
\hline $220 \mathrm{HOOBA1B}$ & 3 \\
\hline $220 \mathrm{H} 006 \mathrm{AlO}$ & 3 \\
\hline $220 H 005 A 18$ & 3 \\
\hline $220 H 004 A 19$ & 3 \\
\hline $320 H 176 A 18$ & 3 \\
\hline $320 \% 177 A 18$ & 3 \\
\hline $320 \mathrm{HI} 7 \mathrm{OAL}$ & 3 \\
\hline $215 \mathrm{HO27A19}$ & 3 \\
\hline $215 \% 026 A 18$ & 3 \\
\hline $215 H 025 \lambda 18$ & 3 \\
\hline $215 \% 024 A 18$ & 3 \\
\hline $215 H 023 \mathrm{AlB}$ & 3 \\
\hline $215 H 022 A 18$ & 3 \\
\hline $211 \mathrm{H} 043 \mathrm{Al} 9$ & 5 \\
\hline $2111042 A 19$ & 5 \\
\hline $32111174 A 19$ & 5 \\
\hline $321 \% 175 A 29$ & 5 \\
\hline $211 \mathrm{HO44A19}$ & 5 \\
\hline $323 \mathrm{H185A19}$ & 5 \\
\hline $323 \mathrm{H} 186 \mathrm{Al} 9$ & $\mathbf{5}$ \\
\hline $323 \mathrm{H} 187 \mathrm{ALS}$ & 5 \\
\hline 214 HO30A19 & 5 \\
\hline $214 \mathrm{H} 029 \mathrm{Al} 9$ & 5 \\
\hline $214 \mathrm{HO28A19}$ & 5 \\
\hline $214 \$ 031 A 19$ & \\
\hline $216 \% 020 A 19$ & 5 \\
\hline 236H019A19 & 5 \\
\hline 216M018A19 & 5 \\
\hline $210 H 015 A 39$ & 5 \\
\hline 218HO14A19 & $\mathbf{s}$ \\
\hline $210 \mathrm{HO} 13 \mathrm{~A} 19$ & 5 \\
\hline 322H189A19 & 5 \\
\hline $322 \mathrm{H} 189 \mathrm{AL} 9$ & 5 \\
\hline $322 \mathrm{HLOOA} 19$ & 5 \\
\hline $216 \mathrm{H} 021 \mathrm{~A} 19$ & 5 \\
\hline 219HO10AD9 & 5 \\
\hline $220 \mathrm{H} 007 \mathrm{A19}$ & 5 \\
\hline & \\
\hline
\end{tabular}

Figure 9.19 - Case 2, Revised and Decelerated Schedule (Continued). 
SAUD \& EBRAHIM ALABDULRAZZAX EST.

P.EPORT DATE 21 SEP95 RUN NO. 205

R.ova \& Docel Schl by Typical Activity \& ES
PRIMAVERA PROJECT PLANAER

SEVEN SEAS, CONSTRUCTION OF 51 HOUSES
Correcting Out-of-Logie ProgresB NS

START DATE 1APR8? FIN DATE 3OMAR89.

DATA DATE LFEBas PAGE NO. 6

\begin{tabular}{|c|c|}
\hline $\begin{array}{l}\text { ACTIVITY } \\
\text { ID }\end{array}$ & $\begin{array}{l}\text { ORIG } \\
\text { DUR }\end{array}$ \\
\hline $213 H 036 A 19$ & 5 \\
\hline 213 H037A19 & 5 \\
\hline $213 \mathrm{H} 032 \mathrm{~A} 19$ & 5 \\
\hline 213H033A19 & $\mathbf{5}$ \\
\hline $213 H 034 A 19$ & 5 \\
\hline $219 \mathrm{HO} 11 \mathrm{Al} 9$ & 5 \\
\hline $219 H 012 A 19$ & 5 \\
\hline $217 \mathrm{HO} 27 \mathrm{~A} 1 \mathrm{9}$ & 5 \\
\hline 217 HO16A19 & 5 \\
\hline 2211H003A19 & 5 \\
\hline 221H0O2A19 & 5 \\
\hline $221 \mathrm{H} 001 \mathrm{AL}$ & 5 \\
\hline $220 \mathrm{H} 009 \mathrm{~A} 19$ & 5 \\
\hline $220 \mathrm{H000A19}$ & 5 \\
\hline $220 H 006 A 19$ & 5 \\
\hline 220 HOO5Alg & 5 \\
\hline $220 H 004 \mathrm{A19}$ & 5 \\
\hline $320 \mathrm{H176A19}$ & 5 \\
\hline $320 H 177 \mathrm{Alg}$ & 5 \\
\hline $3204170 \mathrm{Al} 19$ & 5 \\
\hline $215 \$ 027 A 19$ & 5 \\
\hline $2154026 \mathrm{~A} 19$ & 5 \\
\hline $215 \mathrm{B025A19}$ & 5 \\
\hline 215 HO24A19 & 5 \\
\hline $215 \mathrm{HO} 23 \mathrm{A19}$ & 5 \\
\hline $215 \% 022 \mathrm{~A} 19$ & 5 \\
\hline 2114043221 & 5 \\
\hline $211 H 042 A 22$ & 5 \\
\hline $3218174 A 21$ & 5 \\
\hline $321 \mathrm{H} 175 \mathrm{~A} 21$ & 5 \\
\hline $2110044 A 21$ & 5 \\
\hline $323 \mathrm{H} 185 \mathrm{A2} 1$ & 5 \\
\hline $323 \mathrm{H} 186 \mathrm{~A} 21$ & 5 \\
\hline $323 \mathrm{H} 187 \mathrm{A2} 1$ & 5 \\
\hline $214 H 030 A 21$ & 5 \\
\hline $2144029 A 21$ & 5 \\
\hline $214 \mathrm{HO2}$ BA21 & 5 \\
\hline $224 \mathrm{H} 031 \mathrm{~A} 21$ & 5 \\
\hline $216 \mathrm{HO} 02 \mathrm{~A} 21$ & 5 \\
\hline $216 \mathrm{HO} 29 \mathrm{A2} 1$ & 5 \\
\hline $216 \mathrm{HO} 1 \mathrm{BA21}$ & 5 \\
\hline $218 \mathrm{HO} 15 \mathrm{A21}$ & 5 \\
\hline $218 \mathrm{HO} 14 \mathrm{A2} 2$ & 5 \\
\hline $228 \mathrm{HO} 13 \mathrm{A21}$ & 5 \\
\hline $322 \mathrm{H} 18 \mathrm{BA} 21$ & 5 \\
\hline $322 \mathrm{H} 189 \mathrm{A2} 2$ & 5 \\
\hline $322 \mathrm{H} 190 \mathrm{~A} 21$ & 5 \\
\hline $216 \mathrm{HO} 21 \mathrm{A21}$ & 5 \\
\hline $219 \mathrm{HO} 10 \mathrm{A21}$ & 5 \\
\hline $22 \mathrm{OH} 007 \mathrm{A2} 2$ & 5 \\
\hline 2139035 A21 & 5 \\
\hline $213 \mathrm{H} 036 \mathrm{A21}$ & 5 \\
\hline $213 \mathrm{HO37A21}$ & 5 \\
\hline $213 \mathrm{H} 032 \mathrm{A21}$ & 5 \\
\hline $213 \mathrm{H} 033 \mathrm{A21}$ & 5 \\
\hline $213 H 034 A 21$ & 5 \\
\hline $219 H 011221$ & 5 \\
\hline $219 K 012 A 21$ & 5 \\
\hline 217HO17A21 & 5 \\
\hline $21770016 A 21$ & 5 \\
\hline $221 H 003 A 21$ & 5 \\
\hline $221 \mathrm{H} 002 \mathrm{~A} 21$ & 5 \\
\hline $221 H 002 A 21$ & 5 \\
\hline $220 \mathrm{HOO} 0921$ & 5 \\
\hline $220 \mathrm{HOOSA21}$ & 5 \\
\hline $220 \mathrm{HO06A21}$ & 5 \\
\hline $220 \mathrm{HO05A21}$ & 5 \\
\hline $220 \mathrm{H} 004 \mathrm{~A} 21$ & 5 \\
\hline $320 \mathrm{H176A21}$ & 5 \\
\hline $320 \mathrm{H} 177 \mathrm{A21}$ & 5 \\
\hline $320 \mathrm{H178A21}$ & 5 \\
\hline $215 \mathrm{HO27A21}$ & $\mathbf{5}$ \\
\hline $215 H 02621$ & 5 \\
\hline $215 \mathrm{HO25A21}$ & 5 \\
\hline $215 \mathrm{HO24A21}$ & 5 \\
\hline $215 H 023 A 21$ & 5 \\
\hline $215 \mathrm{H} 022 \mathrm{A2} 1$ & 5 \\
\hline $21114043 A 22$ & 3 \\
\hline $2114042 A 22$ & 3 \\
\hline $321 \mathrm{H} 174 \mathrm{A22}$ & 3 \\
\hline $321 K 175 \mathrm{~A} 22$ & 3 \\
\hline $211 \% 044 \times 22$ & 3 \\
\hline $323 \mathrm{H} 185 \mathrm{B22}$ & 3 \\
\hline $323 \% 166222$ & 3 \\
\hline $323 \mathrm{H} 1 \mathrm{~B} 7 \mathrm{A22}$ & 3 \\
\hline $214 \mathrm{HO} 30 \mathrm{A22}$ & 3 \\
\hline $214 \mathrm{HO29A22}$ & 3 \\
\hline $214 \mathrm{HO28A22}$ & 3 \\
\hline $214 H 031222$ & 3 \\
\hline $216 \mathrm{HO} 20 \mathrm{A2} 2$ & 3 \\
\hline $216 \mathrm{H} 019 \mathrm{A22}$ & 3 \\
\hline $216 \mathrm{HO19A22}$ & 3 \\
\hline $218 \mathrm{H} 015 \mathrm{~A} 22$ & 3 \\
\hline $238 \mathrm{HO} 14 \mathrm{A22}$ & 3 \\
\hline $218 \mathrm{H} 013 \mathrm{A22}$ & 3 \\
\hline $322 \mathrm{H} 189 \mathrm{A2} 2$ & 3 \\
\hline
\end{tabular}

Figure 9.19 - Case 2, Revised and Decelerated Schedule (Continued). 
SAUD \& EBRAHIM ALABDULRAZZAK EST

REPORT DATE 21SEP9S RUN NO, 205

Revd Decel Schl by Typical Activaty \&
PRIMAVERA PRONECT PLANRER

SEVEN SEAS, CONSTRUCTION OF 52 HOUSES

\begin{tabular}{|c|c|}
\hline $\begin{array}{l}\text { ACTIVITY } \\
\text { ID }\end{array}$ & $\begin{array}{l}\text { ORIG } \\
\text { DUR }\end{array}$ \\
\hline $322 \mathrm{~K} 189 \mathrm{A2} 2$ & 3 \\
\hline $3224190 A 22$ & 3 \\
\hline $216 \mathrm{HO2} 1 \mathrm{A22}$ & 3 \\
\hline $219 R 010 A 22$ & 3 \\
\hline $2204007 A 22$ & 3 \\
\hline $213 H 035 A 22$ & 3 . \\
\hline $2134036 \mathrm{AZZ}$ & 3 \\
\hline $2134037 A 22$ & 3 \\
\hline $2134032 A 22$ & 3 \\
\hline $2134033 A 22$ & 3 \\
\hline $213 H 034 A 22$ & 3 \\
\hline 2194011422 & 3 \\
\hline $219 H 012 A 22$ & 3 \\
\hline $217 \mathrm{HO} 17 \mathrm{A22}$ & 3 \\
\hline $2174016 A 22$ & 3 \\
\hline $221 H 003 A 22$ & 3 \\
\hline $2214002 A 22$ & 3 \\
\hline $221 H 0 O 1 A 22$ & 3 \\
\hline $220 \mathrm{HOO} 9 \mathrm{A22}$ & 3 \\
\hline $220 \mathrm{OHOOBA22}$ & 3 \\
\hline $220 \mathrm{HOO} 0 \mathrm{GA22}$ & 3 \\
\hline $220 \mathrm{HOOSA22}$ & 3 \\
\hline $220 \mathrm{H} 004 \mathrm{A22}$ & 3 \\
\hline $320 \mathrm{H176A22}$ & 3 \\
\hline $320 \mathrm{HL77A22}$ & 3 \\
\hline $320 \mathrm{H} 178 \mathrm{A22}$ & 3 \\
\hline $215 \mathrm{H027A22}$ & 3 \\
\hline $215 \mathrm{H} 026222$ & 3 \\
\hline $215 \mathrm{H} 025 \mathrm{A2} 2$ & 3 \\
\hline $215 \mathrm{HO24A22}$ & 3 \\
\hline $215 \mathrm{HO} 23 \mathrm{A22}$ & 3 \\
\hline $215 \% 022 A 22$ & 3 \\
\hline $211 \% 043 A 23$ & 21 \\
\hline $211 \% 042 A 23$ & 21 \\
\hline $32111774 A 23$ & 21 \\
\hline $321 \mathrm{H} 275 \mathrm{~A} 23$ & 21 \\
\hline $211 H 044 A 23$ & 21 \\
\hline $323 H_{185 A 23}$ & 21 \\
\hline 323 स2B6AZ3 & 21 \\
\hline $323 H 18723$ & 21 \\
\hline $2248030 \mathrm{A23}$ & 21 \\
\hline $224 \mathrm{HO29A23}$ & 21 \\
\hline $214 H 02$ BA23 & 21 \\
\hline $214 H 031423$ & 21 \\
\hline $2164020 \mathrm{A23}$ & 21 \\
\hline $2164019 A 33$ & 21 \\
\hline $216 \mathrm{HO1BA23}$ & 21 \\
\hline $2184015 A 23$ & 21 \\
\hline $218 H 014 N 23$ & 21 \\
\hline $218 \mathrm{H} 013 \mathrm{A23}$ & 21 \\
\hline $322 \mathrm{H} 18 \mathrm{BA} 23$ & 21 \\
\hline $322 \mathrm{H} 189 \mathrm{~B} 23$ & 21 \\
\hline $322 \mathrm{H} 190 \mathrm{~A} 23$ & 21 \\
\hline $216 \% 021 A 23$ & 21 \\
\hline $219 H 010 A 23$ & 21 \\
\hline $220 \mathrm{H} 007 \mathrm{A23}$ & 21 \\
\hline $213 \mathrm{HO} 35 \mathrm{A2} 3$ & 21 \\
\hline $213 \mathrm{HO} 036 \mathrm{A23}$ & 21 \\
\hline $213 H 037423$ & 21 \\
\hline $223 H 032 A 23$ & 21 \\
\hline $213 \mathrm{HO} 33 \mathrm{A2} 3$ & 21 \\
\hline $213 \mathrm{HO} 034 \mathrm{A23}$ & 21 \\
\hline $2194011 \mathrm{A23}$ & 21 \\
\hline $219 H 012 A 33$ & 21 \\
\hline $217 H_{0} 27 A 23$ & 21 \\
\hline $217 \mathrm{HO16A23}$ & 21 \\
\hline $221 \mathrm{H} 003 \mathrm{A23}$ & 21 \\
\hline $221 \mathrm{H} 002 \mathrm{A23}$ & 21 \\
\hline 2224001423 & 21 \\
\hline $220 \mathrm{O} 009 \mathrm{AL}_{23}$ & 23 \\
\hline $220 \mathrm{H} 008 \mathrm{B23}$ & 21 \\
\hline $220 \% 006 \mathrm{A23}$ & 21 \\
\hline $220 \mathrm{HOOSA23}$ & 21 \\
\hline $2204004 A 23$ & 21 \\
\hline 3201276223 & 21 \\
\hline $320 K 177 A 23$ & 21 \\
\hline $320 H 278 A 23$ & 21 \\
\hline $215 H 02723$ & 21 \\
\hline $2154026 A 23$ & 21 \\
\hline $215 \% 025 A 23$ & 21 \\
\hline $215 \mathrm{H} 024 A 23$ & 21 \\
\hline 2154023423 & 21 \\
\hline $215 H 022423$ & 21 \\
\hline $2111 \mathrm{HO43A24}$ & 4 \\
\hline $211 \mathrm{H} 042 \mathrm{A24}$ & 4 \\
\hline $321 \mathrm{H} 274 A 24$ & 4 \\
\hline $321 \mathrm{H175A24}$ & 4 \\
\hline $211 H 044224$ & 4 \\
\hline $323 \mathrm{H} 85 \mathrm{A24}$ & 4 \\
\hline $323 \mathrm{HLB}^{3}$ & 4 \\
\hline $323 H 187224$ & 4 \\
\hline $214 \mathrm{H} 030 \mathrm{OA24}$ & 4 \\
\hline 21411029234 & 4 \\
\hline 214 HO2BA24 & 1 \\
\hline & 4 \\
\hline $216 \mathrm{HO2024}$ & \\
\hline
\end{tabular}

ACTIVITY DESCRIPTION

20 ROOF DRAINS

21 ROOF DRAINS

23 ROOF DRAINS

24 ROOF DRAINS

26 ROOF DRAINS

27 ROOF DRAINS

28 ROOF DRAINS

30 ROOF DRAINS

31 ROOF DRAINS

32 ROOF DRAINS

33 ROF DRAINS

35 ROOF DRAINS

36 ROF DRAINS

3 ROOF DRAINS

39 ROOF DRAINS

4 ROOF DRAINS

12 ROOF DRAINS

43 ROOF DRAIFS

44 ROOF DRAINS

45 ROOF DRAINS

46 ROOE DRAINS

48 ROOF DRAINS

49 ROOE DRAINS

ROOF DRAINS

01 SANDLIME BRICKS CLADDING

O2 SANDLIME BRICKS CLADDING

OA SANDLIME BRICKS CLADDING

O5 SANDLIME BRICKS CLADDINO

06 SANDLIME BRICKS CLADDINO

7 SANDLIME BRICKS CLLDDING

SANDLIME BRICKS CLADDING

OANDLIME BRICKS CLADDINO

2 SANDLIME BRICTS CIADDIMO

1 SANDIME BRICRS CLADDING

14 SANDLIME BRICXS CLADDING

15 SANDLIME BRICKS CLADDING

16 SANDLIME BRICKS CLADDING

7 SANDLIME BRTCKS CLADDING

B SANDLIME BRICKS CLADDING

19 9ANDLIME BRICKS CLADDING

SANDLIME BRICKS CLADDING

22 SANDLIME BRICKS CLADDINO

SANDLIME BRICKS CLADDINO

6 SANDLIME BRICKS CLADDING

7 SANDLIME BRICRS CTADDING

9 SANDLIME BRICRS CLADDING

9 SANDLIME BRICKS CLADDING

SANDLIME BRICRS CLADDING

1 SANDLIME BRICKS CLADDING

32 SANDLIME BRICKS CLADDINO

SANDLIME BRYCKS CLADDING

SANDLIME BRICKS CLADDINB

6 SANDITY BRTCKS CLADOING

g SANDLIME BRICKS CLADDTNG

O SANDLIME BRICKS CLADDTNO

2 SANOLIME BRICKS CLADDINO

3 SANDLIME BRICKS CIADDINO

44 SANDLIME BRICKS CLADDING

45 SANOLIME BRICKS CLAODING

7) SANDLIME BRICKs CLADDING

SANDLIME BRICKS CLADDING

9 SANDLIME BRICKS CLADDING

O SANDLIME BRICKS CLADDING

1 SANDLIME GRICKS CLADDING

01 ELECTRICAL MAIN SWITCH BOARD

02 ELECTRICAL, MAIN SWITCH BOARD

O4 ELECTRICAL MAIN GWITCH BOARD

O4 ELECTRICAL MAIN SWITCH BOARD

O5 ELECTRICAL, MAIN SWITCH BOARD

06 ELECTRICAL MAIN SWITCH BOARD

GLECTRICAL MAIN SWITCH BOARD

9 ELECTRICAL MAIN SWITCH BOARD

I ELECTRICAL MAIN SWITCH BOARD

12 ELECTRICAL MAIN SWITCH BOARD

12 ELECTRICAL MAIN SWITCH BOARD
09 SANDLIME BRICKS CLADDINO

4 SANDLIME BRICKS CLADDINO

7 SANDT BRE CLADOING

39 SANDLIKE BRICKS CLADOING

1 SANDLIME BRICKS CLADDINO
Correcteng Out-of-Logie Progreas NSG

START DATE 1APR8? FIN DATE 3OMARG9"

DATA DATE 2 FEgg8 PAGE NO.

LEVELED LEVELED LEVELEO LEVELED TOTAA ........... . . LS ........... FLOAT 14MAY89 16MAY8 21 MUNB

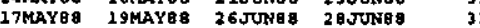

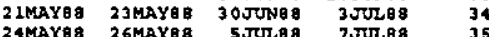
24 MAY88 26 MAY8 5 5 J 31 MAYB 10 JUL8 $1250 L 8$

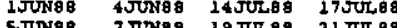
STWN8

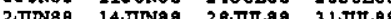
15 JNA8 1 STUNA9 20JNA8 22JUNB B BAUG8 10AUG8 23JUNB8 26JUNBB 13AUUB8B 15AUGB日

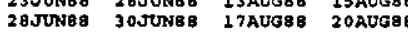
$2 \pi \pi 88$ 4JURB 22AUGB $24 A U G 89$ 6JVL88 9JUR日 29AUGB 30AUG8 10JRR8 12JUR8 15EDB8 4SEPB

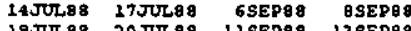

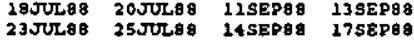

$26 \pi L 88$ 28TULA 29 SEPG 21 SEPB

31 TUL8 2 2AUOA 22 SEPQ9 25 SEPB

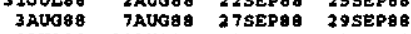

9AUG88 11AUGB 10 CTB B 30CT8

13AUTBS 15AUOA SOCTE B BOCT8B

17AUGBS 20AUGB 9OCTB $110 C T 88$

21AUGB 23AUGB $130 \mathrm{CTB} B$ 160CT88

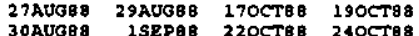

30AUGB 2 1SEPAB 220 CTB 240 CTE8

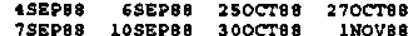

12SEPB B 14 SEPBg 2 NOVB 5 NOVB

3MAR8B 27MAR89 6MAR8 3OMAR89

3MAR8 27 MARE 1 IMARBg 4 APREB

9MAR8 3 3APRE 14 MARSB TAPRBa

14MARE TAPRgB 29MARB 12 APRBg

16MARE9 10APREB 22MARB 16 APRE9

20MARE9 13APREB 27MARB 20 APRE8

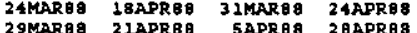

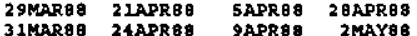

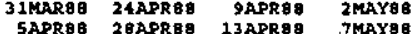

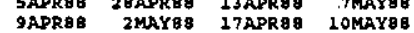

IIAPR98 LMAYBS 21APR9 1 15MAY98

16APR8 9MAY8 25 APRBB 1 GMAY8

19APR8 22MAY9B 30APR8B 23MAY8B

23APRE9 16MAY98 3MAYB 26 MAY89

26APRE9 19HAYB B BMAYE 1 JUNAG

23MAYBQ 11MAYB 5 JUNA

7MAY99 26MAYBg
7 MAYAY

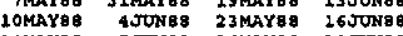

14MAY9B 7 JNN8 26MAY98 $2050 \mathrm{~N} 98$

17MAY8 11 TNR8 31 MAYs 23 JNA8

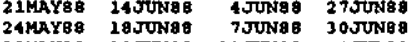

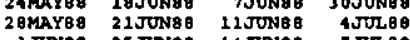

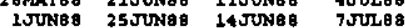

5TNRB 28 JONAB 19 JONB 11 TOLAB

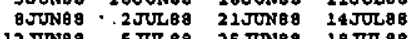

12JUNA 5JUR8 25JUNB L LJUL8

15JUN89 9JULE 28 JONBg 21 JOL9B

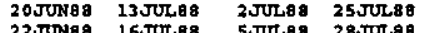

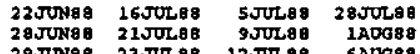

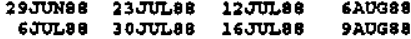

6J0L88 30JUL88 19JULAB 13A0GB9

14JUL8B 9AUOBg 23JUL8B 16 AUGB8

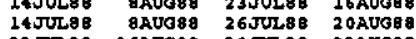

$\begin{array}{lllll}\text { 23NUL88 16AUG89 } & \text { 30JUL8 } & 23 \text { AUGG8 }\end{array}$

$23 \pi R 28$ 16AUGS8 2AUGA $28 A U G B$

$3150 L 89$ 24AUGB 7AUGBS 31 AUOBB

31JULAB 24ADOEO 10AUOB ASEPG

9AUGS 3SEPBB 14AUGS8 7SEP8B

9AUOBO 3SEPBB 17AUGQB 11 SEPBS

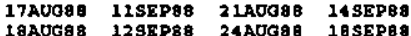

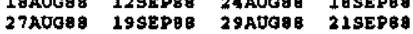

27AUG9O 19SEPB8 1 1SEPEB 25 SEPB

4SEPB B 27SEPB 5 5SEPB 2 2ASEPA

SSEPBB 29 SEPB 9SEP9B $20 \mathrm{CTE9}$

12SEPB 5OCT88 $125 E P B$ SOCTA

3MARBB 7MAR8g 28MAYBS 1JUNA8

OMAR8B 12 MARSB 2JUNB 6 JUNA

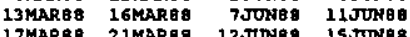

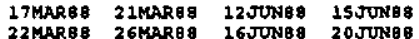

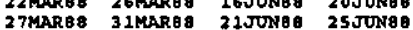

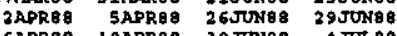

GAPRB8 LAPRE9 30JUNA8 IJULAB

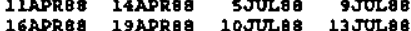

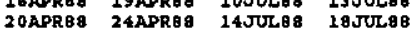

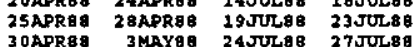

Figure 9.19 - Case 2, Revised and Decelerated Schedule (Continued). 


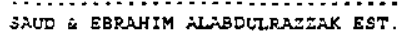

P.EPORT DATE 21 ISEPYS RIN NO. ZOS $10: 31$
PRIMAVERA PROJECT PLANRER

SEVEN SEAS, CONSTRUCTION OF $\mathrm{S}_{1}$ HOUSES corrocting out-of-Logic progres: NSq

START DATE 1APRE7 FIN DATE 3OMAR89.

DATA DATE LFEB8B PAGE No.

\begin{tabular}{|c|c|}
\hline $\begin{array}{l}\text { ACTIVITY } \\
\text { ID }\end{array}$ & $\begin{array}{l}\text { ORIO } \\
\text { DUR }\end{array}$ \\
\hline $216 \mathrm{HO19A24}$ & \\
\hline $216 \mathrm{HO18A24}$ & 4 \\
\hline $2184015 A 24$ & 4 \\
\hline $2194014 A 24$ & 4 \\
\hline $21 \mathrm{aHO13A24}$ & \\
\hline $322 \mathrm{H} 188 \mathrm{A2}$ & 4 \\
\hline $322 \mathrm{H} 189 \mathrm{A2} 4$ & 4 \\
\hline $322 \mathrm{H} 190 \mathrm{A24}$ & 4 \\
\hline $216 \mathrm{HO} 1 \mathrm{AN2} 4$ & \\
\hline $219 H O 10 A 24$ & 4 \\
\hline $220 \mathrm{HO07A24}$ & 4 \\
\hline $213 \mathrm{H} 035 \mathrm{A2} 4$ & 4 \\
\hline $213 \% 03624$ & 4 \\
\hline $2134037 A 24$ & 4 \\
\hline $213 \mathrm{H} 032 \mathrm{A2}$ & 4 \\
\hline $213 \mathrm{HO} 33 \mathrm{A2}$ & 4 \\
\hline $213 H 034 A 24$ & 4 \\
\hline $219 \mathrm{HO}^{2} 1 \mathrm{AZ} 4$ & \\
\hline $219 \mathrm{HO}^{2} 12 \mathrm{A2} 4$ & 4 \\
\hline $217 \mathrm{HO17A24}$ & \\
\hline $217 \mathrm{HO16A24}$ & 4 \\
\hline $221 H 003 A 24$ & 4 \\
\hline $221 \mathrm{HOO2A24}$ & 4 \\
\hline $221 \mathrm{HOO1A24}$ & 4 \\
\hline $220 \mathrm{HOOOA24}$ & 4 \\
\hline $220 H 008 A 24$ & 4 \\
\hline $220 \mathrm{HOOGA24}$ & 4 \\
\hline $220 \mathrm{H} 005224$ & 4 \\
\hline $220 \mathrm{H} 004 \mathrm{A24}$ & 4 \\
\hline $320 \mathrm{H} 176 \mathrm{A2} 4$ & 4 \\
\hline $320 \mathrm{H277A24}$ & \\
\hline $320 \mathrm{H278A24}$ & 4 \\
\hline $215 \mathrm{HO2} 7 \mathrm{A24}$ & 4 \\
\hline $2154026 \mathrm{A2} 4$ & 4 \\
\hline $215 H 025 A 24$ & 4 \\
\hline $215 H 024 A 24$ & 4 \\
\hline $215 H 023 A 24$ & 4 \\
\hline $215 \mathrm{H022A24}$ & 4 \\
\hline $211 \mathrm{H} 043225$ & 7 \\
\hline 213H042A2S & 7 \\
\hline $321 \mathrm{H174A2S}$ & 7 \\
\hline $321 \mathrm{H} 175 \mathrm{~A} 2 \mathrm{~S}$ & 7 \\
\hline $212 \mathrm{HO44A25}$ & 7 \\
\hline $323 \mathrm{H} 285 \mathrm{A25}$ & 7 \\
\hline $323 \mathrm{H} 286 \mathrm{A25}$ & 7 \\
\hline 323 H187A25 & 7 \\
\hline $214 \mathrm{H} 030 \mathrm{A25}$ & 7 \\
\hline 2144029825 & 7 \\
\hline $214 \mathrm{HO} 028 A 25$ & 7 \\
\hline $214 H 031225$ & 7 \\
\hline $216 \mathrm{H} 0202025$ & 7 \\
\hline $216 \mathrm{HO} 19 \times 25$ & $?$ \\
\hline $216 \mathrm{HO1OA2S}$ & 7 \\
\hline 218H015A25 & 7 \\
\hline $218 \mathrm{HO14A25}$ & 7 \\
\hline $218 M 013 A 25$ & 7 \\
\hline $322 \mathrm{H} 198 \mathrm{~A} 25$ & 7 \\
\hline $322 \mathrm{H} 189 \mathrm{~N} 25$ & 7 \\
\hline $322 \mathrm{H} 290225$ & $?$ \\
\hline $216 \mathrm{HO2} 1225$ & 7 \\
\hline $219 \mathrm{HO} 10 \mathrm{A25}$ & 7 \\
\hline $22 \mathrm{OHOO7A25}$ & 7 \\
\hline $213 \mathrm{H} 035 \mathrm{A25}$ & 7 \\
\hline $213 \mathrm{H} 036 \mathrm{A25}$ & 7 \\
\hline 213 H033A25 & 7 \\
\hline $2133032 A 25$ & 7 \\
\hline $213 H 033 A 25$ & 7 \\
\hline $213 \mathrm{H} 034 A 25$ & 7 \\
\hline $219 \mathrm{HO} 11 \mathrm{A25}$ & 7 \\
\hline $2194012 A 25$ & 7 \\
\hline 217 O $017 A 25$ & 7 \\
\hline $317 \mathrm{HO16A25}$ & 7 \\
\hline $221 \mathrm{H} 003 \mathrm{A25}$ & 7 \\
\hline $2211 \mathrm{H} 002 \mathrm{A25}$ & 7 \\
\hline $221 \mathrm{H} 00 \mathrm{OA} 25$ & 7 \\
\hline 220H009A25 & 7 \\
\hline $220 \mathrm{OH} 008 \mathrm{~B} 25$ & 7 \\
\hline $220 \mathrm{HOOGA25}$ & 7 \\
\hline $2204005 A 23$ & $?$ \\
\hline $220 \mathrm{H} 004 \mathrm{A25}$ & 7 \\
\hline $320 \mathrm{~B} 176 \mathrm{~A} 25$ & 7 \\
\hline $320 \mathrm{H177A25}$ & 7 \\
\hline $320 H 178 A 25$ & 7 \\
\hline $215 \mathrm{H} 027 \mathrm{A25}$ & 7 \\
\hline $215 \mathrm{SH} 026 \mathrm{A2S}$ & 7 \\
\hline $215 \mathrm{H} 025 \mathrm{~A} 2 \mathrm{~S}$ & $?$ \\
\hline $215 H 024 A 25$ & 7 \\
\hline $215 \mathrm{H} 023 \mathrm{ALS}$ & 7 \\
\hline $215 H 022 A 25$ & 7 \\
\hline $211 \mathrm{HO} 43 \mathrm{~A} 26$ & 10 \\
\hline $211 \mathrm{H} 042 \mathrm{A26}$ & 10 \\
\hline $321 H 174 A 26$ & 10 \\
\hline $321 \mathrm{H175} 226$ & 10 \\
\hline $211 H 044 A 26$ & 10 \\
\hline $323 \mathrm{H} 185 \mathrm{~A} 26$ & 10 \\
\hline $323 \mathrm{H} 196 \mathrm{~A} 2 \mathrm{G}$ & 10 \\
\hline
\end{tabular}

Figure 9.19 - Case 2, Revised and Decelerated Schedule (Continued). 
SAUD \& SERAHTM MLABDULRAZZAK EST

REPORT DATE 21SEP95 RUN NO. 205

PRIMAVERA PROJECT PLANNER

SEVEN SEAS, CONSTRUCTION OF 51 HOUSES
Corrocting Out-of-Logic Progross NSG

START DATE LAPRB7 FIN DATE 3OMARB9*

DATA DATE LFEB8B PAGE NO.

\begin{tabular}{|c|c|}
\hline $\begin{array}{l}\text { ACTIVITY } \\
\text { ID }\end{array}$ & $\begin{array}{l}\text { ORIG } \\
\text { DUR }\end{array}$ \\
\hline $323 \mathrm{H} 187 \mathrm{~A} 26$ & 10 \\
\hline $2144030 A 26$ & 10 \\
\hline $214 H 029 A 26$ & 10 \\
\hline $21+402 \theta A 26$ & 10 \\
\hline 2144031226 & 10 \\
\hline $2164020 A 26$ & 10 \\
\hline $216 \mathrm{HO19A26}$ & 10 \\
\hline $216 \mathrm{HO18A26}$ & 10 \\
\hline $218 \% 015 A 26$ & 10 \\
\hline $218 H 014 A 26$ & 10 \\
\hline $218 H 013 A 26$ & \\
\hline $322 \mathrm{H188A2} 6$ & 10 \\
\hline $322 \mathrm{H} 189 \mathrm{A2} 2$ & 10 \\
\hline $322 \mathrm{H} 190 \mathrm{2} 26$ & 10 \\
\hline $216 \mathrm{HO21A26}$ & 10 \\
\hline $219 H 010 A 26$ & 10 \\
\hline $220 \mathrm{H} 007 \mathrm{A26}$ & 10 \\
\hline $213 \mathrm{H} 035 \mathrm{~A} 26$ & 10 \\
\hline $213 \mathrm{H} 036 \mathrm{~A} 26$ & 10 \\
\hline $213 \mathrm{HO} 37 \mathrm{~A} 26$ & 10 \\
\hline $213 H 032 A 26$ & 10 \\
\hline $313 \mathrm{H033A26}$ & 10 \\
\hline $213 \mathrm{H} 034 \mathrm{~A} 26$ & 10 \\
\hline $219 H 011 \mathrm{A26}$ & 10 \\
\hline $219 \mathrm{HO} 12 \mathrm{A26}$ & 10 \\
\hline $2174017 A 26$ & 10 \\
\hline $217 \mathrm{HO16A26}$ & 10 \\
\hline $221 \mathrm{H} 003 \mathrm{A2} 6$ & 20 \\
\hline $22140022 A 26$ & 10 \\
\hline $221 \mathrm{H} 001 \mathrm{A2} 6$ & 10 \\
\hline $220 \mathrm{HOOgA26}$ & 10 \\
\hline $220 H 0000226$ & 10 \\
\hline $2204006 \mathrm{A26}$ & 10 \\
\hline $220 \mathrm{H005A26}$ & 10 \\
\hline $2204004 A 26$ & 10 \\
\hline 3204176226 & 10 \\
\hline $32014177 \mathrm{A26}$ & 10 \\
\hline $320 \mathrm{H} 178 \mathrm{2} 26$ & 10 \\
\hline $215 K 027 A 26$ & 10 \\
\hline $215 H 026 A 26$ & 10 \\
\hline $215 \mathrm{HO} 25 \mathrm{A2} 6$ & 10 \\
\hline $215 H 024216$ & 10 \\
\hline $215 H 023 A 26$ & 10 \\
\hline 215 HO22A26 & 10 \\
\hline $211 H 043827$ & 13 \\
\hline $211 H 042 A 27$ & 13 \\
\hline $321 \mathrm{H174A27}$ & 13 \\
\hline $321 H 175 A 27$ & 13 \\
\hline 2114044427 & 13 \\
\hline $323 \mathrm{H} 185 \mathrm{~A} 27$ & 13 \\
\hline $323 \mathrm{H} 186 \mathrm{A2} 7$ & 13 \\
\hline $323 \mathrm{H} 187 \mathrm{A2} 7$ & 13 \\
\hline $214 \mathrm{H030A27}$ & 13 \\
\hline 2144029427 & 13 \\
\hline $214 \mathrm{H028A27}$ & 13 \\
\hline $214 \mathrm{H} 031 \mathrm{~A} 27$ & 13 \\
\hline $216 \mathrm{H} 020 \mathrm{A27}$ & 13 \\
\hline $216 \mathrm{HO} 019 \mathrm{A27}$ & 13 \\
\hline $216 \mathrm{HO1B227}$ & 13 \\
\hline $218 \mathrm{H} 015 \mathrm{A27}$ & 13 \\
\hline $218 H 014 A 27$ & 13 \\
\hline $218 H 013 \mathrm{A27}$ & 33 \\
\hline $322 H 188 A 27$ & 23 \\
\hline $322 \% 189227$ & 23 \\
\hline $322 \mathrm{H} 190 \mathrm{~A} 27$ & 13 \\
\hline $216 \mathrm{H021227}$ & 13 \\
\hline $219 H 010247$ & 13 \\
\hline $220 \mathrm{H} 007 \mathrm{A27}$ & 13 \\
\hline $213 \mathrm{H} 035 \mathrm{~A} 27$ & 13 \\
\hline 2134036227 & 13 \\
\hline $213 \mathrm{H037A27}$ & 13 \\
\hline $213 \mathrm{H} 032 \mathrm{A27}$ & 13 \\
\hline $2134033 A 27$ & 13 \\
\hline 2134034427 & 13 \\
\hline 2194011227 & 13 \\
\hline $239 H 012 \mathrm{A27}$ & 13 \\
\hline 2174017427 & 33 \\
\hline $217 H 016 \mathrm{A27}$ & 13 \\
\hline $2218003 A 27$ & 13 \\
\hline $221 \mathrm{H} 002 \mathrm{A27}$ & 23 \\
\hline $221 H 002 A 27$ & 13 \\
\hline $220 \mathrm{H009A27}$ & 13 \\
\hline $220 \mathrm{H008A27}$ & 23 \\
\hline $220 \mathrm{H} 006 \mathrm{A27}$ & 13 \\
\hline $220 \mathrm{HOOSA27}$ & 13 \\
\hline $220 \mathrm{HOO} 4 \mathrm{A27}$ & 13 \\
\hline $320 H 1762827$ & 13 \\
\hline $320 \mathrm{H} 177 \mathrm{A27}$ & 13 \\
\hline 3204178827 & 13 \\
\hline $215 H 027227$ & 13 \\
\hline 2154026227 & 13 \\
\hline $215 H 025427$ & 13 \\
\hline $215 H 024 A 27$ & 13 \\
\hline 2151023327 & 13 \\
\hline $215 \mathrm{HO22A27}$ & 13 \\
\hline $211 H 043$ A28 & 10 \\
\hline
\end{tabular}

Figure 9.19 - Case 2, Revised and Decelerated Schedule (Continued). 


\begin{tabular}{|c|}
\hline $\begin{array}{l}\text { PEPCRT DATE : } \\
\text { ReYd \& DaCel }\end{array}$ \\
\hline $\begin{array}{c}\text { ACIVITY } \\
\text { ID }\end{array}$ \\
\hline 211 HO42A2B \\
\hline $321 H 174 A 2 B$ \\
\hline $321 \mathrm{H} 175 \mathrm{SA2 \theta}$ \\
\hline $211 \mathrm{HO44A2 \theta}$ \\
\hline $323 \mathrm{H} 185 \mathrm{~A} 2 \mathrm{~B}$ \\
\hline $323 \mathrm{H} 186 \mathrm{A2} 8$ \\
\hline $323 \mathrm{H} 187 \mathrm{A2O}$ \\
\hline $214 \mathrm{H} 030 \mathrm{~A} 2 \mathrm{~B}$ \\
\hline $214 \mathrm{H029A2B}$ \\
\hline $214 \mathrm{HO2BA2 \textrm {O }}$ \\
\hline $2144031 A 28$ \\
\hline $216 \mathrm{H020A28}$ \\
\hline $216 \mathrm{HO} 019 \mathrm{A2} \mathrm{B}$ \\
\hline $216 \mathrm{HO} 2 \mathrm{BA2B}$ \\
\hline $218 \mathrm{HO} 015 \mathrm{AN} 2 \mathrm{~B}$ \\
\hline $218 H 014 A 28$ \\
\hline $218 \mathrm{HO} 13 \mathrm{A2}$ \\
\hline $322 \mathrm{H} 18 \mathrm{BA2} \mathrm{B}$ \\
\hline $322 \mathrm{H} 189 \mathrm{A2B}$ \\
\hline $322 \mathrm{H190A2 \textrm {B }}$ \\
\hline $216 \mathrm{H} 021 \mathrm{AB}$ \\
\hline $219 \mathrm{H} 010 \mathrm{~A} 2 \mathrm{~B}$ \\
\hline $220 \mathrm{H} 007 \mathrm{A2O}$ \\
\hline $213 H 035 A 2 \theta$ \\
\hline $213 \mathrm{HO} 36 \mathrm{A2} \mathrm{B}$ \\
\hline $213 \mathrm{HO} 037 \mathrm{A2O}$ \\
\hline $2134032 A 28$ \\
\hline $223 \% 0332228$ \\
\hline $213 \mathrm{HO} 44 \mathrm{A2}$ \\
\hline $2194011 A 28$ \\
\hline $219 \mathrm{HO} 012 \mathrm{~A} 2 \mathrm{~B}$ \\
\hline $2174017 A 2 \theta$ \\
\hline $217 \mathrm{HO16A2 \textrm {O }}$ \\
\hline $221 \mathrm{H} 003 \mathrm{A2} \mathrm{B}$ \\
\hline $221 \mathrm{HO02A2 \textrm {B }}$ \\
\hline $221 M 001 A 28$ \\
\hline $220 \mathrm{HOO9A2B}$ \\
\hline $220 \% 008228$ \\
\hline $220 \% 006$ A2B \\
\hline $220 \% 005 A 28$ \\
\hline 2204004228 \\
\hline $320 H 176 A 28$ \\
\hline $320 \mathrm{H} 277 A 28$ \\
\hline $320 \mathrm{OH} 7 \mathrm{AA2B}$ \\
\hline $215 \mathrm{H027A2B}$ \\
\hline $215 \mathrm{H} 026 \mathrm{BO}$ \\
\hline 215 H025A28 \\
\hline $2154024 A 28$ \\
\hline $215 H 023$ A2 B \\
\hline $215 \mathrm{H02282 \textrm {B }}$ \\
\hline 2114043229 \\
\hline $2314042 A 29$ \\
\hline $3214174 \times 29$ \\
\hline 3214175429 \\
\hline $2114044 A 29$ \\
\hline $323 \mathrm{~K} 105 \mathrm{AP} 9$ \\
\hline $323 H 186 A 29$ \\
\hline $323 H 187 A 29$ \\
\hline $214 \mathrm{HO} 030 \mathrm{A29}$ \\
\hline $214 H 029 A 29$ \\
\hline $214 \mathrm{H028A29}$ \\
\hline 214 H 31 A29 \\
\hline $216 \mathrm{HO20A29}$ \\
\hline $216 \mathrm{H} 019 \mathrm{A2} 9$ \\
\hline $216 H_{018 A 29}$ \\
\hline $21 \mathrm{HH} 015 \mathrm{~A} 29$ \\
\hline $210 \mathrm{HO} 14 \mathrm{A2} 2$ \\
\hline $218 H 013$ A29 \\
\hline $322 \mathrm{H} 18 \mathrm{BA29}$ \\
\hline $322 \mathrm{H189A29}$ \\
\hline $322 \mathrm{H} 190 \mathrm{~A} 29$ \\
\hline $216 \mathrm{HO2} 1 \mathrm{A2} 9$ \\
\hline $219 \mathrm{HO} 10 \mathrm{2} 2$ \\
\hline $220 \mathrm{H} 007 \mathrm{A29}$ \\
\hline $213 \mathrm{H} 035 \mathrm{~A} 29$ \\
\hline $213 H 036 A_{2} 9$ \\
\hline $2134037 A 29$ \\
\hline $213 \mathrm{HO} 32 \mathrm{A2} 9$ \\
\hline $213 H 033 A 29$ \\
\hline 2135034229 \\
\hline $219 H 011 A 29$ \\
\hline $219 \mathrm{HO} 12 \lambda 29$ \\
\hline $217 \mathrm{H017A29}$ \\
\hline $217 \mathrm{H016A29}$ \\
\hline $221 H 003229$ \\
\hline $221 \mathrm{H} 002 \mathrm{A29}$ \\
\hline $221 \% 001229$ \\
\hline $220 \mathrm{H} 009 \mathrm{AZ} 9$ \\
\hline $220 \mathrm{HOOBA29}$ \\
\hline $220 \% 006 A 29$ \\
\hline $220 \mathrm{HOOSA29}$ \\
\hline $220 \mathrm{HOOA429}$ \\
\hline $320 H 176 A 29$ \\
\hline $320 H 177 A 29$ \\
\hline $320 H 278 A 29$ \\
\hline $215 \mathrm{H} 027 \mathrm{A29}$ \\
\hline
\end{tabular}

Figure 9.19 - Case 2, Revised and Decelerated Schedule (Continued). 


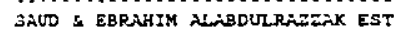

REPCRT DATE IISEPGS RUN NO. 205

Rovd \& Decel Schl by Typical Aetivaty \& ES
PRIMAVERA PROAECT PLANNER

SEVEN SEAS, CONSTRUCTION OF 51 HOUSES

\begin{tabular}{|c|c|}
\hline$\underset{\text { ID }}{\text { ACTIVI }}$ & $\begin{array}{l}\text { ORIG } \\
\text { DUR }\end{array}$ \\
\hline $215 H 026 A 29$ & 7 \\
\hline $215 H 025 A 29$ & 6 \\
\hline $215 H 024 A 29$ & 6 \\
\hline $215 \% 023 A 29$ & 6 \\
\hline $2153022 A 29$ & 6 \\
\hline $211 H 043 A 30$ & 4 \\
\hline 21140422330 & 4 \\
\hline 32111742030 & 4 \\
\hline $321 \% 175 A 30$ & 4 \\
\hline $211 H 044 A 30$ & 4 \\
\hline $323 \mathrm{HLOSA} 3 \mathrm{O}$ & 4 \\
\hline $323 \mathrm{H186A30}$ & 4 \\
\hline $323 \mathrm{H} 187 \mathrm{AA3} \mathrm{O}$ & 4 \\
\hline 2144030230 & 4 \\
\hline $214 H 029 A 30$ & $i$ \\
\hline $214 \mathrm{HO28A30}$ & 4 \\
\hline 2144031230 & 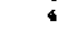 \\
\hline 2164020230 & 4 \\
\hline $216 \mathrm{HO} 90 \mathrm{N3O}$ & $i$ \\
\hline $216 \mathrm{HO} 018 \mathrm{~A} 30$ & 4 \\
\hline $2184015 A 30$ & 4 \\
\hline $218 \mathrm{HO}_{2} 1 \mathrm{AO} \mathrm{O}$ & 4 \\
\hline $228 H 013 A 30$ & 4 \\
\hline $322 \mathrm{H} 188 \mathrm{AO} 3 \mathrm{O}$ & 4 \\
\hline $322 \mathrm{H} 189 \mathrm{3} 30$ & $i$ \\
\hline $322 H 190 A 30$ & 4 \\
\hline $216 \mathrm{H} 021 \mathrm{A3} 3 \mathrm{O}$ & 4 \\
\hline 2194010230 & 4 \\
\hline $220 \mathrm{HOO} 7 \mathrm{~A} 3 \mathrm{O}$ & 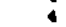 \\
\hline 213 HOSSA3O & 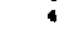 \\
\hline $223 \mathrm{HO} 6 \mathrm{AJ} O$ & 4 \\
\hline $213 \mathrm{HO} 7 \mathrm{A3} 3 \mathrm{O}$ & 4 \\
\hline $213 \mathrm{H032A30}$ & 4 \\
\hline $223 \mathrm{H03} 3230$ & 4 \\
\hline $223 H 0342330$ & 4 \\
\hline 21940112330 & 4 \\
\hline $229 \mathrm{HO} 22 \mathrm{A3O}$ & 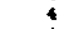 \\
\hline $217 \mathrm{HO} 17 \mathrm{A3O}$ & 4 \\
\hline $2174016 A 30$ & 4 \\
\hline $221 H 003030$ & 4 \\
\hline $221 \mathrm{HO02A30}$ & 4 \\
\hline $2214001 A 30$ & 4 \\
\hline $220 H 009 A 30$ & 4 \\
\hline $220 \mathrm{HOOSA3O}$ & 4 \\
\hline $220 \mathrm{H} 006 \mathrm{~A} 30$ & 4 \\
\hline $220 \mathrm{HOO5A30}$ & 4 \\
\hline $220 H 004 \lambda 30$ & 4 \\
\hline $3204176 A 30$ & 4 \\
\hline $320 \mathrm{H} 177 \mathrm{A3} 0$ & 4 \\
\hline $320 H 1782330$ & 4 \\
\hline 2154027230 & 4 \\
\hline $215 H 026330$ & 4 \\
\hline $215 \mathrm{HO2SA30}$ & 4 \\
\hline $215 H 02 \times A 30$ & 4 \\
\hline $215 H 023 A 330$ & 4 \\
\hline $215 H 022 A 30$ & 4 \\
\hline $211 \mathrm{H} 043 \mathrm{~A} 31$ & 6 \\
\hline $211 \mathrm{H} 042 \mathrm{A31}$ & 6 \\
\hline $321 \mathrm{H174A31}$ & 6 \\
\hline 3211175431 & 6 \\
\hline 211 HO44A31 & 6 \\
\hline $323 \mathrm{H} 05 \mathrm{NA1}$ & 6 \\
\hline $323 \mathrm{H} 1 \mathrm{~B} 6 \mathrm{A3} 1$ & 6 \\
\hline $3234187 A 31$ & 6 \\
\hline 214 H030A31 & 6 \\
\hline $2144029 A 32$ & 6 \\
\hline $214 \mathrm{H} 028 \mathrm{~B} 1$ & 6 \\
\hline 2144031431 & 6 \\
\hline $2164020 A 31$ & E \\
\hline $216 H 019 A 31$ & 6 \\
\hline $216 \mathrm{HO} 19 \mathrm{AB} 1$ & 6 \\
\hline $218 H 015 \times 31$ & 6 \\
\hline $218 \mathrm{HO14A31}$ & 6 \\
\hline $228 H 0132331$ & 6 \\
\hline $322 \mathrm{H} 189 \mathrm{~A} 31$ & 6 \\
\hline $322 \mathrm{H} 189 \mathrm{~A} 3 \mathrm{~L}$ & 6 \\
\hline $322 \mathrm{H} 190 \mathrm{Al} 1$ & 6 \\
\hline $216 \mathrm{H} 0211231$ & 6 \\
\hline $2194020 A 31$ & 6 \\
\hline $220 \mathrm{HOO} 7 \mathrm{A3}$ & 6 \\
\hline 2134035231 & 6 \\
\hline $213 \mathrm{H036231}$ & 6 \\
\hline $213 \mathrm{H} 037 \mathrm{A3} 1$ & 6 \\
\hline $2136032 \mathrm{A31}$ & 6 \\
\hline $213 \mathrm{HO} 33 \mathrm{Al}$ & 6 \\
\hline $213 \mathrm{HO} 44 \mathrm{~A} 31$ & 6 \\
\hline $219 H 011231$ & 6 \\
\hline $219 \mathrm{H} 012 \mathrm{A3}$ & 6 \\
\hline $2174017 A 31$ & 6 \\
\hline 217 HO16A31 & 6 \\
\hline $221 \mathrm{H003A31}$ & 6 \\
\hline $2214002 \sqrt{31}$ & 6 \\
\hline $221 \mathrm{HOOOAN1}$ & 6 \\
\hline 2204009031 & 6 \\
\hline $220 \mathrm{HOOSA31}$ & 6 \\
\hline $220 \mathrm{HOO} 0 \mathrm{A3} 1$ & \\
\hline
\end{tabular}

ACTIVITY DESCRIPTION

7 MARBLT FRAMES

46 MARBLE FRAMES

1 MARBLE FRAMES

O1 WOODEN FRAMES

03 WOODEN FRAMES

O4 WOODEN FRAMES

OS WOODEN FRAMES

O6 WOODEN FRAMES

OA WOODEN FRAMES

09 WOODEN FRAMES

i1 WOODEN FRAMES

12 WOCDEN FRAMES

14 WOODEN FRAMES

16 WOODEN FRAMES

17 WOODEN FRAMES

19 WOODEN FRAMES

19 WOODEN FRAMES

20 WOODEN ERAMES

32 WOODES FRAMES

WOODEN FRAMES

24 WODEN FRAMES

26 WOODEN FRAMES

27 WOODEN FRAMES

28 WOODEN FRAMES

OO WODEN FRAMES

11 WOODEN FRAMES

32 WOODEN FRAMES

33 WOODEN FRAMES

34 WOODEN FRAMES

36 WOODEN FRAMES

37 WOODEN FRAMES

MOODEN FRAMES

40 WOODEN FRAMES

41 WOODEN FRAMES

42 WOODEN FRAMES

43 WOODEN FRAMES

44 WOODEN FRANES

6 MOODEN FRAMES

7 WODEN FRAMES

I9 WOODEN FRAMES

SO WOODE TRAMES

11 WOODEN FRAMES

01 BLOCK WORKS OF CTLASES

O3 BLOCK MORKS OF CHASES

O4 BLOCK WORKS OF CHAASES

OS BLOCK WORTS OF CHASES

06 BLOCK MORKS OF CHASES

O7 BLOCK WORRS OF CHASES

DLCK wORXs of ChAsRs

D B

1 BLOCK wORKs OF CHASEs

13 BLOCK WORKS OF CHASES

14 BLOCK WORXS OF CHASES

15 BLOCK WORKS OF CHASES

16 BLOCK WORXS OF CHASES BLOCK WORKS OF CHASES I BLOCK WORKS OF CHASES 1 BLOCK NORKS OF cHAges I2 BLCK WORK OF CHASES 23 SLOCK WORKS OF CHASES BLOCK YORKS OF CHASES 25 BLOCK MORKS OF CHASES 26 BLOCK WORKS OP CHASES 7 BLOCK WORKS OF CHASES 8LOCK WORKS OF CHASES 29 BLOCK WORKS OF CRASES BLOCK MORKS OF CHASES

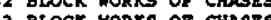
34 BLOCK MORTS OP CHAgEs 35 BLOCK YORKs or chass 36 BLOCX WORKS OF CIASES 37 BLOCK WORKS OF CHASES 38 BLOCK WORKS OF CHASES 39 BLOCK WORKS OF CHASES
40 BLOCK WORKS OF CHASES
49 MARBLE ERAMES

07 WOODEN FRAMES

15 WOODEN TPAMES

02 BLOCK WORTS OF CHASES

2 BLOCK WORKS OF CHASES

17 BLOCK WORKS OF CHASES II BLOCK WORXS OF CHASES
Correct 1 ng Out-of-log 26 Progrese NSq STAPT DATE LAPRB 7 FIN DATE JOMIR39" DATA DATE IFEBBB PAGE NO, LI LEVELED LEVELED LEVELED TOTAL

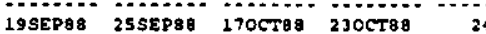
22SEPB 28SEP8 220CTA9 270CTA 27SEPAB 30CT8 250CTA8 $310 \mathrm{CTB}$ 10CT88 60CT8 $290 \mathrm{CTBS}$ 3NOVB 15MARB 15MAR8 19 MARB 23 MARG 22 MARG 20MAR9B 33 MARES 2 MMAR8 27 MARE 23MARE 27 MARB 3 3APR8 29MAR日9 2APRB 7 APR8 2APREQ SAPRB 12APR8 16 APRA 6APRE9 10APRSG 17APR8G 20APRB 1OAPRE8 13APR8Q 21APRE9 25APR9 14APREB 18APR8 26APREg 30APR8 18APR9B 21APRB 1 1MAY99 4 MAY8 23APRAB 26APREB 5MAYBg 9 MAY8

MMAYB MMAYS B TMAY8 15MAYgB 2 MMAYG 9MAY88 13MAY98 24 MAYgB 28 MAYS 12 MAY8 16 MAYS 29 MAYBS 2 JUNB 17 MAY8 21 MAY 98 21MAYB8 24MAY日 BJUN8B $125 U N A 8$ 25MAYB 29MAYA 13JUNBE 16JUNB

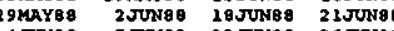
4JUNE8 7JUN8B 22JUNE8 $2650 N 8$ 7JUNB8 11JUN8 27 JUNAB 30 JTNB STJRB (15) 13

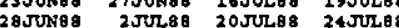

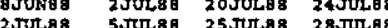

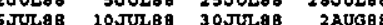

OJTLaB 23JRLB 3AVGa BAUGB

14JUL8 18JUR8 9AUG8B 13AUGB8 $18 \pi U L 8 B$ 21JULEg 14AUOBB 17 AUGB

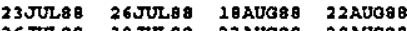
2 GAUGB

3AUGB BAUOB 3 SEPB 6 SEPB

9AUGB 13AUGA 7SEPB 11SEPB

13AUGA 16 AUOAB 12SEPBS 25SEP8

17AUQO 21 AUOQB 17 SEPB 20 SEPBO

21AUGG 24AUO8B 21SEPB8 25SEPG

27AUGBS 30AUGB 26SEP9B 29SEPG9

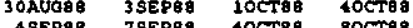

4SEPa 7 7SEPB $40 \mathrm{CTR}$ 8

7SEPA 1 1SEPA 9OCTB 120CTAB

12SEP8B 15 SEPA $230 \mathrm{CTB}$ 260CT88

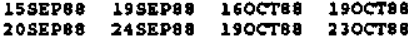

24SEPB 27 SEPB 240 CTB $270 \mathrm{CTAB}$

28SEPB8 20CT8 270CTE $310 \mathrm{CTR}$

20CTB9 50CTaB 310CTR 3NOV8B 6OCTBa 10OCTAB 3NOVB TNOVBS

20MARE9. 26MAR8 21MARE9 27MARBa

23MARBg 3OMARB 26MARB 2APREQ

27MARBg 3APRAB 31MARBO 6APRBg

2APRE9 TAPRE SAPRE

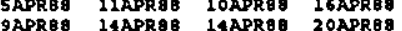

13APRBQ 19APREB 19APRBQ 25APREQ

7APR日9 23APRgB 24APRBB 3OAPR日9

20APRE9 26APR8 2BAPRB 4 MAYA8

SAPREg 1 MAYBE 3 MAYYB 9 MAYBB

2MAYB 4 MAYB 8 BAYYQ 14 MAYB

TMAYBB 2 MAYG 17 MAYB 23 MAYGB

IOMAY9B 16MAYBg 22MAYBg 2 BMAYBB

14MAY8B 19MAY89 26 MAY8 $250 \mathrm{~N} 88$

18MAYB B 24MAY98 IJUNA8 750 N88

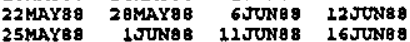

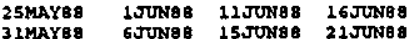

4JUNB G GTNAB 20 JUNaB 26 JUNA

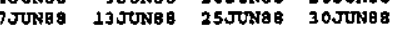

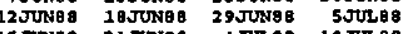

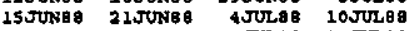

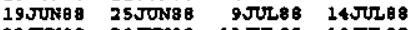

23 JUNA 29 JUNB 13 JULAB 19 JUL8B

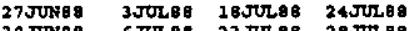

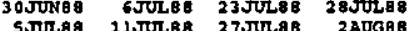

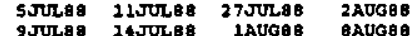

9JULA9 14JULB 1 1AUGB

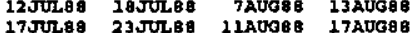

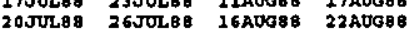

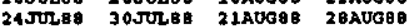

2BJUL8B 3AUGB $27 A$ ACOBB ISEPBB

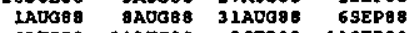

GAUGBS 11AUGB 5 SEPQB $115 E P B 8$

10AUGB 1 16AUGB B 10SEP9B 15SEPB8

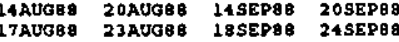

22AUGB 29AUG8 $22 S E P B B$ 28SEPB

Figure 9.19 - Case 2, Revised and Decelerated Schedule (Continued). 


\begin{tabular}{|c|c|}
\hline $\begin{array}{c}\text { ACTIVITT } \\
\text { ID } \\
\ldots\end{array}$ & $\begin{array}{l}\text { ORIO } \\
\text { DUR }\end{array}$ \\
\hline $220 \mathrm{HOOSA31}$ & 6 \\
\hline $220 \mathrm{HOO}+\mathrm{A3} 2$ & 6 \\
\hline $320 \mathrm{H} 176 \mathrm{~A} 31$ & 5 \\
\hline $320 \mathrm{H} 177 \mathrm{A31}$ & 6 \\
\hline $320 \mathrm{H178A31}$ & 6 \\
\hline $215 \mathrm{HO27A31}$ & 6 \\
\hline $215 H 026 A 31$ & 6 \\
\hline $215 H 025 \mathrm{A31}$ & 6 \\
\hline $215 \% 024,31$ & 6 \\
\hline $215 H 023 A 31$ & 6 \\
\hline $2154022 \mathrm{A31}$ & 6 \\
\hline $211 \% 043 A 32$ & a \\
\hline $211 \mathrm{H} 042 \mathrm{A32}$ & 8 \\
\hline $321 \mathrm{H} 174 \mathrm{A32}$ & $\mathbf{9}$ \\
\hline $3214175 A 32$ & 8 \\
\hline $2114044 A 32$ & 9 \\
\hline $3234185 A 32$ & 8 \\
\hline $323 \mathrm{H186A32}$ & $\mathbf{g}$ \\
\hline $323 \mathrm{H} 187 \mathrm{A32}$ & g \\
\hline 2244030232 & 8 \\
\hline $214 H 029 A 32$ & g \\
\hline $214 \mathrm{H} 028 \mathrm{A32}$ & 8 \\
\hline & 8 \\
\hline $216 \mathrm{HO20A32}$ & g \\
\hline $216 \mathrm{HO} 019 \mathrm{A32}$ & g \\
\hline $2164018 A 32$ & 8 \\
\hline $219 \mathrm{HO} 015 \mathrm{SA} 2$ & 8 \\
\hline 22aH014A32 & 8 \\
\hline $239 H 013 A 32$ & 8 \\
\hline $322 \mathrm{H188A32}$ & $\mathrm{g}$ \\
\hline $322 \mathrm{H189A32}$ & $\mathrm{g}$ \\
\hline $322 H 190 A 32$ & a \\
\hline $216 \mathrm{HO21A32}$ & a \\
\hline $219 \mathrm{HO} 10 \mathrm{A32}$ & 8 \\
\hline $220 \mathrm{HOO} 7 \mathrm{A32}$ & 8 \\
\hline 213 H035A32 & g \\
\hline $223 H 036232$ & g \\
\hline $213 \mathrm{H} 037 \mathrm{A32}$ & 8 \\
\hline $2134032 \mathrm{A32}$ & 9 \\
\hline $213 \mathrm{H} 033 \mathrm{A3} 2$ & 8 \\
\hline $213 H 034 A 32$ & 8 \\
\hline $219 \mathrm{HO} 011 \mathrm{~A} 32$ & 8 \\
\hline $219 \mathrm{HO} 012 \mathrm{A32}$ & a \\
\hline $217 \mathrm{HO17A32}$ & 8 \\
\hline $217 \mathrm{HO} 16 \mathrm{A32}$ & a \\
\hline $2214003 A 32$ & 8 \\
\hline $221 \mathrm{HOO} 2 \mathrm{A32}$ & $\mathbf{g}$ \\
\hline $2214001 \mathrm{A32}$ & g \\
\hline $220 \mathrm{H} 009 \mathrm{A3} 2$ & $g$ \\
\hline $220 \mathrm{HOOBA32}$ & 8 \\
\hline $2204006 \mathrm{AOZ}$ & 8 \\
\hline $2204005 \mathrm{A3} 2$ & 8 \\
\hline $220 \mathrm{HOOAA32}$ & g \\
\hline $320 \mathrm{H} 176 \mathrm{A32}$ & B \\
\hline $320 \mathrm{H} 177 \mathrm{A32}$ & $\theta$ \\
\hline $320 \mathrm{H} 178 \mathrm{~A} 32$ & a \\
\hline $215 \mathrm{HO27A32}$ & $B$ \\
\hline $215 \mathrm{H} 026 \mathrm{A32}$ & 8 \\
\hline $215 H 025 A 32$ & 8 \\
\hline $215 \mathrm{HO} 24 \mathrm{~A} 32$ & B \\
\hline $215 \mathrm{H} 023 \mathrm{A3} 2$ & \\
\hline $215 H 022 A 32$ & 8 \\
\hline $211 R 043 A 33$ & 24 \\
\hline $2114042 A 33$ & 24 \\
\hline $321 H 174 A 33$ & 24 \\
\hline $321 \mathrm{H} 175 \mathrm{A3} 3$ & 24 \\
\hline $211 H 044 A 33$ & 24 \\
\hline $323 \mathrm{H} 185 \mathrm{A3} 3$ & 24 \\
\hline $323 \mathrm{H} 186 \mathrm{A3} 3$ & 24 \\
\hline $323 \mathrm{H} 287 \mathrm{A3} 3$ & 24 \\
\hline $214 \mathrm{HO} 30 \mathrm{~A} 33$ & 24 \\
\hline $214 \mathrm{HO} 29 \mathrm{A3} 3$ & \\
\hline $214 \mathrm{HO} 2 \mathrm{OA} 33$ & 24 \\
\hline $2144032 A 33$ & 24 \\
\hline & 24 \\
\hline $\begin{array}{l}216 \mathrm{HO} 019 \mathrm{AB3} 3 \\
216 \mathrm{HO18A33}\end{array}$ & 24 \\
\hline $216 \mathrm{HO} 18 \mathrm{A3} 3$ & 24 \\
\hline $218 \mathrm{HO} 15 \mathrm{A3} 3$ & 24 \\
\hline $218 \mathrm{H} 014 \mathrm{A33}$ & 24 \\
\hline $21 B H 013 A 33$ & 24 \\
\hline $322 \mathrm{H} 188 \mathrm{~B} 33$ & 24 \\
\hline $322 \mathrm{H} 189 \mathrm{A3} 3$ & 24 \\
\hline $322 \mathrm{H} 190 \mathrm{~A} 33$ & 24 \\
\hline $216 \mathrm{HO} 021 \mathrm{A3} 3$ & 24 \\
\hline $219 \mathrm{HO} 10 \mathrm{A33} 3$ & 24 \\
\hline $220 \mathrm{HOOO} 7 \mathrm{A3} 3$ & \\
\hline $213 \mathrm{HO} 035 \mathrm{A3} 3$ & 24 \\
\hline $213 \mathrm{H}_{03} 6 \mathrm{~A} 33$ & 24 \\
\hline $213 \mathrm{HO}^{2} 7 \mathrm{A3} 3$ & 24 \\
\hline $213 \mathrm{HO} 032 \mathrm{A3}$ & 24 \\
\hline $213 \mathrm{H} 033 \times 33$ & 24 \\
\hline $213 K 034 A 33$ & 24 \\
\hline $219 \mathrm{HO} 11 \mathrm{A3} 3$ & 24 \\
\hline $219 \mathrm{HO} 012 \mathrm{A3} 3$ & 24 \\
\hline $2174017 \times 33$ & 24 \\
\hline $227 \mathrm{K016 \textrm {A } 3 3}$ & 24 \\
\hline
\end{tabular}

Figure 9.19 - Case 2, Revised and Decelerated Schedule (Continued). 
SAUD \& SBRAHIM ALABDULRAZZAX EST

REPORT DATE 21 SEP95 RUN NO. 205 Rovd \& Docol Schl by Thical Aetivity \& $\mathbf{E S}$
PRIMAVERA PROJECT PLANNER

SEVEN SEAS, CONSTRUCTION OF 51 HOUSES
Corrocting out-of-Logic Progyobs NSq

START DATE LAPRET FTN DATE JOMARBQ

DATA DATE 1FEBga PAGE NO.

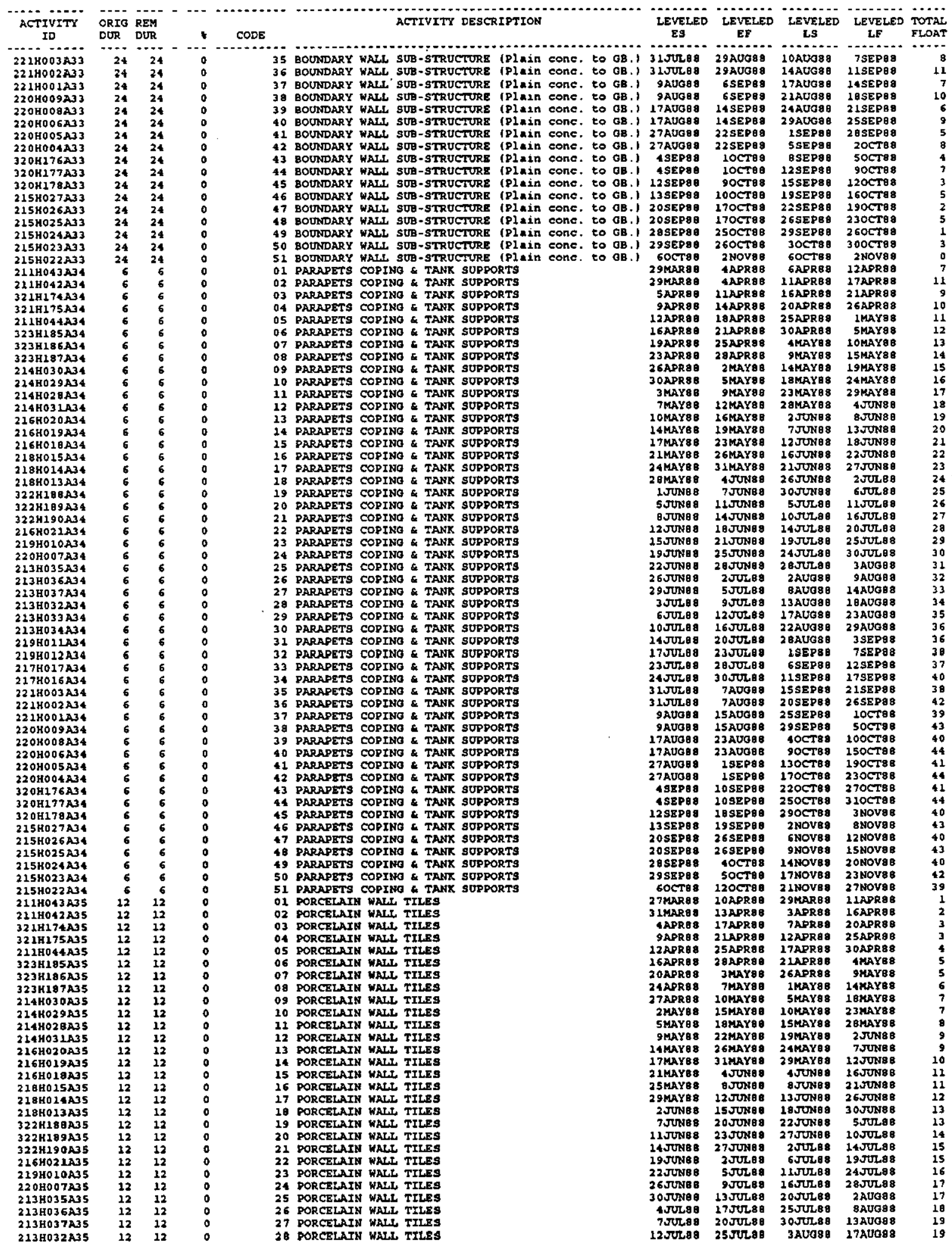

Figure 9.19 - Case 2, Revised and Decelerated Schedule (Continued). 


\begin{tabular}{|c|c|}
\hline $\begin{array}{l}\text { ACTIVITY } \\
\text { ID }\end{array}$ & $\begin{array}{l}\text { ORIO } \\
\text { DUR }\end{array}$ \\
\hline 213 H033A.35 & 12 \\
\hline $213 \mathrm{HO} 3 \mathrm{AA3}$ & 12 \\
\hline $219 H_{011 \mathrm{~A}} 5$ & 12 \\
\hline $219 H 012 A 35$ & 12 \\
\hline 217H017A35 & 12 \\
\hline $2174016 \mathrm{A3} 5$ & 12 \\
\hline $221 \mathrm{H} 003 \mathrm{~A} 35$ & 12 \\
\hline $221 \mathrm{H} 002 \mathrm{A35}$ & 12 \\
\hline $221 \mathrm{HOO} 1 \mathrm{~A} 35$ & 12 \\
\hline $220 \mathrm{HOOSA3S}$ & 12 \\
\hline $220 \mathrm{HOOBA35}$ & 12 \\
\hline $220 \mathrm{H} 006 \mathrm{~A} 35$ & 12 \\
\hline $220 \mathrm{HOOSA35}$ & 12 \\
\hline $220 \mathrm{H} 004 \mathrm{~A} 35$ & 12 \\
\hline $320 \mathrm{H} 176 \mathrm{AS} \mathrm{S}$ & 12 \\
\hline $320 \mathrm{H} 177 \mathrm{~A} 35$ & 12 \\
\hline $320 \mathrm{H178A35}$ & 12 \\
\hline $215 \mathrm{HO} 27 \mathrm{~A} 35$ & 12 \\
\hline $215 H 026 A 35$ & 12 \\
\hline $215 H 025 A 35$ & 12 \\
\hline 215 H024A35 & 12 \\
\hline $215 H 023 A 35$ & 12 \\
\hline $215 H 022 \mathrm{~A} 35$ & 12 \\
\hline $21110043 A 36$ & 10 \\
\hline $2114042 A 36$ & 10 \\
\hline $3214174 A 36$ & 10 \\
\hline $321 \mathrm{H175A36}$ & 10 \\
\hline $211 H 044 A 36$ & 10 \\
\hline $323 H 185 A 36$ & 10 \\
\hline $323 \mathrm{H196A36}$ & 10 \\
\hline $323 \mathrm{H} 197 \mathrm{A3} 6$ & 10 \\
\hline $214 \mathrm{H} 030 \mathrm{~A} 36$ & 10 \\
\hline $214 \mathrm{H} 029 \mathrm{A3} 6$ & 10 \\
\hline $214 \mathrm{HO} 2 \mathrm{BA} 36$ & 10 \\
\hline $214 H 031 A 36$ & 10 \\
\hline $216 \mathrm{H020A36}$ & 10 \\
\hline $216 \mathrm{H019A36}$ & 10 \\
\hline $216 \mathrm{H} 018 \mathrm{A3} 6$ & 10 \\
\hline $220 \mathrm{HO} 015 \mathrm{~A} 36$ & 10 \\
\hline $210 \mathrm{H} 014 \mathrm{~A} 36$ & 10 \\
\hline $218 \mathrm{HO} 013 \mathrm{~A} 36$ & 10 \\
\hline $322 \mathrm{H188A36}$ & 10 \\
\hline $322 \mathrm{H189A36}$ & 10 \\
\hline $322 \mathrm{H} 190 \mathrm{~A} 36$ & 10 \\
\hline $216 \mathrm{HO} 021 \mathrm{A3} 6$ & 10 \\
\hline $219 \mathrm{HO} 10 \mathrm{~A} 36$ & 10 \\
\hline $220 \mathrm{HOO} 7 \mathrm{A3} 6$ & 10 \\
\hline $213 \mathrm{HO} 35 \mathrm{~A} 36$ & 10 \\
\hline $213 \mathrm{H} 036 \mathrm{A36}$ & 10 \\
\hline $213 H 037436$ & 20 \\
\hline $213 \mathrm{H} 032 \mathrm{A3} 6$ & 10 \\
\hline $213 \mathrm{H} 033 \mathrm{~A} 36$ & 10 \\
\hline $213 \mathrm{H} 034836$ & 10 \\
\hline $219 \mathrm{H} 011 \mathrm{A3} 6$ & 20 \\
\hline $219 \mathrm{HO} 12 \mathrm{~A} 36$ & 10 \\
\hline 2174017436 & 10 \\
\hline $2174016 A 36$ & 10 \\
\hline $221 \mathrm{H} 003 A 36$ & 10 \\
\hline $2214002 A 36$ & 10 \\
\hline $2219001 A 36$ & 10 \\
\hline $220 H 009 A 36$ & 10 \\
\hline $220 H 008 A 36$ & 10 \\
\hline $2204006 A 36$ & 10 \\
\hline $220 \mathrm{HOOSA36}$ & 10 \\
\hline $2204004 A 36$ & 10 \\
\hline $320 H 176236$ & 10 \\
\hline $320 \mathrm{H} 177 \mathrm{A3} 6$ & 10 \\
\hline $320 \mathrm{H} 178 \mathrm{A3} 6$ & 10 \\
\hline $215 H 027 A 36$ & 10 \\
\hline $215 \mathrm{HO26A36}$ & 10 \\
\hline $215 H 025 A 36$ & 10 \\
\hline $225 H 024236$ & 10 \\
\hline $215 H 023836$ & 10 \\
\hline $215 H_{022 A 36}$ & 10 \\
\hline 21111043037 & 6 \\
\hline $2111042 A 37$ & 6 \\
\hline $321 \mathrm{H} 174 \mathrm{A3} 7$ & 6 \\
\hline $321 \% 175 A 37$ & 6 \\
\hline $211 \% 044 A 37$ & 6 \\
\hline 323 H165A37 & 6 \\
\hline $323 \mathrm{H186A37}$ & 6 \\
\hline $323 \mathrm{H} 187 \mathrm{A3} 7$ & 6 \\
\hline $214 \mathrm{H} 030237$ & 6 \\
\hline $214 H 029 A 37$ & 6 \\
\hline $214 \mathrm{HO29N37}$ & 6 \\
\hline $214 \mathrm{H} 031 \mathrm{AZ7}$ & 6 \\
\hline $216 \mathrm{H} 020 \mathrm{~A} 37$ & 6 \\
\hline $216 \mathrm{HO} 19 \mathrm{A3} 7$ & 6 \\
\hline $216 \mathrm{H} 018 \mathrm{~A} 37$ & 6 \\
\hline 218H015A3? & 6 \\
\hline $218 \mathrm{HO14A37}$ & 6 \\
\hline $218 \mathrm{HO} 33 \mathrm{~A} 37$ & 6 \\
\hline $322 \mathrm{H} 188 \mathrm{~A} 37$ & 6 \\
\hline $322 \mathrm{H} 189037$ & 6 \\
\hline $322 \mathrm{H190837}$ & 6 \\
\hline $216 \mathrm{H} 021837$ & 6 \\
\hline
\end{tabular}

Figure 9.19 - Case 2, Revised and Decelerated Schedule (Continued). 


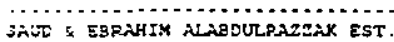

PEPCRT DATE 21SEPSS RUN NO, 205

Revd \& Decol Schi by Typleal Aetivity \& $2 \$$
PRIMAVERA PROJECT PLLANNER

SEVEN SEAS, CONSTRUCTION OF 51 HOUSES
Correcting Out-of-Loge Progress NSq

START DATE IAPRE? FIN DATE JOMAR89*

DATA DATE 1 FEB8 PAGE NO. 15

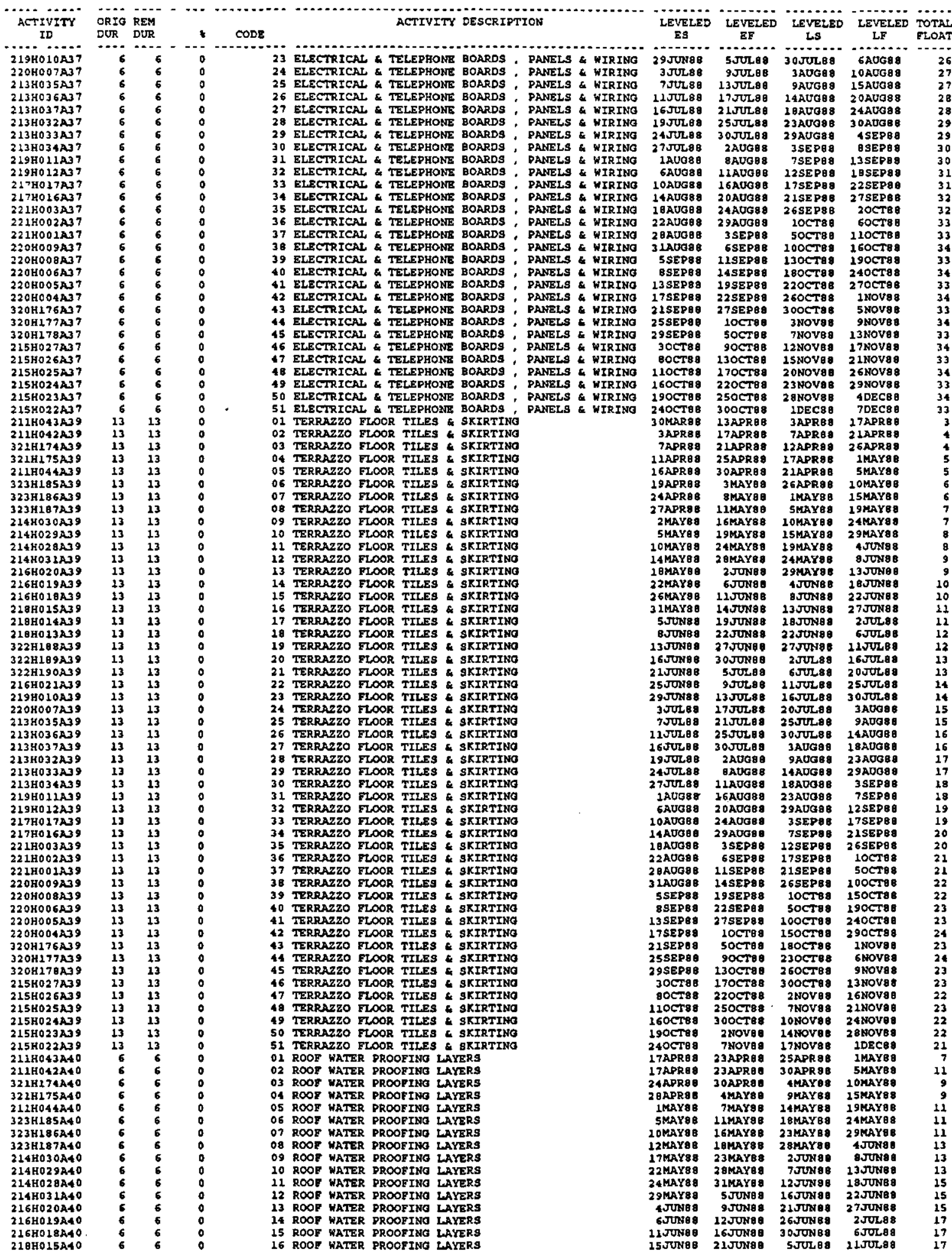

Figure 9.19 - Case 2, Revised and Decelerated Schedule (Continued). 


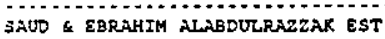

REPORT DATE $215 E P 95$ RUN NO. 205

Rovd \& Decel Schl by Typical Activity \& ES
PRIMAVERA PROJECT PLANNER

SEVEN SEAS, CONSTRUCTION OF 51 HOUSES

\begin{tabular}{|c|c|}
\hline $\begin{array}{c}\text { ACTIVITY } \\
\text { ID }\end{array}$ & $\begin{array}{l}\text { ORIO } \\
\text { DUR }\end{array}$ \\
\hline $218 \mathrm{H} 014240$ & \\
\hline $218 \mathrm{~K} 013 \mathrm{A4O}$ & 6 \\
\hline $322 \mathrm{~K} 28 \mathrm{BA4} 0$ & 6 \\
\hline $3224189 A 40$ & 6 \\
\hline $322 H 190 A 40$ & 6 \\
\hline $216 \mathrm{H} 021 \mathrm{~A} 40$ & 6 \\
\hline $219 \mathrm{HO} 10 \mathrm{A4O}$ & 6 \\
\hline $220 \mathrm{HOO} 7 \mathrm{~A} 4 \mathrm{O}$ & 6 \\
\hline $213 \mathrm{H} 035 \mathrm{A4O}$ & 6 \\
\hline $213 \mathrm{H} 036 \mathrm{~A} 4 \mathrm{O}$ & 6 \\
\hline $213 \mathrm{H} 037 \mathrm{AO}$ & 6 \\
\hline $213 H 032 A 40$ & 6 \\
\hline $213 \mathrm{H} 033 \mathrm{A40}$ & 6 \\
\hline $213 \mathrm{H} 034 \mathrm{~A} 4 \mathrm{O}$ & 6 \\
\hline $219 \mathrm{HO} 011 \mathrm{A4O}$ & 6 \\
\hline $219 \mathrm{HO} 012 \mathrm{A4O}$ & 6 \\
\hline $217 \mathrm{HO17A4O}$ & 6 \\
\hline $217 \mathrm{HO} 016 \mathrm{A4O}$ & 6 \\
\hline $221 \mathrm{H} 003 \mathrm{~A} 4 \mathrm{O}$ & 6 \\
\hline $221 \mathrm{HDO} 2 \mathrm{~A} 40$ & 6 \\
\hline $221 \mathrm{HOO} 2 \mathrm{A4O}$ & 6 \\
\hline 22 OH0O9A4O & 6 \\
\hline $22 \mathrm{OHOOBA4O}$ & 6 \\
\hline $220 \mathrm{HO} 06 \mathrm{A40}$ & 6 \\
\hline $22 \mathrm{OH} 005 \mathrm{A40}$ & 6 \\
\hline $220 \mathrm{H} 004 \mathrm{~A} 4 \mathrm{O}$ & 6 \\
\hline $320 \mathrm{H} 176 \mathrm{A4O}$ & 6 \\
\hline $320 \mathrm{H} 177440$ & 6 \\
\hline $320 \mathrm{H178A40}$ & 6 \\
\hline $215 \mathrm{H} 027 \mathrm{A4O}$ & 6 \\
\hline $215 \mathrm{HO} 6 \mathrm{6A40}$ & 6 \\
\hline $215 \mathrm{HO} 25 \mathrm{A4O}$ & 6 \\
\hline $215 \mathrm{HO} 024 \mathrm{AO}$ & 6 \\
\hline $215 \% 023 \mathrm{~A} 40$ & 6 \\
\hline $215 \mathrm{HO} 022 \mathrm{A4O}$ & 6 \\
\hline $221 \mathrm{HO43A41}$ & 4 \\
\hline $212 \mathrm{HO} 042 \mathrm{~A} 1$ & 4 \\
\hline $321 \mathrm{H1} 74 \mathrm{~A} 41$ & 4 \\
\hline $321 \mathrm{H} 175 \mathrm{~A} 4 \mathrm{I}$ & 4 \\
\hline 211H044A41 & 4 \\
\hline $323 \mathrm{H} 185 \mathrm{AA} 1$ & 4 \\
\hline $323 \mathrm{H} 106 \mathrm{A4} 1$ & 4 \\
\hline $323 \mathrm{H} 187 \mathrm{A4} 1$ & 4 \\
\hline $2144030 A 41$ & 4 \\
\hline 214H029A41 & 4 \\
\hline $214 \mathrm{HO}$ BA4 1 & 1 \\
\hline $214 \mathrm{HO} 1 \mathrm{AA1}$ & 4 \\
\hline $216 \mathrm{HO20A41}$ & 4 \\
\hline $216 \mathrm{HO} 019 \mathrm{A4} 1$ & 4 \\
\hline $216 \mathrm{HO18A4} 1$ & 4 \\
\hline $218 H 015 A 41$ & 4 \\
\hline $21 \mathrm{OH} 014 \mathrm{~A} 4 \mathrm{I}$ & 4 \\
\hline 21부이션 1 & 4 \\
\hline $322 \mathrm{HI} \theta 9 \mathrm{A4} \mathrm{I}$ & 4 \\
\hline $322 \mathrm{H} 1 \mathrm{\theta 9A4} 1$ & 4 \\
\hline $322 \mathrm{H} 190 \mathrm{A4} 1$ & 4 \\
\hline $216 \mathrm{HO2LA41}$ & 4 \\
\hline 219 HO1OA41 & 4 \\
\hline $220 \mathrm{H} 007 \mathrm{As} 1$ & 4 \\
\hline $213 \mathrm{H} 035 \mathrm{A43}$ & 4 \\
\hline $213 \mathrm{H} 036 \mathrm{A4} 1$ & 4 \\
\hline $213 \mathrm{H} 037 \mathrm{AAL}$ & 4 \\
\hline $213 \mathrm{H} 032 \mathrm{A41}$ & 4 \\
\hline $213 \mathrm{H} 033 \mathrm{A4} 1$ & 4 \\
\hline $213 H 034 A 41$ & 4 \\
\hline $219 \mathrm{HO} 12 \mathrm{AL}$ & 4 \\
\hline 219H022A41 & 4 \\
\hline $217 \mathrm{H} 027 \mathrm{A41}$ & 4 \\
\hline $217 \mathrm{H} 026 \mathrm{~A} 41$ & 4 \\
\hline 221H003A41 & 4 \\
\hline $221 \mathrm{H} 002 \mathrm{A41}$ & 4 \\
\hline $221 \mathrm{HOOLA41}$ & 4 \\
\hline 220H009A41 & 4 \\
\hline $220 \mathrm{H} 008 \mathrm{A4} 1$ & 4 \\
\hline $220 \mathrm{H} 006 \mathrm{A41}$ & \\
\hline $220 \mathrm{HOOSA41}$ & 4 \\
\hline $22 \mathrm{OH} 004 \mathrm{A4} 1$ & 4 \\
\hline $320 K 176 A 41$ & 1 \\
\hline $320 \mathrm{H} 177 \mathrm{A41}$ & 4 \\
\hline $320 \mathrm{H} 27 \mathrm{BA4} 1$ & 4 \\
\hline $215 \mathrm{HO} 027 \mathrm{AL}$ & \\
\hline $215 \mathrm{H} 026 \mathrm{A41}$ & 4 \\
\hline $225 \mathrm{H} 025 \mathrm{~A} 41$ & 4 \\
\hline $215 \mathrm{H} 024 \mathrm{A4} 1$ & 4 \\
\hline 215H023A41 & 4 \\
\hline $215 \mathrm{H} 022 \mathrm{A4} 1$ & 4 \\
\hline $211 \mathrm{H} 043 \mathrm{A42}$ & 6 \\
\hline $211 \mathrm{H} 042 \mathrm{~A} 42$ & 6 \\
\hline $321 \mathrm{HI74A2}$ & 6 \\
\hline $321 \mathrm{H175A42}$ & 6 \\
\hline 211 H044A42 & 6 \\
\hline $323 \mathrm{H} 185 \mathrm{~A} 42$ & 6 \\
\hline $323 \mathrm{H} 186242$ & 6 \\
\hline $323 \mathrm{H} 187 \mathrm{~A} 2$ & 6 \\
\hline $214 H 030 A 42$ & 6 \\
\hline $214 \mathrm{H} 029 \mathrm{~A} 42$ & 6 \\
\hline
\end{tabular}

Figure 9.19 - Case 2, Revised and Decelerated Schedule (Continued). 


\begin{tabular}{|c|c|}
\hline $\begin{array}{l}\text { ACTIVITY } \\
\text { ID }\end{array}$ & $\begin{array}{l}\text { ORIG } \\
\text { DUR }\end{array}$ \\
\hline 214 HO29A42 & \\
\hline $2144031 \mathrm{A42}$ & 6 \\
\hline $216 \mathrm{HO2OA42}$ & 6 \\
\hline $216 H 019 A 42$ & 6 \\
\hline $216 \mathrm{HOL18A42}$ & 6 \\
\hline $218 H 015 A 42$ & 6 \\
\hline $218 H 014 A 42$ & 6 \\
\hline $218 \mathrm{HO} 13 \mathrm{A42}$ & 6 \\
\hline $322 \mathrm{H198A42}$ & 6 \\
\hline $322 \mathrm{H} 199 A 42$ & 6 \\
\hline $3224190 \mathrm{A42}$ & 6 \\
\hline $216 \mathrm{HO21A42}$ & 6 \\
\hline $219 \mathrm{HO} 10 \mathrm{A42}$ & 6 \\
\hline $220 \mathrm{H} 007 \mathrm{A42}$ & 6 \\
\hline $213 \mathrm{H} 035 \mathrm{~A} 42$ & 6 \\
\hline $213 \mathrm{H} 036 \mathrm{A42}$ & 6 \\
\hline $213 \mathrm{H} 037 \mathrm{~A} 42$ & 6 \\
\hline $213 \mathrm{H} 032 \mathrm{~A} 42$ & 6 \\
\hline 213H033A42 & 6 \\
\hline $213 \mathrm{HO34A42}$ & 6 \\
\hline $219 \mathrm{HO} 11 \mathrm{A42}$ & 6 \\
\hline $219 \mathrm{HO12A42}$ & 6 \\
\hline $217 \mathrm{HO17A42}$ & 6 \\
\hline $2174016 \mathrm{A4} 2$ & 6 \\
\hline $221 \mathrm{H} 003 \mathrm{A42}$ & 6 \\
\hline $221 \% 002 A 42$ & 6 \\
\hline $2214001 A 42$ & 6 \\
\hline $220 \mathrm{HOO9A42}$ & 6 \\
\hline $2204008 A 42$ & 6 \\
\hline $220 \mathrm{HOOGA42}$ & 6 \\
\hline $220 H 005 A 42$ & 6 \\
\hline $220 \mathrm{HOO4A42}$ & 6 \\
\hline $320 \mathrm{H} 176 \mathrm{A42}$ & 6 \\
\hline $320 \mathrm{H} 177 \mathrm{At2}$ & 6 \\
\hline $320 \mathrm{H} 178 \mathrm{A42}$ & 6 \\
\hline $215 \mathrm{HO} 27 \mathrm{A42}$ & 6 \\
\hline $215 \mathrm{HO26A42}$ & 6 \\
\hline $215 \mathrm{HO} 25 \mathrm{A42}$ & 6 \\
\hline $225 \mathrm{HO} 24 A 42$ & 6 \\
\hline $215 \mathrm{HO} 33 \mathrm{A42}$ & 6 \\
\hline $215 H 022 A 42$ & 6 \\
\hline $2111043 A 43$ & 4 \\
\hline $211 \mathrm{HO}^{2} \mathrm{A4} 3$ & 4 \\
\hline $321 \mathrm{H174A43}$ & 4 \\
\hline $3214175 A 43$ & 4 \\
\hline $211 \mathrm{HO44243}$ & 4 \\
\hline 3233185443 & $i$ \\
\hline $323 \mathrm{H186A43}$ & 4 \\
\hline $323 H 197 A 43$ & 4 \\
\hline $2144030 A 43$ & 4 \\
\hline $214 \mathrm{HO} 29 \mathrm{A43}$ & 4 \\
\hline $214 \mathrm{HO} 2 \mathrm{AA} 43$ & 4 \\
\hline $214 \mathrm{HO} 31 \mathrm{At3}$ & 4 \\
\hline $216 \mathrm{HO2OA43}$ & 4 \\
\hline $216 \mathrm{H} 019 \mathrm{A4} 3$ & 4 \\
\hline $216 \mathrm{HO1BA} 63$ & 4 \\
\hline $210 \mathrm{HO15A43}$ & 4 \\
\hline $210 \mathrm{HO14A43}$ & 4 \\
\hline $218 \mathrm{HO} 13 \mathrm{A4} 3$ & 4 \\
\hline $322 \mathrm{H} 1 \mathrm{BgA4} 3$ & 4 \\
\hline $322 \mathrm{HLOQA43}$ & 4 \\
\hline $322 \mathrm{H} 190 \mathrm{A4} 3$ & 4 \\
\hline $216 \mathrm{HO2} 1 \mathrm{A43}$ & 4 \\
\hline $219 \mathrm{HO} 10 \mathrm{A43}$ & 4 \\
\hline $220 \mathrm{HOO} 0743$ & 4 \\
\hline $213 \mathrm{H} 035 \mathrm{A4} 3$ & 4 \\
\hline $213 \mathrm{HO36A43}$ & \\
\hline $213 H 037 A 43$ & 4 \\
\hline $213 H_{032 A 43}$ & 4 \\
\hline $213 H_{033 \mathrm{~A} 43}$ & 4 \\
\hline $213 H 034243$ & \\
\hline $219 H 011243$ & $i$ \\
\hline $2194012 A 43$ & 4 \\
\hline $217 \mathrm{HO17A43}$ & 4 \\
\hline $2174016 A 43$ & 4 \\
\hline $2219003 \mathrm{A43}$ & 4 \\
\hline $22114002 A 43$ & 4 \\
\hline $221 \mathrm{H} 001 \mathrm{A4} 3$ & 4 \\
\hline $2204009 A 43$ & 4 \\
\hline $2204000 A 43$ & 4 \\
\hline $220 \mathrm{H} 00 \mathrm{GA43}$ & 4 \\
\hline $220 \mathrm{HOOSA43}$ & 1 \\
\hline $2204004 A 43$ & 4 \\
\hline $320 \mathrm{H} 176 \mathrm{A43}$ & 4 \\
\hline 3204277443 & 1 \\
\hline $320 \mathrm{~K}^{278 \mathrm{~A} 43}$ & 4 \\
\hline $215 \mathrm{H} 027 \mathrm{A43}$ & 4 \\
\hline $215 H 026 A 43$ & 4 \\
\hline $215 \mathrm{HO25A43}$ & 4 \\
\hline $215 H 024 A 43$ & 4 \\
\hline $225 H 023 A 43$ & 4 \\
\hline $215 H 022 A 43$ & 4 \\
\hline $2118043 A 44$ & 6 \\
\hline $2111042 A 44$ & 6 \\
\hline $321 H 174 A 44$ & 6 \\
\hline $321 \mathrm{HL75A44}$ & 6 \\
\hline
\end{tabular}

Figure 9.19 - Case 2, Revised and Decelerated Schedule (Continued). 
3AUD \& EBPAHTM ALRBDURRAZZAK EST

REPORT DATE IISEPYS RUN NO. 205

Revd \& Decel Schl by Trpical Activity \& Es
PRIMAVERA PROJECT PLAANER

SEVEN SEAS, CONSTRUCTION OF 51 HOUSES
Corroct 2 ng Out-of-togie Progreas nSq START DATE 1APR.7 FIN DATE 3OMARB9* DATA DATE LFEBga PAGE No. 1a

\begin{tabular}{|c|c|}
\hline $\begin{array}{l}\text { ACTIVITY } \\
\text { ID }\end{array}$ & $\begin{array}{l}\text { ORIG } \\
\text { DUR }\end{array}$ \\
\hline $212 \mathrm{HO} 044 \mathrm{A44}$ & 6 \\
\hline 323 H1O5A44 & 6 \\
\hline $323 \mathrm{H} 186 \mathrm{~A} 44$ & 6 \\
\hline $323 \mathrm{H1O} 7 \mathrm{A44}$ & 6 \\
\hline 214 H030A44 & 6 \\
\hline $214 \mathrm{HO} 29 A 44$ & 6 \\
\hline $214 \mathrm{HO} 28 \mathrm{~A} 44$ & 6 \\
\hline $214 \mathrm{HO} 11 \mathrm{A44}$ & 6 \\
\hline $216 \mathrm{HO20A44}$ & 6 \\
\hline 216H019A44 & 6 \\
\hline $216 \mathrm{HO} 18 \mathrm{~A} 44$ & 6 \\
\hline $2184015 A 44$ & 6 \\
\hline $2194014 A 44$ & 6 \\
\hline $218 \mathrm{HO} 13 \mathrm{A44}$ & 6 \\
\hline $3224188 \mathrm{~A} 44$ & 6 \\
\hline $322 \mathrm{H} 199 \mathrm{A44}$ & 6 \\
\hline $322 \mathrm{H} 190 \mathrm{~A} 44$ & 6 \\
\hline $216 \% 021 \mathrm{A44}$ & 6 \\
\hline $219 \mathrm{HOLOA44}$ & 6 \\
\hline $2204007 A 44$ & 6 \\
\hline $213 \mathrm{H} 035 \mathrm{~A} 44$ & 6 \\
\hline $213 \mathrm{H} 036 \mathrm{A44}$ & 6 \\
\hline $213 \mathrm{H} 037 \mathrm{A44}$ & 6 \\
\hline $213 \mathrm{H} 032 \mathrm{~A} 44$ & 6 \\
\hline $213 \mathrm{H} 033 \mathrm{~A} 44$ & 6 \\
\hline $2134034 \mathrm{A44}$ & 6 \\
\hline $219 \mathrm{HO} 31 \mathrm{~A} 44$ & 6 \\
\hline 219H012A44 & 6 \\
\hline $227 \mathrm{HOL7A44}$ & 6 \\
\hline $217 \mathrm{HO} 26 \mathrm{A44}$ & 6 \\
\hline $221 \mathrm{H} 003 \mathrm{A44}$ & 6 \\
\hline $2214002 A 44$ & 6 \\
\hline $221 \mathrm{H} 001 \mathrm{~A} 44$ & 6 \\
\hline $220 \mathrm{H} 009 \mathrm{A4} 4$ & 6 \\
\hline $220 \mathrm{H} 008 \mathrm{A44}$ & 6 \\
\hline $220 \mathrm{H} 006 \mathrm{A44}$ & 6 \\
\hline $220 \mathrm{H} 005 \mathrm{~A} 44$ & 6 \\
\hline $220 \mathrm{H004A44}$ & 6 \\
\hline $320 \mathrm{H} 176 \mathrm{A44}$ & 6 \\
\hline $320 \mathrm{H} 177 \mathrm{A4} 4$ & 6 \\
\hline $320 \mathrm{H} 178 \mathrm{~A} 44$ & 6 \\
\hline $215 \mathrm{H} 027 \mathrm{A4} 4$ & 6 \\
\hline $215 \mathrm{HO} 26 \mathrm{A4} 4$ & 6 \\
\hline $215 \mathrm{H} 025 \mathrm{A44}$ & 6 \\
\hline $215 H 024 A 44$ & 6 \\
\hline $215 \mathrm{HO} 23 \mathrm{A44}$ & 6 \\
\hline $215 \mathrm{H} 022 \mathrm{A44}$ & 6 \\
\hline $211 \mathrm{H} 043 \mathrm{A45}$ & 2 \\
\hline $211 H 0+2 A 45$ & 2 \\
\hline $321 \mathrm{H} 174 \mathrm{A45}$ & 2 \\
\hline $321 H 175 A 45$ & 2 \\
\hline $211 \mathrm{H} 044 \mathrm{A45}$ & 2 \\
\hline $323 \mathrm{H} 185 \mathrm{AAS}$ & 2 \\
\hline $323 \mathrm{H} 196 \mathrm{A45}$ & 2 \\
\hline 323 H187A45 & 2 \\
\hline $214 H 030 A 45$ & 2 \\
\hline $214 H 029 A 45$ & 2 \\
\hline $214 \mathrm{H} 028 \mathrm{~A} 45$ & 2 \\
\hline $214 \mathrm{H} 031 \mathrm{A45}$ & 2 \\
\hline $216 \mathrm{HO2OA45}$ & 2 \\
\hline $216 \mathrm{HO19A45}$ & 2 \\
\hline $216 \mathrm{H} 018 \mathrm{~A} 45$ & 2 \\
\hline $218 \mathrm{H} 015 \mathrm{A45}$ & 2 \\
\hline $21 \mathrm{BHO14A45}$ & 2 \\
\hline $210 \mathrm{H} 013 \mathrm{A45}$ & 2 \\
\hline $322 \mathrm{H} 188 \mathrm{~A} 45$ & 2 \\
\hline $322 \mathrm{H} 189 \mathrm{~A} 45$ & 2 \\
\hline $322 \mathrm{H} 190 \mathrm{A4} 5$ & 2 \\
\hline $216 \mathrm{HO} 21 \mathrm{~A} 45$ & 2 \\
\hline $219 H 010 A 45$ & 2 \\
\hline $220 \mathrm{HOO} \mathrm{A} 45$ & 2 \\
\hline $2134035 A 45$ & 2 \\
\hline $213 \mathrm{~B} 036 \mathrm{A45}$ & 2 \\
\hline $213 \mathrm{H} 037 \mathrm{A4S}$ & 2 \\
\hline $213 \mathrm{HO} 2 \mathrm{A4S}$ & 2 \\
\hline $223 H 033 A 45$ & 2 \\
\hline $213 \mathrm{HO34A45}$ & 2 \\
\hline 219H011A45 & 2 \\
\hline $219 \mathrm{HO} 12 \mathrm{~A} 4 \mathrm{~S}$ & 2 \\
\hline $2174017 A 45$ & 2 \\
\hline $217 \mathrm{HO} 16 \mathrm{A4} 5$ & 2 \\
\hline $221 \mathrm{H} 003 \mathrm{A4S}$ & 2 \\
\hline $221 H 002 \mathrm{A45}$ & 2 \\
\hline $221 \mathrm{HOOO1A45}$ & 2 \\
\hline $220 \mathrm{HOOOA45}$ & 2 \\
\hline 22 OHOOEA45 & 2 \\
\hline $220 \mathrm{H} 006 \mathrm{A4} 5$ & 2 \\
\hline $220 H 005 A 45$ & 2 \\
\hline $220 \mathrm{H} 004 \mathrm{A45}$ & 2 \\
\hline $320 \mathrm{H} 176 \mathrm{A45}$ & 2 \\
\hline $320 \mathrm{H} 277 \mathrm{A4} 5$ & 2 \\
\hline $320 \mathrm{H} 178 \mathrm{A4} 5$ & 2 \\
\hline $215 \% 027 \mathrm{A45}$ & 2 \\
\hline $215 K 026 A 45$ & 2 \\
\hline 215 HO25A45 & 2 \\
\hline 215 HO24A45 & 2 \\
\hline
\end{tabular}

Figure 9.19 - Case 2, Revised and Decelerated Schedule (Continued). 


\begin{tabular}{|c|c|}
\hline $\begin{array}{l}\text { ACTIVITI } \\
\text { ID }\end{array}$ & $\begin{array}{l}\text { ORIG } \\
\text { DUR }\end{array}$ \\
\hline 215 H023A45 & 2 \\
\hline $215 \mathrm{HO} 22 \mathrm{~A} 45$ & 2 \\
\hline $2111043 \mathrm{A4} 6$ & 7 \\
\hline $211 H 042 A 46$ & 7 \\
\hline $321 \mathrm{H} 174 \mathrm{~A} 46$ & 7 \\
\hline $321 \mathrm{H} 175 \mathrm{~A} 46$ & 7 \\
\hline $2114044 A 45$ & 7 \\
\hline $323 H 285 A 46$ & 7 \\
\hline $323 \mathrm{H} 186 \mathrm{~A} 46$ & 7 \\
\hline $323 \mathrm{H} 187 \mathrm{A4} 6$ & 7 \\
\hline $214 H 030 A 46$ & 7 \\
\hline $214 \mathrm{HO29A46}$ & 7 \\
\hline $214 H 02$ BA4 6 & 7 \\
\hline $214 H 031 A 46$ & 7 \\
\hline $2164020 A 46$ & 7 \\
\hline $216 H 039 A 46$ & 7 \\
\hline $216 \mathrm{H} 019 \mathrm{~A} 46$ & 7 \\
\hline $218 H 015 A 46$ & 7 \\
\hline $218 \mathrm{H014A46}$ & 7 \\
\hline $218 \mathrm{H} 013 \mathrm{~A} 46$ & 7 \\
\hline 3224188846 & $?$ \\
\hline 3224189946 & 7 \\
\hline 322 H190A4 6 & 7 \\
\hline $216 \mathrm{H021A46}$ & 7 \\
\hline $219 H 010 A 46$ & 7 \\
\hline $220 \mathrm{H} 007 \mathrm{A4} 6$ & 7 \\
\hline $213 \mathrm{HO} 35 \mathrm{~A} 46$ & 7 \\
\hline $213 H_{036 A 46}$ & 7 \\
\hline $213 H 037 A 46$ & 7 \\
\hline $213 \mathrm{H} 032 \mathrm{A4} 6$ & 7 \\
\hline $213 H 033 A 46$ & 7 \\
\hline $213 H 034446$ & 7 \\
\hline 219 HO11A46 & 7 \\
\hline $2194012 A 46$ & 7 \\
\hline $217 \mathrm{H} 017 \mathrm{A4} 6$ & 7 \\
\hline $217 \mathrm{HO16A46}$ & 7 \\
\hline $221 H 003 A 46$ & 7 \\
\hline $221 H 002 A 46$ & 7 \\
\hline $2214001 A 46$ & 7 \\
\hline 220 H009A4 6 & ? \\
\hline $22 \mathrm{OHOOOA46}$ & 7 \\
\hline $220 \mathrm{OH} 006 \mathrm{A4} 6$ & 7 \\
\hline $220 \mathrm{HOOSA46}$ & 7 \\
\hline 220 HOO4A46 & 7 \\
\hline $320 \mathrm{H176A46}$ & 7 \\
\hline $320 \mathrm{H} 177 \mathrm{A4} 6$ & 7 \\
\hline $320 \mathrm{H} 178 \mathrm{~A} 46$ & 7 \\
\hline $235 H 027 A 46$ & 7 \\
\hline $215 H 026246$ & 7 \\
\hline 215 P025A46 & 7 \\
\hline $215 H 024 A 46$ & 7 \\
\hline 215 H023A46 & 7 \\
\hline $2154022 A 46$ & 7 \\
\hline $212 H 043 A 47$ & 3 \\
\hline $211 H 042 A 47$ & 3 \\
\hline $321 \mathrm{H} 174 A 47$ & 3 \\
\hline $321 \mathrm{H175A4} 7$ & 3 \\
\hline $211 H 044 A 47$ & 3 \\
\hline $323 H 185 A 47$ & 3 \\
\hline $323 \mathrm{H186A47}$ & 3 \\
\hline $323 H 187 A 47$ & 3 \\
\hline $214 \mathrm{HO} 30 \mathrm{OA} 7$ & 3 \\
\hline $214 \mathrm{HO29A47}$ & 3 \\
\hline $214 H 029 A 47$ & 3 \\
\hline $214 \mathrm{HO} 3$ LA47 & 3 \\
\hline $2164020 A 47$ & 3 \\
\hline $216 \mathrm{HO29A4} 7$ & 3 \\
\hline $216 \mathrm{HO18A47}$ & 3 \\
\hline $218 H 025 A 47$ & 3 \\
\hline $218 \mathrm{H} 014 \mathrm{A4} 7$ & 3 \\
\hline $218 B 013 A 47$ & 3 \\
\hline $322 \mathrm{H} 188 \mathrm{~A} 47$ & 3 \\
\hline $322 \mathrm{H} 189247$ & 3 \\
\hline $322 \mathrm{H} 190 \mathrm{~A} 47$ & 3 \\
\hline $216 H 021 A 47$ & 3 \\
\hline $219 H 010 A 47$ & 3 \\
\hline $220 \mathrm{H} 007 \mathrm{A4} 7$ & 3 \\
\hline $213 H 035 A 47$ & 3 \\
\hline $213 \mathrm{H} 036 \mathrm{A4} 7$ & 3 \\
\hline $213 \mathrm{H} 037 \mathrm{A4} 7$ & 3 \\
\hline $2134032 A 47$ & 3 \\
\hline $213 \mathrm{H} 033 \mathrm{A4} 7$ & 3 \\
\hline $213+034 A 47$ & 3 \\
\hline $219 \mathrm{H011A47}$ & 3 \\
\hline $2194012 A 47$ & 3 \\
\hline 2174017447 & 3 \\
\hline 217 H016A47 & 3 \\
\hline $221 H 003 \mathrm{A4} 7$ & 3 \\
\hline $221 H 002 A 47$ & 3 \\
\hline $221 H 001 A 47$ & 3 \\
\hline $220 H 009 A 47$ & 3 \\
\hline $220 \mathrm{HOOBA4}$ ? & 3 \\
\hline $220 \mathrm{H006A47}$ & 3 \\
\hline $220 \mathrm{H} 005 \mathrm{AA} 7$ & 3 \\
\hline $220 \mathrm{H} 004 \mathrm{~A} 47$ & 3 \\
\hline $320 \mathrm{H} 176 \mathrm{A4} 7$ & 3 \\
\hline
\end{tabular}

Figure 9.19 - Case 2, Revised and Decelerated Schedule (Continued). 


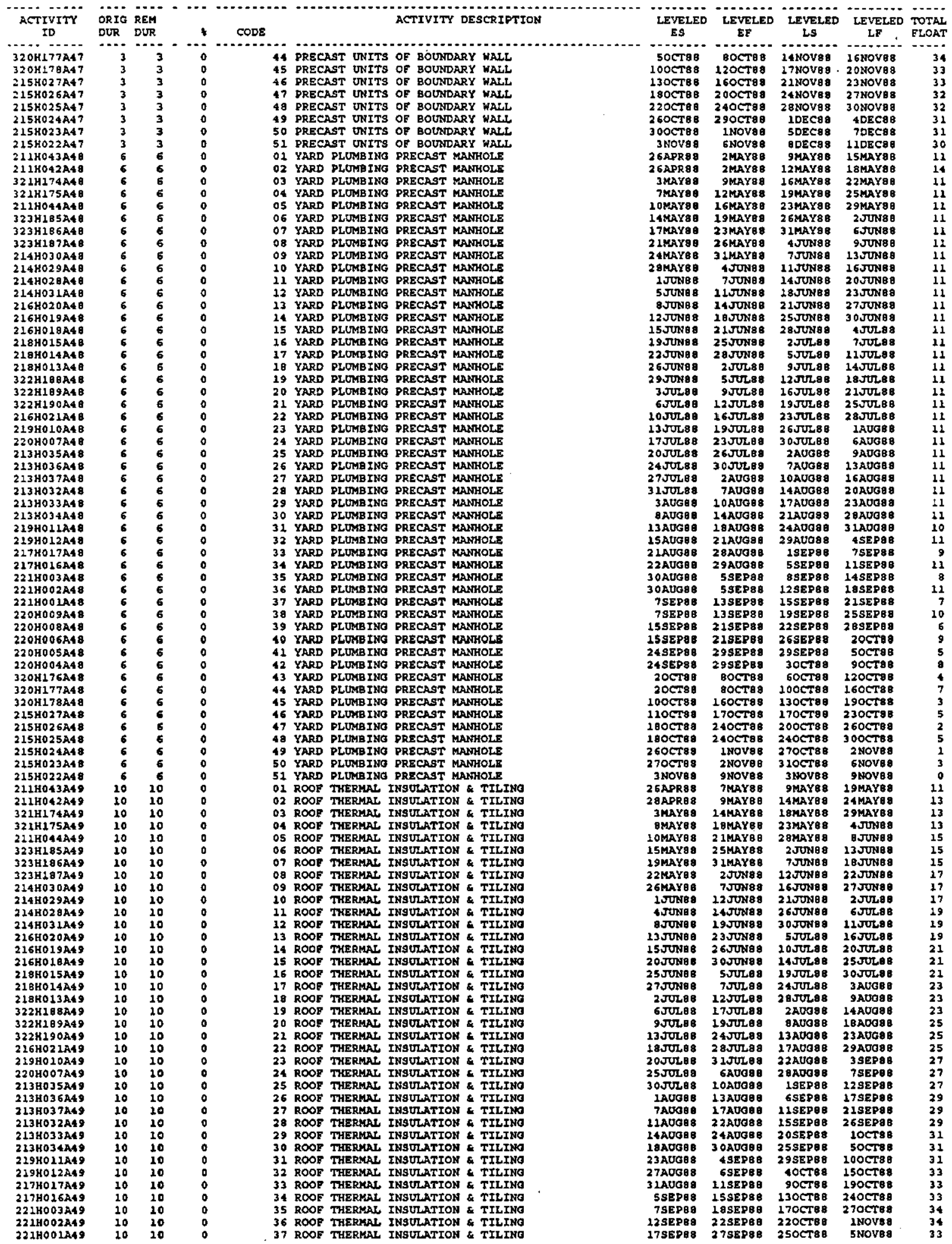

Figure 9.19 - Case 2, Revised and Decelerated Schedule (Continued). 
SAUO \& EBRAHIM ALABDULRAZZAX EST

REPORT DATE 21SEPYS RUN NO. 205 Revont Date $213: 31$
PRIMAVERA PROUECT PLANNER

SEVEN SEAS, CONSTRUCTION OF 51 HOUSES
Correcting Out-of-Logze progress NSq

START DATE 1APR日7 FIN DATE 3OMAR89*

\begin{tabular}{|c|c|}
\hline $\begin{array}{c}\text { ACTIVITY } \\
\text { ID }\end{array}$ & $\begin{array}{l}\text { ORIG } \\
\text { DUR } \\
-.\end{array}$ \\
\hline $220 \mathrm{H} 009 \times 49$ & 10 \\
\hline $2204000 A 49$ & 10 \\
\hline $2204006 \mathrm{A49}$ & 10 \\
\hline $220 \mathrm{HOO5A49}$ & 10 \\
\hline $220 \mathrm{H} 004 \mathrm{A4} 9$ & 10 \\
\hline $320 \mathrm{H} 176 \mathrm{~A} 9$ & 10 \\
\hline $320 \% 177 \mathrm{A49}$ & 10 \\
\hline $320 H 178 A 49$ & 10 \\
\hline 215 HO27A49 & 10 \\
\hline $215 \mathrm{H} 026 \mathrm{A4} 9$ & 10 \\
\hline $215 \mathrm{HO25A49}$ & 10 \\
\hline 2154024449 & 10 \\
\hline $215 \mathrm{KO23A49}$ & 10 \\
\hline $215 H 022 A 49$ & 10 \\
\hline $2114043 A 50$ & 5 \\
\hline $211 H 042 \times 50$ & 5 \\
\hline $321 \mathrm{H174A50}$ & 5 \\
\hline $321 \mathrm{HL} 75850$ & 5 \\
\hline $211 H 044 A 50$ & 5 \\
\hline 323 H1BSASO & 5 \\
\hline $323 \mathrm{~K} 186 \mathrm{~A} 50$ & 5 \\
\hline $323 \mathrm{H1O7A50}$ & 5 \\
\hline $214 \mathrm{HO} O 30 \mathrm{ASO}$ & 5 \\
\hline $214 K 029 A 50$ & 5 \\
\hline $214 H 028 A 50$ & 5 \\
\hline $214 \mathrm{~K} 032 \mathrm{ASO}$ & $\mathbf{5}$ \\
\hline $216 \mathrm{HH} 020 \mathrm{A5O}$ & 5 \\
\hline 216HO19ASO & $\mathbf{5}$ \\
\hline $216 H O 18 A 50$ & 5 \\
\hline $219 B 015 A 50$ & 5 \\
\hline $210 \mathrm{HO} 14 \mathrm{ASO}$ & 5 \\
\hline $218 \mathrm{HO} 13 \mathrm{ASO}$ & 5 \\
\hline $322 \mathrm{H} 18 \mathrm{ASSO}$ & 5 \\
\hline $322 \mathrm{HLQ9ASO}$ & 5 \\
\hline $322 \mathrm{H} 190 \mathrm{~A} 5 \mathrm{O}$ & 5 \\
\hline $216 \mathrm{HO21ASO}$ & 5 \\
\hline $219 \mathrm{HO} 10 \mathrm{ASO}$ & 5 \\
\hline $220 \mathrm{H} 007 \mathrm{ASO}$ & 5 \\
\hline 213 HOSSASO & 5 \\
\hline $213 \mathrm{H} 036 \mathrm{ASO} 0$ & 5 \\
\hline 213 H037A5O & $s$ \\
\hline 213H032A5O & 5 \\
\hline $213 \mathrm{HO} 033 \mathrm{ASO}$ & 5 \\
\hline 213 H034A50 & 5 \\
\hline 219 HOI1A5O & 5 \\
\hline $219 H 012 A 50$ & 5 \\
\hline $217 \mathrm{HO} 17 \mathrm{ASO}$ & 5 \\
\hline 2174026550 & 5 \\
\hline $2224003 A 50$ & 5 \\
\hline $221 H 002 A S O$ & 5 \\
\hline $221 \mathrm{HOOLASO}$ & s \\
\hline $220 \mathrm{H} 009 \mathrm{ASO}$ & s \\
\hline $220 \mathrm{HOOOASO}$ & 5 \\
\hline $220 H 006$ ASO & 5 \\
\hline 220 HOOSASO & 5 \\
\hline $220 \mathrm{HOOAASO}$ & 5 \\
\hline $3204176 A 50$ & 5 \\
\hline 32041777250 & 5 \\
\hline 3204278250 & 5 \\
\hline $215 H 027 A 50$ & 5 \\
\hline $215 H 026 A 50$ & 5 \\
\hline 215H025A5O & 5 \\
\hline 21514024850 & $\mathbf{5}$ \\
\hline 2154023250 & 5 \\
\hline $215 \% 022 A 50$ & 5 \\
\hline 21114043851 & 6 \\
\hline $211 H 042251$ & 6 \\
\hline $321 \% 174 A 51$ & 6 \\
\hline $321 \mathrm{H175A51}$ & 6 \\
\hline $211 \mathrm{HO} 44 \mathrm{A5} 1$ & 6 \\
\hline $323 H 1155 A 51$ & 6 \\
\hline $323 \mathrm{H} 186 \mathrm{AS} 1$ & 6 \\
\hline $323 \mathrm{H} 187 \mathrm{~A} 5 \mathrm{I}$ & 6 \\
\hline $214 H 030 A 51$ & 6 \\
\hline $214 \mathrm{H} 029 \mathrm{~A} 5 \mathrm{I}$ & 6 \\
\hline $214 \mathrm{HO2BA51}$ & 6 \\
\hline 214H03 LAS1 & 6 \\
\hline $216 \mathrm{HO} 020 \mathrm{AS} 1$ & 6 \\
\hline $216 \mathrm{H} 029 \mathrm{AS1}$ & 6 \\
\hline $216 \mathrm{HOL28A51}$ & 6 \\
\hline $2184025 A 51$ & 6 \\
\hline 2184014A51 & 6 \\
\hline 218H013ASI & 6 \\
\hline $3224188 A 51$ & 6 \\
\hline $322 \mathrm{H} 189 \mathrm{ASI}$ & 6 \\
\hline $322 H 190051$ & 6 \\
\hline $216 \mathrm{H} 021 \mathrm{AS} 1$ & 6 \\
\hline $219 \mathrm{HO} 010 \mathrm{A5} 1$ & 6 \\
\hline $220 \mathrm{HOO} 7 \mathrm{AS1}$ & 6 \\
\hline 213 H035AS1 & 6 \\
\hline 213H036A51 & 6 \\
\hline $213 \mathrm{HO} 037 \mathrm{AS} 1$ & 6 \\
\hline $213 \mathrm{H} 032 \mathrm{ASI}$ & 6 \\
\hline 213H033A5I & 6 \\
\hline $213 \mathrm{H} 034 \mathrm{AS} 1$ & 6 \\
\hline 219HO1LAS1 & 6 \\
\hline
\end{tabular}

Figure 9.19 - Case 2, Revised and Decelerated Schedule (Continued). 
GAUD \& EQRAHIM ALABDURRAZZAK EST.

REPORT DATE IISEPYS RUN NO. 205

Rovd \& Decol Schl by Typzeal Aetivity \& ES
PRIMAVERA PROJECT PLANNER

SEVEN SEAS, CONSTRUCTION OF 51 HOUSES
Correct 2 g Out-of-Logle Progreen NS

START DATE IAPRA? FIN DATE 3OMARg9"

DATA DATE LFEgga PAGE NO. 22

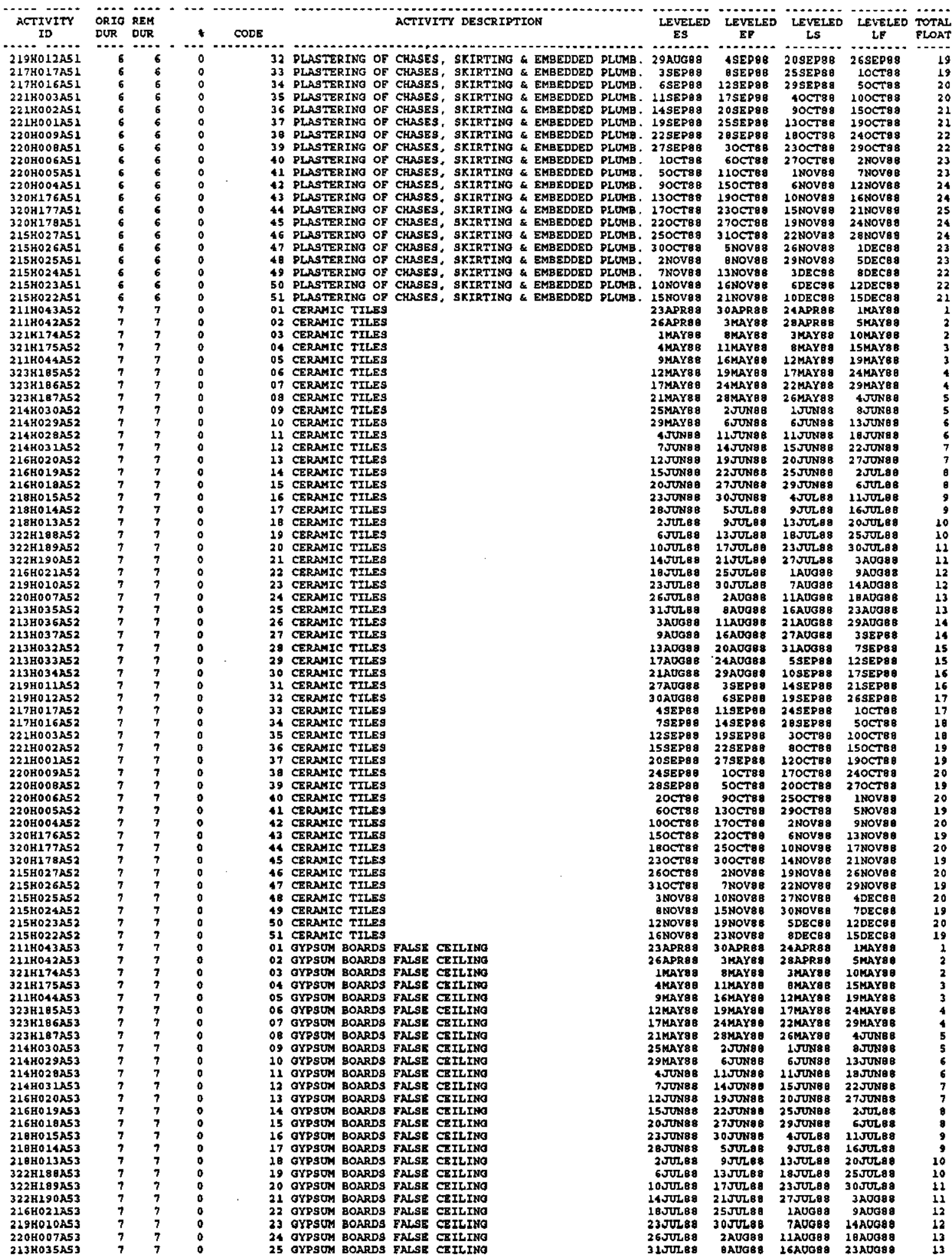

Figure 9.19 - Case 2, Revised and Decelerated Schedule (Continued). 
ZAUT I EBRAHIM ALABDULRAZZAK EST

PEPORT OATE 2LSEP9S RUN NO. 205 P.ord \& Docel $19: 31$ by Typical Activity \& ES

PRIMAVERA PROJECT PLANIER

Correcting out-of-Logie Progrese NSq
SEVEN SEAS, CONSTRUCTION OF 51 hOUSES

START DATE 1ARR87 FIN DATE 30MARB9-

DATA DATE IFEBSB PAGE NO. 23

\begin{tabular}{|c|c|}
\hline $\begin{array}{c}\text { ACTIVITY } \\
\text { ID }\end{array}$ & $\begin{array}{l}\text { ORIO } \\
\text { DUR }\end{array}$ \\
\hline 213 HO36AS3 & \\
\hline 213 HO37ASJ & 7 \\
\hline 213 H032A53 & 7 \\
\hline $2134033 \mathrm{~A} 53$ & $?$ \\
\hline $213 \mathrm{HO34A53}$ & 7 \\
\hline 219 HO11A53 & $?$ \\
\hline $219 K 012 \times 53$ & 7 \\
\hline 217 HO17A53 & 7 \\
\hline $217 \mathrm{HO16A53}$ & 7 \\
\hline 221H003A53 & 7 \\
\hline $221 \mathrm{H} 002 \mathrm{~A} 53$ & 7 \\
\hline $221 H 001 A 53$ & 7 \\
\hline $220 \mathrm{HOOOA53}$ & 7 \\
\hline $220 \mathrm{HOOBA5} 3$ & 7 \\
\hline $220 \mathrm{H} 006 \mathrm{A5} 3$ & 7 \\
\hline $220 H 005 A 53$ & 7 \\
\hline $220 \mathrm{HOO4A53}$ & 7 \\
\hline $320 \mathrm{H176A53}$ & 7 \\
\hline $320 \mathrm{H} 177 \mathrm{AS} 3$ & $?$ \\
\hline $320 \% 170 A 53$ & 7 \\
\hline $215 \% 027 A 53$ & 7 \\
\hline $215 H 026 A 53$ & 7 \\
\hline 215HO2SA53 & $?$ \\
\hline $215 H 024 A 53$ & 7 \\
\hline 215H023A53 & 7 \\
\hline $215 \% 022 A 53$ & 7 \\
\hline 211 H043A54 & 10 \\
\hline $2114042 A 54$ & 10 \\
\hline 321H174AS4 & 10 \\
\hline $3214175 A 54$ & 10 \\
\hline 211H044AS4 & 10 \\
\hline 323 H185A54 & 10 \\
\hline $323 H 186254$ & 10 \\
\hline $323 H 187254$ & 10 \\
\hline 2144030A54 & 10 \\
\hline 214HO29AS4 & 10 \\
\hline 214HO2BAS4 & 20 \\
\hline 214HO31A54 & 10 \\
\hline $216 \mathrm{HO20A54}$ & 10 \\
\hline 216H019A54 & 10 \\
\hline $216 \mathrm{HO} 19 \mathrm{~A} 54$ & 10 \\
\hline $218 H 015 A 54$ & 10 \\
\hline 2LAHO14AS4 & 10 \\
\hline 2184013454 & 20 \\
\hline 322H2BAA54 & 20 \\
\hline $322 \mathrm{H} 1$ B9AS4 & 10 \\
\hline $322 \mathrm{H} 290 \mathrm{AS} 4$ & 10 \\
\hline 216HO21AS4 & 10 \\
\hline $219 \mathrm{HO} 10 \mathrm{AS} 4$ & 20 \\
\hline 220 HOO7AS4 & 10 \\
\hline $213 \mathrm{HO} 35 \mathrm{AS} 4$ & 10 \\
\hline & 10 \\
\hline 213 HO37ASA & 10 \\
\hline $213 \mathrm{HO} 2 \mathrm{AS} 4$ & 10 \\
\hline $213 \mathrm{HO} 33 \mathrm{AS} 4$ & 10 \\
\hline 213 H034AS4 & 10 \\
\hline 219 HOL1ASE & 10 \\
\hline 219HO12AS4 & 10 \\
\hline 217HO17ASA & 10 \\
\hline 217 HO16A54 & 10 \\
\hline $2218003 A 54$ & 10 \\
\hline $22116002 \mathrm{ASA}$ & 10 \\
\hline $221 \% 002 A 54$ & 10 \\
\hline 220 HOOSA54 & 10 \\
\hline $220 \mathrm{HOOOA54}$ & 10 \\
\hline & 10 \\
\hline 220 OHOOSAS4 & 10 \\
\hline $220 \mathrm{HOO4AS4}$ & 10 \\
\hline $320 \mathrm{H176A54}$ & 10 \\
\hline $320 \mathrm{H} 177 \mathrm{ASA}$ & 10 \\
\hline $320 \mathrm{H} 27 \mathrm{BAS} 4$ & 10 \\
\hline $215 \mathrm{H} 027 \mathrm{AS4}$ & 10 \\
\hline $215 \mathrm{HO2} 6 \mathrm{AS4}$ & 20 \\
\hline $215 H 025 A 54$ & 10 \\
\hline $215 H 024 A 54$ & 10 \\
\hline $215 \mathrm{HO} 23 \mathrm{AS4}$ & 10 \\
\hline $2158022 A 54$ & 10 \\
\hline 2118043255 & 22 \\
\hline $211 H 042 A 55$ & 12 \\
\hline $3214174 A 55$ & 12 \\
\hline 321 HL75A55 & 12 \\
\hline 211H044A55 & 12 \\
\hline $323 \mathrm{H} 185 \mathrm{SS}$ & 12 \\
\hline $323 \mathrm{H} 186255$ & 12 \\
\hline $323 \mathrm{H} 187 \mathrm{ASS}$ & 12 \\
\hline 214 H030A53 & 12 \\
\hline $214 \mathrm{HO29A55}$ & 12 \\
\hline 214 HO2BAS5 & 12 \\
\hline $214 \mathrm{HO} 31 \mathrm{ASS}$ & 12 \\
\hline $226 \mathrm{HO} 20 \mathrm{AS5}$ & 12 \\
\hline 216 HO19AS5 & 12 \\
\hline 216HOLOAS5 & 12 \\
\hline 218 HO15A55 & 12 \\
\hline 2194014255 & 12 \\
\hline 210 OHO13A55 & 12 \\
\hline $322 \mathrm{H196A55}$ & 12 \\
\hline
\end{tabular}

Figure 9.19 - Case 2, Revised and Decelerated Schedule (Continued). 
SAUO \& EBRAHIM ALABDULRAZZAK EST

REPORT DATE 21SEPGS RUN NO. 205

Revd \& Decel Sehl by TYpieml Activity \& ES
PRIMAVERA PROJECT PLANNER

SEVEN SEAS. CONSTRUCTION OF 51 HOUSES
Correcting OUE-of-Logie Progxese NSQ

START DATE 1APRE7 FIN DATE JOMARgg*

\begin{tabular}{|c|c|}
\hline $\begin{array}{c}\text { ACTIVITY } \\
\text { ID }\end{array}$ & $\begin{array}{l}\text { ORIO } \\
\text { OUR } \\
\end{array}$ \\
\hline $322 \mathrm{H} 189 \mathrm{~A} 55$ & 12 \\
\hline $322 \mathrm{H} 190 \mathrm{NS5}$ & 12 \\
\hline $216 \mathrm{HO21A55}$ & 12 \\
\hline $219 H 010855$ & 12 \\
\hline $220 \mathrm{HOO7A55}$ & 12 \\
\hline 213 HOJSA.55 & 12 \\
\hline $213 \mathrm{HO} 36 \mathrm{A55}$ & 12 \\
\hline $213 \mathrm{H} 037 \AA 55$ & 12 \\
\hline $213 \mathrm{H} 032 \mathrm{~A} 55$ & 22 \\
\hline * 213H033A55 & 12 \\
\hline $213 \mathrm{HO} 034 \mathrm{A5} 5$ & 12 \\
\hline 219 HO11ASS & 12 \\
\hline $219 \mathrm{HO} 12 \mathrm{~A} 55$ & 12 \\
\hline $217 \mathrm{HO17A55}$ & 12 \\
\hline 217 HO16A55 & 12 \\
\hline $222 \mathrm{H} 003 \mathrm{~A} .55$ & 12 \\
\hline 221H002A55 & 12 \\
\hline $221 H 0021255$ & 12 \\
\hline $220 \mathrm{HOOOA55}$ & 12 \\
\hline $220 \mathrm{H} 008 \mathrm{A5} 5$ & 12 \\
\hline $220 \mathrm{H006} 255$ & 12 \\
\hline $22 \mathrm{OHOOSAS5}$ & 12 \\
\hline $220 \mathrm{HOO} 4 \mathrm{AS5}$ & 12 \\
\hline $320 \mathrm{H} 276 \mathrm{5} 55$ & 12 \\
\hline $320 \mathrm{H} 177 \mathrm{A5} 5$ & 12 \\
\hline $320 \mathrm{HL78A5S}$ & 22 \\
\hline 2154027255 & 12 \\
\hline $215 \mathrm{HO26R55}$ & 12 \\
\hline 215H02SASS & 22 \\
\hline $215 \mathrm{H} 024 \mathrm{ASS}$ & 12 \\
\hline $215 H 023255$ & 12 \\
\hline $215 \mathrm{HO22A55}$ & 12 \\
\hline 21114043256 & 7 \\
\hline $211 \mathrm{H} 042 \mathrm{~A} 56$ & 7 \\
\hline $321 \mathrm{H} 174256$ & 7 \\
\hline $321 H 175 A 56$ & 7 \\
\hline $211 \mathrm{H} 044 \mathrm{~A} 56$ & $?$ \\
\hline $323 \mathrm{H} 185 \mathrm{~A} 56$ & 7 \\
\hline $323 \mathrm{H} 186 \mathrm{AS} 6$ & 7 \\
\hline 323 H187A56 & 7 \\
\hline 2144030856 & 7 \\
\hline $214 \mathrm{H} 029 \mathrm{A5} 6$ & 7 \\
\hline $214 \mathrm{H} 028256$ & 7 \\
\hline $214 H 031 \mathrm{ASG}$ & 7 \\
\hline $216 \mathrm{H} 02$ QAS56 & 7 \\
\hline $216 \mathrm{HO} 19 \mathrm{AS} 6$ & 3 \\
\hline $216 \mathrm{HO} 1 \mathrm{BAS6}$ & 7 \\
\hline $210 \mathrm{H015A56}$ & 7 \\
\hline $218 H 014 A 56$ & 7 \\
\hline $219 \mathrm{HO}^{13} \mathrm{A5} 6$ & 7 \\
\hline $322 \mathrm{H188256}$ & 7 \\
\hline $322 \mathrm{H} 189256$ & $?$ \\
\hline $322 \mathrm{H19OAS6}$ & $?$ \\
\hline $216 \mathrm{H021A56}$ & 7 \\
\hline 219H010A56 & 7 \\
\hline $220 \mathrm{H} 007256$ & 7 \\
\hline 2134035256 & 7 \\
\hline $213 \mathrm{H} 036 \mathrm{A5} 6$ & $?$ \\
\hline 213H037A56 & 7 \\
\hline $213 \mathrm{H} 032 \mathrm{~A} 56$ & 7 \\
\hline $213 \mathrm{H033A56}$ & 7 \\
\hline 2134034256 & 7 \\
\hline $219 H 011 \mathrm{A5} 6$ & 7 \\
\hline $229 \mathrm{H012A56}$ & 7 \\
\hline $217 H 017 A 56$ & 7 \\
\hline 2174016256 & 7 \\
\hline $22111003 A 56$ & 7 \\
\hline $221 H 002 A 56$ & 7 \\
\hline $221 H 001 A 56$ & 7 \\
\hline 220 OOO9A56 & 7 \\
\hline $220 \mathrm{HOOBAS} 6$ & 7 \\
\hline 220HOOGAS6 & 7 \\
\hline $220 \mathrm{HO05A56}$ & 7 \\
\hline $220 \mathrm{H004A56}$ & 7 \\
\hline $320 \mathrm{H} 176 \mathrm{SB} 6$ & 7 \\
\hline $320 H 177256$ & 7 \\
\hline $320 \mathrm{H} 17 \mathrm{GASG}$ & 1 \\
\hline $215 \mathrm{HO} 7 \mathrm{ASE}$ & 7 \\
\hline $215 \mathrm{HO} 6 \mathrm{GASG}$ & 7 \\
\hline $215 \mathrm{HO2SA56}$ & 7 \\
\hline $215 \mathrm{HO24AS6}$ & 7 \\
\hline $215 H 023 A 56$ & 7 \\
\hline 215 H022AS6 & 7 \\
\hline $211 H 043257$ & 7 \\
\hline 211HO42AS? & 7 \\
\hline $321 \mathrm{K174A57}$ & 7 \\
\hline $321 \mathrm{H175A57}$ & 7 \\
\hline 2114044257 & 7 \\
\hline $323 \mathrm{HLOSA57}$ & 7 \\
\hline 3233186457 & 7 \\
\hline $323 \mathrm{H} 187 \mathrm{A5} 7$ & 7 \\
\hline $214 \mathrm{HO3OA57}$ & $?$ \\
\hline $214 K 029 \mathrm{AST}$ & 7 \\
\hline $214 H 028 A 57$ & 7 \\
\hline $2141031 \mathrm{AS7}$ & 7 \\
\hline $216 \% 020 A 57$ & 7 \\
\hline
\end{tabular}

Figure 9.19 - Case 2, Revised and Decelerated Schedule (Continued). 


\begin{tabular}{|c|c|c|}
\hline $\begin{array}{c}\text { ACTIVITY } \\
\text { ID }\end{array}$ & $\begin{array}{l}\text { ORIG } \\
\text { DUR }\end{array}$ & $\begin{array}{l}\text { REM } \\
\text { DUR }\end{array}$ \\
\hline 216HO19AS7 & 7 & 7 \\
\hline 216HO1BAST & 7 & $?$ \\
\hline 219HO15AS7 & 7 & 7 \\
\hline $219 H O 14257$ & 7 & 7 \\
\hline 219 HO13AST & 7 & $?$ \\
\hline $322 \mathrm{H} 2 \mathrm{BBAS} 7$ & 7 & 7 \\
\hline $322 \mathrm{H} 2$ B9AS7 & 7 & 7 \\
\hline 322H190AS7 & 7 & 7 \\
\hline $216 \mathrm{HO21A57}$ & 7 & 7 \\
\hline $219 \mathrm{HO} 10 \mathrm{A5} 7$ & 7 & 7 \\
\hline $220 \mathrm{HO07AS7}$ & 7 & 7 \\
\hline 213 H035A57 & 7 & 7 \\
\hline $213 \mathrm{H} 036 \mathrm{AS} 7$ & 7 & 7 \\
\hline 213 H037AS7 & 7 & 7 \\
\hline 2134032A57 & 7 & 7 \\
\hline $213 \mathrm{H} 033 \mathrm{~A} 57$ & 7 & 7 \\
\hline $213 \mathrm{H} 034 \mathrm{AS} 7$ & 7 & 7 \\
\hline 219H011A57 & 7 & $?$ \\
\hline $219 \mathrm{H} 012 \mathrm{AS} 7$ & 7 & 7 \\
\hline 217 H017A57 & 7 & 7 \\
\hline $2174016 A 57$ & 7 & 7 \\
\hline 221H0O3AS7 & 7 & 7 \\
\hline $221 \mathrm{H} 002 \mathrm{~A} 57$ & 7 & $?$ \\
\hline 221H0O1AS7 & 7 & 7 \\
\hline 220HOOOAS7 & 7 & 7 \\
\hline 220 HOOAAS7 & 7 & 7 \\
\hline $220 H 006 \mathrm{AS} 7$ & 7 & 7 \\
\hline 220 HOOSAS 7 & 7 & 7 \\
\hline $220 \mathrm{HOO4AS7}$ & 7 & 7 \\
\hline $320 \mathrm{H} 176 \mathrm{AS} 7$ & 7 & 7 \\
\hline $320 \mathrm{H} 177 \mathrm{AS} 7$ & 7 & 7 \\
\hline 320H178AS7 & 7 & 7 \\
\hline $215 \mathrm{HO27A57}$ & 7 & 7 \\
\hline $215 \mathrm{H} 026 \mathrm{AS} 7$ & 7 & 7 \\
\hline 215H025A57 & 7 & 7 \\
\hline $215 \mathrm{H} 024 \mathrm{AST}$ & 7 & 7 \\
\hline $215 \mathrm{H} 023 \mathrm{AS} 7$ & 7 & 7 \\
\hline 215 H022AS7 & 7 & 7 \\
\hline $213 \mathrm{HO} 043 \mathrm{AS} 8$ & 6 & 6 \\
\hline 211H042A58 & 6 & 6 \\
\hline $321 \mathrm{H} 174 \mathrm{ASB}$ & 6 & 6 \\
\hline $321 \mathrm{HI} 75 \mathrm{ASB}$ & 6 & 6 \\
\hline $211 \mathrm{HO} 44 \mathrm{ASB}$ & 6 & 6 \\
\hline 323H1ASAS8 & 6 & 6 \\
\hline $323 \mathrm{H} 186 \mathrm{ASB}$ & 6 & 6 \\
\hline $323 \mathrm{H} 287 A 58$ & 6 & 6 \\
\hline $214 \mathrm{HO} 30 \mathrm{ASB}$ & 6 & 6 \\
\hline $214 \mathrm{H} 029 \mathrm{ASB}$ & 6 & 6 \\
\hline $214 \mathrm{H028A5B}$ & 6 & 6 \\
\hline $214 \mathrm{HO} 3$ LA5B & 6 & 6 \\
\hline $216 \mathrm{H} 020 \mathrm{ASB}$ & 6 & 6 \\
\hline $216 \mathrm{HO} 019 \mathrm{ASB}$ & 6 & 6 \\
\hline $216 \mathrm{HO29A5B}$ & 6 & 6 \\
\hline 218H015ASB & 6 & 6 \\
\hline $218 \mathrm{HO14A5B}$ & 6 & 6 \\
\hline $218 \mathrm{HO} 13 \mathrm{ASB}$ & 6 & 6 \\
\hline $322 \mathrm{HIBOASB}$ & 6 & 6 \\
\hline $322 \mathrm{HL} 1$ GASO & 6 & 6 \\
\hline $322 \mathrm{H} 190 \mathrm{ASO}$ & 6 & 6 \\
\hline 216H021A5B & 6 & 6 \\
\hline $219 \mathrm{HO} 010 \mathrm{ASB}$ & 6 & 6 \\
\hline $2204007 A 58$ & 6 & 6 \\
\hline $213 \mathrm{H} 035 \mathrm{SAS}$ & 6 & 6 \\
\hline 213 H036ASO & 6 & 6 \\
\hline 213 H037ASB & 6 & 6 \\
\hline 213 H032ASB & 6 & 6 \\
\hline $213 \mathrm{HO} 033 \mathrm{ASO}$ & 6 & 6 \\
\hline $2234034 \mathrm{ASB}$ & 6 & 6 \\
\hline $219 H 011 A 58$ & 6 & 6 \\
\hline 219 HO12ASB & 6 & 6 \\
\hline $2274017 \mathrm{ASB}$ & 6 & 6 \\
\hline $2274016 A 58$ & 6 & 6 \\
\hline $221 \mathrm{H} 003 \mathrm{ASB}$ & 6 & 6 \\
\hline $2214002 A 58$ & 6 & 6 \\
\hline 221H001ASB & 6 & 6 \\
\hline 2204009 ASB & 6 & 6 \\
\hline 22 OHOOBASB & 6 & 6 \\
\hline $220 \mathrm{HOOGAS8}$ & 6 & 6 \\
\hline $220 \mathrm{HOOSASO}$ & 6 & 6 \\
\hline 220 HOOAAS 8 & 6 & 6 \\
\hline $\begin{array}{l}320 \% 176 A 58 \\
320 H 177458\end{array}$ & $\begin{array}{l}6 \\
6\end{array}$ & $\begin{array}{l}6 \\
6\end{array}$ \\
\hline 320 H178ASQ & 6 & 6 \\
\hline $215 \mathrm{HO27A59}$ & 6 & 6 \\
\hline $215 \mathrm{H026A58}$ & 6 & 6 \\
\hline $215 \mathrm{H}_{025} \mathrm{AS}^{\mathrm{B}}$ & 6 & 6 \\
\hline 215 HO24ASB & 6 & 6 \\
\hline $215 \mathrm{H} 023 \mathrm{~A} 5 \mathrm{~B}$ & 6 & 6 \\
\hline $215 \mathrm{H} 022 \mathrm{ASB}$ & 6 & 6 \\
\hline $211 \mathrm{H} 043 \mathrm{AS} 9$ & 6 & 6 \\
\hline $2114042 \mathrm{AS9}$ & 6 & 6 \\
\hline $321 \mathrm{H174A59}$ & 6 & 6 \\
\hline $321 \mathrm{H} 175 \mathrm{AS} 9$ & 6 & 6 \\
\hline 211H044AS9 & 6 & 6 \\
\hline 323 KLESAS9 & 6 & 6 \\
\hline $323 \mathrm{H} 186 \mathrm{~A} 59$ & 6 & 6 \\
\hline
\end{tabular}

Figure 9.19 - Case 2, Revised and Decelerated Schedule (Continued). 


\begin{tabular}{|c|c|c|c|c|c|c|}
\hline $\begin{array}{c}\text { ACTIVITY } \\
\text { ID }\end{array}$ & $\begin{array}{l}\text { ORIO } \\
\text { DUR }\end{array}$ & $\begin{array}{l}\text { REM } \\
\text { DUR }\end{array}$ & $\therefore$ & CODE & & \\
\hline $323 \% 187459$ & 6 & 6 & 0 & 08 & $R O O F$ & MASTIC \\
\hline $2241030 A 59$ & 6 & 6 & 0 & 09 & $R O O F$ & MASTIC \\
\hline $214 \mathrm{H02} 9 \mathrm{A59}$ & 6 & 6 & 0 & 10 & ROOF & MASTIS \\
\hline $214 \mathrm{H028259}$ & 6 & 6 & 0 & 11 & ROOF & MASTIO \\
\hline $214 H 031 A 59$ & 6 & 6 & 0 & 12 & ROOF & MASTIC \\
\hline $2164020 A 59$ & 6 & 6 & 0 & 13 & ROOF & MASTIC \\
\hline $216 \mathrm{HO} 029 \mathrm{AS9}$ & 6 & 6 & 0 & 14 & ROOF & MASTIC \\
\hline 216 H019AS9 & 6 & 6 & 0 & 15 & ROOF & MASTIC \\
\hline 218 C015ASg & 6 & 6 & 0 & 26 & ROOF & MASTIC \\
\hline .218H014AS9 & 6 & 6 & 0 & 17 & ROOF & MASTIC \\
\hline $218 \mathrm{HO} 13 \mathrm{ASS}$ & 6 & 6 & 0 & 18 & $R O O F$ & MASTIC \\
\hline 322H1BAASS & 6 & 6 & 0 & 19 & $R O O F$ & MASTIC \\
\hline $322 \mathrm{H1} 189 \mathrm{AS} 9$ & 6 & 6 & : & 20 & ROOF & MASTIC \\
\hline $3221190 A 59$ & 6 & 6 & : & 21 & ROOF & Mastic \\
\hline $216 \mathrm{H021 \textrm {AS } 9}$ & $\begin{array}{l}6 \\
6\end{array}$ & $\begin{array}{l}6 \\
6\end{array}$ & $:$ & $\begin{array}{l}22 \\
23\end{array}$ & $\begin{array}{l}\text { ROOF } \\
\text { ROOF }\end{array}$ & MASTIC \\
\hline $\begin{array}{l}219 H 010 A 59 \\
22015007259\end{array}$ & 6 & 6 & 0 & 24 & $\begin{array}{l}\text { ROOF } \\
\text { ROOF }\end{array}$ & $\begin{array}{l}\text { MASTIC } \\
\text { MASTIC }\end{array}$ \\
\hline 213H035AS9 & 6 & 6 & 0 & 25 & ROOF & MASTIC \\
\hline 21313036259 & 6 & 6 & 0 & 26 & ROOF & MASTIC \\
\hline $213 \mathrm{H} 037 \mathrm{AS} 9$ & 6 & 6 & 0 & 27 & ROOF & MASTIC \\
\hline $213 H 032 \AA 59$ & 6 & 6 & 0 & 28 & ROOF & MASTIC \\
\hline $2134033 \AA 59$ & 6 & 6 & 0 & 29 & $R O O F$ & MASTIC \\
\hline $213 H 034 A 59$ & 6 & 6 & 0 & 30 & $R O O F$ & MASTIC \\
\hline 2194011AS9 & 6 & 6 & : & 31 & ROOF & MASTIC \\
\hline $2194012 A 59$ & 6 & 6 & 0 & 32 & ROOF & MASTIC \\
\hline 217H017ASg & 6 & 6 & 0 & 33 & ROOF & MASTIC \\
\hline 217H016AS9 & 6 & 6 & 0 & 34 & ROOF & MASTIC \\
\hline 2214003 A59 & 6 & 6 & 0 & 35 & ROOF & MASTIC \\
\hline $221 \mathrm{H} 002 \mathrm{A59}$ & 6 & 6 & 0 & $\begin{array}{l}36 \\
37\end{array}$ & ROOF & Mastic \\
\hline $\begin{array}{l}2214001 A 59 \\
220 \mathrm{H} 009259\end{array}$ & $\begin{array}{l}6 \\
6\end{array}$ & $\begin{array}{l}6 \\
6\end{array}$ & 0 & $\begin{array}{l}37 \\
38\end{array}$ & ROOF & MASTIC \\
\hline $220 \% 00 B A 59$ & 6 & 6 & 0 & 39 & $R O D F$ & MASTIC \\
\hline $220 H 006$ A59 & 6 & 6 & 0 & 40 & ROOF & MASTIC \\
\hline $220 \mathrm{H} 005 \mathrm{A5} 9$ & 6 & 6 & 0 & 11 & ROOF & MASTIC \\
\hline $220 \mathrm{H004259}$ & 6 & 6 & 0 & 42 & ROOF & MASTIC \\
\hline $320 \mathrm{H176AS9}$ & 6 & 6 & 0 & 43 & ROOF & MASTIC \\
\hline 320H177A59 & 6 & 6 & 0 & 44 & ROOF & MASTIC \\
\hline $320 \mathrm{H}_{178 \mathrm{AS}}$ & 6 & 6 & 0 & 45 & ROOF & MASTIC \\
\hline $2154027 A 59$ & 6 & 6 & 0 & 46 & ROOF & Mastic \\
\hline 215 H026A59 & 6 & 6 & 0 & 47 & ROOF & MASTIC \\
\hline $215 \mathrm{H} 025 A 59$ & 6 & 6 & 0 & 48 & ROOF & MASTIC \\
\hline $215 \mathrm{H} 024 \mathrm{~A} 59$ & 6 & 6 & 0 & 49 & $200 \mathrm{~F}$ & MASTIC \\
\hline 215H023AS9 & 6 & 6 & 0 & 50 & $R O O F$ & MASTIC \\
\hline $215 \mathrm{H} 022 \mathrm{ASg}$ & 6 & 6 & 0 & 51 & $R \infty \mathrm{F}$ & MASTIC \\
\hline $213 \mathrm{HO} 3 \mathrm{~A} 2 \mathrm{OO}$ & 4 & 4 & 0 & 01 & WATER & R TANK \\
\hline $211 \mathrm{HO} 2 \mathrm{~A} 2 \mathrm{AO}$ & 4 & 4 & 0 & 02 & WATER & R TANK \\
\hline $321 \mathrm{H} 274 \mathrm{~A} 60$ & 4 & 4 & 0 & 03 & WATER & R TANR \\
\hline $321 \mathrm{H} 175 \mathrm{~A} 60$ & 4 & 4 & 0 & 04 & WATER & R TANK \\
\hline $211 \mathrm{HO44260}$ & 1 & 4 & 0 & 05 & WATER & R TANR \\
\hline $323 \mathrm{H} 185 \mathrm{~A} 60$ & 4 & 4 & 0 & 06 & WATER & R TANK \\
\hline $323 H 186260$ & 4 & 4 & 0 & 07 & WATER & R TANX \\
\hline $323 \mathrm{K187A60}$ & 4 & 4 & 0 & $O B$ & WATER & R TANK \\
\hline $214 H 030 \mathrm{~A} 60$ & 4 & 4 & 0 & 09 & WATER & R TANK \\
\hline 2144029460 & 4 & 4 & 0 & 10 & WATER & R TANK \\
\hline $214 \mathrm{HO} 28 \mathrm{~A} 60$ & & 4 & 0 & 11 & WATER & R TANK \\
\hline 214H031A60 & 4 & 4 & 0 & 12 & WATER & R TANK \\
\hline $2164020 A 60$ & 4 & 4 & 0 & 13 & WATER & R TANK \\
\hline $2164019 A 60$ & 4 & 4 & 0 & 14 & WATER & R TANK \\
\hline $216 \mathrm{HO18A60}$ & 4 & 1 & 0 & 15 & KATER & R TANR \\
\hline $\begin{array}{l}218 \mathrm{HO14A60} \\
218 \mathrm{HO} 13 \mathrm{~A} 60\end{array}$ & 4 & 4 & 0 & $\begin{array}{l}17 \\
18\end{array}$ & $\begin{array}{l}\text { WATER } \\
\text { WATER }\end{array}$ & $\begin{array}{l}R \\
R \\
\text { R TANNK }\end{array}$ \\
\hline $322 \mathrm{H} 188 \mathrm{~A} 60$ & 4 & 4 & 0 & 19 & WATER & R TANK \\
\hline $322 \mathrm{H} 189 \mathrm{A60}$ & 4 & 4 & 0 & 20 & WATER & R TANR \\
\hline $322 \mathrm{H} 190 \mathrm{~A} 60$ & 4 & 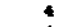 & 0 & 21 & WATER & R TANK \\
\hline $216 \mathrm{HO} 02 \mathrm{LA} 60$ & 4 & 4 & 0 & 22 & MATER & R TANK \\
\hline $219 \mathrm{HO} 0 \mathrm{~A} 60$ & 4 & 4 & 0 & 23 & WATER & R TANR \\
\hline $22 \mathrm{OH} 007 \mathrm{~A} 60$ & 4 & 4 & 0 & 24 & WATER & R TANK \\
\hline $213 \mathrm{H} 035 \mathrm{~A} 60$ & 4 & 4 & 0 & 25 & WATER & R TANK \\
\hline $213 \mathrm{H036A60}$ & 1 & 4 & 0 & 26 & WATER & R TANK \\
\hline 213H037A60 & 4 & 4 & 0 & 27 & WATER & R TANK \\
\hline $213 H 032 A 60$ & 4 & 4 & 0 & 28 & WATER & R TANK \\
\hline 213H033A60 & 4 & 4 & 0 & 29 & WATER & R TANR \\
\hline $213 \mathrm{H} 034460$ & 1 & 4 & 0 & 30 & WATER & R TANK \\
\hline $219 \mathrm{H} 011 \mathrm{~A} 60$ & 4 & 4 & 0 & 31 & WATER & R TANR \\
\hline $219 H 012 \mathrm{~A} 60$ & 4 & 4 & 0 & 32 & WATER & R TANR \\
\hline 217HO17A6O & 4 & 4 & 0 & 33 & WATER & R TANK \\
\hline $2174016 A 60$ & 4 & 4 & 0 & 34 & WATER & R TANK \\
\hline $221 \mathrm{N003A60}$ & 1 & 4 & 0 & 35 & WATER & R TANK \\
\hline $221 H 002 A 60$ & 1 & 4 & 0 & 36 & WATER & R TANK \\
\hline $2214001 \mathrm{A6} 0$ & 4 & 4 & 0 & 37 & MATER & R TANT \\
\hline $\begin{array}{l}220 \mathrm{H} 009 \mathrm{A60} \\
220 \mathrm{H} 00 \mathrm{~A} 60\end{array}$ & 1 & 4 & 0 & 38 & MATER & R TANK \\
\hline $220 \mathrm{H} 008 \mathrm{~g} 6 \mathrm{O}$ & 4 & 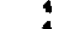 & $:$ & 39 & WATER & R TANK \\
\hline $\begin{array}{l}220 H 006 A 60 \\
220 H 005 A 60\end{array}$ & 4 & 4 & $:$ & $\begin{array}{l}40 \\
41\end{array}$ & WATER & R TANK \\
\hline $\begin{array}{l}220 H 005 A 60 \\
220 H 004 A 60\end{array}$ & 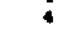 & 4 & 0 & 41 & $\begin{array}{l}\text { WATER } \\
\text { WATER }\end{array}$ & $\begin{array}{l}R \text { TANK } \\
R \text { TANK }\end{array}$ \\
\hline $320 H 176 A 60$ & 4 & 1 & 0 & 43 & MATER & R TANK \\
\hline $320 H 177 A 60$ & 4 & 4 & 0 & 44 & WATER & R TANK \\
\hline $320 \% 178 A 60$ & 4 & 4 & 0 & 45 & MATER & R TANK \\
\hline $215 \% 027 A 60$ & 4 & 4 & 0 & 46 & MATER & R TANK \\
\hline $\begin{array}{r}215 H 026 A 60 \\
2154025 A 60\end{array}$ & 4 & 4 & : & $\begin{array}{l}47 \\
48\end{array}$ & WATER & R TANR \\
\hline $\begin{array}{l}215 H 025 A 60 \\
215 H 024 A 60\end{array}$ & & 4 & 0 & $\begin{array}{l}48 \\
49\end{array}$ & WATER & R TANK \\
\hline $\begin{array}{l}215 \% 024 A 60 \\
215 H 023 A 60\end{array}$ & 4 & 4 & 0 & $\begin{array}{l}49 \\
50\end{array}$ & WATER & R TANK \\
\hline $2154022 A 60$ & 4 & 4 & 0 & 51 & WATER & $\begin{array}{l}R \text { TANK } \\
R \text { TANR }\end{array}$ \\
\hline & & & & & & \\
\hline
\end{tabular}

Figure 9.19 - Case 2, Revised and Decelerated Schedule (Continued). 
SAUD \& इBRAKIM ALABDULRAZZAX EST

REPORT DATE 21 SEP95 RUN NO. 205

Revd a Dacel Schl by TYPical Activity E ES
PRIMAVERA PROJECT PLANNER

SE: TEN SEAS, CONSTRUCTION OF S1 HOUSES
Correct 2 ing out -of-Logie Progrese nSq START DATE LAPRE7 FIN DATE 3OMARA9* DATA OATE LFEBEs PAGE NO. 27

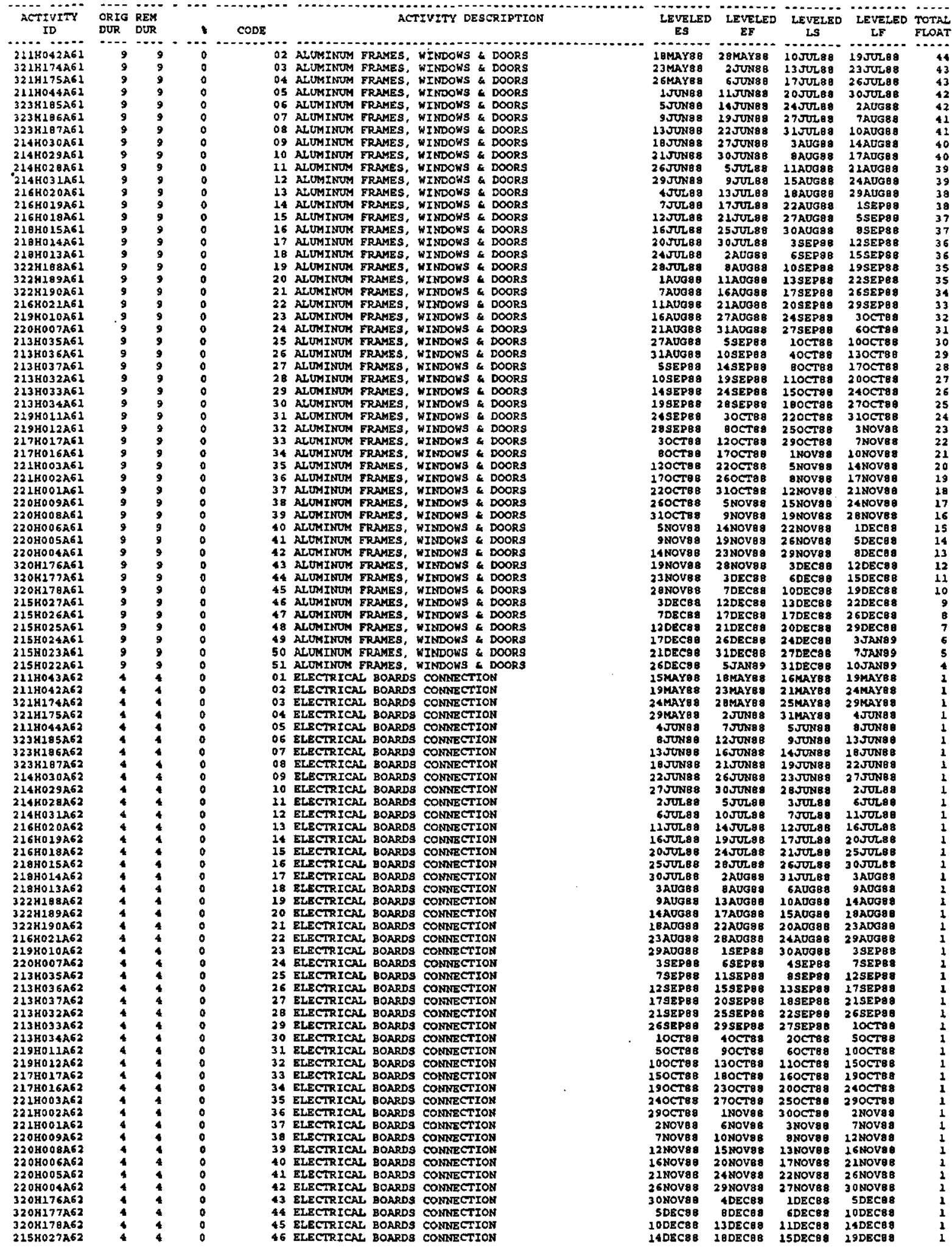

Figure 9.19 - Case 2, Revised and Decelerated Schedule (Continued). 
SAND \& EBRAHIM ALABDULRAZZAX EST.

PEPORT DATE 21SEPSS RUN NO. 205

Revd \& Decel Schi by Typical Activity \& ES
PRIMAVERA PROJECT PLANER

SEVEN SEAS, CONSTRUCTYON OF 51 HOUSES
Correcting Out-of-Logle Progrese NSq

START DATE LAPRB7 FIN DATE 3OMARBg*

DATA DATE LFEB89 PACE NO, 28

\begin{tabular}{|c|c|}
\hline \begin{tabular}{c} 
ACTIVITY \\
ID \\
\hdashline
\end{tabular} & $\begin{array}{l}\text { ORIO } \\
\text { DUR } \\
\end{array}$ \\
\hline $215 \mathrm{HO26A62}$ & 4 \\
\hline $215 \mathrm{H} 025 \mathrm{~A} 62$ & \\
\hline $215 \mathrm{HO24A62}$ & 4 \\
\hline $215 \mathrm{HO23A62}$ & 4 \\
\hline $215 \mathrm{H} 022 \mathrm{~A} 62$ & 4 \\
\hline 211H043A63 & 6 \\
\hline $211 \mathrm{HO42A63}$ & 6 \\
\hline $321 \mathrm{H174A63}$ & 6 \\
\hline $321 \mathrm{H} 175 \mathrm{~A} 63$ & 6 \\
\hline $211 \mathrm{H} 044 \mathrm{~A} 63$ & 6 \\
\hline $323 \mathrm{H} 185 A 63$ & 6 \\
\hline $323 \mathrm{H} 186 \mathrm{~A} 63$ & 6 \\
\hline $323 \mathrm{H} 187 \mathrm{~A} 63$ & 6 \\
\hline $21+H 030 A 63$ & 6 \\
\hline 214 HO29A63 & 6 \\
\hline $214 \mathrm{H} 02 \mathrm{gA} 63$ & 6 \\
\hline $214 \mathrm{H03} 1 \mathrm{~A} 63$ & 6 \\
\hline $2164020 A 63$ & 6 \\
\hline $216 \mathrm{HO19A63}$ & 6 \\
\hline 216H018A63 & 6 \\
\hline 218 HO $15 A 63$ & 6 \\
\hline $218 \mathrm{BH} 014 \mathrm{A63}$ & 6 \\
\hline $210 \mathrm{HO13A63}$ & 6 \\
\hline $322 \mathrm{~K} 188 \mathrm{~A} 63$ & 6 \\
\hline $322 \mathrm{H} 1 \mathrm{~B} 9 \mathrm{~A} 63$ & 6 \\
\hline $322 \mathrm{H190A63}$ & 6 \\
\hline $216 \mathrm{HO} 2 \mathrm{LA63}$ & 6 \\
\hline $219 H O 10 A 63$ & 6 \\
\hline $220 H 007 A 63$ & 6 \\
\hline $213 \mathrm{H} 035 \mathrm{~A} 63$ & 6 \\
\hline 213 H036A63 & 6 \\
\hline $213 \% 037 A 63$ & 6 \\
\hline $213 \mathrm{HO32A63}$ & 6 \\
\hline $213 \mathrm{HO33A63}$ & 6 \\
\hline 213H034A63 & 6 \\
\hline $219 \mathrm{H} 011 \mathrm{A63}$ & 6 \\
\hline $219 H 012 A 63$ & 6 \\
\hline $2174017 A 63$ & 6 \\
\hline $2174016 \mathrm{A63}$ & 6 \\
\hline $221 \% 003 A 63$ & 6 \\
\hline $2214002 A 63$ & 6 \\
\hline $221 \mathrm{H} 001 \mathrm{A63}$ & 6 \\
\hline $220 \mathrm{HOO9A63}$ & 6 \\
\hline $220 \mathrm{HOOBA63}$ & 6 \\
\hline $220 H 006 A 63$ & 6 \\
\hline $220 \mathrm{H} 005 \mathrm{~A} 63$ & 6 \\
\hline $220 \mathrm{H} 004 \mathrm{A63}$ & 6 \\
\hline $320 \mathrm{H} 176 \mathrm{~A} 63$ & 6 \\
\hline $320 H 177 A 63$ & 6 \\
\hline $320 \mathrm{H178A63}$ & 6 \\
\hline $215 \mathrm{H} 027 \mathrm{A63}$ & 6 \\
\hline $215 \mathrm{HO}^{26 \mathrm{~A} 63}$ & 6 \\
\hline $215 \mathrm{H} 025 \mathrm{~A} 63$ & 6 \\
\hline $2154024 A 63$ & 6 \\
\hline $215 H 023 \mathrm{~A} 63$ & 6 \\
\hline $2154022 A 63$ & 6 \\
\hline $211 \mathrm{H} 043 \mathrm{~A} 64$ & 10 \\
\hline $211 \mathrm{HO42A64}$ & 10 \\
\hline $32211274 A 64$ & 10 \\
\hline $321 \mathrm{H175A64}$ & 10 \\
\hline $212 H 044 \mathrm{~A} 64$ & 10 \\
\hline $323 \mathrm{H} 185 \mathrm{~A} 64$ & 10 \\
\hline 323 H286A64 & 10 \\
\hline 323H187A64 & 10 \\
\hline $214 \mathrm{HO} 0 \mathrm{OAE4}$ & 10 \\
\hline 214H029A64 & 10 \\
\hline $224 H 02 \theta A 64$ & 10 \\
\hline 234H03 1A64 & 10 \\
\hline $2164020 A 64$ & 10 \\
\hline 216 H019A64 & 10 \\
\hline $216 \mathrm{KO18A64}$ & 10 \\
\hline 218HO15AG4 & 10 \\
\hline 218H014A64 & 10 \\
\hline $218 \mathrm{OHO13A64}$ & 10 \\
\hline $322 H 188 A 64$ & 10 \\
\hline $322 \mathrm{H} 1 \mathrm{\theta gA64}$ & 10 \\
\hline $322 K 190 A 64$ & 10 \\
\hline $216 \mathrm{H} 021 \mathrm{A64}$ & 10 \\
\hline $219 \mathrm{HO} 10 \mathrm{A64}$ & 10 \\
\hline $220 \mathrm{HOOTAG4}$ & 10 \\
\hline 213H035A64 & 10 \\
\hline $2134036 \mathrm{A64}$ & 10 \\
\hline $213 \mathrm{H} 037 \mathrm{A64}$ & 10 \\
\hline $213 \mathrm{HO} 32 \mathrm{A64}$ & 10 \\
\hline $213 \mathrm{H} 033 \mathrm{~A} 64$ & 10 \\
\hline $213 \mathrm{H} 034 \mathrm{~A} 64$ & 10 \\
\hline $219 \mathrm{H} 011 \mathrm{~A} 64$ & 10 \\
\hline 219H012A64 & 20 \\
\hline 217H017A64 & 10 \\
\hline $217 \mathrm{HO16A64}$ & 10 \\
\hline 221H003A64 & 10 \\
\hline $221 \mathrm{H} 002 \mathrm{~A} 64$ & 10 \\
\hline $221 \mathrm{H} 001 \mathrm{~A} 64$ & 20 \\
\hline 220HOO9A64 & 10 \\
\hline $220 \mathrm{HOOBA64}$ & 10 \\
\hline $220 \mathrm{H} 006 \mathrm{~A} 64$ & 10 \\
\hline
\end{tabular}

Figure 9.19 - Case 2, Revised and Decelerated Schedule (Continued). 
SAUD I EBRAHIM ALABDULRAZZAX EST.

REPORT DATE 21 LEPSS RIN NO. 205

Rovd \& Docel Schl by TYpleal Activity a ES
PRIMAVERA PRONECT PLANNER

SEVEN SEAS, CONSTRUCTION OF 51 HOUSES
Correcting out-of-Logle Progresa NSq START DATE LAPR日7 FIN DATE 3OMARB9 DATA DATE LFEBB PAGE NO. 29

\begin{tabular}{|c|c|c|}
\hline $\begin{array}{c}\text { ACTIVITY } \\
\text { ID }\end{array}$ & $\begin{array}{l}\text { ORIG } \\
\text { DUR } \\
\cdots . .\end{array}$ & $\begin{array}{l}\text { REM } \\
\text { DUR } \\
. . .\end{array}$ \\
\hline 220 HOOSAGA & 10 & 10 \\
\hline $220 \mathrm{H004A64}$ & 10 & 10 \\
\hline $320 H 176 \mathrm{A64}$ & 10 & 10 \\
\hline $320 H 177$ A64 & 10 & 10 \\
\hline $320 H 176 A 64$ & 10 & 10 \\
\hline 215H027A64 & 10 & 10 \\
\hline $215 H 026 \mathrm{AGt}$ & 10 & 10 \\
\hline 215H025A64 & 10 & 10 \\
\hline $215 H 024 A 64$ & 10 & 10 \\
\hline 2 15H023A64 & 10 & 10 \\
\hline $215 H 022 A 64$ & 10 & 10 \\
\hline $2114043 A 65$ & 2 & 2 \\
\hline $211 H 042 A 65$ & 2 & 2 \\
\hline $3214174 A 65$ & 2 & 2 \\
\hline $3214175 A 65$ & 2 & 2 \\
\hline $2114044 A 65$ & 2 & 2 \\
\hline 323 H195A65 & 2 & 2 \\
\hline 323Н186A65 & 2 & 2 \\
\hline $323 \mathrm{H} 187 \mathrm{~A} 6 \mathrm{~S}$ & 2 & 2 \\
\hline $214 \mathrm{H030A65}$ & 2 & 2 \\
\hline $214 H 029 A 65$ & 2 & 2 \\
\hline 214H028A6S & 2 & 2 \\
\hline 214 H031A65 & 2 & 2 \\
\hline $216 \mathrm{H020A65}$ & 2 & 2 \\
\hline 216HO19A65 & 2 & 2 \\
\hline $216 \mathrm{HO1 \theta A65}$ & 2 & 2 \\
\hline 2184015A63 & 2 & 2 \\
\hline 218 B 14 A65 & 2 & 2 \\
\hline $219 H 013 A 65$ & 2 & 2 \\
\hline $322 \mathrm{H} 18 \mathrm{AA} 65$ & 2 & 2 \\
\hline $322 \mathrm{H189A65}$ & 2 & 2 \\
\hline $322 \mathrm{H190A65}$ & 2 & 2 \\
\hline $216 \mathrm{H} 021 \mathrm{~A} 65$ & 2 & 2 \\
\hline $219 H 010 A 65$ & 2 & 2 \\
\hline $220 \mathrm{HOO}$ A65 & 2 & 2 \\
\hline 213H035A65 & 2 & 2 \\
\hline $213 \mathrm{H} 036 \mathrm{A6S}$ & 2 & 2 \\
\hline 213H037A65 & 2 & 2 \\
\hline $213 H 032 A 65$ & 2 & 2 \\
\hline 213H033A6S & 2 & 2 \\
\hline 213H034A65 & 2 & 2 \\
\hline $2194011 \mathrm{A65}$ & 2 & 2 \\
\hline 219 H012A6S & 2 & 2 \\
\hline 217H017A65 & 2 & 2 \\
\hline $217 H 016 A 65$ & 2 & 2 \\
\hline $221 \mathrm{HO} 03 A 65$ & 2 & 2 \\
\hline $221 H 002 A 65$ & 2 & 2 \\
\hline $221 \mathrm{HOO}$ AA65 & 2 & 2 \\
\hline $220 \mathrm{H} 009265$ & 2 & 2 \\
\hline $220 \mathrm{H} 008 \mathrm{~A} 65$ & 2 & 2 \\
\hline $220 \mathrm{HOOGA65}$ & 2 & 2 \\
\hline $220 \mathrm{H} 005 \mathrm{SA6S}$ & 2 & 2 \\
\hline $\begin{array}{l}220 H 004 A 65 \\
320 \mathrm{H} 176 \mathrm{A65}\end{array}$ & 2 & 2 \\
\hline $\begin{array}{l}320 \mathrm{H} 176 \mathrm{AGS} \\
320 \mathrm{H} 177 \mathrm{~A} 5 \mathrm{~S}\end{array}$ & 2 & 2 \\
\hline $320 \mathrm{H} 178 \mathrm{AG5}$ & 2 & 2 \\
\hline $215 \mathrm{H} 027 \mathrm{A65}$ & 2 & 2 \\
\hline $215 \mathrm{H} 026 \mathrm{A6S}$ & 2 & 2 \\
\hline $215 \mathrm{H025A65}$ & 2 & 2 \\
\hline 215 H024A65 & 2 & 2 \\
\hline $215 \mathrm{H} 023 \mathrm{A6S}$ & 2 & 2 \\
\hline $2154022 A 65$ & 2 & 2 \\
\hline 211H043A66 & 3 & 3 \\
\hline $211 \mathrm{HO} 22 \mathrm{AG6}$ & 3 & 3 \\
\hline $321 \mathrm{H174A66}$ & 3 & 3 \\
\hline 321H175A66 & 3 & 3 \\
\hline 211H044A66 & 3 & 3 \\
\hline 323H18SA66 & 3 & 3 \\
\hline 323H186A66 & 3 & 3 \\
\hline $323 \mathrm{H} 187 \not A 66$ & 3 & 3 \\
\hline $214 \mathrm{H} 030 \AA 66$ & 3 & 3 \\
\hline $214 \mathrm{HO29A66}$ & 3 & 3 \\
\hline $214 \mathrm{H} 028 \mathrm{~A} 6 \mathrm{G}$ & 3 & 3 \\
\hline $214 H 0312 A 66$ & 3 & 3 \\
\hline $216 \mathrm{H} 020 A 66$ & 3 & 3 \\
\hline 216H019A66 & 3 & 3 \\
\hline $216 \mathrm{HO} 028 \mathrm{A66}$ & 3 & 3 \\
\hline $210 \mathrm{H} 015 A 66$ & 3 & 3 \\
\hline 21日H014A66 & 3 & 3 \\
\hline $2164023 \mathrm{A66}$ & 3 & 3 \\
\hline $322 \mathrm{H} 288 \mathrm{~A} 66$ & 3 & 3 \\
\hline $322 \mathrm{H189A66}$ & 3 & 3 \\
\hline $322 \mathrm{H190A65}$ & 3 & 3 \\
\hline $216 \mathrm{H} 021 \mathrm{~A} 66$ & 3 & 3 \\
\hline $2194010 \mathrm{A66}$ & 3 & 3 \\
\hline $220 \mathrm{HOO} 7 \mathrm{A66}$ & 3 & 3 \\
\hline 213H035A66 & 3 & 3 \\
\hline 213 H036A66 & 3 & 3 \\
\hline $2134037 \mathrm{AEG}$ & 3 & 3 \\
\hline 213Н032А66 & 3 & 3 \\
\hline $213 H 033 \mathrm{A66}$ & 3 & 3 \\
\hline 213 H034A66 6 & 3 & 3 \\
\hline 2194011266 & 3 & 3 \\
\hline $219 \mathrm{HO} 12 \mathrm{A6} 6$ & 3 & 3 \\
\hline 217 H017A66 & 3 & 3 \\
\hline 217H016A66 & 3 & 3 \\
\hline
\end{tabular}

Figure 9.19 - Case 2, Revised and Decelerated Schedule (Continued). 
SAUD \& EBRAKIM ALADDVLPAZZAK EST

PRPORT DATE 2ISEP9S RUN NO. 205

PRIMAVERA PROTECT PLANNER

SEVEN SEAS, CONSTRUCTION OF S1 HOUSES
Correcting out-of-Logic Progross NSq

START DATE LAPRET FIN DATE 30 MARg9

DATA DATE LFEBgg PAGE NO. 30

\begin{tabular}{|c|c|c|}
\hline $\begin{array}{c}\text { ACTIVITY } \\
\text { ID }\end{array}$ & $\begin{array}{l}\text { ORIG } \\
\text { DUR }\end{array}$ & $\begin{array}{l}\text { REM } \\
\text { DUR }\end{array}$ \\
\hline $221 H 003 A 66$ & 3 & 3 \\
\hline $22112002 A 66$ & 3 & 3 \\
\hline $221 \mathrm{HOO1A66}$ & 3 & 3 \\
\hline $220 \mathrm{HO09A66}$ & 3 & 3 \\
\hline $220 \mathrm{HO08A66}$ & 3 & 3 \\
\hline $220 \mathrm{H} 006 \mathrm{~A} 6 \mathrm{G}$ & 3 & 3 \\
\hline $220 \mathrm{HOOSA66}$ & 3 & 3 \\
\hline $220 \mathrm{HOO} 4 \mathrm{~A} 66$ & 3 & 3 \\
\hline $.320 \mathrm{H176A65}$ & 3 & 3 \\
\hline $320 \mathrm{H} 177 \mathrm{A66}$ & 3 & 3 \\
\hline $320 \mathrm{H179A66}$ & 3 & 3 \\
\hline $215 \mathrm{H027A66}$ & 3 & 3 \\
\hline $215 \mathrm{H} 026 \mathrm{~A} 66$ & 3 & 3 \\
\hline $215 H 025 A 66$ & 3 & 3 \\
\hline $215 H 024 A 66$ & 3 & 3 \\
\hline $215 \mathrm{H023A66}$ & 3 & 3 \\
\hline $215 \mathrm{H} 022 \mathrm{~A} 66$ & 3 & 3 \\
\hline $211 H 043 A 67$ & 3 & 3 \\
\hline $2111042 A 67$ & 3 & 3 \\
\hline $321 \mathrm{H174A67}$ & 3 & 3 \\
\hline $321 \mathrm{H} 175 \mathrm{~A} 67$ & 3 & 3 \\
\hline $2111 \mathrm{HO44A67}$ & 3 & 3 \\
\hline $323 \mathrm{H} 185 \mathrm{~A} 67$ & 3 & 3 \\
\hline $323 \mathrm{H} 196 \mathrm{~A} 67$ & 3 & 3 \\
\hline $323 \mathrm{H} 2 \mathrm{Q7A67}$ & 3 & 3 \\
\hline 214HO3OA67 & 3 & 3 \\
\hline $2144029 A 67$ & 3 & 3 \\
\hline $214 H 02 B A 67$ & 3 & 3 \\
\hline $2144031 A 67$ & 3 & 3 \\
\hline $2164020 A 67$ & 3 & 3 \\
\hline $216 \mathrm{HO19A67}$ & 3 & 3 \\
\hline $2164010 A 67$ & 3 & 3 \\
\hline $218 \mathrm{HO15A67}$ & 3 & 3 \\
\hline $228 \mathrm{HO} 14 \mathrm{AG} 7$ & 3 & 3 \\
\hline 218H013A67 & 3 & 3 \\
\hline $322 H 18$ A67 & 3 & 3 \\
\hline $322 \mathrm{H} 189 \mathrm{A67}$ & 3 & 3 \\
\hline $322 \mathrm{H} 290 \mathrm{~A} 67$ & 3 & 3 \\
\hline $2164021 A 67$ & 3 & 3 \\
\hline 219H010A67 & 3 & 3 \\
\hline $220 \mathrm{HO0} 7 \mathrm{A67}$ & 3 & 3 \\
\hline 213 HO3SA67 & 3 & 3 \\
\hline $213 \mathrm{H} 036 \mathrm{AA} 67$ & 3 & 3 \\
\hline $213 \mathrm{H} 037 \mathrm{A67}$ & 3 & 3 \\
\hline $213 \mathrm{H} 032 \mathrm{AE} 7$ & 3 & 3 \\
\hline $213 \mathrm{HO} 33 \mathrm{A6} 7$ & 3 & 3 \\
\hline $213 \mathrm{H} 034 \mathrm{~A} 67$ & 3 & 3 \\
\hline 219H011A67 & 3 & 3 \\
\hline $219 \mathrm{H} 012 \mathrm{~A} 67$ & 3 & 3 \\
\hline 217H017A67 & 3 & 3 \\
\hline $217 \mathrm{HO16A67}$ & 3 & 3 \\
\hline $221 H 003 A 67$ & 3 & 3 \\
\hline $221 H 002 A 67$ & 3 & 3 \\
\hline $2214001 A 67$ & 3 & 3 \\
\hline $220 \mathrm{H009A67}$ & 3 & 3 \\
\hline 220HOOQA67 & 3 & 3 \\
\hline $220 \mathrm{HOO6A67}$ & 3 & 3 \\
\hline $220 \mathrm{HOOSA67}$ & 3 & 3 \\
\hline $220 \mathrm{HO04A67}$ & 3 & 3 \\
\hline $320 \mathrm{H} 176 \mathrm{~A} 67$ & 3 & 3 \\
\hline $320 H 177 A 67$ & 3 & 3 \\
\hline $320 H 178 A 67$ & 3 & 3 \\
\hline 225H027A67 & 3 & 3 \\
\hline $215 H 026467$ & 3 & 3 \\
\hline 215H025A67 & 3 & 3 \\
\hline 215H024A67 & 3 & 3 \\
\hline $215 \mathrm{HO23A67}$ & 3 & 3 \\
\hline $2154022 A 67$ & 3 & 3 \\
\hline $211 \mathrm{HO43A68}$ & 4 & 4 \\
\hline $221 \% 042 A 6 B$ & 4 & 4 \\
\hline $321 H 174 A 6 B$ & 4 & 4 \\
\hline 321H175A6B & 4 & 4 \\
\hline 211H044A68 & 4 & 4 \\
\hline $323 \mathrm{H} 285 \mathrm{~A} 6 \mathrm{~B}$ & 4 & 4 \\
\hline $323 \mathrm{H186A6B}$ & 4 & 4 \\
\hline $323 \mathrm{H1O7A68}$ & 4 & 4 \\
\hline $214 H 030 A 6 B$ & & 4 \\
\hline 214HO29A69 & 4 & 4 \\
\hline $214 \mathrm{H} 02 \mathrm{gA6 \theta}$ & 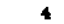 & \\
\hline $2144031 \mathrm{A6g}$ & 4 & 4 \\
\hline $216 \mathrm{HO2OA69}$ & 4 & 4 \\
\hline 216H019A68 & & \\
\hline $216 \mathrm{HO18A68}$ & 4 & 4 \\
\hline $21 \mathrm{aH015A68}$ & 4 & 4 \\
\hline $2184014 \mathrm{Ag}$ & 4 & 4 \\
\hline $2184013 \times 68$ & 4 & 1 \\
\hline $322 \mathrm{H18BA68}$ & 1 & 4 \\
\hline $322 \mathrm{H199A68}$ & 1 & 4 \\
\hline $3224190 A 68$ & 4 & 4 \\
\hline $216 \mathrm{H02} 1 \mathrm{~A} 68$ & 4 & 4 \\
\hline $219 H 010 A 68$ & 1 & 4 \\
\hline $\begin{array}{l}220 \mathrm{H} 007 \mathrm{AGB} \\
2130035 \mathrm{~B}\end{array}$ & 4 & 4 \\
\hline $\begin{array}{l}213 H 035 A 68 \\
213 H 036 A 68\end{array}$ & 4 & 4 \\
\hline $\begin{array}{l}213 H 036 A 68 \\
213 H 037 A 68\end{array}$ & 4 & 4 \\
\hline $\begin{array}{l}213 H 037 A 68 \\
213 H 032 A 68\end{array}$ & 4 & 4 \\
\hline
\end{tabular}

Figure 9.19 - Case 2, Revised and Decelerated Schedule (Continued). 
SAUD \& ERRAHIM ALABDULRAZZAK EST,

REPORT DATE 21SEPS5 RUN NO. 205

Rovd Decel $18: 31$

PRIMAVERA PROTECT PLAMRER

SEVEN SEAS, CONSTRUCTION OF 52 HOUSES
Correcting Out-of-Logie progreas NS START DATE LAPRA7 FIN DATE 3OMAR89* DATA DATE 1FEBE8 PAGE NO. 31

\begin{tabular}{|c|c|c|c|c|}
\hline $\begin{array}{l}\text { ACTrVITY } \\
\text { ID }\end{array}$ & $\begin{array}{l}\text { ORIO } \\
\text { DUR }\end{array}$ & $\begin{array}{l}\text { REM } \\
\text { DUR }\end{array}$ & 4 & CODE \\
\hline $213 \mathrm{H} 033 \mathrm{~A} 68$ & 4 & 4 & 0 & 29 \\
\hline $2134034 A 68$ & 4 & 4 & 0 & 30 \\
\hline 219H011A69 & 4 & 4 & 0 & 31 \\
\hline $219 H 012 A 68$ & 4 & 4 & 0 & 32 \\
\hline $217 \mathrm{H} 017269$ & 4 & 4 & 0 & 33 \\
\hline $217 \mathrm{HO16A69}$ & 4 & 4 & 0 & 34 \\
\hline $221 \mathrm{H} 003 \mathrm{~A} 68$ & 4 & 4 & 0 & 35 \\
\hline $221 H 002 A 68$ & 4 & 4 & 0 & 36 \\
\hline $2219001 \mathrm{~A} 69$ & 4 & 4 & $\therefore$ & 37 \\
\hline $220 \% 009 A 6 a$ & & 4 & 0 & 30 \\
\hline $220 \mathrm{H} 008 \mathrm{~A} 6 \mathrm{9}$ & 4 & 4 & 0 & 39 \\
\hline $220 \mathrm{H} 006 \mathrm{A6a}$ & 4 & 4 & 0 & 40 \\
\hline $220 \mathrm{HO05A68}$ & 1 & 4 & 0 & 41 \\
\hline $220 \mathrm{H} 004 \mathrm{~A} 68$ & 4 & 4 & 0 & 42 \\
\hline $320 \mathrm{H176A68}$ & 4 & 4 & 0 & 43 \\
\hline $320 H 177 A 68$ & 4 & 4 & 0 & 44 \\
\hline $320 \mathrm{H} 178 \mathrm{~A} 6 \mathrm{~B}$ & 4 & 4 & 0 & 45 \\
\hline $215 \mathrm{H} 027 \mathrm{A6O}$ & 4 & 4 & $\circ$ & 46 \\
\hline $\begin{array}{l}215 \mathrm{HO26A68} \\
215 \mathrm{H} 025 \mathrm{~A} 68\end{array}$ & 4 & 4 & $: 0$ & $\begin{array}{l}47 \\
48\end{array}$ \\
\hline $\begin{array}{l}215 \mathrm{H} 025 \mathrm{A68} \\
215 \mathrm{H} 024 \mathrm{A68}\end{array}$ & 4 & 4 & 0 & 48 \\
\hline $215 \mathrm{H} 023 A 68$ & 4 & 4 & 0 & so \\
\hline $215 \mathrm{H}_{022 \mathrm{~A}} \mathrm{~B}$ & & 4 & 0 & 51 \\
\hline 211H043A69 & 6 & 6 & 0 & 01 \\
\hline $\begin{array}{l}211 \mathrm{HO42A69} \\
321 \mathrm{H} 274 \mathrm{~A} 69\end{array}$ & $\begin{array}{l}6 \\
6\end{array}$ & $\begin{array}{l}6 \\
6\end{array}$ & $:$ & $\begin{array}{l}02 \\
03\end{array}$ \\
\hline $\begin{array}{l}321 \mathrm{H} 174 \mathrm{AGg} \\
321 \mathrm{H175A69}\end{array}$ & 6 & 6 & 0 & 04 \\
\hline & 6 & 6 & 0 & os \\
\hline 323 H195A69 & 6 & 6 & 0 & 06 \\
\hline $323 \mathrm{H} 186 A 69$ & 6 & 6 & 0 & 07 \\
\hline 323 H187A69 & 6 & 6 & 0 & 08 \\
\hline $214 H 030 A 69$ & 6 & 6 & 0 & 09 \\
\hline $214 H 029 A 69$ & 6 & 6 & 0 & 10 \\
\hline $2144028 A 69$ & 6 & 6 & 0 & 11 \\
\hline $\begin{array}{l}214 \mathrm{H}_{031 \mathrm{C} 69} \\
216 \mathrm{H020A69}\end{array}$ & $\begin{array}{l}6 \\
6\end{array}$ & $\begin{array}{l}6 \\
6\end{array}$ & $\begin{array}{l}0 \\
0\end{array}$ & $\begin{array}{l}12 \\
13\end{array}$ \\
\hline $216 \mathrm{HO} 19 \mathrm{A69}$ & 6 & 6 & 0 & 14 \\
\hline $216 \mathrm{HO} 18 \mathrm{AB} 9$ & 6 & 6 & 0 & 15 \\
\hline $218 \mathrm{H} 015 \mathrm{~A} 69$ & 6 & 6 & 0 & 26 \\
\hline 218H014A69 & 6 & 6 & 0 & 17 \\
\hline $218 \mathrm{HO} 13 \mathrm{A69}$ & 6 & 6 & 0 & 18 \\
\hline $322 \mathrm{H} 180 \mathrm{~A} 69$ & 6 & 6 & 0 & 19 \\
\hline $322 \mathrm{H} 189 \mathrm{~A} 69$. & 6 & 6 & 0 & 20 \\
\hline $322 \mathrm{H} 190 \mathrm{~A} 69$ & 6 & 6 & $\therefore$ & 21 \\
\hline $216 \mathrm{HO21A69}$ & 6 & 6 & 0 & 22 \\
\hline $219 \mathrm{HO} 10 \mathrm{A69}$ & 6 & 6 & 0 & 23 \\
\hline $220 K 007 A 69$ & 6 & 6 & 0 & 24 \\
\hline 213 HO3SA69 & 6 & 6 & 0 & 25 \\
\hline $213 \mathrm{H} 036 \mathrm{A69}$ & 6 & 6 & 0 & 26 \\
\hline $213 \mathrm{HO37A69}$ & 6 & 6 & : & 27 \\
\hline 213 H032A69 & 6 & 6 & 0 & 28 \\
\hline $213 \mathrm{HO} 33 \mathrm{~A} 69$ & 6 & 6 & 0 & 29 \\
\hline $213 \mathrm{H} 034 A 69$ & 6 & 6 & 0 & 30 \\
\hline 219 H011A69 & 6 & 6 & 0 & 31 \\
\hline 219HO12A69 & 6 & 6 & 0 & 32 \\
\hline $217 \mathrm{HO17A69}$ & 6 & 6 & 0 & 33 \\
\hline $217 H 016269$ & 6 & 6 & 0 & 34 \\
\hline 22 1H003A69 & 6 & 6 & 0 & 35 \\
\hline $221 \mathrm{H} 002 \mathrm{~A} 69$ & 6 & 6 & 0 & 36 \\
\hline $2214001 \mathrm{A69}$ & 6 & 6 & 0 & 37 \\
\hline $220 \mathrm{HOOSAGO}$ & 6 & 6 & 0 & 38 \\
\hline $220 \mathrm{H} 008 \mathrm{AB} 9$ & 6 & 6 & 0 & 39 \\
\hline $220 \mathrm{H006A69}$ & 6 & 6 & 0 & 40 \\
\hline $220 \mathrm{HOOSA69}$ & 6 & 6 & 0 & 41 \\
\hline $220 \mathrm{HOOAA69}$ & 6 & 6 & 0 & 42 \\
\hline 320H176A69 & 6 & 6 & 0 & 43 \\
\hline 32OHL77AG9 & 6 & 6 & 0 & 44 \\
\hline $3204178 \times 69$ & 6 & 6 & 0 & 45 \\
\hline $215 \mathrm{H} 027 \mathrm{A69}$ & 6 & 6 & 0 & 46 \\
\hline $225 \mathrm{H}_{026 \mathrm{~A} 69}$ & 6 & 6 & 0 & 47 \\
\hline $215 H 025 A 69$ & 6 & 6 & 0 & 48 \\
\hline 215H024A69 & 6 & 6 & 0 & 49 \\
\hline $215 \mathrm{H} 023 \mathrm{A69}$ & 6 & 6 & 0 & 50 \\
\hline $215 H 022 A 69$ & 6 & 6 & 0 & 51 \\
\hline $211 \mathrm{HO4} 3 \mathrm{~A} 70$ & 4 & 4 & 0 & 01 \\
\hline $211 H 042 A 70$ & 4 & 4 & 0 & 02 \\
\hline $32111774 A 70$ & 4 & 4 & 0 & 03 \\
\hline $321 K 175 A 70$ & 4 & 4 & 0 & 04 \\
\hline $211 \mathrm{H} 044270$ & 4 & 4 & 0 & 05 \\
\hline $323 \mathrm{HLOSA} 70$ & 4 & 4 & 0 & 06 \\
\hline $323 \mathrm{H} 286 \mathrm{~A} 70$ & 4 & 4 & 0 & 07 \\
\hline $323 \mathrm{H} 287270$ & & 4 & 0 & 08 \\
\hline $214 \mathrm{HO30A70}$ & 4 & 4 & 0 & 09 \\
\hline 2144029970 & 4 & 4 & 0 & 10 \\
\hline $214 \mathrm{H} 028 A 70$ & 4 & 4 & 0 & 12 \\
\hline $214 \mathrm{HO} 31 \mathrm{~A} 70$ & 4 & 4 & 0 & 12 \\
\hline $216 \mathrm{HQ20A70}$ & & 4 & 0 & 13 \\
\hline 216HO19A70 & + & 4 & 0 & 14 \\
\hline $216 \mathrm{HO} 18 \mathrm{AAO}$ & 4 & 4 & 0 & 15 \\
\hline 218 HO15A70 & 4 & 4 & 0 & 16 \\
\hline $218 \mathrm{HO14A7O}$ & 4 & 4 & 0 & 17 \\
\hline 213 HO23A70 & & 4 & 0 & 18 \\
\hline $322 \mathrm{H} 188 \mathrm{~A} 70$ & 4 & 4 & 0 & 19 \\
\hline $322 \mathrm{H} 109 \mathrm{A70}$ & 4 & 4 & 0 & 20 \\
\hline 322H190A70 & 4 & 4 & 0 & 21 \\
\hline $216 \mathrm{HO2} 1 \mathrm{~A} 70$ & 4 & 4 & 0 & 22 \\
\hline
\end{tabular}

Figure 9.19 - Case 2, Revised and Decelerated Schedule (Continued). 
JAUE S EBRAKIM ILABDULRAZZAK EST

REPORT DATE $215 E P 9 S$ RUN NO. 205

Revd \& Decel Sehl by Typeal Actzivity \& Es
PR IMAVERA PROUECT PLANNER

SEVEN SEAS, CONSTRUCTION OF 51 HOUSES
Correcting Out-ot-Logie Progrese NSq

START DATE LAPR87 FIN DATE 3OMAR89"

DATA DATE LFEBGB PAGE NO. 32

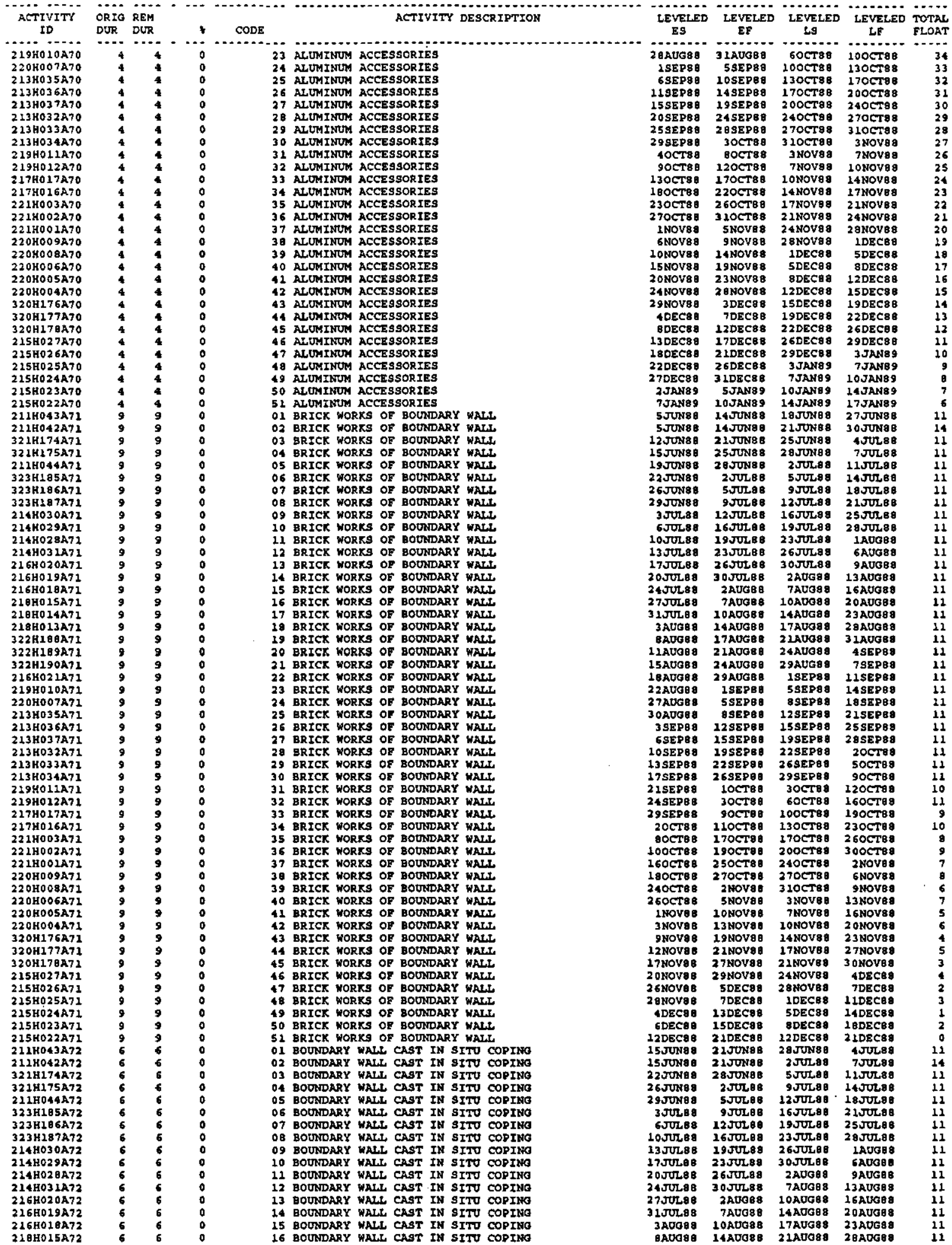

Figure 9.19 - Case 2, Revised and Decelerated Schedule (Continued). 


\begin{tabular}{|c|c|c|}
\hline $\begin{array}{c}\text { ACTIVITY } \\
\text { ID }\end{array}$ & $\begin{array}{l}\text { ORIO } \\
\text { DUR }\end{array}$ & $\begin{array}{l}\text { REM } \\
\text { DUR } \\
. .\end{array}$ \\
\hline $218 \mathrm{HO} 14 \mathrm{A72}$ & 6 & 6 \\
\hline $2184013 A 72$ & 6 & 6 \\
\hline $322 H 188 A 72$ & 6 & 6 \\
\hline $322 \mathrm{H189A72}$ & 6 & 6 \\
\hline $322 \mathrm{H} 190 \mathrm{~A} 72$ & 6 & 6 \\
\hline $216 H 021 \mathrm{~A} 72$ & 6 & 6 \\
\hline $219 \mathrm{HO} 10 \mathrm{~A} 72$ & 6 & 6 \\
\hline $220 \mathrm{OH} 007 \mathrm{A72}$ & 6 & 6 \\
\hline 213 HO035A72 & 6 & 6 \\
\hline $\begin{array}{l}213 \mathrm{H} 036 \mathrm{~A} 72 \\
213 \mathrm{HO} 7 \mathrm{~A} 72\end{array}$ & $\begin{array}{l}6 \\
6\end{array}$ & $\begin{array}{l}6 \\
6\end{array}$ \\
\hline $213 H_{0} 032 A 72$ & 6 & 6 \\
\hline $213 \mathrm{H} 033 \mathrm{A72}$ & 6 & 6 \\
\hline $213 \mathrm{H} 034 \mathrm{~A} 72$ & 6 & 6 \\
\hline $219 H 011 \mathrm{~A} 72$ & 6 & 6 \\
\hline $219 \mathrm{HO} 2 \mathrm{AA72}$ & 6 & 6 \\
\hline 217H017A72 & 6 & 6 \\
\hline 217 H016A72 & 6 & 6 \\
\hline 221H003A72 & 6 & 6 \\
\hline 221H002A72 & 6 & 6 \\
\hline $221 \mathrm{H} 001 \mathrm{~A} 72$ & 6 & 6 \\
\hline $220 \mathrm{HOO9A72}$ & 6 & 6 \\
\hline $220 \mathrm{H} 000 \mathrm{A72}$ & 6 & 6 \\
\hline $220 \mathrm{H} 006 \mathrm{GA72}$ & 6 & 6 \\
\hline $220 \mathrm{OH} 05 \mathrm{5A72}$ & 6 & 6 \\
\hline $22 \mathrm{OH} 004 \mathrm{~A} 72$ & 6 & 6 \\
\hline $320 \mathrm{H}+76 \mathrm{~A} 72$ & 6 & 6 \\
\hline $320 \mathrm{H} 77 \mathrm{~A} 72$ & 6 & 6 \\
\hline $320 \mathrm{H178A72}$ & 6 & 6 \\
\hline $215 \mathrm{H027A72}$ & 6 & 6 \\
\hline $215 H 026 A 72$ & 6 & 6 \\
\hline 21SHO25A72 & 6 & 6 \\
\hline $215 H 024 A 72$ & 6 & 6 \\
\hline $215 \mathrm{H} 023 \mathrm{~A} 72$ & 6 & 6 \\
\hline $215 H 022 A 72$ & 6 & 6 \\
\hline $211 \mathrm{H} 043 \mathrm{~A} 73$ & 1 & 1 \\
\hline $211 \mathrm{HO42A73}$ & 1 & 1 \\
\hline $321 \% 174 A 73$ & 1 & 1 \\
\hline $321 \mathrm{H} 275 \mathrm{~A} 73$ & 1 & 1 \\
\hline $211 H 044 \mathrm{~A} 73$ & 1 & 1 \\
\hline 323 H185A73 & 1 & $\mathbf{1}$ \\
\hline $323 \mathrm{H} 186 \mathrm{~A} 73$ & 1 & 1 \\
\hline $323 K 187 A 73$ & 1 & 2 \\
\hline $214 \mathrm{KO}^{2} 0 \mathrm{~A} 73$ & 1 & 1 \\
\hline $214 \mathrm{HO} 29 \mathrm{~A} 73$ & 1 & 1 \\
\hline $214 \mathrm{HO2}^{28 \mathrm{~A} 73}$ & 2 & 1 \\
\hline $2148031 \mathrm{AA73}$ & 2 & 1 \\
\hline $216 \mathrm{H} 020 \mathrm{~A} 73$ & 1 & 1 \\
\hline $216 \mathrm{H0} 19 \mathrm{~A} 73$ & 1 & 1 \\
\hline $216 \mathrm{HO} 1 \mathrm{GA73}$ & 1 & 1 \\
\hline $218 \mathrm{HO} 15 \mathrm{A73}$ & 1 & 1 \\
\hline 218H014A73 & 1 & 1 \\
\hline 218H013A73 & 1 & 1 \\
\hline $322 \mathrm{H} 18 \mathrm{gA73}$ & 1 & 1 \\
\hline $322 \mathrm{H} 189 \mathrm{~A} 73$ & 1 & 1 \\
\hline $322 \mathrm{H} 190 \mathrm{OA} 73$ & 1 & 1 \\
\hline $216 \mathrm{H} 021 \mathrm{~A} 73$ & 1 & 1 \\
\hline $219 \mathrm{HO} 10 \mathrm{~A} 73$ & 1 & 1 \\
\hline $220 \mathrm{H} 007 \mathrm{A73}$ & 1 & 1 \\
\hline 213 H035A73 & 1 & 1 \\
\hline $213 \mathrm{H}_{036 \mathrm{~A}} \mathrm{3}$ & 1 & 1 \\
\hline $213 \mathrm{~K} 037 \mathrm{A73}$ & 1 & 3 \\
\hline $213 \mathrm{H} 032 \mathrm{~A} 73$ & 1 & 1 \\
\hline $213 \mathrm{H} 033 \mathrm{~A} 73$ & 1 & 1 \\
\hline 213 H034A73 & 1 & 1 \\
\hline $219 \mathrm{HO} 1 \mathrm{AA73}$ & 1 & 1 \\
\hline $219 \mathrm{HO} 12 \mathrm{~A} 73$ & 1 & 1 \\
\hline $217 \mathrm{H017A73}$ & 1 & 1 \\
\hline $217 \mathrm{H} 016 \mathrm{~A} 73$ & 1 & 1 \\
\hline 221H003A73 & 1 & 1 \\
\hline 221H002A73 & 1 & 1 \\
\hline $221 H 001 A 73$ & 1 & 1 \\
\hline $220 \mathrm{HOO}^{2} 73$ & 1 & 1 \\
\hline $220 \mathrm{H008A73}$ & 1 & 1 \\
\hline $220 \mathrm{HO06A73}$ & 1 & 1 \\
\hline $220 \mathrm{HOOSA73}$ & 1 & 1 \\
\hline $220 \mathrm{H004A73}$ & 1 & 1 \\
\hline $320 \mathrm{H} 176 \mathrm{~A} 73$ & 1 & 1 \\
\hline $320 \mathrm{HL77A73}$ & 1 & 1 \\
\hline $320 \mathrm{H} 17 \mathrm{OA}^{2} 3$ & 1 & 1 \\
\hline $2154027 \mathrm{A73}$ & 1 & 1 \\
\hline $215 \mathrm{H} 026 \mathrm{A7} 3$ & 1 & 1 \\
\hline $215 \mathrm{H} 025 \mathrm{~A} 73$ & 1 & 1 \\
\hline $215 \mathrm{HO24A73}$ & 1 & 2 \\
\hline $215 H 023 A 73$ & 1 & 1 \\
\hline $215 \mathrm{H} 022 \mathrm{~A} 73$ & 1 & 1 \\
\hline $211 \% 043 A 74$ & 10 & 10 \\
\hline $211 H 042 A 74$ & 10 & 10 \\
\hline 321H174A74 & 10 & 10 \\
\hline $321 \mathrm{H175A74}$ & 10 & 10 \\
\hline $211 H 044 A 74$ & 10 & 10 \\
\hline 323 H185A74 & 10 & 10 \\
\hline 323 H186A74 & 10 & 10 \\
\hline $323 \mathrm{H} 187 \times 74$ & 10 & 10 \\
\hline $214 \mathrm{HO}^{2} 0 \mathrm{~A} 74$ & 10 & 10 \\
\hline $214 \mathrm{HO29A74}$ & 10 & 10 \\
\hline
\end{tabular}

Figure 9.19 - Case 2, Revised and Decelerated Schedule (Continued). 


\begin{tabular}{|c|c|}
\hline $\begin{array}{l}\text { ACTIVITY } \\
\text { ID }\end{array}$ & $\begin{array}{l}\text { ORIO } \\
\text { DUR }\end{array}$ \\
\hline $214 \mathrm{HO28A74}$ & 20 \\
\hline $214 \mathrm{HO} 31 \mathrm{~A} 74$ & 10 \\
\hline $21615020 A 74$ & 10 \\
\hline $216 \mathrm{HO} 19 \mathrm{AT}$ & 10 \\
\hline $216 \mathrm{HO1OA74}$ & 10 \\
\hline 21AHO15A74 & 10 \\
\hline $218 H 014 A 74$ & 10 \\
\hline $218 \mathrm{BO} 13 \mathrm{~A} 74$ & 10 \\
\hline $322 \mathrm{H} 18 \mathrm{BA74}$ & 10 \\
\hline $322 \mathrm{H} 189974$ & 10 \\
\hline $322 \mathrm{H} 190 A 74$ & 10 \\
\hline $216 \mathrm{HO} 21 \mathrm{~A} 74$ & 10 \\
\hline $219 H 010 \mathrm{~A} 74$ & 10 \\
\hline $220 H 007 A 74$ & 10 \\
\hline $213 H 035 A 74$ & 10 \\
\hline $213 \mathrm{H} 036 \mathrm{~A} 74$ & 10 \\
\hline $2134037 A 74$ & 10 \\
\hline $2134032 \mathrm{~A} 74$ & 10 \\
\hline $2134033 A 74$ & 10 \\
\hline $213 H 034 A 74$ & 10 \\
\hline $219 \mathrm{HO11 \textrm {A } 7 4}$ & 10 \\
\hline $219 H 012 \mathrm{~A} 74$ & 10 \\
\hline 217 H017A74 & 10 \\
\hline $217 \mathrm{HO} 16 \mathrm{~A} 74$ & 10 \\
\hline $221 \% 003 \mathrm{A74}$ & 10 \\
\hline $221 \% 002 A 74$ & 10 \\
\hline $221 H 002 A 74$ & 10 \\
\hline $220 \mathrm{HOOSA74}$ & 20 \\
\hline 2204008874 & 10 \\
\hline $220 \% 006 \mathrm{~A} 74$ & 10 \\
\hline $220 \mathrm{HOO5A74}$ & 10 \\
\hline $2204004 A 74$ & 10 \\
\hline $320 \mathrm{H} 176 \mathrm{A74}$ & 10 \\
\hline $320 \mathrm{H177A74}$ & 10 \\
\hline $320 \mathrm{HA} 78 \mathrm{~A} 74$ & 10 \\
\hline $215 \mathrm{HO}^{27 A 74}$ & 10 \\
\hline $215 H 026 A 74$ & 10 \\
\hline $215 \mathrm{H} 025 \mathrm{~A} 74$ & 10 \\
\hline 215 H024A74 & 10 \\
\hline $215 H 023 A 74$ & 10 \\
\hline $225 H 022 A 74$ & 10 \\
\hline 211H043A75 & 5 \\
\hline $211 H 042 A 75$ & 5 \\
\hline $32111174 A 75$ & 5 \\
\hline 3211175A75 & $\mathbf{5}$ \\
\hline $211 K 044 A 75$ & 5 \\
\hline $323 \mathrm{H} 1 \mathrm{BSA75}$ & 5 \\
\hline $323 \mathrm{HB} 6 \mathrm{AA75}$ & 5 \\
\hline & 5 \\
\hline $\begin{array}{l}2144030 A 75 \\
214 H 029 A 75\end{array}$ & $\mathbf{5}$ \\
\hline $214 H 029 \mathrm{A75}$ & 5 \\
\hline $214 \mathrm{HO} 028 \mathrm{~A} 75$ & 5 \\
\hline $214 \mathrm{H} 03$ LA75 & $\mathbf{s}$ \\
\hline $216 \mathrm{HO20A75}$ & 5 \\
\hline $216 \mathrm{HO} 19 \mathrm{9A5}$ & 5 \\
\hline $216 \mathrm{H} 018 \mathrm{AA75}$ & 5 \\
\hline $218 \mathrm{HO} 25 \mathrm{~A} 7 \mathrm{~S}$ & 5 \\
\hline $218 \mathrm{HO} 24 \mathrm{~A} 75$ & 5 \\
\hline $218 \mathrm{HO} 23 \mathrm{AA5}$ & 5 \\
\hline 322H188A75 & 5 \\
\hline $322 \mathrm{H} 199 \mathrm{~A} 75$ & 5 \\
\hline $322 \mathrm{H} 190 \mathrm{~A} 75$ & 5 \\
\hline $216 \mathrm{H021A75}$ & s \\
\hline $\begin{array}{l}219 H 010 \mathrm{A75} \\
220 \mathrm{OHOO} \mathrm{A} 75\end{array}$ & s \\
\hline $\begin{array}{l}220 H 007 A 75 \\
213 \text { H035A75 }\end{array}$ & $\begin{array}{l}5 \\
5\end{array}$ \\
\hline 213 213035A75 & $\mathbf{s}$ \\
\hline $213 \mathrm{H036A75}$ & 5 \\
\hline $2134037 A 75$ & 5 \\
\hline 213 H032A75 & 5 \\
\hline $2134033 A 75$ & 5 \\
\hline $213 H 034 A 75$ & 5 \\
\hline $219 H 011 \mathrm{~A} 75$ & 5 \\
\hline $2194012 A 75$ & 5 \\
\hline $217 \mathrm{H} 027 \mathrm{A75}$ & 5 \\
\hline 217 HO26A75 & 5 \\
\hline $221 \mathrm{H} 003 \mathrm{~A} 75$ & 5 \\
\hline $221 \mathrm{H} 002 \mathrm{~A} 75$ & 5 \\
\hline $222 \mathrm{H} 001 \mathrm{A75}$ & 5 \\
\hline $22 \mathrm{OH} 009 \mathrm{ATS}$ & 5 \\
\hline $220 \mathrm{H0OBA75}$ & 5 \\
\hline $220 \mathrm{H} 006 \mathrm{AA7}$ & 5 \\
\hline $220 \mathrm{H} 005 \mathrm{~A} 75$ & 5 \\
\hline $220 \mathrm{OHO} 04 \mathrm{ATS}$ & 3 \\
\hline $320 \mathrm{H} 276 \mathrm{~A} 75$ & 5 \\
\hline $320 \mathrm{H} 177 \mathrm{~A} 75$ & \\
\hline $320 \mathrm{H178A75}$ & $\mathbf{5}$ \\
\hline 215H027A75 & 5 \\
\hline 215H026A75 & $\mathbf{5}$ \\
\hline $215 \mathrm{HO} 25 \mathrm{~A} 75$ & 5 \\
\hline $215 \mathrm{HO} 24 \mathrm{~A} 75$ & 5 \\
\hline $215 H 023 A 75$ & $\mathbf{5}$ \\
\hline $215 \mathrm{HO} 22 \mathrm{~A} 75$ & 5 \\
\hline $211 H 043 A 76$ & 2 \\
\hline 211 H042A76 & 2 \\
\hline $321 H 274 A 76$ & 2 \\
\hline $321 H 175 A 76$ & $i$ \\
\hline
\end{tabular}

Figure 9.19 - Case 2, Revised and Decelerated Schedule (Continued). 


\begin{tabular}{|c|c|}
\hline $\begin{array}{c}\text { ACTIVITY } \\
\text { ID }\end{array}$ & $\begin{array}{l}\text { ORIO } \\
\text { DURR }\end{array}$ \\
\hline $2124044 A 76$ & 2 \\
\hline $323 \mathrm{H} 195 \mathrm{~A} 76$ & 2 \\
\hline $323 \mathrm{H1B6A76}$ & 2 \\
\hline 323 स187A76 & 2 \\
\hline $214 H 030 A 76$ & 2 \\
\hline $214 H 029 A 76$ & 2 \\
\hline $214 \mathrm{HO2BA76}$ & 2 \\
\hline $214 \mathrm{HO} 031 \mathrm{A76}$ & 2 \\
\hline $216 \mathrm{H} 020 \mathrm{A76}$ & 2 \\
\hline .216H019A76 & 2 \\
\hline $216 \mathrm{H} 018 \mathrm{~A} 76$ & 2 \\
\hline 218 H015A76 & 2 \\
\hline $218 H 014 A 76$ & 2 \\
\hline $218 H 013 A 76$ & 2 \\
\hline $322 \mathrm{H} 18 \mathrm{QA} 76$ & 2 \\
\hline $322 \mathrm{HIOSA76}$ & 2 \\
\hline $322 \mathrm{HL90A76}$ & 2 \\
\hline $216 H 021 A 76$ & 2 \\
\hline $2194010 \mathrm{~A} 76$ & 2 \\
\hline $22 \mathrm{OH} 007 \mathrm{A76}$ & 2 \\
\hline 213 H035A76 & 2 \\
\hline $213 \mathrm{H} 036 \mathrm{~A} 76$ & 2 \\
\hline $213 H 037 A 76$ & 2 \\
\hline $213 H 032 A 76$ & 2 \\
\hline $2134033 A 76$ & 2 \\
\hline $213 \mathrm{H} 034 \mathrm{A76}$ & 2 \\
\hline $219 \mathrm{H011A76}$ & 2 \\
\hline $219 \mathrm{HO} 2 \mathrm{~A} 76$ & 2 \\
\hline $217 H 017 A 76$ & 2 \\
\hline $217 \mathrm{HO} 016 \mathrm{7} 6$ & 2 \\
\hline $221 \% 003 A 76$ & 2 \\
\hline $221 \mathrm{HO02A76}$ & 2 \\
\hline $221 \mathrm{H0O1A76}$ & 2 \\
\hline 220 H009A76 & 2 \\
\hline $220 \mathrm{HO08A76}$ & 2 \\
\hline $220 \mathrm{H006A76}$ & 2 \\
\hline $2201005 A 76$ & 2 \\
\hline $220 \mathrm{HO04A76}$ & 2 \\
\hline $320 H 176 A 76$ & 2 \\
\hline $320 H 177 A 76$ & 2 \\
\hline $320 \mathrm{H178A76}$ & 2 \\
\hline $215 \mathrm{HO2}^{2} \mathrm{~A} 76$ & 2 \\
\hline $215 K 026 A 76$ & 2 \\
\hline $215 \mathrm{HO} 25 \mathrm{~A} 76$ & 2 \\
\hline $215 H 024 A 76$ & 2 \\
\hline $215 \mathrm{H} 023 \mathrm{~A} 76$ & 2 \\
\hline 215H022A76 & 2 \\
\hline $211 \mathrm{H} 043 \mathrm{~A} 77$ & 5 \\
\hline $21114042 A 77$ & 5 \\
\hline $321 \% 174 A 77$ & 5 \\
\hline $3218175 A 77$ & $\mathbf{s}$ \\
\hline 211H044A77 & 5 \\
\hline $323 \times 185 A 77$ & 5 \\
\hline 323 H186A77 & 5 \\
\hline $323 \mathrm{H} 187 \mathrm{AT7}$ & 5 \\
\hline 214H030A77 & 5 \\
\hline 214 HO29A77 & 5 \\
\hline $214 H 028 A 77$ & 5 \\
\hline $2148031 A 77$ & 5 \\
\hline $216 \mathrm{H020A77}$ & 5 \\
\hline 226H019A77 & 5 \\
\hline 226H018A77 & 5 \\
\hline 218 1915A77 & 5 \\
\hline 21BH014A77 & 5 \\
\hline 210HO13A77 & 5 \\
\hline 322H188A77 & 5 \\
\hline $322 \mathrm{HLB} 9 \mathrm{A77}$ & 5 \\
\hline $322 \mathrm{H190A27}$ & 5 \\
\hline $2161022 \times 77$ & 5 \\
\hline $2194010 A 77$ & 5 \\
\hline $220 \mathrm{H} 007 \mathrm{A77}$ & 5 \\
\hline $213 H 035 A 77$ & $\mathbf{5}$ \\
\hline $213 \mathrm{HO} 036 \mathrm{A77}$ & 5 \\
\hline $213 \mathrm{HO37A77}$ & 5 \\
\hline $2134032 A 77$ & 5 \\
\hline $213 \mathrm{H} 033 \mathrm{A77}$ & 5 \\
\hline $213 \mathrm{H} 034 \mathrm{A77}$ & $\mathbf{s}$ \\
\hline 219HO11A77 & 5 \\
\hline $219 \mathrm{HO212A77}$ & $\mathbf{s}$ \\
\hline $217 \mathrm{H017A77}$ & 5 \\
\hline $217 \mathrm{H016A77}$ & 5 \\
\hline 221H003A77 & 5 \\
\hline $221 \mathrm{H} 002 \mathrm{A77}$ & 3 \\
\hline & 5 \\
\hline 220H009A77 & 5 \\
\hline $220 \mathrm{HOOBA77}$ & 5 \\
\hline $220 \mathrm{H} 006 \mathrm{A77}$ & $\mathbf{5}$ \\
\hline $220 \mathrm{HO05A77}$ & 5 \\
\hline $220 \mathrm{HOO4A77}$ & 5 \\
\hline $320 \mathrm{H176A77}$ & 5 \\
\hline $320 \mathrm{H} 177 A 77$ & 5 \\
\hline $320 \mathrm{H178A7?}$ & 5 \\
\hline $215 H 027 A 77$ & 5 \\
\hline $215 H 026 A 77$ & 5 \\
\hline $215 \mathrm{H} 025 \mathrm{~A} 77$ & 5 \\
\hline $215 \mathrm{H024A77}$ & $\mathbf{s}$ \\
\hline
\end{tabular}

Figure 9.19 - Case 2, Revised and Decelerated Schedule (Continued). 
SALD \& EGRAKIM iLABDULRAzZAK EST.

PEPORT DATE 21SEP95 RUN NO. 205

Revd $18: 32$
PRIMAVERA PROJECT PLANNER

SEVEN SEAS, CONSTRUCTION OF 51 HOUSES
Corrocting Out-of-Logic Progrese NSq

START DATE 1APRE7 FIN DATE 3OMARg9-

DATA DATE 1FEB日B PAGE NO, 36

\begin{tabular}{|c|c|}
\hline $\begin{array}{c}\text { ACTIVITY } \\
\text { ID }\end{array}$ & $\begin{array}{l}\text { ORIG } \\
\text { DUR }\end{array}$ \\
\hline $215 H 023 A 77$ & 5 \\
\hline $225 \mathrm{HO22A77}$ & $\mathbf{5}$ \\
\hline $211 H 043 A 78$ & 3 \\
\hline $211 \mathrm{H042A78}$ & 3 \\
\hline $321 \mathrm{H} 174 \mathrm{~A} 78$ & 3 \\
\hline $321 \mathrm{H} 175 \mathrm{~A} 78$ & 3 \\
\hline $211 \mathrm{H} 044 \mathrm{~A} 78$ & 3 \\
\hline $323 \mathrm{H} 185 \mathrm{~A} 78$ & 3 \\
\hline ' $323 \mathrm{H} 186 \mathrm{AA7}$ & 3 \\
\hline $323 H 187 A 78$ & 3 \\
\hline $214 \mathrm{H030A78}$ & 3 \\
\hline $214 H 029 A 7 B$ & 3 \\
\hline $21+1028 A 78$ & 3 \\
\hline $214 H 032 A 78$ & 3 \\
\hline 216H020A78 & 3 \\
\hline $216 \mathrm{H019A78}$ & 3 \\
\hline $226 \mathrm{H018A7 \theta}$ & 3 \\
\hline $218 H 015 A 7 \mathrm{~g}$ & 3 \\
\hline $210 H 014 A 78$ & 3 \\
\hline 21日H013A78 & 3 \\
\hline $322 \mathrm{H} 188 \mathrm{~A} 78$ & 3 \\
\hline $322 \mathrm{H} 199 \mathrm{~A} 7 \mathrm{~B}$ & 3 \\
\hline $322 \mathrm{H} 190 \mathrm{OA} 7 \mathrm{~B}$ & 3 \\
\hline $216 H 021 A 7 B$ & 3 \\
\hline $219 H 010 A 78$ & 3 \\
\hline $2301007 A 78$ & 3 \\
\hline $223 \mathrm{H} 035 \mathrm{~A} 7 \mathrm{~B}$ & 3 \\
\hline $213 H 036 A 7 B$ & 3 \\
\hline $213 H 037 A 78$ & 3 \\
\hline $213 \mathrm{H} 032 \mathrm{~A} 7 \mathrm{~g}$ & 3 \\
\hline $213 \mathrm{H} 033 \mathrm{~A} 7 \mathrm{~B}$ & 3 \\
\hline $213 \mathrm{H034A78}$ & 3 \\
\hline $2194013 A 7 \mathrm{~g}$ & 3 \\
\hline $219 H 012 A 7 \theta$ & 3 \\
\hline 217H017A78 & 3 \\
\hline $217 \mathrm{H016A78}$ & 3 \\
\hline $221 \mathrm{H003A7 \theta}$ & 3 \\
\hline $2214002 A 78$ & 3 \\
\hline $221 H 002 A 78$ & 3 \\
\hline $220 \mathrm{H0O9A78}$ & 3 \\
\hline $220 \mathrm{HOOBA78}$ & 3 \\
\hline $220 \mathrm{HOO6A78}$ & 3 \\
\hline $220 \mathrm{HOOSA78}$ & 3 \\
\hline $220 \mathrm{HOO4A78}$ & 3 \\
\hline $320 H 176 A 79$ & 3 \\
\hline 320H177A78 & 3 \\
\hline $320 \mathrm{H} 178 \mathrm{~A} 78$ & 3 \\
\hline $225 H 027 A 78$ & 3 \\
\hline $215 \mathrm{H026A79}$ & 3 \\
\hline $215 \mathrm{H} 025 \mathrm{~A} 7 \mathrm{\theta}$ & 3 \\
\hline $215 \mathrm{H} 024 \mathrm{~A} 7 \mathrm{~g}$ & 3 \\
\hline $215 \mathrm{H023A7 \theta}$ & 3 \\
\hline $215 \mathrm{H} 022 \mathrm{~A} 7 \mathrm{~g}$ & 3 \\
\hline $211 \mathrm{H043A79}$ & 4 \\
\hline $221 \mathrm{H} 042 \mathrm{~A} 79$ & 4 \\
\hline $321 \mathrm{H} 174 \mathrm{~A} 79$ & 4 \\
\hline $321 H 175 A 79$ & \\
\hline 211H044A79 & 4 \\
\hline $3233185 \mathrm{A79}$ & 4 \\
\hline $323 \mathrm{H} 186 \mathrm{AA9}$ & 4 \\
\hline $323 \mathrm{H187A79}$ & 4 \\
\hline $214 \mathrm{H} 030 \mathrm{~A} 79$ & 4 \\
\hline $214 H 029 A 79$ & 4 \\
\hline $214 H 02$ BA79 & 4 \\
\hline $214 \mathrm{H031A79}$ & 4 \\
\hline $2164020 A 79$ & 4 \\
\hline $216 \mathrm{HO19A79}$ & \\
\hline 216H018A79 & 4 \\
\hline 219H015A79 & 4 \\
\hline 218Н014А79 & 4 \\
\hline $218 \mathrm{H} 013 \mathrm{~A} 79$ & + \\
\hline $322 \mathrm{H} 18 \mathrm{BA} 79$ & \\
\hline 322H189A79 & 4 \\
\hline $322 H 190 A 79$ & 4 \\
\hline $216 \mathrm{KO21A79}$ & 4 \\
\hline 219 H010A79 & 4 \\
\hline $220 \mathrm{H} 007 \mathrm{~A} 79$ & \\
\hline $213 H 035 A 79$ & 4 \\
\hline 213 H036A79 & 4 \\
\hline $213 H 037 A 79$ & 4 \\
\hline $213 \mathrm{H} 032 A 79$ & 4 \\
\hline $223 H 033 A 79$ & \\
\hline $213 H 034 A 79$ & 4 \\
\hline $219 H 011 \mathrm{A79}$ & 4 \\
\hline $2194012 A 79$ & 4 \\
\hline $217 \mathrm{H} 017 \mathrm{~A} 79$ & 4 \\
\hline $2174016 A 79$ & \\
\hline $221 \% 003 A 79$ & 4 \\
\hline 221H002A79 & \\
\hline $22111001 \mathrm{~A} 79$ & 4 \\
\hline $220 \mathrm{H} 009 \mathrm{~A} 79$ & 4 \\
\hline $220 \mathrm{HOOBA79}$ & 4 \\
\hline 220 H006A79 & 4 \\
\hline 220H005A79 & 4 \\
\hline $220 H 004 A 79$ & 4 \\
\hline $320 \mathrm{H}_{176 A 79}$ & 4 \\
\hline
\end{tabular}

Figure 9.19 - Case 2, Revised and Decelerated Schedule (Continued). 
SAND \& ERRAHIM ALABDULRAZZAK EST.

REPORT LATE IISEP95 RUN NO. 205

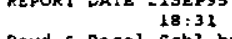

PRIMAVERA PROJECT PLANAER

SEVEN SEAS, CONSTRUCTION OF 51 HOUSES
Corrocting Out-of-Logic Progrens NSq

START DATE LAPRgT FIN DATE 3OMAR89*

DATA DATE 1 FEBas PAGE No.

\begin{tabular}{|c|c|c|}
\hline $\begin{array}{l}\text { ACTIVITY } \\
\text { ID }\end{array}$ & $\begin{array}{l}\text { ORIG } \\
\text { DUR } \\
-\end{array}$ & $\begin{array}{l}\text { REM } \\
\text { DUR }\end{array}$ \\
\hline $320 H 177 A 79$ & 4 & 4 \\
\hline $320 \mathrm{H} 178 \mathrm{~A} 79$ & & \\
\hline $215 H 027 A 79$ & 4 & 4 \\
\hline $215 H 026 A 79$ & 4 & 4 \\
\hline 215H025A79 & & 4 \\
\hline $215 H 024 A 79$ & 4 & 4 \\
\hline $215 \mathrm{H} 023 \mathrm{~A} 79$ & 4 & 1 \\
\hline $\begin{array}{l}215 \mathrm{HO22A79} \\
211 \mathrm{HO43A90}\end{array}$ & $\begin{array}{l}4 \\
1\end{array}$ & 1 \\
\hline $211 H 042 A 80$ & $i$ & 1 \\
\hline $321 \mathrm{H174ABO}$ & 1 & 1 \\
\hline 321H175A90 & 1 & 1 \\
\hline 211 HO44A9O & 1 & 1 \\
\hline 323H185A9O & 1 & 1 \\
\hline 323 H1B6A80 & 1 & 1 \\
\hline $323 H 187 A 80$ & 3 & 1 \\
\hline 214 H03 OA8O & 1 & 1 \\
\hline $214 \% 029 A 80$ & 1 & 1 \\
\hline $214 \mathrm{HO2}$ OA8O & 1 & 1 \\
\hline $214 \mathrm{H} 031 \mathrm{~A} 80$ & 1 & 1 \\
\hline $216 \mathrm{HO2OA80}$ & 1 & 1 \\
\hline $216 \mathrm{HO} 19 \mathrm{A80}$ & 1 & 1 \\
\hline 216H01BA80 & $\mathbf{I}$ & 1 \\
\hline 218HO1SABO & 1 & 1 \\
\hline $218 \mathrm{HO} 14 \mathrm{ABO} 0$ & 1 & 1 \\
\hline 218HO23ABO & 1 & 1 \\
\hline 322H188ABO & 1 & 1 \\
\hline $322 \mathrm{H} 189 \mathrm{AgO}$ & 1 & 2 \\
\hline $322 \mathrm{H} 190 \mathrm{ABO}$ & 1 & 1 \\
\hline $216 \mathrm{HO2} 1 \mathrm{A \theta O}$ & 1 & 1 \\
\hline 219 HOLOABO & 1 & 1 \\
\hline $220{ }^{2} 007 A B O$ & 1 & 1 \\
\hline 213H035A8O & 1 & 1 \\
\hline 213 H036A90 & 1 & 1 \\
\hline 213Н037A90 & 1 & 2 \\
\hline $213 \mathrm{H} 032 \mathrm{ABO}$ & 1 & 1 \\
\hline $213 H 033 \mathrm{ABO}$ & 1 & 1 \\
\hline 213H034A9O & 1 & 1 \\
\hline $219 H 011 \mathrm{AOO}$ & 1 & 1 \\
\hline 219H012ABO & 1 & 1 \\
\hline $217 \mathrm{HO17ABO}$ & 1 & 1 \\
\hline $217 \mathrm{HO} 16 \mathrm{ABO}$ & 1 & 1 \\
\hline 221H003A80 & 1 & 1 \\
\hline $221 \mathrm{H} 002 \mathrm{~A} 80$ & 1 & 1 \\
\hline $221 \mathrm{HOO1A8O}$ & 1 & 1 \\
\hline $220 \mathrm{HOOSABO}$ & $\mathbf{1}$ & 1 \\
\hline 220 HOOBAOO & 1 & 1 \\
\hline $220 \mathrm{HOOGA9O}$ & 1 & 1 \\
\hline $220 \mathrm{HOOSA8O}$ & 1 & 1 \\
\hline $220 \mathrm{HOO4A80}$ & 1 & 1 \\
\hline $320 \mathrm{H176A90}$ & 1 & 1 \\
\hline $320 \mathrm{H} 177 \mathrm{~A} 00$ & 1 & 1 \\
\hline $320 \mathrm{HL78ABO}$ & 1 & 1 \\
\hline 215HO27A9O & 1 & 1 \\
\hline $215 \mathrm{HO} 6 \mathrm{6A80}$ & 1 & 1 \\
\hline $215 H 025 A 30$ & 1 & 1 \\
\hline 215 H024A8O & 1 & 2 \\
\hline 215H023A8O & 1 & 1 \\
\hline $21 \mathrm{SHO} 22 \mathrm{~A} 80$ & 1 & 2 \\
\hline $211 \mathrm{HO} 43 \mathrm{~A} 8 \mathrm{I}$ & 1 & 2 \\
\hline 211H042AO1 & 1 & 2 \\
\hline $321 \mathrm{H174AO1}$ & 1 & 1 \\
\hline $321 \mathrm{H175AO1}$ & 1 & 1 \\
\hline $211 \mathrm{HO44AO1}$ & 1 & 1 \\
\hline $323 \mathrm{H185A81}$ & 1 & 1 \\
\hline $323 H 186 A 81$ & 1 & 1 \\
\hline $323 \mathrm{H197A81}$ & 1 & 1 \\
\hline $214 H 030 A 81$ & 1 & 1 \\
\hline $214 \mathrm{HO29AO1}$ & 1 & 1 \\
\hline 214H028ABI & 1 & 2 \\
\hline $214 \mathrm{HO3LAQ2}$ & 1 & 1 \\
\hline $216 \mathrm{HO2OA91}$ & 1 & 1 \\
\hline 216H019AS1 & 1 & 1 \\
\hline 216H018A91 & 1 & 2 \\
\hline 21日H015ABI & 1 & 1 \\
\hline 218HO14ABI & 1 & 1 \\
\hline $21 \mathrm{BH} 013 \mathrm{AaI}$ & 1 & 1 \\
\hline 322H1日9AB1 & 1 & 1 \\
\hline 322H189AB1 & 1 & 1 \\
\hline $322 \mathrm{~K} 190 \mathrm{ABI}$ & 1 & 2 \\
\hline $216 \mathrm{HO} 2 \mathrm{LAOL}$ & 1 & 1 \\
\hline $219 H 010 A B 1$ & 1 & 1 \\
\hline $2201007 A B 1$ & 1 & 1 \\
\hline 213 H035ABI & 1 & 1 \\
\hline 213 H036AB1 & 1 & 1 \\
\hline $213 H 037 A 81$ & 1 & 1 \\
\hline $213 \mathrm{HO32ABI}$ & 1 & 1 \\
\hline $213 \mathrm{BO} 033 \mathrm{ABI}$ & 1 & 1 \\
\hline $213 H 034 A 81$ & 1 & 1 \\
\hline $219 H 011 A 81$ & 1 & 1 \\
\hline 219H012A81 & 1 & 1 \\
\hline $2174017 A 81$ & 1 & 1 \\
\hline 217H016A81 & 1 & 1 \\
\hline 221H003A81 & 1 & 1 \\
\hline $221 H 002 A B 1$ & 1 & 1 \\
\hline $221 \mathrm{H} 001 \mathrm{ABI}$ & 1 & 2 \\
\hline
\end{tabular}

Figure 9.19 - Case 2, Revised and Decelerated Schedule (Continued). 
SAUD \& EBRAHIM ALABDULRAZZAK EST

REPORT DATE 21SEPSS RUN NO. 205

Rovd \& Decel Schl by Typical Aetivity \& ES
PRIMAVERA PROJECT PLWWNER

SEVEN SEAS, CONSTRUCTION OF 51 HOUSES
Corroct 2 g Out-of-Logle Progrees NSq
START DATE LAPR87 FIN DATE 3OMARg9*

DATA DATE 1FEBg PAGE No. 30

\begin{tabular}{|c|c|}
\hline $\begin{array}{c}\text { ACTIVITY } \\
\text { ID }\end{array}$ & $\begin{array}{l}\text { ORIG } \\
\text { DUR }\end{array}$ \\
\hline 2204009881 & \\
\hline $220 \mathrm{HOOBA81}$ & 1 \\
\hline $220 \mathrm{HOO6A91}$ & 1 \\
\hline $220 H 005 A 81$ & 1 \\
\hline $220 \mathrm{~K} 004 \mathrm{Aal}$ & 1 \\
\hline $320 \mathrm{H176A81}$ & 1 \\
\hline $3204177 A 91$ & 1 \\
\hline $320 H 17 B A 91$ & 1 \\
\hline $215 H 027 A B 1$ & 1 \\
\hline $235 H 026 \mathrm{AB1}$ & 1 \\
\hline $215 \mathrm{H} 025 \mathrm{AOI}$ & 1 \\
\hline $215 H 024 A 81$ & 1 \\
\hline $215 H 023 A 81$ & 1 \\
\hline $225 \mathrm{HO22A81}$ & 1 \\
\hline $2118043 A 82$ & 3 \\
\hline $211 \mathrm{H} 042 \mathrm{A82}$ & 3 \\
\hline $3214174 A 82$ & 3 \\
\hline $321 \mathrm{K175A82}$ & 3 \\
\hline $211 H 044282$ & 3 \\
\hline $323 H 185282$ & 3 \\
\hline $323 \mathrm{H} 196 \mathrm{~A} 82$ & 3 \\
\hline 32ЗН197АВ2 & 3 \\
\hline $21414030 A 82$ & 3 \\
\hline 214H029A82 & 3 \\
\hline $214 H 02$ BA82 & 3 \\
\hline 2144031482 & 3 \\
\hline $216 H 020 A 82$ & 3 \\
\hline $216 \% 029 A 82$ & 3 \\
\hline 216H018A82 & 3 \\
\hline $218 \mathrm{HO15AB2}$ & 3 \\
\hline $218 H O 14 A B 2$ & 3 \\
\hline $218 \mathrm{HOL13AO2}$ & 3 \\
\hline 322H1日8AB2 & 3 \\
\hline $322 \mathrm{HLOSAB2}$ & 3 \\
\hline $3221190 A B 2$ & 3 \\
\hline $216 \mathrm{H} 021 \mathrm{ABO2}$ & 3 \\
\hline 219HO10AB2 & 3 \\
\hline $220 \mathrm{HOOTAB2}$ & 3 \\
\hline $213 \mathrm{HO35A92}$ & 3 \\
\hline $213 \mathrm{HO} 36 \mathrm{AB2}$ & 3 \\
\hline $213 \mathrm{H} 03$ 7A日2 & 3 \\
\hline $213 \mathrm{HO} 2 \mathrm{AAO2}$ & 3 \\
\hline 213H033AR2 & 3 \\
\hline 213 HO34AB2 & 3 \\
\hline 219H012ABZ & 3 \\
\hline $219 \mathrm{HO12AB2}$ & 3 \\
\hline $217 \mathrm{HO17AO2}$ & 3 \\
\hline $217 \mathrm{HO16AB2}$ & 3 \\
\hline $221 H 003 A B 2$ & 3 \\
\hline $221 \mathrm{H} 002 \mathrm{ABZ}$ & 3 \\
\hline 221HOOLAB2 & 3 \\
\hline $22 O H O O 9 A B 2$ & 3 \\
\hline $220 \mathrm{HOOBAB2}$ & 3 \\
\hline $220 \mathrm{H} 006 \mathrm{AB2}$ & 3 \\
\hline $220 \mathrm{HOOSA82}$ & 3 \\
\hline $220 \mathrm{HOOAAS2}$ & 3 \\
\hline $320 H 176 A 92$ & 3 \\
\hline $320 H 277 A 82$ & 3 \\
\hline $320 \% 178 A 82$ & 3 \\
\hline $215 H 027 A 82$ & 3 \\
\hline $215 \mathrm{HO2} 6 \mathrm{A82}$ & 3 \\
\hline $215 \mathrm{H} 025 \mathrm{AB2}$ & 3 \\
\hline $215 H 024 A 82$ & 3 \\
\hline $2255023 A 82$ & 3 \\
\hline $225 H 022 A 82$ & 3 \\
\hline $211 \mathrm{HO} 33 \mathrm{A83}$ & \\
\hline $211 H 042 A 83$ & 4 \\
\hline $321 \mathrm{H174A83}$ & 4 \\
\hline $321 \mathrm{M175A83}$ & 4 \\
\hline $2111044 A 83$ & 4 \\
\hline 323H185A83 & \\
\hline $3233186 \mathrm{AB3}$ & \\
\hline $323 \mathrm{K187A83}$ & 4 \\
\hline $214 H_{03} 0 A 83$ & 4 \\
\hline $214 \mathrm{HO29A83}$ & 4 \\
\hline $214 \mathrm{HO28A83}$ & 4 \\
\hline $214 \mathrm{HO} 31 \mathrm{AB3}$ & \\
\hline $216 \mathrm{HO2OAB3}$ & 4 \\
\hline $216 \mathrm{HO19A03}$ & 4 \\
\hline $216 \mathrm{HO19A \theta 3}$ & 4 \\
\hline $218 \mathrm{HO15AB3}$ & \\
\hline $218 \mathrm{HO14A \theta 3}$ & 4 \\
\hline $218 \mathrm{HO13AB3}$ & 4 \\
\hline $322 \mathrm{HLBOA \theta 3}$ & 4 \\
\hline $322 \mathrm{H189AB3}$ & 4 \\
\hline $322 \mathrm{H190A83}$ & \\
\hline $216 \mathrm{HO2} 1 \mathrm{AB3}$ & \\
\hline $219 H 010 A 83$ & 4 \\
\hline $220 H 007 A 83$ & 4 \\
\hline $213 \mathrm{H} 035 \mathrm{~A} 83$ & 4 \\
\hline $2134036 \mathrm{A83}$ & 4 \\
\hline $213 H 037 A 83$ & 4 \\
\hline $21314032 \mathrm{A83}$ & 4 \\
\hline $213 H 033 A 83$ & 4 \\
\hline $213 H 034 A B 3$ & 4 \\
\hline $219 H 011 \mathrm{Ag3}$ & 4 \\
\hline
\end{tabular}

Figure 9.19 - Case 2, Revised and Decelerated Schedule (Continued). 
SAUD \& EBPAHIM ALABDULRAZZAK EST.

REPORT DATE 21SEP9S RUN NO. 205 Revd \& Decel Schl by Typical Activity \& ES

PR IMAVEPA PPOJECT PLAMNER

SEVEN SEAS, CONSTRUCTION OF 51 HOUSES
Corrocting Out-ol-Logie Progress NSq

START DATE 1APR37 FIN DATE 3OMARB9*

DATA DATE 1FEBaB PAGE NO. 39

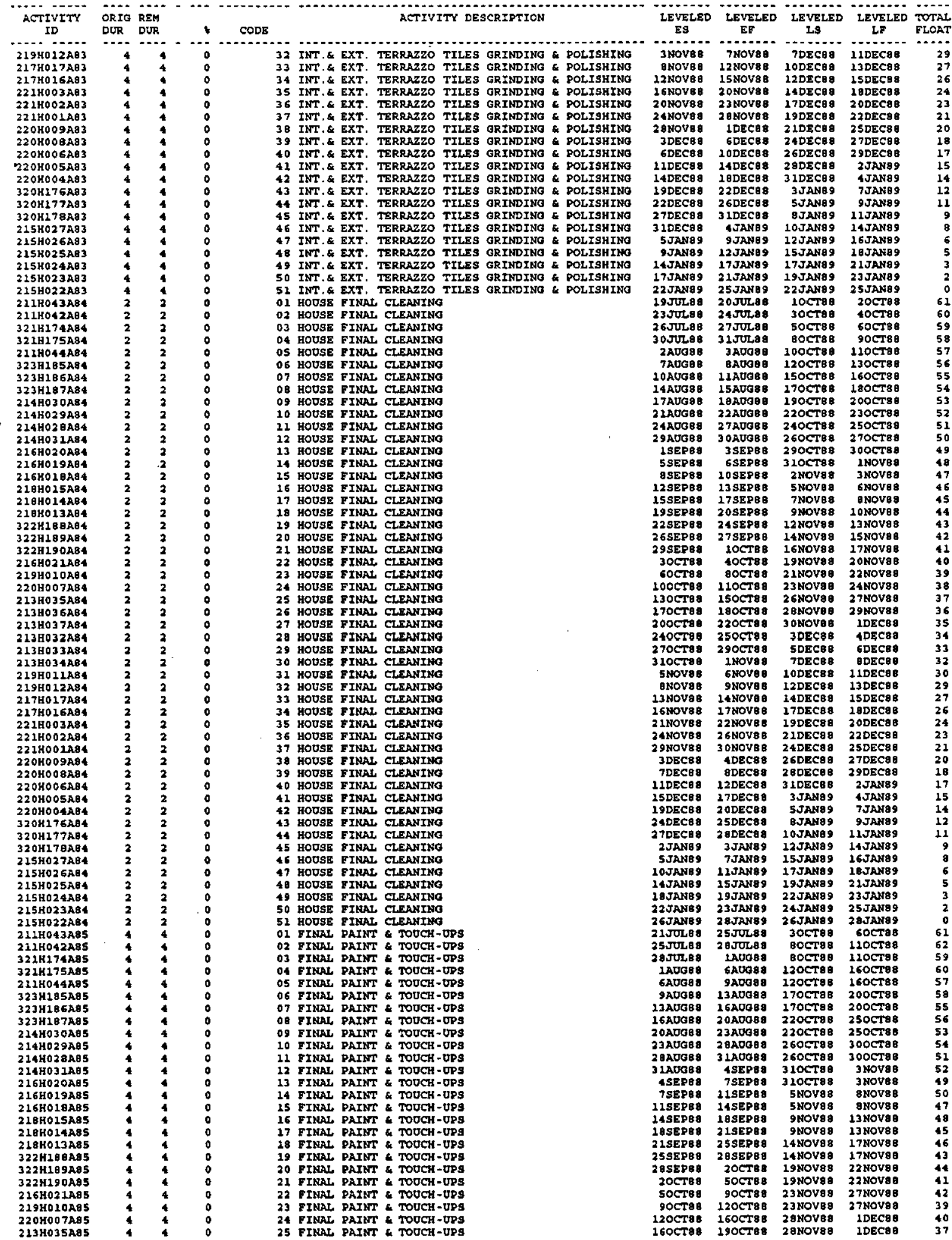

Figure 9.19 - Case 2, Revised and Decelerated Schedule (Continued). 


\begin{tabular}{|c|c|c|c|c|}
\hline $\begin{array}{c}\text { ACTIVITY } \\
\text { ID }\end{array}$ & $\begin{array}{l}\text { ORIO } \\
\text { DUR }\end{array}$ & $\begin{array}{l}\text { REM } \\
\text { DUR } \\
\cdots \cdots\end{array}$ & $\ldots$ & $\begin{array}{c}\text { CODE } \\
-\end{array}$ \\
\hline $213 B 036 A 85$ & 4 & 4 & 0 & 26 \\
\hline $213 \mathrm{HO37A95}$ & 4 & 4 & 0 & 27 \\
\hline $213 \mathrm{H} 032 \mathrm{A \theta 5}$ & & 4 & 0 & 28 \\
\hline $213 \mathrm{H} 033 \mathrm{ABS}$ & & 4 & 0 & 29 \\
\hline $213 \mathrm{HO34AQ5}$ & & 4 & 0 & 30 \\
\hline 219H011ABS & 4 & 4 & 0 & 31 \\
\hline $219 H 012 A 05$ & 4 & 4 & 0 & 32 \\
\hline $217 \mathrm{HO17A \theta 5}$ & 1 & 4 & : & 33 \\
\hline 217 HOL6A8S & 4 & 4 & 0 & 34 \\
\hline $221 \mathrm{H} 003 \mathrm{~A} 85$ & 4 & 4 & 0 & 35 \\
\hline $2214002 \mathrm{~A} 85$ & 4 & 4 & 0 & 36 \\
\hline 221 1H001A85 & 4 & 4 & : & 37 \\
\hline $\begin{array}{l}220 \% 009 A 85 \\
220 H 008 \text { ABS }\end{array}$ & 4 & 4 & $\stackrel{0}{0}$ & $\begin{array}{l}38 \\
39\end{array}$ \\
\hline $220 \mathrm{HOOOAA85}$ & 4 & 4 & 0 & 40 \\
\hline $220 H 005 A 85$ & 4 & 4 & 0 & 41 \\
\hline $220 H 004 A 85$ & 4 & 4 & 0 & 42 \\
\hline $320 \mathrm{H} 176 \mathrm{ABS}$ & 4 & 1 & : & 43 \\
\hline 3204177 Aa 5 & 4 & 4 & 0 & 44 \\
\hline $320 \mathrm{H} 17 \mathrm{AAOS}$ & 4 & 4 & 0 & 45 \\
\hline $2154027 \mathrm{AgS}$ & 4 & 1 & 0 & 46 \\
\hline 215 H026A95 & 4 & 4 & 0 & 47 \\
\hline 215HO25A95 & 4 & 1 & 0 & 48 \\
\hline $\begin{array}{l}215 \mathrm{H} 024 A 85 \\
215 \mathrm{H} 023 \mathrm{AOS}\end{array}$ & 4 & 4 & $:$ & \\
\hline $\begin{array}{l}215 \mathrm{H} 023 \mathrm{AaS} \\
215 \mathrm{HO22 \textrm {ABS }}\end{array}$ & 4 & 4 & 0 & $\begin{array}{l}50 \\
51\end{array}$ \\
\hline $211 \mathrm{HO} 33 \mathrm{AB} 6$ & 0 & 0 & 0 & 01 \\
\hline $211 H 042 A 26$ & 0 & 0 & 0 & 02 \\
\hline $3214174 A B 6$ & 0 & 0 & 0 & 03 \\
\hline $321 \mathrm{H175AB6}$ & 0 & 0 & 0 & 04 \\
\hline $211 H 044 A B 6$ & 0 & $\circ$ & : & os \\
\hline $323 \mathrm{H} 185 \mathrm{ABO} 6$ & 0 & 0 & 0 & 06 \\
\hline $323 \mathrm{H1B6A86}$ & 0 & 0 & 0 & 07 \\
\hline 323Н187AB6 & 0 & 0 & 0 & 08 \\
\hline $214 \mathrm{HO} 30 \mathrm{A8B}$ & 0 & 0 & 0 & 09 \\
\hline $214 H 029 A 86$ & 0 & 0 & 0 & 10 \\
\hline $214 H 028 A 86$ & 0 & 0 & 0 & 11 \\
\hline $214 H 031 \mathrm{AB} 6$ & 0 & 0 & 0 & 12 \\
\hline $2164020 A 88$ & - & 0 & 0 & 13 \\
\hline $216 \mathrm{H} 019 \mathrm{A8} 6$ & 0 & 0 & 0 & 14 \\
\hline $2164018 A 86$ & 0 & 0 & 0 & 15 \\
\hline $210 \mathrm{H} 015 \mathrm{AB} 6$ & 0 & 0 & 0 & 16 \\
\hline $210 \mathrm{H} 014 \mathrm{ABg}$ & 0 & 0 & 0 & 17 \\
\hline $218 \mathrm{HO} 13 \mathrm{AB} 6$ & 0 & 0 & : & 18 \\
\hline 322H18BAB 6 & 0 & 0 & 0 & 19 \\
\hline $322 \mathrm{H199AB6}$ & 0 & 0 & 0 & 20 \\
\hline 322H190A86 & 0 & & 0 & 21 \\
\hline $216 H 021 A 86$ & 0 & : & $\therefore$ & 22 \\
\hline $219 \mathrm{HO} 10 \mathrm{AB} 6$ & $\therefore$ & $:$ & 8 & 23 \\
\hline $220 \mathrm{H} 007 \mathrm{AB} 6$ & $:$ & $\therefore$ & 0 & 24 \\
\hline $213 \mathrm{H} 035 \mathrm{AB} 6$ & 0 & 0 & 0 & 25 \\
\hline $\begin{array}{l}213 \text { 21336A86 } \\
213 \text { Н037АВ6 }\end{array}$ & 0 & 0 & 0 & 26 \\
\hline 213H037AB6 & 0 & 0 & 0 & 27 \\
\hline $\begin{array}{l}213 H 032 A 86 \\
213 H 033 A 86\end{array}$ & 0 & $:$ & 0 & 28 \\
\hline & $:$ & $:$ & $\stackrel{0}{0}$ & $\begin{array}{l}29 \\
30\end{array}$ \\
\hline $\begin{array}{l}213 H 034 A 86 \\
219 H 011 \mathrm{AB6}\end{array}$ & 0 & 0 & 0 & $\begin{array}{l}30 \\
31\end{array}$ \\
\hline $219 \mathrm{HO} 22 \mathrm{AaG}$ & 0 & 0 & 0 & $\begin{array}{l}31 \\
32\end{array}$ \\
\hline $217 \mathrm{HO} 17 \mathrm{ABG}$ & 0 & 0 & 0 & $\begin{array}{l}32 \\
33\end{array}$ \\
\hline 217 H016AB 6 & 0 & 0 & 0 & 34 \\
\hline $222 \mathrm{HO03AB6}$ & 0 & 0 & 0 & 35 \\
\hline $222 \mathrm{HO02AB6}$ & 0 & 0 & 0 & 36 \\
\hline $221 \mathrm{HOOLAB6}$ & 0 & 0 & 0 & 37 \\
\hline $220 \mathrm{HOOSAO}$ & : & 0 & : & 38 \\
\hline $220 \mathrm{HOOBAB} 6$ & 0 & : & : & 39 \\
\hline $220 H 006 A 86$ & 0 & 0 & 0 & 40 \\
\hline $220 \mathrm{HOOSAB6}$ & 0 & 0 & 0 & 41 \\
\hline $220 \mathrm{HH} 004 \mathrm{A8} 6$ & 0 & 0 & 0 & 42 \\
\hline $320 \mathrm{H} 76 \mathrm{Ag} 6$ & 0 & : & 0 & 43 \\
\hline $320 \mathrm{H177A96}$ & 0 & 0 & 0 & 14 \\
\hline $320 \mathrm{H178AB6}$ & 0 & 0 & 0 & 45 \\
\hline $215 \mathrm{HO27ABG}$ & 0 & 0 & 0 & 16 \\
\hline 215 H026A日6 & 0 & 0 & 0 & 47 \\
\hline $215 \mathrm{H}^{25} 5 \mathrm{AB} 6$ & 0 & 0 & 0 & 48 \\
\hline $215 \mathrm{H}_{024 \mathrm{AB}} 6$ & : & 0 & 0 & 49 \\
\hline $215 \mathrm{HO} 23 \mathrm{Ag} 6$ & 0 & 0 & 0 & 50 \\
\hline $22540222 A 86$ & 0 & 0 & 0 & 51 \\
\hline 211H044A91 & 9 & 1 & 89 & 05 \\
\hline 214H03OA91 & 9 & 9 & 0 & 09 \\
\hline $214 \mathrm{H} 029 \mathrm{AO} 1$ & 9 & 9 & 0 & 10 \\
\hline $2144028 \mathrm{Ag} 1$ & 9 & 9 & 0 & 11 \\
\hline $214 \mathrm{HO3} 1 \mathrm{Ag1}$ & 9 & 9 & 0 & 12 \\
\hline $216 \mathrm{HO2OA91}$ & 9 & 9 & 0 & 13 \\
\hline 216Ho19A91 & 9 & 9 & 0 & 14 \\
\hline 216H018A91 & 9 & 9 & 0 & 15 \\
\hline 218H015A91 & 9 & 9 & 0 & 16 \\
\hline $218 \mathrm{HO14A91}$ & 9 & 9 & 0 & 17 \\
\hline $218 \mathrm{HO} 33 \mathrm{AgI}$ & 9 & 9 & 0 & 18 \\
\hline $322 \mathrm{H} 188 \mathrm{AOI}$ & 9 & 9 & 0 & 19 \\
\hline $322 \mathrm{HLB9A91}$ & 9 & 9 & 0 & 20 \\
\hline $322 \mathrm{H} 190 \mathrm{AOI}$ & 9 & 9 & 0 & 21 \\
\hline $216 \mathrm{HO21A91}$ & 9 & 9 & 0 & 22 \\
\hline 219H010A91 & 9 & 9 & 0 & 23 \\
\hline $220 \mathrm{H} 007 \mathrm{AO} 1$ & 9 & $?$ & : & 24 \\
\hline 213H035A91 & ? & 9 & 0 & 25 \\
\hline & & & & \\
\hline
\end{tabular}

Figure 9.19 - Case 2, Revised and Decelerated Schedule (Continued). 


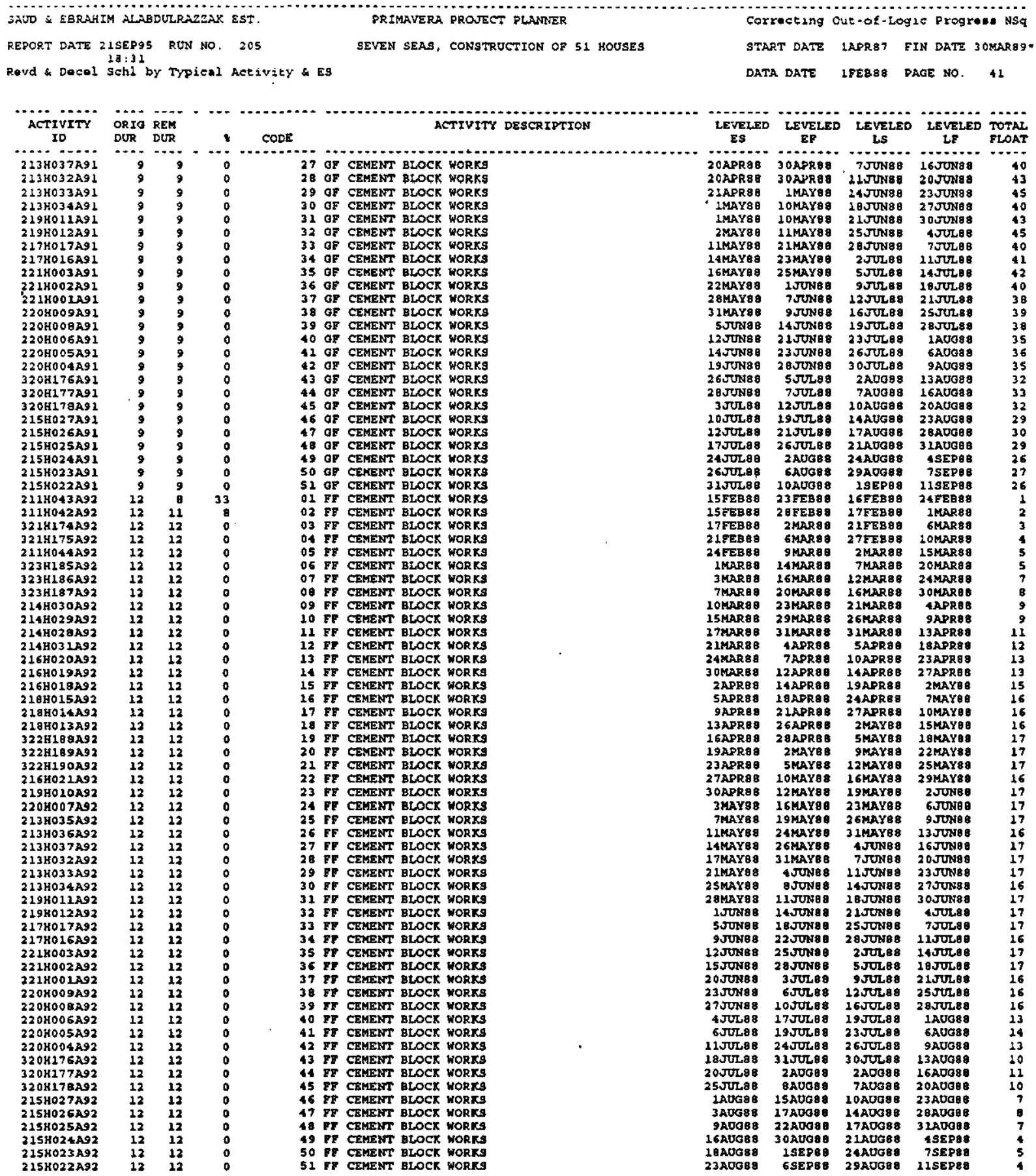

Figure 9.19 - Case 2, Revised and Decelerated Schedule (Continued). 
The California Department of Corrections (CDC) is the owner of a state prison which is being constructed in Susanville, California. The prison construction cost is about $\$ 250$ millions. Construction work is divided into ten bid packages. Heery International, Inc. is the Construction Manager and Hill International, Inc. whom the author worked for is a subconsultant to Heery in charge of claims prevention. McCarthy Western Constructors is the main contractor of bid package three, which comprises housing facilities and thirteen identical guard towers along the circumference of the prison. This case study only concerns the guard towers.

\subsubsection{Guard Towers, the Baseline Schedule}

McCarthy had used logical relationships (start to start) between selected typical activities on each tower subnetwork to stagger the work of the repetitive towers. Alternatively the author deleted these interfaces between the tower subnetworks to apply CPM/LOB. Fig. 9.20 shows the logic diagram of a typical guard tower with the necessary non-repetitive pre-construction activities concerning submittals, approvals, fabrications and deliveries. While only one tower is shown in Fig. 9.20 linked to notice to proceed and delivery activities, notice to proceed and delivery activities were linked as predecessors to all the corresponding activities of each tower.

The towers are numbered 301 to 313 with the first digit referring to the bid package number (three), and the other two to the tower number. According to the general conditions of the contract, the towers are to be constructed in the sequence: $302,303,304,305,306,301,307$, $308,313,309,310,311$ and 312. Further, their construction is constrained by four milestones (MS) whose violation subjects McCarthy to liquidated damages. MS\#8 mandates that towers 302 , 303, 304, 305 and 306 start no earlier than workday 36 and finish no later than workday 252. Similarly MS\#12 constrains towers 301 and 307; MS\#18 constrains towers 308 and 313; and MS\#23 constrains towers 309, 310, 311 and 312. In the CPM/LOB baseline schedule, the milestone requirements are represented by start-no-earlier-than and finish-no-later-than constraints. Fig. 9.21 shows the above information in a linear program associated with calendar dates. It is to be noted that the structural steel design, approval, fabrication and delivery will hold the structural steel erection of all the towers for varying periods if construction of the towers is to start hypothetically at its earliest. The project calendar is shown in Fig. 9.22. 
The application of CPM/LOB shall adopt two completion rates as indicated in Fig. 9.21 by the symbolic dotted broken line. The time available for completion for MS\#8 and MS\#12 towers is $266-213=53$ workdays. This is confirmed by the total floats of MS\#12 towers when the baseline schedule is run without resource leveling and as shown in Fig. 9.23 for tower 307 activities (G07 refers to guard tower 7 and the following two digits refer to the typical activity number). Total floats for MS\#8 towers are 39 workdays, which are 14 days less than those of MS\#12 towers because MS\#8 has to be completed 14 days earlier than MS\#12 (266 - $252=14)$. As shown by the dotted line between workdays 213 and 266 in Fig. 9.21, calculation of the rate of completions for both MS\#8 and MS\#12 towers based only on MS\#12 tower floats shall satisfy the requirement of MS\#8 towers to finish no later than workday 252. Table 9.4 shows the calculation of the minimum rate of squads of the activities of MS\#8 and MS\#12 towers to satisfy the imposed milestone dates. It is noted that the work duration of the squad of typical activity 56 PUNCHLIST is only twelve days which is less than the activity duration (15 days). This yields an overlap of three workdays between activities having the same squad working on them sequentially. Also, per column 10 , this typical activity will control the pace of the progress since it has the lowest normalized independent rate.

After the number of squads on column 8 is entered into the CPM software as resource availability and leveling is performed per the contractual sequence, the early dates of tower 307 activities can serve as dates of a typical first unit for the LOB calculations for the rest of the towers (MS\#18 \& MS\#23 towers). The towers of MS\#18 and MS\#23 are to progress at a lower completion rate than that of MS\#8 and MS\#12 towers and as shown earlier by the symbolic dotted broken line in Fig. 9.21.

Fig. 9.24 shows the leveled early finish dates of the activities of tower 307 associated with workday numbers. At this stage the latest completion dates for the last tower, number 312 , are needed to contrast them with the leveled early finish dates of tower 307 to calculate the available time for completions for MS\#18 and MS\#23 tower activities. This can be easily obtained from the CPM software by running the schedule without leveling. Fig. 9.25 shows the latest completion dates of tower 312 , the last tower.

In order to calculated the minimum number of squads required to complete MS\#18 and MS\#23 towers with lower rates of completions than those of the earlier towers, only typical activities 28, 44 and 56 need to be considered since all the other typical activities can not have fewer than the one squad they already have. However, to find which typical activities will control 
the pace of the progress after tower 307, all typical activities shall be considered. Table 9.5 shows the calculation of the minimum quantity of squads required to complete MS\#18 and MS\#23 towers. It is noted that one squad for each typical activity is enough after tower 307 and that typical activity 56 PUNCHLIST will continue to control the pace of the progress since it has the lowest normalized independent rate: 0.083 tower per day per column 12. Based on that, typical activity 28 , which had two squads until completion of tower 307 , will have only one squad after the completion of tower 307 (after workday 204 per column 5 of Table 9.5). Similarly activity 44 will have one squad after workday 223 and activity 56 PUNCHLIST will have one squad after workday 249.

Fig. 9.26 shows the CPM/LOB baseline schedule for the thirteen towers after running resource leveling with an availability of one squad for all typical activities but two then one squad for typical activities 28,44 and 56 , hence varying rates. The numbers under the column titled CODE are the priority codes. All milestones are satisfied and the completion is on April 13, 1995 which is ahead of May 22, 1995 due to the rounding up of the squad quantities and to the fact that two SQ56's were working partially concurrent on typical activity 56 PUNCHLIST on towers 307 and 308 before day 249 when SQ56 availability was two. As expected the critical path began on the time-critical non-repetitive structural steel activities and concluded with the resourcecritical, typical activity 56 . In between, it passed through time-critical activities on the first tower in sequence, tower 302 . Fig. 9.27 shows the histograms of the squads of selected typical activities. Vertical sight lines are drawn at intervals of one week. It is obvious that work continuity for the squads was well maintained.

\subsubsection{Guard Towers, the Updated Schedule}

McCarthy did schedule updates on the 18th of each month. The author took the actual progress data as of December 18, 1994 and applied it to the baseline schedule to determine the status of the progress based on the squad quantities calculated for the baseline schedule. After scheduling and leveling, it was found that the towers had seriously overrun all their milestones. Fig. 9.28 depicts that showing only uncompleted activities. No significant changes of sequence were noted.

Fig. 9.29 shows four progress regularity charts, one against each milestone. The following terminal tower dates were contrasted with their corresponding baseline dates to prepare 
these charts: tower 306 for MS\#8, tower 307 for MS\#12, tower 313 for MS\#18, and tower 312 for MS\#23. Three groups of typical activities could be identified. Typical activities 02-12 were between their baseline early and late dates on the first two milestones then ahead of their early dates of the last two milestones. Typical activities 14-26 while were behind the first two milestones, were somewhat between their baseline early and late dates of the last two milestones. This should encourage raising the rates of completions of the above two groups of activities relevant only to the first two milestones. The third group of typical activities, 28-56, was consistently behind its baselines throughout the four milestones and therefore should be expedited. Examining the regularity charts along with the logic diagram can identify serious bottlenecks of progress. The start of typical activity 16 , which is a predecessor to 14 , was originally delayed by the delivery of structural steel. It is now a bottleneck. Typical activity 28 is another bottleneck because it did not start on time.

\subsubsection{Guard Towers, The Accelerated Schedule}

It is necessary in such a situation to accelerate the work by choosing a realistic target completion date and calculating the required quantities of squads for each typical activity to achieve it. The target completion date chosen by the author was June 22, 1994 (workday 363), one month after the original completion date of the last tower. Further, it is assumed that the sequence of the towers was to incur the following arbitrary changes: towers 308 and 313 switch sequence, and towers 309 and 311 also switch sequence. These changes do not compromise satisfying any contractual milestone. The new sequence is therefore: $302,303,304,305,306$, $301,307,313,308,311,310,309$ and 312.

Fig. 9.30 lists the uncompleted repetitive activities with their new sequence codes, EF dates, LF dates, and the equivalent workdays. These dates are based on a June 22, 1995 imposed finish target date and resulted from running time scheduling without resource leveling. Obviously, within each typical activity, the difference between the earliest early finish and the latest late finish represents the available time for completions for that typical activity.

Table 9.6 is based on the workday numbers of Fig. 9.30 and shows the calculation of the minimum quantity of squads required to achieve the new target. Based on the independent normalized rate, typical activities $32,50,52,54$, and 56 will be resource-critical activities and will control the pace of the progress. The rounded quantities of squads in column 12 are entered 
into the CPM software and time scheduling then resource leveling are performed to get the accelerated schedule.

Fig. 9.31 shows the accelerated schedule with a projected completion exactly on June 22, 1995 because at least one pacing typical activity had an exact whole number of quantity of squads and did not need rounding up. The new sequence is followed and the critical path flows through the fabrication and delivery of windows, non-repetitive activities F122 and D122; then through the typical activities identified earlier. Typical activities 32, 50, 52 and 54 are resource-critical therefore controlling the pace of the project. Additionally, typical activities 44 and 46 are critical because they are bound between typical activities 32 and 50 and had no float on each tower subnetwork. Typical activity 56 , which has four squads, has only priorities $01,05,09$, and 13 as resource-critical (1st squad) since the other priorities are not constrained in the schedule to complete gradually before June 22,1995 . Had the quantity of squads of this last typical activity been divisible by the number of towers, all priorities would have been resource-critical with zero float. Of course typical activity 56 has to be performed according to the early dates to lessen liquidated damages.

\subsubsection{Guard Towers, Features Validated}

In this case study the following additional features of CPM/LOB have been emphasized and validated as superior characteristics over those provided by the current applications on planning and scheduling repetitive projects:

1. Non-repetitive activities can be readily and effectively incorporated in CPM/LOB. This was demonstrated by the submittal and approval activities and constitutes an advantage over the LOB technique and the Linear Scheduling Methods which do not calculate float and are not quickly adjustable as CPM/LOB.

2. In $\mathrm{CPM} / \mathrm{LOB}$, varying rates of completions throughout the project duration can be effected by varying the quantity of available resources. Each of typical activities 28,44 and 56 had two rates of completions in the baseline schedule. 


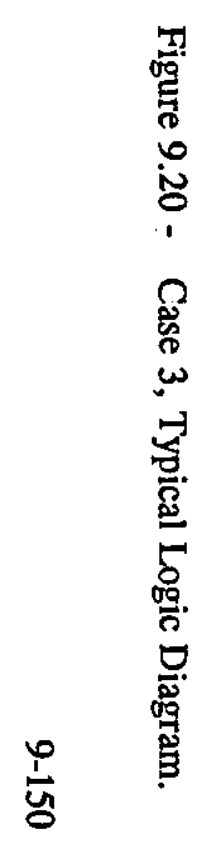

Submittals, Approvals, Fabrications \& Deliveries

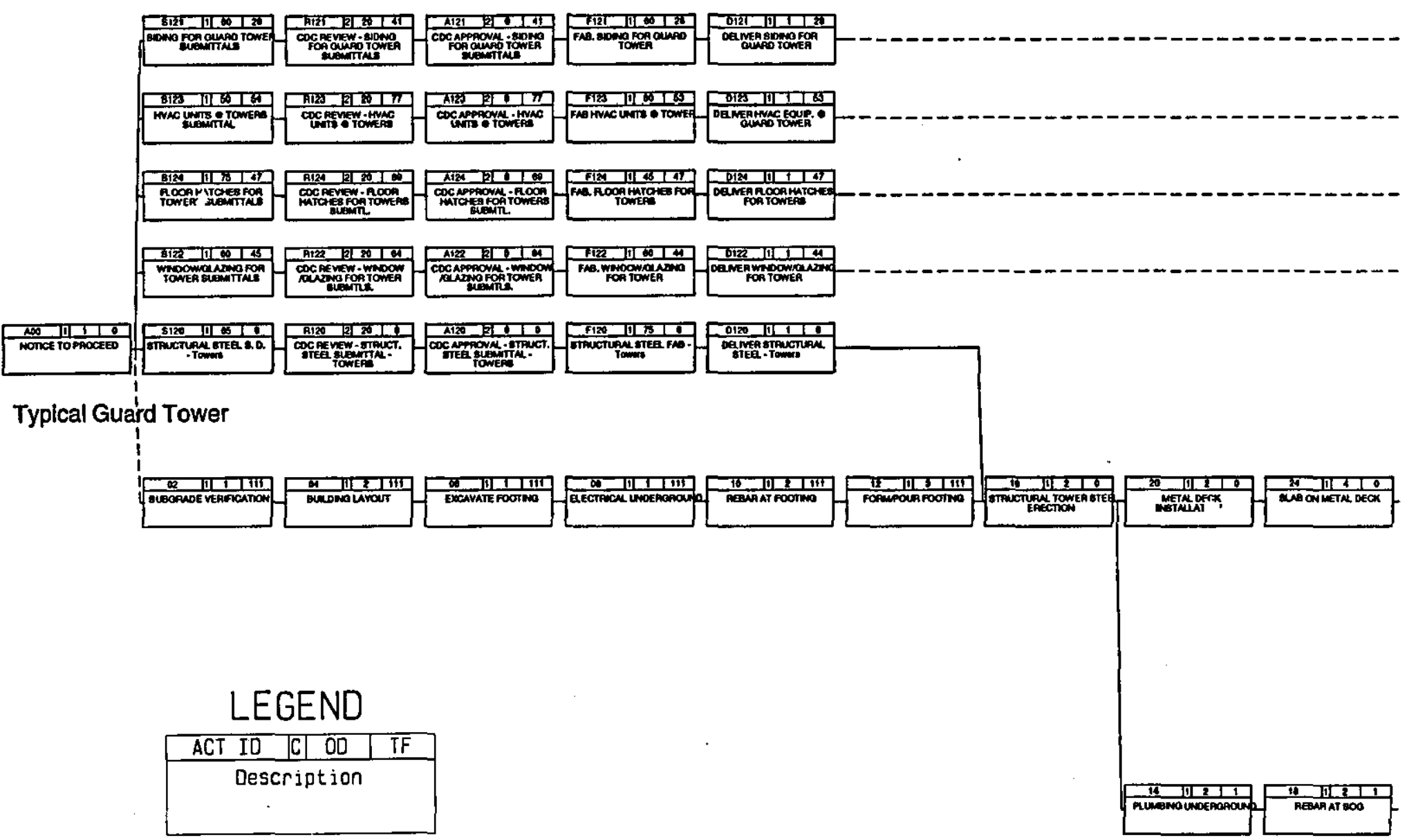




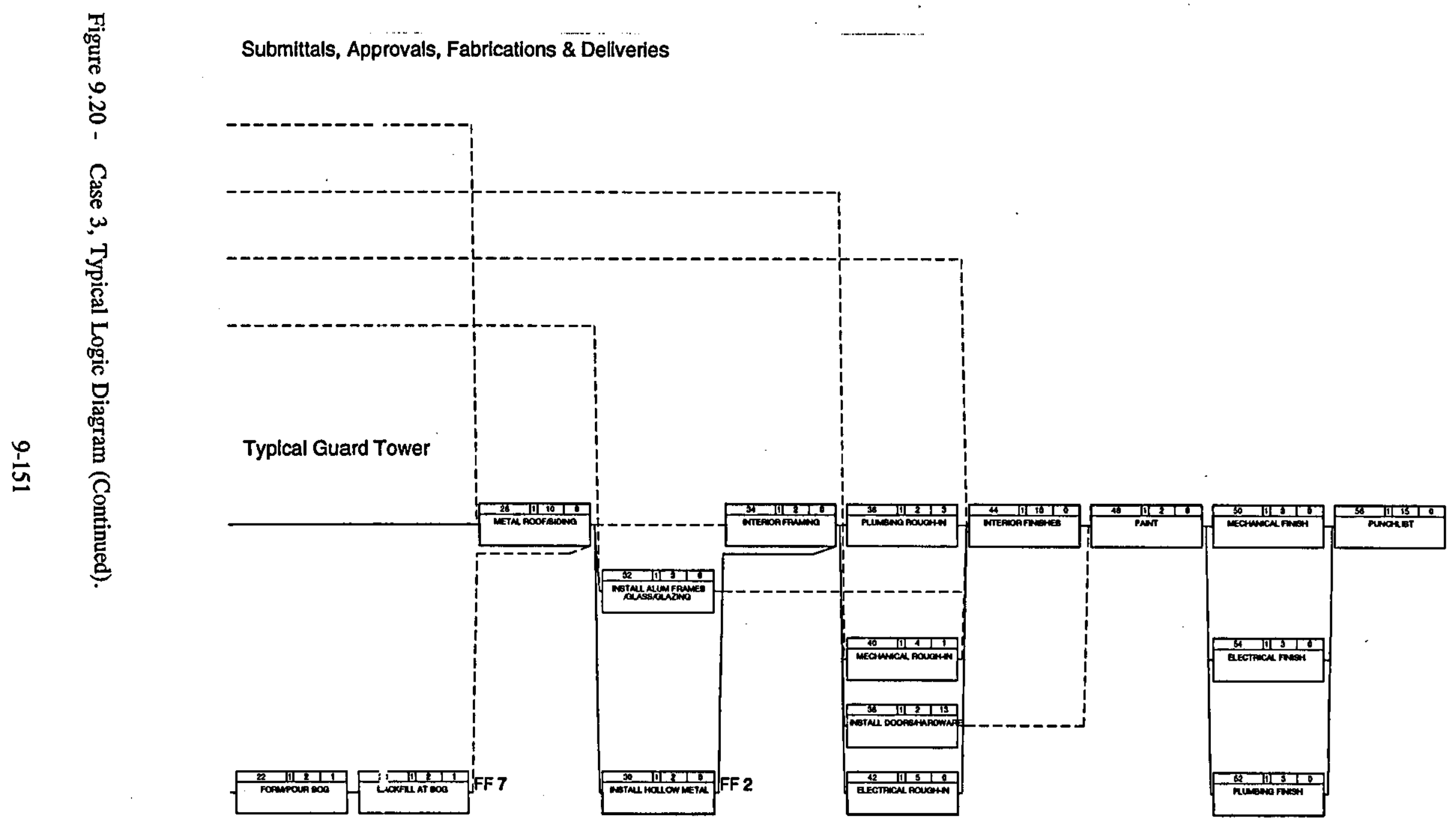




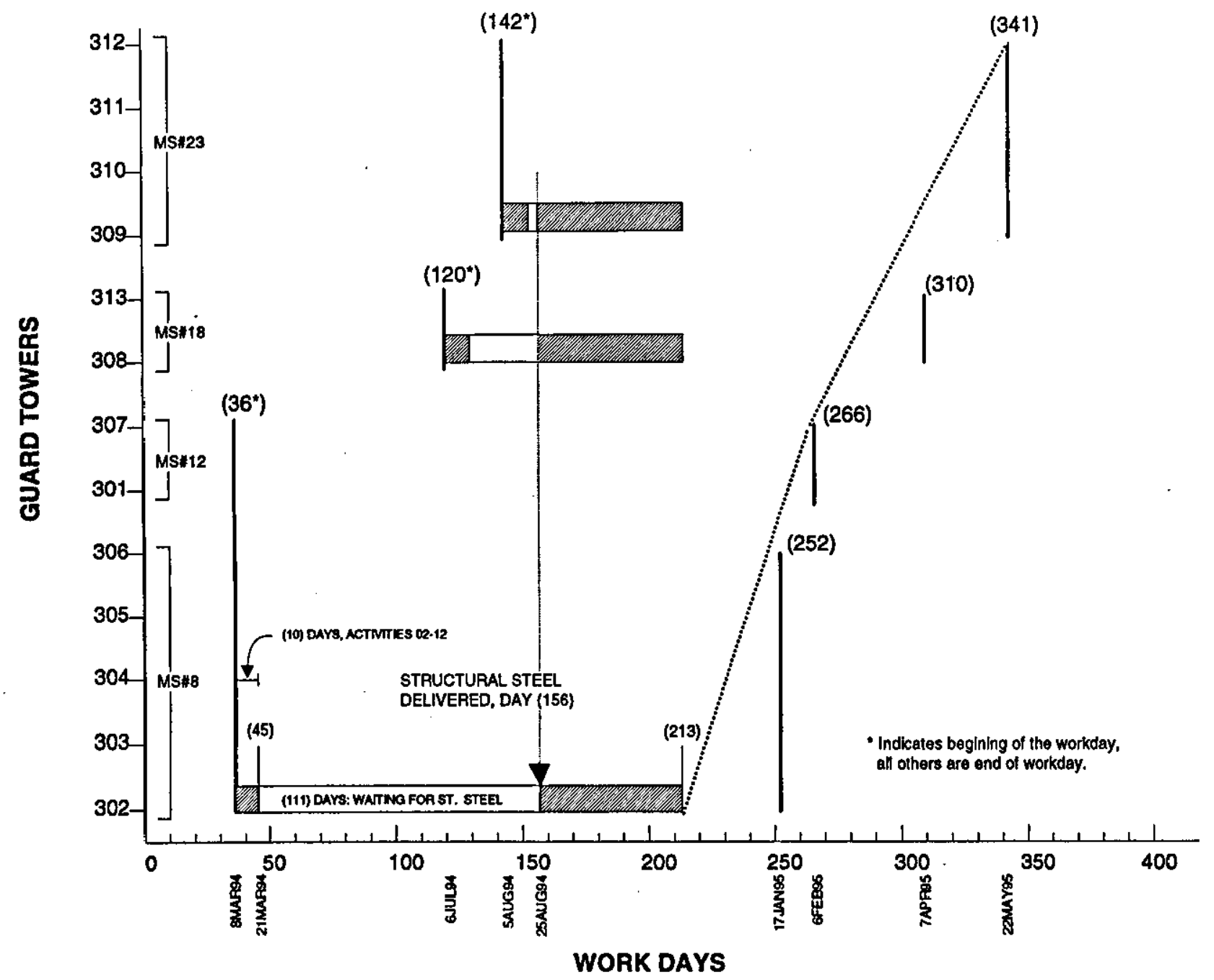

Figure 9.21 - Case 3, Linear Program of Guard Towers. 


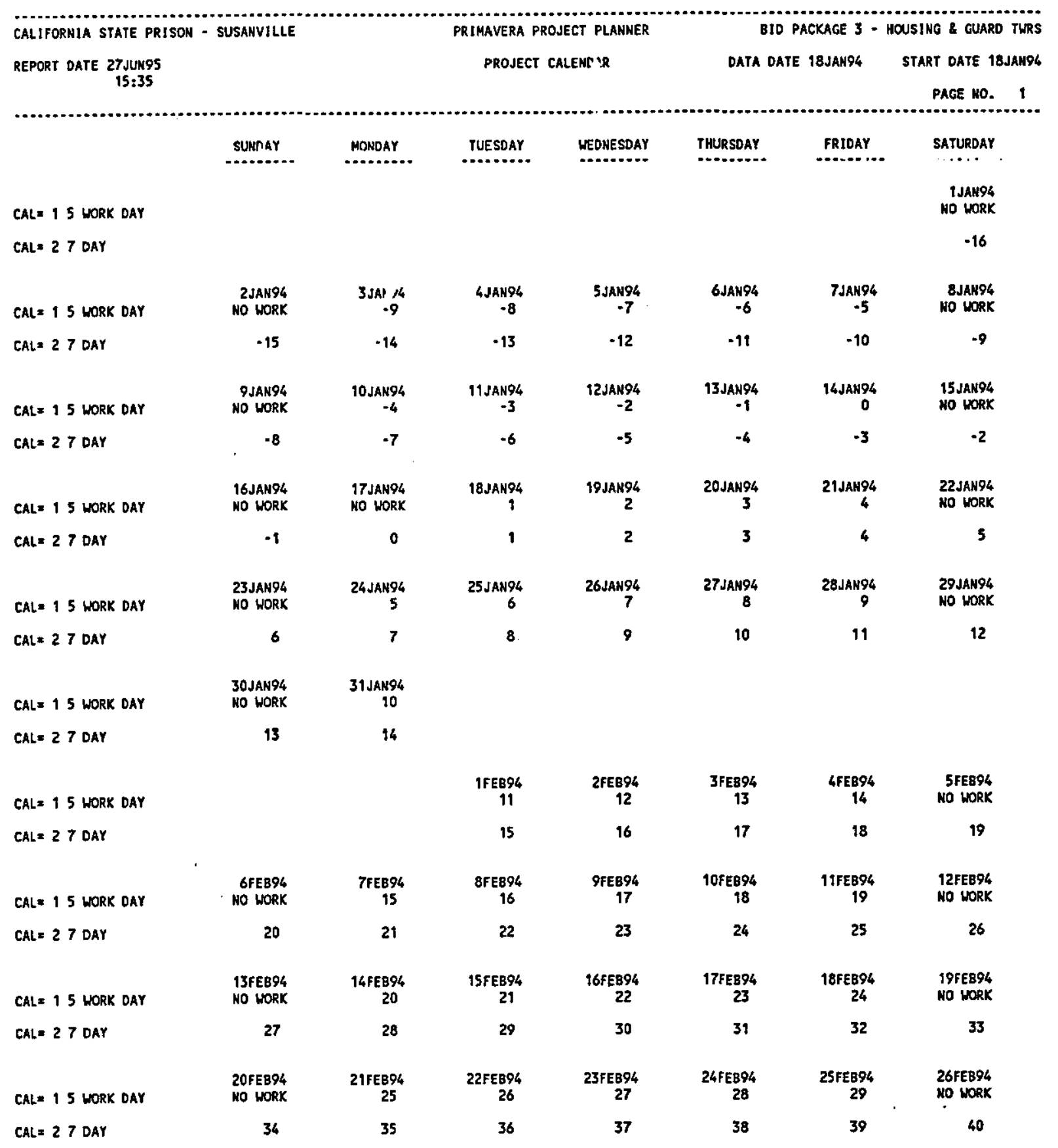

Figure 9.22 - Case 3, Project Calendar. 


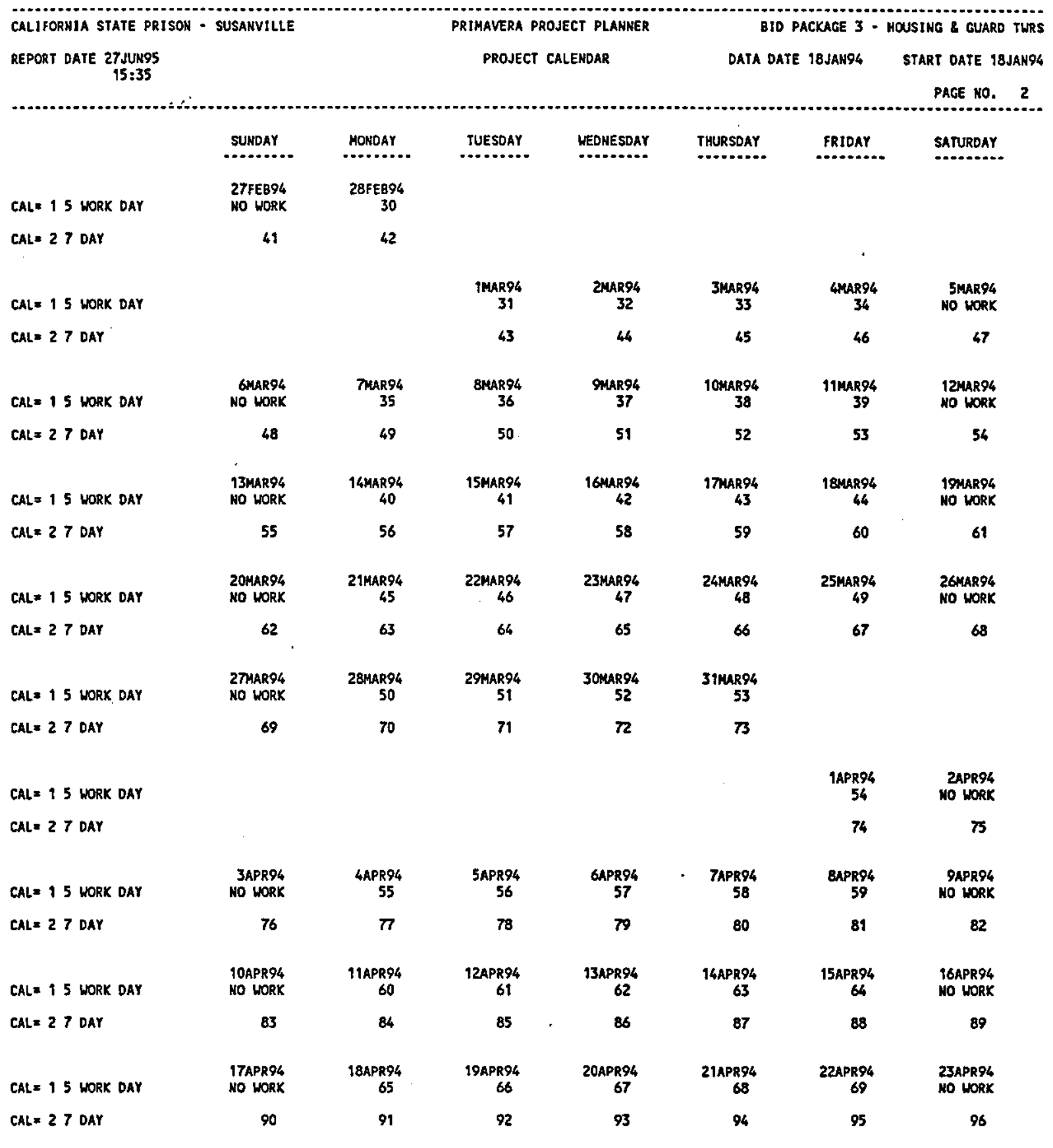

Figure 9.22 - Case 3, Project Calendar (Continued). 


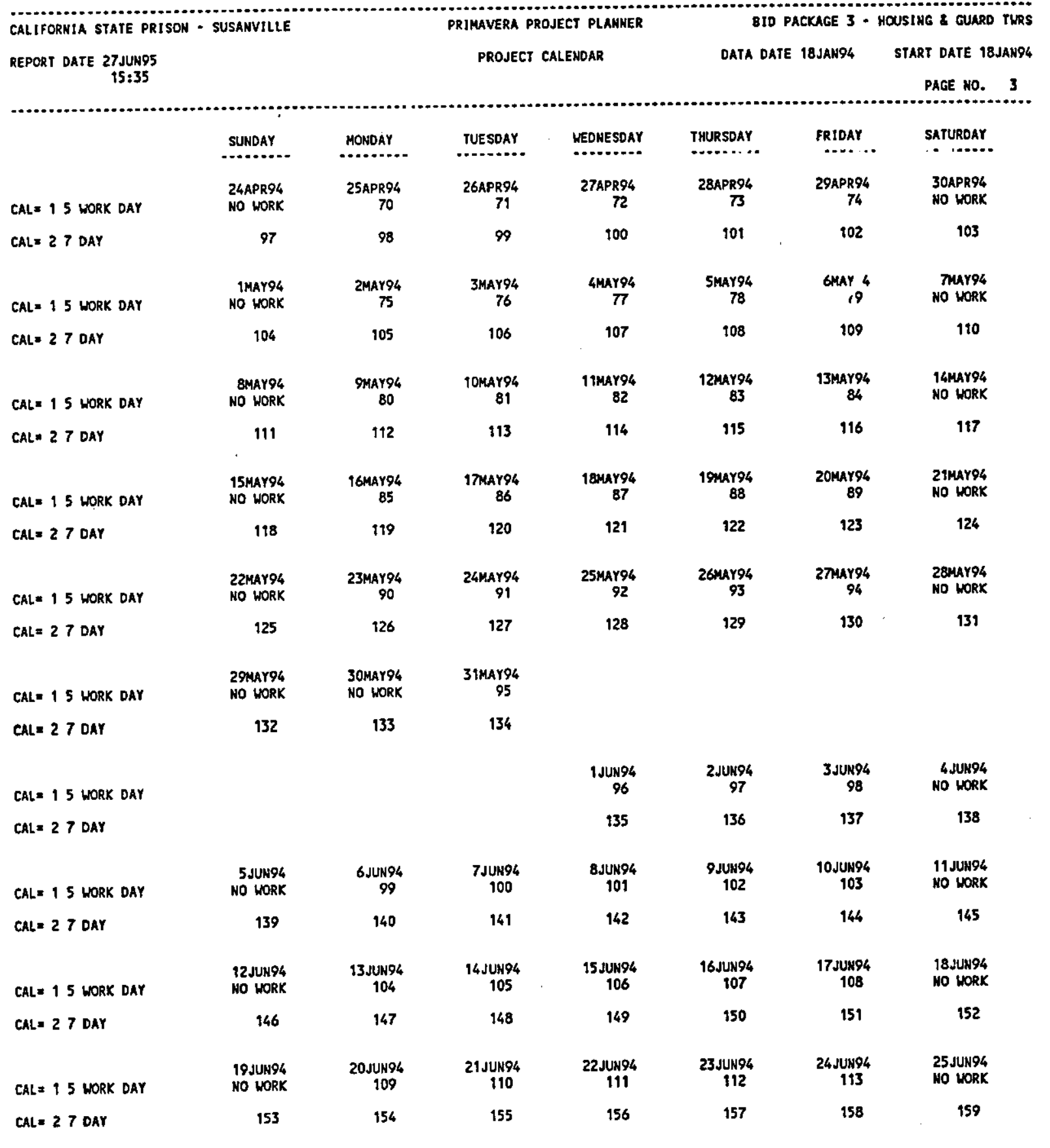

Figure 9.22 - Case 3, Project Calendar (Continued). 


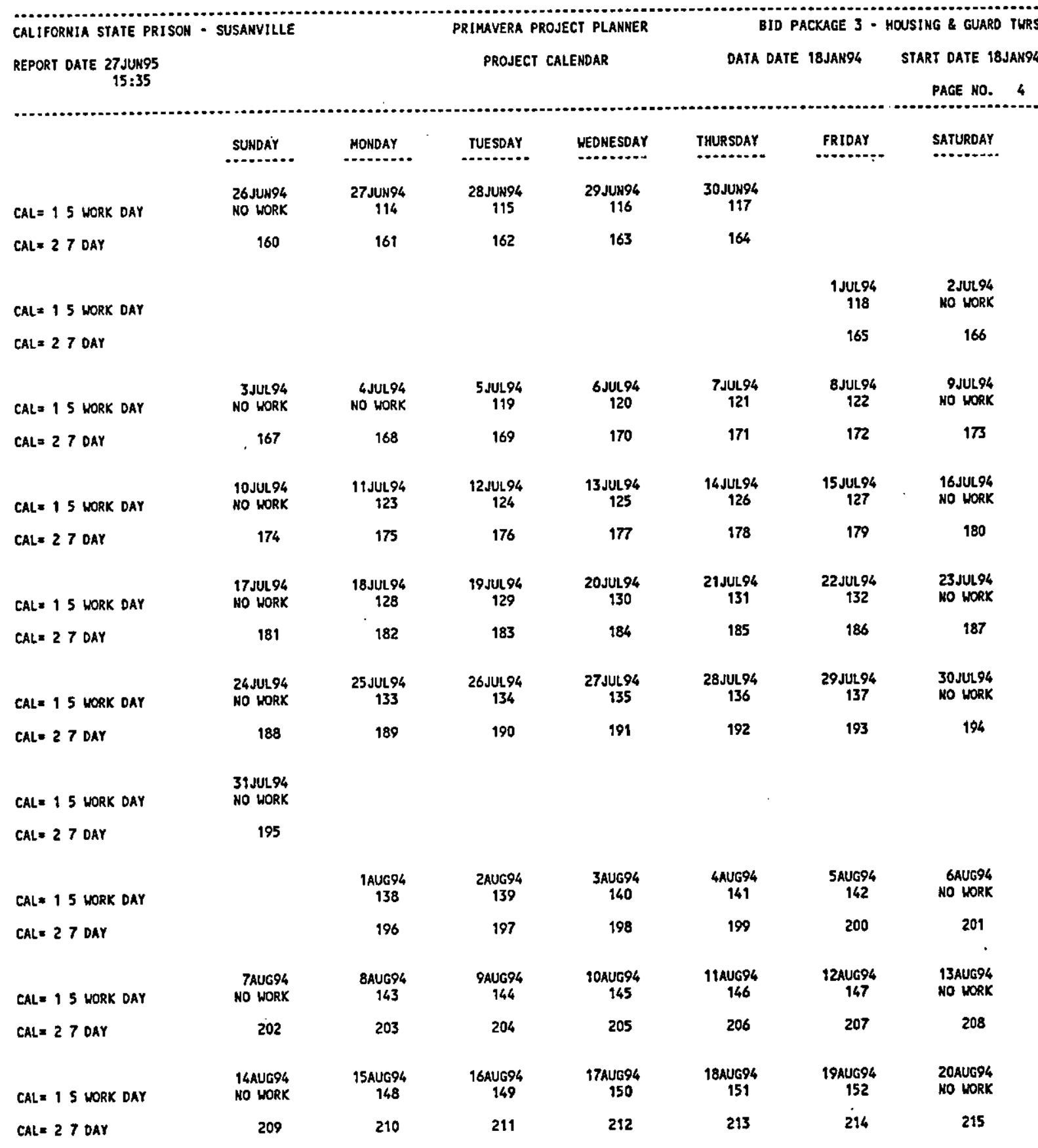

Figure 9.22 - Case 3, Project Calendar (Continued). 


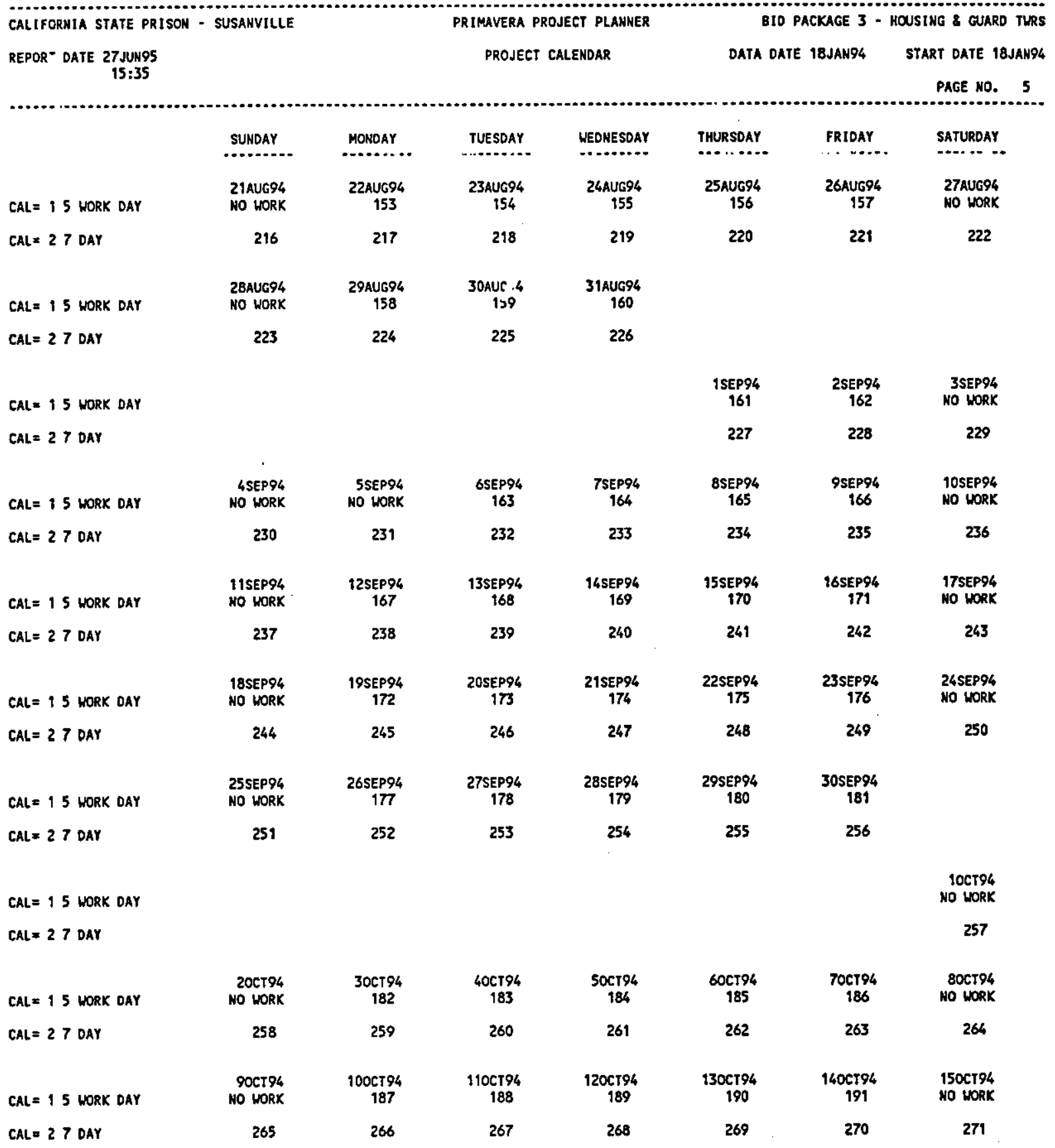

Figure 9.22 - Case 3, Project Calendar (Continued). 


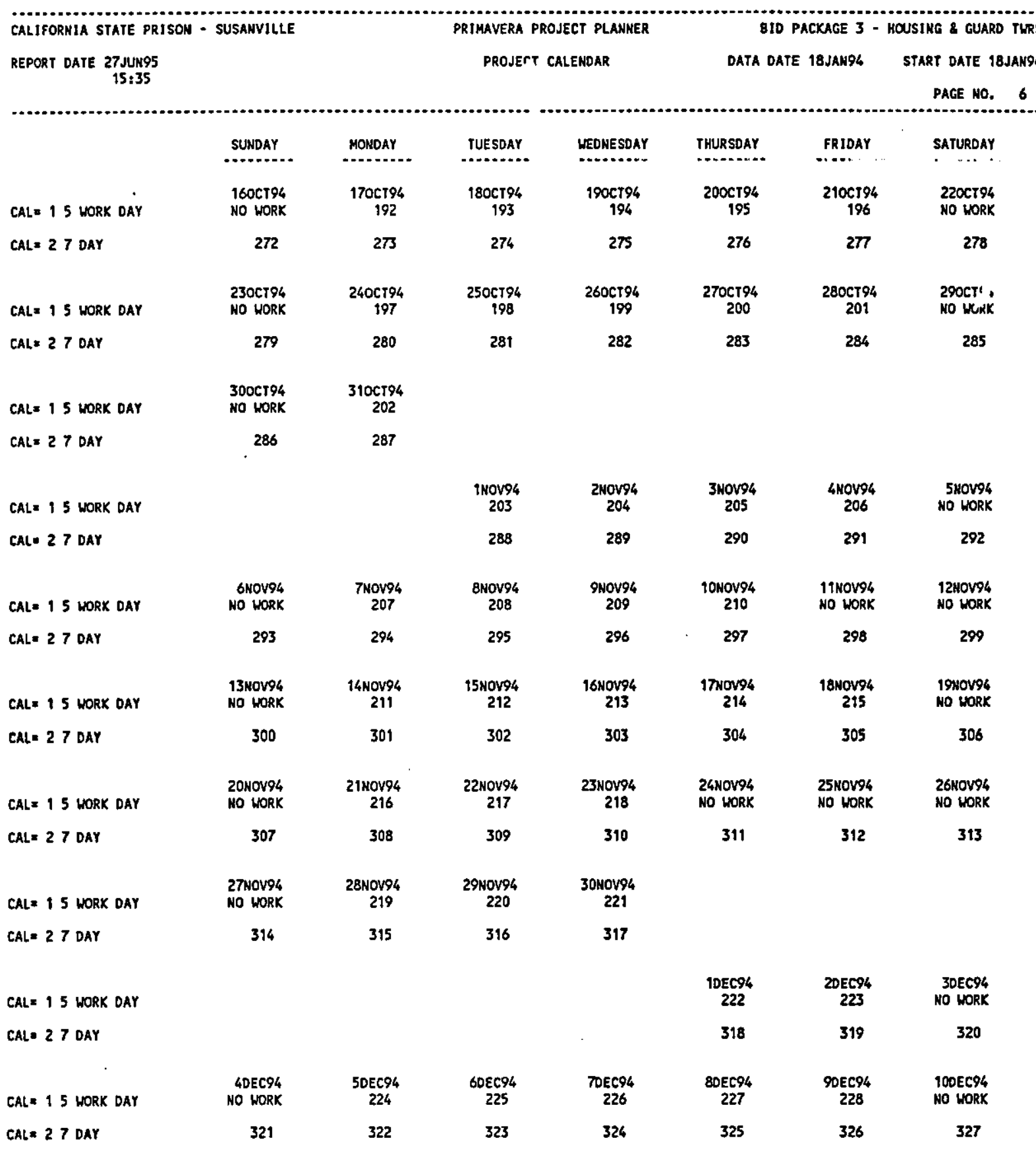

Figure 9.22 - Case 3, Project Calendar (Continued). 


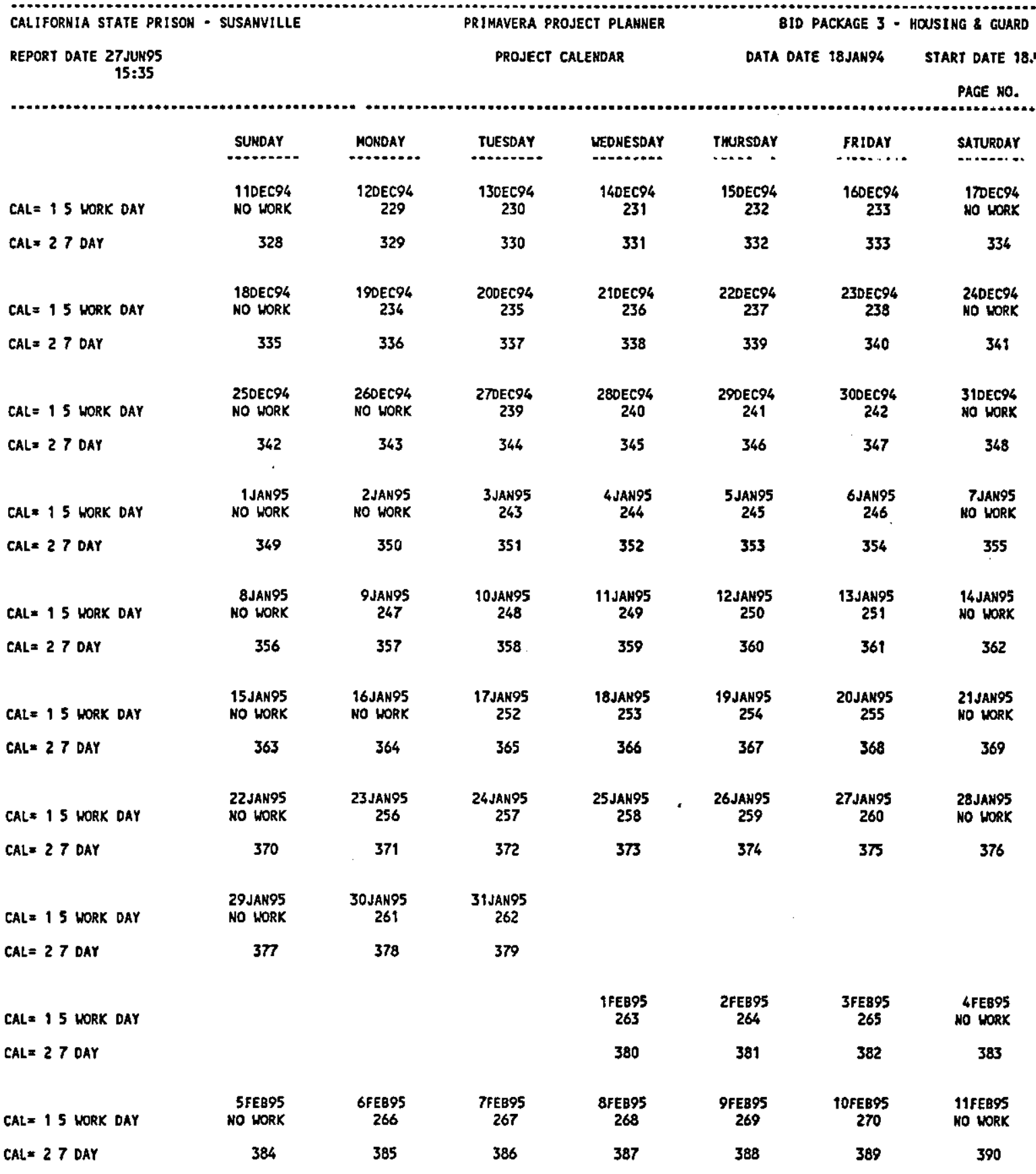

Figure 9.22 - Case 3, Project Calendar (Continued). 


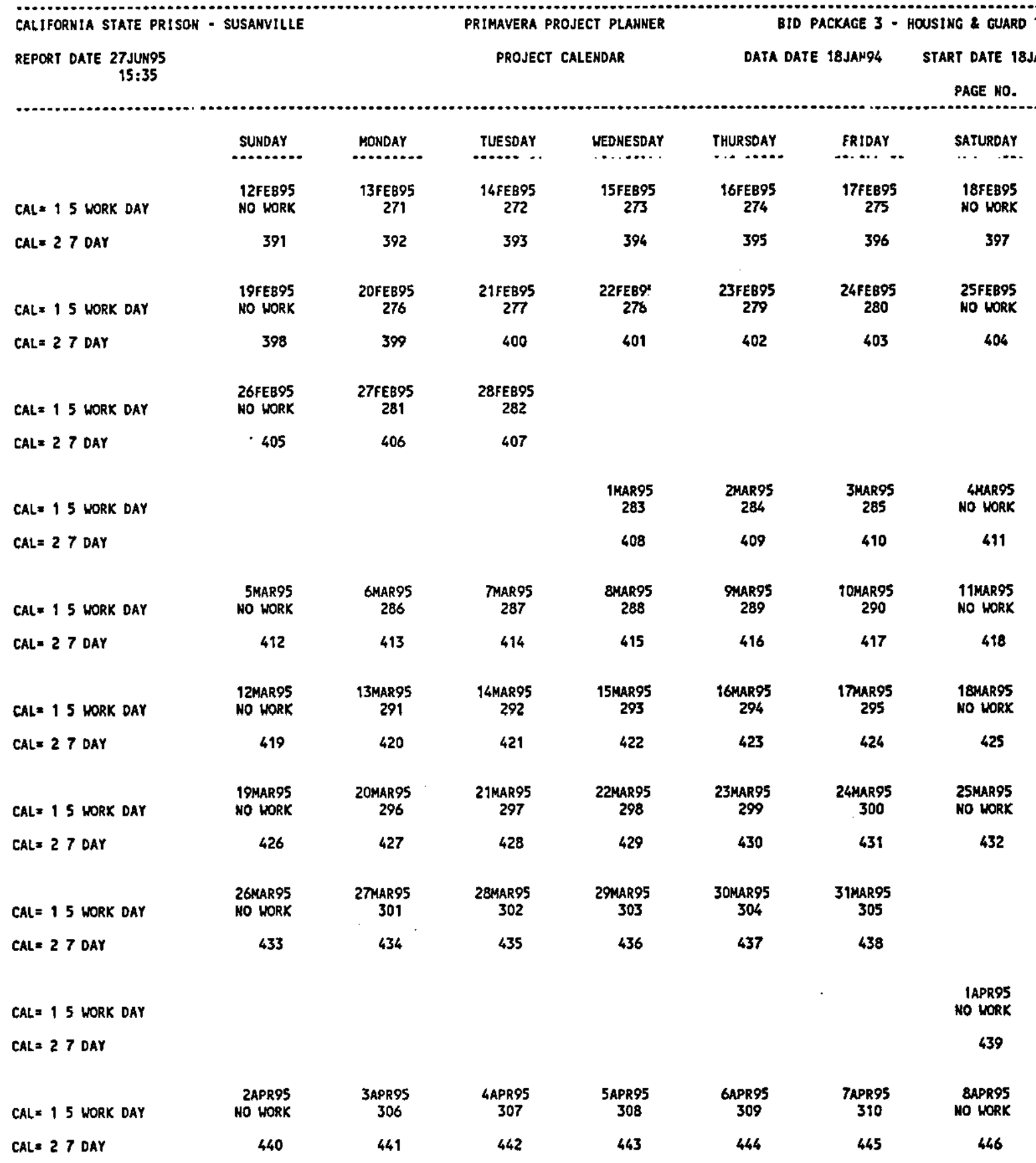

Figure 9.22 - Case 3, Project Calendar (Continued). 


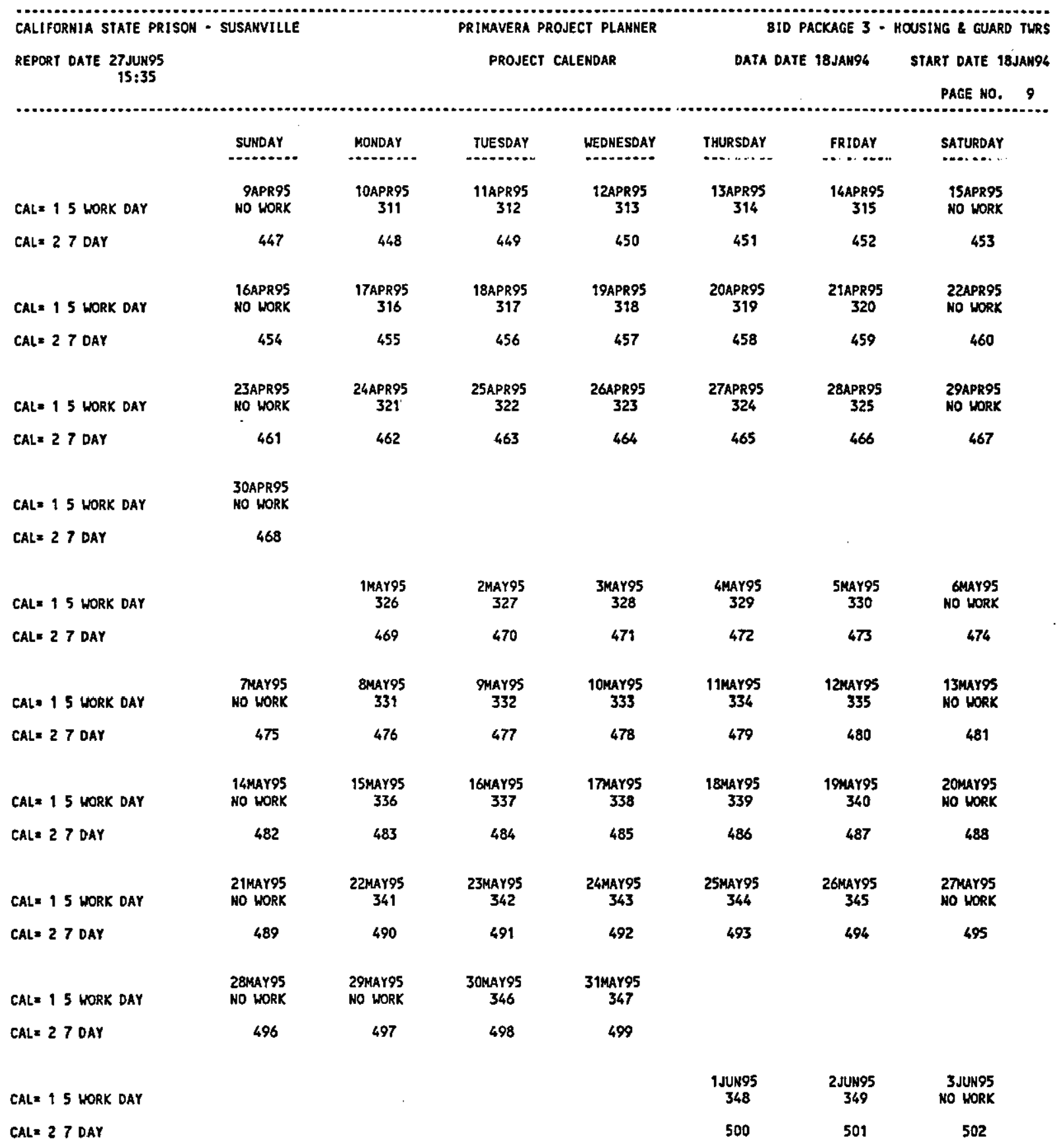

Figure 9.22 - Case 3, Project Calendar (Continued). 


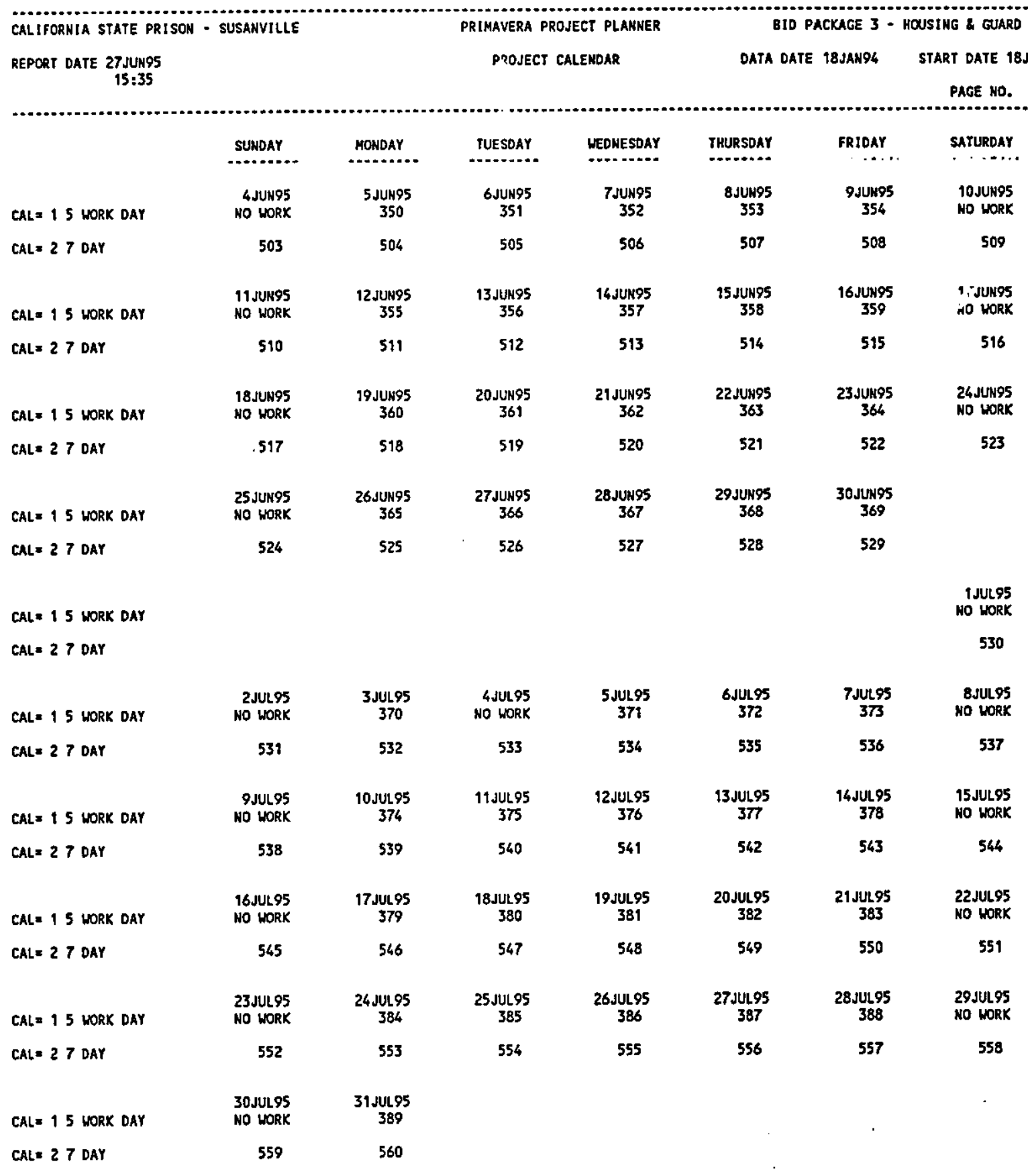

Figure 9.22 - Case 3, Project Calendar (Continued). 


\begin{tabular}{|c|c|c|c|c|c|c|c|c|c|}
\hline \multicolumn{4}{|c|}{ CALIFORNIA STATE PRISON - SUSANVILLE } & \multicolumn{2}{|l|}{ PRIMAVERA PROJECT PLANEER } & \multicolumn{4}{|c|}{ BID PACKAGE 3 - GJARD TOWER } \\
\hline REPORT DATE 1 & $16 \sqrt{6} \cdot 99$ & & 0. 46 & 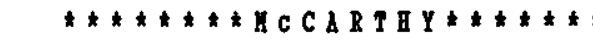 & & ART DATE & 18JaN94 & FIH D.TE 2 & $2212 Y 95 *$ \\
\hline Guard tower 3 & 307 act & ivities & by activit' I & D & & TA DATE & 18JAN94 & PAGE N.. & 1 \\
\hline ICTIVITY & $\begin{array}{l}\text { ORIG } \\
\text { DUR }\end{array}$ & $\begin{array}{l}\text { REAI } \\
\text { DUR CAL }\end{array}$ & 1 & ACTIVITY DESCRIPTIOH & $\begin{array}{l}\text { QAKLYY } \\
\text { START }\end{array}$ & $\begin{array}{l}\text { RARLY } \\
\text { EIMISH }\end{array}$ & STIERT & $\begin{array}{c}\text { LMTR } \\
\text { PINISH }\end{array}$ & $\begin{array}{l}\text { TOMAL } \\
\text { FLOAT }\end{array}$ \\
\hline 60702 & 1 & 11 & 0 & SUBGRADE VERIFICATION & 8MAR94t & * 8HAR94 & 2700794 & $270 \mathrm{CTg}$ & 164 \\
\hline 60704 & 2 & 21 & 0 & BUILDING LAYOOT & 9ILR94 & 10 RaR94 & $280 C 194$ & $3100 \mathrm{~T} 94$ & 164 \\
\hline 6070 & 1 & 11 & 0 & EXCANATE FOOTING & $1112 ! 74$ & 11YAR94 & INOV94 & 2180:94 & 164 \\
\hline 60708 & 1 & 11 & 0 & BLECTRICAL DIDERGROUND & 14LRR94 & 14KAR94 & $2150 \mathrm{V94}$ & $2 \mathrm{HOV} 94$ & 164 \\
\hline 60710 & 2 & 21 & 0 & REBAR AT FOOTING & 15RAR94 & 161LR94 & 3110094 & 4MOV94 & 164 \\
\hline G0712 & 3 & 31 & 0 & PORH/POOR FOOTING & 17RaR94 & 2LUAR94 & 7100794 & $910 \mathrm{V94}$ & 164 \\
\hline 60714 & 2 & 21 & 0 & PLONBING ONDERGROUND & 3010694 & 3120694 & 16 HOV94 & 4 1720V94 & 54 \\
\hline 60716 & 2 & 21 & 0 & STRUCTURAL TONER STEEL ERECTION & 2620694 & $29 A 0694$ & 1010V94 & 414 Nov94 & 53 \\
\hline 60718 & 2 & 21 & 0 & REBAR AT SOC & ISEP94 & 2SEP94 & $18110 V 94$ & 421 NOV94 & 54 \\
\hline 60720 & 2 & 21 & 0 & YETAL DECK INSTALLATTON & 3010694 & 3120694 & 25Nov94 & 4 16Nov94 & 53 \\
\hline 60722 & 2 & 21 & 0 & FORK/POOR SOG & 6SEP94 & 7SEP94 & $22 \mathrm{NON} 94$ & 423 NON94 $_{4}$ & 54 \\
\hline G0724 & 4 & 41 & 0 & SLAB OR IETAL DECK & 1SEP94 & 7SEP94 & 1775ov94 & $22100 \mathrm{Val}$ & 53 \\
\hline G0726 & 2 & 21 & 0 & BACKPILL AT SOG & 8SEP94 & 9SEP94 & 28150V94 & 42910494 & 54 \\
\hline 60728 & 10 & $10 I$ & 0 & YETAL ROOE/SIDING & 8SEP94 & 21SEP94 & 23100994 & 8DEC94 & 53 \\
\hline 60730 & 2 & 21 & 0 & IHSTALL HOLLON KBTAL & 225EP94 & 23SEP94 & $90 R C 94$ & 4 12DEC94 & 53 \\
\hline 60732 & 3 & 31 & 0 & IHST'LLL ALOH FRAKRS/GLASS/GLAZING & 22SEP94 & 26SEP94 & 19DEC94 & 21DRC94 & 59 \\
\hline 60734 & 2 & 21 & 0 & IMTERIOR PRAKIKG & 26SEP94 & 27SPP94 & 13 DEC94 & 140 EC94 & 53 \\
\hline 60736 & 2 & 21 & 0 & PLOKBIMG ROVGB-IY & 28SEP94 & 29STP94 & $200 \mathrm{DC} 94$ & 210EC94 & 56 \\
\hline 60738 & 2 & 21 & 0 & IHSTALL DOORS/HAROWARR & 28SEP94 & 29SEP94 & $5 \mathrm{JAN} 95$ & 6JAK95 & 66 \\
\hline 60740 & 4 & 41 & 0 & IRCHANICAL ROUGE-IH & 28SEP94 & $30 \mathrm{Cr} 94$ & $16 \mathrm{DEC} 94$ & $210 B C 94$ & 54 \\
\hline 60742 & 5 & 51 & 0 & BLECTRICAL ROOGH-IV & 28SEP94 & $40 \mathrm{CT} 94$ & 15DBC94 & 21DEC94 & 53 \\
\hline CO744 & 10 & 101 & 0 & INTERTOR FINISHES & $50 C 194$ & 1800 T94 & $220 E C 94$ & 6JAL195 & 53 \\
\hline 60746 & 2 & 21 & 0 & PAIKT & $1900 \mathrm{Tg}$ & 2000194 & 9JhH95 & 10JAN95 & 53 \\
\hline 60750 & 3 & 31 & 0 & KRCBARICAL FINISH & 2100194 & $250 \mathrm{CT} 94$ & 11JAH95 & 13JAK95 & 53 \\
\hline 60752 & 3 & 31 & 0 & PLOIBIHG FIMISH & $2100 T 94$ & $250<194$ & 11Jג19595 & 13J4W95 & 53 \\
\hline 60754 & 3 & 31 & 0 & BLECTRICAL RINISH & 2100194 & $250 C 194$ & $11 J A 195$ & 13 J4N95 & 53 \\
\hline 60756 & 15 & 151 & 0 & PONCELIST & 2600794 & $16 \% 0 \mathrm{V94}$ & 273 AN95 & 6FEB95* & 53 \\
\hline
\end{tabular}

Figure 9.23 - Case 3, Schedule of Tower 307 Showing $T_{a \mathrm{a}}$ for MS\#8 \& MS\#12 towers under "Total Float" Column. 


\begin{tabular}{|c|c|c|c|c|c|c|c|c|c|}
\hline & & & & & & & & & Normalized \\
\hline & & & & & & & & Independent & Independent \\
\hline & & & Resource & Taa & Rma & Sma & Sma & rate & rate \\
\hline $\begin{array}{ll}\text { ACT } & \text { ID } \\
\end{array}$ & Activity title & Resource & duration & $\mathrm{TF}$ & $\{(7-1\}+(5)$ & $(6) \times(4)$ & rounded & $(8) \div(4)$ & $(9) \times 0.113+(6)$ \\
\hline (1) & (2) & (3) & (4) & (5) & (6) & $(7)$ & $(8)$ & (9) & (10) \\
\hline 60702 & SOBGRADE VERIFICATION & $\$ 802$ & 1 & 164 & 0.037 & 0.037 & 1 & 1.000 & 3.094 \\
\hline 60704 & BOILDING LAYOUT & 3804 & 2 & 164 & 0.037 & 0.073 & 1 & 0.500 & 1.547 \\
\hline 60706 & EXCAVATE POOTING & $\$ 806$ & 1 & 164 & 0.037 & 0.037 & 1 & 1.000 & 3.094 \\
\hline 60708 & BLECTRICAL UNDERGROUND & 3008 & 1 & 164 & 0.037 & 0.037 & 1 & 1.000 & 3.094 \\
\hline 90710 & REBAR AT FOOTING & $\$ 210$ & 2 & 164 & 0.037 & 0.073 & 1 & 0.500 & 1.547 \\
\hline 00712 & FORM/POUR FOOTING & $\$ 212$ & 3 & 164 & 0.037 & 0.110 & 1 & 0.333 & 1.031 \\
\hline 90714 & PLUMBING ONDERGROUND & $\$ 814$ & 2 & 54 & 0.111 & 0.222 & 1 & 0.500 & 0.509 \\
\hline 60716 & STRUCTURAL TOWER STEEL ERECTION & $\$ 816$ & 2 & 53 & 0.113 & 0.226 & 1 & 0.500 & 0.500 \\
\hline 60718 & KEBAR AT SOG & 3018 & 2 & 54 & 0.111 & 0.222 & 1 & 0.500 & 0.509 \\
\hline 60720 & WETAL DECK INSTALLATION & $\$ 220$ & 2 & 53 & 0.113 & 0.226 & 1 & 0.500 & 0.500 \\
\hline G0722 & FORM/POUR SOO & $\overline{822}$ & 2 & 54 & 0.111 & 0.222 & 1 & 0.500 & 0.509 \\
\hline $\mathbf{G 0 7 2 4}$ & SLAB ON METAL DECK & $\$ 224$ & 4 & 53 & 0.113 & 0.453 & 1 & 0.250 & 0.250 \\
\hline 00726 & BACKPILL AT SOG & $\$ 826$ & 2 & 54 & 0.111 & 0.222 & 1 & 0.500 & 0.509 \\
\hline 60728 & NETAL ROOF/SIDING & $\$ 228$ & 10 & 53 & 0.113 & 1.132 & 2 & 0.200 & 0.200 \\
\hline 60730 & ENSTALI HOLLON METAL & 3030 & 2 & 53 & 0.113 & 0.226 & 1 & 0.500 & 0.500 \\
\hline G0732 & TNSTALL ALUM FRAMES/GLASS/GLAZING & $\$ 932$ & 3 & 59 & 0.102 & 0.305 & 1 & 0.333 & 0.371 \\
\hline G0734 & ENTERIOR FRAMING & $\$ 834$ & 2 & 53 & 0.113 & 0.226 & 1 & 0.500 & 0.500 \\
\hline G0736 & PLONBING ROUGH-IN & $\$ 836$ & 2 & 56 & 0.107 & 0.214 & 1 & 0.500 & 0.528 \\
\hline G0738 & INSTALL DOORS/HARDWARE & $\$ 938$ & 2 & 66 & 0.091 & 0.182 & 1 & 0.500 & 0.623 \\
\hline 60740 & MECHANICAL RODGH-IN & $\$ 040$ & 4 & 54 & 0.111 & 0.444 & 1 & 0.250 & 0.255 \\
\hline 60742 & ELECTRICAL ROVGH-IN & $\$ 842$ & 5 & 53 & 0.113 & 0.566 & 1 & 0.200 & 0.200 \\
\hline 00744 & INTERIOR FINISHES & $\$ 844$ & 10 & 53 & 0.213 & 1.132 & 2 & 0.200 & 0.200 \\
\hline 60746 & PAINT & $\$ 946$ & 2 & 53 & 0.113 & 0.226 & 1 & 0.500 & 0.500 \\
\hline 60750 & MECEANICAL FINISH & 3050 & 3 & 53 & 0.113 & 0.340 & 1 & 0.333 & 0.333 \\
\hline 60752 & PLUMBING FINISH & $\$ 052$ & 3 & 53 & 0.113 & 0.340 & 1 & 0.333 & 0.333 \\
\hline 60754 & ELECTRICAL FINISH & 8054 & 3 & 53 & 0.113 & 0.340 & 1 & 0.333 & 0.333 \\
\hline 60756 & PUNCELIST & 3056 & 12 & 53 & 0.113 & 1.358 & 2 & 0.167 & 0.167 \\
\hline
\end{tabular}


CALIFORNIA STATE PRISON - SOSANVILLR

REPORT DATE 16JOL95 RONI NO. 53

Guard tower 307 activities by activity ID -leveled
BID PACRAGE 3 - GUARD TOAER

PRILAVERA PROJECT PLANNER

START DATE 18JAN94 FIN DaTE 22HAY95* DATA DATE 18JAN94 PAGE HO. 1

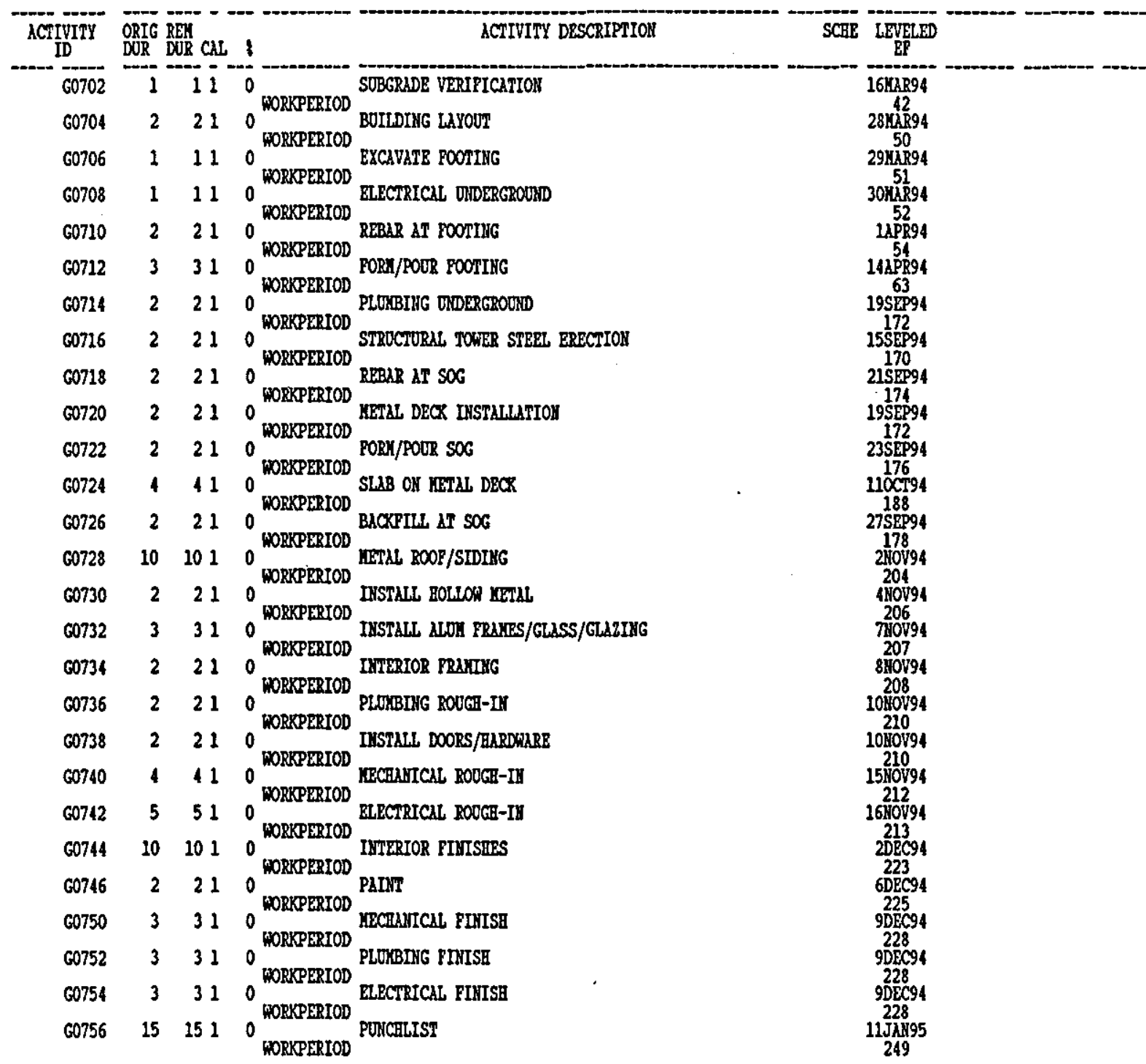

Figure 9.24 - Case 3, Schedule of Tower 307 after Leveling Serves as a First Unit Schedule for Balance of Towers. 
CALIFORNIA STATE PRISON - SUSANVILLE

REPORT DATE 16JUL95 RON NO. 48

Guard tower 312 activities by activity ID
PRIMAVERA PROJECT PLANAER BID PACXAGE 3 - GOARD TOWER

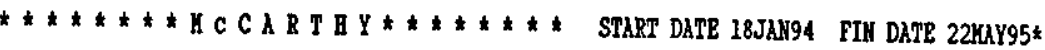
DATA DATE 18 JAN94 PAGR NO. 1

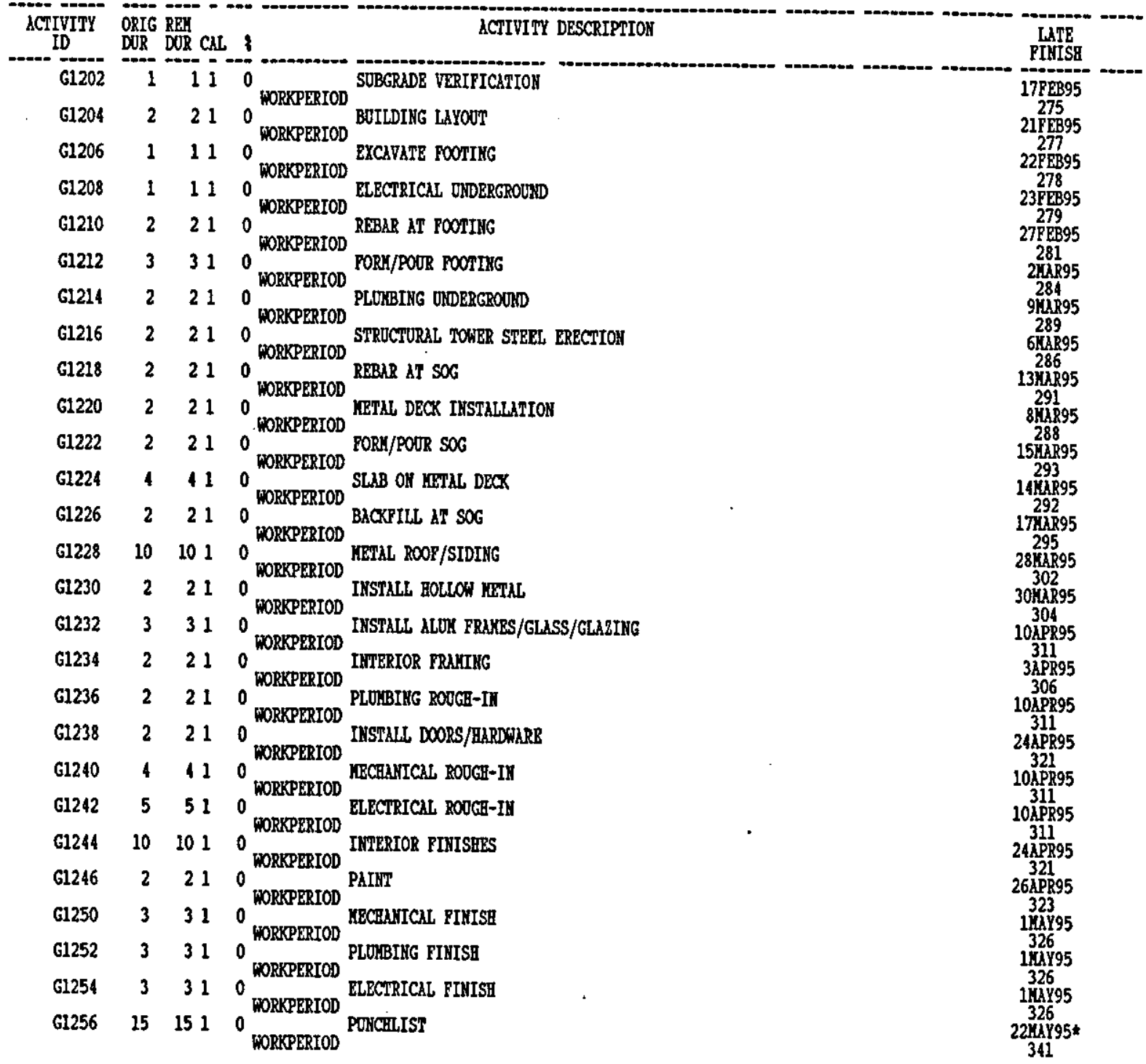

Figure 9.25 - Case 3, Latest Dates of the Last Tower, 312. 


\begin{tabular}{|c|c|c|c|c|c|c|c|c|c|c|c|c|}
\hline \multirow{4}{*}{\multicolumn{2}{|c|}{\begin{tabular}{l|ll}
\multirow{2}{*}{8} & ACT \\
\end{tabular}}} & & & & Tower & Tower & & & & & & Normallzed \\
\hline & & & & & 307 & 312 & & & & & Independent & independent \\
\hline & & & & Resource & leveled & Scheduled & Taa & Rma & Sma & Sma & rate & rate \\
\hline & & Activity titie & Resource & duration & EF & LF & (6) - (5) & $\{7 \cdot 1\} \div(7)$ & $(8) \times(4)$ & rounded & $(10)+(4)$ & $(11) \times 0.065+(8)$ \\
\hline 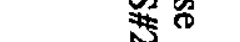 & (1) & (2) & (3) & (4) & (5) & (6) & (7) & (8) & (9) & (10) & (11) & (12) \\
\hline 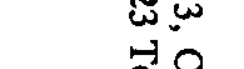 & 60702 & SUBGRADE VERIFICATION & SQ02 & 1 & 42 & 275 & 233 & 0.026 & 0.026 & $I$ & 1.000 & 2.533 \\
\hline$\frac{0}{2}$ & 60704 & BUILDING LAYOUT & $S Q 04$ & $\mathbf{2}$ & 50 & 277 & 227 & 0.026 & 0.053 & 1 & 0.500 & 1.234 \\
\hline 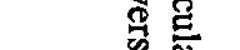 & 90706 & EXCAVATE POOTING & SQ06 & 1 & 51 & 278 & 227 & 0.026 & 0.026 & 1 & 1.000 & 2.467 \\
\hline 莺. & 60708 & ELECTRICAL UNDERGROUND & SQ08 & 1 & 52 & 279 & 227 & 0.026 & 0.026 & 1 & 1.000 & 2.467 \\
\hline 9 & 60710 & REBAR AT FOOTING & SQ10 & 2 & 54 & 281 & 227 & 0.026 & 0.053 & 1 & 0.500 & 1.234 \\
\hline 욤 & G0712 & FORM/POUR FOOTING & SQ12 & 3 & 63 & 284 & 221 & 0.027 & 0.081 & 1 & 0.333 & 0.801 \\
\hline$\vec{z}$ & G0714 & PLUMBING UNDERGROUND & SQ14 & 2 & $\mathbf{1 7 2}$ & 289 & 117 & 0.051 & 0.103 & 1 & 0.500 & 0.636 \\
\hline$\triangleq$ & G0716 & STRUCTURAL TOWER STEEL ERECTION & SQ16 & 2 & 170 & 286 & 116 & 0.052 & 0.103 & 1 & 0.500 & 0.630 \\
\hline$\xi^{\circ}$ & G0718 & REBAR AT SOG & SQ18 & 2 & 174 & 291 & 117 & 0.051 & 0.203 & 1 & 0.500 & 0.636 \\
\hline ? & 60720 & METAL DECK INSTALLATION & $\mathbf{S Q 2 0}$ & 2 & 172 & 288 & 116 & 0.052 & 0.103 & 1 & 0.500 & 0.630 \\
\hline a & 60722 & FORM/POUR SOO & SQ22 & 2 & 176 & 293 & 117 & 0.051 & 0.103 & 1 & 0.500 & 0.636 \\
\hline & 60724 & SLAB ON METAL DECK & SQ24 & 4 & 188 & 292 & 104 & 0.058 & 0.231 & 1 & 0.250 & 0.283 \\
\hline$\cong$ & 60726 & AACKFILL AT SOO & SQ26 & 2 & 178 & 295 & 117 & 0.051 & 0.103 & 1 & 0.500 & 0.636 \\
\hline E. & 60728 & METAL ROOF/SIDING & SQ2B & 10 & 204 & 302 & 98 & 0.061 & 0.612 & 1 & 0.100 & 0.107 \\
\hline 0 & 60730 & FNSTALL HOLLOW METAL & $\mathbf{S Q 3 0}$ & $\mathbf{2}$ & 206 & 304 & 98 & 0.061 & 0.122 & 1 & 0.500 & 0.533 \\
\hline 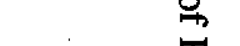 & 60732 & FNSTALLL ALUM FRAMES/GLASS/GLAZZING & SQ32 & 3 & 207 & 311 & 104 & 0.058 & 0.173 & 1 & 0.333 & 0.377 \\
\hline 蛋 & 60734 & FNTERIOR PRAMING & $\mathbf{S 0 3 4}$ & $\mathbf{2}$ & 208 & 306 & 98 & 0.061 & 0.122 & 1 & 0.500 & 0.533 \\
\hline क & 60736 & PLUMBING ROUGE-IN & S036 & 2 & 210 & 311 & 101 & 0.059 & 0.119 & 1 & 0.500 & 0.549 \\
\hline 5 & 60738 & FNSTALL DOORS/EARDWARE & SQ38 & $\overline{2}$ & 210 & 321 & 111 & 0.054 & 0.108 & 1 & 0.500 & 0.603 \\
\hline \&્ & 60740 & MECEANICAI ROUGH-IN & $S \mathbf{S}_{40}$ & 4 & 212 & 311 & 99 & 0.061 & 0.242 & 1 & 0.250 & 0.269 \\
\hline 5 & 60742 & ELECTRICAL ROJGH-IN & SQ42 & 5 & 213 & 311 & 98 & 0.061 & 0.306 & 1 & 0.200 & 0.213 \\
\hline & 60744 & FNIERIOR FINISHES & $\mathbf{S Q 4 4}$ & 10 & 223 & 321 & 98 & 0.061 & 0.612 & 1 & 0.100 & 0.107 \\
\hline 0 & 60746 & PAINI & SQ46 & 2 & 225 & 323 & 98 & 0.061 & 0.122 & 1 & 0.500 & 0.533 \\
\hline$\underline{8}$ & 60750 & MECBANICAI FINISH & 5850 & 3 & 228 & 326 & 98 & 0.061 & 0.184 & 1 & 0.333 & 0.355 \\
\hline $\overrightarrow{0}$ & 60752 & PLUMBING FINISA & $\mathbf{S Q 5 2}$ & 3 & 228 & 326 & 98 & 0.061 & 0.184 & 1 & 0.333 & 0.355 \\
\hline$z$ & 60754 & ELECTRICAL FINISH & $S 054$ & 3 & 228 & 326 & 98 & 0.061 & 0.184 & 1 & 0.333 & 0.355 \\
\hline 灾 & 60756 & PUNCELIST & 3056 & 12 & 249 & 341 & 92 & 0.065 & 0.783 & 1 & 0.083 & 0.083 \\
\hline
\end{tabular}




\begin{tabular}{|c|c|c|}
\hline CALIFORHIA STATE PRISON - SUSANVILLE & PRIHLVERA PROJECT PLANNER & BID PACKAGE 3 - GUARD TONER \\
\hline $\begin{array}{l}\text { REPORT : ATE } 20 \text { SEP95 RON NO. } 87 \\
11: 42 \text { Baseline schedule by Typical sctivity \& ES }\end{array}$ & 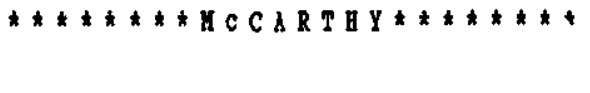 & 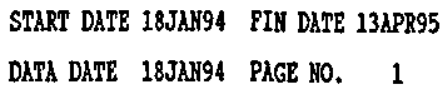 \\
\hline
\end{tabular}

\begin{tabular}{|c|c|c|c|c|c|c|c|c|c|c|}
\hline ACIIVITY & $\begin{array}{l}\text { ORIG } \\
\text { DUR }\end{array}$ & REW & & CODB & ACTIVITY DESCRIPTION & $\begin{array}{l}\text { LEVELED } \\
\text { ES }\end{array}$ & $\begin{array}{l}\text { LEVELLD } \\
\text { EP }\end{array}$ & $\begin{array}{l}\text { LEVELED } \\
\text { LS }\end{array}$ & & OPRL \\
\hline 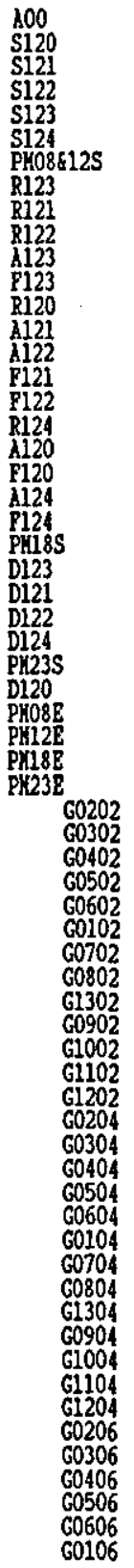 & $\begin{array}{r}1 \\
65 \\
60 \\
60 \\
50 \\
75 \\
0 \\
20 \\
20 \\
20 \\
0 \\
60 \\
20 \\
0 \\
0 \\
60 \\
60 \\
20 \\
0 \\
75 \\
0 \\
45 \\
0 \\
1 \\
1 \\
1 \\
1 \\
1 \\
0 \\
1 \\
0 \\
0 \\
0 \\
0 \\
1 \\
1\end{array}$ & $\begin{array}{rrr}1 & 1 \\
65 & 1 \\
60 & 1 \\
60 & 1 \\
50 & 1 \\
75 & 1 \\
0 & 2 \\
20 & 2 \\
20 & 2 \\
20 & 2 \\
0 & 2 \\
60 & 1 \\
20 & 2 \\
0 & 2 \\
0 & 2 \\
60 & 1 \\
60 & 1 \\
20 & 2 \\
0 & 2 \\
75 & 1 \\
0 & 2 \\
45 & 1 \\
0 & 2 \\
1 & 1 \\
1 & 1 \\
1 & 1 \\
1 & 1 \\
0 & 2 \\
1 & 1 \\
0 & 2 \\
0 & 2 \\
0 & 2 \\
0 & 2 \\
1 & 1 \\
1 & 1 \\
1 & 1 \\
1 & 1 \\
1 & 1 \\
1 & 1 \\
1 & 1 \\
1 & 1 \\
1 & 1 \\
1 & 1 \\
1 & 1 \\
1 & 1 \\
1 & 1 \\
2 & 1 \\
2 & 1 \\
2 & 1 \\
2 & 1 \\
2 & 1 \\
2 & 1 \\
2 & 1 \\
2 & 1 \\
2 & 1 \\
2 & 1 \\
2 & 1 \\
2 & 1 \\
2 & 1 \\
1 & 1 \\
1 & 1 \\
1 & 1 \\
1 & 1 \\
1\end{array}$ & $\begin{array}{l}0 \\
0 \\
0 \\
0 \\
0\end{array}$ & & 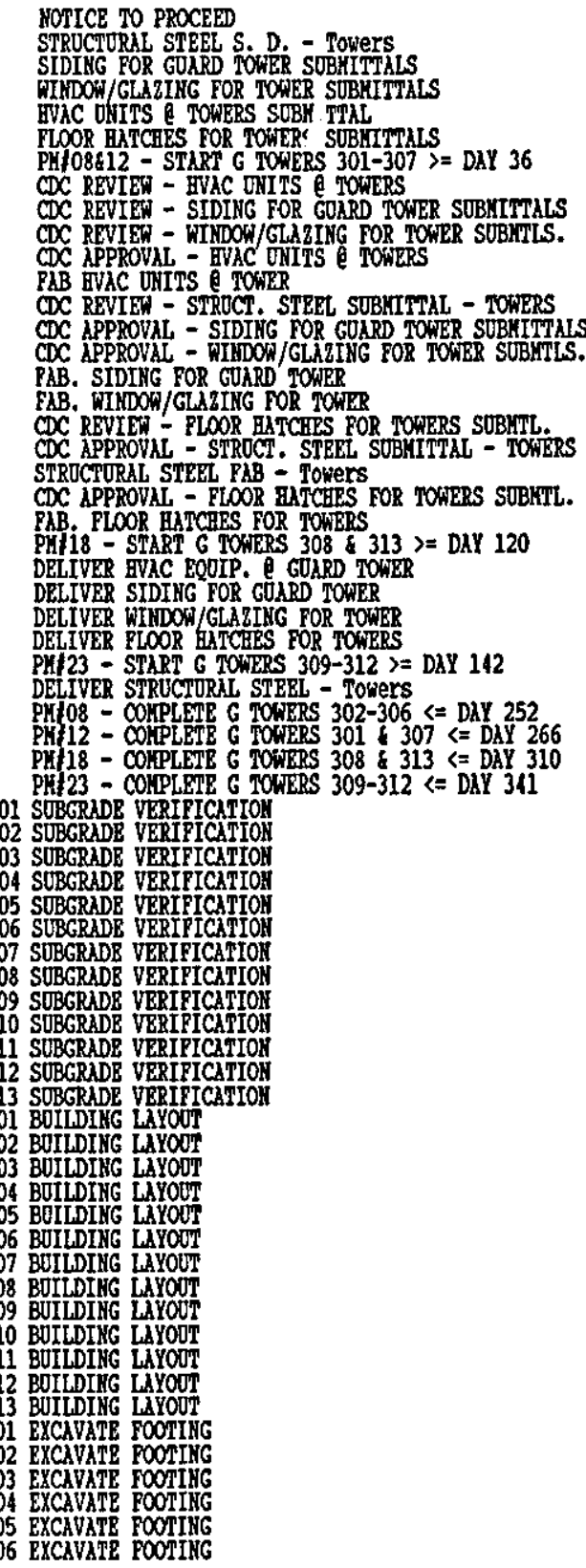 & 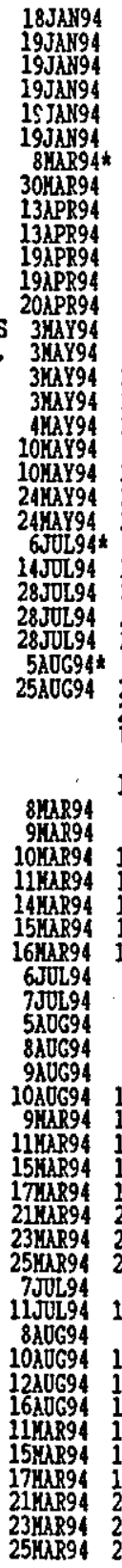 & 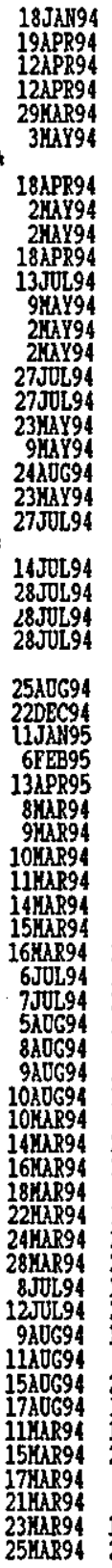 & 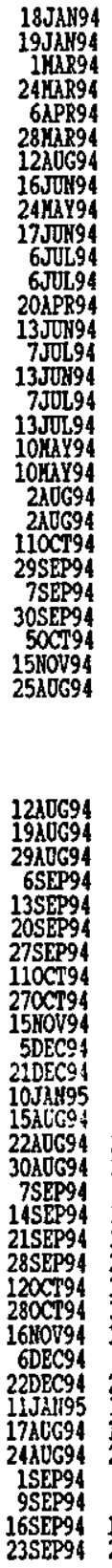 & 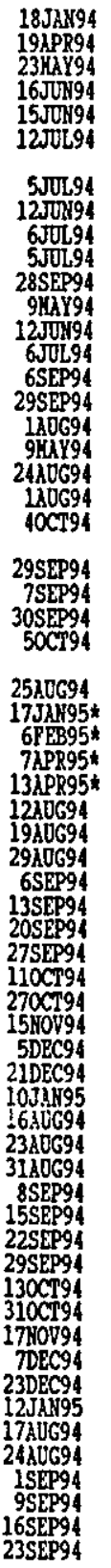 & $\begin{array}{r}0 \\
0 \\
29 \\
46 \\
55 \\
48 \\
157 \\
78 \\
41 \\
65 \\
78 \\
51 \\
0 \\
41 \\
65 \\
28 \\
45 \\
70 \\
0 \\
0 \\
70 \\
48 \\
97 \\
54 \\
28 \\
45 \\
48 \\
102 \\
0 \\
26 \\
26 \\
600 \\
0 \\
111 \\
115 \\
120 \\
124 \\
128 \\
132 \\
136 \\
68 \\
79 \\
70 \\
81 \\
92 \\
103 \\
111 \\
114 \\
118 \\
121 \\
124 \\
127 \\
130 \\
68 \\
78 \\
70 \\
80 \\
90 \\
100 \\
111 \\
114 \\
118 \\
121 \\
124 \\
127\end{array}$ \\
\hline
\end{tabular}

Figure 9.26 - Case 3, Baseline Schedule. 
CALIFORNIA STATE PRISON - SUSANILLE

REPORT DATE 20SEP95 RON KO. 87 Baseline Schedule by Typical Activity \& ES
PRIMLVERA PROJECT PLANKER

BID PACKAGE 3 - GUARD TONER

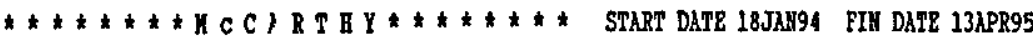
DATA DATE 18JAN94 PAGE HO. 2

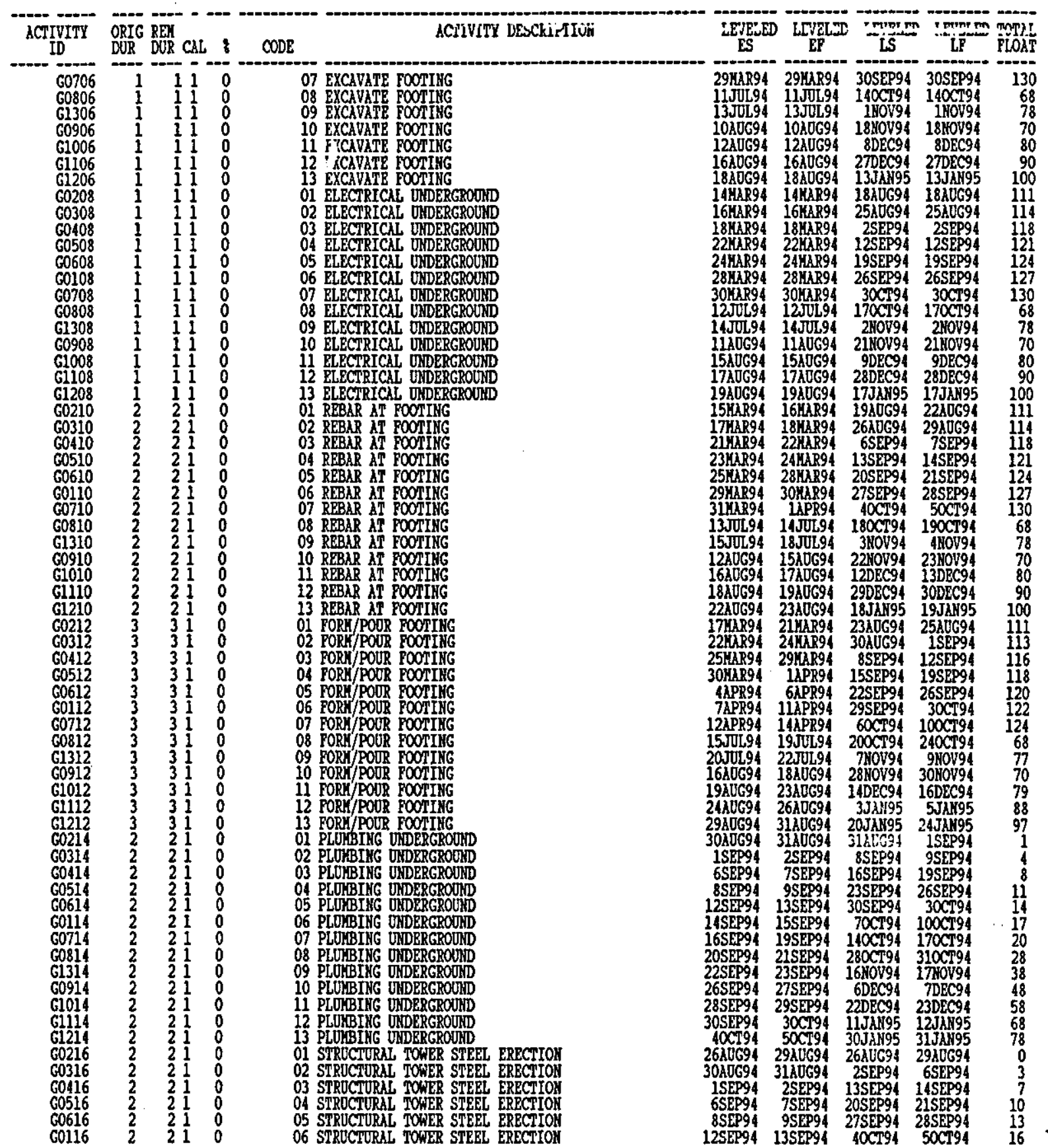

Figure 9.26 - Case 3, Baseline Schedule (Continued). 


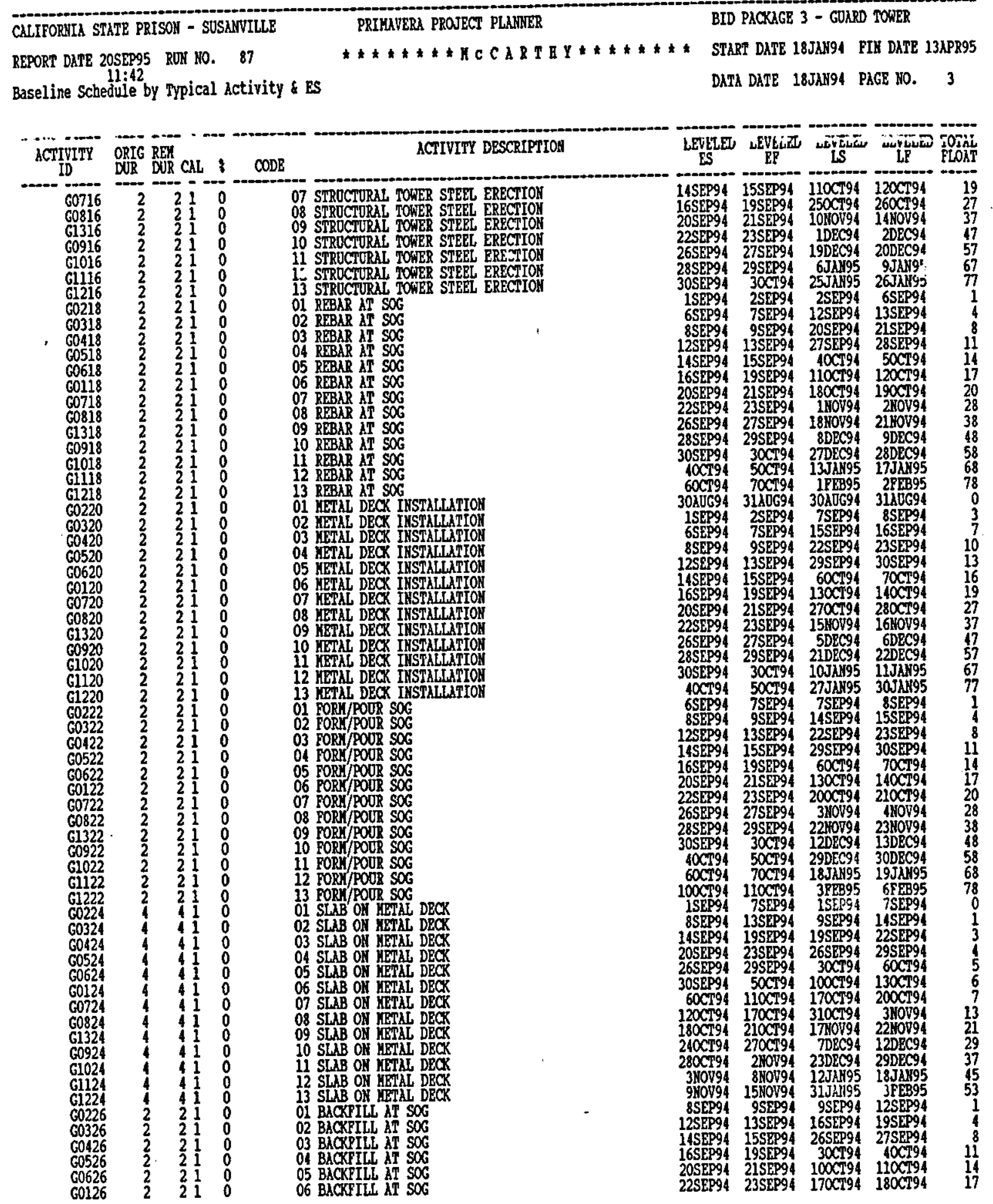

Figure 9.26 - Case 3, Baseline Schedule (Continued). 
CALIFORNIA STATE PRISOH - SUSANVILLB

REPORT DATE 20SEP95 ROH NO. 87

Baseline Schedule by Typical hctivity \& ES
BID PACKAGE 3 - GUARD TOKRR

START DATE 18JגM94 PIR DATR 13APR95 DATA DATE 18JAN94 PAGB NO. :

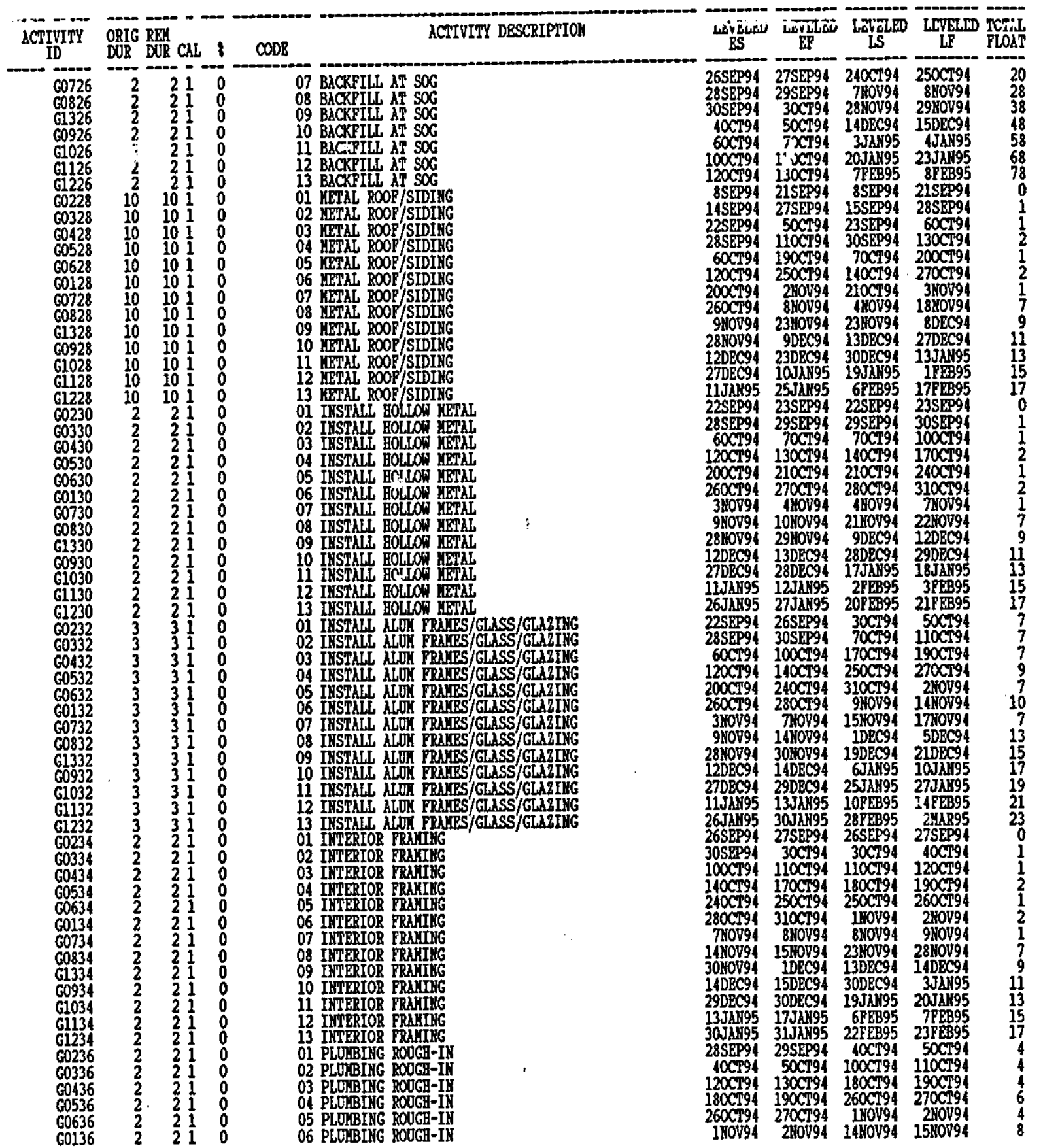

Figure 9.26 - Case 3, Baseline Schedule (Continued). 


\begin{tabular}{|c|c|c|}
\hline CALIFORNIA STATE PRISON - SUSANIILLE & PRIMAVERA PROJECT PLANUER & BID PACKAGE 3 - GUARD TOAER \\
\hline $\begin{array}{l}\text { REPORT DATE } 20 \text { SEP95 } \\
11: 42 \text { RO: } \\
\text { BO. } 87 \\
\text { Baseline Schedule by Typ cal detivity \& ES }\end{array}$ & 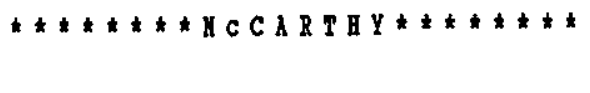 & $\begin{array}{l}\text { START DATE 13JAN94 FIH DATE 13APR95 } \\
\text { DATA DATE 1\}JAM94 PAGE HO. } 5\end{array}$ \\
\hline
\end{tabular}

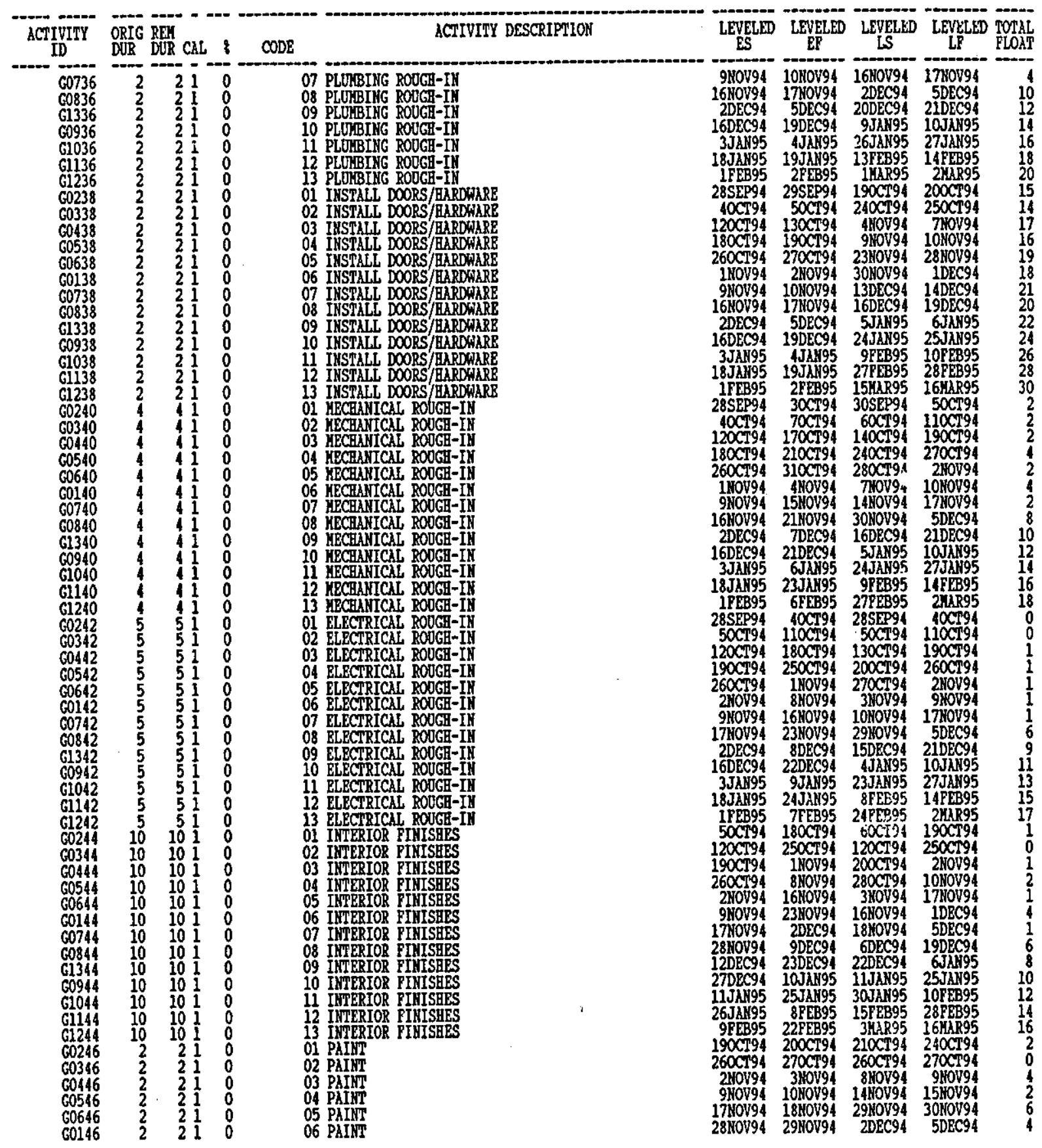

Figure 9.26 - Case 3, Baseline Schedule (Continued). 
CALIFORYIX STATE PRISON - SUSANVILLE

RE.ORT DATE 20SEP95 RON HO. 87

Baseline Schedule by Typical Activity \& ES
PRIMAVER PROJECT PLANEER

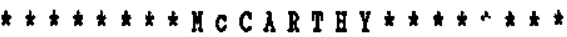

BID PACKAGE 3 - GOARD TOWER

START DATE 18JAN94 RIN DATE 13APR95 DATA DATE 18JAN94 PAGR HO. 6

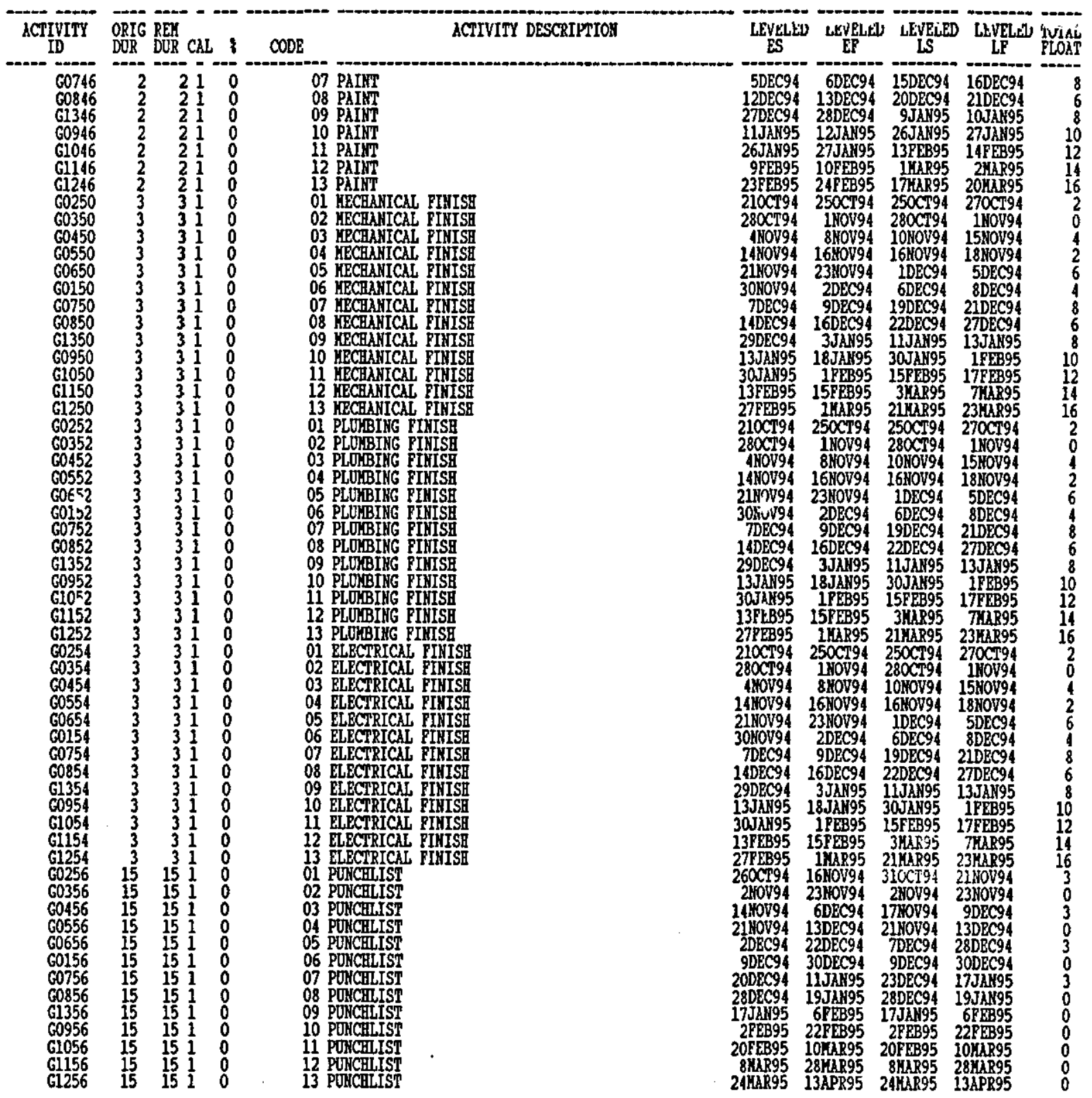

Figure 9.26 - Case 3, Baseline Schedule (Continued). 
Structural Steel Erection Squad - CA16
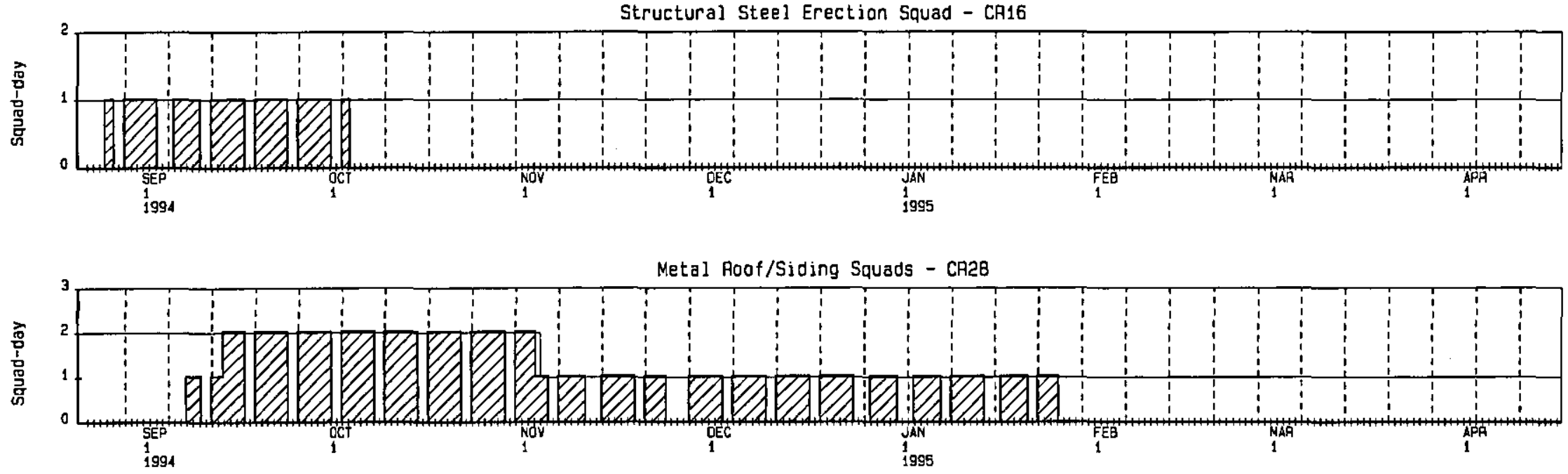

$\stackrel{f}{\frac{7}{1}}$
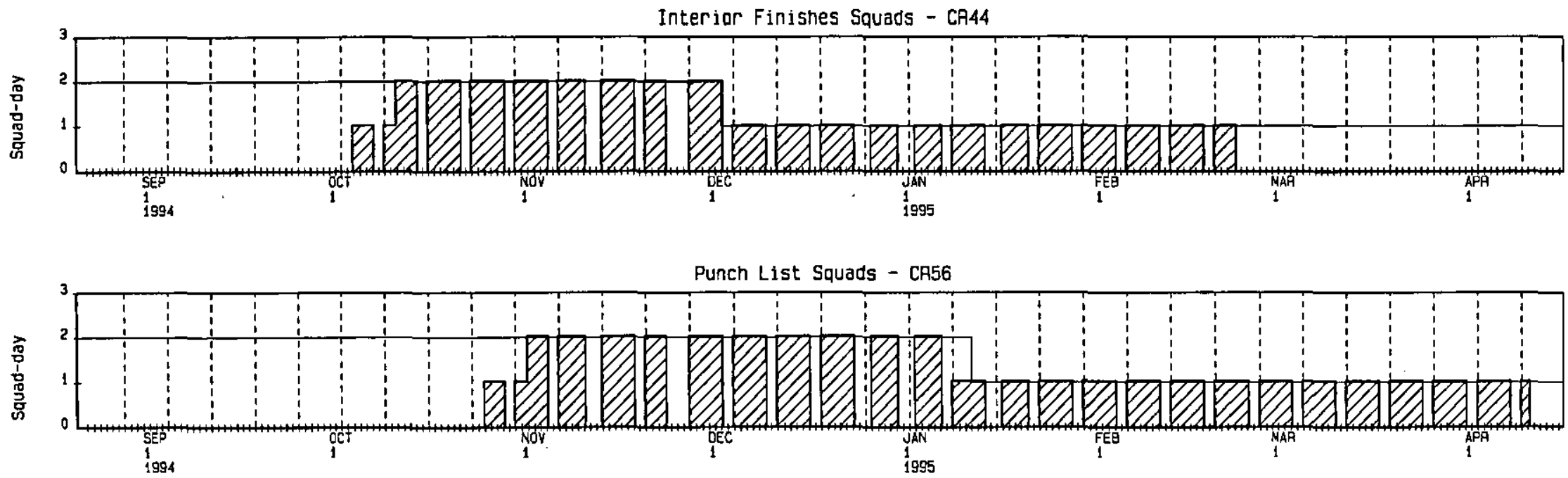

Figure 9.27- Case 3, Squad Histograms Showing Work Continuity. 
CALIFORNIA STATE PRISON - SOSAKVILLE

REPORT DATE 20SEP95 RON NO. 61

Dec. 94 Opdate by TYpical Activity \& ES
PRIKAVERA PROJECT PLANDER

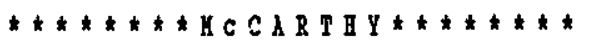

STRRT DATE 18JAN94 FIN DATE 22YAY95* DATA DATE 18DEC94 PAGE NO. 1

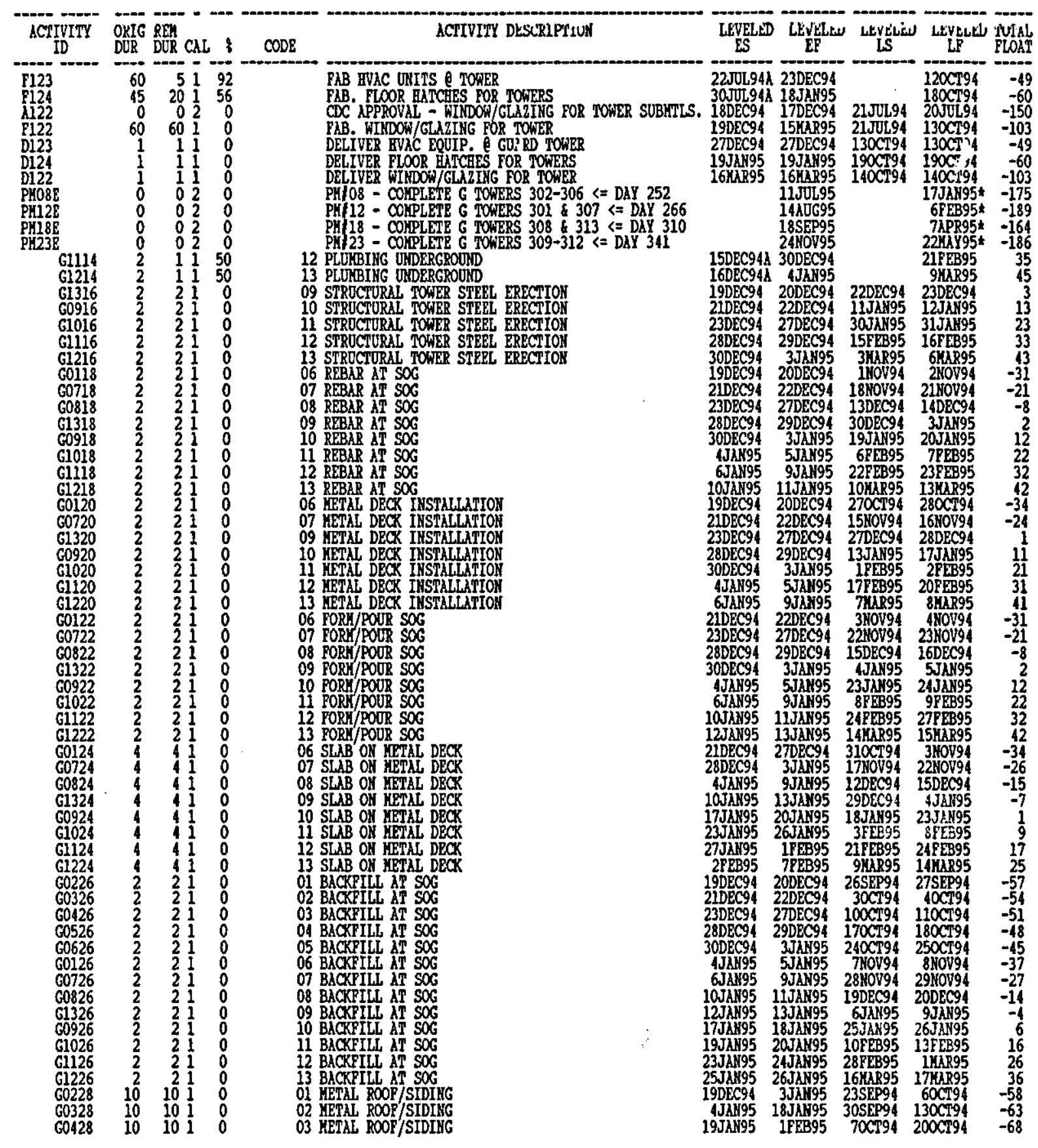

Figure 9.28 - Case 3, Status as of December 18, 1994. 
CALIFORNIA STATE PRISON - SUSANVILLE

REPORT DATE 20SEP95 RON HO. 61 Dec. 94 Opdate by Typical Activity \& ES
PRIMAVERA PROJECT PLANEER

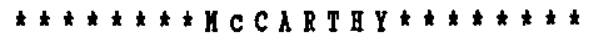

BID PACKAGE 3 - GDARD TOWER

START DATE 18JAK94 PIM DATE 22LAY95* DATA DATE 18DEC94 PAGE NO. 2

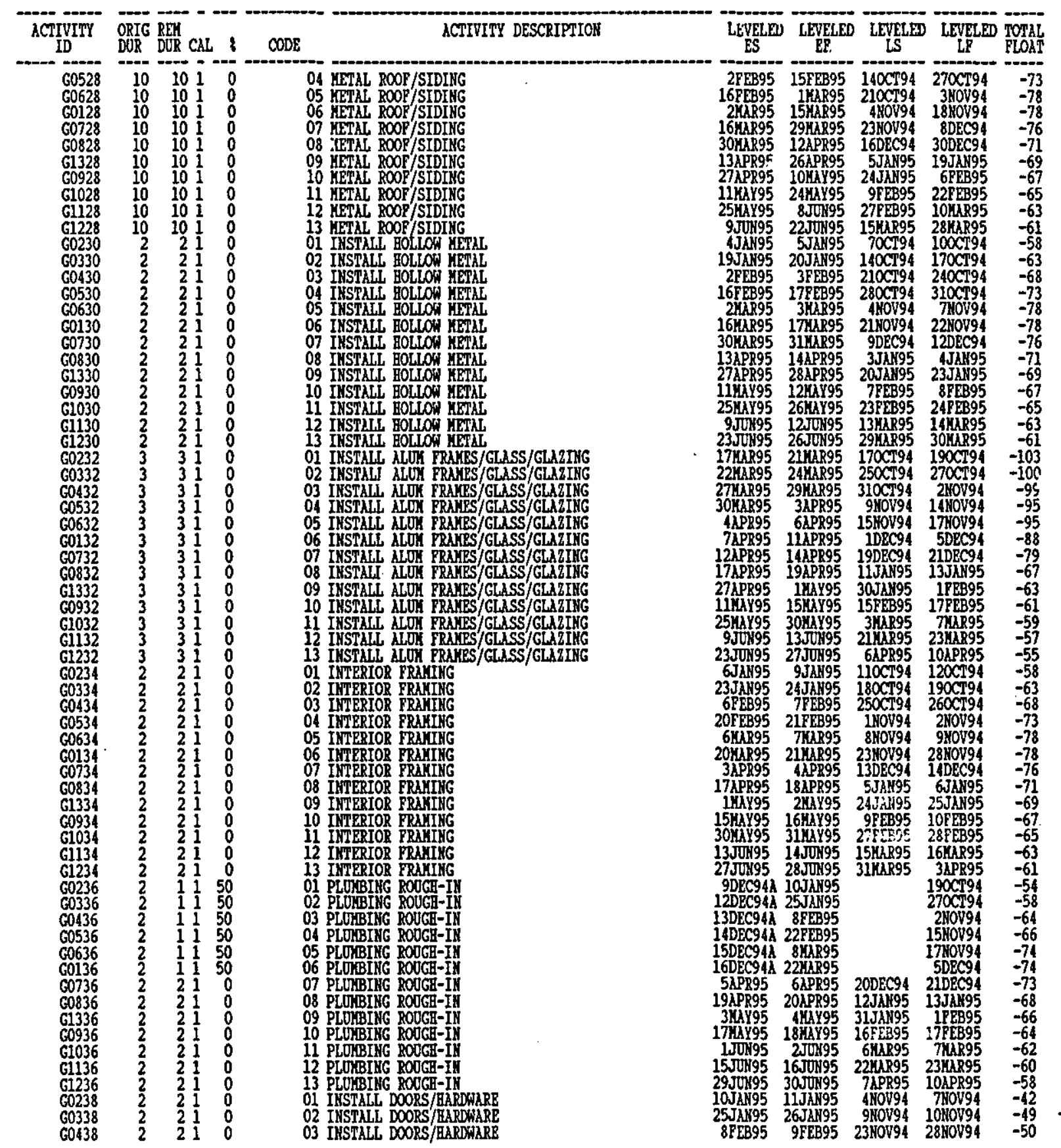

Figure 9.28 - Case 3, Status as of December 18, 1994 (Continued). 
CALIFORHIA STATE PRISON - SUSANILLE

REPORT DATE 20SEP95 RON HO. 61

Dec. 94 Update by Tyuical Activity \& ES
BID PACRAGE 3 - GUARD TONER

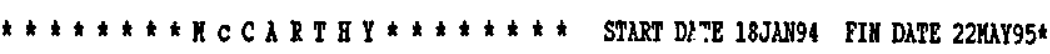
DATA DATF 18DEC94 PAGE HO. 3

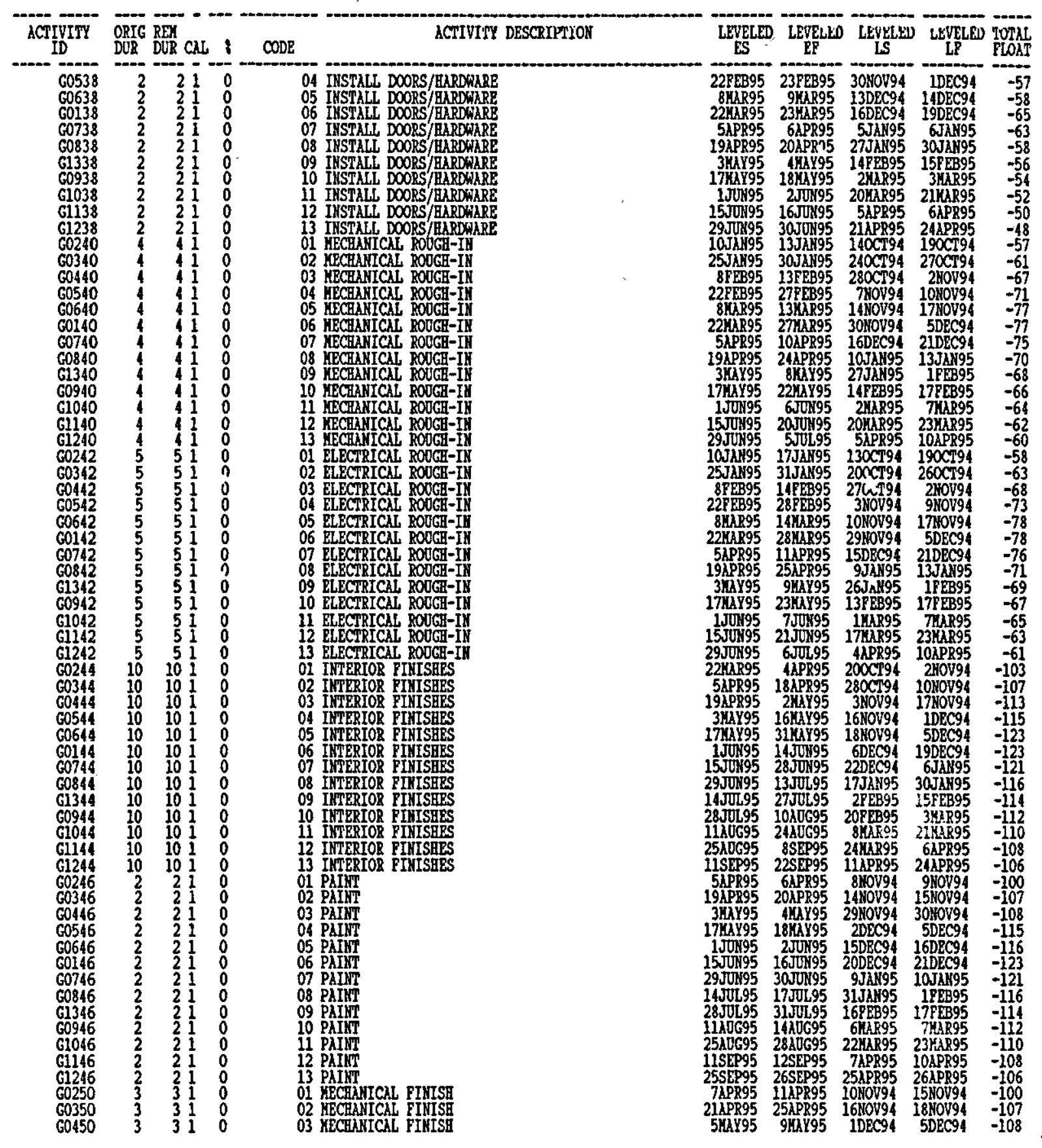

Figure 9.28 - Case 3, Status as of December 18, 1994 (Continued). 
CALIFORNIA STATR PRISON - SUSANVILLE

REPORT DATE 2OSEPS5 RON BO. 61

Dec. 94 Update by Typical sctivity \& ES
BID PACXIGE 3 - GUARE TOWER

PRIMAVERA PROJECT PLANIER

START DATE 18JAN94 FIY DATE 221KAY95* DATA DATE 18DEC94 PAGB HO. 4

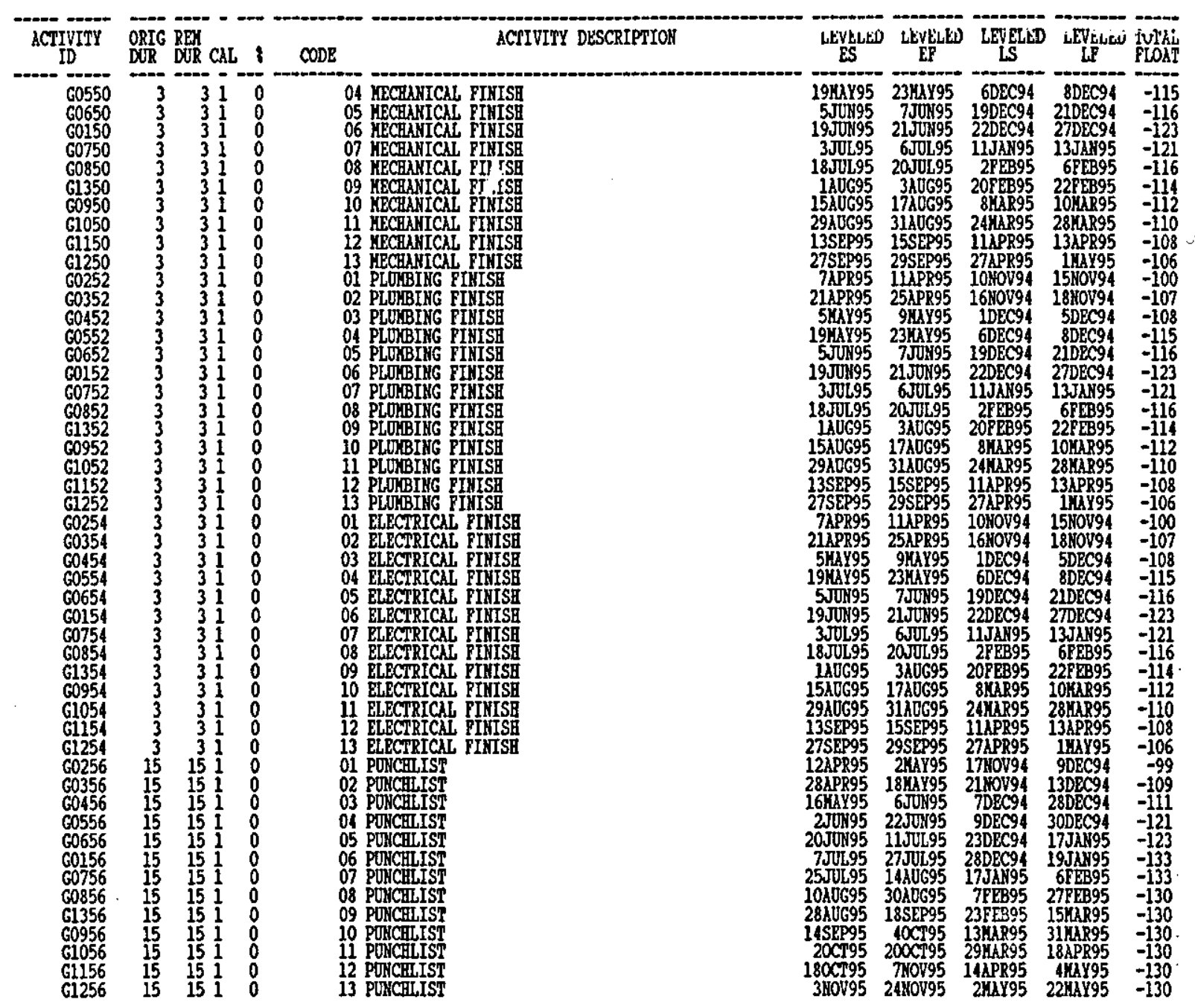

Figure 9.28 - Case 3, Status as of December 18, 1994 (Continued). 


\section{Thirteen Guard Towers}

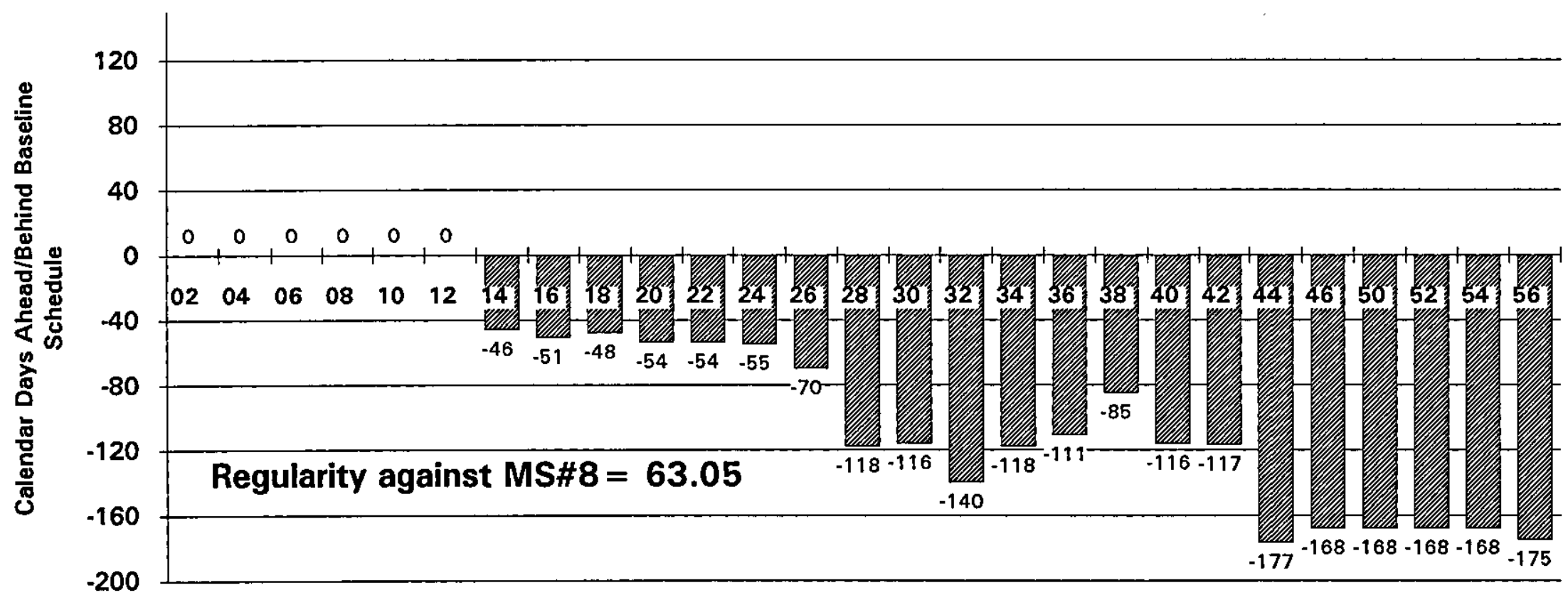

Typical Activities

Figure 9.29 - Case 3, Progress Regularity as of December 18, 1994. 


\section{Thirteen Guard Towers}

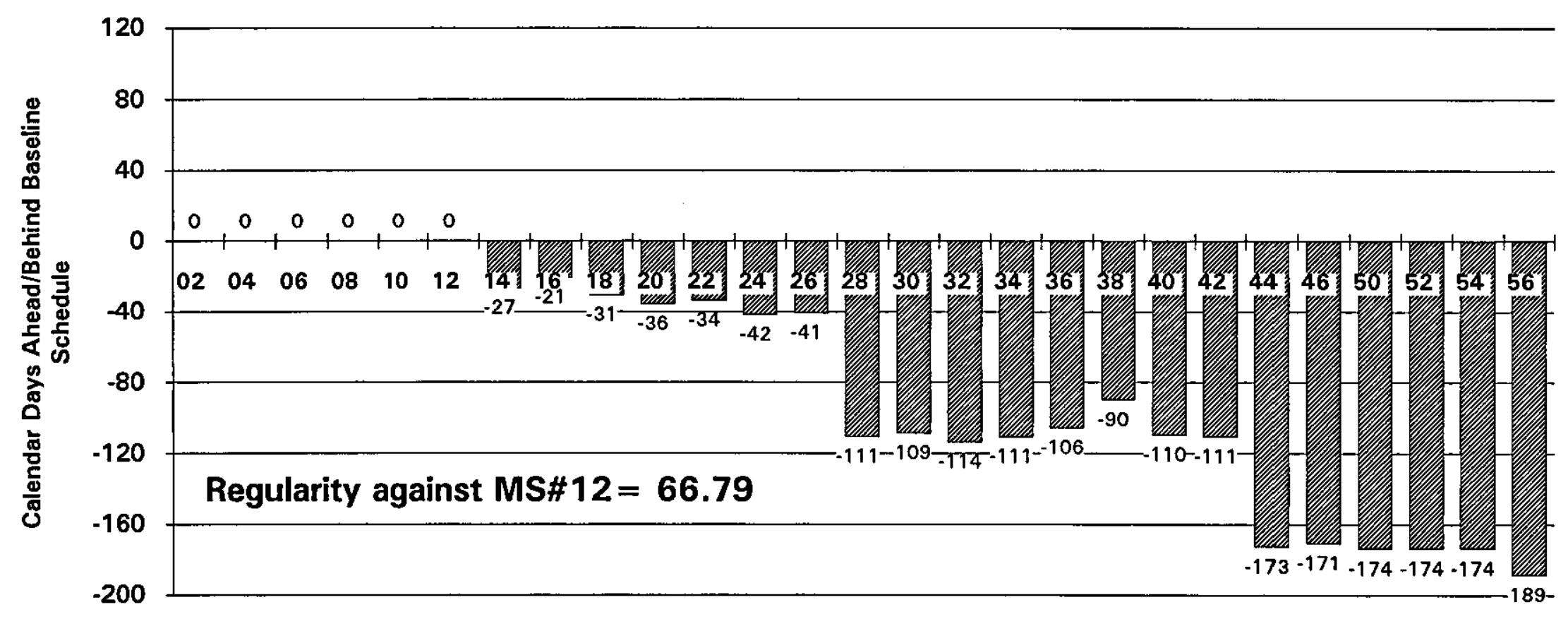

Typical Activities

Figure 9.29 - Case 3, Progress Regularity as of December 18, 1994 (Continued). 


\section{Thirteen Guard Towers}

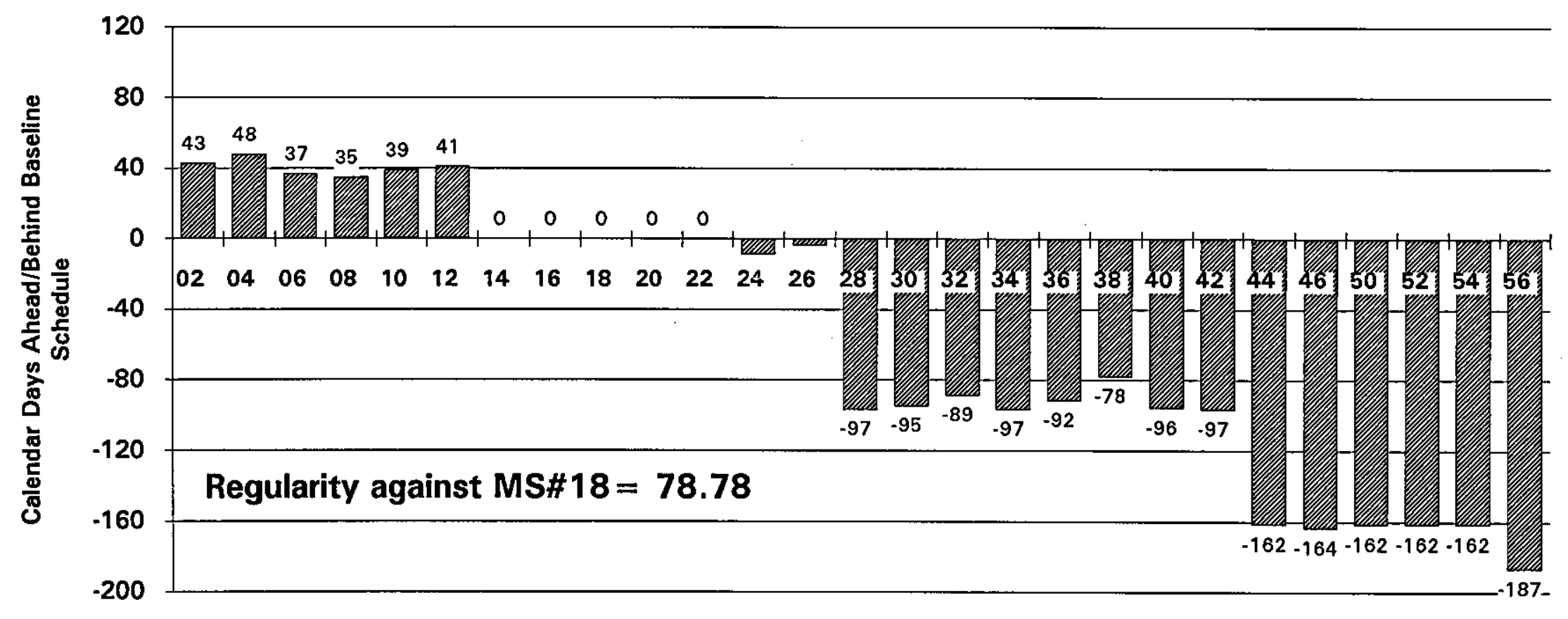

Typical Activities

Figure 9.29 - Case 3, Progress Regularity as of December 18, 1994 (Continued). 


\section{Thirteen Guard Towers}

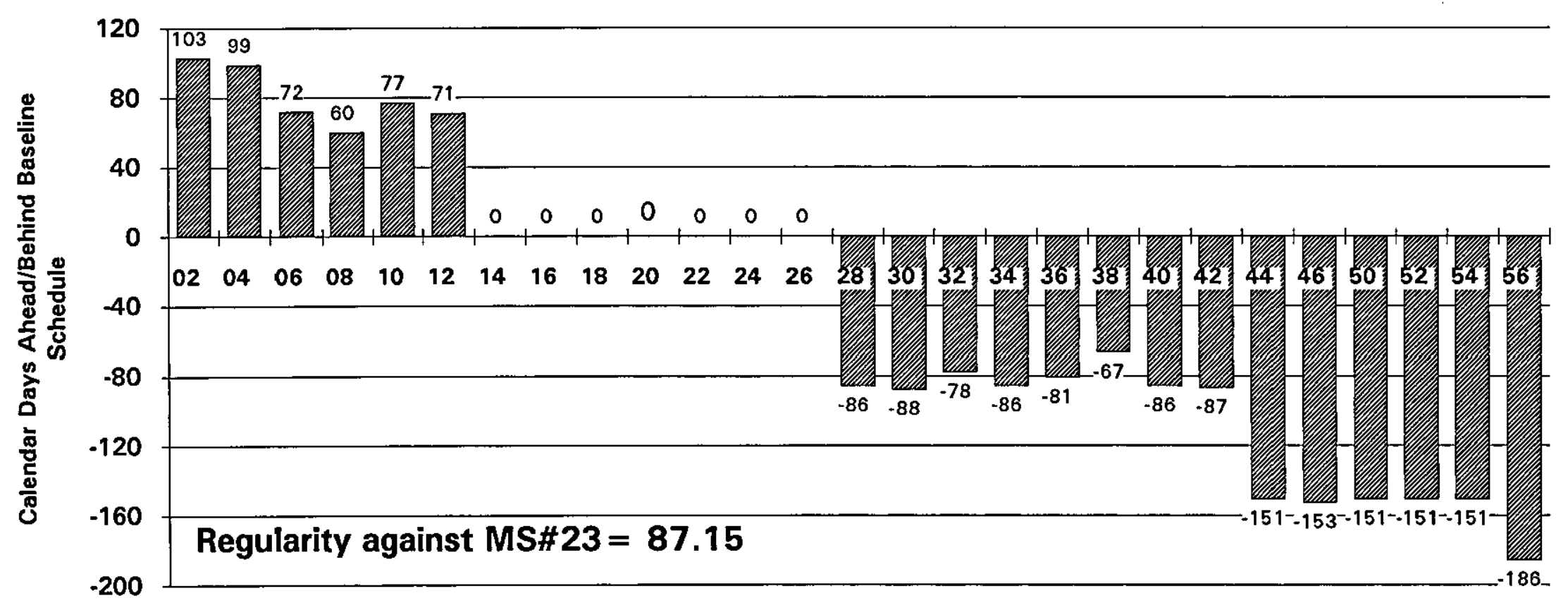

Typical Activities

Figure 9.29 - Case 3, Progress Regularity as of December 18, 1994 (Continued). 
CALIFORNIA STATE PRISON - SOSANVILLE

REPORT DATE 20SEP95 RON NO. 94

ER of current 1st Onits, by EP then Priority
PRIMAVERA PROJECT PLANNER

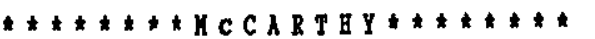

BID PACKAGE 3 - GUARD TOWER

START DATE 18JMY94 FIY DATE 22J0!95* DATK DATE 18DEC94 PAGE MO. 1

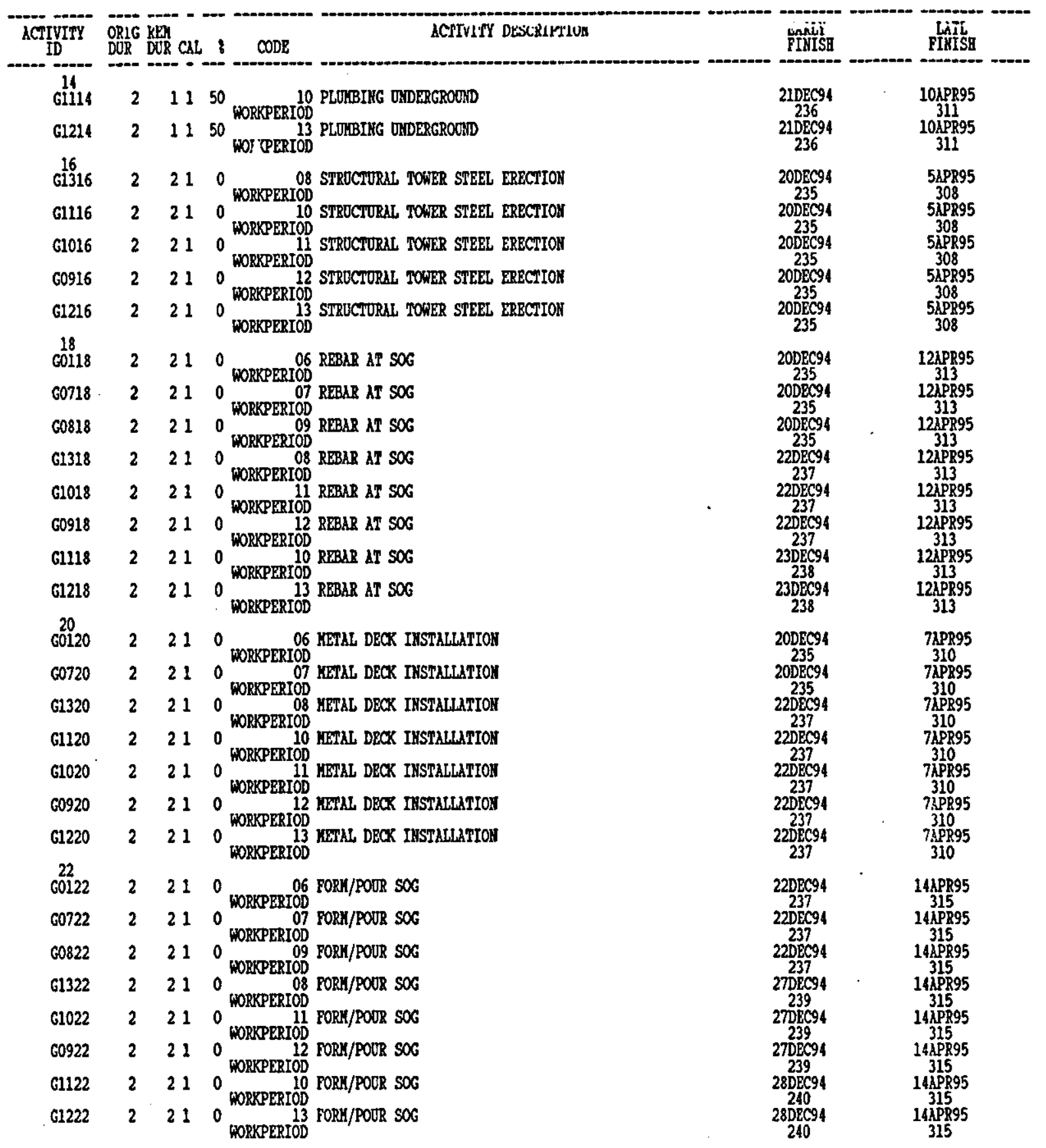

Figure 9.30 - Case 3, Earliest EF and Latest LF for $\mathrm{T}_{\mathrm{aa}}$ Computations. 
CALIFORNIA STRTE PRISOA - SUSAIVILLE

REPORT DATE 20SEP95 RON NO. 94

EF of current ist Onits, by EP then :riority
BID PACKAGE 3 - GUARD TOWER

PRILLVERA PROJECT PLAIRER

START DATE 18JAN94 FIK DATE 22JDN95*

DATA DATE 18DEC94 PAGE d0, 2

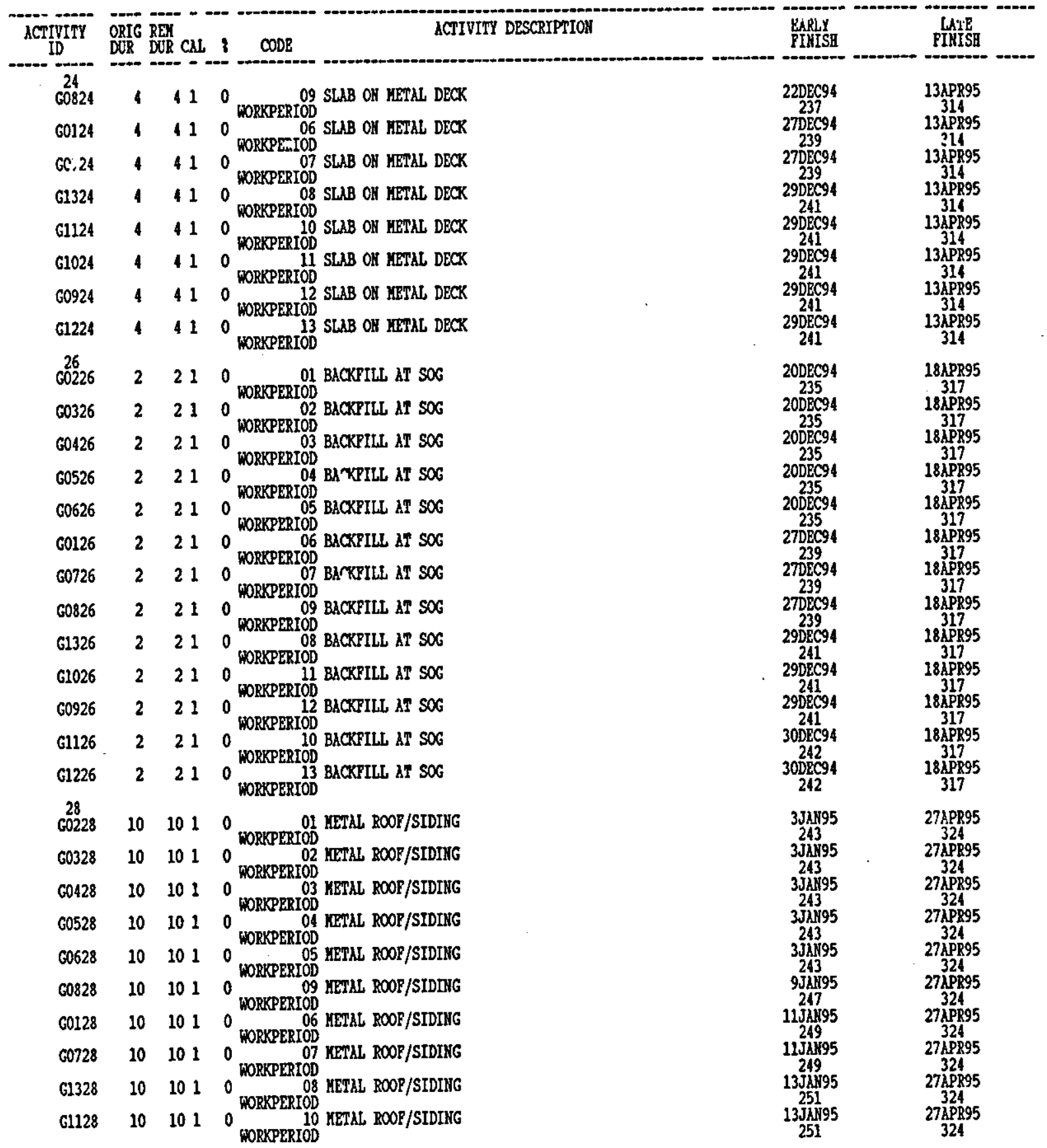

Figure 9.30 - Case 3, Earliest EF and Latest LF for $\mathrm{T}_{2 \mathrm{a}}$ Computations (Continued). 


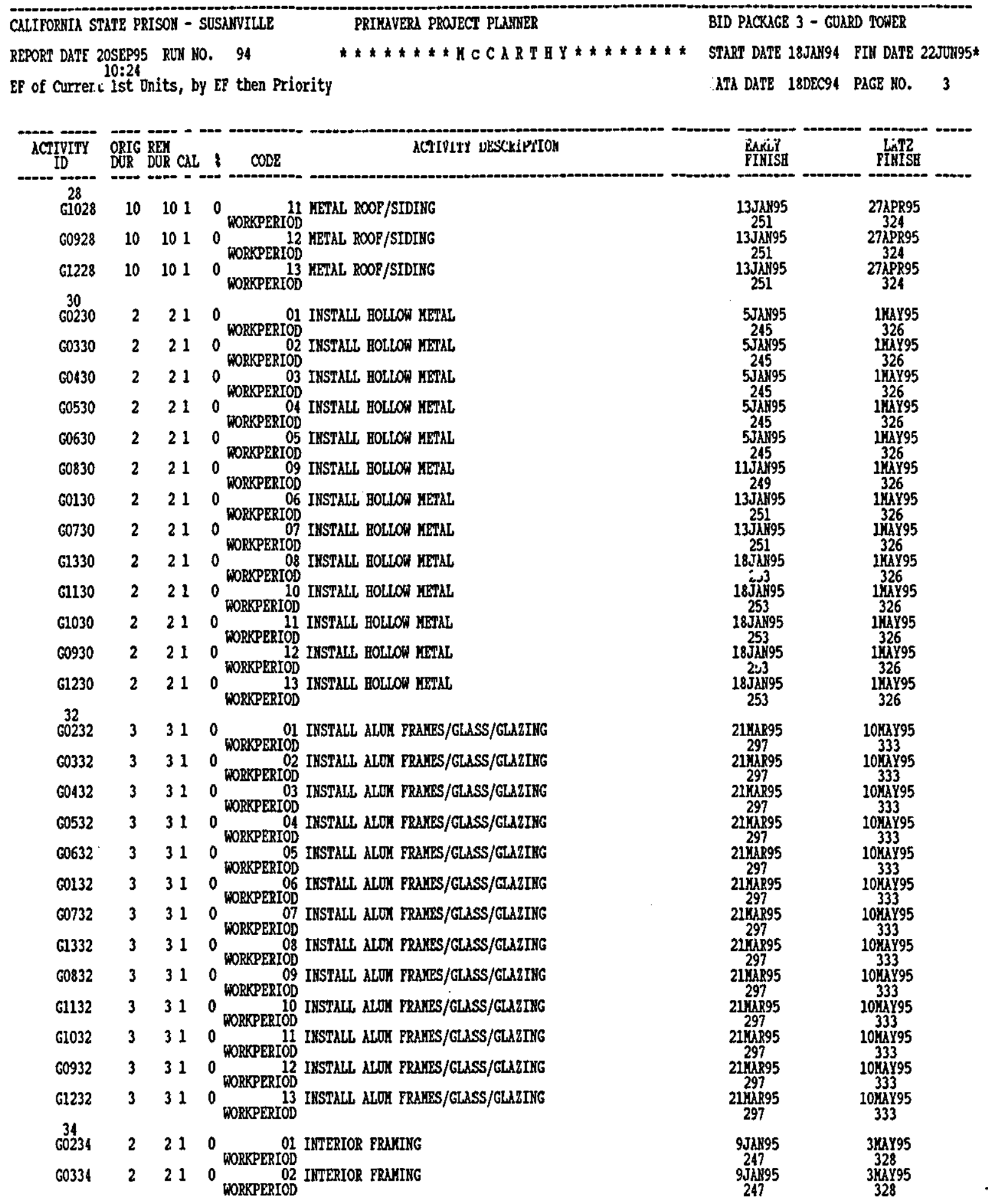

Figure 9.30 - Case 3, Earliest EF and Latest $\mathrm{LF}$ for $\mathrm{T}_{\mathrm{aa}}$ Computations (Continued). 
CALIPORALA STATE PRISON - SOSANVILLE

REPORT DATE 20SEP95 RON HO. 94 EP of Current 1st Onits, by EF then Priority
START DATE 18JAN94 - PIK DATE 22JU195* DATA DATE 18DEC94 PAGE NO.

\begin{tabular}{|c|c|c|c|c|c|c|}
\hline ACTIVITY & $\begin{array}{l}\text { ORIG } \\
\text { DUR } \\
\text { - }\end{array}$ & RDIR & CODE & ACIIVITY DLSCRIPSIUK & $\begin{array}{l}\text { RAKLY } \\
\text { FIKISB }\end{array}$ & FîHİ̈H \\
\hline c0434 & 2 & 21 & 0 MPRPPRPOPD & INTERTOR FRAYING & $\frac{9 J A H 95}{247}$ & $\frac{34 \times 195}{328}$ \\
\hline 60534 & 2 & 21 & 0 WORKPERIOD & INTERIOR FRAYING & $\begin{array}{l}\text { 9JAN95 } \\
247\end{array}$ & $\begin{array}{l}\text { 3HLYY95 } \\
328\end{array}$ \\
\hline C0631 & 2 & 21 & 0 MORKPERIOD & IHTERIC: FRAMING & $\begin{array}{l}\text { 9Jjhis95 } \\
247\end{array}$ & 3).MY 95 \\
\hline G0834 & 2 & 21 & 0 MORKPERTIOD & INTERTOR FRAYIYG & $\begin{array}{l}\text { 13JAM95 } \\
\text { 251 }\end{array}$ & $\begin{array}{c}\text { 3HLY } 95 \\
328\end{array}$ \\
\hline 60134 & 2 & 21 & 0 DORKPERTOD & INTERIOR FRAKING & $\begin{array}{c}18 \mathrm{JAN} 95 \\
253\end{array}$ & $\frac{3 \text { WWYY5 }}{328}$ \\
\hline 60734 & 2 & 21 & 0 MORKPRRIOD & IUTERIOR FRAYING & $\begin{array}{l}\text { 18JAN95 } \\
253\end{array}$ & $\begin{array}{c}3 \% \text { HYY } \\
328\end{array}$ \\
\hline G1334 & 2 & 21 & 0 WORKPERTOD & INTERTOR FRAYIKG & $\begin{array}{l}20 \mathrm{~J} \text { 새 } 95 \\
255\end{array}$ & $\begin{array}{l}3 \text { समYY95 } \\
328\end{array}$ \\
\hline Gl134 & 2 & 21 & 0 TORXPERTOD & IHTERIOR FRAYING & $\begin{array}{l}20 \mathrm{JAN} 95 \\
255\end{array}$ & $\frac{3 \text { HAY95 }}{328}$ \\
\hline G1034 & 2 & 21 & 0 nORKPERTOD & IMTERTIOR PRAYIMG & $\begin{array}{l}20 \mathrm{JuN} 95 \\
255\end{array}$ & 3) 32895 \\
\hline G0934 & 2 & 21 & 0 WORKPRRIOD & INTERIOR FRALING & $\begin{array}{l}\text { 20JAN95 } \\
255\end{array}$ & ${ }_{328}^{3 \text { MUY }}$ \\
\hline G1234 & 2 & 21 & 0 TORXPERIOD & IATEERIOR FRAIIMG & $\begin{array}{l}20 \mathrm{~J} M \mathrm{M} 95 \\
255\end{array}$ & $\frac{3 \text { MYY }}{328}$ \\
\hline $\begin{array}{l}36 \\
60236\end{array}$ & 2 & 11 & $\begin{array}{r}O 1 \\
\text { RKPPRTOD }\end{array}$ & PLUTBIHE ROOGH-IM & 10J4h95 & $10 \mathrm{KAY} 95$ \\
\hline C0336 & 2 & 11 & 50 WORKRERIOD & PLUJBIHG ROUGH-III & ${ }_{248}^{10 J 495}$ & 10.14Y 95 \\
\hline G0436 & 2 & 11 & 50 MORKPERTOD & PLOKBIHE ROOGH-II & $\begin{array}{l}10 \mathrm{JNH} 95 \\
218\end{array}$ & 10)MYK95 \\
\hline 60536 & 2 & 11 & 50 MORKPERTOD & PLDKBING ROOGR-III & $\begin{array}{l}10 \mathrm{Jjh} 195 \\
248\end{array}$ & $\begin{array}{c}10 \text { HY } \\
333\end{array}$ \\
\hline 60636 & 2 & 11 & 50 WOPKPRRTOD & PLDIBIMG ROUGH-II & $\begin{array}{c}10 \text { JAM } 95 \\
248\end{array}$ & $\operatorname{lomaY}_{333}$ \\
\hline 60836 & 2 & 21 & OPRRTOD & PLOYBING BOOGH-III & $\frac{18 \mathrm{JNH} 95}{253}$ & $\log _{333} 95$ \\
\hline 60136 & 2 & 11 & $50{ }^{2}$ & PLOMBIYG ROOGH-II & $\begin{array}{l}\text { 19JNA } 95 \\
254\end{array}$ & $\begin{array}{l}\text { 10).MYY95 } \\
333\end{array}$ \\
\hline 60736 & 2 & 21 & 0 MORKPERTOD & PLOIBISG ROOCE-II & $\begin{array}{l}20 \mathrm{JN} \text { N } 95 \\
255\end{array}$ & $\frac{10 \mathrm{MAY} 95}{333}$ \\
\hline G1336 & 2 & 21 & 0 OOPKPERTOD & PLUIBING ROOGH-IY & 24Juk 95 & ${ }_{333}^{10 \mathrm{LAY} 95}$ \\
\hline G1136 & 2 & 21 & $\begin{array}{l}\text { EKIYU } \\
10 \\
\text { ERTOD }\end{array}$ & PLO:BING ROOGH-III & $\begin{array}{l}\text { 24JuMY95 } \\
257\end{array}$ & $\underset{333}{\operatorname{10maY9} 95}$ \\
\hline G1036 & 2 & 21 & 11 & PLOKBING ROOGH-II & $\begin{array}{l}24 J \text { JNA } 95 \\
257\end{array}$ & $\operatorname{lolax}_{333}$ \\
\hline $\cos 36$ & 2 & 21 & 0 MORKPRERTOD & PLDKBIFG ROUGH-IY & $\begin{array}{c}2 \text { 24JNA95 } \\
257\end{array}$ & 10) \\
\hline 61236 & 2 & 21 & $\begin{array}{l}0 \text { MORKPERIOD } \\
13\end{array}$ & PLEIBIYG RODGH-II & $\begin{array}{c}24 J \mathbf{N} 95 \\
257\end{array}$ & $\underset{333}{10 \mathrm{MAY} 95}$ \\
\hline $\begin{array}{c}38 \\
60238\end{array}$ & 2 & 21 & $0_{\text {MORKPERIOD }}^{01}$ & IHSTALL DOORS/BARDAARR & 11JAM95 & 24\%,XY95 \\
\hline 60338 & 2 & 21 & 0 WORKPERIOD & IXSTALL DOORS/HARDGARE & $\begin{array}{l}11 \text { JuW } \\
249\end{array}$ & 24hAY95 \\
\hline 60438 & 2 & 21 & 0 MORKPIRIOD & IHSTALL DOORS/HARDWARE & $\underset{249}{11 J \text { JN95 }}$ & $\begin{array}{c}24, \mathrm{~h} Y \mathrm{Y} 95 \\
343\end{array}$ \\
\hline G0538 & 2 & 21 & 0 MORKPERIOD & IMSTALL DOORS/BARDWARE & 11 11 Jus 95 & $\frac{24 \operatorname{MAY} 95}{343}$ \\
\hline 60638 & 2 & 21 & 0 MORKPERIOD & INSTALL DCORS/HARDWARE & $\begin{array}{l}\text { 11JuMg95 } \\
249\end{array}$ & $\begin{array}{c}24 \mathrm{~K} Y \mathrm{Y} 95 \\
343\end{array}$ \\
\hline 60838 & 2 & 21 & 0 MORKPERIOD & INSTALL DOORS/HRRDWARE & $\begin{array}{l}18 \text { Jukg } \\
253\end{array}$ & $\frac{24 \mathrm{WAY} 95}{343}$ \\
\hline C0138 & 2 & 21 & 0 WORKPERIOD & IXSTALL DOORS/HARDWARE & $\begin{array}{l}20 \mathrm{JN} N \mathrm{~N} 95 \\
255\end{array}$ & $\underset{343}{24 \max 95}$ \\
\hline
\end{tabular}

Figure 9.30 - Case 3, Earliest EF and Latest LF for $\mathrm{T}_{2 \mathrm{a}}$ Computations (Continued). 
CALIFORNIA STATE PRISON - SUSANVILLE

REPORT DATE 20SEPY5 ROH HO. 94

EF of Current lst Units, by EF then Priority
PRIMAVERA PROSECT PLANNER

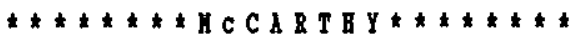

BID PACXIGE 3 - GURRD TOWER

START DATE 18JMM94 PIN DATE 22JUN95* DATA DATE I8DEC94 PAGE HO. 5

\begin{tabular}{|c|c|c|c|c|}
\hline ACTIVITY & $\begin{array}{l}\text { ORIG RRI } \\
\text { DOR DOR CAL }\end{array}$ & ACTIVITY DESCRIPTIOY & $\begin{array}{l}\text { EARLY } \\
\text { FINISH }\end{array}$ & $\stackrel{\text { LIMP }}{\text { PIMISH }}$ \\
\hline $\begin{array}{c}38 \\
60738\end{array}$ & $2 \quad 21$ & 0 DORKPFPTOO IMSTALL DOORS/HARDWARB & 20JAM95 & 24!hy95 \\
\hline G1338 & 21 & 0 OORPXPRPTOD INSTALL DOORS/EARDAIARE & 2455395 & $\begin{array}{l}343 \\
24 \mathrm{HAY} 95 \\
3,33\end{array}$ \\
\hline 61138 & 21 & 0 & 24 24月M959 & $\begin{array}{ll}34 \mathrm{KAY} 95 \\
243\end{array}$ \\
\hline 61038 & 21 & 11 IMSTALL DOORS/HARDALRE & $24 \mathrm{JAM} 95$ & 24 24:AYY95 \\
\hline 60938 & 21 & 12 IHSTALL DOORS/BARDWARE & $24 \mathrm{JNN} 95$ & 24hYM95 \\
\hline G1238 & 21 & ${ }^{0}$ PORKPERTOD & $\begin{array}{l}24 \mathrm{JJM} 95 \\
257\end{array}$ & $\underset{343}{24 \text { KYYY95 }}$ \\
\hline 60240 & 41 & 01 VIRCEANICAL ROOGH-IH & 13JAN95 & $10 \mathrm{MAYY} 95$ \\
\hline 60340 & 41 & 02 MRCBAMICAL ROOGH-IY & 13JMH & $10 \mathrm{kMYY} 95$ \\
\hline 60440 & 11 & RIOD 03 MECHAMICAL $200 \mathrm{CH}-\mathrm{II}$ & 13512195 & $\begin{array}{l}333 \\
1034.4395\end{array}$ \\
\hline 60540 & 41 & O4 YECHAMTCAL RODCH-IM & 13511:95 & $\begin{array}{l}333 \\
103 \mathrm{HAY95} \\
22\end{array}$ \\
\hline 60640 & 41 & 05 KECHANICAL ROOCH-IM & 13J 251195 & 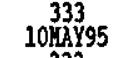 \\
\hline 60840 & 41 & 09 MECHANICAL ROOGH-IH & 20.51 & $\begin{array}{l}333 \\
103 \mathrm{ASY} 95\end{array}$ \\
\hline 60140 & 41 & O6 YECHAHTCAL ROOGB-IN & 24555 & 10:MAY 335 \\
\hline 60740 & 41 & RIOD HECHANICAL RODGH-TH & $245 \mathrm{JuH} / 95$ & $\begin{array}{l}333 \\
103 \mathrm{LAY95}\end{array}$ \\
\hline 61340 & 41 & RIOD 08 HECHAYTCAL ROOGH-IK & 2657 N 2595 & 10HAY95 \\
\hline 61140 & 41 & 10 KECBANICAL ROOGH-IY & 265 J4 2595 & 10:34395 \\
\hline 61040 & 41 & II MECHANICAL ROCGH-IH & $265 \mathrm{NAR} / 195$ & 10333 \\
\hline 60940 & 11 & 12 MECBAMICAL ROOGH-IN & 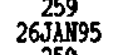 & $\begin{array}{l}333 \\
10 \text { KAY } 95\end{array}$ \\
\hline 61240 & 41 & 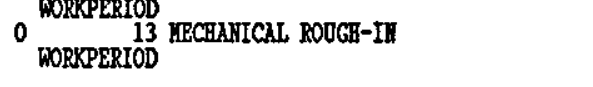 & $\underset{259}{26599}$ & $\begin{array}{c}333 \\
1333 \\
3395\end{array}$ \\
\hline 60242 & 51 & 01 ELPCTRICAL RONGH-IH & $\begin{array}{l}17 J 4 M_{95} \\
252\end{array}$ & ${ }_{333}^{1014 a Y 95}$ \\
\hline 60342 & 51 & 02 ELECTRICAL ROUGH-IF & 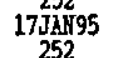 & 10) \\
\hline 60442 & 51 & 03 RLECTRICAL ROOGH-IN & 17JAN 95 & 10), My \\
\hline 60542 & 51 & O4 ELECTRICAL ROOGH-IM & 17JAH 25. & 10 10HYY95 \\
\hline 60642 & 51 & 05 BLPCTRICAL ROUGH-IN & 1752h 255 & 10,41395 \\
\hline 60842 & 51 & O9 RLECTRICAL ROOCH-IN & $23{ }^{252}$ & 10143395 \\
\hline 60142 & 51 & 0 MORKPERIOD ELECTRICAL ROOGH-IK & 25 25]ג 25695 & 10), 333 \\
\hline 60742 & 51 & 07 EIRCTRICAL ROOGH-IH & 25JJKS95 & 10!̣̂Y 95 \\
\hline G1342 & 51 & OS ELECTRICAL ROOCH-IM & $\begin{array}{l}27.8 \\
27 \text { JNA9 }\end{array}$ & 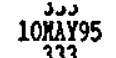 \\
\hline 61142 & 51 & 0 & $27 \mathrm{JNAM9}$ & 10 10MYY95 \\
\hline G1042 & 51 & 0 OOLPKPERTOD BLBCTRICAL ROVGH-IH & 27060 & 10 10:MYM95 \\
\hline 60942 & 51 & 0 & $\begin{array}{l}27 J 3195 \\
260\end{array}$ & ${ }_{333}^{10 \text { RYY }}$ \\
\hline
\end{tabular}

Figure 9.30 - Case 3, Earliest EF and Latest $\mathrm{LF}$ for $\mathrm{T}_{\mathrm{aA}}$ Computations (Continued). 
CALIFORNIA STATE PRISOH - SUSANVILLE

REPORT DATE 20SEP95 RON WO. 94

EF of current lst Units, by EF then Priority
PRIMAVERA PROTECT PLAMIER

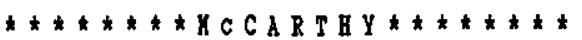

BID PACKAGE 3 - GUARD TOWER

START DATE 18JAN94 PIN DATE 22JUK95* DATK DATE 18DEC94 PAGE HO. 6

\begin{tabular}{|c|c|c|c|c|c|}
\hline SCTIVITY & $\begin{array}{l}\text { ORIG R } \\
\mathrm{DUR}\end{array}$ & DUI CAL \& & ACTIVITY DESCRIPTIOH & $\begin{array}{l}\text { ERRLY } \\
\text { FINISA }\end{array}$ & $\underset{\text { WINISE }}{\text { MTISE }}$ \\
\hline 61242 & 5 & 51 & ${ }^{0}$ WORKPERIOD 13 ELECTRICAL ROUGH-IN & $\begin{array}{l}27 \text { JAH } 95 \\
260\end{array}$ & $\underset{333}{10 \text { MAY95 }}$ \\
\hline 60244 & 10 & 101 & 0 WORKPERIOD INTERIOR PIMISHES & $\begin{array}{l}4 A P R 95 \\
307\end{array}$ & $\begin{array}{l}24 \mathrm{~K} \text { KRY } 95 \\
343\end{array}$ \\
\hline 60344 & 10 & 101 & $\begin{array}{l}02 \\
0 \text { MORKPERIOD INTERIOR PIMTSHES }\end{array}$ & $\begin{array}{l}\text { 4)PR } 95 \\
307\end{array}$ & $24 \frac{\text { KAKY }}{343}$ \\
\hline 60444 & 10 & 101 & 0 O3 INTERTOR FINISHES & 4PRP95 & 24) \\
\hline 60544 & 10 & 101 & 0 DORIPERIOD ILTERIOR FINISHES & $\begin{array}{l}301 \\
4 \text { APR95 } \\
307\end{array}$ & 24 HAY 95 \\
\hline 60644 & 10 & 101 & 0 WORKPERTOD INTERIOR PINISEES & 4APR95 & 24MAY 95 \\
\hline 60114 & 10 & 101 & $\begin{array}{l}0 \text { O6 IITERIOR FIKISHES } \\
\text { SORKPERIOD }\end{array}$ & 4APR95 & 24 IIAY 95 \\
\hline 60744 & 10 & 101 & 0 WORKRERIOD IKTERIOR FIIISHES & 4RPR95 & 24 HAY \\
\hline G1344 & 10 & 101 & $\begin{array}{l}0 \text { O8 INTERTOR FIMISHES } \\
\text { WORKPERIOD }\end{array}$ & $\frac{4 \text { APR95 }}{307}$ & $\frac{24 \pi \text { HY }}{343}$ \\
\hline 60844 & 10 & 101 & 0 O9 INTERIOR RINISHES & 4APR95 & 24LAYY \\
\hline 61144 & 10 & 101 & 0 WORKPBRIOD 10 INTERIOR FIRISHRS & APRPS & 24)KMY 95 \\
\hline G1044 & 10 & 101 & ${ }_{\text {WORKPERIOD }}$ II IMTERIOR PINISHES & 4APR95 & 24 SAMY95 \\
\hline 60944 & 10 & 101 & $\begin{array}{l}12 \text { INTERTOR FINISHES } \\
\text { WORKPERTOD }\end{array}$ & 4 LPPR95 & 24IMYY95 \\
\hline 61244 & 10 & 101 & $\begin{array}{l}13 \\
\text { WORKPERIOD IHTERTOR FINISAES }\end{array}$ & $\begin{array}{l}307 \\
307\end{array}$ & $\begin{array}{l}24 \mathrm{LARY} \\
343\end{array}$ \\
\hline 60246 & 2 & 21 & MORKPERIOD PAIRT & 6APR95 & 26HAY95 \\
\hline 60346 & 2 & 21 & WORKPERIOD PAINT & 6.PPR95 & 261 MYY 95 \\
\hline 60446 & 2 & 21 & WORKPERIOD PAIKT & 61PR95 & $\frac{26) \text { MYY }}{345}$ \\
\hline 60546 & 2 & 21 & WORRPERIOD PAIRT & 6APR95 & 2614KY95 \\
\hline C0646 & 2 & 21 & $\begin{array}{l}\text { NOS PAINT } \\
\text { WORKPERIOD }\end{array}$ & 6APR95 & 26 HAY 345 \\
\hline 60146 & 2 & 21 & WORKPERIOD PAIHT & $\begin{array}{l}309 \\
609\end{array}$ & $\begin{array}{l}345 \\
26 \text { WAY } \\
345\end{array}$ \\
\hline 60746 & 2 & 21 & $\begin{array}{l}07 \text { PAINT } \\
\text { GORKPERIOD }\end{array}$ & 61PR95 & 26 WXY95 \\
\hline G1346 & 2 & 21 & OR PAINP & 6RPR95 & $26 \%$ MYY 95 \\
\hline 60846 & 2 & 21 & DORKPERIOD PAINI & 6APR95 & $\begin{array}{l}345 \\
26 \mathrm{MAY} 95 \\
345\end{array}$ \\
\hline G1146 & 2 & 21 & MORKPERIOD PAIRT & $\begin{array}{l}309 \\
6 \mathrm{APRP} \\
309\end{array}$ & 26134595 \\
\hline G1046 & 2. & 21 & 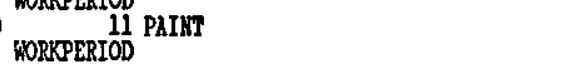 & $\begin{array}{l}309 \\
6 \text { APR95 } \\
309\end{array}$ & $\begin{array}{l}345 \\
26 \% 4 y 95 \\
345\end{array}$ \\
\hline 60946 & 2 & 21 & WORKPERIOD PAIKT & 6APR95 & 2614.995 \\
\hline 61246 & 2 & 21 & $\begin{array}{l}13 \text { PIINT } \\
\text { MORKPERIOD }\end{array}$ & $\begin{array}{c}309 \\
6 A P R 95 \\
\dot{j u g}\end{array}$ & $\begin{array}{l}26 \text { MAY } \\
345\end{array}$ \\
\hline 60250 & 3 & $3 ?$ & WORKPERIOD MECHARICAL FINISH & $\underset{312}{1112 P R 95}$ & $\underset{348}{1 \mathrm{~J} 0 \mathrm{~N} 95}$ \\
\hline 60350 & 3 & $3 \mathrm{~J}$ & $\begin{array}{l}02 \\
\text { MORKPERTOD }\end{array}$ & $\begin{array}{l}11 \text { KPR95 } \\
312\end{array}$ & $\begin{array}{l}1 \text { Jơ } 95 \\
348\end{array}$ \\
\hline 60450 & 3 & 31 & WORKPERIOD YIECHANICAL, FIRISH & ${ }_{312}^{312}$ & $\begin{array}{l}\text { 1. } 30095 \\
348\end{array}$ \\
\hline 60550 & 3 & 31 & $\begin{array}{l}\text { Of KECBAHICAL FIMISH } \\
\text { WORKPERIOD }\end{array}$ & ${ }_{312}^{111 P R 95}$ & $\begin{array}{l}1348 \\
348\end{array}$ \\
\hline
\end{tabular}

Figure 9.30 - Case 3, Earliest EF and Latest LF for $\mathrm{T}_{\mathrm{aa}}$ Computations (Continued). 
CALIFORNIA STATE PRISON - SUSANILLE

REPORT DATE 20SEP95 RON HO. 94

EF of current 1st Units, by EF then Priority
BID PACKAGE 3 - GUARD TOWER

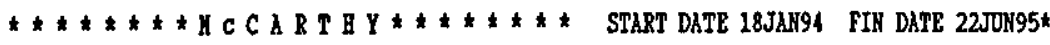
DATA DAPE $180 \mathrm{DC} 94$ PACE NO. 7

\begin{tabular}{|c|c|c|c|c|}
\hline ACTIVITI & $\begin{array}{l}\text { ORIG RER } \\
\text { DUR DOR CAL }\end{array}$ & ACTIVITY DESCRIPTIOH & $\begin{array}{l}\text { EMRLY } \\
\text { PINISB }\end{array}$ & $\begin{array}{l}\text { LATR } \\
\text { PINISB } \\
\text { PIST }\end{array}$ \\
\hline $\begin{array}{l}50 \\
60650\end{array}$ & 31 & 0 O5 MECHANICAL PIHISE & 11APR95 & 1 \\
\hline 60150 & 31 & 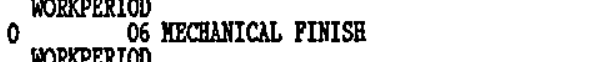 & $\frac{1112 \mathrm{PR} 95}{312}$ & 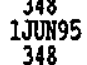 \\
\hline 60750 & 31 & 0 HORKPEKIOD O7 YECHANICAL RIMISH & 11112985 & 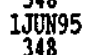 \\
\hline 61350 & 31 & 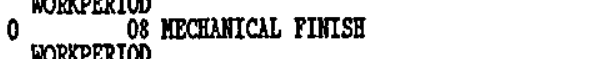 & ${ }_{312}^{1111295}$ & 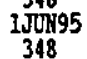 \\
\hline 60850 & 31 & 0 KORAPESIOD MECEANICAL PIRISH & $11113 \mathrm{APR} 95$ & $\begin{array}{l}\frac{1548}{150495} \\
348\end{array}$ \\
\hline G1150 & 31 & 0 WORLPERIOU IO MECHAHTCAL PINISH & ${ }_{312}^{1111 \mathrm{PR} 95}$ & $\begin{array}{l}\int_{348}^{1509} 95 \\
348\end{array}$ \\
\hline 61050 & 31 & 0 WORKPERIOD MECHASTCLL FIMISH & $111 \mathrm{MPR} 95$ & 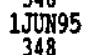 \\
\hline 60950 & 31 & 12 MECGANTCAL PTHISH & $1111 \mathrm{PRg} 95$ & $\begin{array}{l}13095 \\
1348\end{array}$ \\
\hline G1250 & 31 & $\begin{array}{l}0 \text { WORKPERTLO } 13 \text { MECHAHICAL FIRISH } \\
\text { WORPEIOD }\end{array}$ & ${ }_{312}^{1114895}$ & 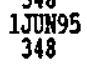 \\
\hline $\begin{array}{l}52 \\
60252\end{array}$ & 31 & 01 PLOIBING PIMTSE & $\operatorname{liMPR95}_{212}$ & 1Juk95 \\
\hline 60352 & 31 & 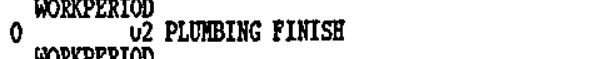 & $1111 \mathrm{PRP} 95$ & 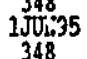 \\
\hline 60452 & 31 & 03 PLOIBIMG PINTSB & $1119 \mathrm{PR} 95$ & $\frac{1.5 W 195}{348}$ \\
\hline 60552 & 31 & 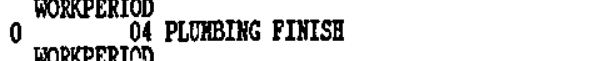 & $11112_{312}^{312}$ & 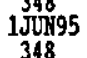 \\
\hline 60652 & 31 & 15 PLOKBING FINISB & $\frac{11 \text { APR95 }}{312}$ & 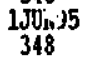 \\
\hline 60152 & 31 & 06 PLOXBIMG FINISB & $\frac{121.895}{312}$ & 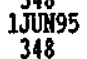 \\
\hline 60752 & 31 & 07 PLUKBIKC FINISH & $\frac{11 \mathrm{pPR} 95}{312}$ & 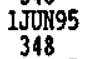 \\
\hline 61352 & 31 & O8 PLUJBING PIKISH & $11 \frac{11295}{313}$ & 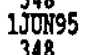 \\
\hline 60852 & 31 & OO PLOKBIHG FINISB & $\frac{11 \mathrm{AR} 95}{312}$ & $\begin{array}{ll}1.30195 \\
348\end{array}$ \\
\hline 61152 & 31 & RIOD PLUKBING FIMTSH & $\frac{119295}{312}$ & 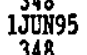 \\
\hline 61052 & 31 & 111 PLDVIBING FINTSB & 1111 PRP95 & $\begin{array}{l}13095 \\
1.048\end{array}$ \\
\hline 60952 & 31 & 12 PLOIBING PINISE & $\frac{119295}{312}$ & 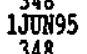 \\
\hline 61252 & 31 & 0 & $\frac{11 \text { APR } 95}{312}$ & $\begin{array}{l}1.3095 \\
1348\end{array}$ \\
\hline 60254 & 31 & OI BLECTRICAL PINISH & $1112 \mathrm{APR} 95$ & $\begin{array}{l}1 \pi 0495 \\
348\end{array}$ \\
\hline 60354 & 31 & 0202 BLECTRICAL FINISH & ${ }_{312}^{11 \text { APR } 95}$ & $\begin{array}{l}130495 \\
348\end{array}$ \\
\hline 60454 & 31 & O3 & ${ }_{11 \mathrm{PPR} 95}^{312}$ & 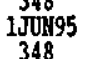 \\
\hline 60554 & 31 & 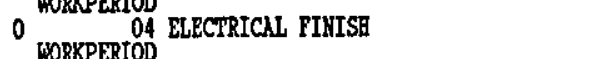 & ${ }_{312}^{11 \text { APR95 }}$ & $\begin{array}{l}1.3095 \\
14048\end{array}$ \\
\hline sn654 & 31 & 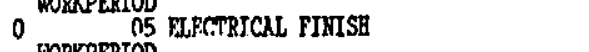 & $11 \mathrm{APR} 95$ & 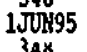 \\
\hline 60154 & 31 & 0 RORKPERIOD O6 ELECTRICAL PINISE & $111 \mathrm{APR} 95$ & 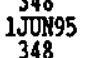 \\
\hline 60754 & 31 & 0 MUNSLA O7 RLEC RICAL PINISE & $11 \mathrm{MPR} 95$ & $\begin{array}{l}1.3095 \\
1348\end{array}$ \\
\hline G1354 & 31 & 0 ODPRPRPTOD ELECT.ICAL PINISE & ${ }_{312}^{1110295}$ & $\begin{array}{l}14095 \\
14048\end{array}$ \\
\hline 60854 & 31 & 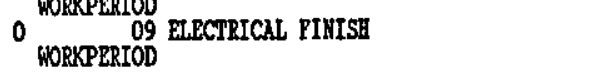 & $\frac{11128}{312}$ & 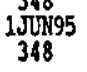 \\
\hline
\end{tabular}

Figure 9.30 - Case 3, Earliest EF and Latest LF for $\mathrm{T}_{2 \mathrm{a}}$ Computations (Continued). 
CALIFORNIA STATE PRISON - SOSAWVILLE

REPORT DATE 20SEP95 RON NO. 94

EF of Current Ist Units, by ER then Priority
PRIMAVERA PROJRCT PLANNER

BID PACRAGR 3 - GUARD TOSAER

$\star * * * * * * *$ M C C A R T B Y $* * * * * * * *$ START DATE 18JaH94 PIN DATE 22JUN95* DATA DATE 18DEC94 PAGE HO. 8

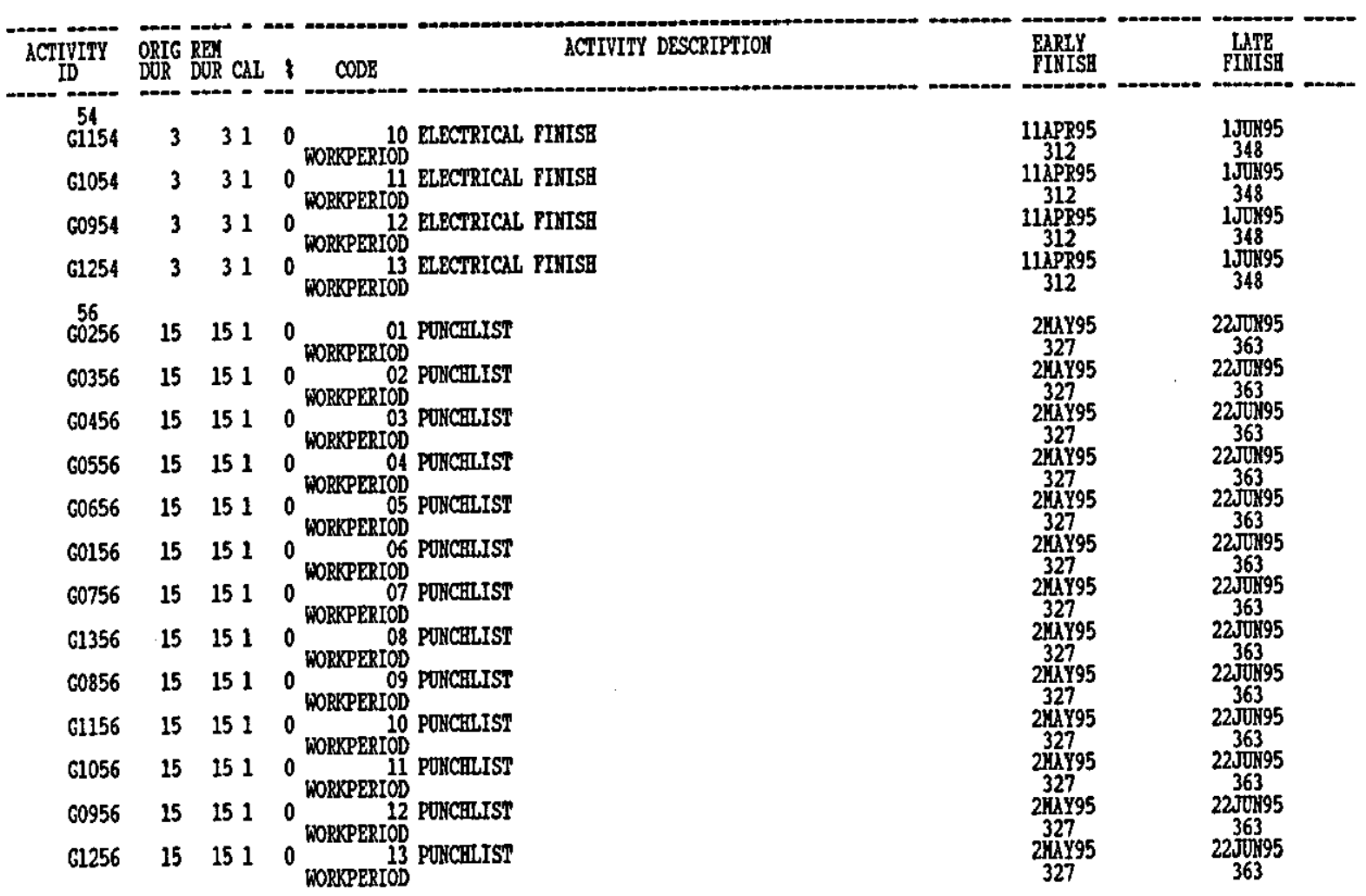

Figure 9.30 - Case 3, Earliest EF and Latest LF for $\mathrm{T}_{\mathrm{aa}}$ Computations (Continued). 


\begin{tabular}{|c|c|c|c|c|c|c|c|c|c|c|c|c|}
\hline & & & & & Current & Last & & & & & & Normalized \\
\hline & & & & & 1st typical & typical & & & & & Independent & Independent \\
\hline Typical & & Quantity & & Resource & activity & actlvity & Taa & Rma & Sma & Sma & rate & rate \\
\hline activity & Activity titie & uncompleted & Resource & duration & EF & LF & $(7) \cdot(6)$ & $\{(3)-1\} \div(8)$ & (9) $\times(5)$ & rounded & $(11)+(5)$ & $(12) \times 0.333+(9)$ \\
\hline (1) & (2) & (3) & (4) & (5) & (6) & (7) & (8) & (9) & (10) & (11) & (12) & (13) \\
\hline 14 & PLOMBING UNDERGROUND & 2 & SQ14 & 2 & 236 & 311 & 75 & 0.013 & 0.027 & 1 & 0.500 & 12.500 \\
\hline 16 & STRUCTURAL TOWER STEEL ERECTION & 5 & SQ16 & 2 & 235 & 308 & 73 & 0.055 & 0.110 & 1 & 0.500 & 3.042 \\
\hline 18 & REBAR AT SOG & 8 & sQ18 & 2 & 235 & 313 & 78 & 0.090 & 0.179 & 1 & 0.500 & 1.857 \\
\hline 20 & GETAL DECR INSTALLATION & 7 & 3220 & 2 & 235 & 310 & 75 & 0.080 & 0.160 & 1 & 0.500 & 2.083 \\
\hline 22 & FORM/POUR SOG & 8 & SQ22 & 2 & 237 & 315 & 78 & 0.090 & 0.179 & 1 & 0.500 & 1.857 \\
\hline 24 & SLAB ON METAL DECK & 8 & $s Q 24$ & 4 & 239 & 314 & 75 & 0.093 & 0.373 & 1 & 0.250 & 0.893 \\
\hline 26 & BACKFILI AT SOG & 13 & 3226 & 2 & 235 & 317 & 82 & 0.146 & 0.293 & 1 & 0.500 & 1.139 \\
\hline 28 & METAL ROOF/SIDINO & 13 & sQ28 & 10 & 243 & 324 & 81 & 0.148 & 1.481 & 2 & 0.200 & 0.450 \\
\hline 30 & INSTALL HOLLON METAL & 13 & sQ30 & 2 & 245 & 326 & 81 & 0.148 & 0.296 & 1 & 0.500 & 1.125 \\
\hline 32 & GNSTALL ALUM FRAMES/GLASS/GLAZIN & 13 & $s Q 32$ & 3 & 297 & 333 & 36 & 0.333 & 1.000 & 1 & 0.333 & 0.333 \\
\hline 34 & INTERIOR FRAMINO & 13 & SQ34 & 2 & 247 & 328 & 81 & 0.148 & 0.296 & 1 & 0.500 & 1.125 \\
\hline 36 & ELTNGING ROUGH-IN & 13 & 8036 & 2 & 248 & 333 & 85 & 0.141 & 0.282 & 1 & 0.500 & 1.181 \\
\hline 38 & INSTALL DOORS / EARDWARE & 13 & $S Q 38$ & 2 & 249 & 343 & 94 & 0.128 & 0.255 & 1 & 0.500 & 1.306 \\
\hline 40 & LEAANICAL ROUGE-IN & 13 & SQ40 & 4 & 251 & 333 & 82 & 0.146 & 0.585 & 1 & 0.250 & 0.569 \\
\hline 42 & ELECTRICAL ROUGE-IN & 13 & $\mathrm{sQ42}$ & 5 & 252 & 333 & 81 & 0.148 & 0.741 & 1 & 0.200 & 0.450 \\
\hline 44 & INTERIOR FINISEES & 13 & $s Q 44$ & 10 & 307 & 343 & 36 & 0.333 & 3.333 & 4 & 0.400 & 0.400 \\
\hline 46 & PAINT & 13 & $s 046$ & 2 & 309 & 345 & 36 & 0.333 & 0.667 & 1 & 0.500 & 0.500 \\
\hline 50 & MECEANICAL PINISH & 13 & $s Q 50$ & 3 & 312 & 348 & 36 & 0.333 & 1.000 & 1 & 0.333 & 0.333 \\
\hline 52 & PLUMBING FINISH & 13 & $S Q 52$ & 3 & 312 & 348 & 36 & 0.333 & 1.000 & 1 & 0.333 & 0.333 \\
\hline 54 & ELECTRICAL FINISE & 13 & $S Q 54$ & 3 & 312 & 348 & 36 & 0.333 & 1.000 & 1 & 0.333 & 0.333 \\
\hline 56 & PUNCHLIST & 13 & SQ56 & 1.2 & 327 & 363 & 36 & 0.333 & 4.000 & 4 & 0.333 & 0.333 \\
\hline
\end{tabular}


CALIFORHIA STATE PRISON - SOSANILLE

REPORT DATE 20SEP95 RON NO. 98 Dec. 94 Acceleration, by Typical detivity \& BS
BID PACKAGE 3 - GUARD TONER

PRILUVERA PROJECT PLANNER

START DATE 18JAN94 FIN DATE 22JWN95*

DATA DATE 18DEC94 PAGE HO. 1

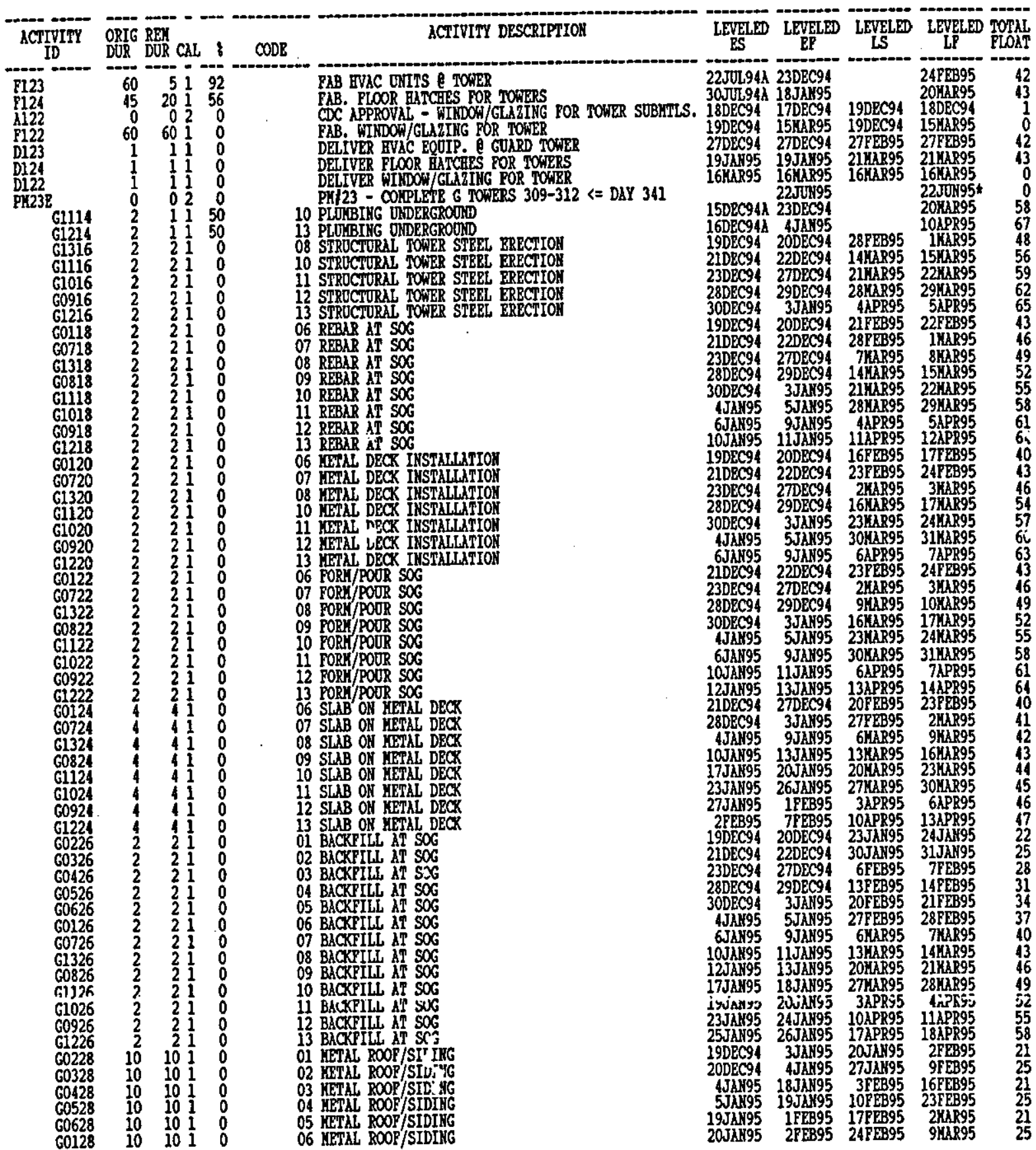

Figure 9.31 - Case 3, Accelerated Schedule. 
CALIPORHL STATE PRISON - SUSANVILLE

REPORT DATE 20SEP95 RON NO. 98

Dec. 94 Acceleration, by Typical Activity \& BS
BID PACKAGE 3 - CUARD TOAER

PRIYLUERA PROJECT PLANMER

START DATE 18JAH94 FIM DATE 22JON95*

DATA DATE 18DRC94 PAGE HO. 2

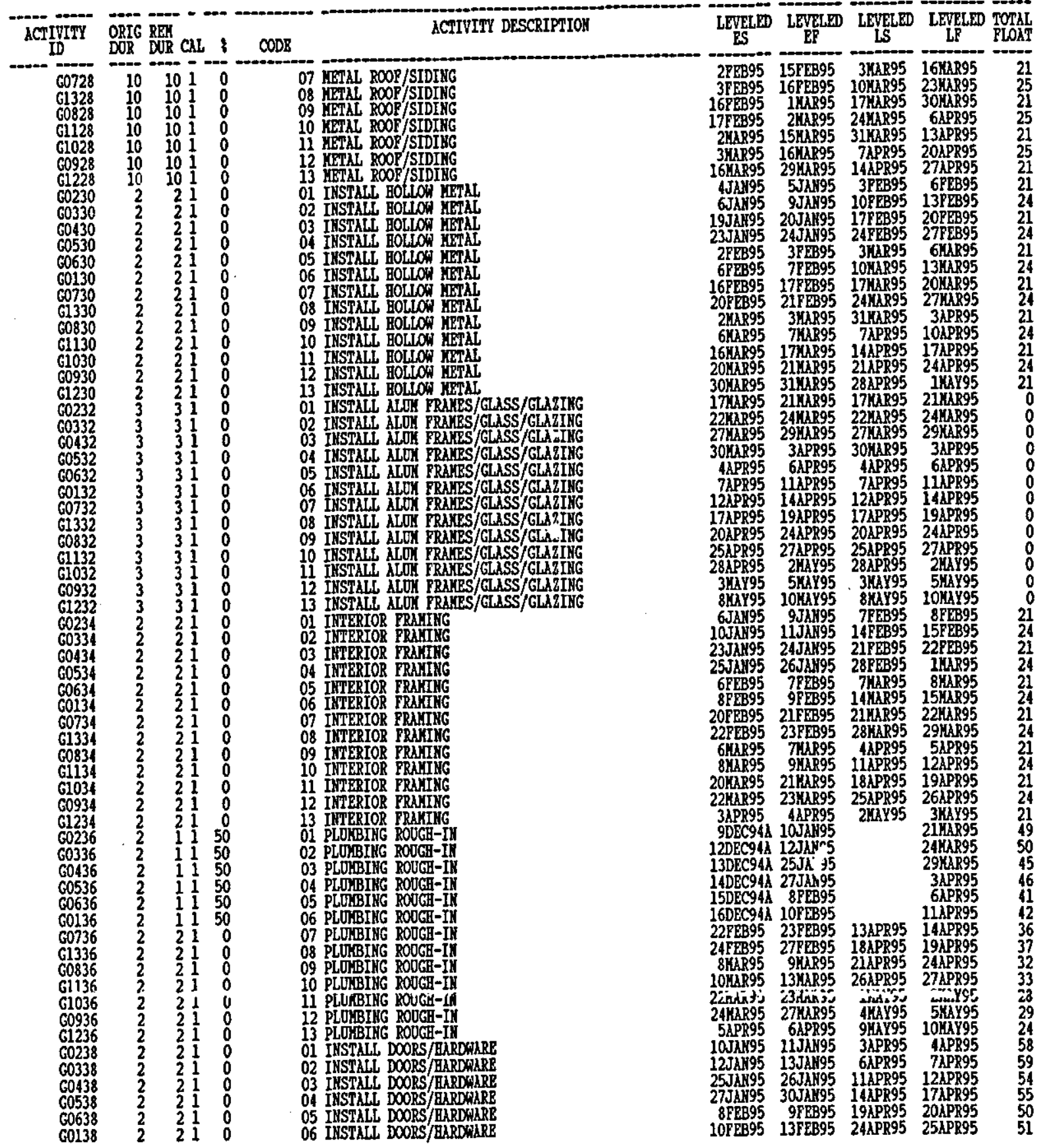

Figure 9.31 - Case 3, Accelerated Schedule (Continued). 
CALIFORNIA STATE PRISON - SUSAWILLP

REPORT DATE 20SEP95 RON HO. 98 $10: 54$

Dec. 94 dcceleration, by Typical sctivity \& ES
PRIKAVERA PROJECT PLANRER

BID PACKAGE 3 - GUARD TOWER

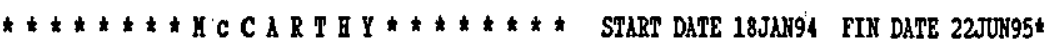
DATA DATE 18DEC94 PAGE NO. 3

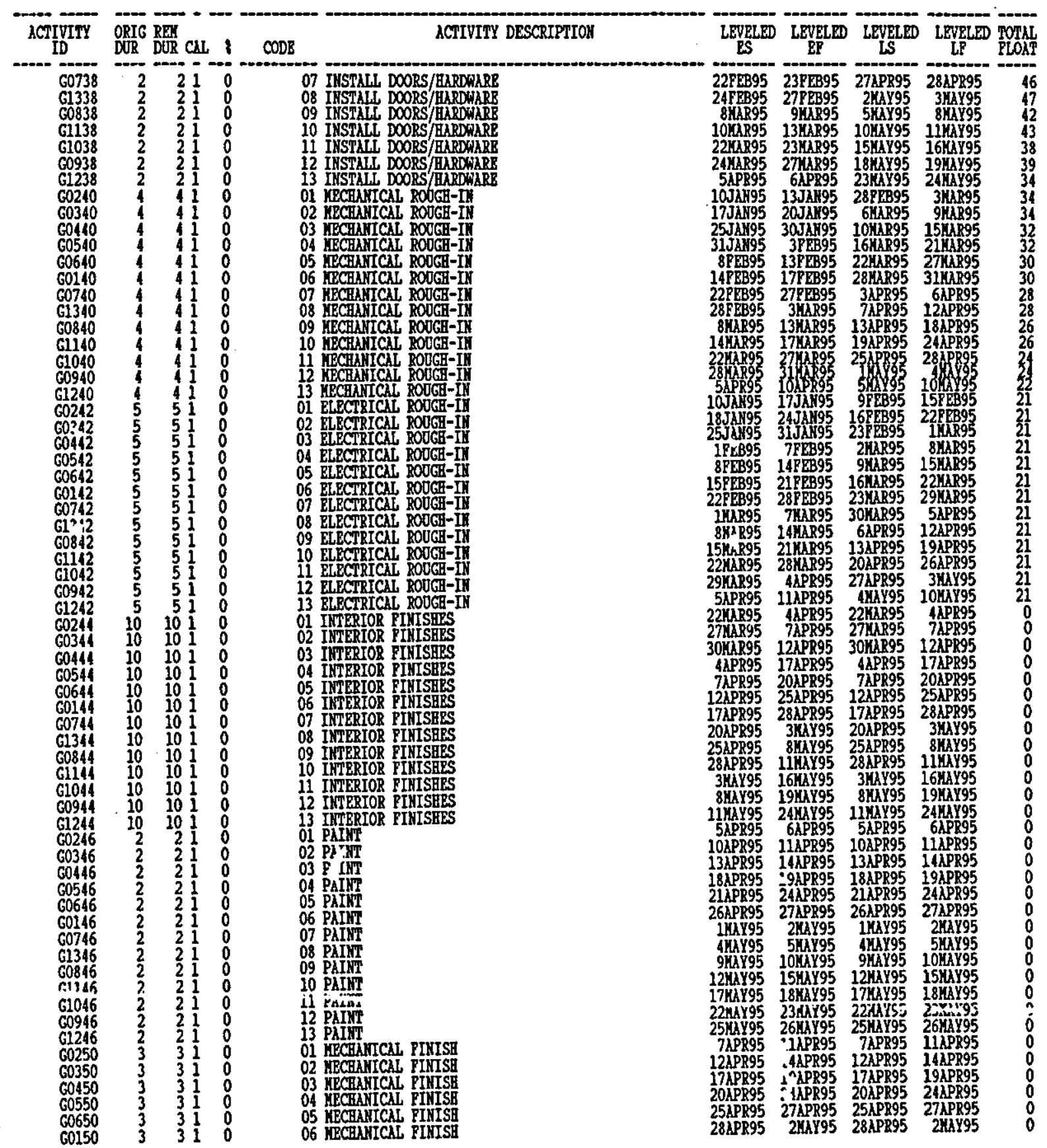

Figure 9.31 - Case 3, Accelerated Schedule (Continued). 
REPORT DATB $205 E P 95$ RON 10.98

$\star * * * * * * *$ K С C A R T H Y $* * * * * * * *$ START DATE 18JAN94 FIN DATE 22JON95* Dec. 94 Acceleration, by Typical Activity \& $\mathrm{BS}$

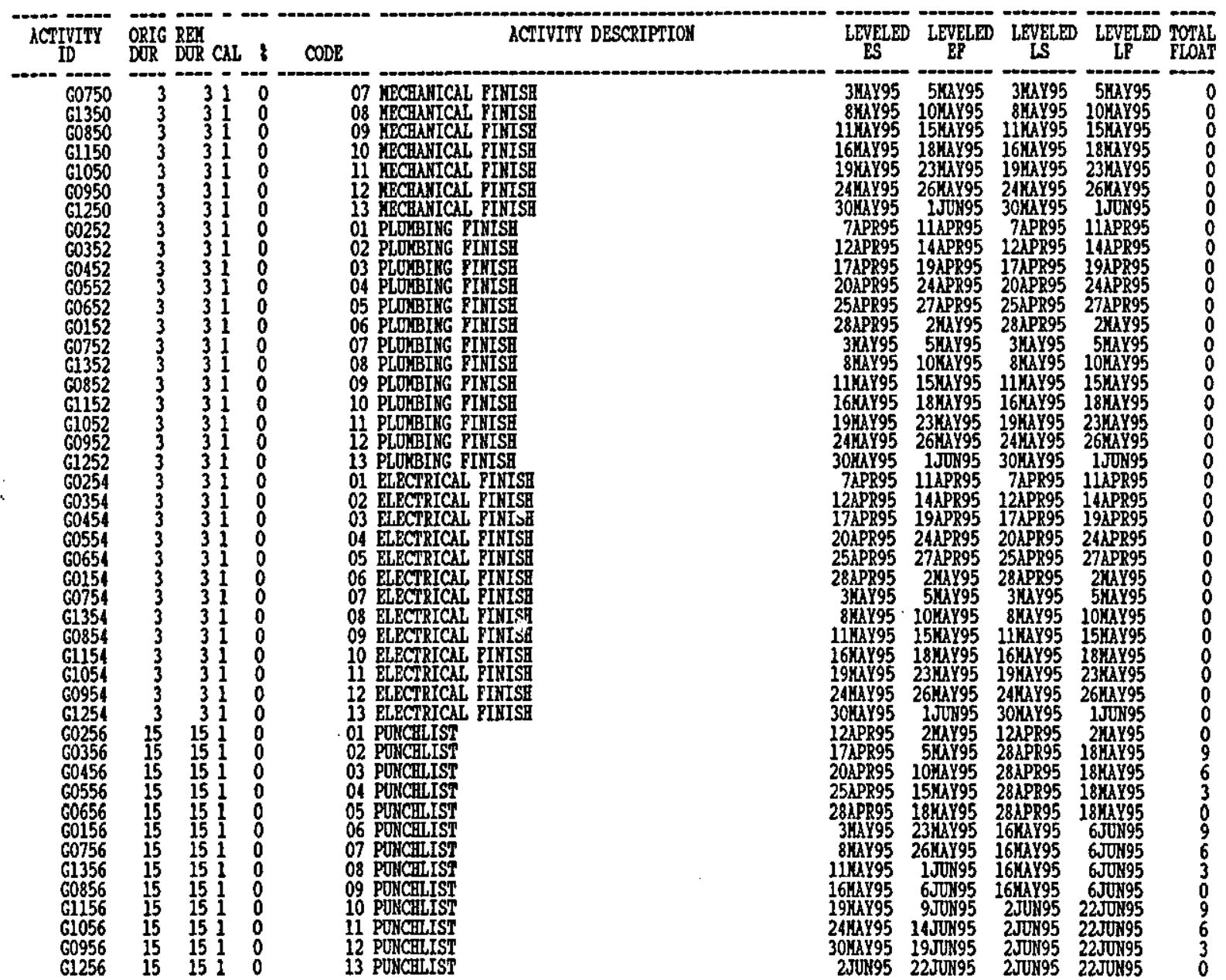

Figure 9.31 - Case 3, Accelerated Schedule (Continued). 


\section{CHAPTER 10}

\section{CONCLUSIONS AND SUGGESTIONS FOR FUTURE RESEARCH}

\subsection{Objectives Satisfied}

The following is a summary of the objectives achieved listed in the order they were first stated in section 1.3:

1. In section 4.2, past and current attempts of integrating CPM and LOB were identified and evaluated.

2. In section 5.2 and 5.3, the practical applications of LOB and CPM were investigated.

3. Chapter 6 showed the development of the new methodology that fully integrated CPM and LOB. Six examples were offered to test and verify the capabilities claimed.

4. In chapter 9, the effectiveness of CPM/LOB was further tested and validated on three real life projects.

\subsection{Test of the Principal Hypothesis}

The examples in chapters 6,7 and 8 along with the three case studies in chapter 9 , have proven unequivocally true the hypothesis stated in section 1.5: CPM and LOB were integrated and resulted in a more powerful tool than either one of them applied independently. Further it was confirmed that they retained their powerful features and counteracted their weaknesses. Within CPM/LOB, it was possible to react promptly to changes in sequence of work and to maintain work continuity. $\mathrm{LOB}$ in the environment of $\mathrm{CPM} / \mathrm{LOB}$ was capable of handling complicated branching networks and incorporating float in the calculations. With CPM/LOB it was possible to determine the right combination of team resources and identify the critical path of small as well as large projects even before running the CPM software thereby offering a better insight to a repetitive project than any of CPM or LOB applied independently. The user enjoys a considerable advantage in assessing the impacts of delays and resource-driven effects, which should help 
proving delays and sensible utilization of float times. In case study $3, \mathrm{CPM} / \mathrm{LOB}$ was proved capable of incorporating discrete activities along with repetitive activities.

\subsection{Test of the Secondary Hypotheses}

Section 1.6 stated the secondary hypotheses that were predicated on an expected successful integration of CPM and LOB to identify, quantify and overcome the effects of: common activities, out-of-logic progress and excessive quantities of uncompleted repetitive activities. The following summarizes how the secondary hypotheses were proven true:

1. The effect of the common activities was defined and a formula was derived to quantify it in section 7.2 which included an example. Additionally, the baseline schedule of case study 2 isolated the effect of the common activity and quantified it after fixing all other variables.

2. Out-of-logic progress was identified and quantified through an example in section 7.4. It was addressed again and overcome in case study 2 , sections 9.3 .2 and 9.3.3.

3. The problem of excessive quantities of partly completed activities was discussed in section 7.5 and the suggested solution was to split the said activity. No numeric example was given per se, however, the solution was of a very close nature to splitting the GF \& FF CEMENT BLOCK WORK activity in case study 2 .

\subsection{Conclusions}

This research succeeded in integrating CPM and LOB in an original methodology that manifested and validated its invulnerability to changes in operations sequence, and its capability of maintaining practical continuity for the team-resources. CPM/LOB marks the beginning of LOB's gain from future developments of CPM commercial packages. Its application is so flexible that it greatly facilitates experimentation with alternative resource and sequence strategies. 
It should be realized now that practical methods in use which utilize LOB alone, or resource-based relationships in CPM for planning and scheduling projects with significant repetition can be seen as inefficient.

This work further claims the original exposure and improvement of several aspects in the management of repetitive projects. Namely, the effect of common activities, out-of-logic and outof-sequence progress, excessive quantities of uncompleted activities, progress regularity, stage handovers, and merger. Examples were given to justify the claim.

Because CPM/LOB uses heuristic-based resource leveling which maintains a dynamic link among single repetitive activities, it leaves less float to dispute between the parties, especially when resource strategies change, let alone implied resource strategies that leave float for potential future utilization.

Contrary to previous beliefs by Peer (1974), Birrel (1980), Johnston (1981), Jaafari (1984), Rahbar and Rowings (1992), and Hegazy et al. (1993), CPM/LOB proved that resource leveling is capable of maintaining work continuity.

CPM/LOB negated, for the first time, the notion that the slowest rate activity determines the critical path hence control the pace of a repetitive project. This opinion was expressed by Peer (1974), Carr and Meyer (1974), and Humphries (1993). CPM/LOB revealed that due to the existence of float, activity completion rates must be normalized (as shown in the three case studies) before using the lowest rate as an indication of resource criticality. The critical path starts with the longest chain of activities preceding the slowest normalized rate activity. It then goes throughout that typical activity and to the longest chain of activities succeeding it to the end of the whole project.

CPM was criticized for giving no indication of production rates (Arditi and Albulak 1986; Rahbar and Rowings 1992; Humphries 1993). In CPM/LOB, as well as resource-based relationship CPM networks, this issue was considered and a single resource of one day duration was allocated to the last day of each typical activity. This resource was called "completion" and was separately aggregated for each typical activity to plot completions against time. Fig. 6.7 showed that. 
Due to multiple team-resource allocations to repetitive activities, not all the squads of a critical typical activity should be critical. This is especially true when there is no requirement for gradual completions or handovers. Moreover, critical activities in the unit network may not be critical activities in the overall project schedule because in repetitive construction, resources are the major parameter that determines criticality. Activity durations before and after those critical resources determine the rest of the critical path.

Breaking activities into single squad tasks can be done if CPM/LOB is to be utilizes at the trade subcontractor level of detail. This will lead to less overall project duration due to the elimination of unnecessary sequential leveling of long-duration summary squads as opposed to overlapping short-duration individual or specialty squads.

Completion dates could be slightly earlier than target dates when rounding up the quantity of minimum resources required to complete on target. This does not happen if the resource requirement of the dominating activity or activities is already an integer and does not need rounding up. Nevertheless, earlier completion dates could still occur due to the utilization of more than one squad on a typical activity. This is so because the mathematical computation of the required minimum quantity of resources assumes these squad completions follow a straight inclined line, whereas they could be anywhere between that and completely concurrent depending on their predecessors and resources availability. The discussion in section 7.3 should illustrate that.

A repetitive activity can be executed with a range of quantities of team-resources. Arriving at the right combination of resources for all the repetitive activities is a trial and error process (Perera and Ramaswamy 1980) until finally satisfying the project target completion date. These combinations are many; on a small typical network of ten activities where each activity can be executed by one of two possible quantities of a single team-resource, there are more than one thousand possible combinations of resources to investigate in attempting to arrive at the target completion date:

(Range of qunatities of resources) $)^{(\text {number of opical activites) }}=$ possible combinations

$(2)^{10}=1,024$ Combinations for resource leveling 
The integration of CPM and LOB reduces these to a few then the float utilization concept in CPM/LOB narrows these down to a single feasible combination without trial and error. A lot of time and effort is therefore saved.

CPM/LOB was applied by the author on several real projects: electrical substations, houses, guard towers, precast bridges, and schools. Due to space limitations only three of these projects have been included as case studies. The results of the practical application were most satisfying.

The author refrained from designing a fully automated computer program for CPM/LOB to discourage possible laxative and cavalier applications. Some planners and schedulers submit to the limitations of computer programs and therefore move the process of planning and scheduling away from the prudence of the indispensable human interaction.

Because CPM/LOB is versatile and relatively free of mathematical complexities, it is expected to be attractive to constructors of repetitive projects. The many researchers interested in repetitive construction planning and scheduling should also find this work interesting.

\subsection{Suggestions for Future Research}

CPM/LOB could be more useful if the following recommendations could be incorporated to show the extreme efficiency of the methodology:

1. Identifying preferential logic relationships in CPM from absolute logic relationships in the baseline schedule thereby giving the scheduler the option of overriding the preferential logic when necessary. A clear definition of scope for each activity would be instrumental in achieving that.

2. Enabling the plotting of resource flows on bar charts and linked bar charts to track critical resources besides critical activities toward achieving a comprehensive and quick identification of the critical path.

3. Enabling cumulative resource curves (flow lines) in a step-line format. 
4. Resource smoothing was tried experimentally on a non-team resource that was needed by various repetitive activities. It is worth it to investigate and confirm if it tends to compromise the continuity of repetitive activities.

5. Enabling changes in resource availabilities, calendars, start constraints, etc. referenced to a specific or a variable quantity of typical activities, e.g. step up the resource availability after the completion of the 10th unit, or typical activity "excavation" should not lead "concrete foundations" by more than 10 units in the rainy season. Also enabling pre-defined quantity buffers despite expected changes in sequence.

6. Introducing float for resources within a single CPM activity, e.g. a five day activity needs a one day resource on any of its five days, hence the resource has four days of float.

7. Enabling resource leveling to start an activity even if its resource requirements were not available throughout its projected duration, then leaving it partway to a subsequently available activity.

8. Automating the preparation of the regularity chart for speedy analysis. It should be mentioned that in order to facilitate the identification of bottlenecks, no activity should be placed after its successor in a regularity chart.

9. In order to avoid situations where some resources become in short supply while others are abundant, increasing resources should be thought of in terms of marginal returns on increments in completion rates. Whether accelerating or decelerating, the balance between resource quantities has to be maintained to reduce the cost of resources.

10. There is no one planning technique that will work for all types of construction (Schlick 1981). Repetitive projects can be divided into two categories as far as typical activity durations are concerned; "typical repetitive", where typical activities have identical durations in all units (e.g. housing projects) and; "nontypical repetitive", where typical activity durations vary from unit to unit or section to section (e.g. highway construction). The variation in duration can be 
due to differences in quantities, weather conditions and the learning curve effect (Voster and Bafna 1992; Moselhi and El-Rayes 1993). Location-time diagrams and dynamic programming based techniques are tailored to both types of projects, however, LOB type techniques (including CPM/LOB) are more suitable to typical repetitive projects. Forbes (1971) had mentioned that within the LOB application on housing projects, little differences in work content were coupled with a wide range of manpower output hence any attempt at precise timing of the work was unrealistic.

Nevertheless, the flexibility of CPM/LOB should encourage future research work to extend its utilization to nontypical repetitive projects by firstly incorporating the effect of the learning curve and secondly, the effect of significant variations in work content.

Russell and Wong (1993) removed the computer-generated learning curve effects from their model (REPCON) because users did not like delegating such judgement to a machine. Nevertheless, this author is of the opinion that once a system offers realistic and flexible solutions, users start to trust it. It is therefore recommended that the learning effect be reflected in activity durations driven by the degree of learning of the squad that happens to work on them. Obviously the degree of learning is dependent on the number of repetitions.

11. Expert Systems' solutions are not current alternatives to CPM/LOB but this author recommends incorporating the methodology of CPM/LOB in future AI works, certainly in those utilizing commercial planning and scheduling software.

Perhaps enhancing CPM/LOB with expert systems could promote an option of resource scheduling per the natural rhythm and dynamic buffers that account for sequence changes automatically rather than by logic relationships.

12. Neural Networks can probably be applied on non-heuristic resource leveling to aid the scheduler in arriving at an optimized combination of resources for sophisticated requirements. Ahuja et al. (1994) did some work on productivity by Neural Networks. 
13. It is worthwhile to point out that the industry has always been interested in simple solutions. 


\section{APPENDIX}

\section{A.1 Publications from this Thesis}

1. Suhail, S. A. (1993). "Out-of-logic progress," Cost Engineering, American Association of Cost Engineers, International, Vol. 35, No. 4, April, pp. 23-28.

2. Suhail, S. A., and Neale, R. H., (1994). "CPM/LOB: New methodology to integrate CPM and line of balance," Journal of Construction Engineering and Management, American Society of Civil Engineers, Vol. 120, No. 2, September, pp. 667-684. 


\section{A.2 References}

1. A guide to the project management body of knowledge (PMBOK). (1994). Exposure Draft, the Project Management Institute Standards Committee, Upper Darby, Pennsylvania, USA, August.

2. Ahuja, H. N., Dozzi, S. P. and Abourizk, S. M. (1994). Project management, techniques in planning and controlling construction projects, 2nd Ed., John Wiley \& Sons, Inc., New York.

3. Al Sarraj, Z. M. (1990). "Formal development of the line-of-balance," Journal of Construction Engineering and Management, ASCE, Vol. 116, No. 4, December, pp. 689-704.

4. Arditi, D. (1976). "Problems in the process of introducing network analysis," Proceedings of the Sth INTERNET Congress, Birmingham, UK, September, pp. 143-151.

5. Arditi, D., and Albulak, M. Z. (1979). "Comparison of network analysis with line of balance in a linear repetitive construction project," Proceedings of the 6th INTERNET Congress, Vol. 2, Garmisch-Partenkirchen, W. Germany, September, pp. 13-25.

6. Arditi, D., and Albulak, M. Z. (1986). "Line-of-balance scheduling in pavement construction," Journal of Construction Engineering and Management, ASCE, Vol. 112, No. 3, September, pp. 411-424.

7. Arditi, D., and Patel, B. (1989). "Impact analysis of owner-directed acceleration," Journal of Construction Engineering and Management, ASCE, Vol. 115, No. 1, March, pp. 144-157.

8. Army programs, Line of balance. (1968). US Army Materials Command, Army Material Command Pamphlet No. 11-5, Washington, DC.

9. ASCE authors' guide to journals, books, and reference publications. (1993). ASCE, February. 
10. Ashley, D. B. (1980). "Simulation of repetitive-unit construction," Journal of the Construction Division, ASCE, Vol. 106, No. CO2, June, pp. 185-194.

11. Baram, G. E., (1994). "Delay analysis-issues not for granted," Proceedings of the 38th Annual Meeting, AACE, International, San Francisco, California, June 12-22, pp. DCL.5.1-DCL.5.9.

12. Barrett, M. L. and Beerel, A. C. (1988). Expert systems in business: A practical approach, Ellis Horwood Ltd., Chichester, UK.

13. Behling, J. H. (1978). Guidelines for preparing the research proposal, University Press of America, R. F. Publishing, Inc., Washington, DC, USA.

14. Brech, E. F. L. (1971). Construction management in principle and practice, Longman, UK.

15. Birrell, G. S. (1980). "Construction planning-beyond the critical path," Journal of Construction Division, ASCE, Vol. 106, No. CO3, September, pp. 389-407.

16. Callahan, M. T., Quackenbush, D. G., and Rowings, J. E. (1992). Construction project scheduling, 1st Ed., McGraw-Hill, Inc., New York.

17. Calvert, R. E. (1970). Introduction to building management, Newness-Butterworths, London.

18. Carr, R. I., and Meyer, W. L. (1974). "Planning construction of repetitive building units, "Journal of the Construction Division, ASCE, Vol. 100, No. CO3, September, pp. 403-412.

19. Cass, D. J. (1976). "Bridging the gap on network methodologies available for construction management," Proceedings of the 5th INTERNET Congress, Introductory Volume, Birmingham, UK, September, pp. 38-46. 
20. Chang, D. Y. and Carr, R. I. (1987). "RESQUE: A resource oriented simulation system for multiple resource constrained processes, " Proceedings of Project Management Institute Annual Symposium, Milwaukee, Wisconsin, 2-7 October, pp. 4-19.

21. Chrzanowski, Jr., E. N., and Johnston, D. W. (1986). "Application of linear scheduling," Journal of Construction Engineering and Management, ASCE, Vol. 112, No. 4, December, pp. 476-491.

22. Clough, R. H., and Sears, G. A. (1991). Construction project management, 3rd Ed., John Wiley \& Sons, Inc., New York.

23. Cormican, D. (1985). Construction management: Planning and finance, Construction Press, UK.

24. Darlow, M. J. (1968). "Investigations into line of balance scheduling for house building," Project report, Department of Civil Engineering, Loughborough University of Technology, UK.

25. Dawood, N. N. (1994). "Discussion on 'DBID: Analogy-based DSS for bidding in construction,' by O. Moselhi and T. Hegazy," Journal of Construction Engineering and Management, ASCE, Vol. 120, No. 4, December, pp. 894-896.

26. de Leon, G. P. (1986). "Float ownership: Specs treatment," Cost Engineering, AACE, Vol. 28, No. 10, October, pp. 12-15.

27. Digman, L. A. (1967). "PERT/LOB: Life-cycle technique," The Journal of Industrial Engineering, Vol. 18, No. 2, February, pp. 154-158.

28. Echeverry, D., Ibbs, C. W. and Kim, S. (1991). "Sequencing knowledge for construction scheduling," Journal of Construction Engineering and Management, ASCE, Vol. 117, No. 1, March, pp. 118-130.

29. Enrico, J. (1995). Lecturer, University of Southern California, personal discussion, AACE Southern California Section dinner meeting, January 12. 
30. Faris, R. K. (1994). Executive Vice President, Primavera Systems, Inc., personal discussion, Project Management Institute-Orange County, dinner meeting, July 12.

31. Finck, N. E. (1965). "Line of balance gives the answer," Systems and Procedures Journal, Vol. 16, No. 4, July- August, pp. 8-14.

32. Fleming, Q. W. (1994). Personal communication, September 17.

33. Forbes, W. S. (1971). "Flow charts to control progress on housing sites," Building Research Station Digest, No. 134, Building Research Establishment, Watford, England, October, pp. 244-250.

34. Gilyutin, 1. (1993). "Managing resources for a construction project in a mixed (PCMainframe) environment, " Project Management Journal, Project Management Institute, Vol. XXIV, No. 2, June, pp. 34-40.

35. Goodman, L. J., and Love, R. N. (1980). Project planning and management: An integrated approach, Pergamon Press, New York.

36. Halpin, D. W. and Leland, S. R. (1992). Planning and analysis of construction operations, John Wiley \& Sons, USA.

37. Halpin, D. W. and Woodhead, R. W. (1976). Design of construction and process operations, John Wiley \& Sons, USA.

38. Harbert, J. A. (1976). "Development of the combined PERT and LOB (CPL) chart," Proceedings of the 5th INTERNET Congress, Vol. Friday, Birmingham, UK, pp. 241246.

39. Harper, D. J. (1983). "An investigations into the feasibility of using a resource-oriented planning technique in non-repetitive construction," Project report, Department of Civil Engineering, Loughborough University of Technology, UK.

40. Harris, F. C. and McCaffer, R. (1977). Modern construction management, Crosby Lockwood Staples, London, England. 
41. Harrison, F. L. (1985). Advanced project management, 2nd Ed., Gower, UK.

42. Hegazy, T. and Moselhi, O. (1994). "Closure of Discussion on 'DBID: Analogy-based DSS for bidding in construction,' by O. Moselhi and T. Hegazy," Journal of Construction Engineering and Management, ASCE, Vol. 120, No. 4, December, pp. 896-897.

43. Hegazy, T., Moselhi, O. and Fazio, P. (1993). "BAL: An algorithm for scheduling and control of linear projects," AACE Transactions of the 37th Annual Meeting, Dearborn, Michigan, USA, July 11-14, pp. C.8.1-C.8.14.

44. Heyel, C. (1973). The encyclopedia of management, 2nd Ed., Van Nostrand Reinold Co., New York.

45. Heyel, C. (1979). The VNR concise guide to industrial management, Van Nostrand Reinold Co., New York.

46. Hinkley, P. (1989). "Planning and control of repetitive construction," Project Management Journal, Project Management Institute, Vol. XX, No. 2, June, pp. 41-47.

47. Householder, J. L., and Rutland, H. E. (1990). "Who owns float," Journal of Construction Engineering and Management, ASCE, Vol. 116, No. 1, March, pp. 130133.

48. Howel, A. W. (1983). "Notes on Loughborough University computer program, resourceoriented scheduling," Loughborough University of Technology, England, December.

49. Hsu, Kuo-chun Murphy (1991). "Automated line-of-balance scheduling technique," Master of Science, Technical Report, Dept. of Civil Eng., Ohio State University, USA.

50. Humphries, L. L. (1993). "RPM - repetitive production method scheduling technique," AACE Transactions of the 37th Annual Meeting, Dearborn, Michigan, USA, July 11-14, pp. C.11.1-C.11.5. 
51. Iannone, A. L. (1967). Management program planning and control with PERT, MOST, and $L O B$, Prentice-Hall, Inc., Englewood Cliffs, NJ, USA.

52. Jaafari, A. (1984). "Criticism of CPM for project planning analysis," Journal of Construction Engineering and Management, ASCE, Vol. 110, No. 2, June, pp. 222-323.

53. Jacobs, O. L. R. (1967). An introduction to dynamic programming, the theory of multistage decision processes, Chapman and Hall Ltd., London.

54. Johnston, D. W. (1981). "Linear scheduling method for highway construction," Journal of the Construction Division, ASCE, Vol. 107, No. CO2, June, pp. 247-261.

55. Kartam, N. A. and Levitt, R. E. (1990). "Intelligent planning of construction projects," Journal of Computing in Civil Engineering, ASCE, Vol. 4, No. 2, April, pp. 155-176.

56. Kavanagh, D. P. (1985). "SIREN: A repetitive construction simulation model," Journal of Construction Engineering and Management, ASCE, Vol. 111, No. 3, September, pp. 308-323.

57. Khisty, C. J. (1970). "The application of the 'Line of Balance' technique to the Construction Industry," Indian Concrete Journal, July, pp. 297-300 and 319-320.

58. Lang, G. and Heiss, G. D. (1984). A practical guide to research methods, 3rd. Ed., University Press of America.

59. Laudon, K. C. and Laudon, J. P. (1991). Management information systems, a contemporary perspective, 2nd Ed., Macmillan Publishing Company, New York.

60. Line of balance technology. (1966). US Navy Material Command, NAVEMAT 1851 (Rev. 4-62), (Formerly NAVEXOS) (Reviewed and approved November 1, 1966).

61. Levine, H. A., Aliberti, E. M. and Ford, B. P. (1976). "The application of line of balance on an international project," Proceedings of the 5th INTERNET Congress, Vol. Thursday, Birmingham, UK, pp. 251-258. 
62. Levine, H. A. (1994a). "Resource leveling and roulette: Games of chance - part 1," $P M$ Network, Project Management Institute, April, PP. 25-27.

63. Levine, H. A. (1994b). "Resource leveling and roulette: Games of chance - part 2," $P M$ Network, Project Management Institute, July, PP. 23-25.

64. Levitt, H. P. (1968). "Computerized line of balance," The Journal of Industrialized Engineering, Vol. 19, No. 2, February, pp. 61-66.

65. Levitt, R. E., Kartam, N. A. and Kunz, J. C. (1988). "Artificial intelligence techniques for generating construction project plans," Journal of Construction Engineering and Management, ASCE, Vol. 114, No. 3, September, pp. 329-343.

66. Logcher, R. D. (1989). "Discussion of 'Concurrent delays in construction projects,' by Zeki M. Kraiem and James E. Diekmann." Journal of Construction Engineering and Management, ASCE, Vol. 115, No. 2, June, pp. 335-337.

67. Lorterapong, P. (1994), "A fuzzy heuristic method for resource-constrained project scheduling, "Project Management Journal, Project Management Institute, Vol. XXV, No. 4, December, pp. 12-18.

68. Lumsden, P. (1968). The line-of-balance method, Pergamon Press, London.

69. Lutz, J. D. and Halpin, D. W. (1992). "Analyzing linear construction operations using simulation and line of balance," Transportation Research Record, Number 1351, pp. 4856, ISSN 031-1981.

70. Lutz, J. D. (1990). "Planning linear construction projects using simulation and line of balance," PhD Dissertation, Purdue University, December.

71. Mangin, J. C., Dufau, J. and Miramond, M. (1979). "Scheduling methods for repetitive tasks in second stage building construction, "Proceedings of the 6th INTERNET Congress, Vol. 3, Garmisch-Partenkirchen, W. Germany, September, pp. 287-295. 
72. Mansur, F. (1990). "The line of balance method," Major Technical Report, Centre for Building Studies, Concordia University, Montreal, Canada.

73. Matthews, M. (1994). "Resource scheduling: Incorporating capacity into schedule construction," Project Management Journal, Project Management Institute, Vol. XXV, No. 2, June, pp. 44-54.

74. Mawdesley, M. J., et al. (1989). "Time chainage charts for scheduling linear projects," Proceedings of the 6th Conference on Computing in Civil Engineering, ASCE, Atlanta, Georgia, USA, September 11-13, pp. 613-620.

75. McCahill, D. F. and Bernold, L. E. (1993). "Resource-oriented modeling and simulation in construction," Journal of Construction Engineering and Management, ASCE, Vol. 119, No. 3, September, pp. 590-606.

76. McCuen, R. H., Johnson, P. A. and Davis, C. (1993). Dynamic communication for engineers, The American Society of Civil Engineers, New York.

77. McKim, R. A. (1993). "Neural network applications to cost engineering," Cost Engineering, AACE, Vol. 35, No. 7, July, pp. 31-35.

78. Melin, J. W., and Whiteaker, B. (1981). "Fencing a bar chart," Journal of the Construction Division, ASCE, Vol. 107, No. CO3, September, pp. 497-507.

79. Menasche, L. (1984). Writing a research paper, University of Pittsburgh Press, Pennsylvania, USA.

80. Morad, A. A. and Beliveau, Y. J. (1991). "Knowledge-based planning system," Journal of Construction Engineering and Management, ASCE, Vol. 117, No. 1, March, pp. 1-12.

81. Morad, A. A. and Beliveau, Y. J. (1994). "Geometric-based reasoning system for Project Planning," Journal of Computing in Civil Engineering, ASCE, Vol. 8, No. 1, January, pp. 52-71. 
82. Moselhi, O., and El-Rayes, K. (1993). "Scheduling of repetitive projects with cost optimization," Journal of Construction Engineering and Management, ASCE, Vol. 119, No. 4, December, pp. 681-697.

83. Moselhi, O., and Nicholas, M. J. (1990). "Hybrid expert system for construction planning and scheduling," Journal of Construction Engineering and Management, ASCE, Vol. 116, No. 2, June, pp. 221-237.

84. Moselhi, O., Hegazy, T., and Fazio, P. (1991). "Neural networks as tools in construction," Journal of Construction Engineering and Management, ASCE, Vol. 117, No. 4, December, pp. 606-625.

85. Moselhi, O, and Lorterapong, P. (1993). "Scheduling of multi-story buildings in a constraint environment," Proceedings of the 5th International Conference on Computing in Civil and Building Engineering, ASCE, Anaheim, California, USA, June, pp. 18221829.

86. Neale, R. H., and Neale, D. E. (1989). Construction planning, 1st Ed., Thomas Telford Ltd., London.

87. Neale, R.H., and Raju, B. (1988). "Line-of-balance planning by spreadsheet," Building Technology and Management, India, January, pp. 22-27.

88. Nemhauser, G. L. (1966). Introduction to dynamic programming, John Wiley \& Sons, Inc., New York.

89. O'Brien, J. J. (1969). Scheduling handbook, McGraw-Hill Book Co., Inc., New York, NY, pp. 246-255.

90. O'Brien, J. (1975). "VPM scheduling for high-rise buildings," Journal of the Construction Division, ASCE, Vol. 101, No. CO4, December, pp. 895-905.

91. O'Brien, J., et al. (1985). "Network scheduling variations for repetitive work, " Journal of Construction Engineering and Management, ASCE, Vol. 111, No. 2, June, pp. 105 116. 
92. Oxley, R. and Poskitt, J. (19 ). "Modern site control methods suitable for large projects," The Practice of Site Management, 2nd Ed., The Institute of Building, UK, pp. 70-73.

93. Oxley, R. and Poskitt, J. (1971). Management techniques applied to the construction industry, Pitman Press, UK.

94. Pedersen, H. (1972). "Network planning of repetitive processes in housing construction industry," Proceeding of the 3rd INTERNET Congress, Book II, Stockholm, Sweden, May, pp. 381-392.

95. Peer, S. (1974). "Network analysis and construction planning," Journal of the Construction Division, ASCE, Vol. 100, No. CO3, September, pp. 203-210.

96. Peer, S. and Selinger, S. (1976). "CPT - New approach to construction planning," Proceedings of the CIB W-65 Symposium on Organization and Management of Construction, US National Academy of Sciences, Washington, DC, USA, May 19-20, pp. IV-156-IV-163.

97. Perera, S. (1981). "The chain bar-chart for project planning and control," National Development, June/July, pp. 68-72.

98. Perera, S. (1982). "Network planning of projects comprising repetitive activities, " IAHS Conference on Impact of Economy and Technology, Vienna, Austria, November 15-18, pp. 927-985.

99. Perera, S. (1983). "Resource sharing in linear construction," Journal of Construction Engineering and Management, ASCE, Vol. 109, No. 1, March, pp. 102-111.

100. Perera, S., and Ramaswamy, G. S. (1980). "Planning of housing construction using control charts," Housing Science, IAHS, Florida, USA, Vol. 4, No. 5, pp. 447-458.

101. Person, J. C. (1991). "Who owns the float," Construction Briefings, Federal Publications, Inc., No. 91-7, June, pp. 1-12. 
102. "PERT/LOB-A plan for all seasons." (1968). The Accountant, Vol. CLVIII, No. 6489, London, pp. 91-92.

103. Pilcher, R. (1976). Principles of construction management, 2nd Ed., McGraw-Hill Book Company (UK) Ltd.

104. Pilcher, R. (1992). Principles of construction management, 3rd Ed., McGraw-Hill Book Company Europe.

105. Popescu, C. (1979). "A planning method for linear projects," Proceedings of Project Management Institute Annual Symposium, Atlanta, Georgia, October 11, pp. 265-273.

106. Popescu, C. (1991). "The pitfalls of CPM resource scheduling," Proceedings of Project Management Institute Annual Symposium, Dallas, Texas, September 28 - October 2, page 265-270.

107. Primavera Project Planner reference manual; release 4.0. (1990). Primavera Systems, Inc., Bala Cynwyd, PA, USA.

108. Primavera Project Planner reference manual; release 5.0. (1991). Primavera Systems, Inc., Bala Cynwyd, PA, USA.

109. Programming house building by line of balance. (1968). National Building Agency, England.

110. Rahbar, F. F., and Rowings, J. E. (1992). "Repetitive activity scheduling process," AACE Transactions of the 36th Annual Meeting, Orlando, USA, pp. O.5.1-0.5.8.

111. Reda, R. M. (1990). "RPM: Repetitive project modeling," Journal of Construction Engineering and Management, ASCE, Vol. 116, No. 2, June, pp. 316-330.

112. Rist, P. T. (1972). "Cascade charts in construction," Proceedings of the 3rd INTERNET Congress, Book II, Stockholm, Sweden, May, pp. 250-261. 
113. Roesch, W. (1972). "Network planning and velocity-diagrams in housing construction industry," Proceedings of the 3rd INTERNET Congress, Book II, Stockholm, Sweden, May, pp. 381-388.

114. Russell, A. D., (1985), "Microcomputers, management, and high-rise construction: The next step," Canadian Journal of Civil Engineering, Vol. 12(2), pp. 396-414.

115. Russell, A. D., and Wong, W. C. M. (1993). "New generation of planning structures," Journal of Construction Engineering and Management, ASCE, Vol. 119, No. 2, June, pp. 196-214.

116. Russell, A. D., and Caselton, W. F. (1988). "Extensions to linear scheduling optimization," Journal of Construction Engineering and Management, ASCE, Vol. 114, No. 1, March, pp. 36-52.

117. Russell, A. D., and McGowan, N. (1993). "Linear scheduling - a practical implementation," Proceedings of the 5th International Conference on Computing in Civil and Building Engineering, ASCE, Anaheim, California, USA, Vol. 1, June, pp. 279-286.

118. Schlick, H. (1981). "Discussion of 'Construction planning-beyond the critical path,' by George S. Birrell." Journal of the Construction Division, ASCE, Vol. 107, No. CO2, June, p. 412.

119. Selinger, S. (1980). "Construction planning for linear projects," Journal of the Construction Division, ASCE, Vol. 106, No. CO2, June, pp. 195-205.

120. Shaked, O. and Warszawski, A. (1992). "CONSCHED: Expert system for scheduling of modular construction projects, " Journal of Construction Engineering and Management, ASCE, Vol. 118, No. 3, September, pp. 488-506.

121. Shaked, O. and Warszawski, A. (1995). "Knowledge-based system for construction planning of high-rise buildings," Journal of Construction Engineering and Management, ASCE, Vol. 121, No. 2, June, pp. 172-182. 
122. Shoderbek, P. P., and Digman, L. A. (1967). "Third generation, PERT/LOB," Harvard Business Review, Vol. 45, NO. 5, Sept.-Oct., pp. 100-110.

123. Short, J. W. (1993). "Using schedule variance as the only measure of schedule performance," Cost Engineering, AACE, Vol. 35, No. 10, October, pp. 35-39.

124. Singh, A. (1993). "Earned value analysis interface with line of balance," Proceedings of the 24th Annual Seminar/Symposium, Project Management Institute, San Diego, USA, October 1-7, pp. 193-197.

125. Smith, R. V. (1984). Graduate research, a guide for students in the sciences, ISI Press, Philadelphia, Pennsylvania, USA.

126. Sobhy, A. I. (1995). Lecturer, Civil Engineering Dept., California State University, Long Beach, personal discussions.

127. Soliman, N. H. (1987). "Planning and control of repetitive unit projects," Master of Science, Technical Report, Civil Engineering Dept., Oklahoma State University, May.

128. Stradal, O., and Cacha, J. (1982). "Time space scheduling method," Journal of the Construction Division, ASCE, Vol. 108, No. CO3, September, pp. 445-457.

129. Suh, K. (1993). "A scheduling system for repetitive-unit construction using line-ofbalance technology," $\mathrm{PhD}$ dissertation, Illinois Institute of Technology, Chicago, Illinois, May.

130. Teplitz, C. J. and Amor, J. P. (1993). "Improving CPM's accuracy using learning curves", Project Management Journal, Project Management Institute, Vol. XXIV, No. 2, December, pp. 15-19.

131. Thabet, W. Y. and Beliveau, Y. J. (1994). "HVLS: Horizontal and vertical logic scheduling for multistory projects," Journal of Construction Engineering and Management, ASCE, Vol. 120, No. 4, December, pp. 875-892. 
132. Thompson, P. A. (1981). Organization and economics of construction, McGraw Hill Book Company Ltd., UK.

133. Trauner, T. J., Jr. (1990). Construction delays, documenting causes, winning claims, recovering costs, R. S. Means Company, Inc., Kingston, MA, USA, pp. 171 and 187 :

134. Trimble, E. G. (1984). "Resource-oriented scheduling," Project Management Journal, Project Management Institute, Vol. 2, No. 2, May, pp. 70-74.

135. Trimble, E. G. (1984). "Pilot study of resource oriented construction planning, and other planning considerations," Final report, Civil Engineering Department, Loughborough University of Technology, England, December.

136. Trimble, E. G. and Harries, T. W. (1974). "Factors which affect success in network applications," Proceedings of the 4th INTERNET Congress, Paris, France, pp. 439-446.

137. Turban, E. (1968). "The line of balance-a management by exception tool," The Journal of Industrial Engineering, Vol. 19, No. 9, September, pp. 440-448.

138. Voster, M. C., and Bafna, T. (1992). "Discussion of 'Formal development of line of balance technique,' by Z. M. Al Sarraj," Journal of Construction Engineering and Management, ASCE, Vol. 118, No. 1, March, pp. 210-211.

139. Westmeyer, P. (1981). A guide for use in planning and conducting research projects, Charles C. Thomas, Springfield, Illinois, USA.

140. Wickwire, J. M. et al. (1975). "Project scheduling, principles, applications and problems," The Briefing Papers Collection, Federal Publications, Inc., Vol. 3, 19731975, No. 75-5, October, pp. 233-251.

141. Wickwire, J. M., Driscoll, T. J. and Hurlbut, S. B. (1991). Construction scheduling: Preparation, liability, and claims, Wiley Law Publications, John Wiley and Sons, New York, p. 262. 
142. Woodworth, B. M. (1989). "Is resource-constrained project management software reliable," Cost Engineering, AACE, Vol. 31, No. 7, July, pp. 7-11.

143. Yamamoto, K. and Wada, K. (1993). "Resource constrained time-space scheduling model for pipeline laying work, " Proceedings of the 5th International Conference on Computing in Civil and Building Engineering, ASCE, Anaheim, California, USA, June, pp. 17941801.

144. Zack, J. G. Jr. (1991). "Schedule 'games' people play, and some suggested 'remedies'," AACE Transactions of the 35th Annual Meeting, Seattle, USA, June 23-26, pp. K.2.1K.2.6.

145. Zack, J. G. Jr. (1993). "Claimsmanship: Current perspective," Journal of Construction Engineering and Management, ASCE, Vol. 119, No. 3, September, pp. 480-497.

146. Zuwaylif, F. H. (1979). General applied statistics, 3rd Ed., Addison-Wesley Publishing Co., Reading, Massachusetts, USA. 
